\title{
A New Version of Unified Field Theory---- Stochastic Quantum Space Theory on Particle Physics and Cosmology
}

\section{Zhi-Yuan Shen (Retired DuPont Fellow)}

(e-mail: zyshen@comcast.net)

\begin{abstract}
Stochastic quantum space theory is a new version of unified field theory. It starts with three postulations: 1, Gaussian probability assigned to every discrete point separated by Planck length $L_{P}$ in the space; 2, prime numbers is intrinsically correlated to elementary particles and cosmology; 3 , vacuon is defined as movable geometry point in space. A framework is built with dozens of theoretical results agreed with experimental data and 25 predictions for experimental verification. Then three versions of basic equations are established based on Einstein field equations without the original stress-energy tensor and the redefined gauge tensor multiplied with Gaussian probability. The first version for gravity with the merit of no singularity has the same form of Einstein field equations with the stress-energy tensor automatically regenerated from derivatives of Gaussian probability multiplied to the redefined gauge tensor. The second version is for electromagnetic force. Comparison of these two versions naturally explains the tremendous strength difference between electrostatic force and gravitational force in macroscopic scale and the equality of these two forces at $71 L_{P}$. The third version as primary basic equations for the new version of unified field theory provides solutions for all elementary particles and interactional forces including gravity and things on upper levels.

Keywords: Unified field theory, space structure, elementary particles, Gaussian Probability, prime numbers, sporadic groups, GUT, dark matter, dark energy, cosmos inflation, multiverse, anthropic principle, general relativity, primary basic equations.
\end{abstract}

\section{Section 1: Introduction}

This paper is the continuation and extension of the author's previous paper [1], which was published in Chinese. For people not familiar with Chinese language, a brief review of the previous paper is included in this paper.

Stochastic Quantum Space (SQS) theory initially was intended to be a theory of space. It turns out as a unified field theory including particle physics and cosmology.

In essence, SQS theory is a mathematic theory. Its results are interpreted into physics quantities by using three basic physics constants, $h, c, G$ or equivalently $L_{P}, t_{P}, E_{P}\left(M_{P}\right)$. In principle no other physics inputs are needed.

SQS theory is based on three fundamental postulations, Gaussian Probability Postulation, Prime Numbers Postulation, Vacuon Postulation, which serve as the first principle of SQS theory.

Based on three fundamental postulations, SQS theory built a framework.

Based on Einstein's general relativity equations for vacuum and redefined gauge tensors attached to probability, SQS theory established the basic equations including two parts. The microscopic part is the primary basic equations for elementary particles, interactions and things on upper levels. The macroscopic part as the averaged version includes two sets of basic equations, one set for gravity and the other set for electromagnetic force.

SQS theory provides twenty five predictions for verifications.

The basic ideas of SQS theory are summarized as the following:
1. Vacuum is the ultimate unified field. Particles are exitantions and interactions are ripples in the vacuum.

2. Space is a stochastic continuum with Gaussain probability distribution function assigned at discrete points separated by Planck length. The basic equations for unified field theory is to assign Gaussian probalbility to garge tansors of the Einstein field equations for vacuum.

3. Cosmology and particle physics are intrinsically correlated with mathematics, in which prime numbers play the central role.

SQS theory laid down the foundations and built a framework. There are many open areas for physicists and mathematicians to explore and contribute.

\section{Section 2: Gaussian Probability Assignment}

According to Stochastic Quantum Space (SQS) theory, space is stochastic and continuous with grainy structure in Planck scale.

The Planck length is:

$$
L_{P}=\sqrt{\frac{h G}{2 \pi c^{3}}}=1.61625 \times 10^{-35} \mathrm{~m} .
$$

Based on $L_{P}$, Planck time $t_{P}$, Planck energy $E_{P}$ and Planck mass $M_{P}$ are defined as:

$$
\begin{aligned}
& t_{P}=\frac{L_{P}}{c}=\sqrt{\frac{h G}{2 \pi c^{5}}}=5.39123 \times 10^{-44} \mathrm{~s} . \\
& E_{P}=\frac{h c}{L_{P}}=\sqrt{\frac{2 \pi h c^{5}}{G}}=1.22905 \times 10^{10} \mathrm{~J} .
\end{aligned}
$$




$$
M_{P}=\frac{h}{L_{P} c}=\sqrt{\frac{2 \pi h c}{G}}=1.367498 \times 10^{-7} \mathrm{~kg} .
$$

In which $h, c$ and $G$ are Planck constant, speed of light in vacuum and Newtonian constant of gravitation, respectively.

Postulation 2.1A: Gaussian Probability Postulation. The relation between different points in space is stochastic in nature. Gaussian probability distribution function is assigned to each discrete point $x_{i}$ separated by Planck length. In 1-dimensional case, the Gaussian probability at point $x$ is:

$$
\begin{aligned}
& p\left(x ; x_{i}\right)=\frac{1}{\sqrt{2 \pi} \sigma} e^{-\frac{\left(x-x_{i}\right)^{2}}{2 \sigma^{2}}} ; x=-\infty, \cdots, 0, \cdots, \infty ; \\
& x_{i}=-\infty, \cdots,-2,-1,0,1,2, \cdots, \infty .
\end{aligned}
$$

The distance between adjacent discrete points is normalized to $L_{P}=1$.

Explanation: The Gaussian Probability Postulation serves as the first fundamental postulation of SQS theory. It represents the stochastic nature of space and also represents the quantum nature of space without sacrificing space as a continuum. The $p\left(x ; x_{i}\right)$ serves as the value at point $x$ from the Gaussian probability distribution function centered at discrete point $x_{i}$.

Postulation 2.1 is for 1-dimensional case as the foundation for 3-dimensional case.

The Standard Deviation (SD) $\sigma$ of Gaussian probability is selected to let the numerical factor in front of exponential term in (2.2) equal to 1 :

$$
\sigma=\frac{1}{\sqrt{2 \pi}}=0.398942280401433 .
$$

The reason of selecting such specific value for $\sigma$ will be explained later.

Substituting (2.3) into (2.2) yields:

$$
\begin{aligned}
& p\left(x ; x_{i}\right)=e^{-\pi\left(x-x_{i}\right)^{2}} ; x=-\infty, \cdots, 0, \cdots, \infty ; \\
& x_{i}=-\infty, \cdots,-2,-1,0,1,2, \cdots, \infty
\end{aligned}
$$

Postulation 2.1B: In the 3-dimensional space, (2.2) is extended as:

$$
\begin{aligned}
& p\left(x, y, z ; x_{i}, y_{j}, z_{k}\right)=\frac{1}{(2 \pi)^{3 / 2} \sigma^{3}} e^{-\frac{\left(x-x_{i}\right)^{2}+\left(y-y_{j}\right)^{2}+\left(z-z_{k}\right)^{2}}{2 \sigma^{2}}} ; \\
& x, y, z=-\infty, \cdots, 0, \cdots, \infty ; x_{i}, y_{j}, z_{k}=-\infty, \cdots,-2,-1,0,1,2, \cdots, \infty .
\end{aligned}
$$

The values of $\sigma$ are determined by the roots of the following equation:

$$
(2 \pi)^{3 / 2} \sigma^{3}-1=0 \text {. }
$$

Equation (2.6) has three roots:

$$
\sigma_{0}^{\prime}=\sigma=\frac{1}{\sqrt{2 \pi}}, \quad \sigma_{1}^{\prime}=\frac{e^{i 2 \pi / 3}}{\sqrt{2 \pi}}, \sigma_{2}^{\prime}=\frac{e^{i 4 \pi / 3}}{\sqrt{2 \pi}} .
$$

Substituting $\sigma=\sigma_{0}^{\prime}=1 / \sqrt{2 \pi}$ into (2.5) yields:

$$
\begin{aligned}
& p\left(x, y, z ; x_{i}, y_{j}, z_{k}\right)=e^{-\pi\left[\left(x-x_{i}\right)^{2}+\left(y-y_{j}\right)^{2}+\left(z-z_{k}\right)^{2}\right]} ; \\
& x, y, z=-\infty, \cdots, 0, \cdots, \infty ; x_{i}, y_{j}, z_{k}=-\infty, \cdots,-2,-1,0,1,2, \cdots, \infty
\end{aligned}
$$

In (2.8), only the real root of $\sigma_{0}^{\prime}=\sigma=1 / \sqrt{2 \pi}$ is used. The meaning of all three roots will be discussed later.

Definition 2.1: The Gaussian sphere centered at discrete $\operatorname{point}_{\left(x_{i}, y_{j}, z_{k}\right)}$ is defined as its surface represented by the following equation:

$$
\left(x-x_{i}\right)^{2}+\left(y-y_{j}\right)^{2}+\left(z-z_{k}\right)^{2}=R^{2} \text {. }
$$

The radius of Gaussian sphere is defined as:

$$
R=\frac{1}{2 \sqrt{2}}=0.353553390593274 \text {. }
$$

Explanation: The 3-dimensional Gaussian probability distribution of (2.8) has spherical symmetry like a sphere with blurred boundary. The Gaussian sphere is defined with a definitive boundary. It plays an important role for the structure of space as shown in Section 21.

For the 1-dimensional case, according to (2.4), the unitarity of probability distribution function $p\left(x ; x_{i}\right)$ with respect to continuous variable $x$ is satisfied for any discrete point $x_{i}$ :

$$
\int_{-\infty}^{\infty} p\left(x ; x_{i}\right) d x=\int_{-\infty}^{\infty} e^{-\pi\left(x_{i}-x\right)^{2}} d x=1 .
$$

In general, the unitarity of probability $p\left(x ; x_{i}\right)$ with respect to

\begin{tabular}{|c|c|c|c|}
\hline$x\{L,-1\}$ & $S(x)$ & $S(x)$ & $x(L,-1)$ \\
\hline 0 & 1.08643481121331 & 1.08643481121331 & 1 \\
\hline 0.0625 & 1.07985384105429 & 1.07985384105829 & 0.9375 \\
\hline 0.125 & 1.06111370929117 & 1.06111370029117 & 0.875 \\
\hline 0.1875 & 1.03306956928642 & 1.03306956928642 & 0.8125 \\
\hline 0.24998715673026 & 1 & 1 & 0.75001284326974 \\
\hline 0.25 & $0,999993025315 \geq 88$ & 0.999993025315288 & 0.75 \\
\hline 0.28001283726974 & 0.999989050630757 & 0.999989050630757 & 0.74998716273026 \\
\hline 0.3125 & 0.960920567019865 & 0.966920567019865 & 0.6875 \\
\hline 0.375 & 0.938896290708834 & 0.938886290708834 & 0.625 \\
\hline 0.4375 & 0.920156022639425 & 0920156022639425 & 0.5625 \\
\hline 0.5 & 0.913579138156117 & 0.913579138156117 & 0.5 \\
\hline
\end{tabular}
discrete variable $x_{i}$ is not satisfied.

Definition 2.2: $\boldsymbol{S}$-Function. Define the summation of $p\left(x ; x_{i}\right)$ with respect to $x_{i}$ as the $S(x)$-function:

$$
S(x)=\sum_{x_{i}=-\infty}^{\infty} p\left(x ; x_{i}\right)=\sum_{x_{i}=-\infty}^{\infty} e^{-\pi\left(x-x_{i}\right)^{2}} .
$$

Theorem 2.1: $S$-function $S(x)$ satisfies periodic condition:

$$
S(x-1)=S(x) \text {. }
$$

Proof: According to (2.11):

$S(x-1)=\sum_{x_{i}=-\infty}^{\infty} e^{-\pi\left(x-1-x_{i}\right)^{2}}=\sum_{x_{i}=-\infty}^{\infty} e^{-\pi\left[x-\left(x_{i}+1\right)\right)^{2}}=\sum_{x_{j}=-\infty}^{\infty} e^{-\pi\left(x-x_{j}\right)^{2}}=S(x)$.

QED

The values of $S(x)$ in the region $0 \leq x \leq 1$ are listed in Table 2.1 and shown in Fig. 2.1.

Table 2.1: The Values of $S(x)$ in Region $0 \leq x \leq 1$

In the region $[0,1]$, except two points at $x \approx 0.25$ and $x \approx 0.75$, in general $S(x)$ defined by (2.11) does not satisfy unitarity requirement, which has important implications. Theorem 2.2: $S(x)$ satisfies the following symmetrical condition: 
Z. Y. SHEN

$$
S(x)=S(1-x), 0 \leq x \leq 1 .
$$

Proof: According to (2.11):

$$
S(1-x)=\sum_{x_{i}=-\infty}^{\infty} e^{-\pi\left(1-x-x_{i}\right)^{2}}=\sum_{x_{i}=-\infty}^{\infty} e^{-\pi\left[x+\left(x_{i}-1\right)\right]^{2}}=\sum_{x_{j}=-\infty}^{\infty} e^{-\pi\left(x-x_{j}\right)^{2}}=S(x) \cdot \quad \text { QED }
$$

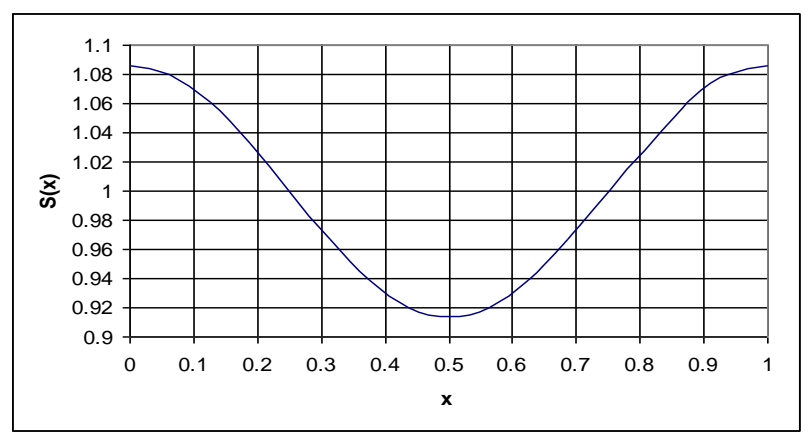

Fig.2.1 $S(x)$ curve in region $0 \leq x \leq 1$

Definition 2.3: $\Delta S$-Function. Define the $\Delta S$-function as: $\Delta S(x)=S(x)-1$.

Numerical calculation found that $\Delta S(x)$ satisfies the following approximately anti-symmetrical condition:

$$
\Delta S(x) \approx-\Delta S(0.5-x), 0 \leq x \leq 0.5 .
$$

The symmetry of $S(x)$ with respect to $x=0.5$ in region $[0,1]$ given by (2.13) is exact. The anti-symmetry of $\Delta S(x)$ with respect to $x=0.25$ in region $[0,0.5]$ given by $(2.15)$ is approximate with a deviation less than $10^{-5}$. The deviation is tiny, but its impact is significant. It plays a pivotal role for SQS theory, which will be shown later.

Numerical calculation found that at the center $x=0.25$ of the region $[0,0.5]$ :

$$
S(0.25)=0.999993025315288 \text {. }
$$

(2.16) indicates that, $S(0.25)$ has a deviation of $\sim 7 \times 10^{-6}$ from 1 required by the unitarity. Numerical calculation found a point $x_{c}$ in region $[0,0.5]$ satisfying unitarity:

$$
\begin{aligned}
& S\left(x_{c}\right)=1, \\
& \int_{0}^{x_{c}}[S(x)-1] d x-\int_{x_{c}}^{0.5}[1-S(x)] d x=0, \\
& x_{c}=0.24998715627302645 .
\end{aligned}
$$

On the $x$-axis, $x_{c}$ is located at the left side of $x=0.25$. It extends the region of $S(x)<1$ and shrinks the region of $S(x)>1$. The special point $x_{c}$ has a profound effect on elementary particles and unifications of interactions, which will be given in later sections.

Definition 2.4: Based on $x_{c}$, three other special points $x_{a}$,

$$
x_{b}, x_{d} \text { are defined: }
$$$$
x_{d}=0.5-x_{c}=0.25001284372697355 \text {, }
$$

$$
\begin{aligned}
& \int_{0}^{x_{a}}[S(x)-1] d x-\int_{x_{c}}^{0.25}[1-S(x)] d x=0, \\
& x_{a}=5.181994687988211 \times 10^{-10}, \\
& \int_{0}^{x_{b}}[S(x)] d x-\int_{x_{c}}^{0.25}[S(x)] d x=0, \\
& x_{b}=1.1821861791847719 \times 10^{-5} .
\end{aligned}
$$

The physics meaning of four special points, $x_{a}, x_{b}, x_{c}, x_{d}$, will be given later.

In 3-dimensional case, according to (2.8), the unitarity of $p\left(x, y, z ; x_{i}, y_{j}, z_{k}\right)$ with respect to continuous variables $x, y, z$ is satisfied for any discrete point $\left(x_{i}, y_{j}, z_{k}\right)$ :

$$
\begin{aligned}
& \int_{-\infty}^{\infty} d x \int_{-\infty}^{\infty} d y \int_{-\infty}^{\infty} d z p\left(x, y, z ; x_{i}, y_{j}, z_{k}\right)=\int_{-\infty}^{\infty} d x \int_{-\infty}^{\infty} d y \int_{-\infty}^{\infty} d z e^{-\pi\left[\left(x-x_{i}\right)^{2}+\left(y-y_{j}\right)^{2}+\left(z-z_{k}\right)^{2}\right]} \\
& =\int_{-\infty}^{\infty} e^{-\left(x-x_{i}\right)^{2}} d x \int_{-\infty}^{\infty} e^{-\left(y-y_{j}\right)^{2}} d y \int_{-\infty}^{\infty} e^{-\left(z-z_{k}\right)^{2}} d z=1
\end{aligned}
$$

In general, the unitarity of probability $p\left(x, y, z ; x_{i}, y_{j}, z_{k}\right)$ with respect to discrete variables $x_{i}, y_{j}, z_{k}$ is not satisfied.

Definition 2.5: Define the summation of the probability

$$
\begin{aligned}
& p\left(x, y, z ; x_{i}, y_{j}, z_{k}\right) \text { with respect to } x_{i}, y_{j}, z_{k} \text { as: } \\
& S_{3}(x, y, z)=\sum_{x_{i}=-\infty}^{\infty} \sum_{y_{j}=-\infty}^{\infty} \sum_{i=-\infty}^{\infty} p\left(x, y, z ; x_{i}, y_{j}, z_{k}\right)=\sum_{x_{i}=-\infty}^{\infty} \sum_{y_{j}=-\infty}^{\infty} \sum_{z_{k}=-\infty}^{\infty} e^{-\pi\left[\left(x-x_{i}\right)^{2}+\left(y-y_{j} j^{2}+\left(z-z_{k}\right)^{2}\right]\right.} .
\end{aligned}
$$

Theorem 2.3: $S_{3}(x, y, z)$ can be factorized into three factors:

$$
\begin{aligned}
& S_{3}(x, y, z)=\sum_{x_{i}=-\infty}^{\infty} \sum_{y_{j}=-\infty}^{\infty} \sum_{z_{k}=-\infty}^{\infty} e^{-\pi\left[\left(x-x_{i}\right)^{2}+\left(y-y_{j}\right)^{2}+\left(z-z_{k}\right)^{2}\right]} \\
& =\left[\sum_{x_{i}=-\infty}^{\infty} e^{-\left(x-x_{i}\right)^{2}}\right]\left[\sum_{y_{j}=-\infty}^{\infty} e^{-\left(y-y_{j}\right)^{2}}\right]\left[\sum_{z_{k}=-\infty}^{\infty} e^{-\left(z-z_{k}\right)^{2}}\right]=S(x) S(y) S(z) .
\end{aligned}
$$

Proof: The three-fold summation in (2.25) includes terms for all possible combinations of $e^{-\left(x-x_{i}\right)^{2}}, e^{-\left(y-y_{j}\right)^{2}}, e^{-\left(z-z_{k}\right)^{2}}$. The three multiplications in (2.25) include the same terms. They are only different in processing, the results are the same.

QED

By its definition and (2.12), (2.25), $S_{3}(x, y, z)$ satisfies the following periodic conditions:

$$
\begin{aligned}
& S_{3}(x-1, y, z)=S_{3}(x, y, z), \\
& S_{3}(x, y-1, z)=S_{3}(x, y, z), \\
& S_{3}(x, y, z-1)=S_{3}(x, y, z) .
\end{aligned}
$$

Definition 2.6: Planck cube is defined as a cube with edge lengths $L_{P}=1$ and with discrete point $\left(x_{i}, y_{j}, z_{k}\right)$ at its center or its corner.

The values of $S_{3}(x, y, z)$ at 125 points in a Planck cube with discrete points at its corner are calculated from (2.24) and listed in Table 2.2. 
Table 2.2: $\quad S_{3}(x, y, z)$ Values at 125 Points in a Planck Cube $\left(x_{i}, y_{j}, z_{k}\right.$ truncated at $\left.\pm \mathbf{1 0 0 0}\right)$

\begin{tabular}{|c|c|c|c|}
\hline$z=0 \& z=1$ & $x=0 \& x=1$ & $x=0.25 \& x=0.75$ & $x=0.5$ \\
\hline$y=0 \& y=1$ & 1.28236311585946 & 1.18033236651257 & 1.0783345471798 \\
\hline$y=0.25 \& y=0.75$ & 1.18033236651257 & 1.08641965618562 & 0.99253725580835 \\
\hline$y=0.5$ & 1.0783345471798 & 0.99253725580835 & 0.90676765516773 \\
\hline$z=0.25 \& z=0.75$ & $x=0 \& x=1$ & $x=0.25 \& x=0.75$ & $x=0.5$ \\
\hline$y=0 \& y=1$ & 1.18033236651257 & 1.08641965618562 & 0.99253725580835 \\
\hline$y=0.25 \& y=0.75$ & 1.08641965618562 & 0.999979076091801 & 0.913566394347662 \\
\hline$y=0.5$ & 0.99253725580835 & 0.913566394347662 & 0.834621020415 \\
\hline$z=0.5$ & $x=0 \& x=1$ & $x=0.25 \& x=0.75$ & $x=0.5$ \\
\hline$y=0 \& y=1$ & 1.0783345471798 & 0.99253725580535 & 0.90676765516773 \\
\hline$y=0.25 \& y=0.75$ & 0.99253725580535 & 0.913566394347662 & 0.834621020415 \\
\hline$y=0.5$ & 0.90676765516773 & 0.834621020415 & 0.762497670698562 \\
\hline
\end{tabular}

Theorem 2.4: Probability Conservation Theorem. The average value of $S_{3}(x, y, z)$ over a Planck cube equals to unity:

$$
\int_{\text {PlanckCube }} S_{3}(x, y, z) d v=\int_{0}^{1} d x \int_{0}^{1} d y \int_{0}^{1} d z S_{3}(x, y, z)=1 .
$$

Proof: Substitute (2.24) into left side of (2.27):

$$
\begin{aligned}
& \int_{\text {PlanckCube }} S_{3}(x, y, z) d v=\int_{0}^{1} d x \int_{0}^{1} d y \int_{0}^{1} d z S_{3}(x, y, z) \\
= & \int_{0}^{1} d x \int_{0}^{1} d y \int_{0}^{1} d z\left[\sum_{x_{i}=-\infty}^{\infty} \sum_{y_{j}=-\infty}^{\infty} \sum_{z_{k}=-\infty}^{\infty} e^{-\pi\left[\left(x-x_{i}\right)^{2}+\left(y-y_{j}\right)^{2}+\left(z-z_{k}\right)^{2}\right]}\right] \\
= & \sum_{x_{i}=-\infty}^{\infty} \sum_{y_{j}=-\infty}^{\infty} \sum_{z_{k}=-\infty}^{\infty} \int_{0}^{1} d x \int_{0}^{1} d y \int_{0}^{1} d z e^{-\pi\left[\left(x-x_{i}\right)^{2}+\left(y-y_{j}\right)^{2}+\left(z-z_{k}\right)^{2}\right]} .
\end{aligned}
$$

Change variables as:

$$
x^{\prime}=x_{i}-x, y^{\prime}=y_{j}-y, z^{\prime}=z_{k}-z .
$$

Substituting (2.29) into (2.28) and changing integrations' upper and lower limits accordingly yield:

$$
\begin{aligned}
& \int_{V} S_{3}(x, y, z) d v=\int_{0}^{1} d x^{\prime} \int_{0}^{1} d y^{\prime} \int_{0}^{1} d z^{\prime} S_{3}\left(x^{\prime}, y^{\prime}, z^{\prime}\right) \\
& =\sum_{x_{i}=-\infty}^{\infty} \sum_{y_{j}=-\infty}^{\infty} \sum_{z_{k}=-\infty}^{\infty} \int_{x_{i}-1}^{x_{i}} d x^{\prime} \int_{y_{j}-1}^{y_{j}} d y^{\prime} \int_{z_{k}-1}^{z_{k}} d z^{\prime} e^{-\pi\left[\left(x^{\prime}-x_{i}\right)^{2}+\left(y^{\prime}-y_{j}\right)^{2}+\left(z^{\prime}-z_{k}\right)^{2}\right]} \\
& =\int_{-\infty}^{\infty} d x^{\prime} \int_{-\infty}^{\infty} d y^{\prime} \int_{-\infty}^{\infty} d z^{\prime} e^{-\pi\left[\left(x^{\prime}-x_{i}\right)^{2}+\left(y^{\prime}-y_{j}\right)^{2}+\left(z^{\prime}-z_{k}\right)^{2}\right]}=1 .
\end{aligned}
$$

QED

Probability Conservation Theorem is important. It proved that, even though in general $S_{3}(x, y, z)$ does not satisfy unitarity requirement, but it does satisfy unitarity requirement in terms of average over a Planck cube. The conservation of probability means that, the event carriers of probability are moving around but they cannot be created or annihilated.

Lemma 2.4.1: The average value of $S(x)$ over region

$[0,1]$ equals to unity:

$$
\int_{0}^{1} S(x) d x=1 \text {. }
$$

Proof: Substitute (2.11) into the left side of (2.30):

$$
\int_{0}^{1} S(x) d x=\int_{0}^{1} \sum_{x_{i}=-\infty}^{\infty} e^{-\pi\left(x-x_{i}\right)^{2}} d x=\sum_{x_{i}=-\infty}^{\infty} \int_{0}^{1} e^{-\pi\left(x-x_{i}\right)^{2}} .
$$

Change variables as:

$$
x^{\prime}=x_{i}-x,
$$

Substituting (2.32) into (2.31) and changing integration's upper and lower limits accordingly yield:

$$
\int_{0}^{1} S(x) d x=\sum_{x_{i}=-\infty}^{\infty} \int_{x_{i}-1}^{x_{i}} e^{-\pi x^{\prime 2}} d x^{\prime}=\int_{-\infty}^{\infty} e^{-\pi x^{\prime 2}} d x=1 . \quad \text { QED }
$$

Lemma 2.4.2: Planck cube with volume $V=1$ (length normalized to $\left.L_{P}=1\right)$ is divided into two parts $V_{1}$ and $V_{2}$ :

$$
\begin{aligned}
& V_{1}+V_{2}=V=1, \\
& \int_{V} d v=V=1, \int_{V_{1}} d v=V_{1}, \int_{V_{2}} d v=V_{2} .
\end{aligned}
$$

Theorem 2.4 leads to the following equation:

$$
\int_{V_{1}}\left[S_{3}(x, y, z)-1\right] d v=\int_{V_{2}}\left[1-S_{3}(x, y, z)\right] d v .
$$

Proof: According to (2.27) and (2.33):

$$
\int_{V_{1}} S_{3}(x, y, z) d v+\int_{V_{2}} S_{3}(x, y, z) d v=\int_{V_{1}+V_{2}} S_{3}(x, y, z) d v=1=V=V_{1}+V_{2}=\int_{V_{1}} d v+\int_{V_{2}} d v .
$$

Moving the terms on left and right sides yields (2.34).

QED

\section{Section 3: Unitarity}

Unitarity is a basic requirement of probability. As shown in Section 2, the unitarity with respect to discrete variables and continuous variables for Gaussian probability are contradictory. In this section, three schemes are presented to solve the unitarity problem.

Scheme-1: To treat all points in space equally.

For Scheme-1, Gaussian probabilities are not only assigned to discrete points but to every point in the continuous space. As a result, (2.4) becomes:

$$
\begin{aligned}
& p\left(x ; x^{\prime}\right)=e^{-\pi\left(x-x^{\prime}\right)^{2}} ; \\
& x=-\infty, \cdots, 0, \cdots, \infty ; x^{\prime}=-\infty, \cdots, 0, \cdots, \infty .
\end{aligned}
$$

The summations in $S_{3}(x, y, z)$ of (2.24) introduced in Section 2 become integrations:

$$
\begin{aligned}
& S_{3}(x, y, z)=\sum_{x_{i}=-\infty}^{\infty} \sum_{y_{j}=-\infty}^{\infty} \sum_{z_{k}=-\infty}^{\infty} p\left(x, y, z ; x_{i}, y_{j}, z_{k}\right) \Rightarrow \iint_{-\infty-\infty}^{\infty} \int_{-\infty}^{\infty} p\left(x, y, z ; x^{\prime}, y^{\prime}, z^{\prime}\right) d x^{\prime} d y^{\prime} d z^{\prime} \\
& =\int_{-\infty}^{\infty} e^{-\pi\left(x-x^{\prime}\right)^{2}} d x^{\prime} \int_{-\infty}^{\infty} e^{-\pi\left(y-y^{\prime}\right)^{2}} d y^{\prime} \int_{-\infty}^{\infty} e^{-\pi\left(z-z^{\prime}\right)} d z^{\prime}=S(x) S(y) S(z)=1 .
\end{aligned}
$$

The unitarity problem is solved.

For Scheme-1, the discrete points are no longer special and all points in the space are on an equal footing. But Scheme-1 does not represent the space for SQS theory, instead, it represents the space for quantum mechanics. To introduce Scheme-1 is for comparison purpose. It indicates that, the uniform space does not have unitarity problem. The unitarity problem is caused by space grainy structure, which SQS theory must deal with. 


\section{Z. Y. SHEN}

In Appendix 1, A Fourier transform is applied to probability $p(x)$ of (3.1) to convert it into $k$-space. According to (A1.2), the corresponding Gaussian probability function $P(k)$ in $k$-space is:

$$
P(k)=\frac{1}{2 \pi} e^{-\frac{k^{2}}{4 \pi}} .
$$

The standard deviation of $P(k)$ is $\sigma_{k}=\sqrt{2 \pi}$. Multiplying (A1.6) with $\hbar$ yields:

$$
\left(\hbar \sigma_{k}\right) \cdot \sigma=\Delta p_{x} \cdot \Delta x=\hbar .
$$

In (3.4), $\Delta x$ and $\Delta p_{x}$ are 1-dimensional displacement and momentum difference, respectively. The $\hbar$ on right side is two times greater than the minimum value $\hbar / 2$ from Heisenberg uncertainty principle. The increased uncertainty is due to the asymmetry of $\sigma=1 / \sqrt{2 \pi}$ and $\sigma_{k}=\sqrt{2 \pi}$.

The wave function corresponding to $P\left(k ; x^{\prime}\right)$ of (A1.1) is:

$$
\begin{aligned}
& \psi\left(k ; x^{\prime}\right)=\sqrt{P(k)} Q\left(k ; x^{\prime}\right)=\frac{1}{2 \pi} e^{-\frac{k^{2}}{8 \pi}} e^{i k x^{\prime}} ; \\
& k=-\infty, \cdots, 0, \cdots, \infty ; x^{\prime}=-\infty, \cdots, 0, \cdots, \infty .
\end{aligned}
$$

Notice that, the wave function (3.5) has following features: 1. The relation between $P\left(k ; x^{\prime}\right)$ and $\psi\left(k ; x^{\prime}\right)$ is consistent with quantum mechanics:

$$
P\left(k ; x^{\prime}\right)=\psi^{*}\left(k ; x^{\prime}\right) \psi\left(k ; x^{\prime}\right) .
$$

2. $\psi\left(k ; x^{\prime}\right)$ is not an eigenstate of $k$. The magnitude $\sqrt{P(k)}$ of $\psi\left(k ; x^{\prime}\right)$ serves as distribution function for $k$.

Before explore other schemes, a discussion for the essence of probability unitarity is necessary. Probability is associated with events. In Section 2, Table 2.2 data show that, in the vicinity of Planck cube's center $(x=0.5, y=0.5, z=0.5)$, the sum of probabilities $S_{3}(x, y, z)<1$. Because the set of events at these points are incomplete; some events are missing. These missing evens cause the sum of local probabilities less than one. In the vicinity of the Planck cube corners $\left(x_{i}, y_{j}, z_{k}\right)$, $S_{3}(x, y, z)>1$, because the set of events over there includes some events belong to other places. These excessive evens cause the sum of local probabilities greater than one. In other words, events associated with their probabilities move around inside Planck cube causing the unitarity problem. To move these events back to where they belong will solve the discrete unitarity problem. But it distorts the Gaussian probability distribution and jeopardizes the unitarity with respect to continuous variables based on Gaussian probability distribution.

To solve the problem requires some new concept. Traditionally, unitarity is local, which requires the sum of probability equals to unity at each point in space. $S_{3}(x, y, z) \neq 1$ is caused by events moving around. The foundation for local unitarity no longer exists. A generalized unitarity is proposed:
1. Recognize the fact that events associated with probabilities move around;

2. Follow the moving events for probability unitarity. According to Theorem 2.4, the Probability Conservation Theorem, generalized unitarity is not contradictory to the traditional unitarity for the Planck cube as a whole entity. But it does change the rules inside the Planck cube. For the microscopic scales, as the events inside Planck cube are concerned, generalized unitarity is necessary. For the macroscopic scale including many Planck cubes, the local unitarity is still valid in the average sense.

The following two schemes are based on generalized unitarity.

\section{Scheme-2: Unitarity via probability transportation on complex planes}

The complex planes are inherited from the 3-dimensional Gaussian probability. Consider a Planck cube centered at a discrete point $\left(x_{i}=0, y_{j}=0, z_{k}=0\right)$ as shown in Fig. 3.1.

According to (2.5), the 3 -dimensional Gaussian probability is:

$$
p(x, y, z ; 0,0,0)=\frac{1}{(2 \pi)^{3 / 2} \sigma^{3}} e^{\frac{x^{2}+y^{2}+z^{2}}{2 \sigma^{2}}} .
$$

Normalize the three values of the standard deviations $\sigma_{0}^{\prime}, \sigma_{1}^{\prime}$,

$$
\begin{aligned}
& \sigma_{2}^{\prime} \text { of (2.7) as: } \\
& \sigma_{0}=\sqrt{2 \pi} \sigma_{0}^{\prime}=1, \sigma_{1}=\sqrt{2 \pi} \sigma_{1}^{\prime}=e^{i 2 \pi / 3}, \sigma_{2}=\sqrt{2 \pi} \sigma_{2}^{\prime}=e^{-i 2 \pi / 3} .
\end{aligned}
$$

In which two of them $\sigma_{1}$ and $\sigma_{2}$ are complex numbers. To keep the probabilities as real numbers related to $\sigma_{1}$ and $\sigma_{2}$, it is necessary to extend the $x$-axis, $y$-axis, $z$-axis into three complex plans $\dot{x}$-plane, $\dot{y}$-plane, $\dot{z}$-plane, respectively.

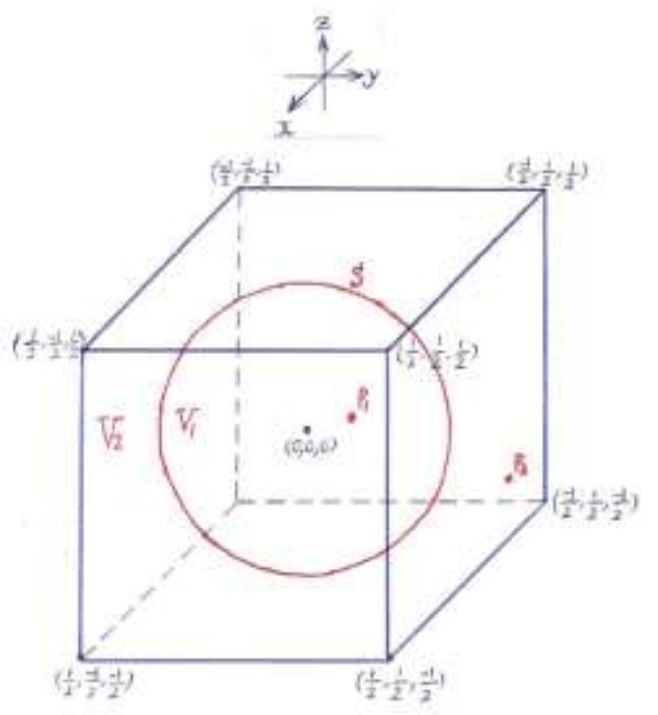

Fig. 3.1 The Planck cube with center at a discrete point $\left(x_{i}=0, y_{j}=0, z_{k}=0\right)$.

Definition 3.1: Define three complex planes associated with $x$-axis, $y$-axis, $z$-axis: 


$$
\begin{aligned}
& \dot{\xi}_{1,2}=(x-\alpha) \sigma_{1,2} \xi=(x-\alpha) e^{ \pm i \vartheta} \xi, \\
& \dot{\psi}_{1,2}=(y-\beta) \sigma_{1,2} \psi=(y-\beta) e^{ \pm i \vartheta} \psi, \\
& \dot{\zeta}_{1,2}=(z-\gamma) \sigma_{1,2} \zeta=\zeta(z-\gamma) e^{ \pm i \vartheta} \zeta . \\
& \vartheta=\frac{2 \pi}{3}=120^{\circ} .
\end{aligned}
$$

In which $\alpha, \beta, \gamma$ and $\xi, \psi, \zeta$ are real parameters.

Explanation: In (3.9a), $\dot{\xi}_{1,2}$ represents two straight lines on complex $\dot{x}$-plane intercepting to the real $x$-axis at $x=\alpha$ with angles of $\pm \vartheta= \pm 120^{\circ}$. Continuously change the value of $\alpha, \dot{\xi}_{1}$ and $\dot{\xi}_{2}$ sweep across $x$-axis to construct the complex $\dot{x}$-plane. Every point on the complex $\dot{x}$-plane is the intersection of two straight lines defined by (3.9a). The $\dot{y}$-plane and $\dot{z}$-plane associated with $y$-axis and $z$-axis are constructed in the same way.

On the complex $\dot{x}$-plane, three straight lines $\dot{\xi}_{1}, \dot{\xi}_{2}$ and $x$ axis intercept at $x=\alpha=0$ with 3 -fold rotational symmetry as shown in Fig. 3.2. The 3 -fold rotational symmetry has its physics significance, which will be discussed later.

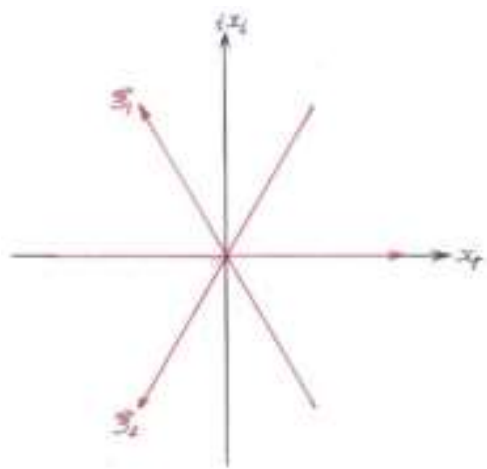

Fig.3.2 Three straight lines with 3-fold rotational symmetry on complex $\dot{x}$-plane.

Rule 3.1: In order to keep the values of $p\left(x ; x_{i}\right)$, $p\left(x, y, z ; x_{i}, y_{j}, z_{k}\right)$ and $S(x), S_{3}(x, y, z)$ as real numbers, in the Gaussian probability exponential part, spatial variables $x, y, x$ in the numerator choice their path according to (3.9) matching the $\sigma$ value in denominator to keep these values always equal to real numbers.

Explanation: The validity of Rule 3.1 to $p\left(x ; x_{i}\right)$, $p\left(x, y, z ; x_{i}, y_{j}, z_{k}\right)$ is obvious. Its validity for $S(x)$, $S_{3}(x, y, z)$ needs explanation. According to the definition of $S(x)$ :

$$
S(x)=\sum_{x_{i}=-\infty}^{\infty} p\left(x ; x_{i}\right)=\sum_{x_{i}=-\infty}^{\infty} e^{-\pi\left(x-x_{i}\right)^{2}} .
$$

All terms of $S(x)=\sum_{x_{i}=-\infty}^{\infty} p\left(x ; x_{i}\right)$ except $p(x ; 0)$ have their "tail" in region $[-0.5,+0.5]$, which are equivalent to the "tails" of $p(x ; 0)$ in regions of $[-\infty,-0.5]$ and $[+0.5,+\infty]$ :

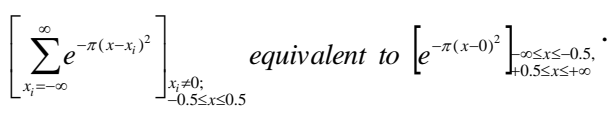

$S(x)$ in region $[-0.5,0.5]$ can be viewed as a single probability distribution function $p(x ; 0)$ with "multireflections" at the two boundaries of region [-0.5, 0.5]. Fig. 3.3 shows an example for the $x_{i}=0$ term along with two adjacent terms $x_{i}=1$ and $x_{i}=-1$ with their "tails" in region $[-0.5,0.5]$. In essence, probability transportation via complex plane for $p\left(x ; x_{i}\right)$ is also valid for $S(x)$. According to

Theorem 2.3, $S_{3}(x, y, z)=S(x) S(y) S(x)$, the same argument is valid for $S_{3}(x, y, z)$ as well.

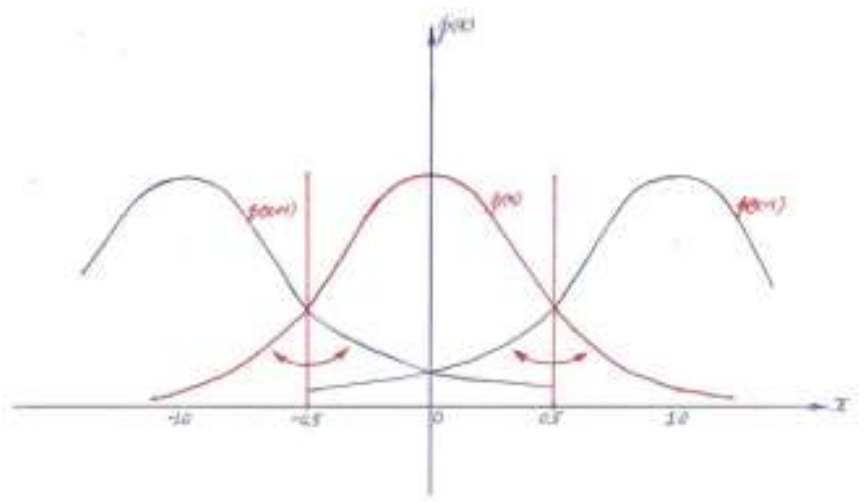

Fig. 3.3 Three adjacent Gaussian probability distribution functions show the "tails".

For double check, let's look it the other way, consider the $p\left(x ; x_{i}\right)$ term in $S(x)$ :

$$
p\left(x ; x_{i}\right)=e^{-\pi \frac{\left(x-x_{i}\right)^{2}}{\sigma^{2}}} .
$$

In which $x_{i}$ is a real number and $x$ is a complex number. As long as the point corresponding to $\left(x-x_{i}\right)$ is on the lines defined by (3.9a), $p\left(x ; x_{i}\right)$ is a real number, and so is $S(x)$.

In the Planck cube centered at discrete point $\left(x_{i}=0, y_{j}=0, z_{k}=0\right.$ ) as shown in Fig.3.1, a closed surface $S$ is defined by $\left[S_{3}(x, y, z)-1\right]=0$, which divides Planck cube in two parts $V_{1}$ and $V_{2}$. In the inner region $V_{1},\left[S_{3}(x, y, z)-1\right]_{V_{1}}>0$; in the outer region $V_{2},\left[S_{3}(x, y, z)-1\right]_{V_{2}}<0$. By means of probability transportation, the excessive events associated with probabilities in the inner region $V_{1}$ transport to the outer region $V_{2}$. According to Theorem 2.4 and lemma 2.4.2, $S_{3}(x, y, z)$ satisfies generalized unitarity.

Since $S_{3}\left(x_{1}, y_{1}, z_{1}\right)=S(x) S(y) S(z)$ and $S(x), S(y), S(z)$ have the same type of exponential expression, exploring one, $S(x)$, is sufficient. (2.13) shows that, in region $[0,1], S(x)$ is symmetry with respect to $x=0.5$, to explore $S(x)$ in the half region $[0,0.5]$ is sufficient. In region [0, 0.5], (2.15) shows 
that $\Delta S(x)=S(x)-1$ is approximately anti-symmetry with respect to $x=0.25$. In the meantime, let's treat it as exactly anti-symmetry and consider the difference later.

In Scheme-2, probabilities along with events transport back and forth to satisfy the discrete and continuous unitarity requirements alternatively.

Fermions and bosons are essentially different particles with different properties. Their probability transportations are different. It turns out that, bosons without mass take the straight real path along the real axis; while Dirac type fermions take the zigzagging path on the complex plane.

The following rules of probability transportation are for Dirac type fermions.

Rule 3.2: The probability transportation rules for fermions are as follows.

1. Consider two points $x_{1},\left(0 \leq x_{1} \leq 0.25\right), x_{2}=0.5-x_{1}$ $\left(0.25 \leq x_{2} \leq 0.5\right)$ along the real $x$-axis, as shown in Fig. 3.4. The excessive probability $S\left(x_{1}\right)>1$ at $x_{1}$ transports along a set of complex lines $\dot{\xi}_{1}$ and $\dot{\xi}_{2}$ to $x_{2}$ where probability having deficient $S\left(x_{2}\right)<1$. The path length is:

$$
l=2\left(x_{2}-x_{1}\right)
$$

The factor 2 in (3.12) comes from:

$$
1 /|\cos ( \pm \vartheta)|=1 /\left|\cos \left( \pm 120^{\circ}\right)\right|=2 \text {. }
$$

The probability transportation makes $S\left(x_{1}\right)=1$ and $S\left(x_{2}\right)=1$ to satisfy unitarity with respect to discrete variable $x_{i}$. But it distorts the Gaussian probability with respect to continuous variable $x$.

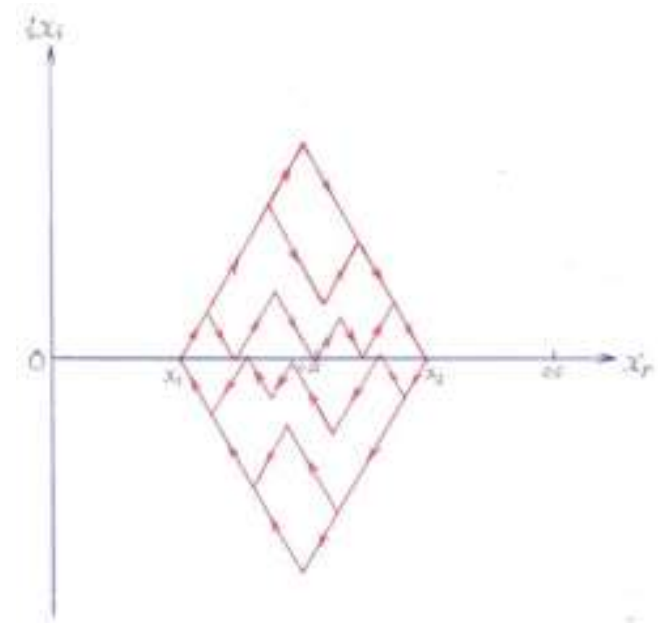

Fig 3.4 Transporting paths with the same loop lengths and different routs on complex plane.

2. To reinstall the Gaussian probability distribution, it transports back from $x_{2}$ to $x_{1}$ along another set of complex lines $\dot{\xi}_{1}$ and $\dot{\xi}_{2}$ via another path with the same path length $l=2\left(x_{2}-x_{1}\right)$ as shown in Fig. 3.4. The two paths form a closed loop with loop length:

$$
L=2 l=4\left(x_{2}-x_{1}\right)
$$

The probability following its event goes back and forth between $x_{1}$ and $x_{2}$ around closed loops.

3. The path length of (3.12) and the loop length of (3.14) are valid for all zigzagging paths shown in Fig.3.4. The multi-path nature has its physics significance, which will be discussed in later sections

The repetitive probability transportations along closed loops cause oscillating between two points $x_{1}$ and $x_{2}$. In this way, the two types of local unitarity are satisfied alternatively, and the generalized unitarity is always satisfied. It provides a kinematic scenario for the oscillation. The dynamic mechanism and driving force of the oscillation will be discussed in Scheme-3.

As mentioned in Section 2, the anti-symmetry of $\Delta S(x) \approx-\Delta S(0.5-x)$ is only an approximation. In general, the unitarity by probability transportation is not exact. The tiny difference between $\Delta S\left(x_{1}\right)$ and $\left[-\Delta S\left(x_{2}\right)\right]$ provides a slight chance for probability transportation path to go off loop. The off loop path goes to other places with different values of $x_{1}$ and $x_{2}$ corresponding to other particles, which provide the mechanism for interactions between particles and transformation of particles.

This is the scenario of probability transportation on the complex $\dot{x}$-plan associated with $x$-axis. The same is for the complex $\dot{y}$-plane and $\dot{z}$-plane associated with $y$-axis and $z$ axis.

For Scheme-2, the three real axes in 3-dimensional real space are extended to three complex planes with 6 independent variables instead of 3 . The extended space with three complex planes is an abstract space. For SQS theory, the real space is 3-dimensional. The essence of complex plane is to add the phase angle to real spatial parameters. The physics meaning of the phase angle will be discussed later. Scheme-3: Unitarity in curved 3-dimensional space.

According to general relativity, in 3-dimensional curved space, the distance between point $P(x, y, z)$ and discrete point $P_{d}\left(x_{i}, y_{j}, z_{k}\right)$ is the geodesic length:

$$
L_{G}\left(P ; P_{d}\right)=L_{G}\left(x, y, z ; x_{i}, y_{j}, z_{k}\right)
$$

According to (A2.2) in Appendix 2, geodesic length $L_{G}\left(p ; p_{d}\right)$ is determined by following differential equation:

$$
\frac{d^{2} x^{a}}{d s^{2}}+\Gamma_{b c}^{a} \frac{d x^{b}}{d s} \frac{d x^{c}}{d s}=0, P \text { to } P_{d} .
$$

In which, $\Gamma_{b c}^{a}$ is Christoffel symbol of second type. Taking $L_{G}^{2}\left(P ; P_{d}\right)$ to replace $\left[\left(x-x_{i}\right)^{2}+\left(y-y_{j}\right)^{2}+\left(z-z_{k}\right)^{2}\right]$ in $(2.24)$ yields:

$$
S_{3}(P)=\sum_{x_{i}=-\infty}^{\infty} \sum_{y_{j}=-\infty}^{\infty} \sum_{z_{k}=-\infty}^{\infty} e^{-\pi L_{G}^{2}\left(P(x, y, z) ; P_{d}\left(x_{i}, y_{j}, z_{k}\right)\right)} .
$$

As mentioned previously, at point $P_{1}\left(x_{1}, y_{1}, z_{1}\right)$ in $V_{1}$ shown in Fig. 3.1, there are excessive events associated with 
$\left[S_{3}\left(P_{1}\right)-1\right]_{V_{1}}>0$; at point $P_{2}\left(x_{2}, y_{2}, z_{2}\right)$ in $V_{2}$, there are

deficient events associated with $\left[S_{3}\left(P_{2}\right)-1\right]_{V_{2}}<0$. For scheme3 , the probability transportation from $P_{1}$ to $P_{2}$ takes its geodesic path:

$$
L_{1 \rightarrow 2}\left(P_{1}, P_{2}\right)=L_{1 \rightarrow 2}\left(P_{1}\left(x_{1}, y_{1}, z_{1}\right) ; P_{2}\left(x_{2}, y_{2}, z_{2}\right)\right) \text {. }
$$

To adjust gauge tensor $g_{a b}(x, y, z)$ along the path $L_{1 \rightarrow 2}\left(P_{1}, P_{2}\right)$ in curved space, the unitarity of probability $\left[S_{3}\left(P_{1}\right)-1\right]_{V_{1}}=0$ at $P_{1}$ and $\left[S_{3}\left(P_{2}\right)-1\right]_{V_{2}}=0$ at $P_{2}$ are satisfied. But the Gaussian probability is distorted. Then the gained probability at $P_{2}$ transports back to $P_{1}$ takes the geodesic path:

$$
L_{2 \rightarrow 1}\left(P_{2}, P_{1}\right)=L_{2 \rightarrow 1}\left(P_{2}\left(x_{2}, y_{2}, z_{2}\right) ; P_{1}\left(x_{1}, y_{1}, z_{1}\right)\right) \text {. }
$$

It goes back to $P_{1}$ to reinstall Gaussian probability. The transportations via $L_{1 \rightarrow 2}\left(P_{1}, P_{2}\right)$ and $L_{2 \rightarrow 1}\left(P_{2}, P_{1}\right)$ finish one cycle of oscillation. The process goes on and on. In this way, the local unitarity requirement with respect to discrete variables and continuous variables of Gaussian probability are satisfied alternatively, and the generalized unitarity is always satisfied. This is the scenario of probability oscillation in 3-dimesional curved space.

Hypothesis 3.1: To adjust the gauge tensor $g_{a b}(x, y, z)$ properly makes geodesic paths $L_{1 \rightarrow 2}\left(P_{1}, P_{2}\right)$ such that $\left[S_{3}\left(P_{1}\right)-1\right]_{V_{1}}=0$ and $\left[S_{3}\left(P_{2}\right)-1\right]_{V_{2}}=0$ are satisfied. To adjust the gauge tensor $g_{a b}(x, y, z)$ properly makes geodesic paths $L_{2 \rightarrow 1}\left(P_{2}, P_{1}\right)$ such that the Gaussian probability is reinstalled. The adjusted $g_{a b}(x, y, z)$ determines the space curvature inside the Planck cube.

Explanation: According to Hypothesis 3.1, the alternative unitarity of Gaussian probability with respect to discrete variables and continuous variables is not only the driving force for probability oscillation, but also serves as the driving force to build the curved space inside Planck cube. This is the expectation from SQS theory.

Let' go back to the 1-dimension case.

Definition 3.2: $S$-Equation. Define the $S$-equation along the $x$-axis as:

$$
S(x)-1=\sum_{x_{i}=-\infty}^{\infty} e^{-\pi\left(x-x_{i}\right)^{2}}-1=0 .
$$

Explanation: $S$-equation is the origin of a set of secondary $S$ equations serving as the backbone of SQS theory. It plays a central role to determine particles parameters on their models, which will be discussed in later sections.

Theorem 3.1: Along the $x$-axis, the 1-dimensional unitarity requires:

$$
S(x)-1=\sum_{x_{i}=-\infty}^{\infty} e^{-\pi(x)\left(x-x_{i}\right)^{2}}-1=0 \text { for all } x .
$$

The only way to satisfy $S(x)-1=0$ for all $x$ is that $\pi(x)$ is a function of $x$ as a running constant.
Proof: In Section 2, (2.17) show that,

$S\left(x_{c}\right)=S(0.24998715627302645)=1$. For all other points in region [1,0.5], $S\left(x \neq x_{c}\right) \neq 1$. In order to satisfy $S(x)-1=0$ for all $x$, something in the $S(x)$ must be adjustable. There are only two constants $e$ and $\pi$ in $S(x)$. In which $e$ as a mathematical constant does not depend on geometry, while $\pi$ does. Therefore, the only way to satisfy unitarity of $S(x)=1$ for all $x$ is that $\pi(x)$ is a function of $x$ as a running constant. QED Explanation: For SQS theory, Theorem 3.1 plays a central role for the models and parameters of elementary particles, which will be demonstrated in later sections.

In the 1-dimensional case, what does $\pi(x)$ mean? The answer is: $\pi(x)$ carrying information in curved 3dimensional space around point $x, \pi(x)<\pi$ indicates space having positive curvature corresponding to attraction force; $\pi(x)>\pi$ indicates space having negative curvature corresponding to repulsive force. The real examples will be given later.

In Table 3.1, the values of $\pi(x)$ calculated from (3.21) are listed along with the types of space curvatures and

\begin{tabular}{|c|c|c|}
\hline$\pi$ (Unit: Planck length) & $\pi(x)^{\circ}$ & Curvature (force) \\
\hline 8 & $\rightarrow \infty$ & Negative (repulstive) \\
\hline$x_{2}=5.18199+087988211 \times 10^{-i 1}$ & $\geq 36.736312443671079>\pi$ & Negative (regularve) \\
\hline$x_{4}=1.182186179184772 \times 10^{-2}$ & $\geq 20.3701945579133>\pi$ & Negative (repulsive) \\
\hline 0.0625 & $=4.82665804324767>\pi$ & Negative (repuisive) \\
\hline 0.1250 & $=3.877102924420037>\pi$ & Negatsve (repulstve) \\
\hline 0.1875 & $-341425510042990>\pi$ & Negative (repulsive) \\
\hline$x_{z}=024998715627302645$ & $-3.141592653589793=\pi$ & Zero (nono) \\
\hline 0.2500 & $-3.141548838186024<\pi$ & Positive (antraction) \\
\hline$x_{d}=0.25001284372697355$ & $-3.141505026927053<\pi$ & Positive (attraction) \\
\hline 0.3125 & $-2.970546532407703<\pi$ & Positive (attraction) \\
\hline 0.3750 & $-2.864592498254391<\pi$ & Positive (attraction) \\
\hline 0.4375 & $-2806341913386171<\pi$ & Positive (artraction) \\
\hline 0.5000 & $-27877195311894243<\pi$ & Positive (attraction) \\
\hline
\end{tabular}
corresponding forces.

Table 3.1: $\pi(x)$ as A Function of $x$ Calculated from (3.21) $\left(x_{i}\right.$ truncated at \pm 1000000 )

Notes: * The precision of values for $\pi\left(x<1.2 \times 10^{-5}\right)$ is limited by 16 -digid numerical calculation. The lower limits are listed.

The attraction force is the ordinary gravitational force. The repulsive force means that, in the vicinity of discrete point gravity reverses its direction. This is one of predictions provided by SQS theory, which is important in many senses. For one, the repulsive force prevents forming singularity, which solves a serious problem for general relativity. For another, without repulsive force to balance the attraction force, space cannot be stable. The others will be given later. Theorem 3.2: At discrete points $x=x_{i}$, the unitarity equation of (3.20) requires:

$$
\pi\left(x_{i}\right) \rightarrow \infty \text {, for } x_{i}=-\infty, \cdots,-2,-1,0,1,2, \cdots, \infty \text {. }
$$




\section{Z. Y. SHEN}

Proof: Consider the opposite. If $\pi\left(x_{i}\right)$ is not infinity, When the summation index $x_{i} \rightarrow \infty$,

$$
S\left(x_{i}\right)=\sum_{x_{i}=-\infty}^{\infty} e^{-\pi\left(x_{i}\right)\left(x_{i}-x_{i}\right)^{2}}=\sum_{x_{i} \rightarrow-\infty}^{\infty} e^{-\pi\left(x_{i}\right) \times 0} \rightarrow \infty .
$$

equation (3.20) cannot be satisfied. The opposite, i.e. $\pi\left(x_{i}\right) \rightarrow \infty$ must be true.

QED

Theorem 3.2 is a mathematic theorem with physics significance, which will be presented later.

For Scheme-2, probability oscillation is to satisfy alternative unitarity, which does not provide the dynamic mechanism and the driving force. For scheme-3, the repulsive and attraction forces provide the dynamic mechanism and the driving force for oscillation. At $x=x_{1}$ where $\pi\left(x_{1}\right)>1$, the repulsive force pushes the event associated with its probability towards $x_{2}$. When it arrived $x=x_{2}$ where $\pi\left(x_{2}\right)<1$, the attractive force pulls it back to $x_{1}$. In this way, the oscillation continues. The dynamic scenario provides the mechanism of oscillation, which is originated from space curvature.

As mentioned in Scheme-2, the approximation of antisymmetry of (2.15) provides a slight chance for transportation off loop representing interactions, which is also valid for Scheme-3.

For Scheme-3, the curvature patterns make the Planck scale grainy structure.

As a summary, Table 3.2 shows a brief comparison of three schemes.

Table 3.2: Summary of the Features for Three Schemes

\begin{tabular}{|c|c|c|c|c|}
\hline Scherne & 3- dimension space & Additiona! & $\begin{array}{c}\text { Quarntum prace grainy } \\
\text { characteristics }\end{array}$ & $\begin{array}{l}\text { Types of } \\
\text { uniturity }\end{array}$ \\
\hline -Ifor QM & real Euclidian & nowe & nobe & regular \\
\hline-2 for $S Q S$ & complex Euclidian & 3 complex planes & Plance scaie grans & generalizad \\
\hline-3 for $5 Q 5$ & Curved real & none & Planck scale grains & geberalized \\
\hline
\end{tabular}

The three schemes are three manifestos of the vacuum state. Scheme-1 corresponds to the quantum mechanics vacuum state. Schemes-2 and Scheme-3 are SQS vacuum states at a level deeper than quantum mechanics.

The probability oscillation in Scheme-2 is the same as in Scheme-3. It implies that Scheme-2 is equivalent to Scheme3. Moreover, in Scheme-2, three complex planes have 6 independent real variables; in Scheme-3, the symmetrical $3 \times 3$ gauge matrix of $g_{a b}$ spatial part also has 6 independent components. The correlation indicates that, the complex planes of Scheme-2 are closely linked to curved space of Scheme-3. It confirms that, the three complex planes associated with three real axes are some type of abstract expression of the curved 3-dimensional real space. For SQS theory, there is no additional dimension or dimensions beyond the real 3-dimensional space in existence.

In reference [2], Penrose demonstrated the correlation between Riemann surface and the topological manifoldtorus. According to Penrose, $\sigma_{0}, \sigma_{1}, \sigma_{2}$ of (3.8) are three branch points of the complex function $\left(1-z^{3}\right)^{1 / 2}$ on the Riemann surface:

$$
z=\sigma_{0}=1, z=\omega=\sigma_{1}=e^{i \frac{2 \pi}{3}}, z=\omega^{2}=\sigma_{2}=e^{i \frac{4 \pi}{3}}
$$

As shown in Fig 3.5(a), The Riemann surface for $\left(1-z^{3}\right)^{1 / 2}$ has branch points of order 2 at $1, \omega, \omega^{2}$ and another one at $\infty$. Penrose showed that, for Riemann surface's two sheets each with two slits, one from 1 to $\infty$ and the other from $\omega$ to $\omega^{2}$, these are two topological cylindrical surfaces glued correspondingly giving a torus as shown in Fig. 3.5(b). On the torus surface, there are four tiny holes $h_{1}, h_{\infty}, h_{\omega}, h_{\omega^{2}}$ representing $1, \infty, \omega, \omega^{2}$ on the Riemann surface, respectively. The four tiny holes on torus have important physics significance, which well be discussed in later sections.
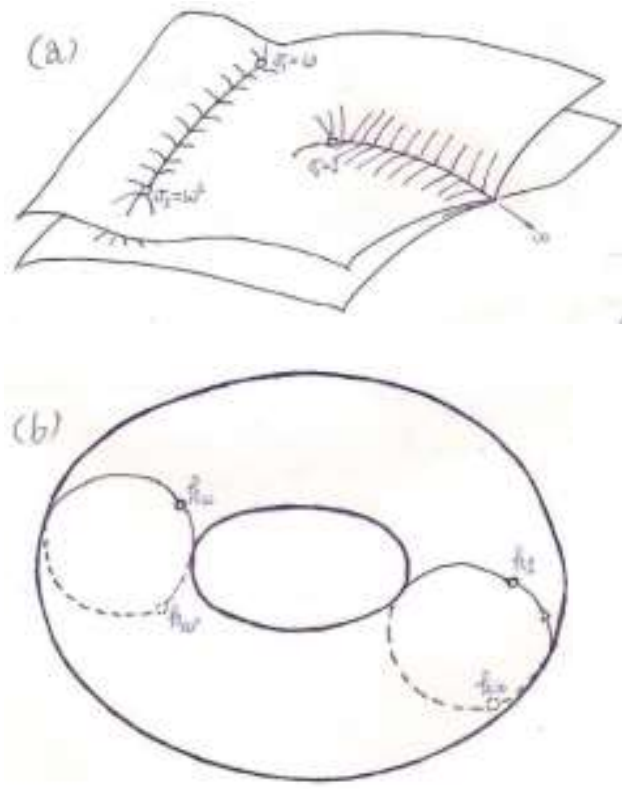

Fig. 3.5 (a) Four branch points and two glued cuts on two sheets of Riemann surface; (b) Four tiny holes on torus surface.

For SQS theory, the correspondence of Riemann surface and torus is very important. It plays a pivotal rule for constructing the topological models for quarks, leptons, and bosons with mass and much more, which will be discussed in later sections.

\section{Section 4: Random Walk Theorem and Converting Rules}

Random walk process is based on stochastic nature of space. It plays an important role for SQS theory. In this section, the Random Walk Theorem is proved and converting rules are introduced serving as the key to solve many hierarchy problems.

Definition 4.1: Short Path and Long Path. In 3dimensional space, there are two types of paths between two discrete points. The "short path" $L$ from point 
$\left(x_{i}, y_{j}, z_{k}\right)$ to point $\left(x_{i^{\prime}}, y_{j^{\prime}}, z_{k^{\prime}}\right)$ is defined as the straight distance between them.

$$
L=\sqrt{\left(x_{i^{\prime}}-x_{i}\right)^{2}+\left(y_{j^{\prime}}-y_{j}\right)^{2}+\left(z_{k^{\prime}}-z_{k}\right)^{2}} .
$$

The "long path" $\hat{L}$ from point $\left(x_{i}, y_{j}, z_{k}\right)$ to point

$\left(x_{i}, y_{j^{\prime}}, z_{k^{\prime}}\right)$ is defined as step-by-step zigzagging path in lattice space with Planck length $L_{P}$ as step length $l_{i}=L_{P}$.

$$
\hat{L}=\sum_{i=1}^{N} l_{i}=N L_{P}, N=l+m+n .
$$

The random walk from point $\left(x_{i}, y_{j}, z_{k}\right)$ to point

$\left(x_{i^{\prime}}, y_{j^{\prime}}, z_{k^{\prime}}\right)$ takes $l, m, n$ steps along $\pm x, \pm y, \pm z$

directions, respectively.

Theorem 4.1: Random Walk Theorem. Short path $L$ and long path $\hat{L}$ are correlated by the random walk formula:

$$
\hat{L}=L^{2} \text {; or } L=\sqrt{\hat{L}} \text {. }
$$

$L$ and $\hat{L}$ are normalized with respect to Planck lengt $L_{P}$, both are numbers.

Proof: According to (2.8), the probability from point

$$
\begin{aligned}
& \left(x_{i}, y_{j}, z_{k}\right) \text { to point }\left(x_{i^{\prime}}, y_{j^{\prime}}, z_{k^{\prime}}\right) \text { is: } \\
& p\left(x_{i}, y_{j}, z_{k} ; x_{i^{\prime}}, y_{j^{\prime}}, z_{k^{\prime}}\right)=e^{\left.-\pi\left(x_{i}-x_{i^{\prime}}\right)^{2}+\left(y_{j^{\prime}}-y_{j^{\prime}}\right)^{2}+\left(z_{k}-z_{k^{\prime}}\right)^{2}\right]}=e^{-\pi L^{2}} .
\end{aligned}
$$

Take a random walk from $\left(x_{i}, y_{j}, z_{k}\right)$ to $\left(x_{i^{\prime}}, y_{j^{\prime}}, z_{k^{\prime}}\right)$ with $l, m$, $n$ steps along $\pm x, \pm y, \pm z$ directions, respectively. The probability of reaching the destination is:

$$
p\left(x_{i}, y_{j}, z_{k} ; x_{i^{\prime}}, y_{j^{\prime}}, z_{k^{\prime}}\right)=\prod_{l} e^{-\pi \cdot 1^{2}} \prod_{m} e^{-\pi \cdot 1^{2}} \prod_{n} e^{-\pi \cdot 1^{2}}=e^{-\pi(l+m+n)}=e^{-\pi \hat{L}},
$$

$\hat{L}=l+m+n$.

Combining (4.3) and (4.4) yields $\hat{L}=L^{2}$.

Obviously, Random Walk Theorem is based on Gaussian Probability Postulation introduced in Section 2. As a precondition, the standard deviation $\sigma$ of 3-dimensional Gaussian probability must take the values to make the factor in front of exponential term equal to 1. Otherwise, Random Walk Theorem does not hold. It means that, the only parameter $\sigma$ in the first fundamental postulation of SQS theory is determined.

Random Walk Theorem provides the foundation for conversions, which are governed by a set of converting rules. Physics quantities can be converted by applying these converting rules, which serve as the way to dealing with hierarchy problems.

Definition 4.2: The converting factor for short path and long path is defined as:

$$
N=L / L_{P} \text {. }
$$

Lemma 4.1: $L, \hat{L}$ and $N$ are related as:

$$
\hat{L}=N L=N^{2} L_{P} \text {. }
$$

Proof: According to Theorem 4.1, the lengths $L$ and $\hat{L}$ in (4.2) are normalized with respect to $L_{P}$. Let $L_{P}$ appears in (4.2):

$$
\frac{\hat{L}}{L_{P}}=\left(\frac{L}{L_{P}}\right)^{2}=\left(\frac{L}{L_{P}}\right) \frac{L}{L_{P}}=N \frac{L}{L_{P}} .
$$

Multiplying $L_{P}$ to both sides of (4.7) yields:

$$
\hat{L}=N L \text {. }
$$

According to (4.5), substituting $L=N L_{P}$ into (4.8a) yields:

$$
\hat{L}=N^{2} L_{P}
$$

(4.8a) plus (4.8b) is (4.6).

The basic unit of length in Theorem 4.1 and Lemma 4.1 as well as the step length of random walk is $L_{P}$, which indicate the importance of Planck length.

According to SQS theory, physics quantities at different scales have different values determined by converting factors, which are governed by converting rules originated from Random Walk Theorem.

Definition 4.3: The conversion factors for general purpose are defined as follows.

\section{For bosons without mass:}

$$
N=\lambda / L_{P} \text {. }
$$

$\lambda$ is the wavelength of the boson.

\section{For particles with mass:}

$$
N=\lambda_{C} / L_{P} \text {. }
$$

$\lambda_{C}$ is the Compton wavelength of the particle:

$$
\lambda_{C}=\frac{h}{M c} \text {. }
$$

$M$ is the mass of that particle and $c$ is the speed of light in vacuum.

Conversion rules for general purpose are given as follows.

\section{For length:}

$$
\hat{L}=N L=N^{2} L_{P} .
$$

$\hat{L}, L$, and $L_{P}$ are long path, short path, and Planck length, respectively.

\section{For time interval:}

$$
\hat{t}=N t=N^{2} t_{P} \text {. }
$$

$\hat{t}, t$, and $t_{P}$ are long path time interval, short path time interval, and Planck time, respectively.

\section{For energy and mass:}

$$
\begin{aligned}
& \hat{E}=E / N=E_{P} / N^{2} . \\
& \hat{M}=M / N=M_{P} / N^{2} .
\end{aligned}
$$

$\hat{E}, E$, and $E_{P}$ are long path energy, short path energy, and Planck scale energy, respectively. $\hat{M}, M$, and $M_{P}$ are long path mass, short path mass, and Planck mass, respectively.

Take the ratio of electrostatic force to gravitational force between two electrons as an example to show how converting rules work.

According to Coulomb's low, the electrostatic force between two electrons separated by a distance $r$ is: 
Z. Y. SHEN

$$
f_{E}=\frac{e^{2}}{4 \pi \varepsilon_{0} r^{2}} .
$$

In which, $e$ is the electrical charge of electron, $\varepsilon_{0}$ is permittivity of free space.

According to Newton's gravity low, the gravitational force between two electrons separated by a distance $r$ is:

$$
f_{G}=G \frac{M_{e}^{2}}{r^{2}} .
$$

In which, $G$ is Newtonian gravitational constant, $M_{e}$ is electron mass.

According to (4.16) and (4.17), the ratio of electrostatic force to gravitational force between two electrons is:

$$
R_{E / G}=\frac{f_{E}}{f_{G}}=\frac{e^{2}}{4 \pi \varepsilon_{0} G M_{e}^{2}} .
$$

According to (4.15) and (2.1d):

$$
\begin{aligned}
& M_{e}=M_{P} / N_{e}, \\
& M_{P}=\sqrt{\frac{2 \pi h c}{G}}, \text { or } G=\frac{2 \pi h c}{M_{P}^{2}} .
\end{aligned}
$$

$N_{e}$ is the converting factor for electron. $M_{P}$ is Planck mass. Substituting (4.15) and (2.1d) into (4.18) yields:

$$
R_{E / G}=\frac{f_{E}}{f_{G}}=\frac{e^{2}}{4 \pi \varepsilon_{0} G M_{e}^{2}}=\frac{1}{4 \pi^{2}} \frac{e^{2}}{2 \varepsilon_{0} h c}\left[\frac{M_{P}}{M_{e}}\right]^{2}=\frac{\alpha}{4 \pi^{2}} N_{e}^{2} .
$$

In (4.19), $\alpha$ is the fine structure constant. At electron mass scale:

$$
\alpha\left(M_{e}\right)=\frac{e^{2}}{2 \varepsilon_{0} h c}=\frac{1}{137.05999084},
$$

In which, $\alpha=1 / 137.035999084(51)$ is cited from 2010PDG (p.126) according to references [3] and [4]. Electron converting factor is:

$$
N_{e}=\frac{M_{P}}{M_{e}}=1.501197 \times 10^{23} .
$$

Substituting (4.20) and (4.21) into (4.19) yields:

$$
R_{E / G}=4.164905 \times 10^{42} \text {. }
$$

$R_{E / G}$ is one of many hierarchy problems in physics. By applying conversion rules not only solves the hierarchy problem but also reveals its origin and mechanism. On the right side of (4.19), the first factor is electrically originated:

$$
\frac{\alpha}{4 \pi^{2}}=1.84811744 \times 10^{-4} \text {. }
$$

The second factor $N_{e}^{2}$ is mass originated:

$$
N_{e}^{2}=\left[\frac{M_{P}}{M_{e}}\right]^{2}=\left(1.501197 \times 10^{23}\right)^{2}=2.253593 \times 10^{46} .
$$

According to Random Walk Theorem and Lemma 4.1, converting factor $N_{e}$ is equal to the ratio of long path over short path. Keep this in mind, the $N_{e}^{2} \sim 10^{46}$ factor can be explained naturally. For a pair of electron, the electrostatic force is inversely proportion to the square of the straight distance $r$ (short path) between them; while the gravitational force actually is inversely proportional to the square of the zigzagging long path $\hat{r}=N_{e} r$ between them. In terms of force mediators, photon takes the short path, while graviton takes the long path. According to SQS theory, this is the mechanism of tremendous strength difference between electrostatic and gravitational forces, which is originated from random walk.

It is the first time to show that Random Walk Theorem and the long path versus short path as well as the conversing rules are real and useful. There are more examples along this line in later sections.

Once the mechanism is revealed, there are more insights to come.

Rule 4.1: Electron's converting factor $N_{e}(l)$ is a running constant as a function of length scale $l$ (in this case, $l$ is the distance between two electrons) with different behaviors in two ranges.

Range-I: For the length scale $l \geq \lambda_{e, C}$ :

$$
N_{e}(l)=N_{e}=\frac{M_{P}}{M_{e}}=\frac{\lambda_{e, C}}{L_{P}}=\text { const. for } l \geq \lambda_{e, C} .
$$

Range-II: For the length scale $l_{\min }=71 L_{P}<l<\lambda_{e C}$ :

$$
N_{e}(l)=N_{e} \frac{l}{\lambda_{e, C}}=\frac{M_{P}}{M_{e}}\left[\frac{l}{\lambda_{e, C}}\right]=\frac{l}{L_{P}} \text { for } l_{\min }=71 L_{P}<l<\lambda_{e C} .
$$

In (4.25), $\lambda_{e, C}$ is the Compton wavelength of electron, $l_{\min }=71 L_{P}<l<\lambda_{e C}$ is the lower limit of $l$ in Range-II, which will be explained in Section 16 .

Explanation: The reason for $N_{e}\left(l \geq \lambda_{e, C}\right)=$ const. in Range-

I is obvious. Otherwise, if $N_{e}\left(l \geq \lambda_{e, C}\right)$ is not a constant, then electron mass in macroscopic scale varies with distance, which is obviously not true. Range-II needs some explanation. According to Lemma 4.1, $N=L / L_{P}$, in this case $N_{e}=l / L_{P},(4.25 \mathrm{~b})$ is explained. Fig. 4.1 shows the variation of $N_{e}(l)$, the $N_{e}(l)$ versus $l$ profile is made of two straight lines. In Range-I, $N_{e}(l)$ is a flat straight line with zero slop. In Range-II, $N_{e}(l)$ is a straight line with slop $1 / L_{P}=1$. Two straight lines intersect at $l=\lambda_{e, C}$. It shows a peculiar behavior of $N_{e}(l)$. Most physics running constants vary asymptotically toward end. This one is different. The straight line with slop $1 / L_{P}=1$ on left suddenly stops at $l=\lambda_{e, C}$ and changes course to the flat straight line on right. At two straight lines' intersecting point, the first order derivative is not continuous. The mechanism of such peculiar behavior will be explained in Section 16 .

There is another factor $\alpha /\left(4 \pi^{2}\right)$ in $R_{E / G}$, in which $\alpha=\alpha(M)$ is a running constant. The variation of $\alpha(M)$ 
makes $R_{E / G}(l)$ different from $N_{e}^{2}(l)$. It rounds the corner of $R_{E / G}(l)$ versus $l$ curve at intersecting point show in Fig. 4.1.

The $R_{E / G}(l)$ for two electrons given by (4.19) is just an example. It can be extended to other charged particles. For instance, two protons separated by a distance $r$, the ratio of electrostatic force to gravitational force is:

$$
\begin{aligned}
& \left.R_{E / G}\right|_{\text {prot }}=\frac{e^{2}}{4 \pi \varepsilon_{0} G M_{\text {prot }}^{2}}=\frac{1}{4 \pi^{2}} \frac{e^{2}}{2 \varepsilon_{0} h c} N_{\text {prot }}^{2}=\frac{\alpha\left(M_{\text {proo }}\right)}{4 \pi^{2}} N_{\text {prot }}^{2} ; \\
& N_{\text {prot }}=M_{P} / M_{\text {prot }}
\end{aligned}
$$

In which, $M_{\text {prot }}, N_{\text {prot }}$, and $\alpha\left(M_{\text {prot }}\right)$ are mass, converting factor and fine structure constant at proton energy scale, respectively.

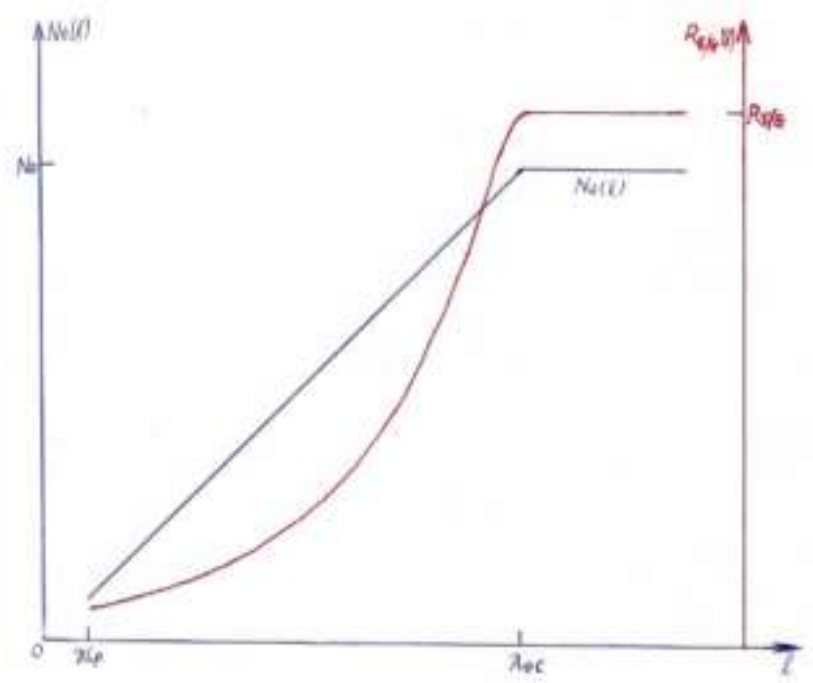

Fig. $4.1 N_{e}(l)$ and $R_{E / G}(l)$ versus distance $l$ curves. (Scales are not in proportion.)

Substituting data into (4.26) and ignoring the difference between $\alpha\left(M_{\text {prot }}\right)$ and $\alpha\left(M_{e}\right)$ of (4.20) yields the ratio for protons:

$$
\left.R_{E / G}\right|_{\text {prot }} \approx 1.235343 \times 10^{36}
$$

The conversion rules introduced in this section are subject to more verifications. Other applications of converting rules will be presented in later sections.

\section{Section 5: Apply to Quantum Mechanics and Special Relativity}

In this section, the converting rules introduced in Section 4 are applied to some examples in quantum mechanics and special relativity.

According to Feynman path integrals theory [5], the state $\psi\left(x_{2} ; t_{2}\right)$ at point $x_{2}$ and time $t_{2}$ is related to the initial state $\psi\left(p_{1} ; t_{1}\right)$ at point $x_{1}$ and time $t_{1}$ as:

$$
\begin{aligned}
& \psi\left(x_{2} ; t_{2}\right)=\int_{x_{1}}^{x_{2}} K(2,1) \psi\left(x_{1} ; t_{1}\right) d l, t_{2}>t_{1} ; \\
& K(2,1)=\sum_{x_{1} \rightarrow x_{2}}^{\text {allpaths }} A e^{\frac{i}{h} S(x(t))}=\sum_{x_{1} \rightarrow x_{2}}^{\text {allpaths }} A e^{\frac{i^{\prime 2} h_{t_{1}} L\left(x, x_{i} ; t\right) d t}{} .}
\end{aligned}
$$

In which $A$ is a constant, $S(x(t))$ is the action, $L(x, \dot{x} ; t)$ is the Lagrangian, $x_{1}, x_{2}, x$ and $x(t)$ are 3-dementional coordinates with simplified notations. The integral in (5.1a) and summation in (5.1b) include "all possible paths" from point $x_{1}$ to point $x_{2}$.

Assuming the particle is a photon with visible lights wavelength of $\lambda \sim 10^{-7} \mathrm{~m}$, it travels with speed $c$ from $x_{1}$ to $x_{2}$ separated by distance $L=1 \mathrm{~m}$. The photon traveling through $L=1 \mathrm{~m}$ once takes time $\Delta t=L / c \approx 3.3 \times 10^{-9} \mathrm{~s}$. The obvious question is: How does photon have time to travel so many times through "all possible paths" between $x_{1}$ and $x_{2}$ ? Theorem 4.1 and Lemma 4.1 provide the answer. According to (4.9), the converting factor for photon with wavelength $\lambda \sim 10^{-7} \mathrm{~m}$ is:

$$
N=\lambda / L_{P} \sim 10^{28} .
$$

According to (4.12), the photon's long path wavelength is:

$$
\hat{\lambda}=N \lambda \sim 10^{28} \lambda \sim 10^{21} \mathrm{~m} \sim 10^{5} \text { lightyears . }
$$

The $N \sim 10^{28}$ tremendous difference between long path wavelength $\hat{\lambda}$ and wave length $\lambda$ is originated from the Random Walk Theorem. From SQS theory viewpoint, the "all possible paths" in (5.1) of Feynman path integrals theory are covered by photon's long path wavelength $\hat{\lambda} \sim 10^{28} \lambda \sim 10^{21} \mathrm{~m}$. It is sufficient for the photon to go through "all possible paths" via many billions of billions different routes from $x_{1}$ to $x_{2}$.

According to (4.13), for the photon with wavelength $\lambda \sim 10^{-7} \mathrm{~m}$, the long path time interval $\Delta \hat{t}$ is much longer than its short path time interval $\Delta t$ :

$\Delta \hat{t}=N \Delta t \sim 10^{28} \times\left(3.3 \times 10^{-9}\right) \approx 3.3 \times 10^{19} s>\Delta t \approx 3.3 \times 10^{-9} s$.

Photon has sufficient time to travel through "all possible paths" including many billions of billions routes from $x_{1}$ to $x_{2}$. This is the explanation of SQS theory for Feynman path integrals theory.

But there is a question. If the photon with wavelength $\lambda \sim 10^{-7} \mathrm{~m}$ really travels through the long path $\hat{\lambda} \sim 10^{28} \lambda \sim 10^{21} \mathrm{~m}$, for a stationary observer, it only takes time interval of $\Delta t \approx 3.3 \times 10^{-9} s<<\Delta \hat{t} \approx 3.3 \times 10^{19} \mathrm{~s}$. The question is: What is photon speed seen by a stationary observer? If the stationary observer sees the long path, the speed is indeed superluminal. But according to SQS theory, the wave pattern of a particle such as photon is established step by step with step length $L_{P}$ during its zigzagging long path journey. The short path is the folded version of the long 


\section{Z. Y. SHEN}

path. For an ordinary photon, the folded long path is hidden in its wave pattern. The stationary observer sees neither the hidden long path nor the superluminal speed. In case the photon's long path shows up from hiding that is another story. It will be discussed later.

The explanation for Feynman's path can be used to explain other similar quantum phenomena such as the double-slot experiment for a single particle and quantum entanglements.

Take the double slots experiment for a single photon as an example. Experiments have proved that, when the light source emits one phone at a time, the interference pattern still shows up. As mentioned previously, a photon with wavelength $\lambda \sim 10^{-7} \mathrm{~m}$ has its long path wavelength $\hat{\lambda} \sim 10^{28} \lambda \sim 10^{21} \mathrm{~m}$ and superluminal speed for vacuons (in Section 18 , vacuon is defined as a geometrical point in space) to travel along the long path, which provide the condition to let the vacuons pass through two slits enormous times to form the interference pattern. Fig.5.1 shows the double-slit interference pattern for a single photon.

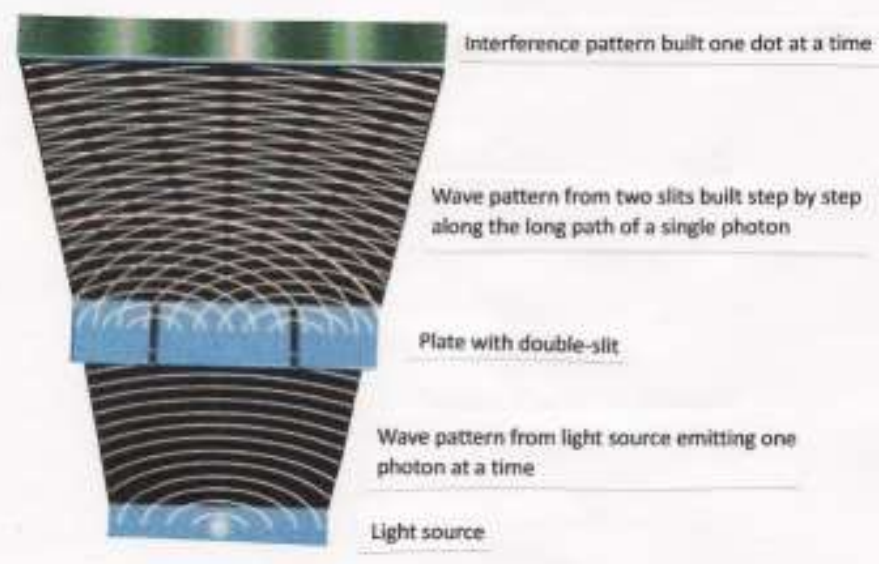

Fig.5.1 The double-slit interference pattern for a single photon.

The single photon's long path builds the wave pattern step by step in the space between the plate with double-slit and the screen. The two waves come from two slits to form the interferential pattern on the screen just like the regular double-slit interference pattern. The single photon strikes on the screen at a location according to probability determined by the interference wave pattern's magnitude square. When more photons strike on screen, the interference pattern gradually shows up. The long path provides the condition for a single photon's wave pattern to interfere with itself. It is possible because of the long path's extremely long length and vacuons' superluminal speed, which allow the vacuons pass through two slits so many times. In this sense, a single photon does pass through two slits.

According to the converting factor $N=\lambda / L_{P}=c / f L_{P}$ based on the Random Walk Theorem, as photon's frequency $f$ and energy increase, $N=c / f L_{P}$ decreases. The difference between long path and short path decreases accordingly. As a result, the wave pattern becomes coarser and more random. In other words, the wave-particle duality is a changing scenario with energy, the particle nature is enhanced and the wave nature is diluted with increasing energy.

The tremendous difference between short path and long path is related to special relativity. A stationary observer sees the photon having wavelength $\lambda$. The photon traveling along its short path with a speed $v$ less than $c$ and very close to $c$, according to Lorentz transformation:

$$
\lambda=\hat{\lambda} \sqrt{1-(v / c)^{2}}, \frac{\hat{\lambda}}{\lambda}=\frac{1}{\sqrt{1-(v / c)^{2}}} .
$$

From SQS theory perspective, $\lambda$ and $\hat{\lambda}$ are photon's short path wavelength and long path wavelength originated from Random Walk Theorem. From special relativity perspective, $\lambda$ versus $\hat{\lambda}$ is the result of Lorentz transformation. These two apparently different scenarios are two sides of the same coin. The key concept is to recognize photon traveling along its short path with a speed $v$ less than $c$ and very close to $c$. It is a deviation from special relativity.

Substituting $N=\hat{\lambda} / \lambda$ into (5.4) yields:

$$
N=\frac{1}{\sqrt{1-(v / c)^{2}}}=\frac{1}{\sqrt{1-\beta^{2}}}=\gamma .
$$

$\beta$ and $\gamma$ are the standard notations in special relativity. As shown by (5.5), converting factor $N$ is closely related to $\beta$ and $\gamma$ of special relativity.

Substituting photons' converting factor $N=\lambda / L_{P}$ from (4.9) into (5.5) yields:

$$
\frac{\lambda}{L_{P}}=\frac{c}{L_{P} f}=\frac{1}{\sqrt{1-(v / c)^{2}}},
$$

Solving (5.6) for photon's speed $v$ as a function of frequency $f$ or wavelength $\lambda$ yields:

$$
\begin{aligned}
& v(f)=c \sqrt{1-\left(f L_{P} / c\right)^{2}}, \\
& v(\lambda)=c \sqrt{1-\left(L_{P} / \lambda\right)^{2}} .
\end{aligned}
$$

Photon's speed varying with its frequency or wavelength means dispersion. (5.7) is the dispersion equation of photon. The speed of photon decreases with increasing frequency. The constant $c$ is not the universal speed of photons, instead, it is the speed limit of photon with frequency approaching zero. This is a modification of special relativity proposed by SQS theory.

According to the Gaussian Probability Postulation, space has periodic structure with Planck length $L_{P}$ as spatial period. It is well known that, wave traveling in periodic structure has (5.7) type dispersion. Look at it the other way: Dispersion is caused by the fact that photon interacts with space. For SQS theory, space is a physics substance.

The dispersion effect of visible lights is extremely small. It is negligible in most cases. According to (5.7), the speed $v$ of a photon with wavelength $\lambda \sim 10^{-7} \mathrm{~m}$ deviates from $c$ in the 
order of $\sim 10^{-56}$. On the other hand, for $\gamma$ - ray with extremely high energy, the dispersion effect is detectable. It serves as a possible way for verification.

On May 9th, 2009, NASA's Fermi Gamma-Ray Space Telescope recorded a $\gamma$-ray burst from source GRB090510 $[6,7,8]$. The observed data are given as follows.

Low energy $\gamma$-ray: energy $E_{1}=1 \times 10^{4} \mathrm{eV}=1.602 \times 10^{-15} \mathrm{~J}$, wavelength $\lambda_{1}=1.24 \times 10^{-10} \mathrm{~m}$.

High energy $\gamma$-ray: energy $E_{2}=3.1 \times 10^{10} \mathrm{eV}=4.967 \times 10^{-9} \mathrm{~J}$, wavelength $\lambda_{2}=3.999 \times 10^{-17} \mathrm{~m}$.

Distance to $\gamma$-ray source: $L_{O}=7.3 \times 10^{9} \mathrm{ly}=6.906 \times 10^{25} \mathrm{~m}$

Observed time delay (after CBM trigger) for the high energy $\gamma$-ray: $\Delta t_{O}=0.829 \mathrm{~s}$.

According to (5.7b), the SQS theoretical value for time delay is:

$$
\Delta t_{T}=L\left[\frac{1}{v_{2}}-\frac{1}{v_{1}}\right]=L \frac{v_{1}-v_{2}}{v_{1} v_{2}}=\frac{L}{c} \frac{\sqrt{1-\left(L_{P} / \lambda_{1}\right)^{2}}-\sqrt{1-\left(L_{P} / \lambda_{2}\right)^{2}}}{\sqrt{1-\left(L_{P} / \lambda_{1}\right)^{2}} \sqrt{1-\left(L_{P} / \lambda_{2}\right)^{2}}} \approx \frac{L}{2 c}\left[\left(\frac{L_{P}}{\lambda_{2}}\right)^{2}-\left(\frac{L_{P}}{\lambda_{1}}\right)^{2}\right] .
$$

The approximation is due to $L_{P} / \lambda_{1}<<1, L_{P} / \lambda_{2}<<1$.

Substituting observed data and $L=L_{O}$ into (5.8) yields:

$$
\Delta t_{T 1} \approx \frac{L_{O}}{2 c}\left[\left(\frac{L_{P}}{\lambda_{2}}\right)^{2}-\left(\frac{L_{P}}{\lambda_{1}}\right)^{2}\right]=1.881 \times 10^{-20} s .
$$

Substituting observed data and $L=\hat{L}_{O}=N L_{O}=\left(\lambda / L_{P}\right) L_{O}$ into (5.8) yields:

$$
\Delta t_{T 2} \approx \frac{L_{O}}{2 c}\left[N_{2}\left(\frac{L_{P}}{\lambda_{2}}\right)^{2}-N_{1}\left(\frac{L_{P}}{\lambda_{1}}\right)^{2}\right]=\frac{L_{O}}{2 c}\left[\frac{L_{P}}{\lambda_{2}}-\frac{L_{P}}{\lambda_{1}}\right]=0.047 \mathrm{~s} .
$$

$\hat{L}_{O}$ is the long path of $L_{O}, N_{1}=\lambda_{1} / L_{P}$ and $N_{2}=\lambda_{2} / L_{P}$ are converting factors for $\lambda_{1}$ and $\lambda_{2}$, respectively. The dispersion equation corresponding to (5.9b) according to some other theories is:

$$
v(f)=c \sqrt{1-\left(L_{P} / \lambda\right)}=c \sqrt{1-\left(f L_{P} / c\right)} .
$$

(5.7) and (5.10) can be expressed as one equation:

$$
v(f)=c \sqrt{1-\left(L_{P} / \lambda\right)^{n}}=c \sqrt{1-\left(f L_{P} / c\right)^{n}} ; n=1, \quad n=2 .
$$

In which, $n=1$ is for (5.10) and $n=2$ is for (5.7).

Superficially, the observed data seem to favor the result of (5.9b) and $n=1$ for (5.11). Actually it is not true. After extensive analysis, the authors of $[6,7,8]$ concluded: “... even our most conservative limit greatly reduces the parameter space for $n=1$ models. ... makes such theories highly implausible (models with $n>1$ are not significantly constrained by our results)."

The observation data from GRB090510 neither confirm nor reject dispersion equation (5.7). In fact, for the distance of $L_{O} \sim 7.3 \times 10^{9} l y$, to verify (5.7) directly requires the high energy $\gamma$-ray burst with energy level around $E_{2} \sim 10^{20} \mathrm{eV}$, which is a very rare event.

Quantum mechanics supports non-locality. For a pair of entangled photons separated by an extremely long distance, their quantum states keep coherent. Measure one photon's polarization, the other one "instantaneously" change its polarization accordingly. Einstein called it: "Spooky action at a distance."

SQS theory does not support non-locality. For a pair of entangled photons, SQS theory provides the following understanding and explanation.

1. There is a real physical link between entangled photons.

They are linked by the long path. In case of entanglement, the long path shows up from hiding with energy extracting from entangled photons.

2. The transmission of information and interaction between two entangled photons does not occur instantaneously, instead, it takes time. Even though the time interval is extremely short, but it is not zero. For ordinary photons, the long path is folded to form photon's wave pattern, the stationary observer only sees the short path with photon speed of $v \approx c$ given by (5.7a). For a pair of entangled photons, the long path shows up serving as the link. A stationary observer now sees the long path and superluminal speed. The speed of signal transmitting along the long path between two entangled photons is

$$
\hat{v}=N v \approx N c=\left[\frac{\lambda}{L_{P}}\right] c>>c .
$$

For visible light with wavelength $\lambda \sim 10^{-7} \mathrm{~m}$, according to (5.12), $\hat{v} \approx N c \sim 10^{28} c$. This is why territorial entanglement experimenters found that the interaction seems instantaneous. Actually it is not. The interaction between entangled photons is carried by a signal transmitting alone the long path with superluminal speed of (5.12). Recently, Salart et al report their testing results: the speed exceeds $10^{4} c$ [9]. Indeed, it is superluminal.

3. In the entanglement system, two entangled photons and the link connecting them have the same wavelength to keep the system coherent.

Entanglement provides a rare opportunity to peep at the long path. It is worthwhile to take a close look.

According to (4.6) of Lemma 4.1 based on the Random Walk Theorem, the relations of photon wavelength $\lambda$, long path wavelength $\hat{\lambda}$, converting factor $N$ and Planck length $L_{P}$ are:

$$
\begin{aligned}
& \lambda=N L_{P}, N=\lambda / L_{P}, \\
& \hat{\lambda}=N \lambda=N^{2} L_{P}=\lambda^{2} / L_{P} .
\end{aligned}
$$

The relations given by (5.13) serve as the guideline to deal with photons entanglement.

Postulation 5.1: For a pair of entangled photons, the entanglement process must satisfy energy conservation law and (5.13) relations. Under these conditions, a pair of entangled photons' original wavelength $\lambda_{0}$ changes to $\lambda>\lambda_{0}$ and the original long path wavelength $\hat{\lambda}_{0}$ changes to $\hat{\lambda}>\hat{\lambda}_{0}$ according to the following formulas:

$$
\begin{aligned}
& L=2 N_{\text {link }} \lambda, \\
& d=L / 2=N_{\text {link }} \lambda, N_{\text {link }}=d / \lambda,
\end{aligned}
$$




$$
\begin{aligned}
& N=\lambda / L_{P}, \\
& \hat{\lambda}=N \lambda=N^{2} L_{P}=\lambda^{2} / L_{P} .
\end{aligned}
$$

Explanation: The distance between two entangled photons is $d$. The link has two tracks, one track for one direction and the other for opposite direction. The total length of two tracks is $L=2 d$. According to SQS theory, photon's geometrical model is a closed loop with loop length of $2 L_{P}$. In the entanglement system, two entangled photons and the link connecting them share a common loop. The link's double-track structure is necessary to close the loop. $N=\lambda / L_{P}$ is converting factor for photons with wavelength $\lambda, N_{\text {link }}=d / \lambda$ is the number of wavelengths in one track. Conservation of energy requires total energy for entanglement system kept constant:

$$
\begin{aligned}
& \frac{2 h c}{\lambda_{0}}=\frac{2 h c}{\lambda}+\frac{N_{\text {link }}}{N+N_{\text {link }}} \frac{2 h c}{\lambda}, \\
& \frac{1}{\lambda_{0}}=\frac{1}{\lambda}+\frac{1}{1+N / N_{\text {link }}} \frac{1}{\lambda} .
\end{aligned}
$$

In which, $h$ is Planck constant. The term on (5.15a) left side is the energy of two photons with original wavelength $\lambda_{0}$. On (5.15a) right side, the first term is the energy of two photons with elongated wavelength $\lambda>\lambda_{0}$, the second term is the energy extracted from two photons to build the link. Substituting $N_{\text {link }}$ and $N$ from (5.14) into (5.15b) yields the formula to determine the elongated wavelength $\lambda$ :

$$
\frac{\lambda}{\lambda_{0}}=1+\frac{1}{1+\frac{\lambda^{2}}{L_{P} d}}=1+\frac{1}{1+\frac{\hat{\lambda}}{d}}
$$

A 16-digit numerical calculation is used to solve (5.16)

\begin{tabular}{|c|c|c|c|c|c|c|}
\hline $\begin{array}{c}d \\
(w)\end{array}$ & \multicolumn{2}{|c|}{$\begin{array}{c}\text { Wavelength change ratio } \\
\qquad / / \lambda_{6}\end{array}$} & \multicolumn{2}{|c|}{$\begin{array}{l}\text { Long path wavelength } \\
\qquad(\mathrm{m})\end{array}$} & \multicolumn{2}{|r|}{$d / \hat{k}$} \\
\hline $1 \times 10^{2}$ & \multicolumn{2}{|c|}{$1+1.11076512 \times 10^{-17}$} & \multicolumn{2}{|c|}{$6.18716164 \times 10^{51}$} & \multicolumn{2}{|c|}{$1.61625 \times 10^{-11}$} \\
\hline $1 \times 10^{2}$ & \multicolumn{2}{|c|}{1.00000016} & \multicolumn{2}{|c|}{$6.18710364 \times 10^{2}$} & \multicolumn{2}{|c|}{0,00000016} \\
\hline $1 \times 10^{21}$ & \multicolumn{2}{|l|}{1.00000162} & \multicolumn{2}{|c|}{$6.18718164 \times 10^{\text {II }}$} & \multicolumn{2}{|c|}{0.00000162} \\
\hline $1 \times 10^{7}$ & \multicolumn{2}{|l|}{1.00001616} & \multicolumn{2}{|c|}{$6.18736163 \times 10^{21}$} & \multicolumn{2}{|c|}{0.00001616} \\
\hline $1 \times 10^{2}$ & \multicolumn{2}{|l|}{1.00016155} & \multicolumn{2}{|c|}{$6.18016083 \times 10^{21}$} & \multicolumn{2}{|c|}{0.00016157} \\
\hline $1 \times 10^{3}$ & \multicolumn{2}{|l|}{1.00160847} & \multicolumn{2}{|c|}{$6.2070814 \times 10^{21}$} & \multicolumn{2}{|c|}{0.00161106} \\
\hline $1 \times 10^{-7}$ & \multicolumn{2}{|l|}{1.01543303} & \multicolumn{2}{|c|}{$6.3796036 \times 10^{21}$} & \multicolumn{2}{|c|}{0.01567494} \\
\hline $1 \times 10^{2}$ & \multicolumn{2}{|l|}{1.11504048} & \multicolumn{2}{|c|}{$7.69259153 \times 10^{21}$} & \multicolumn{2}{|c|}{0.12995519} \\
\hline $1 \times 10^{3}$ & \multicolumn{2}{|l|}{1.43852913} & \multicolumn{2}{|c|}{$1.28035023 \times 10^{3}$} & \multicolumn{2}{|c|}{0.7810363} \\
\hline \multicolumn{2}{|c|}{$\begin{array}{l}d=1.39211137 \times 10^{3} \mathrm{~m} \\
=1471494 \times 10^{2} \text { Lighres }\end{array}$} & \multicolumn{2}{|c|}{$\lambda / \lambda_{\mathrm{a}}=1+\frac{1}{2}$} & \multicolumn{2}{|c|}{$\begin{array}{l}\lambda=1.5 i_{3}=1.5 \times 10^{3} \cdot \mathrm{w} \\
j=d=1.39211137 \times 10^{3} \mathrm{~m}\end{array}$} & $\begin{array}{l}d(\hat{\lambda}=1 \\
d / \hat{\lambda}_{3}=2.25\end{array}$ \\
\hline
\end{tabular}
for $\lambda$ as a function of $d$ for $\lambda_{0}=1 \mathrm{~mm}=10^{-3} \mathrm{~m}$. The results are listed in Table 5.1 .

Table 5.1: Entangled Photons Wavelength $\lambda(d)$ as a Function of $d$ for $\lambda_{0}=10^{-3} \mathrm{~m}$

The data listed in Table 5.1 show some interesting features.

1. Maximum entanglement distance: Solve (5.16) for $d$ :

$$
d=\frac{\lambda-\lambda_{0}}{2 \lambda_{0}-\lambda} \hat{\lambda}=\frac{\lambda-\lambda_{0}}{2 \lambda_{0}-\lambda}\left(\frac{\lambda^{2}}{L_{P}}\right) .
$$

It shows that, the distance $d$ between two entangled photons increases with increasing wavelength $\lambda$. At the wavelength $\lambda=2 \lambda_{0}$, the distance $d \rightarrow \infty$. It seems no limitation for $d$. But that is not the case. Because another requirement is involved: The link as an integrated part of entanglement system must have the same wavelength of two photons. Otherwise, there is no coherency. In this case, $\lambda=2 \lambda_{0}$ corresponds to $h f=\frac{1}{2} h f_{0}$. A half

$(1-1 / 2=1 / 2)$ of each photon's energy is extracted out to build the link. According to SQS theory, photon's model is a closed loop with loop length of $2 L_{P}$, which corresponds to two wavelengths and two long path wavelengths inside the photon to build its wave pattern. The half energy extracted from two entangled photons is only sufficient to provide two wavelengths and two long path wavelengths for the link. Under such circumstance, the only way to build the link with infinite length is to infinitively elongate the long path wavelength as well as the wavelength in the link, which make them very different from two entangled photons'. It is prohibited by violating coherency requirement. So the entanglement distance $d$ does have its limit. In fact, only one case satisfies both requirements: energy conservation and quantum coherency. The unique case is $d=\hat{\lambda}$. According to (5.16), $d=\hat{\lambda}$ yields $\lambda=1.5 \lambda_{0}=\left(1+\frac{1}{2}\right) \lambda_{0}=\frac{3}{2} \lambda_{0}$ corresponding to $h f=\frac{2}{3} h f_{0}$. A third $(1-2 / 3=1 / 3)$ of each photon's energy is extracted out to build the link. The total energy is just right to make the original two wavelengths and two long path wavelengths in each photon becoming three wavelengths and three long path wavelengths for the entangled system, in which two are kept for each photon itself and one extracted out to build one track with length $d=\hat{\lambda}$. In this way, both requirements are satisfied and self-consistent. Hence, there is a maximum distance $d_{\max }$ between two photons to keep entangled, which is determined by (5.17) with $\lambda=\frac{3}{2} \lambda_{0}=1.5 \lambda_{0}$ :

$d_{\max }=\left[\frac{\lambda-\lambda_{0}}{2 \lambda_{0}-\lambda} \hat{\lambda}\right]_{\lambda=1.5 \lambda_{0}}=\hat{\lambda}=\left(\frac{\lambda^{2}}{L_{P}}\right)=\left(\frac{3}{2}\right)^{2} \hat{\lambda}_{0}=\left(2+\frac{1}{4}\right) \lambda_{0}=2.25 \hat{\lambda}_{0} \cdot$

When $d>d_{\text {max }}$, the link breaks down and two entangled photons are automatically de-coherent even without any external interference. The data for $\lambda_{0}=10^{-3} \mathrm{~m}$ are listed in the bottom row of Table 5.1.

2. Energy balance: At the maximum distance $d=d_{\max }$, $\lambda / \lambda_{0}=1+\frac{1}{2}=\frac{3}{2}$ corresponds to $h f=\frac{2}{3} h f_{0}$. It indicates 
that, one third of each photon's energy is extracted out to build the link. Because the double-track link extracts energy from tow photons, $2\left(1-\frac{2}{3}\right) h f_{0}=\frac{2}{3} h f_{0}$, the link has the same energy of each photon's energy. The link acts like another photon with the some energy and the same wavelength of each entangled photon. In other words, at the maximum entanglement distance $d=d_{\max }$, the entanglement system is seemingly made of three photons, in which two are entangled real photons and the third one makes the link to connect them. It serves as evidence that, the link is a physics substance with energy. At shorter distance $d<d_{\max }$, the extracted energy gradually increases to build the link and to push the link for expansion. At distance beyond maximum distance, $d>d_{\max }$, the entanglement system breaks automatically, because it lacks sufficient energy to maintain the over expanded link. In this way, both requirements are satisfied and everything is consistent. The key is to recognize the long path serving as the physics link for entanglement.

3. Entanglement red shift: The wavelength $\lambda(d)$ increases with increasing distance $d$. The red shift is caused by the fact that, a portion of the entangled photons energy is extracted out to build the physics link. It is the energy conservation law in action. According to (5.16), the red shift continuously increases with increasing distance. The maximum red shifted wavelength at $d=d_{\max }$ is.

$$
\lambda_{\max }=\left(1+\frac{1}{2}\right) \lambda_{0}=1.5 \lambda_{0} .
$$

The entanglement red shift happens gradually. For a pair of photons separated by distance much shorter than the maximum distance, the tiny red shift is very difficult to detect. As listed in Table 5.1, for a pair of photons with wavelength $\lambda=10^{-3} \mathrm{~m}$ at distance $d=10^{6} \mathrm{~m}$, the relative red shift is only $\sim 10^{-16}$. For entangled photons with visible light wavelength $\lambda \sim 10^{-7} \mathrm{~m}$, the red shift is many orders of magnitude less than $\sim 10^{-16}$. This is why entanglement experiments with limited distance haven't found the red shift effect yet. But it is out there.

Otherwise, the physics link energy has nowhere to come from.

4. De-coherent blue shift: When a pair of entangled photons is de-coherent, the outcomes depend on the decoherence location. If the location is right at the middle $d / 2$, the physics link is broken evenly and each photon gets back equal share of the link energy to resume original wavelength corresponding to a blue shift. According to (5.16), the two de-coherent photon's wavelength is shortened from $\lambda$ to $\lambda_{0}<\lambda$ causing the blue shift.

$$
\frac{\lambda_{0}}{\lambda}=\frac{1+\frac{\lambda^{2}}{L_{P} d}}{2+\frac{\lambda^{2}}{L_{P} d}}=\frac{1+\frac{\hat{\lambda}}{d}}{2+\frac{\hat{\lambda}}{d}}
$$

At $d=d_{\max }=\hat{\lambda}$, the blue shift is:

$$
\left[\frac{\lambda_{0}}{\lambda}\right]_{d=\hat{\lambda}}=\frac{1+1}{2+1}=\frac{2}{3} .
$$

In terms of frequency, the blue shift is:

$$
\left[\frac{f_{0}}{f}\right]_{d=\hat{\lambda}}=\left[\frac{\lambda}{\lambda_{0}}\right]_{d=\hat{\lambda}}=\frac{3}{2}=1.5 .
$$

If the de-coherent location is at the close vicinity of one photon, this one does not gain energy to change its wavelength and shows no blue shift. The other one gets all energy of the link and has the maximum de-coherent blue shift to the wavelength $\lambda^{\prime}{ }_{0}$ shorter than the original wavelength $\lambda_{0}$. According to energy balance of (5.15):

$$
\frac{h c}{\lambda^{\prime}{ }_{0}}=\frac{h c}{\lambda}+\frac{N_{\text {link }}}{N+N_{\text {link }}} \frac{2 h c}{\lambda}=\frac{h c}{\lambda}+\frac{2 h c}{\left[1+\frac{\lambda^{2}}{L_{P} d}\right] \lambda}
$$

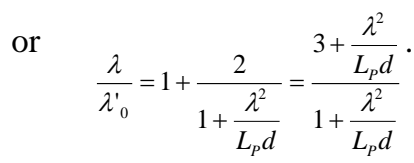

For photon at distance $d$ from de-coherent location, its wavelength is shortened to $\lambda_{0}^{\prime}$ :

$$
\frac{\lambda_{0}^{\prime}}{\lambda}=\frac{1+\frac{\lambda^{2}}{L_{P} d}}{3+\frac{\lambda^{2}}{L_{P} d}}=\frac{1+\frac{\hat{\lambda}}{d}}{3+\frac{\hat{\lambda}}{d}} .
$$

For de-coherence at locations between $d / 2$ and $d$, the blue shift for the far away one is between the two values given by (5.20) and (5.23). At the maximum entanglement distance $d=d_{\max }=\hat{\lambda}$, according to (5.23), the maximum blue shift in terms of frequency is:

$$
\frac{f_{0, \max }^{\prime}}{f}=\left[\frac{\lambda}{\lambda^{\prime}{ }_{0}}\right]_{d=\hat{\lambda}}=\frac{3+1}{1+1}=2 .
$$

$f^{\prime}{ }_{0, \max }$ is the blue shifted frequency of the photon at the distance $d=d_{\max }$ from the de-coherence location. For decoherence at locations between $d / 2$ and $d$, the blue shift is between the two values given by (5.22) and (5.24). The de-coherent blue shift happens suddenly with a large frequency increase, which is relatively easy to detect, but the problem is the uncertainty of de-coherent timing. The above analyses show that, entangled photons are connected by a physics link, interactions and information between them are transmitted with superluminal speed $\hat{v}=N v \approx N c=\left(\lambda / L_{P}\right) c>>c$. It is much faster than $c$ but not infinite. From SQS theory standpoint, the physics link and the non-infinite superluminal speed serve as the 


\section{Z. Y. SHEN}

foundation for locality. After all, Einstein was right: No spooky action at a distance.

Conclusion 5.1: Entanglement has limited distance. The distance between entangled particles cannot be infinitely long.

Proof: Conclusion 5.1 is not based on Postulation 5.1. It is based on basic principle. Consider the opposite. If a pair of entangled particles is separated by infinite distance, the physics link between them must have nonzero energy density, energy per unite length. Then the total energy of the link equals to infinity. That is impossible, the opposite must be true.

QED

Explanation: According to Conclusion 5.1, the maximum entanglement distance $d_{\max }$ given by (5.18) serves only as an upper limit. Whether a pair of entangled photons can be separated up to $d_{\max }$ or not, it also depends on other factors. For entangled photons with very long wavelength, their quantum has very low energy. As the link stretched very long, the energy density becomes lower than the vacuum quantum noise. The link could be broken causing de-coherence with distance shorter than the maximum distance $d_{\max }$. The other factor is external interferences causing do-coherence, which is well known and understood.

According to SQS theory, photons travel along the short path with speed of $v \approx c$ with dispersion given by (5.7); the signals between entangled photons transmit along the long path with superluminal speed $\hat{v}=N v \approx N c$ of (5.12). These are the conclusions derived from converting rules introduced in Section 4. The key concept is the long path, which is defined by (4.12) based the converting factor and originated from the Random Walk Theorem. If the existence of long path is confirmed, so are these conclusions as well as its foundation.

If photon's long-path is confirmed, the non-locality of quantum mechanics must be abandoned. Moreover, long path is based on converting rule. If it is confirmed meaning photon does have dispersion. Special relativity should been revised as well.

The dispersion equation of (5.7) is not the final version. In Section 25, a generalized dispersion equations will be introduced, in which the Planck length in (5.7) is replaced by longer characteristic lengths. It makes easier for experimental verifications.

In this section, special relativity is revised. For most practical cases, the revision for photon's speed in vacuum is extremely small, but its impact are huge such as the introduction of superluminal speed $\hat{v}=N v \approx N c=\left(\lambda / L_{P}\right) c>>c$. Is it inevitable? Let's face the reality: Experiment carried out by Salart et al [9] proved that, the speed of signal transmitting between two entangled photons exceeds $10000 c$. It leaves us only two choices: One is to introduce non-infinite superluminal speed as we did in this section; the other is to accept "spooky action at a distance". Obviously, the second choice is much harder for physicists to swallow. Therefore, the superluminal speed is indeed inevitable. Besides, the superluminal speed introduced in this section is within special relativity framework. The key concept is that, the long path and the superluminal speed are hidden, they only show up in very special cases such as entanglement.

The converting factor seemingly has two different meanings: One is from random walk; the other is from Lorentz transformation. Actually, they are duality. Such duality is common in physics. One well known example is wave-particle duality. The mechanism of the random walkLorentz duality is not clear, which is a topic for further work; and so it the mechanism of the wave-particle duality. In fact, the long path concept digs into the mechanism of waveparticle duality down to a deeper level: The vacuons' movement builds the wave-pattern step by step.

\section{Section 6: Electron.}

$$
\begin{aligned}
& \text { Define the } D S \text {-function } D S(x)=\frac{1}{2}\{[S(x)-1]-[1-S(0.5-x)]\} \text { as: } \\
& \qquad D S(x)=\frac{1}{2}\left[\sum_{x_{i}=-\infty}^{\infty} e^{-\pi\left(x_{i}-x\right)^{2}}+\sum_{x_{i}=-\infty}^{\infty} e^{-\pi\left(x_{i}-0.5+x\right)^{2}}\right]-1 \cdot \quad \text { (6.1) }
\end{aligned}
$$

According to its definition, $D S(x)$ is symmetrical with respect to $x=0.25$ in region $[0,0.5]$ :

$$
D S(0.5-x)=D S(x) ; 0 \leq x \leq 0.5 \text {. }
$$

$D S(x)$ satisfies the periodic condition:

$$
D S(x+0.5)=D(x) \text {. }
$$

Fig. 6.1 shows $D S(x)$ versus $x$ curve in region [0,0.25]. The other part in region $[0.25,0.5]$ is the mirror image of this part with respect to $x=0.25$.

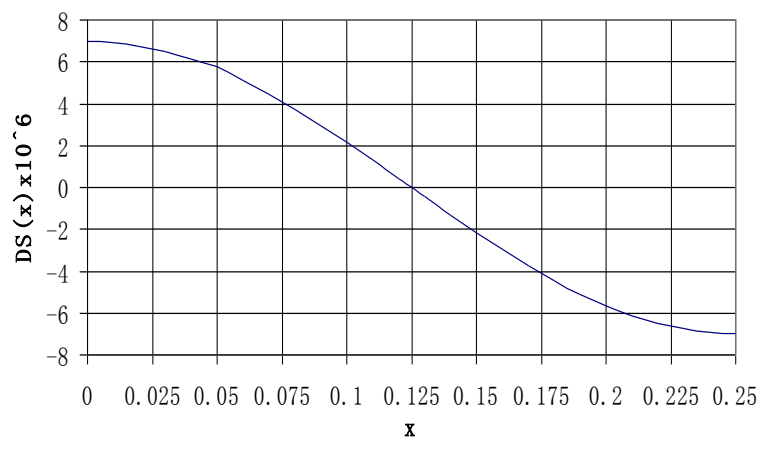

Fig. 6.1 $D S(x)$ versus $x$ curve in region $[0,0.25]$.

Definition 6.1: Define the $D S$-equation as a member of the $S$ equation family:

$D S(x)=\frac{1}{2}\left[\sum_{x_{i}=-\infty}^{\infty} e^{-\pi\left(x_{i}-x\right)^{2}}+\sum_{x_{i}=-\infty}^{\infty} e^{-\pi\left(x_{i}-0.5+x\right)^{2}}\right]-1=0$ 
In region $[0,0.5], D S(x)=0$ has two roots: $x_{1}=0.125$, $x_{2}=0.375$. According to (3.12), the path length of probability transportation from $x_{1}$ to $x_{2}$ via complex $\dot{x}$ plane is:

$$
l_{e}=2\left(x_{2}-x_{1}\right) L_{P}=0.5 L_{P} .
$$

In (6.5), $L_{P}$ appears as the unit length hidden in (3.12). The reason for the factor 2 in (6.5) has been explained mathematically in Section 3. Physically, according to the spinor theory proposed by Pauli, electron as Dirac type fermion has two components, which move in the zigzagging path called "zitterbewegung" phenomenon [10].

According to (3.14), the loop length corresponding to path length for $x_{1}$ and $x_{2}$ is:

$$
L_{e}=2 l_{e}=L_{P} \text {. }
$$

$D S(x)=0$ means that the probabilities compensation between excess and deficit is exact. The oscillation between $x_{1}=0.125$ and $x_{2}=0.375$ does not decay, which corresponds to a stable fermion. Electron is the only free standing stable elementary fermion, which neither decays nor oscillates with other particles. It is the most probable candidate for this particle.

Assuming the resonant condition for the lowest excitation in a closed loop with loop length $L_{e}$ is:

$$
L_{e}=\lambda_{C}=\frac{h}{\hat{M} c} .
$$

In which, $\hat{M}$ and $\lambda_{C}$ are the mass and Compton wavelength of the particle, respectively. Substituting (6.6) into (6.7) and solving for the mass of this particle yield:

$$
\hat{M}=\frac{h}{L_{P} c}=1.367498 \times 10^{-7} \mathrm{~kg} .
$$

It is recognized that $\hat{M}=M_{P}$ is the Planck mass. According to 2010 PDG data, the mass of electron is:

$$
M_{e}=9.10938215(45) \times 10^{-31} \mathrm{~kg} \text {. }
$$

$\hat{M}$ is $\sim 10^{23}$ time heavier than $M_{e}$, which is one of the hierarchy problems in physics. It can be resolved by applying conversion rule. According to (4.10), the converting factor for electron is:

$$
N_{e}=\frac{\lambda_{e, C}}{L_{P}}=\frac{h}{M_{e} L_{P} c} .
$$

The mass of (6.8) after conversion is:

$$
M=\frac{\hat{M}}{N_{e}}=\frac{h /\left(L_{P} c\right)}{h /\left(M_{e} L_{P} c\right)}=M_{e} .
$$

The particle is identified as electron. Of cause, this is a trivial case, but it serves as the basic reference for nontrivial cases given later.

The reason for miscalculating the mass with $\sim 10^{23}$ times discrepancy is mistakenly using Compton wavelength $\lambda_{C}$ in
(6.7). In reality, the resonant condition in Planck scale closed loop should be:

$$
\begin{aligned}
& L=m \lambda_{P} ; m=1,2,3, \cdots . \\
& \lambda_{P}=\lambda_{C} / N=L_{P} .
\end{aligned}
$$

$N$ is the converting factor for that particle. $\lambda_{P}=L_{P}$ is defined as the Planck wavelength. The number $m$ in (6.12) is related to the spin of particle. For electron, $m=1$ corresponds to spin $h / 2$. In general, the spin of a particle equals to $m h / 2$. Odd $m$ corresponds to fermions, and even $m$ corresponds to bosons. $m$ is the first numerical parameter introduced by SQS theory.

Electron as a Dirac type fermion, its trajectory has two types of internal cyclic movements, one contributes to its spin and the other one does not. In (6.12), the loop with length $L=m \lambda_{P}$ is the main loop celled loop-1 and the other loop is loop-2. The dual loop structure of electron corresponds to two components. The dual loop structure is not only for electron but also for other Dirac type fermions, which will be discussed in later sections.

The basic parameters for electron are listed below. Mass:

$M_{e}=9.10938215(45) \times 10^{-31} \mathrm{~kg}=0.510998910(13) \mathrm{MeV} / \mathrm{c}^{2}$

Compton wavelength:

$$
\lambda_{e C}=h /\left(M_{e} c\right)=2.42631022 \times 10^{-12} \mathrm{~m} .
$$

Converting factor:

$$
N_{e}=M_{P} / M_{e}=1.501197 \times 10^{23} .
$$

Loop parameters:

$$
x_{1}=0.125, x_{2}=0.375, l_{e}=0.5, L_{e}=1 .
$$

At $x_{1}=0.125, x_{2}=0.375, S\left(x_{1}\right)-1=1-S\left(x_{2}\right)$, the probability compensation is exact corresponding to electron as a stable particle. At other locations, the probability compensation is not exact corresponding to unstable particles.

Electron is unique. Its mass servers as basic unit used for calculating other fermion's mass. The general formula to determine $\left(x_{2}-x_{1}\right)$ for fermion with mass $M$ is:

$$
\frac{4\left(x_{2}-x_{1}\right)}{\left[4\left(x_{2}-x_{1}\right)\right]_{e}}=\frac{4\left(x_{2}-x_{1}\right)}{1}=4\left(x_{2}-x_{1}\right)=\frac{M_{e}}{M} .
$$

The reason for (6.18) is that, loop length $L=4\left(x_{2}-x_{1}\right)$ is inversely proportional to mass.

According to (6.18), the values of $x_{1}$ and $x_{2}$ of the fermion with mass $M$ are:

$$
\begin{aligned}
& x_{1}=0.25-\frac{x_{2}-x_{1}}{2}=0.25-\left(\frac{M_{e}}{8 M}\right), \\
& x_{2}=0.5-x_{1} .
\end{aligned}
$$

Along the $x$-axis, according to (2.19) and (2.20), the region between two special points $x_{c}$ and $x_{d}$ is: $\left[x_{c}, x_{d}\right]=[0.24998715627302645,0.25001284372697355]$. 


\section{Z. Y. SHEN}

Inside region $\left[x_{c}, x_{d}\right]$, both $S\left(x_{1}\right)<1$ and $S\left(x_{2}\right)<1$, probability transportation for unitarity does not make sense.

Rule 6.1: The special points $x_{c}$ sets a mass upper limit $M_{\text {Max }}$ for stand alone fermions:

$$
M_{\text {Max }}=\frac{0.125}{0.25-x_{c}} M_{e}=4.97323432 \mathrm{GeV} / \mathrm{c}^{2} \text {. }
$$

A fermion with mass heavier than $M_{\text {Max }}$ cannot stand alone. It must associate with an anti-fermion as companion to form a boson state.

Rule 6.2: The $x_{1}$ and $x_{2}$ inside region $\left[x_{c}, x_{d}\right]$ belong to gauge bosons with spin $\hbar$.

Rule 6.3: The region $\left[-x_{c}^{\prime}, x_{c}^{\prime}\right]$ belongs to scalar bosons with spin 0. Point $x_{c}^{\prime}$ is defined as:

$$
\begin{aligned}
& x_{c}^{\prime}=0.25-x_{c}=0.25-0.24998715627302645 . \\
& =1.284372697355 \times 10^{-5}
\end{aligned}
$$

The meaning and the effectiveness of these rules will be given in later sections.

This section serves as the introduction of electron for SQS theory. It will be followed by later sections in much more details.

\section{Section 7: $D S$-Function on $k$-Plane as Particles Spectrum}

In Section 6, the $D S(x)$ as a function of $x$ is defined as:

$$
D S(x)=\frac{1}{2}\left[\sum_{x_{i}=-\infty}^{\infty} e^{-\pi\left(x_{i}-x\right)^{2}}+\sum_{x_{i}=-\infty}^{\infty} e^{-\pi\left(x_{i}-0.5+x\right)^{2}}\right]-1 .
$$

Taking Fourier transformation to convert $D S(x)$ into $D S_{k}(k)$ on complex $k$-plane yields:

$$
\begin{aligned}
& D S_{k}(k)=\frac{1}{2 \pi} \int_{-\infty}^{\infty} D S(x) e^{i k x} d x=\frac{1}{2 \pi} \int_{-\infty}^{\infty}\left\{\frac{1}{2} \sum_{j=-\infty}^{\infty}\left[e^{-\pi(j-x)^{2}}+e^{-\pi(j-0.5+x)^{2}}\right]-1\right\} e^{i k x} d x \\
& =\frac{1}{4 \pi} e^{-\frac{k^{2}}{4 \pi}}\left\{\sum_{j=-\infty}^{\infty}\left[e^{i j k}+e^{-i(j-0.5) k}\right]\right\}-\delta(k) .
\end{aligned}
$$

Summation index $x_{i}$ in (6.1) is replaced by index $j$ in (7.1) for simplicity. In (7.1), $k$ is the wave-number on complex $k$ plane. Normalize $k$ with respect to $2 \pi$ as:

$$
k=k /(2 \pi) \text {. }
$$

In terms of $k$, the $D S_{k}(k)$ function (7.1) becomes:

$$
D S_{k}(k)=\frac{1}{4 \pi} e^{-\pi k^{2}}\left\{\sum_{j=-\infty}^{\infty}\left[e^{i 2 \pi j k}+e^{-i 2 \pi(j-0.5) k}\right]\right\}-\delta(k) \text {. }
$$

$D S_{k}(k)$ and $D S_{k}(k)$ are the $D S$-functions on the complex $k$ plane and $k$-plane, respectively. Because $x$ and $x_{i}$ in (6.1) are normalized with respect to Planck length $L_{P}$ as numbers.
In the Fourier transformation process, $x k$ and $x_{i} k$ are also numbers, so $k$ and $k$ are normalized with respect to $1 / L_{P}$.

Definition 7.1: The real part $k_{r}$ and imaginary part $k_{i}$ of $k$ are related to particle's complex mass as:

$$
k=k_{r}+i k_{i}=\frac{M}{M_{e}}+i \frac{M_{i}}{M_{e}}
$$

$M$ and $M_{i}$ are the real mass and the imaginary mass of the particle, respectively. $M_{e}$ is electron's mass serving as the basic mass unit.

Explanation: Definition 7.1 is based on the concept that, $k$ plane serves as the spectrum of particles. According to (7.4), particle's mass $M$ and its decay time $t$ are:

$$
\begin{aligned}
& M=k_{r} M_{e}, \\
& t=\frac{\lambda_{e C}}{c k_{i}} .
\end{aligned}
$$

In which, $M_{e}$ and $\lambda_{e C}$ are the mass and Compton wavelength of electron, respectively.

Formula (7.5a) is derived from real part of (7.4). Formula (7.5b) is derived from imaginary part of (7.4) as:

$$
k_{i}=\frac{M_{i}}{M_{e}}=\frac{\lambda_{e C} M_{i} c}{h}=\frac{\lambda_{e C} E_{i}}{h c}=\frac{\lambda_{e C} h f_{i}}{h c}=\frac{\lambda_{e C}}{c t} .
$$

$E_{i}$ and $f_{i}$ are imaginary part of energy and frequency of the particle, respectively.

Numerical calculations of $D S_{k}(k)$ found the following results.

$1,\left|D S_{k}(k=1)\right|=0, k=1$ is a root of $\left|D S_{k}(k)\right|=0$. According

to $(7.5 \mathrm{a})$ and $(7.5 \mathrm{~b})$ :

$$
M=M_{e}, t \rightarrow \infty .
$$

$\left|D S_{k}(k)\right|=0$ at $k=1$ corresponds to electron as a fermion.

2, $\left|D S_{k}(k=0)\right| \rightarrow \infty, k=0$ is a pole of $\left|D S_{k}(k)\right|$. According to $(7.5 \mathrm{a})$ and $(7.5 \mathrm{~b})$ :

$$
M=0, t \rightarrow \infty \text {. }
$$

$\left|D S_{k}(k=0)\right| \rightarrow \infty, k=0$ corresponds to photon as a boson.

Rule 7.1: In general, the local minimum of $\left|D S_{k}(k)\right|$ corresponds to a fermion, while the local maximum of $\left|D S_{k}(k)\right|$ corresponds to a boson. At the local minimum or local maximum of $\left|D S_{k}(k)\right|, k$ with real value corresponds to a stable particle, $k$ with complex value corresponds to an unstable particle.

Explanation: Rule 7.1 is the generalization of $\left|D S_{k}(k=1)\right|=0$ for electron as a fermion and $\left|D S_{k}(k=0)\right| \rightarrow \infty$ for photon as a boson.

Consider the factor $\frac{1}{4 \pi} e^{-\pi k^{2}}$ in (7.3): 


$$
\frac{1}{4 \pi} e^{-\pi k^{2}}=\frac{1}{4 \pi} e^{-\pi\left(k_{r}+i_{i}\right)^{2}}=\frac{1}{4 \pi} e^{-\pi\left(k_{r}^{2}-k_{i}^{2}\right)} e^{-2 i \pi k_{r} k_{i}}
$$

In (7.9), for $k_{r}>4$ and $k_{i}<<k_{r}, \frac{1}{4 \pi} e^{-\pi\left(k_{r}^{2}-k_{i}^{2}\right)}<10^{-22}$, which drastically suppresses the magnitudes of local minimum and local maximum of $\left|D S_{k}(k)\right|$ and makes numerical calculation difficult. In (7.3), the $\delta$-function $\delta(k)$ does not contribute except for $k=0$. Let's disregard the factor $e^{-\pi k^{2}} /(4 \pi)$ and drop the $\delta(k)$ term to define the simplified version of $D S_{k}(k)$ as:

$$
D S_{k}(k)=\sum_{j=-\infty}^{\infty}\left[e^{i 2 \pi j k}+e^{-i 2 \pi(j-0.5) k}\right] .
$$

In terms of the $k$ value at local minimum or local maximum of $\left|D S_{k}(k)\right|$, the error caused by simplification is evaluated in Appendix 3, which is negligible in most cases.

The simplified $D S_{k}(k)$ of (7.10) is taken for technical reasons. It does not mean ignoring the importance of the factor $e^{-\pi k^{2}} /(4 \pi)$ and the term $\delta(k)$ in the original $D S_{k}(k)$ of (7.3). In fact, the factor $e^{-\pi k^{2}} /(4 \pi)$ serves as the suppression factor for the original $D S_{k}(k)$ of (7.3). The suppression factor plays an important role in Section 15 for unifications. In addition, as the suppression factor value decreases to extremely low level, the magnitudes of the local minimums and local maximums are suppressed too much and no longer distinguishable from the background noise. This scenario may relate to the early universe with extremely high temperatures. The term $\delta(k)$ in the original $D S_{k}(k)$ of (7.3)

comes from the unitarity term " 1 " in $D S(x)$ of (6.1). In Section 9, $D S_{k}(k)$ is extended based on the extension of the $\delta(k)$ term. Then the extended version is Fourier transformed back to the complex $\dot{x}$-plane, a number of new things show up, which will be discussed in Section 9 .

$\left|D S_{k}(k)\right|$ serves as particles spectrum with fermions at local minimum and bosons at local maximum. Particle's mass and decay time can be calculated from $k=k_{r}+i k_{i}$ according to (7.5). The summation index $j$ in (7.10) must be truncated at integer $n$. The rules for truncation are:

For odd $n: D S_{k}(k)=\sum_{j=-(n-1) / 2}^{(n-1) / 2}\left[e^{i 2 j \pi k}+e^{-i(j-0.5) \pi k}\right]$,

For even $n: D S_{k}(k)=\sum_{j=-(n / 2-1)}^{n / 2}\left[e^{i 2 j \pi k}+e^{-i(j-0.5) \pi k}\right]$,

or $D S_{k}(k)=\sum_{j=-n / 2}^{n / 2-1}\left[e^{i 2 j \pi k}+e^{-i(j-0.5) \pi k}\right]$.

The numerical parameter $n$ assigned to particles is from the mass ratio:

$$
\frac{p}{n}=\frac{M}{M_{e}}=k_{r} .
$$

For $\left|D S_{k}(k)\right|$ serving as spectrum, the number $n$ for truncation in (7.12) must be integer, if the $n$-parameter in (7.12) is not an integer, multiplication is taken to convert it into an integer for the truncation in (7.11).

In (7.12), $n$ and $p$ are the second and third numerical parameters introduced after the first one of $m$ introduced in Section 6. For a particle, the set of three numerical parameters $m, n, p$ plays important roles for particles models and parameters, which will be explained in later sections.

As examples, (7.11) is used to calculate the parameters of muon and taon. The results of 16-digit numerical calculation are listed in Table 7.1. In which, the reason for taking the values of numerical parameters $m, n, p$ will be given in later

\begin{tabular}{|c|c|c|}
\hline Name of particle & Stucs & Taen \\
\hline$m$ & 18 & 42 \\
\hline (in) $\times$ mesituple & $\left(29 \frac{1}{4}\right) \times 4=117$ & 120 \\
\hline$R^{2}$ & 6048 & 417270 \\
\hline$\left|D S_{*}(k)\right|$ & $1.9951094704733 \times 10^{-11}$ & $1.13655267172921 \times 10^{2}$ \\
\hline t. & 206.76923076923077 & 3477,25 \\
\hline$k_{*}^{*}$ & $3.683739 \times 10^{-11}$ & $2.7850308715 \times 10^{-4}$ \\
\hline Calculated mass $\left(\mathrm{W} / \mathrm{C}\left(c^{2}\right)\right.$ & 105.658851544615 & 1776.8709397975 \\
\hline Mess $\left(3 d a k c^{2}\right)$ from 2010-PDG & $105.0583608 \pm 00000038$ & $177682=0.16$ \\
\hline Relative discrepancy of mass ${ }^{t+}$ & $4588 \times 10^{-4}$ & $1.742 \times 10^{-7}$ \\
\hline
\end{tabular}
sections.

Table 7.1: The Calculated Parameters of Muon and Taon

* The listed $k_{i}$ value corresponds to particle's lifetime.

** The relative discrepancy of mass is calculated with the medium value of 2010-PDG data.

For the truncated $\left|D S_{k}(k)\right|$ of (7.11), the locations of local minimums and local maximums depend on the value of $n$, which must be given beforehand. In other words, different $n$ values give different mass values for different particles. Fortunately, the $n$ value of a particle can be determined by other means. For instance, quarks' $n$ is selected from a set of prime numbers and it is tightly correlated to strong interactions. It can be determined within a narrow range and in many cases uniquely. The details will be given in later sections.

Look at the spectrum from another perspective, $\left|D S_{k}(k)\right|$ actually provides a dynamic spectrum for all particles. As the value of $n$-parameter increases, the locations of local maximums and minimums change accordingly corresponding to different particles. It is conceivable that, for the full range of $n$-parameter, $\left|D S_{k}(k)\right|$ serves as the spectrum of all

elementary particles. Whether it includes composite particles or not, which is an interesting open issue.

Using 16-digit numerical calculations found that, for a given value of $k_{r}$ such as $k_{r}=206.76923076923077$ for muon, there are a series of local minimums located at different values of $k_{i}$. Table 7.2 shows twenty three $k_{i}$ values for muon over a narrow range from $k_{i}=3.68348 \times 10^{-15}$ to 


\section{Z. Y. SHEN}

$k_{i}=3.68387 \times 10^{-15}$. There are 6 local minimums

corresponding to 6 possible decay times.

Table 7.3 shows $\left|D S_{k}(k)\right|$ profile as a function of $k_{i}$ over a broad range of $k_{i}$ alone $k_{r}=206.76923076923077$ line.

Table 7.2 Muon Decay Data in Narrow Range*

\begin{tabular}{|c|c|c|c|}
\hline$k_{2}$ & $\begin{array}{l}\text { Possible decay times } \\
(5)\end{array}$ & $\mid P S_{*}(k)$ & Notes \\
\hline $3.68348 \times 10^{-11}$ & & $1.99520905111587 \times 10^{-17}$ & \\
\hline $3.68349 \times 10^{-45}$ & $2.197182 \times 10^{-4}$ & $1.99517210965912 \times 10^{-4}$ & Iocal min \\
\hline $3.68353 \times 10^{-11}$ & & $1.99517393942542 \times 10^{-19}$ & \\
\hline $3.68357 \times 10^{-17}$ & & $199519606835691 \times 10^{-11}$ & \\
\hline $3.68358 \times 10^{-12}$ & $2.197129 \times 10^{-6}$ & $1.9951518104956 \times 10^{-11}$ & local min \\
\hline $3.68360 \times 10^{-12}$ & & $1.99520338799526 \times 10^{-10}$ & \\
\hline $3.68361 \times 10^{-16}$ & & $1.99517943019693 \times 10^{-71}$ & \\
\hline $3.68362 \times 10^{-2}$ & & $1.99515730132801 \times 10^{-11}$ & \\
\hline $3.68364 \times 10^{-24}$ & & $1.99511304359522 \times 10^{-4}$ & \\
\hline $3.68365 \times 10^{-12}$ & $2.197087 \times 10^{-1}$ & $199510572281382 \times 10^{\text {t1 }}$ & local min \\
\hline $3.68366 \times 10^{-12}$ & & $199513517240078 \times 10^{-4}$ & \\
\hline $3.68367 \times 10^{-25}$ & & $1.99512419288313 \times 10^{-21}$ & \\
\hline $3.68369 \times 10^{-12}$ & $2.197063 \times 10^{-7}$ & $19951094704733 \times 10^{-t}$ & Iocal min \\
\hline $3.68375 \times 10^{-11}$ & & $199515372853247 \times 10^{-10}$ & \\
\hline $3.68376 \times 10^{-21}$ & & $1.99513159950205 \times 10^{-4}$ & \\
\hline $3.68377 \times 10^{-16}$ & & $1.9951004704733 \times 10^{-11}$ & \\
\hline $3.68378 \times 10^{\text {-11 }}$ & & $1.99506521242083 \times 10^{-11}$ & \\
\hline $3.68380 \times 10^{-11}$ & $2.196998 \times 10^{-6}$ & $199503942634126 \times 10^{-4}$ & local min \\
\hline $3.68381 \times 10^{-25}$ & & $199506155540554 \times 10^{-4}$ & \\
\hline $3.68382 \times 10^{-21}$ & & $1.99510581353914 \times 10^{-41}$ & \\
\hline $3.08383 \times 10^{-11}$ & & $1.99512794260845 \times 10^{-14}$ & \\
\hline $3.68384 \times 10^{-12}$ & $2.196974 \times 10^{-4}$ & $1.99509474900511 \times 10^{-11}$ & local min \\
\hline $3.68387 \times 10^{-25}$ & & $1.99510206389579 \times 10^{-4}$ & \\
\hline
\end{tabular}

* The parameters $m, n, p$ and $k_{r}$ are the same as those listed in Table 7.1.

Table 7.3 $\left|D S_{k}(k)\right|$ over Broad Range for Muon*

\begin{tabular}{|c|c|c|c|}
\hline$h_{1}$ values & Decay times (s) & $|D S .(k)|$ base line valoes & Regions \\
\hline 0 & $x$ & $2.1268050972575 \times 10^{-11}$ & \multirow{6}{*}{$\begin{array}{l}\text { Region-I: } \\
\text { Thelaseline of } \\
\left|D S_{,}(t)\right| \text { is fat in the } \\
\text { region. }\end{array}$} \\
\hline $1 \times 10^{-2}=$ & $8.0933 \times 10^{2}$ & $2.1268050972575 \times 10^{-11}$ & \\
\hline $1 \times 10^{-2}$ & $8.0933 \times 10^{1}$ & $2.1208050972575 \times 10^{-24}$ & \\
\hline $1 \times 10^{-3}$ & 8.0933 & $2.1268050972575 \times 10^{-21}$ & \\
\hline $1 \times 10^{-3}$ & $8.0933 \times 10^{-4}$ & $2.1268050972575 \times 10^{-11}$ & \\
\hline $1 \times 10^{-2}$ & $8.0933 \times 10^{-2}$ & $2.1268050972575 \times 10^{-21}$ & \\
\hline $1 \times 10^{-111}$ & $8.0933 \times 10^{-5}$ & $2.12672631175312 \times 10^{-111}$ & \multirow{6}{*}{ 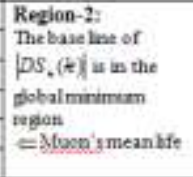 } \\
\hline $1 \times 10^{-57}$ & $8.0933 \times 10^{-4}$ & $2.1264633210293 \times 10^{-11}$ & \\
\hline $1 \times 10^{-2}$ & $8.0933 \times 10^{-4}$ & $2.12309300162024 \times 10^{-24}$ & \\
\hline $1 \times 10^{-11}$ & $8.0933 \times 10^{-4}$ & $2,09081707910007 \times 10^{-151}$ & \\
\hline $3.683739 \times 10^{111}$ & $2.197034 \times 10^{4 *}$ & $1.9951094704733 \times 10^{911}$ & \\
\hline $1 \times 10^{-14}$ & $8.0033 \times 10^{-2}$ & $1.774222482075355 \times 10^{-11}$ & \\
\hline $1 \times 10^{20}$ & $8.0933 \times 10^{-1}$ & $2.16317641738313 \times 10^{-11}$ & \multirow{12}{*}{ 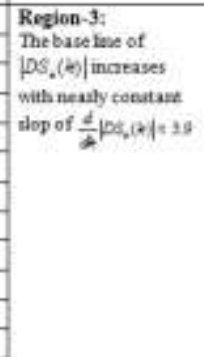 } \\
\hline $1 \times 10^{-2}$ & $8.0033 \times 10^{-7}$ & $3.73754270925124 \times 10^{-20}$ & \\
\hline $1 \times 10^{-2}$ & $8.0033 \times 10^{-5}$ & $391167532090745 \times 10^{-5}$ & \\
\hline $1 \times 10^{-11 / 2}$ & $8.0933 \times 10^{-11}$ & $3.92917701690788 \times 10^{-4}$ & \\
\hline $1 \times 10^{-4}$ & $8.0033 \times 10^{-12}$ & $3.93002796320018 \times 10^{-7}$ & \\
\hline $1 \times 10^{-1}$ & $8.0933 \times 10^{-11}$ & $3.93110299813939 \times 10^{-t}$ & \\
\hline $1 \times 10^{-1}$ & $8.0933 \times 10^{-34}$ & $3.9311199539999 \times 10^{-5}$ & \\
\hline $1 \times 10^{-1}$ & $8.0933 \times 10^{-12}$ & $3.99111623523555 \times 10^{-4}$ & \\
\hline $1 \times 10^{-2}$ & $8.0033 \times 10^{-15}$ & $3.93106959859394 \times 10^{-2}$ & \\
\hline $1 \times 10^{-7}$ & $8.0933 \times 10^{-21}$ & $3.93139011942916 \times 10^{2}$ & \\
\hline $1 \times 10^{-3}$ & $8.0933 \times 10^{-11}$ & $4.01392578494715 \times 10^{-4}$ & \\
\hline $1 \times 10^{-2}$ & $8.0933 \times 10^{-717}$ & $2.07639142123463 \times 10^{1}$ & \\
\hline $1 \times 10^{-2}$ & $8.0933 \times 10^{-25}$ & $4.14040248344403 \times 10^{12}$ & \multirow{2}{*}{$\begin{array}{l}|D S,(A)| \text { base int } \\
\text { increasesrapidy }\end{array}$} \\
\hline 1.0000000 & $1.0933 \times 10^{-21}$ & $1.79334761526856 \times 10^{111}$ & \\
\hline
\end{tabular}

* The parameters $m, n, p$ and $k_{r}$ are the same as those listed in Table 7.1.

** 2010-PDG listed muon's mean life $\tau=(2.197034 \pm 0.000021) \times 10^{-6} s$.

As shown in Table 7.3 muon $k_{i}$ values from $k_{i}=0$ to $k_{i}=1$ divided into three regions. In Region-1 $\left(0 \leq k_{i}<10^{-18}\right)$, average values of $\left|D S_{k}(k)\right|$ as base line keep constant: . In Region-2 $\left(10^{-18} \leq k_{i} \leq 10^{-14}\right),\left|D S_{k}(k)\right|$ base line is in the global minimum region. Region-2 is the effective region of muon's decay activities. In which, $k_{i}=3.683739 \times 10^{-15}$ corresponds to muon's mean life of $\tau=2.197034 \times 10^{-6} s$. In Range-3 $\left(10^{-14}<k_{i} \leq 1\right),\left|D S_{k}(k)\right|$ base line increases monotonically.

Table 7.4 listed some samples of local minimums distribution at 11 locations, which are used to estimate the average value of the separation between two adjacent local minimums.

Table 7.4: Samples of Local Minimums of $\left|D S_{k}(k)\right|$ for Muon at $k_{r}=206.76923076923077 *$

\begin{tabular}{|c|c|c|c|}
\hline $\begin{array}{c}\text { Starting } k_{1} \text { value } \\
k_{1}\end{array}$ & $\begin{array}{c}\text { Endiag } k \text { value } \\
\qquad k, 1\end{array}$ & $\begin{array}{l}\text { Number of } \\
\text { local mins }\end{array}$ & $\begin{array}{l}\text { Separation of } 2 \text { adjacent } k_{1} \\
\Delta k=\left(k_{2}-k_{1}\right)(11-1)\end{array}$ \\
\hline $10^{-15}+11 \times 10^{-21}$ & $10^{-11}+263 \times 10^{-11}$ & 11 & $2.52 \times 10^{-25}$ \\
\hline $5 \times 10^{-11}+7 \times 10^{-31}$ & $5 \times 10^{-11}+317 \times 10^{\text {सा }}$ & 11 & $3.1 \times 10^{-2}$ \\
\hline $10^{-17}+2 \times 10^{-11}$ & $10^{-17}+395 \times 10^{-91}$ & 11 & $393 \times 10^{-5}$ \\
\hline $5 \times 10^{-17}+27 \times 10^{-11}$ & $5 \times 10^{-15}+445 \times 10^{-11}$ & 11 & $418 \times 10^{-50}$ \\
\hline $10^{-15}+46 \times 10^{-41}$ & $10^{-16}+431 \times 10^{-11}$ & 11 & $3.85 \times 10^{-11}$ \\
\hline $5 \times 10^{-11}+20 \times 10^{-21}$ & $5 \times 10^{217}+425 \times 10^{-11}$ & 11 & $4.05 \times 10^{-5}$ \\
\hline $10^{-12}+25 \times 10^{-11}$ & $10^{-12}+305 \times 10^{-21}$ & 11 & $2.8 \times 10^{-2}=$ \\
\hline $5 \times 10^{-i 1}-10 \times 10^{-21}$ & $5 \times 10^{-21}+542 \times 10^{-11}$ & 11 & $555 \times 10^{-5}$ \\
\hline $10^{-16}+18 \times 10^{-1 / 2}$ & $10^{-2+}+543 \times 10^{-21}$ & 11 & $525 \times 10^{-51}$ \\
\hline $10^{-2 D}-35 \times 10^{-21}$ & $10^{-16}+363 \times 10^{-11}$ & 11 & $3.28 \times 10^{-5}$ \\
\hline $10^{-2}+3 \times 10^{-2}$ & $10^{-1}+452 \times 10^{-21}$ & 11 & $4.49 \times 10^{-3}$ \\
\hline \multicolumn{3}{|c|}{ Average value of $\Delta t_{\text {f }}$} & $\begin{aligned} \overline{\Delta \hbar}=3909 \times 10^{-2}+1.641 \times 10^{-2} \\
-1.389 \times 10^{-2}\end{aligned}$ \\
\hline
\end{tabular}

* The parameters $m, n, p$ and $k_{r}$ are the same as those listed in Table 7.1.

A distinctive feature of these theoretical results is that, along a $k_{r}=$ const straight line, $\left|D S_{k}(k)\right|$ has a series of local minimums corresponding to a series of possible decay times for a particle such as muon. Does it make sense? From the theoretical viewpoint, it does. According to the first fundamental postulation, SQS is a statistic theory in the first place. A series of $\left|D S_{k}(k)\right|$ local minimums corresponding to a serious of possible decay times should be expected. On the practical side, muon's mean life having a definitive value $\tau=2.197034 \times 10^{-6} s$ is for large numbers of muons as a group. For an individual muon, $\tau$ is the statistical average value of many possible decay times, it by no means must decay exactly at $t=\tau$.

As shown in Table 7.4, the 121 local minimums are taken as samples from $k_{i}=1 \times 10^{-18}$ to $k_{i}=1 \times 10^{-12}$ with $10^{-21}$ as variation step. It shows that, local minimums behavior randomly. The average separation between two adjacent minimums is calculated from these samples as $\overline{\Delta k_{i}}=3.909 \times 10^{-20}+1.641 \times 10^{-20}$, which roughly kept constant over a broad region. These data is used to estimate the total number of local minimums in Region-2 between $k_{i, 1}=1 \times 10^{-18}$ and $k_{i, 2}=1 \times 10^{-14}$ as: 


$$
N=\frac{k_{i, 2}-k_{i, 1}}{\overline{\Delta k_{i}}}=2.558 \times 10^{5} .
$$

Region-2 with decay time from $t=8.0933 \times 10^{-7} \mathrm{~s}$ to $t=8.0933 \times 10^{-3} s$ is the effective region of muon's decay activity. There are $N=2.558 \times 10^{5}$ local minimums in this region, each one corresponds to a possible decay time. The locations of local minimums determine the values of possible decay times. Besides Region-2, there are local minimums in Region-1 and in part of Region-3, which will be discussed later.

By counting all local minimums of $\left|D S_{k}(k)\right|$, in principle, the theoretical mean life $\tau$ of muon can be calculated by extensive number crunching. But it requires a tailor made program. In the meantime, let's take a rough estimate.

According to (7.5b), the separation $\Delta t$ of two adjacent possible decay times and corresponding decay time's density (number of possible decays per unit time) $\Delta N$ are:

$$
\begin{aligned}
& \Delta t=\left|\Delta\left[\frac{\lambda_{e C}}{k_{i} c}\right]\right|=\frac{\lambda_{e C} \Delta k_{i}}{k_{i}^{2} c} . \\
& \Delta N=\frac{1}{\Delta t}=\frac{k_{i}^{2} c}{\lambda_{e C} \Delta k_{i}} .
\end{aligned}
$$

In the $k_{i}$ domain, the local minimums have roughly even distribution as shown in Table 7.4. In the time domain, because of the inverse relation $\Delta t \propto 1 / k_{i}^{2}$ of (7.14a), the local minimum of $\left|D S_{k}(k)\right|$ in the $k_{i}$ domain corresponds to the temporal response as the local maximum in the time domain. As shown by (7.14a), the local maximums in time domain are unevenly distributed caused by the ${k_{i}^{2}}^{2}$ factor in denominator of $\Delta t$.

The effective Region-2 is divided into four sub-regions: Region-2a: $10^{-18} \leq k_{i}<10^{-17}$ with center at $k_{i}=5 \times 10^{-18}$; Region-2b: $10^{-17} \leq k_{i}<10^{-16}$ with center at $k_{i}=5 \times 10^{-17}$; Region-2c: $10^{-16} \leq k_{i}<10^{-15}$ with center at $k_{i}=5 \times 10^{-16}$; Region-2d: $10^{-15} \leq k_{i} \leq 10^{-14}$ with center at $k_{i}=5 \times 10^{-15}$.

The values of $k_{i}, t, \Delta t, \Delta N$ and $t \Delta N$ at center of each sub-regions calculated according to (7.14) and Table 7.4 are listed in Table 7.5.

Table 7.5: Parameters in the Center of Four Sub-Regions

\begin{tabular}{|l|l|l|l|l|}
\hline Sub-region & Reqion-2a & Region-2b & Region-2c & Region-2d \\
\hline$k_{i}$ & $5 \times 10^{-11}$ & $5 \times 10^{-1}$ & $5 \times 10^{-2 t}$ & $5 \times 10^{-1}$ \\
\hline$t(s)$ & $1.619 \times 10^{-3}$ & $1.619 \times 10^{-4}$ & $1.619 \times 10^{-3}$ & $1.619 \times 10^{-5}$ \\
\hline$\Delta t(s)$ & $1.004 \times 10^{-5}$ & $1.353 \times 10^{-7}$ & $1.311 \times 10^{-7}$ & $1.787 \times 10^{-11}$ \\
\hline$\Delta V$ & $9.94 \times 10^{4}$ & $7.39 \times 10^{8}$ & $7.627 \times 10^{6}$ & $5.596 \times 10^{10}$ \\
\hline$t \Delta V(s)$ & $1.613 \times 10^{2}$ & $1.196 \times 10^{3}$ & $1.235 \times 10^{4}$ & $9.058 \times 10^{4}$ \\
\hline
\end{tabular}

The values at the center of each sub-region are treated as the average values for that sub-region. Take $N_{j} / \sum_{j=a}^{d} N_{j}$ as the probability for muon decay in $j(j=a, b, c, d)$ sub-region, muon's mean life is roughly estimated as:

$$
\tau \approx \bar{t}=\frac{\sum_{j=a}^{d} N_{j} t_{j}}{\sum_{j=a}^{d} N_{j}}=1.838 \times 10^{-6} s .
$$

The value of $\tau \approx \bar{t}$ is $83.7 \%$ of muon's measured mean life $\tau=2.197034 \times 10^{-6} s$, which is in the ballpark. Since only the activity in Region- 2 is counted, the $16.3 \%$ discrepancy is understandable. The ballpark agreement shows that, the spectrum does contain the information of mean lifetime in the muon's case and Region- 2 is the effective region.

The rough estimation is based on the assumption that, Region-2 is the effective region for muon' decay activity. The effects of other two regions are not taken into account, which need justification.

The local minimums are not restricted in Region-2, they extended to Region-1 from $k_{i} \sim 10^{-19}$ to $k_{i} \sim 10^{-23}$. According to $(7.14 \mathrm{~b})$, the decay time density $\Delta N$ is proportional to $k_{i}^{2}$, in Region-1, $\Delta N$ value decreases rapidly as $k_{i}$ value decreasing. For instance, the $\Delta N$ value at the boundary of Region-1 and Region- $2, k_{i}=10^{-18}$, is roughly less than $10^{-7}$ of the $\Delta N$ value at the center of Region- 2 where muon's mean life is close by. In other words, muon rarely decays in Region-1 with extremely low probability.

Prediction 7.1: The probability of muon decay time longer than $t=8.0933 \times 10^{-3} s$ corresponding to $k_{i}=1 \times 10^{-18}$ is less than $10^{-7}$ of the probability of muon decay at $t \approx \tau=2.197034 \times 10^{-6} \mathrm{~s}$ corresponding to $k_{i} \approx 3.684 \times 10^{-15}$.

Explanation: According (7.14b), $\Delta N$ is proportional to $k_{i}^{2}$. The ratio of decay probabilities at $k_{i}=1 \times 10^{-18}$ and at $k_{i} \approx 3.684 \times 10^{-15}$ is estimated as:

$$
\left[\frac{1 \times 10^{-18}}{3.684 \times 10^{-15}}\right]^{2}=7.368 \times 10^{-8}<10^{-7} \text {. }
$$

For Region-1 with $k_{i}<1 \times 10^{-18}$, the ratio of decay possibilities is much less than $7.368 \times 10^{-8}$, which can be estimated the same way. So the rough estimation of $\tau \approx \bar{t}$ disregarding Region-1 is justified.

The local minimums are also extended in Region-3 with rapidly increasing density. However, it does not mean that muon decays more frequently in Region-3. In fact, muon decays rarely in Region-3, which needs explanation. $\left|D S_{k}(k)\right|$ serves as spectrum with fermion at local minimum. In the spectrum, the tendency for muon as a fermion to reach minimum value of $\left|D S_{k}(k)\right|$ actually is in two senses, locally and globally. The former was considered, now it's the time to consider the latter. In Region-1 and Region-2, as shown in Table 7.3, the base line of $\left|D S_{k}(k)\right|$ is almost flat with minor 


\section{Z. Y. SHEN}

variations. The vast numbers of local minimums with different densities compete for the possible decay time. The base line of $\left|D S_{k}(k)\right|$ increases monotonically in Region-3 and the bottom values of local minimums increase with it. In most part of Region-3, the bottom values of local minimums are higher than the base line level in Region-1 and Region-2. The turning point is probably at the vicinity of $k_{i}=1 \times 10^{-13}$

corresponding to $t=8.0933 \times 10^{-8} \mathrm{~s}$. Muons have very low probability for decay times shorter than $t=8.0933 \times 10^{-8} \mathrm{~s}$ despite the fact that the values of $\Delta N$ are many orders of magnitudes larger than those in Region-2. The abrupt drop of decay probability in Region-3 is caused by the local minimums disqualified in the global sense, because their bottom values are higher than the base line in Region-1 and Region-2. So the rough estimation of $\tau \approx \bar{t}$ disregarding Region-3 for muon is also justified.

Moreover, according to $(7.14 \mathrm{a})$, the time separation $\Delta t$ is proportional to the inverse of $k_{i}^{2}$. In Region-3, as $k_{i}$

increases, $\Delta t$ decreases rapidly. At certain point, the extremely crowded local minimums in time domain are overlapped and no longer distinguishable. In fact, they disappear by submerging into the background noise.

Prediction 7.2: Muon has zero probability to decay at times shorter than $t_{\min }=2 \times 10^{-13} \mathrm{~s}$.

Explanation: The disappearance of local minimums in Region-3 happens at the point that, separation $\Delta t$ becomes shorter than the width of the response in time domain for muon. At that point, individual response in time domain is no longer distinguishable. Muon's decay is caused by weak interaction mediated by gauge bosons $W^{ \pm}$or $Z^{0}$ with mean lifetime of $\tau_{W, Z}=2 \times 10^{-25} \mathrm{~s}$. The muon's decaying process must complete before its mediators' decay, which roughly determines the width of individual response in time domain. The criterion is $\Delta t \leq \tau_{W, Z}$. According to (7.5b) and (7.14a), the $t_{\min }$ for muon is:

$$
t_{\min }=\frac{\lambda_{e C}}{c k_{i}}=\frac{\lambda_{e C}}{c} \sqrt{\frac{c \Delta t}{\lambda_{e C} \overline{\Delta k_{i}}}}=\sqrt{\frac{\lambda_{e C} \Delta t}{c \overline{\Delta k_{i}}}} \leq \sqrt{\frac{\lambda_{e C} \tau_{W, Z}}{c \overline{\Delta k_{i}}}} \approx 2.075 \times 10^{-13} s^{.}
$$

In which $\overline{\Delta k_{i}}=3.909 \times 10^{-20}$ is the medium average value cited from Table 7.4.

As another example, electron's $\left|D S_{k}(k)\right|$ profile over broad range is sown in Table 7.6. The reason for taking such values of numerical parameters $m, n, p$ for electron will be given in later sections.

The distinctive features of electron's $\left|D S_{k}(k)\right|$ profile over broad range are the disappearance of Region-2 and Region-1 becoming global minimum region with only one local minimum of $\left|D S_{k}(0)\right|=0$ at $k_{i}=0$ corresponding to $t=\infty$. It is consistent with the fact that electron is stable.
This is an important check point to verify that, the rough estimation of mean life based on the global minimum concept for muon is correct. It also increases the credibility of $\left|D S_{k}(k)\right|$ serving as particles spectrum with information of decay times and in some way related to mean life. But nuon and electron are just two examples, which are by no means sufficient to draw a conclusion. The real correlation between $\left|D S_{k}(k)\right|$ as spectrum and particle's mean life is still an open issue. More works along this line are needed.

As illustrated in this section, $D S_{k}(k)$ as a member of the $S$-equation family has rich physics meanings. In general, $\left|D S_{k}(k)\right|$ serving as particle mass spectrum is conditional. It subjects to a prior knowledge of numerical parameters. Even though, it does provide useful information. More importantly, $D S_{k}(k)$ serves as the base for an extended version, which reveals more physics significance. Details will be given in later sections.

Table 7.6 $\left|D S_{k}(k)\right|$ over Broad Range for Electron at $k_{r}=1$

\begin{tabular}{|c|c|c|c|}
\hline$k_{f}$ values & $\begin{array}{c}t \\
\text { (seconds) }\end{array}$ & DS. $(k)$ base line values & Notes \\
\hline 0 & $x$ & 0 & \multirow{9}{*}{ 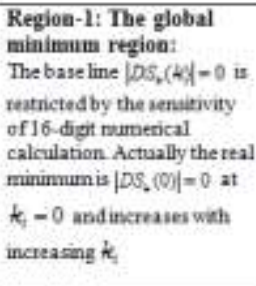 } \\
\hline $1 \times 10^{-21}$ & $8.0933 \times 10^{2}$ & 0 & \\
\hline $1 \times 10^{-21}$ & $8.0933 \times 10^{2}$ & 0 & \\
\hline $1 \times 10^{-21}$ & 8.0933 & 0 & \\
\hline $1 \times 10^{-32}$ & $8.0933 \times 10^{-5}$ & 0 & \\
\hline $1 \times 10^{-19}$ & $8.0933 \times 10^{6}$ & 0 & \\
\hline $1 \times 10^{-211}$ & $8.0933 \times 10^{-2}$ & 0 & \\
\hline $1 \times 10^{-15}$ & $8.0033 \times 10^{-4}$ & 0 & \\
\hline $1 \times 10^{-16}$ & $8.0933 \times 10^{8}$ & 0 & \\
\hline $1 \times 10^{-25}$ & $8.0933 \times 10^{-6}$ & $3.111035630811 \times 10^{-12}$ & \multirow{18}{*}{$\begin{array}{l}\text { Region-3: } \\
\text { The base line of }\left|D 5_{6}(t)\right| \\
\text { mcreases with neady } \\
\text { constant dop of } \\
\frac{d}{d k}\left|D S_{i}(k)\right|=3\end{array}$} \\
\hline $1 \times 10^{-16}$ & $8.0933 \times 10^{2}$ & $3.14195502486876 \times 10^{-3}$ & \\
\hline $1 \times 10^{-25}$ & $8.0933 \times 10^{-7}$ & $3.14193139834189 \times 10^{-21}$ & \\
\hline $1 \times 10^{-12}$ & $8.0933 \times 10^{-4}$ & $3.14159809516859 \times 10^{-21}$ & \\
\hline $1 \times 10^{-15}$ & $8.0933 \times 10^{-1}$ & $3.14159809280567 \times 10^{-14}$ & \\
\hline $1 \times 10^{-11}$ & $8.0933 \times 10^{-11}$ & $3.14159254166692 \times 10^{-210}$ & \\
\hline $1 \times 10^{-7}$ & $8.0933 \times 10^{\text {.II }}$ & $3.14159265268899 \times 10^{-14}$ & \\
\hline $1 \times 10^{-4}$ & $8.0933 \times 10^{-21}$ & $3.14159260828006 \times 10^{-2}$ & \\
\hline $1 \times 10^{2}$ & $8.0933 \times 10^{-1 / 4}$ & $3.14159215974996 \times 10^{-2}$ & \\
\hline $1 \times 10^{-1}$ & $8.0933 \times 10^{-d}$ & $3.14158771874684 \times 10^{-4}$ & \\
\hline $1 \times 10^{-2}$ & $8.0933 \times 10^{-50}$ & $3.1415433060844 \times 10^{-2}$ & \\
\hline $1 \times 10^{-1}$ & $8.0933 \times 10^{-1 / 1}$ & $3.14109922504247 \times 10^{-4}$ & \\
\hline $1 \times 10^{-7}$ & $80933 \times 10^{-11}$ & $3.13666301504589 \times 10^{-7}$ & \\
\hline $1 \times 10^{-2}$ & $8.0933 \times 10^{-7}$ & $3.09275736951894 \times 10^{-4}$ & \\
\hline $1 \times 10^{-1}$ & $8.0933 \times 10^{-20}$ & $2.69597308951354 \times 10^{-1}$ & \\
\hline 1.0000000 & $8.0933 \times 10^{\text {-11 }}$ & 0.956786081736228 & \\
\hline $1 \times 10^{1}$ & $8.0933 \times 10^{212}$ & 0.99999999999997 & \\
\hline $1 \times 10^{2}$ & $8.0933 \times 10^{-3}$ & 1 & \\
\hline
\end{tabular}

In this section, muon and taon are used as examples for $D S_{k}(k)$ serving as particles spectrum on the complex $k$-plane. More details of muon and taon will be given in later sections.

\section{Section 8: Electron Torus Model and Trajectories}

As mentioned in Section 6, electron has two-loop structure. Loop-1 is the primary loop with loop length $L_{1}$. Loop-2 perpendicular to loop-1 is the secondary loop with 
loop length $L_{2}$. Loop-2 center rotates around loop-1 circumference to forms a torus surface. According to SQS theory, all Dirac type fermions' models are based on torus. Torus is a genus-1 topological manifold with one center hole and four tiny holes $h_{1}, h_{\infty}, h_{\omega}, h_{\omega^{2}}$ corresponding to four branch points on Riemann surfaces described in Section 3.

To begin with, torus as a topological manifold has neither definitive shape nor determined dimensions. The four tiny holes $h_{1}, h_{\infty}, h_{\omega}, h_{\omega^{2}}$ without fixed location can move around on torus surface. To represent a particle such as electron, the torus model must have definitive shape and determined dimensions, and the location of four tiny holes must be fixed as well. To determine these geometrical parameters, additional information is needed, which comes from SQS theory first principle.

Fig.8.1 shows the torus serving as electron model. There are three circles on $x-y$ cross section shown in Fig. 8.1a. The two solid line circles represent the inner and outer edges of torus, and the dot-dashed line circle represents loop- 1 and the trace of rotating loop-2 center. In Fig. 8.1b, the right and left circles shown torus two cross sections are cut from line $O_{1} G$ and line $O_{1} H$ on $x-y$ plane, respectively.

According to SQS theory, a set of three numerical parameters, $m, n, p$ is assigned to each fermion defined as:

$$
\begin{aligned}
\frac{n}{m} & =\frac{L_{2}}{L_{1}}, \\
\frac{p}{n} & =\frac{M}{M_{e}},
\end{aligned}
$$

In which, $M$ and $M_{e}$ are the mass of the fermion and electron, respectively.

For electron, its original $m, n, p$ parameters are selected as:

$$
m=2, n=1, p=1 \text {. }
$$

Substituting (8.2) into (8.1) yields:

$$
\begin{aligned}
& \frac{L_{2}}{L_{1}}=\frac{1}{2}, \\
& \frac{M}{M_{e}}=\frac{1}{1} .
\end{aligned}
$$

The torus surface is divided into two halves as shown in Fig.8.1b. The outer half has positive curvature and the inner half has negative curvature. According to $S$-equation of (3.20), unitarity requires:

$$
S(x)-1=\sum_{j=-N}^{N} e^{-\pi(x)(j-x)^{2}}-1=0 .
$$

In (8.4), the original summation index $x_{i}$ is replaced by $j$ for simplicity. The lower and upper summation limits are truncated at $j= \pm N$ for numerical calculation. A sufficient large $N$ is selected for $S(x)-1$ to converge. As discussed in Section 6, the two points on real $x_{r}$-axis of Fig.3.4 representing electron are:

$$
x_{1}=0.125 \text {, and } x_{2}=0.375 \text {. }
$$

Substituting (8.5) into (8.4) and solving for $\pi(x)$ yields:

$$
\begin{aligned}
& \pi\left(x_{1}=0.125\right)=3.877102924420037>\pi ; \\
& \pi\left(x_{2}=0.375\right)=2.864592498254391<\pi .
\end{aligned}
$$

$\pi\left(x_{1}\right)$ and $\pi\left(x_{2}\right)$ serve as the messengers to transfer information from $S$-equation to torus model. $\pi\left(x_{1}\right)>\pi$ corresponds to negative curvature on the inner half of torus; and $\pi\left(x_{2}\right)<\pi$ corresponds to positive curvature on the outer half of torus.

The distance between two loops' centers is $d$, which is the radius of loop-1. For electron, loop-1 circumference equals to one Planck wavelength, $L_{1}=\lambda_{P}=L_{P}=1$, which corresponds to $d=L_{1} / 2 \pi=1 / 2 \pi$. For convenience, let's set $d=1$ as the reference length for other lengths on the torus models, and consider its real value later.

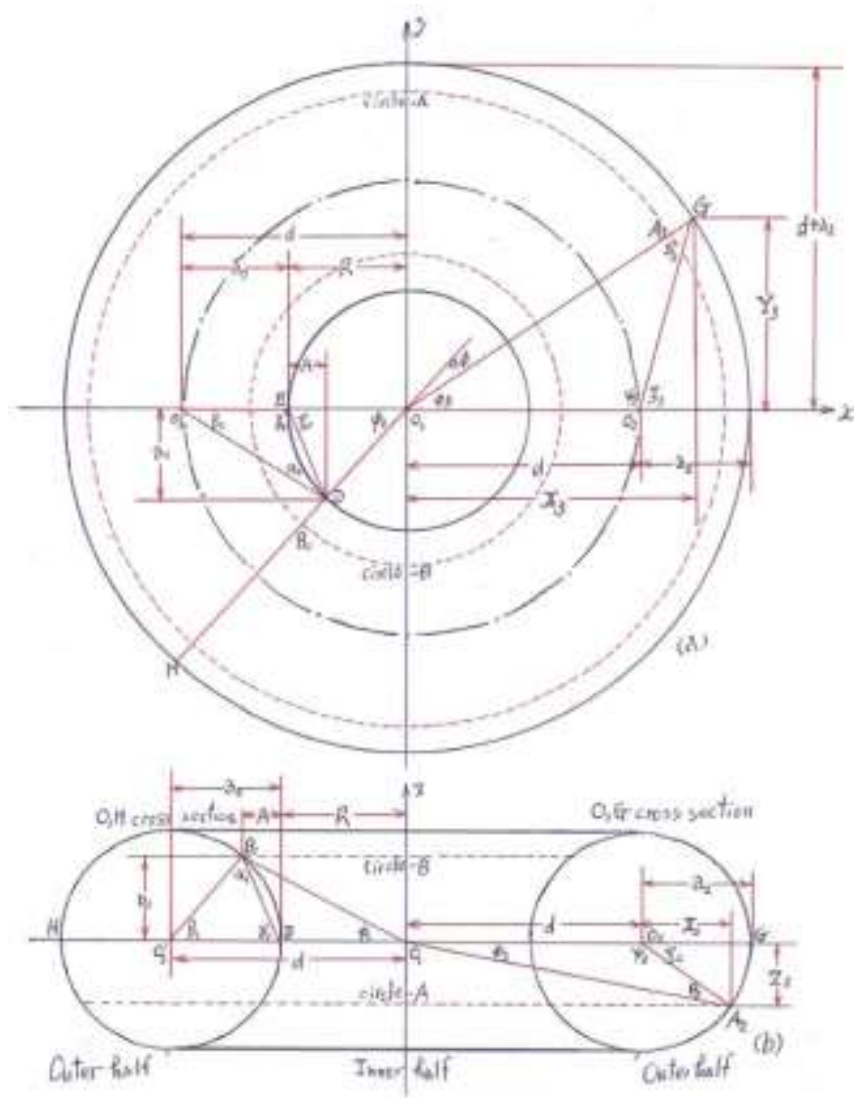

Fig.8.1 Electron torus model: (a) $x-y$ cross section; (b) Right is cross section along line $O_{1} G$, left is cross section along line $O_{1} H$.

According to (8.3a) and $d=1$, the radius of loop-2 for electron is determined as:

$$
a_{2}=\frac{L_{2}}{L_{1}} d=\frac{1}{2} d=0.5 \text {. }
$$

The two dimensions of torus as electron model are determined as $d=1, a_{2}=0.5$. The next step is to fix the 


\section{Z. Y. SHEN}

locations for four tiny holes $h_{1}, h_{\infty}, h_{\omega}, h_{\omega^{2}}$ shown in Fig. $3.5 \mathrm{~b}$.

In fact, the electron torus model is shared with its antiparticle, the positron. For the four tiny holes $h_{1}, h_{\infty}, h_{\omega}, h_{\omega^{2}}$, two of them belong to electron and the other two belong to positron. The values of $\pi\left(x_{2}\right)$ and $\pi\left(x_{1}\right)$ determine the locations of two characteristic points $A, B$ for electron.

$\pi\left(x_{2}\right)=2.864592498254391<\pi$ of $(8.6 \mathrm{~b})$ corresponds to the torus outer half with positive curvature like a sphere. On the $O_{1} G$ cross section at the right of Fig $8.1 \mathrm{~b}$, the location of point $A_{2}$ at $x=X_{2}, z=Z_{2}$ with origin at cross section center $\mathrm{O}_{2}$ is determined by $\pi\left(x_{2}\right)$ according to the following formulas:

$$
\begin{aligned}
& \frac{\int_{-\xi_{2}}^{\xi_{2}} \sqrt{\left(a_{2} \sin t\right)^{2}+\left(b_{2} \cos t\right)^{2}} d t}{2 Z_{2}}-\frac{\pi}{\pi\left(x_{2}\right)}=0, b_{2}=a_{2}, \\
& \cos \xi_{2}=\frac{X_{2}}{a_{2}}, \\
& \left(\frac{X_{2}}{a_{2}}\right)^{2}+\left(\frac{Z_{2}}{b_{2}}\right)^{2}-1=0, b_{2}=a_{2} .
\end{aligned}
$$

As shown in Fig. 8.1b, point $A_{2}$ and two loops' centers $\mathrm{O}_{1}, \mathrm{O}_{2}$ form a triangle $\mathrm{A}_{2} \mathrm{O}_{1} \mathrm{O}_{2}$. The three inner angles $\psi_{2}$, $\phi_{2}, \theta_{2}$ of triangle $\mathrm{A}_{2} \mathrm{O}_{1} \mathrm{O}_{2}$ are determined by:

$$
\begin{aligned}
& \tan \xi_{2}=\frac{Z_{2}}{X_{2}}, \psi_{2}=180^{\circ}-\xi_{2}, \\
& \tan \phi_{2}=\frac{Z_{2}}{d+X_{2}}, \\
& \theta_{2}=\xi_{2}-\phi_{2} .
\end{aligned}
$$

On $x-y$ plane shown by Fig. 8.1a, the location of point $G$ at $x=X_{3}, y=Y_{3}$ with origin at $O_{1}$ is determined by angle $\phi_{3}$ from $\pi\left(x_{2}\right)$ according to following formulas:

$$
\begin{aligned}
& \frac{\left(d+a_{2}\right) \phi_{3}}{\left(d+a_{2}\right) \sin \phi_{3}}-\frac{\pi}{\pi\left(x_{2}\right)}=0, \\
& X_{3}^{2}+Y_{3}^{2}=\left(d+a_{2}\right)^{2} .
\end{aligned}
$$

The three inner angles $\psi_{3}, \phi_{3}, \theta_{3}$ of triangle $G O_{1} O_{2}$ are determined by:

$$
\begin{aligned}
& \tan \xi_{3}=\frac{Y_{3}}{X_{3}-d}, \psi_{3}=180^{\circ}-\xi_{3}, \\
& \tan \phi_{3}=\frac{Y_{3}}{X_{3}}
\end{aligned}
$$$$
\theta_{3}=\xi_{3}-\phi_{3} \text {. }
$$

$\pi\left(x_{1}\right)=3.877102924420037>\pi$ of (8.6a) corresponds to the inner half of torus with negative curvature like a saddle surface with sinusoidal variation. The parameters of saddle surface are determined by $\pi\left(x_{1}\right)$ according to following formulas:

$$
\begin{aligned}
& \frac{\int_{0}^{2 \pi} \sqrt{1+\left[2 A_{m} \cos 2 t\right]^{2}} d t}{2 \pi}-\frac{\pi\left(x_{1}\right)}{\pi}=0, \\
& \frac{A}{A_{m}}=\frac{\int_{0}^{2 \pi} \sqrt{\left(a_{1} \sin t\right)^{2}+\left(b_{1} \cos t\right)^{2}} d t}{2 \pi \times 1}, b_{1}=a_{1} .
\end{aligned}
$$

$A_{m}$ and $A$ are the amplitudes of saddle sinusoidal variation on circles with radius 1 and radius $a_{1}$, respectively. The locations of points $B_{1}$ and point $D$ are determined by the following simultaneous equations:

$$
\begin{aligned}
& (R-A)^{2}+a_{1}^{2}-R^{2}=0, R=d-a_{2}=1-a_{2}, \\
& \left(\frac{a_{2}-A}{a_{2}}\right)^{2}+\left(\frac{b_{1}}{b_{2}}\right)^{2}-1=0, b_{1}=a_{1}, b_{2}=a_{2} .
\end{aligned}
$$

Equation (8.13a) represents a circle with radius $R$ centered at $O_{1}$. The location of point $D$ at $x=-(R-A), y=-a_{1}$ with origin at $O_{1}$ is determined. Equation (8.13b) represents the circle with radius $a_{2}$ centered at $O_{2}^{\prime}$ on the $O_{1} H$ cross section in Fig.8.1b. The location of point $B_{1}$ at $x=\left(a_{2}-A\right), y=b_{1}$ with origin at $O_{2}^{\prime}$ is determined.

In the $O_{1} H$ cross section in Fig. 8.1b, the three inner angles $\alpha_{1}, \beta_{1}, \gamma_{1}$ of triangle $B_{1} D O_{2}^{\prime}$ and angle $\phi_{1}$ are determined by:

$$
\begin{aligned}
& \tan \beta_{1}=\frac{b_{1}}{a_{2}-A}, \\
& \tan \gamma_{1}=\frac{b_{1}}{A}, \\
& \alpha_{1}=180-\beta_{1}-\gamma_{1}, \\
& \tan \phi_{1}=\frac{b_{1}}{R+A} .
\end{aligned}
$$

On the $x-y$ plane shown by Fig. 8.1a, The three inner angles $\alpha_{0}, \beta_{0}, \gamma_{0}$ of triangle $D E O^{\prime}{ }_{2}$ and angle $\phi_{0}$ are determined by:

$$
\begin{aligned}
& \tan \xi_{0}=\frac{a_{1}}{A}, \gamma_{0}=180-\xi_{0}, \\
& \tan \beta_{0}=\frac{a_{1}}{a_{2}+A}, \\
& \alpha_{0}=\xi_{0}-\beta_{0}, \\
& \tan \phi_{0}=\frac{a_{1}}{R-A} .
\end{aligned}
$$

According to the torus model and two characteristic points $A, B$ determined by $\pi\left(x_{1}\right)$ and $\pi\left(x_{2}\right)$ from the $S$-equation, electron parameters calculated with the above formulas are listed in Table 8.1.

In Table 8.1, notice that:

$$
\begin{aligned}
& \xi_{2}=\phi_{3}=42.24442009^{\circ}, \\
& \theta_{2}=\xi_{2}-\phi_{2}=\phi_{3}-\phi_{2}=28.45987086^{\circ}, \\
& \phi_{0}=\beta_{1}=55.257547^{\circ}
\end{aligned}
$$




$$
\phi_{1}=\beta_{0}=29.88107155^{\circ} .
$$

Let's consider the meanings of (8.16a). $\xi_{2}$ is the angle at the center of loop-2 between line $\mathrm{O}_{2} \mathrm{G}$ and line $\mathrm{O}_{2} \mathrm{~A}_{2}$ as shown in Fig.8.1b, which serves as the initial phase angles of cyclic movements along loop-2. $\phi_{3}$ is the angle at the center of loop- 1 between the $x$-axis and line $O_{1} G$ on $x$ - $y$ cross section shown in Fig.8.1a, which serves as the initial phase angles of cyclic movements along loop-1. $\xi_{2}=\phi_{3}$ means that the two cyclic movements around loop-2 and loop-1 are synchronized in phase. (8.16b) indicates that the phase angles' differences of $\phi_{3}-\phi_{2}$ and $\xi_{2}-\phi_{2}$ both equal to

$\theta_{2}=28.45987086^{\circ}$, which is close to the Weinberg angle $\theta_{W}$.

This is the first hint that, the characteristic points such as point $A$ and the triangle $\mathrm{A}_{2} \mathrm{O}_{1} \mathrm{O}_{2}$ have something to do with particle's interaction parameters. (8.16c) and (8.16d) indicate that, the some types of synchronizations as (8.16a) and (8.16b) hold between angles $\phi_{0}$ and $\beta_{1}$ as well as between $\phi_{1}$ and $\beta_{0}$ in the inner half of torus shown by Fig.8.1a and Fig.8.1b on left side.

Table 8.1: Parameters for Electron Torus Model*

\begin{tabular}{|c|c|c|c|}
\hline \multicolumn{3}{|c|}{ 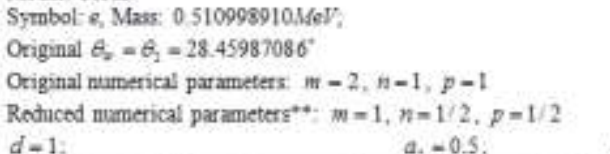 } & Charge: \& \\
\hline $\begin{array}{l}\text { Torus inner-half } \\
x_{1}=0.125, \pi\left(x_{1}\right)=3 \\
R=d-a_{2}=0.5 \\
A_{n}=0.52342698, A\end{array}$ & $\begin{array}{l}\text { uegative elorvature) } \\
87710292 \\
1=0.21505573\end{array}$ & \multicolumn{2}{|c|}{$\begin{array}{l}\text { Torus outer-half (positnve curvature) } \\
x_{2}=0.375, \pi\left(x_{2}\right)=2.8645925\end{array}$} \\
\hline $\begin{array}{l}\text { Frisugle } B, D O_{1}^{\prime} \text { on } \\
O, H \text { cross section }\end{array}$ & $\begin{array}{l}\text { Triangle } \mathrm{DEO}_{2} \text { on } \\
x-y \text { cross section }\end{array}$ & $\begin{array}{l}\text { Triangle } A_{2}, O_{,} O_{3} \text { on } \\
O_{1} G \text { cross section }\end{array}$ & $\begin{array}{l}\text { Truangle } \mathrm{GO}_{1} \mathrm{O}_{2} \text { on } \\
x-y \text { cross section }\end{array}$ \\
\hline$b_{1}=0.41086101$ & $a_{1}=0.41086101$ & $\begin{array}{l}X_{2}=0.3701418 \\
Z_{I}=0.33614736\end{array}$ & $\begin{array}{l}X_{3}=1.11042541 \\
Y_{3}=1.00844207\end{array}$ \\
\hline$\alpha_{\mathrm{s}}=62.3712265^{\circ}$ & $\alpha_{0}=32.49015495$ & $\theta_{2}=28.4598708 \sigma^{\circ}$ & $\theta_{2}=41.50653225^{\circ}$ \\
\hline$\beta_{2}=55.257547^{\circ}$ & $\beta_{0}=29.88107155^{\circ}$ & $\theta_{2}=13.78454923^{\circ}$ & $\phi_{1}=42.24442009^{\prime}$ \\
\hline$y_{1}=62.3712265^{\circ}$ & $y_{0}=117.6287735^{\circ}$ & $y=137.75557991^{*}$ & $\varphi_{1}=96.24904766^{\circ}$ \\
\hline$A=29.88107155^{\circ}$ & $A_{1}=55.253547^{\circ}$ & $s=42.24442009^{\circ}$ & $\zeta=83.75095234^{\circ}$ \\
\hline
\end{tabular}

* All data are from 16-digit numerical calculations, only 8-digit after the decimal point is presented.

** The reduced numerical parameters are the original numerical parameters divided by $m$.

These types of synchronizations are interpreted as the geometrical foundation of electron's stability. It is the first conclusion drawn from electron's torus model.

The torus model represents electron, it must have all electron parameters expressed in geometrical terms. This is the job a model supposed to do. But the torus has only two geometrical parameters $d$ and $a_{2}$ to determine its shape and size, which are by no means sufficient to represent all parameters. $\pi\left(x_{1}\right)$ and $\pi\left(x_{2}\right)$ come to help. They serve as the messengers to transfer information from $S$-equation to torus model to define the locations of characteristic points and the triangles associated with them. In this way, the torus model with defined characteristic points and triangles is capable to represent all parameters of electron. The details will be given later.

For the standard model, particle is represented by a point. A point carries no information except its location. That is why twenty some parameters are handpicked and put in for standard model. For SQS theory, parameters are derived from the first principle and represented by geometrical model. In which, two messengers $\pi\left(x_{1}\right), \pi\left(x_{2}\right)$, the characteristic points and triangles play pivotal roles.

The torus model provides a curved surface to support the trajectory of electron's internal movement. Electron internal movement includes three types: (1) cyclic movement along loop-1; (2) cyclic movement along loop-2; (3) sinusoidal oscillation along trajectory. Fig. 8.2a and Fig. 8.2b show the projections of electron's trajectory on $x-y$ plane and $x-z$ plane, respectively. On $x-y$ plane shown in Fig.8.2a, the top trajectory is for electron, and the bottom trajectory is for positron. Because these two trajectories are symmetrical, to explain the one for electron is sufficient to understand the other.
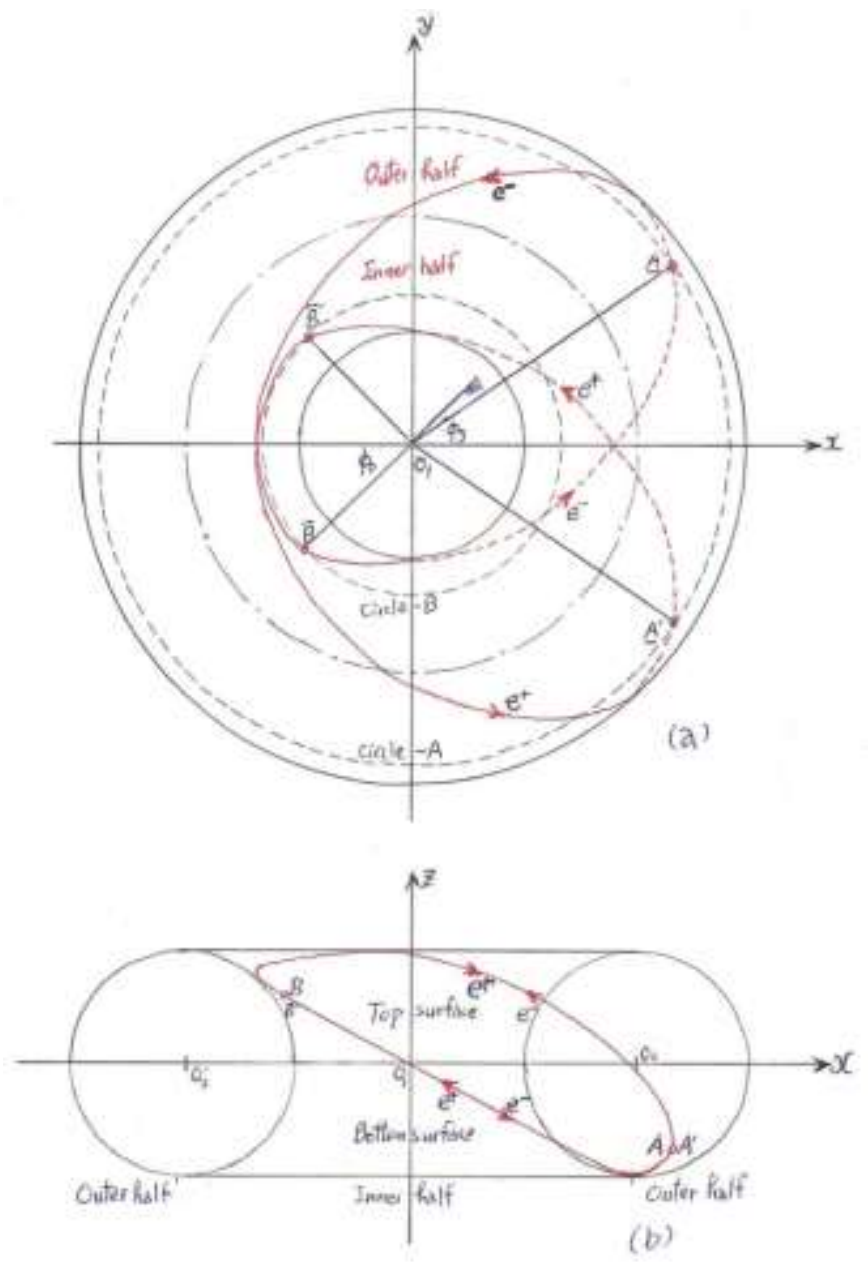

Fig. 8.2 Electron and positron trajectories on torus model: (a) Projection on $x-y$ plane; (b) Projection on $x-z$ plane. 


\section{Z. Y. SHEN}

The trajectory is a closed loop. It can start anywhere on the loop as long as it comes back to close the loop. Let's look at trajectory starting at point $\underline{A}$ on torus outer half bottom surface represented by the short dashed curve shown in Fig. 8.2a. It passes through the torus outer edge and goes to the upper surface shown by solid curve. It passes the top center line getting into the inner half and reaches point $\bar{B}$ on torus inner half top surface to complete its first half journey. The second half journey starts from point $\bar{B}$. At the torus inner edge, it goes back to the bottom surface shown by dashed curve. It passes through the bottom center line and comes back to point $\underline{A}$ to complete a full cycle. The trajectory repeats its journey again and again. The $x$ - $z$ plane projection of the trajectory is shown in Fig. 8.2b.

The trajectory shown in Fig.8.2 is a rough sketch. Its exact shape is determined by two geodesics on the torus surface. One from point $\underline{A}$ to point $\bar{B}$; the other from point $\bar{B}$ back to point $\underline{A}$ to close the trajectory loop. The characteristic points $\underline{A}$ and $\bar{B}$ not only carry the parameters information to define the triangles but also serve as the terminals for the two geodesics to form the trajectory.

Notice that, in Fig.8.1 and Fig. 8.2a, the three points $A, O_{1}$, $B$ are not aligned. The difference between two angles $\phi_{0}$ and $\phi_{3}$ is:

$\Delta \phi=\phi_{0}-\phi_{3}=55.257547^{\circ}-42.24442009^{\circ}=13.01312691^{\circ}$.

$\Delta \phi$ is the angle deviated from $180^{\circ}$ representing a perfect alignment of three points $A, O_{1}, B$. It is important to point out that, $A$ and $B$ are not fixed points. Instead, they define two circles, circle- $A$ and circle- $B$, with radius $O_{1} A$ and $O_{1} B$, respectively. The trajectory may start at a point on circle- $A$ halfway through a point at circle- $B$ and comes back to point $A$. The trajectory is legitimate as long as it kept the same angle of $\angle A O_{1} B$ :

$$
\angle A O_{1} B=180^{\circ}-\Delta \phi=166.98687309^{\circ} .
$$

There are many trajectories on torus surface with the same angle $\angle A O_{1} B$ given by (8.18), all of them contain the same information carried by $\pi\left(x_{1}\right)$ and $\pi\left(x_{2}\right)$. These trajectories spread over torus entire surface. As shown in later sections, trajectories are discrete in nature and the number of trajectories is countable, which form a set of discrete trajectories on torus surface. At a given time, electron is represented by a trajectory. As time passing by, it jumps to other trajectories. The scenario is dynamic and stochastic. Physically, jumping trajectories on the same torus surface corresponds to emitting and absorbing a virtual photon by the electron.

For the $x-y$ projection shown in Fig. 8.2a, the trajectory on the bottom for positron goes through two characteristic points $\underline{A}^{\prime}$ and $\bar{B}^{\prime}$ with anti-clockwise direction. As shown in Fig.8.2b, the $x-z$ projections of two trajectories are coincided with opposite directions: anti-clockwise for electron and clockwise for positron.

In essence, the $S$-equation determine the value of $\pi\left(x_{1}\right)$ and $\pi\left(x_{2}\right)$ from $x_{1}$ and $x_{2} ; \pi\left(x_{2}\right)$ and $\pi\left(x_{1}\right)$ determine the location of characteristic points $A$ and $B$ on torus model; Points $A, B$ and two geodesics between them define a trajectory on torus surface; Rotating points $A$ and $B$ defines circle- $A$ and circle- $B$ along with a set of discrete trajectories on torus model.

The sinusoidal oscillation along trajectory path is represented by a term in two ad hoc equations. Fig. 8.3 shows two orthogonal differential vectors $\rho d \varphi$ and $a_{2}^{\prime} d \theta$ :

$$
\begin{aligned}
& \frac{\rho d \varphi}{a^{\prime}{ }_{2} d \theta}=\frac{\sqrt{d^{2}-a_{2}^{\prime 2}}}{a_{2}^{\prime}}, \\
& \rho=d+a_{2}^{\prime} \cos \theta .
\end{aligned}
$$

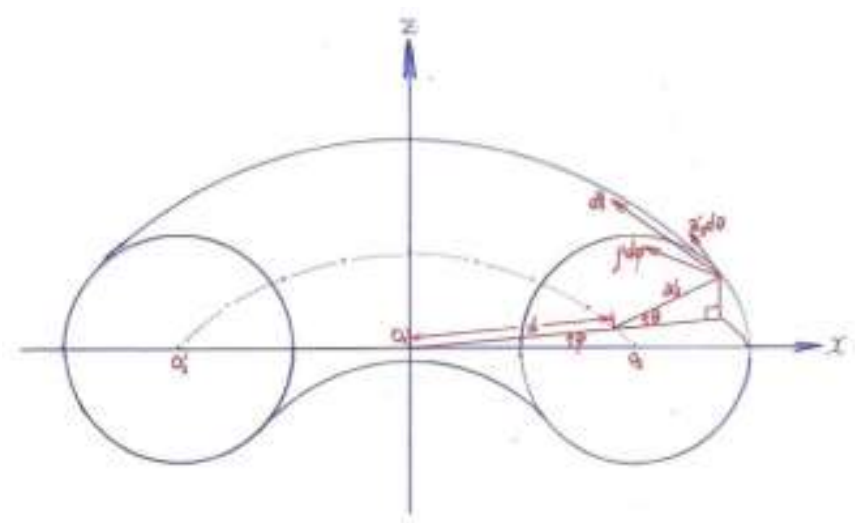

Fig. 8.3 Differential vectors on torus model.

The oscillation on trajectory is represented by a sinusoidal term:

$$
\begin{aligned}
& a_{2}^{\prime} \sin (\alpha \theta), \\
& \alpha=\frac{2 p}{m}=2\left(\frac{p}{n}\right)\left(\frac{n}{m}\right)=2\left(\frac{M}{M_{e}}\right)\left(\frac{L_{2}}{L_{1}}\right) .
\end{aligned}
$$

In which, $M$ and $M_{e}$ are the mass of the particle and electron, respectively. For electron $M / M_{e}=p / n=1$, $L_{2} / L_{1}=n / m=1 / 2$ and $\alpha=1,(8.20 \mathrm{a})$ becomes:

$$
a_{2}^{\prime} \sin (\alpha \theta)=a_{2}^{\prime} \sin (\theta) \text {. }
$$

As shown by (8.20), the sinusoidal oscillation term $a_{2}^{\prime} \sin (\alpha \theta)$ is related to mass, it is called the "mass term". Adding the mass term of $(8.20 \mathrm{c})$ to the numerator on right side of (8.19a) yields:

$$
\begin{aligned}
\frac{\rho d \varphi}{a_{2}^{\prime} d \theta} & =\frac{\sqrt{d^{2}-a_{2}^{\prime 2}}+a_{2}^{\prime} \sin \theta,}{a_{2}^{\prime}}, \\
\text { or } \quad \rho d \varphi & =\left[\sqrt{d^{2}-a_{2}^{\prime 2}}+a_{2}^{\prime} \sin \theta\right] d \theta .
\end{aligned}
$$

According to Fig. 8.3 and (8.21b), the combined differential vector length is:

$$
d l=\sqrt{\left(a_{2}^{\prime} d \theta\right)^{2}+(\rho d \varphi)^{2}}=\sqrt{a_{2}^{\prime 2}+\left[\sqrt{d^{2}-a_{2}^{\prime 2}}+a_{2}^{\prime} \sin \theta\right]^{2}} d \theta .
$$

Take the integral of (8.22) from 0 to $2 \pi$ : 


$$
L=\int_{0}^{2 \pi} d l=\int_{0}^{2 \pi} \sqrt{a_{2}^{\prime 2}+\left[\sqrt{d^{2}-a_{2}^{\prime 2}}+a_{2}^{\prime} \sin (\theta)\right]^{2}} d \theta .
$$

According to (8.21b) and (8.19b), the differential angle along the $\varphi$-direction is:

$$
d \varphi=\frac{\sqrt{d^{2}-a_{2}^{\prime 2}}+a_{2}^{\prime} \sin \theta}{\rho} d \theta=\frac{\sqrt{d^{2}-a_{2}^{\prime 2}}+a_{2}^{\prime} \sin \theta}{d+a_{2}^{\prime} \cos \theta} d \theta .
$$

Take the integral of $d \varphi$ from 0 to $2 \pi$ :

$$
\varphi=\int_{0}^{2 \pi} d \varphi=\int_{0}^{2 \pi} \frac{\sqrt{d^{2}-a_{2}^{\prime 2}}+a_{2}^{\prime} \sin \theta}{d+a_{2}^{\prime} \cos \theta} d \theta
$$

Definition 8.1: Define the Angle Tilt $(A T)$ equation and the Phase Sync $(P S)$ equation as:

1. AT-equation:

$$
\frac{1}{2 \pi d} \int_{0}^{2 \pi} \sqrt{a_{2}^{\prime 2}+\left[\sqrt{d^{2}-a_{2}^{\prime 2}}+a_{2}^{\prime} \sin \theta\right]^{2}} d \theta-\frac{d}{2 a_{2}^{\prime}}=0
$$

2. PS-equation:

$$
\frac{1}{2 \pi} \int_{0}^{2 \pi} \frac{\sqrt{d^{2}-a_{2}^{\prime 2}}+a_{2}^{\prime} \sin \theta}{d+a_{2}^{\prime} \cos \theta} d \theta-1=0 .
$$

In (8.26a), the factor 2 in the denominator of second term comes from Section 3:

$$
\left|\frac{1}{\cos ( \pm \vartheta)}\right|=\left|\frac{1}{\cos \left( \pm 120^{\circ}\right)}\right|=2 \text {. }
$$

$\vartheta= \pm 120^{\circ}$ is the separation angle of three lines on the complex plane shown in Fig.3.2. yields:

For $d=1$, solving the two equations of (8.26) for $a_{2}^{\prime}$

$$
a_{2}^{\prime}=0.491817117322215 \text {. }
$$

$A T$ and $P S$ are two independent equations with one unknown $a_{2}^{\prime}$. Both equations are satisfied simultaneously with the same solution $a_{2}^{\prime}=0.491817117322215$. It indicates that they are self consistent and mean something.

$a_{2}^{\prime}<a_{2}$ means that, the torus original circular cross section is distorted. To keep loop lengths ratio $L_{2} / L_{1}=n / m$ unchanged, the original cross section parameters, $a_{2}$ and $b_{2}=a_{2}$ must be changed accordingly, which makes the torus cross section elliptical.

Definition 8.2: The Modification Factors $(M F)$ of the $f$ modification are defined as:

$$
\begin{aligned}
& f_{a}=\frac{a_{2}^{\prime}}{a_{2}}, \\
& f_{b}=\frac{b_{2}^{\prime}}{b_{2}} .
\end{aligned}
$$

For electron, $a_{2}=0.5, b_{2}=0.5, a_{2}^{\prime}=0.491817117322215$, and $b_{2}^{\prime}$ is determined by:

$$
\begin{aligned}
& \int_{0}^{2 \pi} \sqrt{\left(a_{2}^{\prime}\right)^{2} \sin (t)+\left(b_{2}^{\prime}\right)^{2} \cos ^{2}(t)} d t=\int_{0}^{2 \pi} \sqrt{a_{2}^{2} \sin ^{2}(t)+b_{2}^{2} \cos ^{2}(t)} d t \\
& b_{2}^{\prime}=0.508116460019755 .
\end{aligned}
$$

Explanation: In essence, the $f$-modification is introduced to satisfy (8.26) and to keep loop-2 length $L_{2}$ unchanged as shown by (8.29a). It is important to keep loop length ratio $L_{2} / L_{1}=n / m$ unchanged, because it is related to interactions.

According to Definition 8.2, the modification factors of electron are calculated as:

$$
\begin{aligned}
& f_{a}=\frac{a_{2}^{\prime}}{a_{2}}=0.9836342346444303, \\
& f_{b}=\frac{b_{2}^{\prime}}{b_{2}}=1.01623292003951 .
\end{aligned}
$$

After the $f$-modification, the geometrical parameters are changed accordingly. The rules are to keep the initial phase angles unchanged as the originals:

$$
\begin{aligned}
& \xi_{2}^{\prime}=\xi_{2}, \\
& \phi_{3}^{\prime}=\phi_{3}, \\
& \beta_{1}^{\prime}=\beta_{1}, \\
& \phi_{0}^{\prime}=\phi_{0} .
\end{aligned}
$$

The other geometrical parameters of the modified torus model change accordingly. The rules are: (1) To keep the initial phase angles given by (8.31) unchanged; (2) The torus cross section becomes elliptical with $a_{2}^{\prime}$ and $b_{2}^{\prime}$ given by (8.27) and (8.29b), respectively. The rest is from geometry.

The modified point $\mathrm{A}_{2}^{\prime}$ and triangle $\mathrm{A}_{2}^{\prime} \mathrm{O}_{1} \mathrm{O}_{2}$ related angles are determined by:

$$
\begin{aligned}
& \psi_{2}^{\prime}=180^{\circ}-\xi_{2}^{\prime}=180^{\circ}-\xi_{2}, \\
& \tan \phi_{2}^{\prime}=\frac{Z_{2}^{\prime}}{d+X_{2}^{\prime}}, \theta_{2}^{\prime}=\xi_{2}^{\prime}-\phi_{2}^{\prime}, \\
& X_{2}^{\prime}=\frac{1}{\sqrt{\left(1 / a_{2}^{\prime}\right)^{2}+\left(1 / b_{2}^{\prime}\right)^{2} \tan ^{2}\left(\xi_{2}^{\prime}\right)}}, \\
& Z_{2}^{\prime}=X_{2}^{\prime} \tan \xi_{2}^{\prime} .
\end{aligned}
$$

The modified point $G^{\prime}$ and triangle $G^{\prime} O_{1} O_{2}$ related angles are determined by:

$$
\begin{aligned}
& \tan \xi_{3}^{\prime}=\frac{Y_{3}^{\prime}}{X_{3}^{\prime}-d}, \psi_{3}^{\prime}=180-\xi_{3}^{\prime}, \\
& \phi_{3}^{\prime}=\phi_{3}, \theta_{3}^{\prime}=\xi_{3}^{\prime}-\phi_{3}^{\prime}, \\
& X_{3}^{\prime}=\frac{d+a_{2}^{\prime}}{\sqrt{1+\tan ^{2}\left(\phi_{3}^{\prime}\right)}}, \\
& Y_{3}^{\prime}=X_{3}^{\prime} \tan \phi_{3}^{\prime} .
\end{aligned}
$$

The modified point $B_{1}^{\prime}$ at $x=X_{1}^{\prime}, y=Z_{1}^{\prime}$ with origin at $O^{\prime}{ }_{2}$ and triangle $B_{1}^{\prime} D^{\prime} O^{\prime}{ }_{2}$ related angles are determined by:

$$
\begin{aligned}
& \tan \gamma_{1}^{\prime}=\frac{Z_{1}^{\prime}}{a_{2}^{\prime}-X_{1}^{\prime}}, \\
& \beta_{1}^{\prime}=\beta_{1}, \alpha_{1}^{\prime}=180^{\circ}-\beta_{1}^{\prime}-\gamma_{1}^{\prime}, \\
& \tan \phi_{1}^{\prime}=\frac{Z_{1}^{\prime}}{d-X_{1}^{\prime}}, \\
& X_{1}^{\prime}=\frac{1}{\sqrt{\left(1 / a_{2}^{\prime}\right)^{2}+\left(1 / b_{2}^{\prime}\right)^{2} \tan ^{2}\left(\beta_{1}^{\prime}\right)}}, \\
& Z_{1}^{\prime}=X_{1}^{\prime} \tan \beta_{1}^{\prime} .
\end{aligned}
$$

The modified point $D^{\prime}$ at $x=-X_{0}^{\prime}, y=-Y^{\prime}{ }_{0}$ with origin at $O_{1}$ and triangle $D^{\prime} E^{\prime} O_{2}^{\prime}$ related angles are determined by: 
Z. Y. SHEN

$$
\begin{aligned}
& \tan \beta_{0}^{\prime}=\frac{Y_{0}^{\prime}}{d-X_{0}^{\prime}}, \\
& \tan \xi_{0}^{\prime}=\frac{Y_{0}^{\prime}}{R-X_{0}^{\prime}}, \quad \gamma_{0}^{\prime}=180^{\circ}-\xi_{0}^{\prime}, \\
& \alpha_{0}^{\prime}=\xi_{0}^{\prime}-\beta_{0}^{\prime}, \\
& \phi_{0}^{\prime}=\phi_{0}, \\
& X_{0}^{\prime}=\frac{R}{\sqrt{1+\tan ^{2}\left(\phi_{0}^{\prime}\right)}}, \\
& Y_{0}^{\prime}=X_{0}^{\prime} \tan _{\phi_{0}} .
\end{aligned}
$$

\begin{tabular}{|c|c|c|c|}
\hline \multicolumn{4}{|c|}{ Electron (Modified as Effective) } \\
\hline \multicolumn{4}{|c|}{$\begin{array}{l}\text { Model: Tous } \\
\text { Symbol: e, Mass: 0.510996911uer, }\end{array}$} \\
\hline \multicolumn{4}{|c|}{ Weinberg angle: $\theta_{\eta}=\theta_{2}=28.47948454^{\circ}$} \\
\hline \multicolumn{4}{|c|}{ Original usmerical parameters, $\mathrm{n}=2, n=1, p=1$} \\
\hline \multicolumn{4}{|c|}{ Reduced mumerical parameters ${ }^{* *}, m=1, n=1 / 2, p=1 / 2$} \\
\hline \multicolumn{4}{|c|}{ Modification factor. $f_{2}=0.98363423, f_{b}=1.01623292$} \\
\hline \multicolumn{2}{|c|}{ Original: } & \multicolumn{2}{|c|}{$a_{2}=0.5, b_{2}=0.5$} \\
\hline \multicolumn{2}{|c|}{ Modified as effective: } & \multicolumn{2}{|c|}{$a_{2}^{\prime}=0.49181712, b_{2}=0.50811646$} \\
\hline \multicolumn{2}{|c|}{$\begin{array}{l}\text { Tonas inner-half (negative curvature) } \\
x_{1}=0.125, r\left(x_{1}\right)=3.87710292 \\
R=0.50818288 \\
A_{n}=0.52342698, A=0.21505573\end{array}$} & \multicolumn{2}{|c|}{$\begin{array}{l}\text { Tonus outer-haif (positive curvature) } \\
x_{2}=0.375, z\left(x_{2}\right)=2.8645925\end{array}$} \\
\hline $\begin{array}{l}\text { Triangie } B_{1}^{\prime} D^{\prime} O_{2}^{\prime} \text { on } \\
O_{1} H^{\prime} \text { cross section }\end{array}$ & $\begin{array}{l}\text { Triangle } D E^{\prime} O_{1} \text { on } \\
x-y \text { cross section }\end{array}$ & $\begin{array}{l}\text { Triangle } A_{2}^{\prime}, O, O, \text { on } \\
O_{1} G^{\prime} \text { Cross Section }\end{array}$ & $\begin{array}{l}\text { Triangle } G^{\prime} O_{1} O_{2} \text { on } \\
x-y \text { Cross Section }\end{array}$ \\
\hline$b_{1}=0.41086101$ & $a_{1}=0.41086101$ & $X_{2}^{\prime}=0.36939205$ & $X_{3}=1.10436775$ \\
\hline$X_{1}^{\prime}=0.28545244$ & $X_{0}^{\prime}=0.2396076$ & $Z_{2}^{\prime}=0.33546647$ & $T_{3}=1.00294077$ \\
\hline$Z_{1}^{\prime}=0.41303564$ & $Y_{y}^{\prime}=0.41758506$ & & \\
\hline$a_{1}^{\prime}=61.17939213^{\circ}$ & $\alpha_{0}^{\prime}=31.92323081^{2}$ & $\theta_{1}=28.47948454^{2}$ & $\theta_{1}=41.81466473^{2}$ \\
\hline$\beta_{1}^{\prime}=55.257547^{z}$ & $\beta_{0}^{\prime}=30.44799569^{2}$ & $\phi_{2}^{\prime}=13.76493556^{2}$ & $\phi_{3}^{\prime}=42.24442009^{\prime}$ \\
\hline$\gamma_{\mathrm{L}}^{\prime}=63.56306087^{\circ}$ & $y_{0}^{\prime}=117.6287735^{2}$ & $\psi_{2}^{\prime}=137.75557991^{2}$ & $\psi_{3}^{\prime}=9594091518^{\circ}$ \\
\hline$\phi_{1}=30.06432177^{2}$ & $\phi_{0}^{\prime}=55.257547^{2}$ & $\xi_{2}=42.24442009^{=}$ & $\xi_{3}=84.05908482^{2}$ \\
\hline
\end{tabular}

The modified data for electron are listed in Table 8.2. In which the effective parameters after $f$-modification are marked with ' sign.

Table 8.2: Modified Parameters for Electron Torus Model

* All data are from 16-digit numerical calculations, only 8-digit after the decimal point is presented.

** The reduced numerical parameters are the original numerical parameters divided by $m$.

After modification, despite the change of $\theta_{W}^{\prime}=\theta_{2}^{\prime}=28.47948454^{\circ}$ from original $\theta_{W}=\theta_{2}=28.45987086^{\circ}$, as shown in Table 8.2, three out of four synchronizations still hold with one slightly off:

$$
\begin{aligned}
& \xi_{2}^{\prime}=\phi_{3}^{\prime}=42.24442009^{\circ}, \\
& \theta_{2}^{\prime}=\xi_{2}^{\prime}-\phi_{2}^{\prime}=\phi_{3}^{\prime}-\phi_{2}^{\prime}=28.47948454^{\circ}, \\
& \phi_{0}^{\prime}=\beta_{1}^{\prime}=55.257547^{\circ}, \\
& \phi_{1}^{\prime}=30.06432177^{0} \approx \beta_{0}^{\prime}=30.44799569^{\circ} .
\end{aligned}
$$

It indicates that electron stability is persistent and robust.

To understand the meaning of $f$-modification, in the $A T$ equation, let's set the mass term $a_{2}^{\prime} \sin \theta=0$ to see its effect, (8.26a) and (8.28) become:

$$
\begin{aligned}
& \frac{1}{2 \pi d} \int_{0}^{2 \pi} \sqrt{a_{2}^{\prime 2}+\left[\sqrt{d^{2}-a_{2}^{\prime 2}}\right]^{2}} d \theta-\frac{d}{2 a_{2}^{\prime}}=\frac{d}{2 \pi d} \int_{0}^{2 \pi} d \theta-\frac{d}{2 a_{2}^{\prime}}=1-\frac{1}{2 a^{\prime}{ }_{2}}=0 \\
& a_{2}^{\prime}=0.5 d=0.5=a_{2}, b_{2}^{\prime}=0.5=b_{2} ; f_{a}=f_{b}=1 .
\end{aligned}
$$

$f_{a}=f_{b}=1$ means no $f$-modification. It clearly shows that the effect of $f$-modification is caused by the added mass term of $a_{2}^{\prime} \sin \theta$, which represents the mass effect.

In the standard model, particle acquires mass through symmetry broken. Likewise, in SQS theory, the mass term of $a_{2}^{\prime} \sin \theta$ breaks the 3 -fold symmetry with $\vartheta=120^{\circ}$ on the complex plane. This analogue plus the simultaneous satisfaction of two independent equations with the same solution $a_{2}^{\prime}$ give some legitimacy to $A T$-equation and $P S$ equation despite their ad hoc nature.

Let's look at the geometrical meaning of the $f$ modification. As shown in Section 3, the angle separates three lines on complex plane is:

$$
\vartheta=\frac{2 \pi}{3}=120^{\circ} \text {. }
$$

The $f$-modification causes the angle having a slight tilt from $\vartheta$ to $\vartheta^{\prime}$ :

$\frac{\cos \vartheta^{\prime}}{\cos \vartheta}=\frac{\cos \left(180^{\circ}-\arctan \left(\frac{\sqrt{d^{2}-a_{2}^{\prime 2}}}{a_{2}^{\prime}}\right)\right)}{\cos \left(120^{\circ}\right)}=0.9836342346444315=f_{a}$

$\vartheta^{\prime}=180^{\circ}-\arctan \left(\frac{\sqrt{d^{2}-a_{2}^{\prime 2}}}{a_{2}^{\prime}}\right)=119.460085550422^{\circ}$,

$\Delta \vartheta=\vartheta-\vartheta^{\prime}=0.539914449578^{\circ}$.

$\Delta \vartheta$ is the tilting angle deviated from $\vartheta=120^{\circ}$. It indicates that, original $\vartheta=120^{\circ}$ 3-fold symmetry is slightly broken by tilting angle $\Delta \vartheta$ for electron having mass.

After $f$-modification, $A T$-equation and $P S$-equation are satisfied simultaneously. It indicates that, the two cyclic movements of two loops and the sinusoidal oscillation along the trajectory are synchronized perfectly for electron as a stable particle.

Numerical calculations found that, $A T$-equation of (8.26a) has only one root $a_{2}^{\prime}$ given by (8.27) with the $f_{a}$ value given by $(8.30 \mathrm{a})$. On the other hand, $P S$-equation of $(8.26 \mathrm{~b})$ has a series of roots. Start from $f_{a}=0.9836342346444303$, varying its value with $10^{-16}$ steps calculate the values of $\phi\left(f_{a}\right)$ as a function of $f_{a}$ :

$$
\begin{aligned}
& \phi\left(f_{a}\right)=\frac{1}{2 \pi} \int_{0}^{2 \pi} \frac{\sqrt{d^{2}-a_{2}^{\prime 2}}+a_{2}^{\prime} \sin \theta}{d+a_{2}^{\prime} \cos \theta} d \theta-1, \\
& a_{2}^{\prime}=f_{a} a_{2}=0.5 f_{a} .
\end{aligned}
$$

A sample of numerical calculated results are listed in Table 8.3.

In Table 8.3, $\left[\delta \phi=\phi-\phi_{0}\right]=0$ means phase precisely synchronized, and $\left[\delta \phi=\phi-\phi_{0}\right] \neq 0$ means off sync. The 
results of Table 8.3 are interpreted as that, electron's torus model is dynamic and stochastic in nature. It changes its loop-2 tilting angle constantly corresponding to different $f_{a}$, $f_{b}$ and $a_{2}^{\prime}, b_{2}^{\prime}$ values representing different torus surfaces. Electron's trajectory changes accordingly. The tilting angle changes discretely, so does the trajectory, which means that trajectories are quantized. At a given time, electron is represented by a trajectory on a torus surface. As time passing by, it jumps to other trajectories on another torus surface. It is a stochastic scenario of jumping trajectories on different torus surfaces. Physically, it corresponds to interactions such as emitting and absorbing a photon. As mentioned previously, jumping trajectories on the same torus surface corresponds to emitting and absorbing a virtual photon by electron.

Table 8.3: Some Roots of Equation (8.26b)

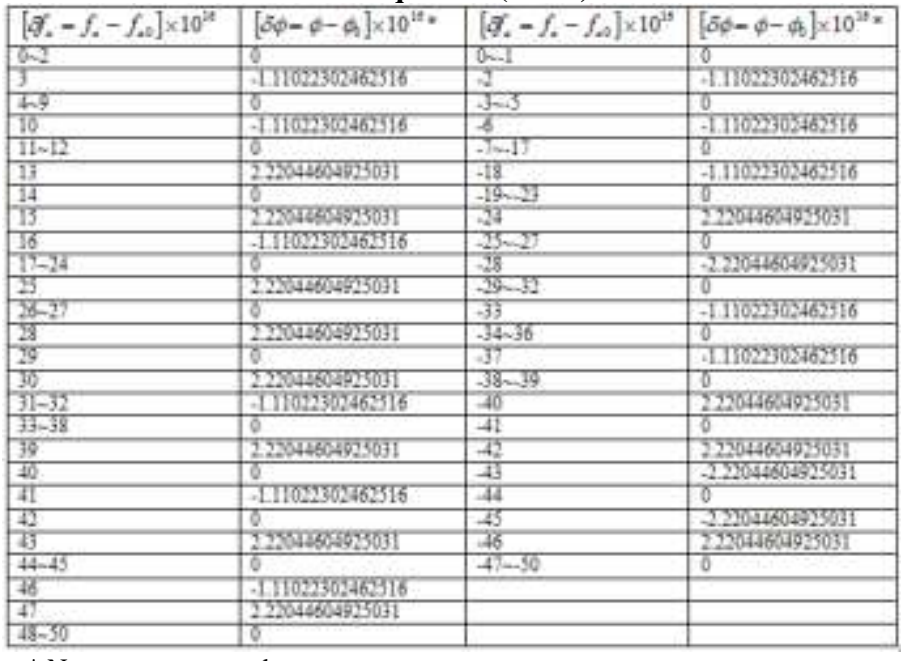

* Note: $\phi=\phi\left(f_{a}\right)$ and $\phi_{0}=\phi\left(f_{a 0}=0.9836342346444303\right)$.

As shown in Table 8.3, $P S$-equation has 23 roots in region $\delta f_{a}=0 \rightarrow 5 \times 10^{-15}$ corresponding root density of:

$$
D=\frac{23}{5 \times 10^{-15}}=4.6 \times 10^{15} \text {. }
$$

The root density $D$ roughly kept constant in the effective region $\left[f_{a}, 1\right]$. For orders of magnitude estimation, the total number of roots for $P S$-equation in region $\left[f_{a}, 1\right]$ is:

$$
N^{\prime}=\left.\Delta f_{a}\right|_{0.984 \rightarrow 1} \times D=(1-0.9836) \times\left(4.6 \times 10^{15}\right)=7.544 \times 10^{13} \text {. }
$$

Since the root density $D$ is roughly the same in the other effective region $\left[1,1+\left(1-f_{a}\right)\right]$, the total number of roots for $P S$-equation in region $\left[\left(1-f_{c}\right), 1+\left(1-f_{a}\right)\right]$ is:

$$
N=\left.2 \Delta f_{a}\right|_{0.984 \rightarrow 1} \times D=1.509 \times 10^{14} .
$$

As mentioned previously, there is a set of discrete trajectories on the same surface of a torus surface. Now on top of it, there is another set of discrete trajectories on $N=7.544 \times 10^{13}$ different torus surfaces caused by $f$ modifications. At a given time, the real trajectory is the one randomly chosen from these two sets of discrete trajectories.
In other words, electron trajectories are dynamic and stochastic in nature, which spread like clouds around the torus surfaces. The term "electron clouds" was used to describe electron's behavior around a nucleon according to quantum mechanics wave function. Here the clouds appear in a deeper level, which should not be a surprise.

As shown in Fig. 3.4 of Section 3, the loop on the complex plane connecting $x_{1}$ and $x_{2}$ has many different paths with the same loop length. That scenario is consistent with the different trajectories with the same length on different torus surfaces and different locations. It shows the consistency of the theory.

In Table 8.3, the step of $\delta f_{a}$ variations and step of $\delta a^{\prime}{ }_{2}$ and $\delta b_{2}^{\prime}$ variations are in the order of $10^{-16}$ to $10^{-15}$ Planck length corresponding to $10^{-51}$ to $10^{-50}$ meters. The step of torus surface variations is extremely tiny. As the torus' loop2 tilts, the electron's trajectory jumps from one torus surface to the other. In fact, this dynamic picture is expected from quantum theory. The three types of movement for electron described in this section all are deterministic in nature. Without trajectory jumping, the deterministic movements are contradictory to the uncertainty principle. Moreover, the Gaussian Probability Postulation of SQS theory is stochastic in the first place. The trajectory jumping is ultimately originated from the Gaussian probability assigned to discrete points in space. The $\left[\delta \phi=\phi-\phi_{0}\right]$ fluctuating data listed in

Table 8.3 is an indication of the stochastic nature of SQS theory, even though the $P S$-equation of $(8.26 \mathrm{~b})$ is not derived from the first principle.

Fig 8.4 shows the right side of Fig. 8.1b in details. Points $A, F, O_{2}$ define a right triangle $\mathrm{AFO}_{2}$, which contains two additional right triangles: $A F K$ and $\mathrm{FKO}_{2}$. The triangle $A \mathrm{FO}_{2}$ is identified as the Glashow-Weinberg-Salam triangle, $G W S$-triangle for short. In the $c=\hbar=1$ unit system, the sides of $G W S$-triangle are related to electroweak coupling parameters:

$$
F K=e, A F=g, F O_{2}=g^{\prime}, A O_{2}=\sqrt{g^{2}+g^{\prime 2}} .
$$

$e$, and $g, g^{\prime}$ are electric charge and two weak coupling constants, respectively. The following formulas are from geometry:

$$
\begin{aligned}
& \sin \theta_{W}=\frac{e}{g}=\frac{g^{\prime}}{\sqrt{g^{2}+g^{\prime 2}}}, \\
& \cos \theta_{W}=\frac{e}{g^{\prime}} .
\end{aligned}
$$

Combining (8.43a) and (8.43b) yields:

$$
\sin \theta_{W} \cos \theta_{W}=\frac{e}{\sqrt{g^{2}+g^{\prime 2}}} .
$$

Formula (8.44) is used extensively in later sections as the criterion to construct the model for other fermions.

According to 16-digit numerical calculation, the original and effective Weinberg angles of electron are: 
Z. Y. SHEN

Original: $\quad \theta_{W e \mathbf{O}}=\theta_{2}=28.4598708641138^{\circ}$,

Effective: $\theta_{W e M}=\theta_{2}^{\prime}=28.479484537808^{\circ}$.

One of SQS theory final goals is that, all parameters of an elementary particle should be derived from its model. To identify the GWS-triangle with Weinberg angle in the torus model is a step toward the final goal. Some other characteristic triangles will be introduced in later sections.

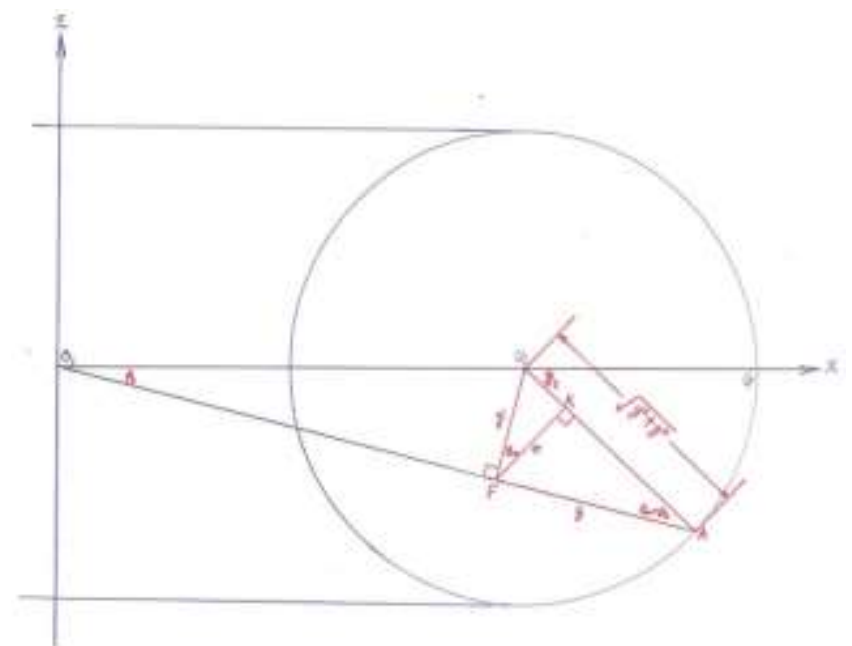

Fig. 8.4 Glashow-Weinberg-Salam triangle.

From Einstein's unified field theory viewpoint, everything including all elementary particles and interactions are originated from geometry. For SQS theory, the model plays that role. Torus as a genus-1 topological manifold has one center hole, its shape and size are arbitrary to begin with. In order for the torus model to represent a particle with its parameters, additional steps must be taken. Take electron as an example. As the first step, the shape and dimensions of torus are determined by loop-2 to loop-1 length ratio of $L_{2} / L_{1}=n / m=1 / 2$ and $L_{1}=2 \pi d$. The second step is to fix the locations of characteristic points $A$ and $B$ on torus surface by utilizing the curvature information carried by $\pi\left(x_{2}\right)$ and $\pi\left(x_{1}\right)$ from the $S$-equation. In this way, the triangles such as the $G W S$-triangle are determined and the parameters are determined as well. The process shows mathematics at work. The mathematics at work viewpoint will be enhanced further in later sections.

Recall in Section 3, the four tiny holes $h_{1}, h_{\infty}, h_{\omega}, h_{\omega^{2}}$ served as four branch points $1, \infty, \omega, \omega^{2}$ on the Riemann surface. Moreover, the way Penrose built the torus is to glue a pair of slits on two sheets of Riemann surface together [2]. In fact, there are infinite sheets of Riemann surface corresponding to a general form of (3.23):

$$
\begin{aligned}
& z=\sigma_{0}=e^{i n 2 \pi}, z=\omega=\sigma_{1}=e^{i\left(\frac{2 \pi}{3}+n 2 \pi\right)}, z=\omega^{2}=\sigma_{2}=e^{i\left(\frac{4 \pi}{3}+n 2 \pi\right)}, \\
& n=0, \pm 1, \pm 2, \pm 3 \cdots .
\end{aligned}
$$

These sheets can be combined into pairs to build many genus-1 torus surfaces, which serve as the topological base of many torus surfaces with slightly different parameters $a_{2}^{\prime}$ and $b^{\prime}{ }_{2}$ derived from $P S$-equation as discussed earlier. After all, there are enormous numbers of torus surfaces provided by (8.46) for trajectory to jump on. This argument gives more credit to the ad hoc $P S$-equation.

Moreover, the torus with four tiny holes shown in Fig. $8.5 \mathrm{a}$ is topologically equivalent to a pair of trousers with a large hole in their waistband shown in Fig. 8.5b. The four tiny holes on torus with their edge extended outwards form four tubes as the four ports. According to [11], if the loops around trousers shrink to points, the trousers with four ports degenerate to a Feynman diagram with one closed loop and four branch lines shown in Fig. 8.5c. Feynman diagram is correlated to interactions. Therefore, the triangles such as $G W S$-triangle defined by characteristic points carry interactions information are natural.

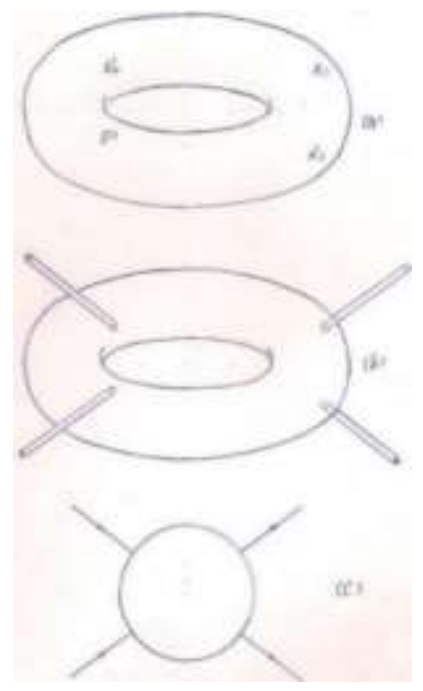

Fig. 8.5 (a) Torus with four tiny holes; (b) Four tiny holes' edge extended into four tubes; (c) Degenerated into a Feynman diagram with one loop.

In summary, electron's torus model is built on three bases:

1. Loop lengths ratio $L_{2} / L_{1}=n / m=1 / 2$ and masses ratio $M / M_{e}=p / n=1 / 1$ are determined by a set of three numerical parameters, $m=2, n=1, p=1$.

2. The 3-dimensional Gaussian probability's $\sigma_{0}, \sigma_{1}, \sigma_{2}$ plus $\infty$ are identified as four branch points on the Riemann surface, which are topologically equivalent to four tiny holes on torus.

3. The four tiny holes on torus correspond to characteristic points $A, B$ and $A^{\prime}, B^{\prime}$. Their locations are fixed according to the information carried by $\pi\left(x_{1}\right)$ and $\pi\left(x_{2}\right)$, which are the solutions of the 1dimensional $S$-equation.

In the three bases, No.2 and No.3 are originated from SQS theory first fundamental postulation, the Gaussian Probability Postulation. No.1 is a set of three numerical parameters. It is related to the second fundamental postulation of SQS theory, which will be introduced in later 
section. These are the only things needed to build the model for a particle such as electron to carry all its parameters. It shows the power and the simplicity of the first principle of SQS theory.

The electron torus model introduced in this section serves as the basic building block. It is not the final version. The details will be given in Section 12 .

\section{Section 9: Complex $\dot{x}$-Plane and Fine Structure Constant}

$D S_{k}(k)$ of (7.1) is the Fourier transformation of $D S(x)$ of (6.1):

$$
D S_{k}(k)=\frac{1}{4 \pi} e^{-\frac{k^{2}}{4 \pi}}\left\{\sum_{j=-\infty}^{\infty}\left[e^{i j k}+e^{-i(j-0.5) k}\right]\right\}-\delta(k) .
$$

$D S_{k}(k)$ serves as particles spectrum. The local minimums of $\left|D S_{k}(k)\right|$ correspond to fermions and the local maximums of $\left|D S_{k}(k)\right|$ correspond to bosons. In this section, $D S_{k}(k)$ is extended as $E D S_{k}(k)$. Then $E D S_{k}(k)$ is Fourier transformed back to the complex $\dot{x}$-plane and compared with $D S(x)$ to find some physics implications.

Definition 9.1: Define the $E D S_{k}(k)$ function as the extension of $D S_{k}(k)$ function

$$
E D S_{k}(k)=\frac{1}{4 \pi} e^{-\frac{k^{2}}{4 \pi}}\left\{\sum_{j=-\infty}^{\infty}\left[e^{i k}+e^{-i(j-0.5) k}\right]\right\}-e^{-\frac{k^{2}}{4 \pi}} \sum_{j=-\infty}^{\infty}\left[\delta\left(k-4 j^{\prime} \pi\right)+\delta\left(k-4\left(j^{\prime}-0.5\right) \pi\right)\right] .
$$

Explanation: In the $\operatorname{EDS}_{k}(k)$, the original term $\delta(k)$ in $D S_{k}(k)$ of (7.1) is extended by the second summation terms with two sets of $\delta$-functions. The $j^{\prime}=0$ term in the second summation, $\left.\delta\left(k-4 j^{\prime} \pi\right)\right|_{j^{\prime}=0}=\delta(k)$ is the original delta function $\delta(k)$ in $D S_{k}(k)$, and all the other terms in the second summation are newly added delta functions. The extension adds a series of additional local maximums for $\left|E D S_{k}(k)\right|$ representing bosons.

Look at (9.1) closely, the added $\delta$-functions also affect fermions in (7.1). For instance, $k=2 \pi(k=k / 2 \pi=1)$ in $D S_{k}(k)$ is a root of $\left|D S_{k}(k)\right|=0$ represents electron as a fermion. In $E D S_{k}(k)$, the $j^{\prime}=1, k=2 \pi$ term

$$
\begin{aligned}
& \left.\delta\left(k-4\left(j^{\prime}-0.5\right) \pi\right)\right|_{j^{\prime \prime}=1, k 2 \pi}=\delta(0) \rightarrow \infty \text { causes } \\
& \left|E D S_{k}(k=2 \pi)\right|_{j^{\prime \prime}=1}=\left|E D S_{k}(k=1)\right|_{j^{\prime \prime}=1} \rightarrow \infty . \text { It represents a boson. }
\end{aligned}
$$

Using Fourier transform to transfer $\operatorname{EDS}_{k}(k)$ back to the complex $\dot{x}$-plane:

$$
E D S_{\dot{x}}(x)=\int_{-\infty}^{\infty} E D S_{k}(k) e^{-i k x} d k
$$

Substituting (9.1) into (9.2) yields the $E D S_{\dot{x}}(x)$-function on the complex $\dot{x}$-plane:

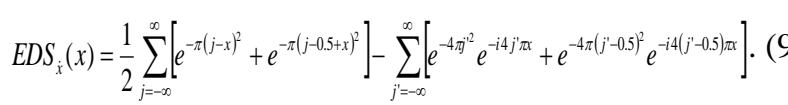

In the $\operatorname{EDS}_{\dot{x}}(x)$, the first summation is $D S(x)+1$ as expected; the second summation includes the unitarity term: $\left\lfloor e^{-4 \pi j^{\prime 2}} e^{-i 4 j^{\prime} \pi x}\right]_{j^{\prime}=0}=1$. The other terms in the second summation correspond to bosons representing interactions, which are originated from delta functions added in $\operatorname{EDS}_{k}(k)$.

Numerical calculations found that:

In general on $\dot{x}$-plane:

$$
E D S_{\dot{x}}(x) \approx-E D S_{\dot{x}}(0.5-x) .
$$

On the real $x$-axis:

$$
\operatorname{EDS}_{\dot{x}}(x) \approx 1-S(x) \text {. }
$$

Errors of approximations are around $10^{-15}$ and $10^{-5}$ for (9.4a) and $(9.4 \mathrm{~b})$, respectively.

Definition 9.2: Define the $S S$-function and $S S$-equation on the complex $\dot{x}$-plane as:

$S S(x)=E D S_{\dot{x}}(x)+E D S_{\dot{x}}(0.5-x)$

$$
\begin{aligned}
& =\frac{1}{2} \sum_{j=-\infty}^{\infty}\left[e^{-\pi(j-x)^{2}}+e^{-\pi(j-0.5+x)^{2}}\right]-\sum_{m=-\infty}^{\infty}\left[e^{-4 \pi g^{2}} e^{-i 4 j \pi x}+e^{-4(j-0.5)^{2} \pi x} e^{-i 4(j-0.5)) x}\right] \\
& +\frac{1}{2} \sum_{j=-\infty}^{\infty}\left[e^{-\pi\left[[j-(0.5-x)]^{2}\right.}+e^{-\pi\left(j(j-0.5+(0.5-x))^{2}\right.}\right]-\sum_{j=-\infty}^{\infty}\left[e^{-4 \pi j^{2}} e^{-i 4 j \pi(0.5-x)}+e^{-4 \pi(j-0.5)^{2}} e^{-i 4(j-0.5) \pi(0.5-x)}\right]
\end{aligned}
$$

According to (9.4a) and (9.5a), $S S(x) \approx 0$. The values of $|S S(x)|$ fluctuate around $10^{-15} \sim 10^{-17}$ and occasionally equal to zero, which are the roots of $S S(x)=0$.

As shown in Section 6, $D S(x)=0$ is a real equation on the real $x$-axis. It has a root at $x_{1}=0.125$ on the $x$-axis corresponding to electron. On the other hand, $S S(x)=0$ is a complex equation and $x_{1}=0.125$ is not its root. Instead, a root of $S S(x)=0$ is found by numerical calculations at:

$$
\begin{aligned}
& \dot{x}_{1}=x_{1}^{\prime} e^{i \theta_{W}}, \\
& x_{1}^{\prime}=0.12408112557821315, \\
& \theta_{W}=28.4598708641138^{\circ} .
\end{aligned}
$$

$\theta_{W}=28.4598708641138^{\circ}$ is electron original Weinberg angle of (8.45a) before $f$-modification. $x_{1}^{\prime}=0.12408112557821315$ is slightly less than $x_{1}=0.125$. According to (6.19a), $x_{1}^{\prime}=0.12408112557821315$ correlates to the mass $M_{e}^{\prime}$ slightly less than $M_{e}$ :

$$
\frac{M_{e}^{\prime}}{M_{e}}=\frac{1}{8\left(0.25-x_{1}^{\prime}\right)} .
$$

As shown in Appendix-4, charged particle mass subjects to electromagnetic modification. According to (A4.5) and (9.7): $\frac{M_{e}^{\prime}}{M_{e}}=\frac{M_{e}-M_{E M}}{M_{e}}=1-\alpha=\frac{1}{8\left(0.25-x_{1}^{\prime}\right)}=0.992702647430687^{\circ}$ 
Z. Y. SHEN

In which $\alpha$ is "fine structure constant" of electron. Solving (9.8) for $\alpha$ yields:

$$
\alpha^{-1}=\left[1-\frac{1}{8\left(0.25-x_{1}^{\prime}\right)}\right]^{-1}=137.03599908345 .
$$

According to references [3,4], 2010-PDG (p.126) provides the experimental data:

$$
\alpha^{-1}=137.035999084(51) \text {. }
$$

The relative deviation of SQS theoretical value and 2010PDG data medium value is

$$
\delta=4.013 \times 10^{-12} .
$$

$|S S(x)|$ is also used for calculating the $\alpha^{-1}$ values for electron quantum states with fractional charges. According to (8.44) with assumption of $\sqrt{g^{2}+g^{\prime 2}}=$ const , the Weinberg angle $\theta_{W, F}$ for particles with fractional charges are determined by:

$$
\frac{\sin \theta_{W, F} \cos \theta_{W, F}}{\sin \theta_{W} \cos \theta_{W}}=F
$$

$F=1 / 3, F=2 / 3$, are for fractional charges, $e / 3,2 e / 3$, respectively. Formula (9.12) and $\theta_{W}=28.4598708641138^{\circ}$ are used to calculate the values of $\theta_{W, F}$.

The definition of fine structure constant $\alpha$ is:

$$
\alpha=\frac{e^{2}}{2 \varepsilon_{0} h c} .
$$

According to (9.13), $\alpha$ is proportion to $e^{2}$. For the electron states with fractional charges $e / 3,2 e / 3,(9.8)$ and (9.9) are changed accordingly as.

$$
\begin{aligned}
& 1-F^{2} \alpha=\frac{1}{8\left(0.25-x_{1}^{\prime}\right)} . \\
& \alpha^{-1}=F^{2}\left[1-\frac{1}{8\left(0.25-x_{1}^{\prime}\right)}\right]^{-1} .
\end{aligned}
$$

\begin{tabular}{|c|c|c|c|c|}
\hline Charge & $x_{1}^{2}$ & $\theta_{r}$ of $\theta_{r, r}$ & $\alpha^{-7}$ & $\delta^{*}$ \\
\hline$e$ & 0.12408112557821315 & $28.4598708641138^{\circ}$ & 137,03599908345 & $4.103 \times 10^{-12}$ \\
\hline e:3 & 0.1248985656362798 & $8.10928341940384^{\prime}$ & 137035999082998 & $7321 \times 10^{-2}$ \\
\hline 2673 & 0.124599327239738625 & $1697967408852128^{\circ}$ & 137.035999083326 & $4918 \times 10^{-12}$ \\
\hline
\end{tabular}

The SQS theoretical values of $\alpha^{-1}, \theta_{W}$ and $\theta_{W, F}$ for electron states with different charges from 16-digit numerical calculations are listed in Table 9.1.

Table 9.1: $\alpha, \theta_{W}, \theta_{W, F}$ for Electron with Different Charges

* Note: $\delta$ is the relative deviation from 2010-PDG medium value of

$$
\alpha^{-1}=137.035999084 \text {. }
$$

In fact, the electron fractional charge states did show up in the quantum Hall effect experiments.

The $\alpha$ effect on mass is originated from electromagnetic interaction. It is consistent with the fact that $D S(x)$ does not include interactions and $S S(x)$ does. It also explains why $x_{1}=0.125$ on the real $x$-axis does not require mass correction with $\alpha$ and $x_{1}^{\prime}=0.12408112557821315$ with phase angle $\theta_{W}=28.4598708641138^{\circ}$ on the complex $\dot{x}$ plane does.

The values listed in Table 9.1 are not unique. In fact, $S S(x)=0$ has a series of roots corresponding to a series of different $\alpha$ values. The multi-value behavior reflects the fact that $\alpha$ is a running constant and the stochastic nature of SQS theory. The details will be discussed in later sections.

The $E D S_{\dot{x}}(x)$ function introduced in this section is not only used to define $S S(x)$ function but also has other important applications, which will be given in Section 15.

\section{Section 10: Muon and Taon Torus Model and Parameters}

Muon and taon belong to the second and third generations of lepton family. Their torus model is similar to electron torus model except that the $x-z$ cross section is elliptical for the original version. Instead of one radius $a_{2}$ for the circular cross section of electron torus model, the elliptical cross section has two radii $a_{2}$ and $b_{2}$. To determine the parameter $b_{2}$ requires an additional equation. The option taken in this section is to keep the original (before $f$-modification) Weinberg angle the same for all three charged leptons:

$$
\theta_{W O}=\theta_{W e O} \text {. }
$$

$\theta_{W e O}$ is the original Weinberg angle for electron, $\theta_{W O}$ is the original Weinberg angle for muon or taon. According to (10.1a) and (8.45a), the original angle $\theta_{2}=\theta_{W O}=\theta_{W e O}$ for muon and taon is determined:

$$
\theta_{2}=\theta_{W O}=\theta_{W e O}=28.4598708641138^{\circ} \text {. }
$$

The original numerical parameters $m, n, p$ for muon and taon are selected as:

Muon: $\quad m=18, n=29 \frac{1}{4}, \quad p=6048$;

Taon: $\quad m=42, n=120, \quad p=417270$.

The reasons for selecting such values of $m, n, p$ will be given in later sections.

The values of $x_{1}$ and $x_{2}$ for muon and taon are calculated according to (6.19):

$$
\begin{aligned}
& x_{1}=0.25-\frac{M_{e}}{8 M}=0.25-\frac{n}{8 p}, \\
& x_{2}=0.5-x_{1} .
\end{aligned}
$$

In (10.3a), $M_{e} /(8 M)=n /(8 p)$ is according to $M / M_{e}=p / n$ of (8.1b). Substitute the values of $p$ and $n$ given by (10.2) into (10.3) yields:

Muon:,;

$$
x_{1}=0.249395461309524, x_{2}=0.250604538690476 \text {; }
$$

Taon:

$x_{1}=0.249964052052628, x_{2}=0.250035947947372$. 
Substituting $x_{1}$ and $x_{2}$ of (10.4) into the $S$-equation (3.20) and solving for $\pi\left(x_{1}\right)$ and $\pi\left(x_{2}\right)$ yield:

Muon:

$\pi\left(x_{1}\right)=3.143615677542496, \pi\left(x_{2}\right)=3.13949118154063967$;

Taon:

$\pi\left(x_{1}\right)=3.141671482326853, \pi\left(x_{2}\right)=3.14142622651424$.

Most formulas of electron torus model to determine characteristic point $A$, point $B$ locations and other geometrical parameters in Section 8 are valid for muon and taon except some differences caused by the cross section change from circular to elliptical.

The formula to calculate loop length ratio $L_{2} / L_{1}=n / m$ is:

$$
\frac{\int_{0}^{2 \pi} \sqrt{\left(a_{2} \sin t\right)^{2}+\left(b_{2} \cos t\right)^{2}} d t}{2 \pi d}=\frac{L_{2}}{L_{1}}=\frac{n}{m}
$$

For the torus outer half, formulas $(8.8 \mathrm{~b}),(8.9 \mathrm{a})$ through (8.9c), (8.10a) through $(8.10 b),(8.11 a)$ through $(8.11 c)$ are also valid for muon and taon. The changes are (8.8a) and (8.8c), in which $b_{2}=a_{2}$ is replaced by $b_{2} \neq a_{2}$.

For the torus inner half, formulas (8.12a), (8.13a), (8.14a) through $(8.14 \mathrm{~d}),(8.15 \mathrm{a})$ through $(8.15 \mathrm{~d})$ are valid for muon and taon. The changes are: in $(8.12 \mathrm{~b}), b_{1}=a_{1}$ is replace by $b_{1} \neq a_{1}$; in (8.13b), $b_{1}=a_{1}$ and $b_{2}=a_{2}$ are replaced by $b_{1} \neq a_{1}$ and $b_{2} \neq a_{2}$.

For the $f$-modification, (8.26a) and (8.26b) are for electron. For other fermions including muon and taon, they are generalized as:

$A T$-equation:

$$
\frac{1}{2 \pi d} \int_{0}^{2 \pi} \sqrt{a_{2}^{\prime 2}+\left[\sqrt{d^{2}-a_{2}^{\prime 2}}+a_{2}^{\prime} \sin (\alpha \theta)\right]^{2}} d \theta-\frac{d}{2 a_{2}^{\prime}}=0 ;
$$

$P S$-equation:

$$
\frac{1}{2 \pi} \int_{0}^{2 \pi} \frac{\sqrt{d^{2}-a_{2}^{\prime 2}}+a_{2}^{\prime} \sin (\alpha \theta)}{d+a_{2}^{\prime} \cos \theta} d \theta-1=0 .
$$

Mass term's $\alpha$ :

$$
\alpha=\frac{2 p}{m}=2\left(\frac{n}{m}\right)\left(\frac{p}{n}\right)=2\left(\frac{L_{2}}{L_{1}}\right) \frac{M}{M_{e}} .
$$

The $\cos \theta$ in the denominator of $P S$-equation does not change, because it is originated from geometry relation of (8.19b) and has nothing to do with mass.

The rest of formulas for the $f$-modification, (8.28a), (8.28b), (8.29a), (8.31a) through (8.31d), (8.32a) through $(8.32 \mathrm{~d}),(8.33 \mathrm{a})$ through $(8.33 \mathrm{~d}),(8.34 \mathrm{a})$ through $(8.34 \mathrm{e})$, (8.35a) through (8.35f), angle tilt formulas (8.38a) through $(8.38 \mathrm{c})$ and $(8.39)$ all are valid for muon and tuaon without change. The GWS-triangle and related formulas (8.40), (8.41) and (8.42) are also valid for muon and tuaon.

Table 10.1 and 10.2 list the calculated parameters for muon and taon, respectively. In these tables, the parameters with the ' mark are effective, i.e. after the $f$-modification and

\begin{tabular}{|c|c|c|c|}
\hline \multicolumn{4}{|c|}{ 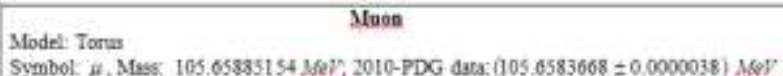 } \\
\hline \multicolumn{4}{|c|}{ Reliaive deviation $t=4.388 \times 10^{-1}$} \\
\hline \multicolumn{4}{|c|}{ Weinberg angle: $\theta_{5}-\theta_{1}=29.6163361^{*}$ : } \\
\hline \multicolumn{4}{|c|}{ Original ammerical pacameters: $w-18, n=29 \frac{1}{4}, p=6048$} \\
\hline \multicolumn{4}{|c|}{ Redaced numerical parameterst: $n=1, n=1 \frac{5}{8}, p=336$} \\
\hline \multicolumn{4}{|c|}{$d=1$} \\
\hline \multicolumn{4}{|l|}{$f_{2}=0.91340563$} \\
\hline \multicolumn{2}{|c|}{$f_{1}=1.00850309$} & \multicolumn{2}{|c|}{ Effective $a_{1}^{\prime}=0.49181712 ; \hat{b}_{2}^{\prime}=2.42734896$} \\
\hline \multicolumn{2}{|c|}{$\begin{array}{l}\text { Torus imer half (negative curvature) } \\
x_{1}=0.24939546, \pi\left(x_{1}\right)=3.14361568 \\
R=0.50815285 \\
A_{\mathrm{a}}=0.02538227, A=0.00619876\end{array}$} & \multicolumn{2}{|c|}{$\begin{array}{l}\text { Torus outer half (positive curvature) } \\
x_{2}=0.25060454, \pi\left(x_{2}\right)-3.13949118\end{array}$} \\
\hline $\begin{array}{l}\text { Trangle } B_{i} D^{Y O} \\
\text { on } \\
O_{H} H^{\prime} \text { cress sectioa }\end{array}$ & $\begin{array}{l}\text { Triamgit } D \mathrm{ECO}_{2} \mathrm{~cm} \\
x-y \text { Cross Section }\end{array}$ & $\begin{array}{l}\text { Triangle } \bar{S}_{2} \partial_{1} O_{2} \\
\text { de } \\
O_{2} G^{\prime} \text { ctoss section }\end{array}$ & $\begin{array}{l}\text { Triandie } G O O_{2} \text { on } \\
x-y \text { cross section }\end{array}$ \\
\hline$b_{2}=0,36+16569$ & $a_{1}=0.07539057$ & $x_{4}^{\prime}=0.47589704$ & $X_{2}=1.48 B 8238$ \\
\hline$X_{1}=048715818$ & $x_{p}=050135792$ & $Z_{1}=0.61259653$ & $Y_{y}=0.09445633$ \\
\hline$z_{1}^{\prime}=0.33331729$ & $Y_{2}^{\prime}=0.08300648$ & & \\
\hline$\alpha_{1}^{\prime}=\$ 6.42057154^{*}$ & $\alpha_{0}^{\prime}=75,8485164 T$ & $\theta_{2}=296163361^{\circ}$ & $\theta_{2}=730639579^{\prime}$ \\
\hline$\beta_{1}=34.38022705$ & $f_{1}=9.4510823 \gamma^{\prime}$ & $\phi_{2}=22.54174704^{*}$ & $\phi 1,=3.63018464^{\prime \prime}$ \\
\hline$y_{1}=89.19920141^{\circ}$ & $y_{0}=94.70040116^{2}$ & $\gamma_{2}^{\prime}=127.84191686^{\prime}$ & $V_{2}=169.06341956^{\prime}$ \\
\hline$\phi_{1}=33.0215193 z^{\prime}$ & $\phi_{0}=9.40080232^{*}$ & $C_{2}=5215808314^{*}$ & $\xi,=10.93658044^{4}$ \\
\hline
\end{tabular}
the parameters without the ' mark are original, i.e. before the $f$-modification.

\begin{tabular}{|c|c|c|c|}
\hline \multicolumn{4}{|c|}{ 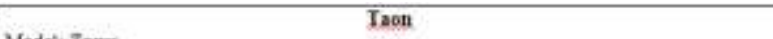 } \\
\hline \multirow{2}{*}{\multicolumn{4}{|c|}{ 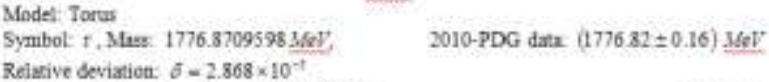 }} \\
\hline & & & \\
\hline \multicolumn{4}{|c|}{ Weinberg angle: $\theta_{5}-\theta_{2}=29.1877623 y^{*} \quad$ Charge: $e$} \\
\hline \multicolumn{4}{|c|}{ Original mumerical parameters $n=42, n=120, P=417270$} \\
\hline \multirow{2}{*}{\multicolumn{4}{|c|}{ Reduced mumerical parceneterst- $\quad \pi=1,4=2 \frac{6}{7}, p=9935$}} \\
\hline \multicolumn{3}{|c|}{$d=1$} & \\
\hline \multicolumn{2}{|l|}{$f_{a}=0.94733344$} & \multirow{2}{*}{\multicolumn{2}{|c|}{$\begin{array}{l}\text { Ongimal: } a_{1}=0.5208362, b_{2}=439440915 \\
\text { Effective: } a_{2}^{\prime}=0.49340555, b_{1}^{\prime}-4.40264701\end{array}$}} \\
\hline \multicolumn{2}{|c|}{$f_{3}-1.00187+62$} & & \\
\hline \multicolumn{2}{|c|}{$\begin{array}{l}\text { Toerus imner half (negatrie curvatine) } \\
x_{1}=0.24996405, r\left(x_{3}\right)=3.14167148 \\
R=0.50659445 \\
A_{n}=0.00500924, d=0.00078376\end{array}$} & \multicolumn{2}{|c|}{$x_{1}=0.25003595 ; \pi\left(x_{1}\right)=3.14142623$} \\
\hline $\begin{array}{l}\text { Triangle } B_{1} D^{\prime} O_{2} \\
\text { on } \\
O_{1} H^{\prime} \text { cross sectioes }\end{array}$ & $\begin{array}{l}\text { Trimgle } D^{\prime} E O_{z} \text { at } \\
x-y \text { cross sectuon }\end{array}$ & $\begin{array}{l}\text { Triangle } A_{2} O_{1} O_{7} \\
\text { ol } \\
O_{1} G \text { roos section }\end{array}$ & $\begin{array}{l}\text { Triangle } \mathrm{G} O \mathrm{O}_{2} \text { on } \\
x-y \text { cross section }\end{array}$ \\
\hline$b,-0,24098647$ & $a_{1}=0.02739495$ & $X_{1}^{4}=0488+4459$ & $x_{3}=1.49316821$ \\
\hline$X_{1}=0.49274155$ & $x_{0}=0.50576582$ & $Z_{2}^{\prime}=0.62275234$ & $Y_{2}^{\prime \prime}=0.02662385$ \\
\hline$Z_{1}=0.22833091$ & $x_{0}=0.02896323$ & & \\
\hline$\alpha_{1}^{\prime}=6530414182^{\prime}$ & $a_{0}^{\prime}=3500741245$ & $e_{2}=29.18776233^{4}$ & $\theta_{1}=2.06863135^{2}$ \\
\hline$\rho_{1}=24.86247320^{\circ}$ & $\beta_{1}-3.3538249 T^{\prime}$ & $\phi_{2}=2270401238^{\circ}$ & $\phi \phi_{1}=1.02150092^{*}$ \\
\hline$P_{1}=89.83338092^{2}$ & $Y_{1}=91.63876258^{2}$ & $\gamma_{2}=128.10822529^{\circ}$ & $\gamma_{1}=176,90986773$ \\
\hline$\phi_{t}=24.23381334^{*}$ & $6,=3.27752510^{\circ}$ & $\sum_{2}=51.89177471^{*}$ & $8 i=3,09013227^{*}$ \\
\hline
\end{tabular}

Table 10.1: The Calculated Parameters of Muon Torus Model*

Table 10.2: The Calculated Parameters of Taon Torus Model*

* All data are from 16-digit numerical calculations, only 8-digit after the decimal point is presented.

** The reduced numerical parameters are the original numerical parameters divided by $m$.

The synchronization related angles in Table 10.1 are:

$\xi_{2}^{\prime}=52.15808314^{\circ} \neq \phi_{3}^{\prime}=3.63018464^{\circ}$,

$\theta_{2}^{\prime}=29.6163361^{\circ}=\xi_{2}{ }_{2}-\phi_{2}^{\prime} \neq \phi_{3}^{\prime}-\phi_{2}^{\prime}=-18.91156239^{\circ},(10.8 \mathrm{~b})$

$\phi_{0}^{\prime}=9.40080232^{\circ} \neq \beta_{1}^{\prime}=34.38022705^{\circ}$,

$\phi_{1}^{\prime}=33.02151938^{\circ} \neq \beta^{\prime}{ }_{0}=9.45108237^{\circ}$.

The synchronization of two loops cyclic movements for electron described in Section 8 no longer holds for muon. It indicates that, muon is not a stable particle. In fact, muon has 
Z. Y. SHEN

a mean life of $\tau=(2.197034 \pm 0.000021) \times 10^{-6} \mathrm{~s} \quad(2010-\mathrm{PDG}$ data).

The synchronization related angles in Table 10.2 are:

$\xi_{2}^{\prime}=51.89177471^{\circ} \neq \phi_{3}^{\prime}=1.02150092^{\circ}$,

$\theta_{2}^{\prime}=29.18776233^{\circ}=\xi_{2}^{\prime}-\phi_{2}^{\prime} \neq \phi_{3}^{\prime}-\phi_{2}^{\prime}=-21.68251145^{\circ}$,

$\phi_{0}^{\prime}=3.27752516^{\circ} \neq \beta^{\prime}{ }_{1}=24.86247726^{\circ}$,

$\phi_{1}^{\prime}=24.23381334^{\circ} \neq \beta_{0}^{\prime}=3.35382497^{\circ}$.

The synchronization of two loops cyclic movements for electron described in Section 8 no longer holds for taon. It indicates that, taon is not a stable particle. In fact, taon has a mean life of $\tau=(2.906 \pm 0.001) \times 10^{-13} \mathrm{~s}$ (2010-PDG data).

The parameters listed in Table 10.1 and Table 10.2 for muon and taon are calculated according to the formulas in Section 8 for electron with modifications introduced in this section, in which some of them are optional and subject to verification. If some of them are replaced by other options, related parameters should be changed accordingly.

The characteristic points, the trajectory, the circle- $A$, circle- $B$, the tilt angle $\Delta \vartheta$ breaking $\vartheta=180^{\circ} 3$-fold symmetry, the jumping trajectories, the torus model with four tiny holes equivalent to trousers with a large hole in the waistband and 4 ports degenerated to Feynman diagram, these and related issues discussed in Section 8 for electron are also valid for muon and taon.

The torus models for muon and taon introduced in this section serve as the basic building blocks, which are not the final version. The final version of models will be introduced in Section 12.

\section{Section 11: Quarks Model and Parameters}

Quarks torus model has elliptical $x-z$ cross section. The formulas for muon and taon in Section 10 are valid for quarks with exception that formula (10.1) is replaced by following formulas for quarks with fractional charges.

For up-type quarks:

$$
\frac{\sin \theta_{2 O, u} \cos \theta_{2 O, u}}{\sin \theta_{\text {WeO }} \cos \theta_{\text {WeO }}}-\frac{2}{3}=0, \theta_{2 O, u}=16.9796740885213^{\circ},
$$

For down-type quarks:

$$
\frac{\sin \theta_{2 O, d} \cos \theta_{2 O, d}}{\sin \theta_{W e O} \cos \theta_{W e O}}-\frac{1}{3}=0, \theta_{2 O, d}=8.10928341940384^{\circ} .
$$

In which, $\theta_{2 O, u}$ and $\theta_{2 O, d}$ are original values of the angle $\mathrm{O}_{1} \mathrm{~A}_{2} \mathrm{O}_{2}$ as shown in Fig. 8.1 before $f$-modification for up type and down type quarks, respectively. Formulas (11.1) is based on an assumption: $\sqrt{g^{2}+g^{\prime 2}}=$ const , which is optional.

There is another difference. The top quark is different from the other quarks. Because its mass exceeds the upper limit set by (6.21), top quark's model is spindle type torus with covered center hole as shown in Fig.11.1. The inner half of spindle shape torus also has positive curvature, which is consistent with top quark's $\pi\left(x_{1}\right)<\pi$. This difference makes top quark's inner half two triangles with different definitions and different physics meanings.

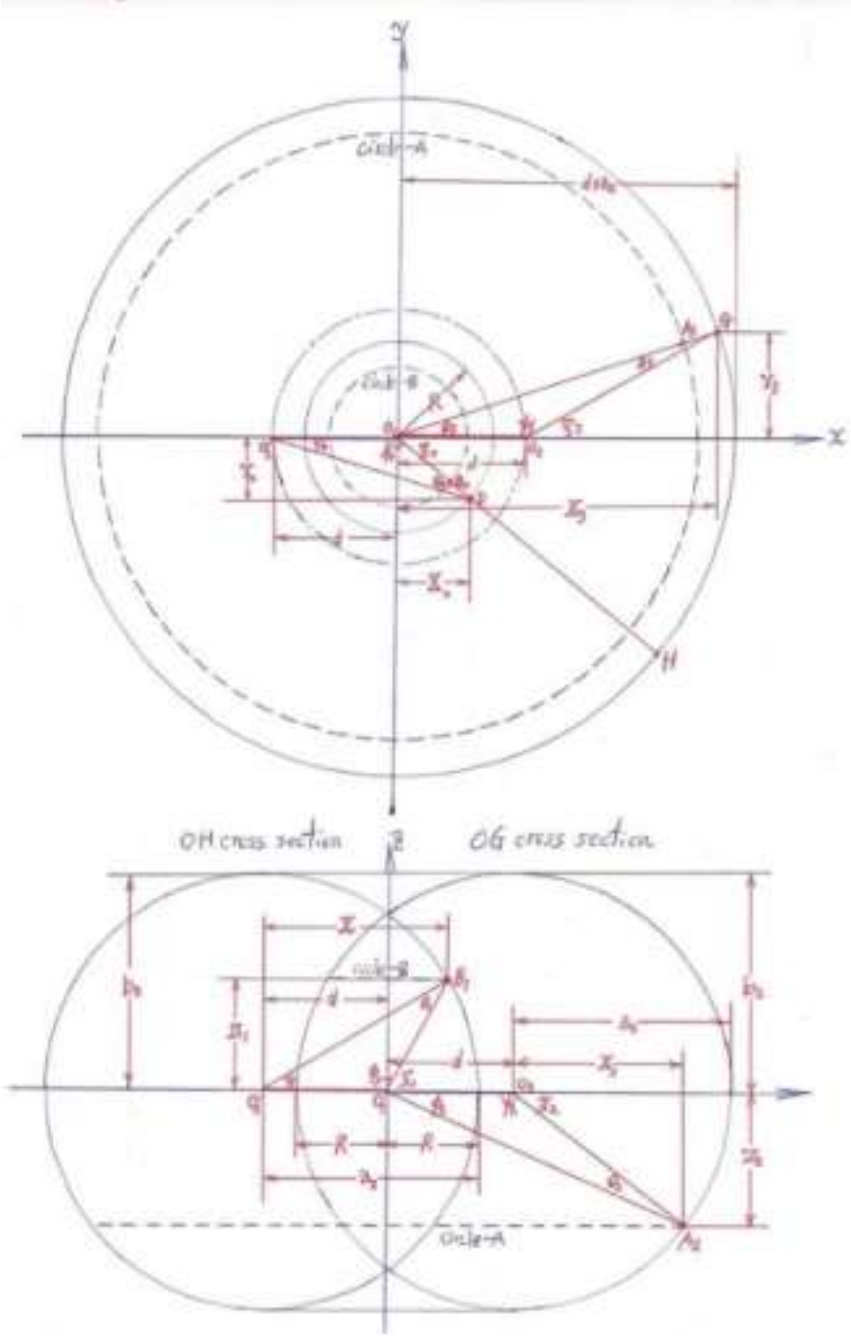

Fig. 11.1 Spindle type torus model for top quarks.

As shown in Fig.11.1, the location of points $D$ and $B$ are determined by $\pi\left(x_{1}\right)<\pi$ the same way as points $G$ and $A$ determined by $\pi\left(x_{2}\right)<\pi$.

On $x-y$ cross section:

$$
\begin{aligned}
& \frac{R \xi_{0}}{R \sin \xi_{0}}-\frac{\pi}{\pi\left(x_{1}\right)}=0, R=a_{2}-d, \\
& X_{0}^{2}+Y_{0}^{2}-R^{2}=0 .
\end{aligned}
$$

On $O_{1} H$ cross section:

$$
\begin{aligned}
& \frac{\int_{-\psi_{1}}^{\nu_{1}} \sqrt{\left(a_{2} \sin t\right)^{2}+\left(b_{2} \cos t\right)^{2}} d t}{2 Z_{1}}-\frac{\pi}{\pi\left(x_{1}\right)}=0, b_{2} \neq a_{2}, \\
& \cos \psi_{1}=\frac{X_{1}}{a_{2}}, \\
& \left(\frac{X_{1}}{a_{2}}\right)^{2}+\left(\frac{Z_{1}}{b_{2}}\right)^{2}-1=0, b_{2} \neq a_{2} .
\end{aligned}
$$

In Fig.11.1a, the triangle $\mathrm{DO}_{1} \mathrm{O}_{2}$, related angles are: 


$$
\begin{aligned}
& \tan \xi_{0}=\frac{Y_{0}}{X_{0}}, \\
& \tan \psi_{0}=\frac{Y_{0}}{d+X_{0}}, \\
& \phi_{0}=180^{\circ}-\xi_{0} . \\
& \theta_{0}=\xi_{0}-\psi_{0} .
\end{aligned}
$$

In Fig.11.1b, the triangle $\mathrm{B}_{1} \mathrm{O}_{1} \mathrm{O}^{\prime}{ }_{2}$ related angles are:

$$
\begin{aligned}
& \tan \psi_{1}=\frac{Z_{1}}{X_{1}}, \\
& \tan \xi_{1}=\frac{Z_{1}}{X_{1}-d}, \\
& \phi_{1}=180^{\circ}-\xi_{1}, \\
& \theta_{1}=\xi_{1}-\psi_{1} .
\end{aligned}
$$

The generalized $A T$ - and $P S$-equations of (10.7) are applicable to all quarks except the top quark. The top quark's model must have $a_{2}^{\prime}>d=1$ to qualify as the spindle type torus. The $f$-modification reduces $a_{2}>d=1$ to $a_{2}^{\prime} \approx 0.5<d=1$, that is not valid for spindle type torus. The effectiveness of $f$ modification for top quarks is limited to the $a_{2}^{\prime}>d=1$ part, which does not includes the root for the $A T$-equation.

Before going further, one question must be answered:

How many quarks are there?

Postulation 11.1: Quarks with the same flavor and different colors are different elementary particles. There are eighteen quarks in three generations.

Explanation: Elementary particles are distinguished from each other according to their different intrinsic parameters. Quarks with the same flavor and different colors have at least two different intrinsic parameters: one is color and the other is mass. To recognize them as different elementary particles is inevitable and legitimate.

According to Postulation 11.1, there are eighteen different quarks instead of six, in which six flavors each has three colors as shown in Table 11.1. Postulation 11.1 has important impacts beyond quarks, which will be shown in later sections.

\section{Postulation 11.2: Prime Numbers Postulation. Prime} numbers are intrinsically correlated to elementary particles' parameters as well as cosmic space structure and cosmic evolution.

Explanation: Prime Numbers Postulation serves as the second fundamental postulation with importance next to the first fundamental postulation of Gaussian probability. It provides a principle. The details are given by corresponding rules.

Definition 11.1: A pair of two consecutive odd prime numbers with average value equal to even number is defined as an even pair. A pair of two consecutive odd prime numbers with average value equal to odd number is defined as an odd pair.

The numerical $m$-parameters of 18 quarks are selected by

\begin{tabular}{|c|c|c|c|c|c|c|c|c|c|c|}
\hline Generstions & \multicolumn{3}{|c|}{ Fins: } & \multicolumn{3}{|c|}{ Second } & \multicolumn{3}{|c|}{ Thind } & Ended \\
\hline Up-typequaska & ע. & $u_{4}$ & $u_{z}$ & $c$ & $c_{t}$ & $c_{3}$ & t, & $t_{i}$ & $t_{3}$ & Nowe \\
\hline$w_{<}$xi primes & 1 & 7 & 13 & 19 & 29 & 37 & 43 & 53 & 61 & 71 \\
\hline$\left(w_{<}+w_{-\infty}\right)^{1 / 2}$ & 2 & 6 & 12 & 18 & 26 & 34 & 42 & 50 & 60 & 69 \\
\hline$m_{\text {drot }}$ as primes & 3 & 5 & 11 & 17 & 23 & 31 & 41 & 47 & 59 & 67 \\
\hline $\begin{array}{l}\text { Downtype } \\
\text { quake }\end{array}$ & $d$, & $d_{x}$ & $d_{3}$ & 1 , & $s_{2}$ & $I_{2}$ & 3. & $b_{1}$ & $\bar{k}$ & Nopec \\
\hline
\end{tabular}
the following rule.
Rule 11.1: The eighteen least odd prime numbers including 1 are assigned as the $m$-parameters of eighteen quarks as shown in Table 11.1. The $m$-parameters of eighteen quarks are paired of up-type and down-type for each color. All nine pairs are even pairs.

Table 11.1: 18 Prime Numbers Assigned to 18 Quarks $m$-Parameters*

* The $m$-parameters listed are their magnitude; the signs are defined by (11.6).

Conclusion 11.1: There are only three generations of quarks.

Proof: As shown in Table 11.1, for the nine pairs of quarks in three generations, their $m$-parameters: $1 \& 3,5 \& 7$, $11 \& 13,17 \& 19,23 \& 29,31 \& 37,41 \& 43,47 \& 53$, $59 \& 61$ all are even pairs. The next prime numbers pair of $67 \& 71$ is not an even pair, which violates Rule 11.1 . The fourth generation quarks are prohibited based on the Prime Numbers Postulation and the prime numbers table.

QED

In fact, no quarks beyond three generations have found in experiments.

The numerical parameters $n$ and $p$ of quarks are selected in the following rules.

Rule 11.2: The quarks' $n$-parameters are selected from prime numbers. The values of quarks $n$-parameter are closely related to strong interactions among them, which will be discussed in Section 13.

Rule 11.3: For a quark, the $p$-parameter is determined by $p / n=M / M_{e}$, in which, $M$ and $M_{e}$ are the mass of the quark and the mass of electron, respectively. The ratio $2 \mathrm{p} / \mathrm{m}$ equals to an integer.

The reasons for such rules will be explained later.

Definition 11.2: The signs of numerical parameters $m, n, p$ for fermions and anti-fermions with different handedness are defined as:

Fermion with right handedness:

$$
m>0, n>0, p>0 \text {, }
$$

Fermion with left handedness:

$$
m<0, n<0, p<0,
$$

Anti-fermion with right handedness:

$$
m>0, n<0, p<0,
$$

Anti-fermion with left handedness:

$$
m<0, n>0, p>0,
$$

Explanation: According to definition 11.2, for all four cases, the ratios $p / n$ for mass are always positive as they should be. Loop ratios are different: $n / m>0$ for fermions and $n / m<0$ for anti-fermions, which serve as the mathematical distinction for fermions and antifermions. For all fermions, $m>0$ represents right handedness, and $m<0$ represents left handedness. 
Z. Y. SHEN

The verifications and applications of Definition 11.2 will be given later.

The geometry parameters of quarks calculated by using above formulas and rules are listed in Table 11.2. In which, for up, down, strange, charm, bottom quarks, the parameters with the ' mark are effective, i.e. after the $f$-modification, and the parameters without the " mark are original, i.e. before the $f$-modification. All parameters for top quarks listed in Table 11.2 are original.

Table 11.2: Calculated Parameters for 18 Quarks*

\begin{tabular}{|c|c|c|c|}
\hline \multicolumn{4}{|c|}{$\mathrm{t}_{\mathrm{p}}$ Qmark (Red) } \\
\hline \multirow{2}{*}{\multicolumn{4}{|c|}{ 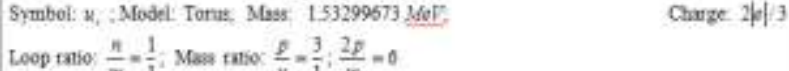 }} \\
\hline & & & \\
\hline \multicolumn{4}{|c|}{$d_{d=1}$ का 1 o 1 w } \\
\hline \multicolumn{2}{|c|}{$f_{x}=0.51975039$} & \multicolumn{2}{|c|}{ Origina: $o_{2}=094025636, \theta_{3}=105233579$} \\
\hline \multicolumn{2}{|l|}{$f_{b}=132838739$} & \multicolumn{2}{|c|}{ Effective $\alpha_{1}=0.49181711, s_{1}=1.39290038$} \\
\hline \multirow{3}{*}{\multicolumn{2}{|c|}{$\begin{array}{l}\text { Torus innet half (negative cirvature) } \\
x_{1}=0.20033339 ; \pi\left(a_{1}\right)=3.30834046 \\
R=d-a_{2}^{\prime}=0.50818289 \\
A_{n}=0.23488901, t=0.0542077\end{array}$}} & \multirow{3}{*}{\multicolumn{2}{|c|}{\begin{tabular}{|} 
Tocus outer half (posinive curvatume) \\
$x_{1}=0.29166657 ; \pi\left(x_{1}\right)=3.01906376$
\end{tabular}}} \\
\hline & & & \\
\hline & & & \\
\hline $\begin{array}{l}\text { Triasgie } B_{1} D \cdot O_{7} \\
\text { on } \\
O_{1} F^{\prime} \text { coses section }\end{array}$ & $\begin{array}{l}\text { Triangle } D E O_{1} \text { co } \\
x-y \text { cros section }\end{array}$ & $\begin{array}{l}\text { Triangle } 6, O_{1} O_{2}=0 \\
O, G \text { crow Sertion }\end{array}$ & $\begin{array}{l}\text { Thungle } \mathrm{C}^{\circ} \mathrm{O}_{0} \mathrm{O}_{2} \text { ail } \\
x \rightarrow y \text { eross setion }\end{array}$ \\
\hline \multirow{3}{*}{$\begin{array}{l}b_{1}=0.35106281 \\
x_{1}=0.48716955 \\
z_{2}^{\prime}=0.19172397\end{array}$} & $a_{1}=0.05374169$ & \multirow{3}{*}{$\begin{array}{l}X_{2}=0+7893757 \\
Z_{2}=031733200\end{array}$} & \multirow{3}{*}{$\begin{array}{l}X_{3}=1.31864343 \\
\gamma_{2}=0.69763731\end{array}$} \\
\hline & $x_{0}-0.00438505$ & & \\
\hline & $y_{\gamma}^{\prime}=0.50816394$ & & \\
\hline$\alpha_{1}=69.96667512^{\circ}$ & $\alpha_{0}^{\prime}=1791576026$ & $\theta_{2}=21.41537445^{5}$ & $\theta_{3}=37.57022938^{\circ}$ \\
\hline$\tilde{F}_{1}=21.48195453^{+}$ & $E_{1}=26.8368+825^{4}$ & $\phi_{1}=1210987699^{*}$ & $\phi_{1}=27.88138+23^{4}$ \\
\hline$y_{1}^{\prime}=85.61137009^{*}$ & $y_{0}^{\prime}=135.24737145^{\circ}$ & $\psi_{2}^{\prime}=146.47474856^{\circ}$ & $\nabla_{1}^{\prime}=114.54833635$ \\
\hline$\phi_{1}=20.49848254^{\circ}$ & $\phi_{0}=90.69474291^{*}$ & $5 y=13.52525164^{*}$ & $3_{3}^{\prime}=65.45 \mathrm{t} 6 \mathrm{t}+162^{\prime}$ \\
\hline \multicolumn{4}{|c|}{ Ip Quark (Grem) } \\
\hline \\
\hline \multirow{2}{*}{\multicolumn{4}{|c|}{$\operatorname{Lopp}_{d=1}$ raxio: $\frac{n}{m}-\frac{3}{7}:$ Mass ratio: $\frac{f}{n}-\frac{17.5}{3} ; \frac{2 F}{w}=5$}} \\
\hline & & \multirow{2}{*}{\multicolumn{2}{|c|}{ Originat: $a_{7}=0.41134294, b,-0.445+6026$}} \\
\hline \multicolumn{2}{|c|}{$f_{a}=1.19563769$} & & \\
\hline \multicolumn{2}{|c|}{$f_{1}=0.30865799$} & \multicolumn{2}{|c|}{ Effoctive $a_{2}^{1}=0.49181712, b_{2}=0.36021836$} \\
\hline \multicolumn{2}{|c|}{$\begin{array}{l}\text { Toras inter ball (negative curvature) } \\
x_{2}=0.22857143 ; r\left(x_{1}\right)=3.22077538 \\
R=d-a=0.50813288 \\
A=0.10024719, A=0.02672217\end{array}$} & \multicolumn{2}{|c|}{$\begin{array}{c}\text { Torns outr half (poitove curvalute) } \\
x_{2}=0.27142857: t\left(x_{2}\right)=3.07390694\end{array}$} \\
\hline $\begin{array}{l}\text { Trimgle } B_{1}^{\prime} D O_{2}^{\prime} \text { on } \\
Q_{2} H^{\prime} \text { cross section. }\end{array}$ & $\begin{array}{l}\text { Trimgle } D E O_{+}^{\prime} \text { do } \\
x-y \text { cross section. }\end{array}$ & $\begin{array}{l}\text { Triargle } A_{4}^{2} O_{2} O_{2} \text { on } \\
O G G \text { cross Section }\end{array}$ & $\begin{array}{l}\text { Trimate } G \mathrm{O}_{\mathrm{p}} \mathrm{O}_{2} \text { on } \\
x-y \text { cross sectioe }\end{array}$ \\
\hline$b_{0}=0.15733829$ & $a_{3}-0.17534628$ & $x_{7}=0.42045431$ & $x_{;}=139581171$ \\
\hline$x_{1}=0.42900000$ & $x_{j}=0.48511385$ & $Z_{2}^{1}=0.18698924$ & $r_{3}=0.5265242$ \\
\hline$z_{1}^{\prime}=0.17616191$ & $Y_{y}=0.15137503$ & & \\
\hline$\alpha_{1}^{\prime}=57.30085269^{\circ}$ & $\alpha_{2}=64.95150732$ & $\theta_{2}=16.46943341^{2}$ & $\theta_{t}=3239534835^{\circ}$ \\
\hline$\rho_{1}=22.32471133^{\circ}$ & $F_{1}-16.38318211^{*}$ & $\phi_{2}=7.49535646^{\circ}$ & $\phi_{3}=20.6673467 \mathrm{r}^{2}$ \\
\hline$y_{1}^{1}=7037640398^{*}$ & $Y_{0}=98.0650105 T$ & $v_{2}=156.03516013^{*}$ & $v_{1}=126.93380494^{2}$ \\
\hline$\phi_{1}=1714576477^{\circ}$ & $d_{c}=17.33002113^{t}$ & $K_{2}=23,96483957$ & $\zeta_{1}=53.06619506$ \\
\hline \multicolumn{4}{|c|}{$\begin{array}{c}\text { T Quark (Bh̆ae) } \\
\end{array}$} \\
\hline Symbot: $u_{y} ;$ Model: I & Corus Ntass 33214929 & $2 \mathrm{MeV}$ & Churge: $2 k / 3$ \\
\hline Losp ratio. $\frac{n}{2}-\frac{5}{13}$ & Mass ratio: $\frac{E}{n}-\frac{325}{5}$ & $2 \vec{z}=5$ & \\
\hline$d=1 \quad m \quad 13$ & 43 & w. & \\
\hline$f_{s}=1.39909987$ & & Originat: $a_{1}=0.36727$ & \\
\hline$f_{i}=0.64573563$; & & Effective $a_{2}=0.4913$ & $12, H_{2}=025931049$ \\
\hline Torts imer baff & aegative curratare) & Tonas outer has & sositive curvature) \\
\hline$x_{2}=0.23078923 ; x 6$ & & & \\
\hline$R=d-a=0.508182$ & & & \\
\hline$A_{n}=0.15101126, A$ & -0.02774201 & & \\
\hline $\begin{array}{l}\text { Triangle } B_{1} D^{\prime} O_{2}^{\prime} \text { on } \\
O_{1} H^{\prime} \text { ctos sectoon }\end{array}$ & $\begin{array}{l}\text { Triangle } D \bar{E} \sigma_{1} \text { ch } \\
x-y \text { cros section }\end{array}$ & $\begin{array}{l}\text { Trisalele } A_{2} O_{1}^{\prime} O_{2} \text { ob } \\
O_{1} G^{\prime} \text { cross section }\end{array}$ & $\begin{array}{l}\text { Triangle } \mathrm{F} \mathrm{O}_{\mathrm{O}} \mathrm{O}_{2} \text { on } \\
x-y \text { cros section }\end{array}$ \\
\hline$b_{3}=014203952$ & $a_{1}=0.17169936$ & $X_{1}=0.03150795$ & $x_{2}=1.40493771$ \\
\hline$x_{1}^{\prime \prime}=033700996$ & $x_{y}=0.48911414$ & $z_{1}^{\prime}=0.16364752$ & $Y_{2}=0.50106558$ \\
\hline$z_{1}^{\prime}=0.16001608$ & $Y_{7}=0.13790283$ & & \\
\hline$\alpha_{1}^{1}=100.76044282^{\circ}$ & $\alpha_{1}^{\prime}=67.02147615$ & $\theta_{2}=16.46136568^{\circ}$ & $\theta_{1}=31.43971429^{2}$ \\
\hline$\beta_{\mathrm{t}}=22+6350957^{\circ}$ & $8,-15 \cdot 1057797 T$ & $\phi_{2}=6.75554513^{\prime}$ & $f_{i}=19.65028338^{\circ}$ \\
\hline$y_{1}=56.77604761^{\circ}$ & $r_{t}=97.37274400^{\circ}$ & $v_{j}^{\prime}=156.78308919^{\circ}$ & $v_{1}=123.91000232^{\prime}$ \\
\hline$\phi_{4}=14630 \mathrm{t} 182^{\circ}$ & $\phi_{0}-15.74548316^{\circ}$ & $S_{2}^{\prime}=23.2169108 \mathrm{r}^{*}$ & $\zeta_{3}-51.06999768$ \\
\hline
\end{tabular}

Tp Quark Summary+*

\begin{tabular}{|c|c|c|c|}
\hline \multicolumn{4}{|c|}{ Tp Quark Summary*t } \\
\hline \multicolumn{4}{|c|}{ 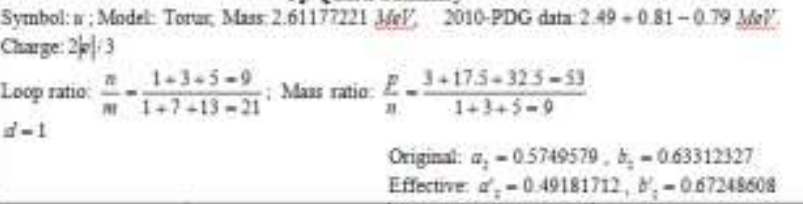 } \\
\hline $\begin{array}{l}\text { Triengle } \mathrm{B}_{1}^{\prime} \mathrm{D}^{\prime} \mathrm{O}_{2} \text { on } \\
\mathrm{O}_{2} \mathrm{~F}^{2} \text { cress sectiou }\end{array}$ & $\begin{array}{l}\text { Trimgle } D E O_{2}^{\prime} \text { on } \\
\text { xy y etode section }\end{array}$ & $\begin{array}{l}\text { Triangle } A_{2}^{\prime} O_{2} O_{2} \text { on } \\
O_{1} G \text { cross Section }\end{array}$ & $\begin{array}{l}\text { Triangle } \mathrm{C}^{\prime} \mathrm{O}, \mathrm{O}_{1} \text { on } \\
x-y \text { croes sectben }\end{array}$ \\
\hline$a_{3}^{\prime}=85.98933354^{\prime}$ & $\alpha_{1}=499630212 f^{2}$ & $\theta_{2}=18.11540785$ & $\theta_{2}=33.80293067$ \\
\hline$\beta_{1}=22.09005849^{2}$ & $\theta_{2}=19.44193672$ & $\phi_{2}=8.73692619^{*}$ & $\phi_{1}=22.33300477$ \\
\hline$y_{1}=7192060795^{\circ}$ & $Y_{2}=110.59504203^{\circ}$ & $V_{2}^{\prime}=153.09766596^{\circ}$ & $V_{1}=123,46406455^{2}$ \\
\hline \multicolumn{4}{|c|}{ Dewn Quark (Red) } \\
\hline \multicolumn{4}{|c|}{ 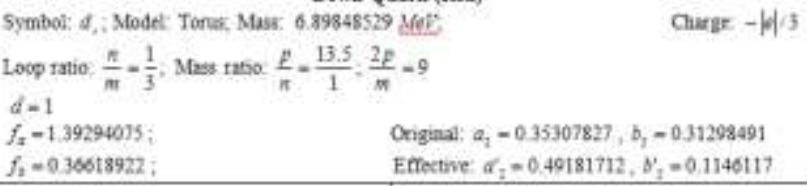 } \\
\hline \multicolumn{2}{|c|}{$\begin{array}{l}\quad \text { Torus inder half (negative curtimure) } \\
x_{1}=0.24074074 ; \pi\left(x_{2}\right)=3.17423947 \\
R=d-a=0.50818283 \\
A=0.10233596, A=0.00939183\end{array}$} & \multicolumn{2}{|c|}{\begin{tabular}{|c|} 
Torus outer half (ponitive curvature) \\
$x_{2}=0.25925926 ; \approx\left(x_{2}\right)=3.11101398$
\end{tabular}} \\
\hline $\begin{array}{l}\text { Triangle } S_{1}, D^{\prime} O_{2} \text { on } \\
\text { O, } \mathrm{H}^{\prime} \text { eros section }\end{array}$ & $\begin{array}{l}\text { Triangit } D E O O_{7}^{\prime} \text { on } \\
x-y \text { crose sectooe }\end{array}$ & $\begin{array}{l}\text { Triange } A^{\prime}, O_{1}^{\prime} O_{2}, \infty \\
O_{1} G^{\prime} \text { eross Sectica }\end{array}$ & $\begin{array}{l}\text { Triangle } G O O_{i}, \text { on } \\
x-y \text { coses nectooe }\end{array}$ \\
\hline $\begin{array}{l}b_{4}=0.07170849 \\
x_{1}=0.36641451 \\
z_{1}=0.07645056\end{array}$ & $\begin{array}{l}a_{1}=0.10883328 \\
x_{7}=0.50030522 \\
y_{y}=0.08627344\end{array}$ & $\begin{array}{l}X_{z}=0.37839865 \\
z_{z}=0.07321179\end{array}$ & $\begin{array}{l}x_{3}=1.4433404 \\
y_{3}=0.35753096\end{array}$ \\
\hline$\alpha_{3}^{\prime}=136.84638764^{\prime}$ & $\alpha_{t}=7530668026$ & $\vec{\theta}_{2}=7.90984532^{\circ}$ & $\theta_{1}=24.7042024$ \\
\hline$\beta_{1}=11.78540743^{4}$ & $\rho_{s}=9.80585437^{*}$ & $\phi_{2}=304033172^{*}$ & $\delta_{1}=13.86655617^{4}$ \\
\hline$r_{1}^{\prime}=31.36820493^{*}$ & $\gamma_{s}=94.83746537$ & $\nabla_{2}=169.04982296 ?$ & $\varphi_{1}^{\prime}=141.42924143$ \\
\hline$\phi_{1}=688024196$ & $4,-9.77693075^{\circ}$ & $\xi^{4}=10.95017704^{*}$ & $5,-38.57075857$ \\
\hline \multicolumn{4}{|c|}{ 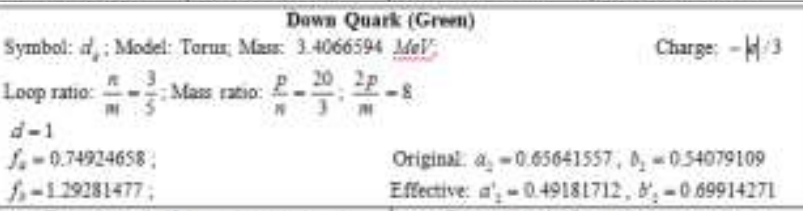 } \\
\hline \multicolumn{2}{|c|}{ 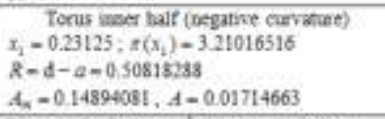 } & \multicolumn{2}{|c|}{$\begin{array}{c}\text { Torvs ouser half (pontive curvatiest) } \\
x_{1}=0.26575 ; \pi\left(x_{1}\right)=3.0817935\end{array}$} \\
\hline $\begin{array}{l}\text { Triatele } S_{1}^{\prime} D^{\prime} O_{2}^{\prime}, 00 \\
O_{1} H^{\top} \text { eross section }\end{array}$ & $\begin{array}{l}\text { Triande } D^{\prime} E \sigma_{1} \infty \\
x-y \text { crose section }\end{array}$ & $\begin{array}{l}\text { Trangle } f_{2} O_{1}^{\prime} O_{2} \text { on } \\
q G \text { cross sectose }\end{array}$ & $\begin{array}{l}\text { Truagle } G O, O_{2} \text { on } \\
x-y \text { dos sectioe }\end{array}$ \\
\hline$b_{4}=0.12279755$ & $a_{5}=0.10718500$ & $X_{z}=0.48509771$ & $X_{3}=1.40695468$ \\
\hline $\begin{array}{l}x_{1}^{\prime}=0.48738753 \\
Z_{1}^{\prime}=0.09962256\end{array}$ & $\begin{array}{l}x_{l}=0.48282194 \\
y_{l}=0.15853333\end{array}$ & $z ;=0.1151 \gamma_{443}$ & $Y_{3}=0.49598069$ \\
\hline$a_{3}^{\prime}=82.83529795^{\circ}$ & $\alpha_{1}-6396911946$ & $\theta_{2}=8.92155435^{*}$ & $e_{1}=3121233724$ \\
\hline$\beta_{1}=1087353138^{\circ}$ & $\rho_{1}=17.04215066$ & $\phi_{2}=4,43460801^{\circ}$ & $\phi_{1}=19.41861079$ \\
\hline$r_{1}^{1}=8229117127^{*}$ & $Y_{1}^{\prime}=99.08872988^{\circ}$ & $\psi_{y}=166.64383764^{\prime}$ & $\psi_{1}=12936905197$ \\
\hline$\phi_{1}=10.3503137 \mathrm{t}^{\circ}$ & $\phi_{1}=18.17745976^{-1}$ & $\zeta_{2}-13.35616236^{\circ}$ & $\xi,=50.6309430 y$ \\
\hline \multicolumn{4}{|c|}{ 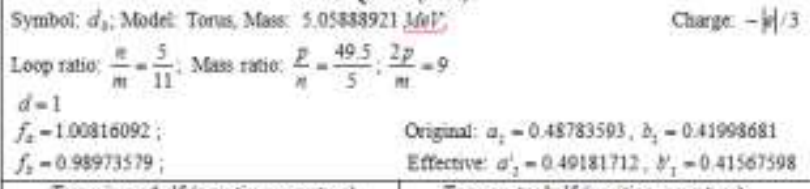 } \\
\hline $\begin{array}{r}\text { Totus inder balf } \\
x_{3}=0.23737374 ; \pi( \\
R=d-a=0.5051328 \\
A_{m}=0.12045909, A\end{array}$ & $\begin{array}{l}\text { negative cursature) } \\
6.18609446\end{array}$ & $\begin{array}{r}\text { Torus outer half }(\mathrm{p} \\
x_{7}=0.26262020 ; r(x\end{array}$ & $\begin{array}{l}\text { osetive curvature }) \\
7)=3.1004(453)\end{array}$ \\
\hline $\begin{array}{l}\text { Triangle } S_{1} D^{\prime} O_{2}^{\prime} \text { an } \\
O, H^{\prime} \text { cross section }\end{array}$ & $\begin{array}{l}\text { Trimgie } D^{\prime} E O^{\prime} \text {, an } \\
x y \text { crous section }\end{array}$ & $\begin{array}{l}\text { Triangle } A_{2}^{\prime} O_{1}^{\prime} O_{2} \text { on } \\
O_{;} G^{\prime} \text { croas Section. }\end{array}$ & $\begin{array}{l}\text { Inimgle } G O_{1} O_{1} \text { on } \\
\text { sy crose section }\end{array}$ \\
\hline$B_{1}=0.09436711$ & $a_{1}=0.11234662$ & $X_{2}^{\prime}=0.47690246$ & $x_{3}=1.43330999$ \\
\hline $\begin{array}{l}x_{1}^{\prime}=0.47875746 \\
Z_{1}^{\prime}=0.09504712\end{array}$ & $\begin{array}{l}x_{r}=0.4950595 \\
\gamma_{0}=0.11147332\end{array}$ & $z_{2}=0.10159139$ & $y_{1}=0.41309107$ \\
\hline$\alpha_{1}=8657764229^{2}$ & $\alpha_{2}=71.19723555$ & $\theta_{2}=8.0905951^{\circ}$ & $\theta_{1}=27.57359093$ \\
\hline$\theta_{1}^{\prime}=1122816929^{\prime}$ & $\rho_{0}=12.46700365^{5}$ & $\theta_{2}=393499463^{*}$ & $\sigma_{2}=16.0995572 \mathrm{r}^{\circ}$ \\
\hline$y_{1}^{1}=82.19418842^{\circ}$ & $Y_{1}=963350258^{\circ}$ & $\psi_{\gamma}^{\prime}=167.97441027^{\prime}$ & $W_{1}=13632685180^{\circ}$ \\
\hline$\phi_{2}=10.33476624^{*}$ & $\phi \phi_{1}=12.6712516^{\prime}$ & $\zeta_{2}^{\prime}=12.02558973^{\prime}$ & $5_{3}=43.67314814$ \\
\hline
\end{tabular}




\begin{tabular}{|c|c|c|c|}
\hline $\begin{array}{l}\text { Symboi: } d \text {, Model: To } \\
\text { Ouege: }-H / 3 \\
\text { Loop ratio: } \frac{n}{n}=\frac{1-3}{3+5} \\
d=1\end{array}$ & 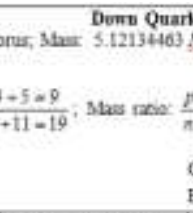 & 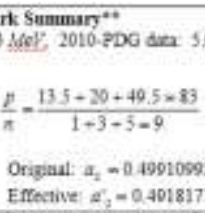 & $\begin{array}{l}.05=0.75-0.953 .6 V \\
22, b_{2}=0.4245876 \\
72, r_{1}=0.40051013\end{array}$ \\
\hline 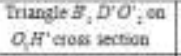 & $\begin{array}{l}\text { Triangle } D E \sigma_{4} \text { an } \\
x-y \text { cour sectiva }\end{array}$ & $\begin{array}{l}\text { Triangie } \gamma_{2} \mathrm{O}_{2} O_{2} \text { en } \\
Q_{1} \sigma \text { ctosin Section }\end{array}$ & $\begin{array}{l}\text { Triangle } \mathrm{Cr} \mathrm{O}_{2} \text { on } \\
x-y \text { coss nection }\end{array}$ \\
\hline$\alpha_{1}=101.75310000^{\circ}$ & $\alpha_{1}=7012436176$ & $\theta_{1}=8.90733159^{\circ}$ & $\theta_{1}=27.83004352$ \\
\hline $\bar{b}_{1}=11.2957027^{\circ}$ & $f_{n}=13.10503125$ & $\phi_{2}=3,80331145^{*}$ & $\phi_{1}=16.46157472^{\prime}$ \\
\hline$Y_{1}=66951118321^{\circ}$ & $Y=-96.77050702^{2}$ & $\gamma_{2}=16783935696^{\circ}$ & $\psi_{1}=135,70334175$ \\
\hline 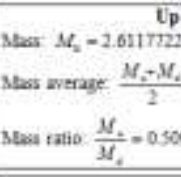 & 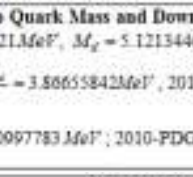 & 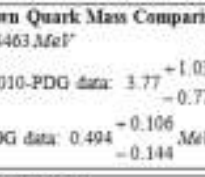 & Met \\
\hline 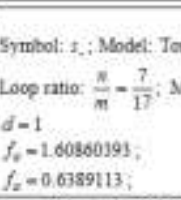 & $\begin{array}{l}\text { Strasge Qua } \\
\text { orus, Mass: } 90.59250670 \\
\text { Man catio: } \frac{R}{\pi}-\frac{124 J}{7} ; \frac{2}{}\end{array}$ & 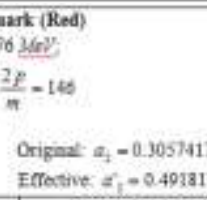 & $\begin{array}{l}\text { Charge }-|k| 3 \\
174, b_{2}=0.3054689 \\
1736, v_{1}=032593701\end{array}$ \\
\hline 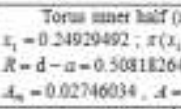 & 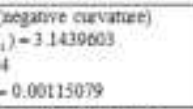 & $\begin{array}{r}\text { Tonis vuter bate } \\
x_{z}-0.25030506 ; x(x\end{array}$ & $\begin{array}{l}\text { (pointive curvature) } \\
\left.x_{y}\right)=3.1391 \text { ts }\end{array}$ \\
\hline $\begin{array}{l}\text { Triangle } B_{1} D \mathrm{O}_{2} \text { on } \\
0_{2} \mathrm{~A}^{\prime} \text { cross sectioe }\end{array}$ & 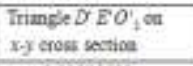 & $\begin{array}{l}\text { Trimple } C_{2} O_{1} O_{1} \text { or } \\
G, G \text { coss Secoin }\end{array}$ & $\begin{array}{l}\text { Trimple } G O O_{2} \text { od } \\
\text { y-y cross section }\end{array}$ \\
\hline $\begin{array}{l}b_{1}=0.04381350 \\
x_{1}=0.404042462 \\
z_{1}=0.06910532\end{array}$ & $\begin{array}{l}a_{j}=0.03593698 \\
x_{p}=0.50734029 \\
x_{p}^{\prime}=0.02924768\end{array}$ & $\begin{array}{l}x_{2}=0.47295351 \\
z_{2}=0,098590+4\end{array}$ & $\begin{array}{l}X_{5}=1.46333796 \\
Y_{3}=0.10182905\end{array}$ \\
\hline$\alpha_{1}^{\prime}=91.17604404^{\circ}$ & $\alpha_{0}=84.95281722$ & $\theta_{1}=7.1600328^{\prime}$ & $\theta_{1}=7.86100955$ \\
\hline$\beta_{1}=8.18540036^{\circ}$ & $\theta_{0}=3.397 .48512^{\circ}$ & $\phi_{2}=3.4435067^{*}$ & $\phi_{1}-3,91.1960 \mathrm{~m}^{*}$ \\
\hline$f_{1}=30.6385556^{\circ}$ & $r^{\prime} x^{\prime}=91.64969746^{6}$ & $v_{x}=16939196051^{+}$ & $\gamma_{1}=168.22136968^{\prime}$ \\
\hline$\phi_{1}=757607431^{\prime}$ & $\phi_{1}=1.2993949^{2}$ & $\xi_{z}=10,60813949^{\prime}$ & $\xi_{1}=11.77863032$ \\
\hline $\begin{array}{l}\text { 5ymat: } s_{e} ; \text { Sodel: Th } \\
\text { Loop satio; } \frac{n}{m}=\frac{11}{2}, \\
d=1 \\
f_{z}=1.45595178 \\
f_{b}=0.77841862\end{array}$ & $\begin{array}{l}\text { Stratse Qu } \\
\text { torus, Mas: } 105776774 \\
\text { Mas ratio } \frac{E}{n}=\frac{2277}{11}\end{array}$ & $\begin{array}{l}\text { Otignal: } a_{1}=0.342502 \\
\text { Effiective: } \alpha_{2}=0.49181\end{array}$ & $\begin{array}{l}253, A_{2}=059673505 \\
1712, V_{1}=0.45450967\end{array}$ \\
\hline 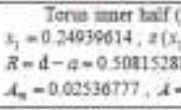 & $\begin{array}{l}\text { (petative curvature) } \\
\text { (j) }=3.14361337 \\
88 \\
=0.0010838\end{array}$ & $\begin{array}{r}\text { Torus sulet balf } \\
f_{1}=0.25060386 ;: 0\end{array}$ & $\begin{array}{l}\text { (poditive Cruatare) } \\
\left(\gamma_{3}\right)=3.13049347\end{array}$ \\
\hline $\begin{array}{l}\text { Triande } B_{1} D \sigma_{1} \text { an } \\
O H_{1} \text { cross section }\end{array}$ & $\mid \begin{array}{l}\text { Triangle } D E O_{1}^{\prime} \text { en } \\
\text { xy ars sortive }\end{array}$ & $\begin{array}{l}\text { Triangle } d_{2}^{\prime}, O_{1} O_{2} \text { ot } \\
O G^{\prime} \text { croes Section }\end{array}$ & $\begin{array}{l}\text { Trinde } C^{\prime} O_{0} O_{1} \text {, ab } \\
x y \text { coses sectoon }\end{array}$ \\
\hline $\begin{array}{l}\delta_{1}=0.04743466 \\
x_{1}=0.48658076 \\
z_{1}^{\prime}=0.08700298\end{array}$ & $\begin{array}{l}a_{j}=0.03773618 \\
x_{y}=0.50734522 \\
x_{p}=0.03916647\end{array}$ & $\begin{array}{l}X_{1}^{\prime}=0.48187592 \\
Z_{2}^{\prime}=0.09290415\end{array}$ & $\begin{array}{l}x_{1}=1.43832706 \\
x_{2}=0.09440493\end{array}$ \\
\hline$\alpha_{1}^{\prime}=36.51940954^{\prime}$ & $\alpha_{3}-81.96072169$ & $\theta_{2}=7.32508775^{\circ}$ & $\theta_{3}=7.3024952^{\prime}$ \\
\hline$f_{1}=7.90969623^{\circ}$ & $f^{n},-33831075^{\circ}$ & $\phi_{2}=3,58737434^{*}$ & $\phi_{x}=3.623 .067 \gamma^{\prime}$ \\
\hline$z_{1}^{\prime}=85.57089423^{\prime}$ & $f^{\prime} t_{0}=91.64511101^{\prime}$ & $V_{z}=16908753792^{2}$ & $V_{1}=16906929800^{2}$ \\
\hline$\phi_{1}=7.50100052^{\circ}$ & $\phi_{0}=3,290022202^{*}$ & \begin{tabular}{l|l|}
$\xi_{2}=10.91246208^{\circ}$ \\
\end{tabular} & $C_{1}=10.93070198^{\circ}$ \\
\hline $\begin{array}{l}\text { Symbol: } s_{k} ; \text { Model: Io } \\
\text { Loop ratio: } \frac{n}{m}-\frac{13}{31} ; A \\
d=1 \\
f_{2}=1.63243321 \\
f_{2}=0.6505010\end{array}$ & $\begin{array}{l}\text { Strange Qu } \\
\text { focus Manr: } 110.385263 \\
\text { Mas ratio: } \frac{D}{18}-\frac{333}{13}\end{array}$ & $\begin{array}{l}\text { Origimal: } a_{7}-0,30127 a \\
\text { Effoctive: } a_{1}^{\prime}=0.4918\end{array}$ & $\begin{array}{l}\text { ass, } b_{2}=0.52250032 \\
81712, b_{2}=0.339945+2\end{array}$ \\
\hline 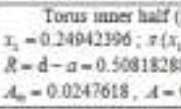 & 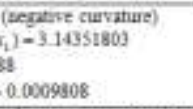 & $\begin{array}{r}\text { Ters outer haif } \\
x_{2}=0.25057604 ; \pi(x)\end{array}$ & $\begin{array}{l}\text { (positive curvanue) } \\
\left(x_{s}\right)=3.19958799\end{array}$ \\
\hline $\begin{array}{l}\text { Tningle } B_{2} D Q_{2} \text { un } \\
O_{1} H \text { 'crose section }\end{array}$ & $\begin{array}{l}\text { Irimgle } \mathrm{DEO}_{2} \mathrm{Em} \\
x-y \text { erow netioe }\end{array}$ & $\begin{array}{l}\text { Tringle } A_{1}^{\prime} O_{1} O_{1} \text { co } \\
Q \sigma^{\prime} \text { cross Section }\end{array}$ & $\begin{array}{l}\text { Triangle } G O O, O=\text { m } \\
\text { ty y ctoas section }\end{array}$ \\
\hline $\begin{array}{l}B_{3}=0.04212705 \\
X_{1}=0.48199034 \\
z_{2}=0.0076157\end{array}$ & $\begin{array}{l}a_{i}=0.03700574 \\
x_{0}=0.50706954 \\
r_{i}=0.0269166\end{array}$ & $\begin{array}{l}x_{1}=0.47450032 \\
z_{2}=0.03862555\end{array}$ & $\begin{array}{l}x_{7}=1.48896167 \\
x_{2}=0.09225751\end{array}$ \\
\hline$a_{1}^{\prime}=9028346766^{\prime}$ & $a_{x}^{\prime}-85.35383375$ & $\theta_{2}=7.13194595^{\prime}$ & $\theta_{1}=7.19940951^{\prime}$ \\
\hline$B_{1}=798558854^{\circ}$ & $F_{6}=3.128081 \mathrm{I}^{2}$ & $\phi_{2}=3.4339332^{2}$ & $\phi_{1}=3.54556923$ \\
\hline$y_{1}=81.73094349^{2}$ & $Y_{2}=915 t 828515$ & $\psi_{1}=169,42712087^{2}$ & $\psi_{1}=10931502126$ \\
\hline$\phi_{t}=7.43676 t 7^{2}$ & $8,=309617031^{*}$ & $\zeta_{1}=1057287913^{\circ}$ & $5,-10.63497874^{\circ}$ \\
\hline
\end{tabular}

\begin{tabular}{|c|c|c|c|}
\hline \multirow{2}{*}{\multicolumn{4}{|c|}{ 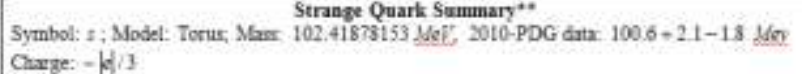 }} \\
\hline & & & \\
\hline \multirow{2}{*}{\multicolumn{4}{|c|}{ Loop ratio: $\frac{n}{m}-\frac{7+11-13-31}{17+21+31-71} ;$ Mass ratio: $\frac{E}{n}-\frac{1241+2277+2821-6330}{7+11+13-31}$}} \\
\hline \multirow{2}{*}{\multicolumn{3}{|c|}{ Original: $a_{3}=0.31650761, b_{i}=0.54156459$}} & \\
\hline & & \multirow{2}{*}{\multicolumn{2}{|c|}{$\begin{array}{l}\text { Original : } a_{3}=0.31650761, b_{4}=0.54156459 \\
\text { Effective } a_{2}=0.4918172, b_{2}=0.37579737\end{array}$}} \\
\hline & & & \\
\hline $\begin{array}{l}\text { Triangle } B_{1}^{\prime} D O_{2}^{\prime} \text { on } \\
Q_{2} H^{\prime} \text { aces sectiod }\end{array}$ & 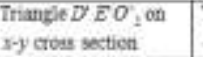 & $\begin{array}{l}\text { Trimigle } A_{1}^{\prime} O_{3} O_{1} \infty \\
O_{3} G \text { cosos Section }\end{array}$ & $\begin{array}{l}\text { Triesple } \mathrm{C}^{\prime} \mathrm{OP}_{2} \text { ea } \\
x-y \text { cross section }\end{array}$ \\
\hline$\alpha_{2}^{\prime}=8932632708^{\prime}$ & $\alpha^{\prime},-85,09114415^{\prime}$ & $\theta_{2}=7.200555549^{+}$ & $\theta_{1}=7.43552475^{\prime}$ \\
\hline$F_{1}-502069514^{*}$ & $\ddot{f}_{1}=130455 \times 97^{\circ}$ & $\phi_{a}=1.48927141^{*}$ & $\sigma_{1}=1.09591220^{\circ}$ \\
\hline$y_{1}^{\prime}=82.64677777$ & $Y_{1}^{\prime}=91.60429787$ & $\psi_{2}^{\prime}=169.3021731^{\prime}$ & $V_{1}^{\prime}=168.86856299^{\prime}$ \\
\hline \multirow{2}{*}{\multicolumn{4}{|c|}{ 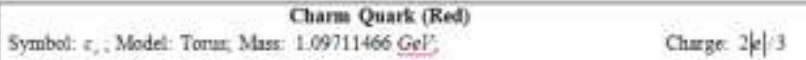 }} \\
\hline & & & \\
\hline \multicolumn{4}{|c|}{ Loop ratio: $\frac{n}{m}-\frac{7}{19} ;$ Mass ratio: $\frac{E}{n}-\frac{15009}{7}: \frac{2 p}{n}-1582$} \\
\hline \multicolumn{4}{|c|}{ 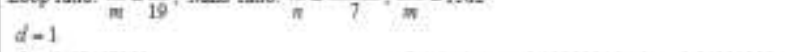 } \\
\hline \multicolumn{2}{|l|}{$f_{z}=-167467953$} & \multirow{2}{*}{\multicolumn{2}{|c|}{$\begin{array}{l}\text { Originat: } a_{1}=0.13583946, b_{2}=0.540 .21392 \\
\text { Effective } a_{1}^{\prime}=0.40151712, b_{1}=0.21347422\end{array}$}} \\
\hline \multicolumn{2}{|l|}{$f_{2}=0,40442168:$} & & \\
\hline \multicolumn{2}{|c|}{ 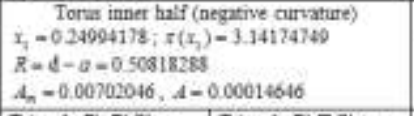 } & \multicolumn{2}{|c|}{$x_{7}=0.25005822: \pi\left(x_{7}\right)=314135027$} \\
\hline $\begin{array}{l}\text { Trimglo } B_{1}^{\prime} D^{\prime} \sigma_{2} \text { on } \\
Q_{1} H^{\prime} \text { coss section }\end{array}$ & \begin{tabular}{|l} 
Tringle $D E O_{1}^{\prime}$ on \\
$x-y$ coss section
\end{tabular} & $\begin{array}{l}\text { Trisingle } A_{1}^{\prime} O_{3} O_{4} 00 \\
O G^{\prime} \text { aces Setion }\end{array}$ & $\begin{array}{l}\text { Trimgle } \mathrm{GO}_{0} \mathrm{O}_{2} \text { an } \\
x \rightarrow y \text { tross sectioe }\end{array}$ \\
\hline$b_{1}-0.0252659$ & $a_{5}=0.01592798$ & $x ;=0.38550010$ & $x_{2}=1.49147183$ \\
\hline$x_{1}=0.5525042$ & $x_{0}=0.50000695$ & $2 ;=0.13546549$ & $\Gamma_{1}=0.03209436$ \\
\hline $2 ;-0.05552750$ & $r_{r}-0.00934507$ & & 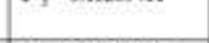 \\
\hline$\alpha_{1}=10395097758^{\circ}$ & $\alpha_{3}^{\prime}=83.38479759^{\circ}$ & $\theta_{2}=13.7020193^{*}$ & $\theta_{i}=2.50356000^{\circ}$ \\
\hline$\beta_{1}=10.70179659^{\circ}$ & $\hat{\beta}_{0}=1.0833615 g^{\prime}$ & $\phi_{2}=5.5828292^{\prime \prime}$ & $\psi_{1}=123275286$ \\
\hline$y_{1}^{4}=6534722583^{*}$ & $x_{0}=90.52634082^{2}$ & $\psi_{2}=100.05515023^{*}$ & $\psi_{1}=17620308705^{\prime}$ \\
\hline$\phi_{1}=8.87971373^{\circ}$ & $Q_{0}=1.05363163^{\circ}$ & $\zeta_{2}=1934434972$ & $\xi_{i}=3.72031295^{\circ}$ \\
\hline \multicolumn{4}{|c|}{ Carm Q } \\
\hline \multicolumn{4}{|c|}{ Symbol: $s_{y}$; Model: Torus, Mass: $1.37816406 \mathrm{GaV}$} \\
\hline \multirow{2}{*}{\multicolumn{4}{|c|}{ Loop ratio: $\frac{n}{n}-\frac{23}{29} ;$ Masu aztio: $\frac{D}{n}-\frac{62031}{23} ; \frac{2 F}{m}-4278$}} \\
\hline & & & \\
\hline \multicolumn{2}{|c|}{$f_{s}=1.88103598$} & \multicolumn{2}{|c|}{ Otiginal: $\alpha_{2}=0.26146077, \sigma_{2}-1.1753136$} \\
\hline \multicolumn{2}{|l|}{$f_{i}-0.88852712$} & \multicolumn{2}{|c|}{ Effective: $\sigma_{2}=0.49181712, b_{2}=1.04+29801$} \\
\hline 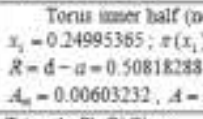 & 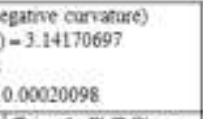 & $\begin{array}{r}\text { Torus obter late } \\
x_{2}=0.25004635 ; x 0\end{array}$ & $\begin{array}{l}\text { posinive curvanure) } \\
\text { 4) }=3.14139076\end{array}$ \\
\hline 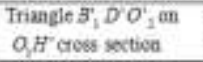 & $\begin{array}{l}\text { Thange } D \equiv O_{1} \text {, on } \\
x-y \text { acos section }\end{array}$ & $\begin{array}{l}\text { Trisede } d_{2}^{\prime} O_{1}^{\prime} O_{2} \text { an } \\
O_{1} G^{\prime} \text { aos Section }\end{array}$ & $\begin{array}{l}\text { Triascle } C^{\prime} O \rho_{2} \text { co } \\
x-y \text { ctous section }\end{array}$ \\
\hline$b_{1}-0.04507401$ & $a_{1}=0.01722845$ & $x_{7}=0.48342215$ & $x_{3}=1.49152951$ \\
\hline$x_{1}^{x}=0,45012930$ & $x=0.00304459$ & $2 ; z=019212854$ & $F_{3}=0.02929228$ \\
\hline $2,=000543592$ & $Y_{e}=0.01185476$ & & \\
\hline$\alpha_{1}^{\prime}-\$ 1.1170 .236^{*}$ & $\alpha_{i}-8795124719$ & $\theta_{2}=14.2946944^{*}$ & $\theta_{1}=2.28530676^{\circ}$ \\
\hline$\beta_{1}^{8}=10.00145794^{*}$ & $\theta_{1}=1.38040174^{\circ}$ & $\delta_{2}=737959975^{*}$ & $\phi_{1}=1.12509209^{\prime}$ \\
\hline$y_{1}^{\prime}=8388150971^{*}$ & $r_{1}=90.60835107$ & $V_{2}=158.32570655^{\circ}$ & $V_{1}=176.58954115$ \\
\hline$\phi_{1}=962160726^{\circ}$ & $\phi_{1}=133670214^{\prime}$ & $5_{3}-21.67429315^{5}$ & $5,-3.41045885$ \\
\hline & Charm Q & re (Blue) & \\
\hline Symboil: $c_{3} ;$ Mosel: Tor & ras, Mass $1.34239414 \mathrm{~S}$ & & Charge: $2 p \mid / 3$ \\
\hline Loop ratio: $\frac{\pi}{n}=\frac{29}{37}, \mathrm{M}$ & Cass ratio: $\frac{E}{n}=\frac{76183}{70}:=$ & $\frac{2 p}{n}=$ & \\
\hline$d=1 ;$ & $n 29$ & & \\
\hline$f_{a}=1.893119 ;$ & & Original: $a_{4}=0.259$ & $b_{z}=1.16086964$ \\
\hline$f,-0.88500306$ & & Effortive $a_{1}^{1}=0.49$ & $12,5_{3}-1,0280697$ \\
\hline $\begin{array}{r}\text { Torus inner balf } f 0 \\
x_{1}=0.24995242 ; \pi\left(x_{1}\right. \\
R=d-d=0.50818288 \\
L_{n}-0.00014251, \lambda-\end{array}$ & ogdive curvatice) & $\begin{array}{l}\text { Tocus odfer hi } \\
x_{3}=0.25004758 \text {; }\end{array}$ & $\begin{array}{l}\text { positive curvatiae) } \\
=31413655\end{array}$ \\
\hline $\begin{array}{l}\text { Triangle } B^{\prime}, D^{\prime} O_{1}^{\prime} \text { un } \\
O_{1} H^{\prime} \text { acoss sotion }\end{array}$ & $\begin{array}{l}\text { Trimgle } \mathrm{DEO}^{\prime} \text {, on } \\
x-y \text { aros sectioe }\end{array}$ & $\begin{array}{l}\text { Trumple } A_{z} \sigma_{1}, O \\
\text { on } \\
O, G \text { cross Section }\end{array}$ & 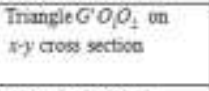 \\
\hline$b_{3}=0.04615009$ & $a_{1}=0.01743585$ & $X_{1}^{\prime}=0.45318876$ & $x_{;}=1.49152351$ \\
\hline & $x=0.5050+188$ & & $r_{1}=0.02959618$ \\
\hline & $F_{0}=0.01197042$ & & \\
\hline$a_{1}^{\prime}=81.0522684^{*}$ & $\alpha_{4}=82.93127035$ & $\theta_{2}-14.27744210^{\circ}$ & $\theta_{1}=23090329 \mathrm{I}^{2}$ \\
\hline$B_{\mathrm{t}}=10.08108219$ & $\varepsilon_{1}=1,3938 \times 663^{\circ}$ & $\phi_{2}=736565041^{\circ}$ & $\sigma_{1}=1.13676067^{\circ}$ \\
\hline$y_{1}=88.83664941^{\circ}$ & $y_{1}=90.67487302^{2}$ & $V_{3}=158.35690745^{5}$ & $V_{1}=176.554200072^{\prime}$ \\
\hline$\phi_{1}=9.69521631^{\circ}$ & $6 t_{2}=1.34974604^{\circ}$ & $G_{2}=21.04309257$ & $5:=3.4579938^{\circ}$ \\
\hline
\end{tabular}


Z. Y. SHEN

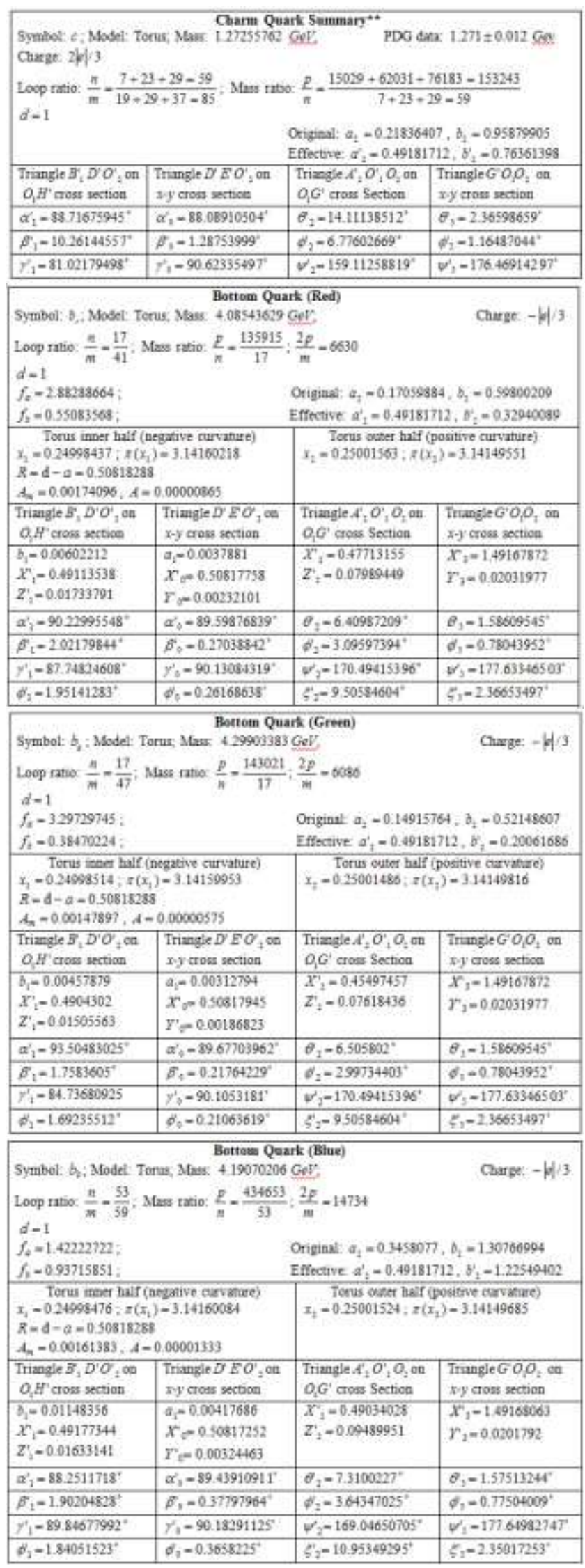

Symbol: $b$; Modet: Torus, Mass: $4.19172406 \mathrm{GeV} \quad 2010-\mathrm{PDG}$ data $4.187=0.010 \mathrm{GeV}$ Charge: $-|p| / 3$

Loop ratio: $\frac{n}{m}-\frac{17+17+53=87}{41+47+59=147} ;$ Mast ratio: $\frac{E}{n}-\frac{135915+143021-434653=713589}{17+17+53-87}$ $d=1$

Origimal: $a_{2}=0.22185473, b_{2}=0.9090527$

Effective: $a_{2}^{\prime}=0.49181712, b_{2}=0.58517059$

\begin{tabular}{|c|c|c|c|}
\hline $\begin{array}{l}\text { Triangle } B^{\prime}, D^{\prime} O^{\prime} ; \text { ot } \\
Q_{1} H^{\prime} \text { cross section }\end{array}$ & $\begin{array}{l}\text { Triangle } D E O_{4} \text {, on } \\
x-y \text { eross section }\end{array}$ & $\begin{array}{l}\text { Triangle } A_{7} O_{3}, O_{7} \text { on } \\
o, G \text { cross Section }\end{array}$ & $\begin{array}{l}\text { Tringle } G O^{\prime} O_{\text {, on }} \\
x-y \text { ctoss section }\end{array}$ \\
\hline$\alpha_{1}=90.66198584^{*}$ & $\alpha_{n}=89.57163904^{\prime}$ & $\theta_{2}=6.74279893^{\circ}$ & $\theta_{1}=1.58244 t_{11}$ \\
\hline$\beta_{1}=1.89406907^{*}$ & $\beta_{s}=0.28867012^{*}$ & $\phi_{1}=324559607$ & $\phi_{3}=0.77863971^{*}$ \\
\hline$\gamma_{1}^{\prime}=87.44394508^{\circ}$ & $\gamma_{t}^{\prime}=90.13969085^{\circ}$ & $V_{2}^{\prime}=170.01160499^{\prime \prime}$ & $\psi_{3}=177.63891918$ \\
\hline
\end{tabular}

Symbol $1_{n} ;$ Model: Spindle type torus; Mass: 171.19127784 GeVt;

Loop ratio: $\frac{n}{m}=\frac{283}{43} ;$ Mass ratio: $\frac{p}{n}=\frac{94808679}{283} ; \frac{2 p}{m}=4409706$

Carge: $2 p \mid / 3$

$d=1$

Originat: $a_{2}=105327498, b_{2}=10.16538325$

\begin{tabular}{l|l}
\hline Torus inase half (positive curvature) & Torus outer half (positive curvature)
\end{tabular}

$x_{1}=0.24999963 ; \pi\left(x_{2}\right)=3.14155011 \quad x_{2}=0.25000037 ; \pi\left(x_{2}\right)=3.1415475$ ?

$R=|a-a|=0.05327498$

\begin{tabular}{l|l|l|l} 
Triangle $B_{1} O_{1} O_{2}$ on & Triangle $D O_{1} O_{2}^{\prime}$ on & Triangle $\alpha_{2} O_{2} O_{2}$ on & Triangle $G O_{1} O_{2}$ on
\end{tabular}

\begin{tabular}{l|l|l|l}
\hline,$H$ cross section & $x-y$ cross section & $Q, G$ cross section & $x-y$ cross section
\end{tabular}

\begin{tabular}{l|l|l|l}
\hline$X_{1}=1.04929973$ & $X_{0}=0.05327281$ & $X_{1}=1.04906252$ & $X_{3}=2.05318657$
\end{tabular}

\begin{tabular}{l|l|l|l}
$Z_{1}=0.88234854$ & $Y_{0}=0.00043021$ & $Z_{2}=0.90824121$ & $Y_{1}=0.01905347$
\end{tabular}

\begin{tabular}{|l|l|l|l|}
\hline$Q_{1}=46.74178431^{*}$ & $Q_{2}=0.49033701^{*}$ & $B_{2}=16.97967409^{*}$ & $\theta_{1}=0.50475284^{*}$
\end{tabular}

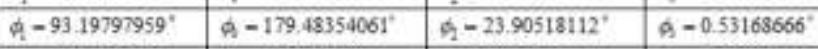

\begin{tabular}{|l|l|l|l|}
\hline$V_{f}+40.0602361^{*}$ & $V_{f}=0.02612238^{\circ}$ & $v_{2}=139.11514479^{*}$ & $\psi_{1}=178.9635605$ \\
\hline
\end{tabular}

\begin{tabular}{|c|c|c|c|}
\hline $5-86.80202041^{*}$ & $5-0.51645939^{\circ}$ & $4=40,33485521^{2}$ & $\zeta=1.036+395$ \\
\hline
\end{tabular}

Top Quark (Green)

Symbol: $t_{i} ;$ Model: Spindle type torus, Mass: $170.9746143 \mathrm{GeV}$;

Loop ratio: $\frac{n}{m}=\frac{331}{53}$, Mass ratio: $\frac{p}{n}=\frac{110748959}{331} ; \frac{2 p}{m}=4179206$

d' -1 ;

Original $a_{f}-1.02059277, b_{-}-9.64029008$

\begin{tabular}{c|c} 
Torus imer balf (positive curvature) & Torus outer lalf (positive curvature)
\end{tabular}

$x_{1}=0.24999963 ; \pi\left(x_{1}\right)=3.14155011 \quad x_{2}=0.25000037 ; \pi\left(x_{1}\right)=3.14154756$

$R=|1-a|=0.02059277$

\begin{tabular}{|l|l|l|l|}
\hline Triangle $B_{2} O_{1} O_{2}^{\prime}$ on & Trumgle $D O_{1} O_{1}^{\prime}$ an & Trangle $A_{2} O_{1} O_{4}$ on & Trangle $G O_{2} O_{2}$ on
\end{tabular}

\begin{tabular}{l|l|l|l}
$O, F$ crosa section & $x y$ cross section & $Q_{1}, G$ cross section & $x-y$ cross section
\end{tabular}

\begin{tabular}{l|l|l|l}
\hline$X_{1}=1.01690285$ & $X_{0}=0.02059193$ & $X_{2}=1.01608235$ & $X_{1}=2.02050577$
\end{tabular}

\begin{tabular}{l|l|l|l}
$Z_{1}=0 . \$ 1902000$ & $Y_{0}=0.00018502$ & $Z_{2}=0.84309001$ & $Y_{3}=0.01875053$
\end{tabular}

\begin{tabular}{|l|l|l|l|}
\hline$\theta_{1}-49.96951438^{\circ}$ & $\theta_{5}=0.50602919^{*}$ & $\theta_{2}-16.97967409^{*}$ & $\theta_{1}=0.52092419^{\prime}$
\end{tabular}

\begin{tabular}{|l|l|l|l|}
\hline$\phi-91.1822964^{\prime}$ & $\phi_{0}=179.4835504^{\prime}$ & $\phi_{3}-22.68775201^{\circ}$ & $\phi_{-}-0.53169617^{\prime}$
\end{tabular}

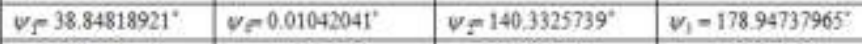

\begin{tabular}{|l|l|l|l|}
\hline$\zeta r^{88} \$ 177036^{\circ}$ & $\$ r 0.5164496^{\circ}$ & $\$ r^{39} .6674261^{\circ}$ & $\$ r 1.05262035^{\circ}$ \\
\hline
\end{tabular}

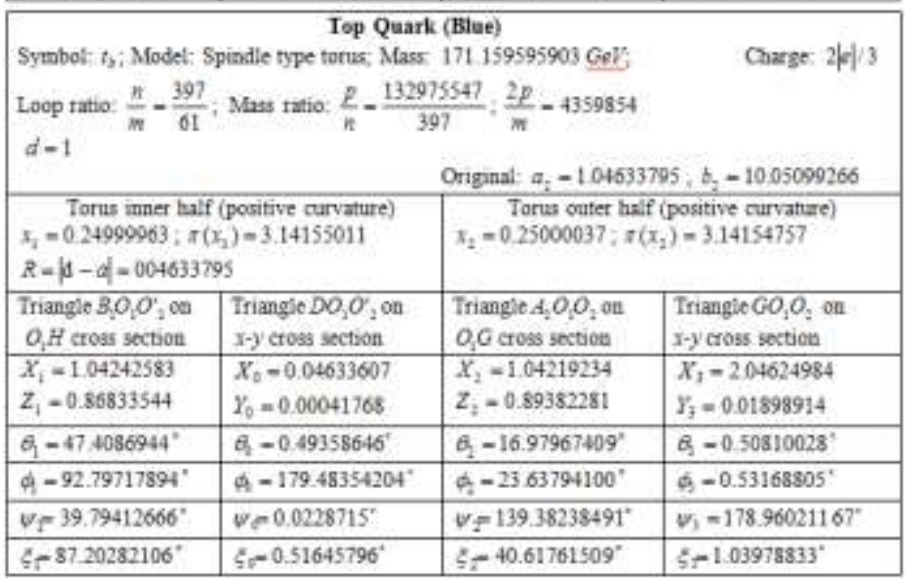




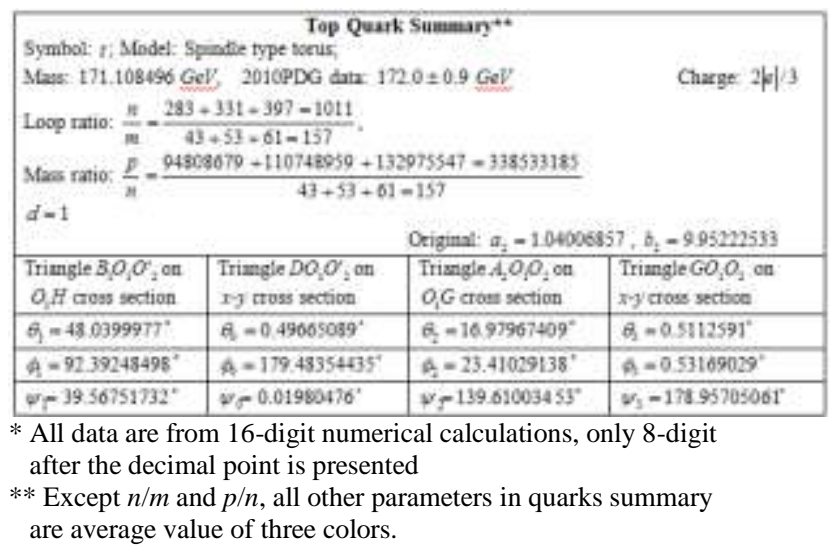

The mass values for six quarks as the average values of three colors for each flavor listed in Table11.2 are all within 2010-PDG data error ranges. The PDG data are not from direct measurements; they are extracted from experimental data of baryons made of quarks. So the agreements are indirect.

The three inner angles of the triangle $B_{1}^{\prime} D^{\prime} O_{2}^{\prime}$ for six quarks are listed in Table 11.3, which is averaged over three colors for each flavor cited from the summary tables of Table 11.2 .

Table 11.3: Three Inner Angles $\alpha_{1}, \beta_{1}, \gamma_{1}$ of Triangle $B^{\prime}{ }_{1} D^{\prime} O^{\prime}{ }_{2}$

\begin{tabular}{|l|l|l|l|}
\hline Quarks name & \multicolumn{1}{|c|}{$\alpha_{1}$} & \multicolumn{1}{c|}{$\beta_{1}$} & \multicolumn{1}{c|}{$\gamma_{1}$} \\
\hline Up & $85.98933354^{\circ}$ & $22.09005849^{\circ}$ & $71.92060796^{\circ}$ \\
\hline Down & $101.75310909^{\circ}$ & $11.2957027^{\circ}$ & $66.95118821^{\circ}$ \\
\hline Strange & $89.32632708^{\circ}$ & $8.02689514^{\circ}$ & $82.64677777^{\circ}$ \\
\hline Charm & $88.71675945^{\circ}$ & $10.26144557^{\circ}$ & $81.02179498^{\circ}$ \\
\hline Bottom & $90.66198584^{\circ}$ & $1.89406907^{\circ}$ & $87.44394508^{\circ}$ \\
\hline Top & Triangle $B_{1}^{\prime} E_{1}^{\prime} O_{2}^{\prime}$ is not valid for spindle torus due to $\pi\left(x_{1}\right)<\pi$. \\
\hline
\end{tabular}

According to 2010-PDG (pp. 146-151) experimental data, in the Cabibbo-Kobayashi-Maskawa ( $C K M)$ triangles, the three inner angles of the unitarity triangle are:

$$
\begin{gathered}
\alpha=89.0^{\circ}+4.4^{\circ}, \\
-4.2^{\circ}, \\
\beta=21.15^{\circ}+0.904^{\circ}, \\
-0.879^{\circ}, \\
\gamma=73^{\circ}+22^{\circ} . \\
-25^{\circ}
\end{gathered}
$$

Other five $C K M$-triangle all are elongated.

Comparing Table 11.3 to 2010-PDG data shows close similarities:

1. The $B_{1}^{\prime} D^{\prime} O_{2}{ }_{2}$ triangle of up quark is very close to the unitarity triangle given by (11.7). In fact, the SQS theoretical values of two angles $\alpha_{1}$, and $\gamma_{1}$ are within PDG data error ranges. The relative deviation of $\beta_{1}=22.09005849^{\circ}$ from $2010-\mathrm{PDG}$ medium value $\beta=21.15^{\circ}$ is $4.3 \times 10^{-2}$ at its error range's upper edge.

2. The experimental data show that, except for the unitarity triangle of (11.7), five other $C K M$-triangles are elongated. In Table 11.3, except for $B_{1}^{\prime} D^{\prime} O_{2}^{\prime}$ triangle of the up quark, other four quarks' $B_{1}^{\prime} D^{\prime} O^{\prime}{ }_{2}$ triangles are elongated and the one for top quark is not valid.

3. Required by unitarity of probability, the side between angle $\beta$ and angle $\gamma$ of $C K M$-triangle is normalized to unity. The side $O_{2}^{\prime} D^{\prime}$ of triangle $B_{1}^{\prime} D^{\prime} O_{2}^{\prime}$ is normalized to unity for the other two sides representing probabilities.

According to SQS theory, there are fifteen $B_{1}^{\prime} D^{\prime} O^{\prime}{ }_{2}$ triangles comparing to five $C K M$-triangles for five flavored quarks except the top quark. This difference may provide an important clue for the question regarding $C K M$-triangle: Is the unitarity $C K M$-triangle really a triangle? This is a serious question. If the answer is no, the standard model must be revised. As shown by (11.7), two angles $\alpha$ and $\gamma$ have large error ranges, and the sum of three inner angles medium values equals to $183.15^{\circ}$ instead of $180^{\circ}$. From SQS theory standpoint, the problem can be naturally resolved by recognized the fact that, there are eighteen quarks with different flavors as well as different colors. As a result, the unitarity $C K M$-triangle isn't a single triangle, it is a set of three triangle corresponding to three different colored quarks $u_{r}, u_{g}, u_{b}$. As listed in Table 11.2, three up quarks $u_{r}, u_{g}$, $u_{b}$ have $\gamma_{1, u r}=88.61137029^{\circ}, \gamma_{1, u g}=70.37440598^{\circ}$, $\gamma_{1, u b}=56.77604761^{\circ}$, respectively. The large error range of $\gamma=73^{\circ}+22^{\circ}-25^{\circ}$ give by $(11.7 \mathrm{c})$ is the result of attempting to combine three different triangles into one. The same argument is applicable to angles $\alpha$ and $\beta$. So the large error ranges of $C K M$-triangle data have a reasonable explanation based on Postulation 11.1.

There are other reasons to identify triangles $B_{1}^{\prime} D^{\prime} O_{2}^{\prime}$ as the $C K M$-triangles. Quarks are represented by their torus models and characteristic points carry information from the $S$-equation to torus model. In principle, all parameters including the $C K M$-triangles should be derived from the model. Moreover, if the angles are kept the same, the triangles are similar. As one side is normalized, the other two sides of the similar triangles also represent the same information. In this way, the converting probabilities among different quarks via weak interactions indicated by the other two sides of the $C K M$-triangle should be transferred to the $B_{1}^{\prime} D^{\prime} O_{2}^{\prime}$ triangle as well. For all these reasons, the $B_{1}^{\prime} D^{\prime} O^{\prime}{ }_{2}$ triangles are identified as the $C K M$-triangles. It is another step towards the final goal: All physics parameters of an elementary particle are derived from its model.

The generalized $A T$ - and $P S$-formulas of (10.7) are used to calculate the angle tilt and phase sync data for fifteen quarks listed in Table 11.4. Three top quarks are excluded, because for them the $f$-modification is not fully applicable. The data for three charged leptons are listed for comparison. 
Z. Y. SHEN

Table 11. 4: Phase Sync Data for 15 Quarks and 3 Charged Leptons*

\begin{tabular}{|c|c|c|c|c|c|}
\hline Panticis & $\begin{array}{l}\text { P5 value at } \\
A T=0\end{array}$ & $\begin{array}{l}\text { F5 valote } \\
\text { vamation }\end{array}$ & Particle & $\begin{array}{l}P S \text { value at } \\
A T=0\end{array}$ & $\begin{array}{l}\text { PS vinat } \\
\text { vaniation }\end{array}$ \\
\hline$d_{\text {. }}$ & 0 & $\begin{array}{l}+4.4 \times 10^{-16} \\
+2.22 \times 10^{-16}\end{array}$ & ע. & 0 & $\begin{array}{l}+221 \times 10^{-9} \\
-222 \times 10^{-4}\end{array}$ \\
\hline$d_{r}$ & 0 & $\begin{array}{l}+122 \times 10^{-21} \\
-2.22 \times 10^{-18}\end{array}$ & $u_{t}$ & 0 & $\begin{array}{l}+2.22 \times 10^{-11} \\
-2.22 \times 10^{414}\end{array}$ \\
\hline$d_{b}$ & $\overline{0}$ & $\begin{array}{l}+2.22 \times 10^{-1} \\
-0\end{array}$ & $u_{b}$ & 0 & $\begin{array}{l}+2.22 \times 10^{10} \\
-1.11 \times 10^{-11}\end{array}$ \\
\hline ti & $8.85 \times 10^{-71}$ & $\begin{array}{l}+4.44 \times 10^{-1 / 6} \\
-4.4 \times 10^{-16}\end{array}$ & c. & $-1.13 \times 10^{14}$ & $\begin{array}{l}+2.22 \times 10^{-1} \\
-3.39 \times 10^{-16}\end{array}$ \\
\hline$s_{x}$ & 0 & $\begin{array}{l}+6.66 \times 10^{-11} \\
-4.45 \times 10^{-4}\end{array}$ & $c_{r}$ & 0 & $\begin{array}{l}+0.56 \times 10^{-19} \\
-4.4 \times 10^{-4}\end{array}$ \\
\hline si & $1.11 \times 10^{-11}$ & $\begin{array}{l}+1.11 \times 10^{41} \\
-1.11 \times 10^{-41}\end{array}$ & $c_{b}$ & $-192 \times 10^{-14}$ & $\begin{array}{l}+155 \times 10^{-11} \\
-1.11 \times 10^{-44}\end{array}$ \\
\hline $\bar{b}$ & $-126 \times 10^{-4}$ & $\begin{array}{l}-4.44 \times 10^{-14} \\
-44 \times 10^{-21}\end{array}$ & $e$ & 0 & $\begin{array}{l}-222 \times 10^{-4} \\
-2.22 \times 10^{4}\end{array}$ \\
\hline$b_{t}$ & $-3.69 \times 10^{-4}$ & $\begin{array}{l}+4.4 \times 10^{-11} \\
-2.22 \times 10^{44}\end{array}$ & $\mu$ & $9.55 \times 10^{-1}$ & $\begin{array}{l}+5.77 \times 10^{-1} \\
-2.22 \times 10^{-4}\end{array}$ \\
\hline$b_{2}$ & $+22 \times 10^{44}$ & $\begin{array}{l}+2.22 \times 10^{-1} \\
-2.22 \times 10^{-18}\end{array}$ & $t$ & $2.12 \times 10^{-4}$ & $\begin{array}{l}+0 \\
-7,7 \pi \times 10^{-16}\end{array}$ \\
\hline
\end{tabular}

* 1. The data are from 16-digit numerical calculations. Only three effective digits are listed.

2. The listed $f_{a}$ vary in $1 \times 10^{-15}$ steps within range of $\pm 100 \times 10^{-15}$.

The features of these results are summarized as follows:

1. Electron, three up quarks and three down quarks have perfect phase synch among two loops' cyclic movements and the sinusoidal oscillation of the mass term indicated in Table 11.4 as " $P S$ values at $A T=0$ " equal to zero. Their angle tilt equation (10.7a) and phase sync equation (10.7b) are satisfied simultaneously. The perfect synchronization is interpreted as electron, up quarks and down quarks are stable fermions. In fact, these three types of particles are stable and serve as the building blocks of all atoms and molecules in the real world.

2. The other particles listed in Table 11.4 namely muon, taon, and strange, charm, bottom quarks are not perfectly synced indicated by their " $P S$ values at $A T=0$ " equal to nonzero values. According to the same reason, it can be interpreted as they are not stable particles. In fact, muon, taon, and all hadrons composited with strange, charm, bottom quarks are unstable and subject to decay.

3. All fifteen quarks and three charged leptons have fluctuation phase variations noted as the " $P S$ value variation" in Table 11.4. It means that all these particles have the trajectory jumping behavior similar to electron's trajectory jumping behavior described in Section 8.

Formulas of (8.38) are used to calculated the tilted angle $\Delta \vartheta$ deviated from $\vartheta=120^{\circ}$. The $\Delta \vartheta$ data along with $a_{2}$, $a_{2}^{\prime}$ and $f_{a}$ for three charged leptons and fifteen quarks are listed in Table 11.5. Three top quarks are excluded, because the $f$-modification is not fully applicable.

It is interesting to find out that, for the fifteen quarks despite of their more than three orders of magnitude mass differences, the values of $\Delta \vartheta=0.53990^{\circ} \pm 0.00001^{\circ}$ are within $10^{-5}$ degree, which corresponds to the values of $\vartheta=120^{\circ}-\Delta \vartheta$ within the same range. This is possible because despite their very different mass and $a_{2}$ values, the $f$-modification is capable to bring back the $a_{2}{ }_{2}$ values within a very narrow range of $a_{2}^{\prime}=0.4918172 \pm 0.0000001$. These results are related to the $S U(3)$ group symmetry associated with quark's flavors and colors, which will be discussed in Section 21 and Section 24.

Table 11.5: Calculated $f_{a}, a_{2}^{\prime}, \Delta \vartheta$ Data for 3 Charged

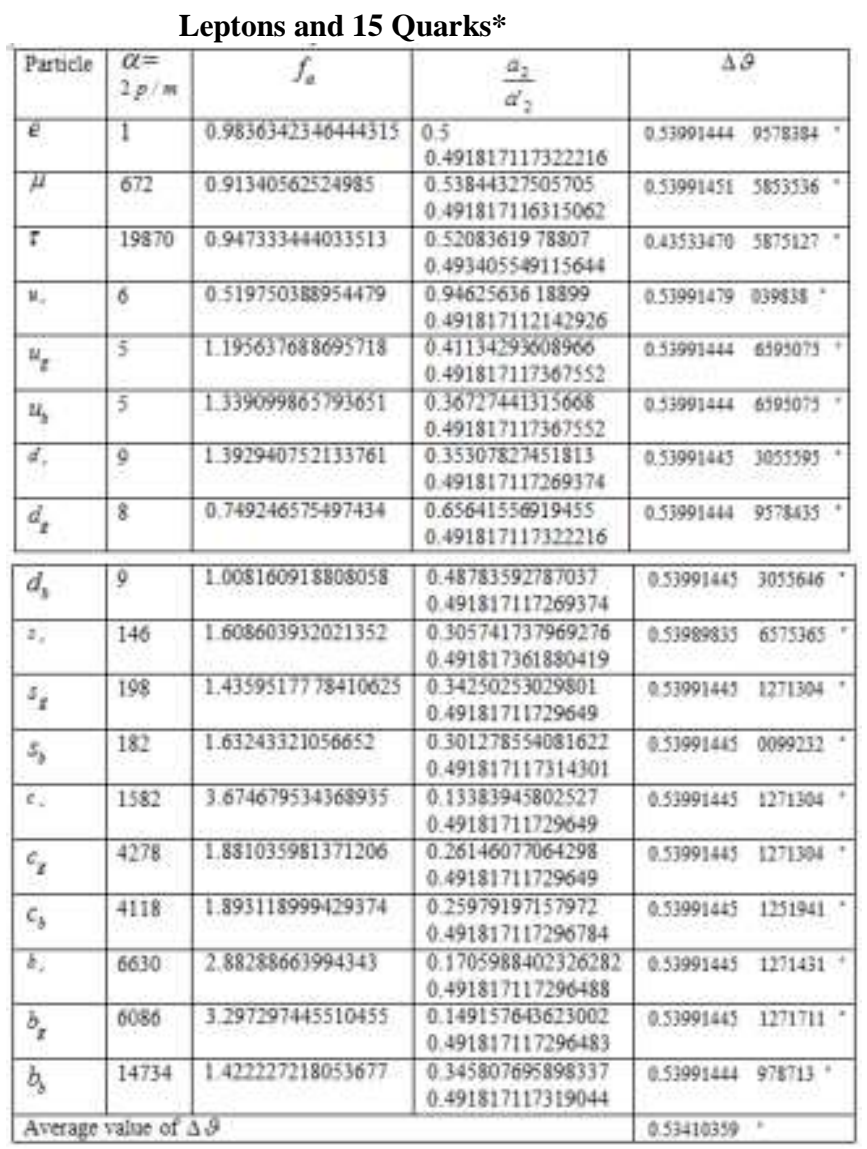

*. The data for leptons are based on trefoil type model in Section 12.

The results shown in Table 11.4 and Table 11.5 indicate that, even though the $A T$ - and $P S$-equations are ad hoc equations, they catch the essence of these particles.

Postulation 11.1 is important for SQS theory. To recognize quarks of same flavor with different colors as different particles plays pivotal roles in many areas. There are at least two facts to support Postulation 11.1. As mentioned previously, the large error ranges of $\alpha$ and $\gamma$ for the unitarity triangle shown in (11.7) can be explained naturally by three up quarks with different colors as three particles instead of one. It serves as evidence. The other evidence is quarks mass values. As shown in the PDG data book, most of the weighted average curves for quarks' mass have more than one peaks corresponding to a flavored quark made of multicomponents with different mass values. According to Postulation 11.1, the multi-peak behavior corresponds to quarks with the same flavor and different colors having different masses. Moreover, compared to the 2008-PDA data, the 2010-PDA data show more evidences of multi-peak behavior for quarks mass curves. This argument is also supported by other evidence. In the PDG data book, most 
weighted average mass curves for hadrons made of quarks (anti-quarks) with different flavors show similar multi-peak behavior as they should be. Quarks with different flavors having different mass values are recognized as different elementary particles, with the same reason, so are quarks with different colors having different mass values.

Experiments found that, a hadron is composed of pointlike constituents named "partons". There are three valence partons identified as three quarks, $u, u, d$ as the constituents of proton. According to Postulation 11.1, proton is composed of nine quarks: $u_{r}, u_{g}, u_{b}$ for an $u$ quark, $u_{r}, u_{g}, u_{b}$ for another $u$ quark, $d_{r}, d_{g}, d_{b}$ for the $d$ quark. The question is: How the nine quarks show up in a proton? There are two possible options.

Option-1: There are three smaller point-like constituents inside a valence parton simultaneously. If this is the case, a flavored quark's mass equals to the sum of three constituents quarks. It is contradictory to fact that, as shown by quark multi-peak weighted average mass curve, a flavored quark's mass equals to the average of constituents' mass. So this option is ruled out.

Option-2: For a quark with the same flavor and different colors such as $u_{r}, u_{g}, u_{b}$ each one takes turns to show up. At a given time, only one out of three shows up. A flavored quark's mass equals to the average of its three constituent colored quarks' mass. It fits the multi-peak weighted average mass curve well. This option is accepted. But it raises a question: Does each colored quark show up with different time intervals? If the answer is yes, then the flavored quark's mass equals to the weighted average of three constituents mass. In this way, the average mass for favored quark and the theoretical value $\beta_{1}=22.09005849^{\circ}$ listed in Table 11.3 should be re-calculated to include the weighed factors. The results with weighted factors proportional to the reciprocal of three colors' mass values are as follows. Weighted up quark mass value:

$$
M_{u}=2.3276313 \mathrm{MeV} / \mathrm{c}^{2},
$$

Weighted up quark $\beta_{1}$ value:

$$
\beta_{1}=21.93059933^{\circ} \text {. }
$$

Both results are within 2010PDG data error ranges.

The importance of Postulation 11.2 and Rule 11.1 has been shown by Conclusion 11.1. In fact, Postulation 11.2 as the second fundamental Postulation of SQS theory has many important impacts far beyond quarks, which will be given in later sections.

\section{Section 12: Trefoil Type Model for Charged Leptons}

In this section, a broad view is taking to look at leptons. Based on Prime Numbers Postulation and intrinsic relation between leptons and quarks, a new type of model with torus as building blocks is introduced for charged leptons.
In Section 11, nine even pairs of prime numbers are assigned as the $m$-parameters for nine pairs of up type and down type quarks as listed in Table 11.1.

Postulation 12.1: The original (before reduction) $m$ parameter of a lepton is an even number equal to the average value of the $m$-parameters of associated up type quark and down type quark.

Explanation: In fact, this is the unstated reason in Section 8 and Section 10 to select 2, 18, and 42 for the original $\mathrm{m}$ parameters of electron, muon and taon, respectively.

$$
\begin{aligned}
& m_{e}=\left(m_{u r}+m_{d r}\right) / 2=(1+3) / 2=2, \\
& m_{\mu}=\left(m_{c r}+m_{s r}\right) / 2=(19+17) / 2=18, \\
& m_{\tau}=\left(m_{t r}+m_{b r}\right) / 2=(43+41) / 2=42,
\end{aligned}
$$

\begin{tabular}{|c|c|c|c|c|c|c|c|c|c|c|}
\hline \multirow{2}{*}{$\begin{array}{c}\text { Generations } \\
\text { Colors }\end{array}$} & \multicolumn{3}{|c|}{$1^{\text {It }}$ generation } & \multicolumn{3}{|c|}{$2^{\text {nd }}$ generation } & \multicolumn{3}{|c|}{$3^{\text {td }}$ generanion } & \multirow[t]{2}{*}{ Fnd } \\
\hline & red & green & blue & red & mem & Boue & red & grcen & blue & \\
\hline$m_{\infty}$ & 1 & 7 & 13 & 19 & 29 & 37 & 43 & 53 & $6 !$ & 71 \\
\hline Up type quarks & is, & $\mathrm{n}_{t}$ & $u_{k}$ & $c_{r}$ & $c_{f}$ & $c_{i}$ & $t_{4}$ & $t_{t}$ & $t_{3}$ & probilibitent \\
\hline Leptons & $e$ & 9 & $?$ & $\mu$ & $?$ & 7 & $\tau$ & 7 & 7 & prohibited \\
\hline Neutrmos & $v_{\text {, }}$ & 7 & 7 & $v_{4}$ & 7 & 7 & $v_{r}$ & 7 & $?$ & Drobibited \\
\hline$\frac{m_{\mathrm{ce}}+m_{\mathrm{mar}}}{2}$ & $\begin{array}{l}2 \\
\text { even }\end{array}$ & $\begin{array}{l}6 \\
\text { even }\end{array}$ & $\begin{array}{l}12 \\
\text { event }\end{array}$ & $\begin{array}{l}18 \\
\text { even }\end{array}$ & $\begin{array}{l}26 \\
\text { even }\end{array}$ & $\begin{array}{l}\text { It } \\
\text { even }\end{array}$ & $\begin{array}{l}42 \\
\text { even }\end{array}$ & $\begin{array}{l}50 \\
\text { event }\end{array}$ & $\begin{array}{l}60 \\
\text { even }\end{array}$ & $\begin{array}{l}69 \\
\text { कda }\end{array}$ \\
\hline Denn type quara & di, & $d_{z}$ & $d_{k}$ & 8. & $s_{t}$ & $I_{1}$ & b. & $b_{t}$ & b) & peohibited \\
\hline in deve & 3 & 5 & II & 17 & 23 & 31 & 41 & 47 & 59 & 67 \\
\hline
\end{tabular}

According to Postulation 12.1, the results for six leptons are listed in Table 12.1. The $m$-parameters of eighteen quarks are also listed for reference.

Table 12.1: Leptons and Quarks with Assigned $m$-Parameters*

* The $m$-parameters listed are their magnitude; signs are defined by (11.6).

Conclusion 12.1: There are only three generations of quarks and leptons. The fourth generation is prohibited.

Proof: In the "End" column of Table 12.1, the average of two $m$-parameters, $67 \& 71$, is an odd number,

$(67+71) \div 2=69$. According to Postulation 12.1, the

fourth generation leptons are prohibited. According to Conclusion 11.1, the fourth generation quarks are prohibited. QED

Conclusion 12.1 is the extension of Conclusion 11.1 based on the Prime Numbers Postulation and the intrinsic relation between quarks and leptons.

On the experiment side, according to 2010-PDG data, the number of light neutrino types from direct measurement of invisible $Z$ width is $2.92 \pm 0.05$. The number from $e^{+} e^{-}$ colliders is $2.9840 \pm 0.0082$. Both results show no trace of fourth generation neutrino existence. These experimental data support Conclusion 12.1.

Notice that, there are vacant cells marked with "?" in Table 12.1. The question is: Are there any undiscovered leptons? In the three generations, there are twelve lepton vacancies, in which six are $e, \mu, \tau$ type, and the other six are $v_{e}, v_{\mu}, v_{\tau}$ type. If these vacancies correspond to undiscovered leptons, the six $e, \mu, \tau$ type would be charged leptons with mass ranging from a few $\mathrm{MeV} / \mathrm{c}^{2}$ to a few thousands $\mathrm{MeV} / \mathrm{c}^{2}$. That is impossible, because charged particles in such mass range should be discovered already. 


\section{Z. Y. SHEN}

The neutrinos $v_{e}, v_{\mu}, v_{\tau}$ are intrinsically associated with their companions leptons, $e, \mu, \tau$ respectively. If there are no undiscovered charged leptons, so are no undiscovered neutrinos associated with them.

To fill the vacancies with undiscovered leptons isn't the only way. The other way is that, these vacancies serve as a hint for new structure of existing leptons.

The first generation fermions are divided into four categories including two types of leptons $e$ and $v_{e}$, and two

flavors of quarks each with three colors, $u_{r}, u_{g}, u_{b}$ and $d_{r}$, $d_{g}, d_{b}$. The second and third generations have the same structure. Should leptons also have colors? This is the initial thought inspired by the vacancies in Table 12.1.

The basic idea is that, leptons' new model has three branches. Each branch separately is a torus model. The three branches combine to form the new model.

Leptons' torus model has spin $\hbar / 2$. The new model made of three torus should also have spin $\hbar / 2$. There are two options to deal with the spin problem.

Option-1. Let two branches have spin $+\hbar / 2$, and one branches has spin $-\hbar / 2$. The sum of three branches spin is $\hbar / 2+\hbar / 2+(-\hbar / 2)=\hbar / 2$. But this option makes the new model lost three-fold circular symmetry. More seriously, the opposite spin in one branch abruptly reverses loop-1 movement direction, which violates the requirement for smooth trajectory. It is not acceptable.

Option-2. Let each branch has spin $\hbar / 6$. It can be done by selecting the reduced $m$-parameter $m=1 / 3$ for each branch. According to SQS theory, the lepton's spin equals to $m \hbar / 2$. For the new model as a whole entity, the reduced $m$-parameter add up to $m=1 / 3+1 / 3+1 / 3=1$ corresponding to the spin $\hbar / 2$. This option is accepted.

Next step is to find out how the three torus branches and three trajectories are combined. According to Penrose [12], there are two types of topological structures with three branches. The trefoil-knot-type shown in Fig.12.1(a) is a single loop self-knotted to form a trefoil structure. It fits the job to combine three trajectories on three torus models into one trajectory on the trefoil type model. The Borromean-ringtype structure shown in Fig. 12.1(b) is irrelevant to leptons model, because its three loops do not combine into one.

Fig.12.2 shows the $x-y$ plan cross section, in which the three loop-1 circles shown by dot-dashed lines touch each other tangentially from one circle to the other circle with continuous first order derivatives. In this way, loop-1 goes smoothly from one branch to the other. The total length of combined loop-1 equals precisely the sum of three branches' loop-1 lengths representing $\hbar / 6+\hbar / 6+\hbar / 6=h / 2$ spin for electron as a whole entity.

Fig.12.2 shows how the three branch trajectories combined into a trefoil trajectory. As mentioned in Section 8, on the electron torus surface, point $A$ and point- $B$ in Fig.8.2 actually represent two circles, circle- $A$ and circle- $B$. A trajectory may start at a point on circle- $A$ and halfway through at a point on circle- $B$ to keep the angle $\angle A O_{1} B$ :

$$
\angle A O_{1} B=180^{\circ}-\Delta \phi=166.98687309^{\circ} .
$$

This rule is originated from the $S$-equation and strictly related to $\pi\left(x_{1}\right), \pi\left(x_{2}\right)$ to determine curvatures of the torus model. To construct the trefoil trajectory, (8.18) is used to determine the location of point $-B$ from the location of point $-A$ for each branch.
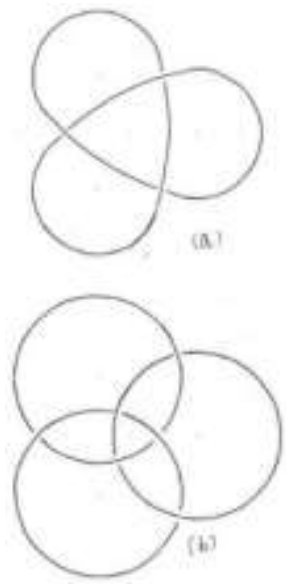

Fig.12.1: (a) Trefoil-knot-type; (b) Borromean rings type.

The other rules for the trefoil trajectory are:

1. The trefoil trajectory must go through points- $A$ and point- $B$ of three branches to satisfy the requirements of $\pi\left(x_{1}\right)$ and $\pi\left(x_{2}\right)$ for each branch.

2. The trajectory is the geodesics between adjacent point- $A$ and point $-B$ on trefoil type model surface.

3. The three branches of trefoil trajectory have the same shape separated by $120^{\circ}$ for the 3 -fold circular symmetry.

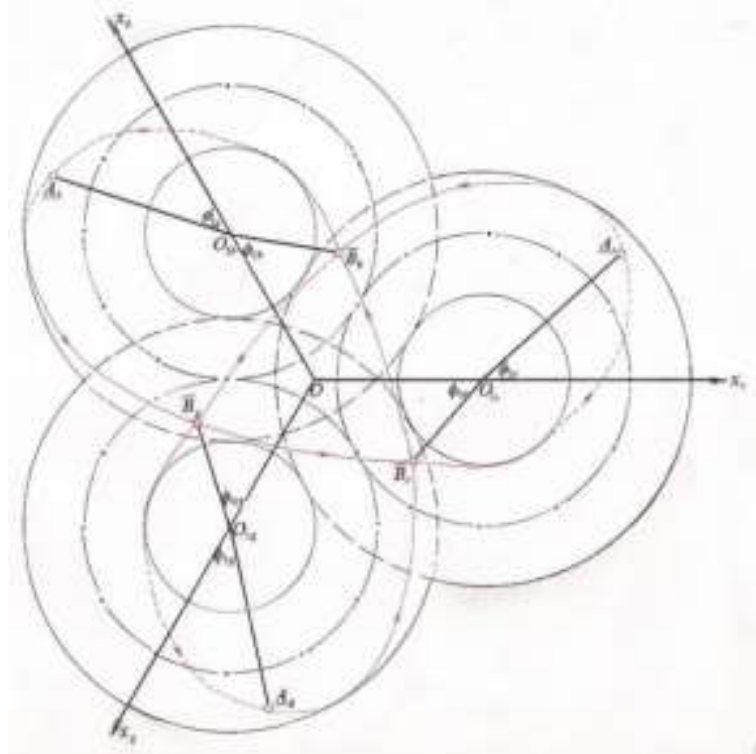

Fig. 12.2: The cross section and the projection of trefoil trajectory for electron on $x-y$ plane. 
In Fig. 12.2, the trajectory on top surface is shown by the solid curve and on bottom surface is shown by the dashed curve.

Trajectory for electron goes anti-clockwise through six characteristic points and back to close one cycle:

$$
\underline{A}_{r} \rightarrow \bar{B}_{b} \rightarrow \bar{B}_{g} \rightarrow \underline{A}_{g} \rightarrow \bar{B}_{r} \rightarrow \bar{B}_{b} \rightarrow \underline{A}_{b} \rightarrow \bar{B}_{g} \rightarrow \bar{B}_{r} \rightarrow \underline{A}_{r} .
$$

Indeed, the trajectory is a trefoil type closed loop with the correct topological structure and the 3 -fold circular symmetry.

The Weinberg angle $\theta_{W}^{\prime}=28.47948454^{\circ}$ is the same for all three branches as well as for electron as a whole entity. It needs explanation. As mentioned in Section 8, Weinberg angle is a phase shift between loop-1 and loop-2 periodic movements:

$$
\theta_{W}=\theta_{2}=\xi_{2}-\phi_{2}
$$

For the trefoil trajectory, $\theta_{W}=\theta_{2}$ repeats three times at three locations, $\underline{A}_{r}, \underline{A}_{g}, \underline{A}_{b}$. The repetition means the same phase shift kept no change along trajectory at three locations. Therefore, the three angles should not be added up to $3 \theta_{W}$. Look at it the other way, the combined trajectory is the same one on the original genus- 1 torus surface, which is reconfigured to fit the genus- 3 manifold. The combined trajectory has one Weinberg angle $\theta_{W}^{\prime}=\theta_{2}^{\prime}=28.47948454^{\circ}$ corresponding to the charge of $e$ for electron.

The trajectory shown in Fig. 12.2 is a samples selected from two sets of discrete possible trajectories. The jumping trajectories described in Section 8 for electron torus model are also valid for the trefoil type model. As long as the trajectories meet all rules, they are legitimate. In other words, the "electron clouds" is also a visualized description of electron behavior for the trefoil type model. The same is true for the trajectories on trefoil type models of muon and taon.

Introducing the trefoil type model solves the vacancies problem in Table 12.1. Table 12.2 shows the vacancies in Table 12.1 are filled with leptons' branches.

Table 12.2: The $m$-Parameters of Quarks and Leptons with 3

\begin{tabular}{|c|c|c|c|c|c|c|c|c|c|}
\hline Generation & \multicolumn{3}{|c|}{$1^{\text {st }}$ generation } & \multicolumn{3}{|c|}{$2^{\mathrm{nd}}$ generation } & \multicolumn{3}{|c|}{$3^{\text {td }}$ generation } \\
\hline Cator & red & green & Blate & $\mathrm{red}$ & giten & Wrue & Ied & sueen & blue \\
\hline$m_{\psi}$ & 1 & 7 & 13 & 19 & 29 & $37=$ & 43 & 53 & $6 \mathrm{t}$ \\
\hline Up type quark & 4. & $\mathrm{N}_{t}$ & s, & c, & $c_{r}$ & $c_{t}$ & t. & ${ }_{r}{ }_{r}$ & t, \\
\hline Leptan & $e, v_{m}$ & $e_{t}, v_{v e}$ & $e_{s,}, v_{n}$ & $\mu, v_{j}$ & $\mu_{t}, V_{s}$ & $\mu_{3}, \nu_{\rho}$ & $t, \ldots v_{7}$ & $r_{r}, V_{z}$ & $F_{2}, V_{n}$ \\
\hline$m_{l e q} *$ & $2(1)$ & $6(1)$ & $12(1)$ & $18(1)$ & $26(1)$ & $34(1)$ & $42(1)$ & $50(1)$ & $60(1)$ \\
\hline Dows type quark & d, & $d_{t}$ & $d_{b}$ & s, & $x_{t}$ & I, & b. & $b_{t}$ & $b_{1}$ \\
\hline$w_{\text {dws }}$ & 3 & 5 & 11 & 17 & 23 & 31 & 41 & 47 & 59 \\
\hline
\end{tabular}
Branches *

* The $m$-parameters listed are their magnitude; their signs are defined by (11.6).

** The number in parenthesis is the reduced $m$-parameter.

For three generations of charged leptons, the formulas given by (12.1) of Postulation 12.1 are generalized for the original $m$-parameters (before reduction) of trefoil type model's each branch and as a whole entity based on the original $m$-parameters of corresponding up type quark $m_{\text {uptype }, q_{i, j}}$ and down type quark $m_{\text {downtyp } q_{i, j}}$.
For each branch:

$$
m_{i, j}=\frac{1}{3}\left[\frac{m_{\text {uptype } q_{i, j}}+m_{{\text {downtyp } q_{i, j}}}}{2}\right], i=1,2,3, j=r, g, b .
$$

For lepton as a whole entity:

$$
m_{i}=\sum_{j=r, g, b} m_{i, j}, i=1,2,3 \text {. }
$$

The factor $1 / 3$ in (12.4a) is introduced to make each branch with spin $\hbar / 6$. The index $i=1,2,3$ is for three generations and the index $j=r, g, b$ is for three branches.

The rule to select the $n$-parameters for the trefoil type model is to make the loop ratio $n / m$ identical for all three branches. The formulas to determine the original $n$ parameters (before reduction) of trefoil type model each branch and as a whole entity are based on the $m$-parameters of (12.4) and the torus model original $n$-parameter $n_{\text {torus }}$.

For each branch:

$$
n_{i, j}=\frac{1}{3}\left[\frac{m_{i, j}}{m_{i, r}}\right] n_{t o r u s}, \quad i=1,2,3, j=r, g, b,
$$

For lepton as a whole entity:

$$
n_{i}=\sum_{j=r, g, b} n_{i, j}, i=1,2,3 \text {. }
$$

The rule to select the $p$-parameters for the trefoil type model is to make the mass ratio $p / n$ identical for all three branches. The formulas to determine the original $p$ parameters of the trefoil model each branch and as a whole entity are based on the $m$-parameters of (12.4) and the torus model original $p$-parameter $p_{\text {torus }}$.

For each branch:

$$
p_{i, j}=\frac{1}{3}\left[\frac{m_{i, j}}{m_{i, r}}\right] p_{t o r u s}, i=1,2,3, j=r, g, b,
$$

For lepton as a whole entity:

$$
p_{i}=\sum_{j=r, g, b} p_{i, j}, i=1,2,3 \text {. }
$$

The original numerical parameters are reduced to make the $m$-parameter for the trefoil type model as a whole entity equals to 1 corresponding to spin $\hbar / 2$. The way of reduction is that, the original $m, n, p$ are divided by the original $m$ for each branch.

In Table 12.3, the numerical parameters calculated according to formulas (12.4), (12.5), (12.6) are listed for each branch as well as for lepton as a whole entity for electron, muon and taon with trefoil type model.

As shown in Table 12.3, all reduced numerical parameters $m, n, p$ are identical for three branches. It indicates that, the trefoil type model three branches are mathematically identical. After reduction, their differences in the original parameters no long show up. 


\section{Z. Y. SHEN}

Table 12.3: Numerical Parameters for Three Generations of Charged Leptons

\begin{tabular}{|c|c|c|c|c|c|c|c|}
\hline \multirow[b]{2}{*}{ Name } & \multirow[t]{2}{*}{ Branch } & \multicolumn{2}{|c|}{ m-paramete } & \multicolumn{2}{|c|}{ n-paramete } & \multicolumn{2}{|c|}{ 3-parameter } \\
\hline & & Otiginal & Reduced & Original & Reduced & Original & Reduced \\
\hline \multirow{4}{*}{ Electron } & Red & 23 & $1 / 3$ & $1 / 3$ & $1 / 6$ & $1 / 3$ & 16 \\
\hline & Green & $6 / 3$ & $1 / 3$ & $3 / 3$ & 16 & $3 / 3$ & 16 \\
\hline & Hlue & 123 & $1 / 3$ & $6 \sqrt{3}$ & 1.6 & $6 \sqrt{3}$ & 16 \\
\hline & Whate & $6+2 / 3$ & 1 & $3+1 / 3$ & 12 & $3+1 / 3$ & $1 / 2$ \\
\hline \multirow{4}{*}{ Muon } & zed & $18 / 3$ & $1 / 3$ & $(29+1 / 4) 3$ & $(\mathrm{t}+5.8)^{3}$ & $6048 / 3$ & $336 / 3$ \\
\hline & Oreen & 263 & $1 / 3$ & $(42+1 / 4) 7$ & $(1+328) 5$ & $8736 / 3$ & 336,3 \\
\hline & Blut & 343 & $1 / 3$ & $(55+1 / 4) / 3$ & $(\mathrm{I}+5,8) \sqrt{3}$ & $11424 / 3$ & 3363 \\
\hline & Whote & 26 & 1 & $42+1 / 4$ & $1+5 / 3$ & 8736 & 336 \\
\hline \multirow{4}{*}{ Tran } & Ind & $42 / 3$ & $1 / 3$ & 1203 & $(2+6,7) 3$ & 417270.3 & $9935 / 3$ \\
\hline & Groen & $50 / 3$ & $1 / 3$ & $(1+2+67) \sqrt{3}$ & $(2+6.7)^{3}$ & $496750 / 3$ & 9935,3 \\
\hline & Bhar & $60 \sqrt{3}$ & $1 / 3$ & $(171+3 / 7) 3$ & $(2+6,7) 3$ & $\$ 96100 \sqrt{3}$ & 9935,3 \\
\hline & Whate & $50+2 / 3$ & 1 & $144+16 / 21$ & $2+67$ & $503373+1 / 3$ & 9935 \\
\hline
\end{tabular}

The calculated parameters for electron, muon and taon with trefoil type model are listed in Table 12.4, Table 12.5 and Table 12.6, respectively. In which, the parameters with the ' mark are effective, i.e. after $f$-modification and the parameters without the ' mark are original, i.e. before $f$ modification.

The generalized $A T$-equation and $P S$-equation of (10.7) are also valid for leptons' trefoil model.

As listed in Table 12.4, Table 12.5 and Table 12.6, except the original numerical parameters differences, all reduced numerical parameters as well as other parameters for electron, muon and taon trefoil type model are the same of those for their torus model listed in Table 8.2, Table 10.1 and Table 10.2. The consistence is expected. The torus models and trajectories serve as the building blocks for trefoil type models and trajectories. The three torus models and three trajectories combine into one trefoil type model and one trefoil trajectory. In the combination process, the only thing changed is their dimensions shrunk to one third. Therefore, all angles as well as all normalized lengths are kept the same.

There is an apparent problem. As listed in the tables, the values of $p / n$ ratio for each branch are the same for the lepton as a whole entity. Since $p / n$ ratios equal to mass ratios: $p / n=M / M_{e}$, the question is: Does the mass of each branch equal to the mass of the lepton? Of cause not, but it deserves an explanation. As shown by $(10.7 \mathrm{c})$ for the generalized $A T$-equation and $P S$-equation, the mass term is:

$$
\sin (\alpha \theta)=\sin \left(\frac{2 p}{m} \theta\right)=\sin \left(2 \frac{n}{m} \frac{p}{n}\right)=\sin \left(2 \frac{L_{2}}{L_{1}} \frac{M}{M_{e}} \theta\right)
$$

The mass term is oscillating along the entire trefoil trajectory. There is no way to define another mass term for each branch different from the one for the whole trefoil model. The situation is similar to the Weinberg angle discussed earlier. The $p / n=M / M_{e}$ as mass ratio is not for each branch separately; it is for the lepton as a whole entity.

It must emphasis that, lepton trefoil type model as a whole entity represents the lepton. The red, green, blue three branches are not separated particles. This is the major differences between leptons' colors and quarks' colors.
Table 12.4: Parameters for Electron with Trefoil Type Model*

\begin{tabular}{|c|c|c|c|c|}
\hline \multicolumn{5}{|c|}{$\begin{array}{l}\text { Model. Trefonl bpe } \\
\text { Spmbol e, Mass: } 0.51099891 M \sigma V \\
\text { Weinberg angle: } \theta_{n}=\theta_{1}=28.47948454^{*}\end{array}$} \\
\hline \multicolumn{5}{|c|}{ Numencal prameters } \\
\hline Rad branch & \multicolumn{4}{|c|}{ 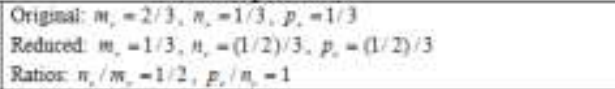 } \\
\hline Greet brasch & \multicolumn{4}{|c|}{ 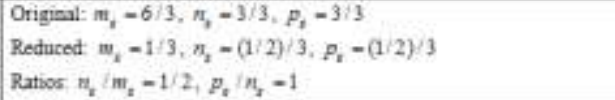 } \\
\hline Blue branch & \multicolumn{4}{|c|}{$\begin{array}{l}\text { Original: } w_{3}=12: 3, n_{3}=6 / 3, p_{3}=6 / 3 \\
\text { Reduced: } w_{3}=1 / 3, n_{2}=(1 / 2) / 3, p_{2}=(1 / 2) / 3 \\
\text { Ratios. } n_{7} / m_{3}-1 / 2, p_{3} / n_{3}-1\end{array}$} \\
\hline Whose Entity & \multicolumn{4}{|c|}{$\begin{array}{l}\text { Otigual: } m=2, n=1, p=1 \\
\text { Reduced: } m=1, n=1 / 2, p=1 / 2,2 p / m=1 \\
\text { Ratbos: } n / m=1 / 2, p / n=M, / M,=1\end{array}$} \\
\hline \multicolumn{5}{|c|}{ Parmeters fot thee branches as well as for electron as whole entity } \\
\hline \multicolumn{5}{|c|}{$\begin{array}{l}d-1 \text { for electron, } d=1 / 3 \text { for each brants" } \\
\text { Modification fattoe: } f_{n}=0.98363423, f_{3}=1.01023292\end{array}$} \\
\hline \multicolumn{3}{|c|}{$\begin{array}{l}\text { Torus imer-haif (ueganve curvahure) } \\
I_{1}=0.125, x\left(x_{1}\right)=3.87710292 \\
R=0.50815288 \\
A_{m}=0.52342698, A=0.21505973\end{array}$} & \multicolumn{2}{|c|}{$\begin{array}{l}\text { Tonis outer-half (postive curvanure) } \\
x_{z}=0.375, \pi\left(x_{z}\right)-2.8645925\end{array}$} \\
\hline \multicolumn{2}{|c|}{$\begin{array}{l}\text { Triangle } B_{2}^{\prime} D^{\prime} O_{2} \\
\text { on } \\
Q_{1} H^{\prime} \text { eross section }\end{array}$} & $\begin{array}{l}\text { Triangle } D^{\prime} E^{\prime} O_{2}^{\prime} \text { on } \\
\text { xy cross section }\end{array}$ & $\begin{array}{l}\text { Triangle } A_{1}^{\prime} O_{2} O_{2} \mathrm{ce} \\
O_{1} G^{\prime} \text { Cross Section }\end{array}$ & $\begin{array}{l}\text { Triangle } \mathrm{GO}_{0} \mathrm{O}_{2} \text { in } \\
\text { xy Cross Section }\end{array}$ \\
\hline \multirow{3}{*}{\multicolumn{2}{|c|}{$\begin{array}{l}d_{2}=0.41088101 \\
x_{3}=0.28645244 \\
z_{1}=0.41303564\end{array}$}} & $a_{1}=0.41086101$ & $X_{2}^{\prime}=0.36939205$ & $x_{3}=1.10636775$ \\
\hline & & $x_{\sin } 0.2396076$ & $Z_{z}^{\prime}=0.33546647$ & $r_{3}=1.00094077$ \\
\hline & & $Y_{\mathrm{j}}=0.41758506$ & & \\
\hline \multicolumn{2}{|c|}{$\alpha_{1}^{\prime}=61.17939213^{*}$} & $a_{1}^{\prime}=31.9232308 r^{r}$ & $\theta_{2}=28.47948 t 54^{4}$ & $\theta_{3}=41.8146647 y^{\prime}$ \\
\hline \multicolumn{2}{|c|}{$\beta_{1}=55.257547^{\circ}$} & $B_{s}=30.44799569^{\prime}$ & $\phi_{z}=13.76493556^{\circ}$ & $\phi_{1}=42.24442009^{\circ}$ \\
\hline \multicolumn{2}{|c|}{$y_{1}^{\prime}=6356306057^{\prime}$} & $Y_{s}=117.6287735$ & $\gamma_{1}^{\prime}=137.75557991^{*}$ & $\psi_{1}=95.94091518^{\prime}$ \\
\hline \multicolumn{2}{|c|}{$\phi_{1}=30.0643217 \%$} & $\varphi_{t}=55257547^{\circ}$ & $5 z^{\prime}=42.2442009^{\prime}$ & $\xi_{1}=8+05908482^{*}$ \\
\hline
\end{tabular}

Table 12.5: Parameters of Muon with Trefoil Type Model*

\begin{tabular}{|c|c|c|c|c|}
\hline \\
\hline \multirow{2}{*}{\multicolumn{5}{|c|}{ 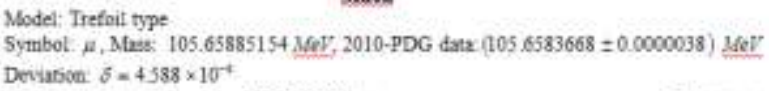 }} \\
\hline \multirow{2}{*}{\multicolumn{5}{|c|}{ 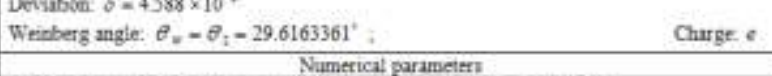 }} \\
\hline & & & & \\
\hline Red branch & \multicolumn{4}{|c|}{ 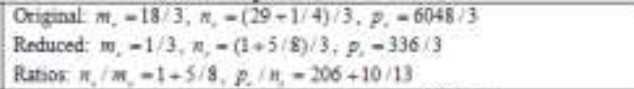 } \\
\hline Geen branch & \multicolumn{4}{|c|}{ 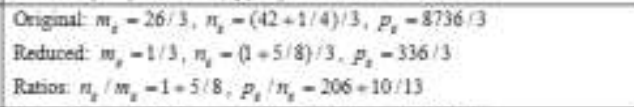 } \\
\hline Blue tranch & \multicolumn{4}{|c|}{$\begin{array}{l}\text { Origual: } m_{0}=34 / 3, n_{0}=(55-1 / 4) / 3, p_{2}=11424 / 3 \\
\text { Reduced: } m_{3}=1 / 3, n_{3}=(1+5 / 8) / 3, p_{2}=336 / 3 \\
\text { Ranos: } n_{3} / m_{2}=1-3 / 8, p_{3} / n_{3}=206+10 / 13\end{array}$} \\
\hline Whoie Entity & \multicolumn{4}{|c|}{$\begin{array}{l}\text { Original } m=26, n=42+1 / 4, p=8736 \\
\text { Reduced: } m=1, n=1+5 / 8, p=336,2 p / m=672 \\
\text { Ratios: } n / m=1+5 / 8, p / n=M, / M=206+10 / 13\end{array}$} \\
\hline \multicolumn{5}{|c|}{ Parmeters for thee bramenes al well as for muen as wbole entity } \\
\hline \multicolumn{3}{|c|}{$\begin{array}{l}f_{2}=0.91340563 \\
f_{3}=1.0085030 \%\end{array}$} & \multicolumn{2}{|c|}{$\begin{array}{l}\text { Originat: } a_{2}=0.53844328, b_{1}=2.40688502 \\
\text { Enfective } a_{1}^{t}=0.49181712, b_{2}^{\prime}=2.42734896\end{array}$} \\
\hline \multicolumn{3}{|c|}{$\begin{array}{l}\text { Toris inner half (negative cuevasure) } \\
x_{1}=0.24939546, \pi\left(x_{1}\right)=3.14361568 \\
R=0.50818285 \\
A_{a}=0.02538227, A=0.00619876\end{array}$} & \multicolumn{2}{|c|}{$\begin{array}{l}\text { Torva outer hate (pesitive carvanares } \\
x_{2}=0.25060454, \pi\left(x_{i}\right)=3.1394918\end{array}$} \\
\hline \multicolumn{2}{|c|}{$\begin{array}{l}\text { Triangle } B_{1} D^{\prime} O_{2} \text { and } \\
O_{1} H^{\prime} \text { cross section. }\end{array}$} & $\begin{array}{l}\text { Triangte } D E^{\prime} O_{r} \text { an } \\
x-y \text { Crose Sectoo }\end{array}$ & $\begin{array}{l}\text { Iriangle } A_{2}^{\prime} O_{1}^{\prime} O_{2} \text { en } \\
O_{1} O^{\prime} \text { eroses section }\end{array}$ & $\begin{array}{l}\text { Triangle } \mathrm{G}^{\prime} \mathrm{O}_{2} \text { an } \\
x-y \text { cross section }\end{array}$ \\
\hline \multirow{3}{*}{\multicolumn{2}{|c|}{$\begin{array}{l}b_{1}=0.36416560 \\
X_{2}=0.48715818 \\
Z_{1}^{\prime}=0.33331729\end{array}$}} & $a_{1}=0.07539057$ & $X_{2}^{\prime}=0.47589704$ & $X_{3}=1.4888238$ \\
\hline & & $x_{0}^{n}=0.50135792$ & $Z_{2}=0.01259683$ & $r_{3}=0.09445033$ \\
\hline & & $Y_{\gamma}=0.05300048$ & & \\
\hline \multicolumn{2}{|c|}{$a_{1}^{\prime}=56.42057154^{*}$} & $a_{6}^{\prime}=258485164 T$ & $\theta_{7}=29.6163361^{\circ}$ & $\theta_{3}=7.30639579$ \\
\hline \multicolumn{2}{|c|}{$\beta_{1}=34.38022705^{5}$} & $\beta_{6}=9.45108237^{\circ}$ & $\phi_{2}=2254176704^{\circ}$ & $\phi_{1}^{\prime}-3.63018+64^{2}$ \\
\hline \multicolumn{2}{|c|}{$Y_{1}^{\prime}=89.1992014 r^{r}$} & $y^{\prime},=94.70040110^{\circ}$ & $V_{2}=127.84191686^{\circ}$ & $\psi_{1}=169.06341956$ \\
\hline \multicolumn{2}{|c|}{$\phi_{1}=33.02151938^{\circ}$} & $\phi_{2}=9.40080232^{\prime}$ & $\sum_{3}=52.15838314^{*}$ & $\Sigma_{1}=10.93659044^{2}$ \\
\hline
\end{tabular}


Table 12.6: Parameters of Taon with Trefoil Type Model*

\begin{tabular}{|c|c|c|c|c|}
\hline \multicolumn{5}{|c|}{ 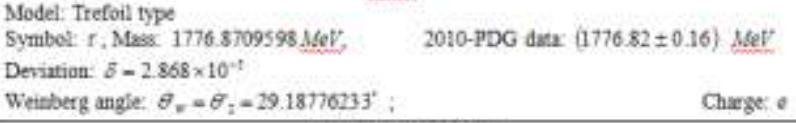 } \\
\hline \multicolumn{5}{|c|}{ Numerical parametrms } \\
\hline Red beanch & \multicolumn{4}{|c|}{ 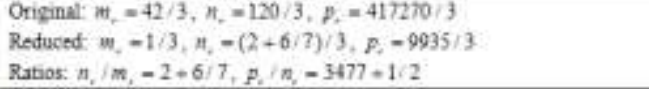 } \\
\hline Green branch & \multicolumn{4}{|c|}{ 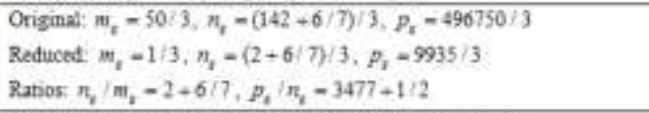 } \\
\hline Btac branch & \multicolumn{4}{|c|}{$\begin{array}{l}\text { Original: } m_{6}=60 / 3, n_{2}=(17 t+3 / 7) / 3, p_{2}=596100 / 3 \\
\text { Reduced: } m_{3}=1 / 3, n_{2}=(2+6 / 7) / 3, p_{3}=9935 / 3 \\
\text { Ratios: } n_{2} / m_{3}=2+6 / 7, p_{2} / n_{3}=3477+1 / 2\end{array}$} \\
\hline Wasie Entity & \multicolumn{4}{|c|}{$\begin{array}{l}\text { Original: } m=50+2 / 3, n=144+16 / 21, p=503373+1 / 3 \\
\text { Reduced } m=1, n=2-6 / 7, p=9935,2 p / m=19870 \\
\text { Ratios: } n / m=2+6 / 7, p / n=M_{2} ; M_{q}=3477+1 / 2\end{array}$} \\
\hline \multicolumn{5}{|c|}{ Parameters for thrce bramches as well as for taon as whole enary } \\
\hline $\begin{array}{ll}f_{a}=0.94733344 ; & \text { Original } a_{1}=0.5208362, b_{4}=4.39440915 \\
f_{2}=1.00187462 ; & \text { Effective: } a_{2}^{\prime}=0.49340555, b_{2}^{\prime}=4.40264701\end{array}$ & $\begin{array}{ll}2=1 \\
4 ;\end{array}$ & 3 for each branch" & $\begin{array}{l}\text { Original: } a_{1}=0.520836 \\
\text { Effective: } a_{2}^{\prime}-0.49340\end{array}$ & $\begin{array}{l}62, b_{2}=4.39440915 \\
555, b_{z}^{\prime}=4.40264701\end{array}$ \\
\hline \multicolumn{3}{|c|}{$\begin{array}{l}\text { Tonts imer half (nezative curvature) } \\
x_{1}=0.24996405, \pi\left(x_{3}\right)=3.14167148 \\
R=0.50659445 \\
A_{x}=0.00500924, A=0.00075376\end{array}$} & 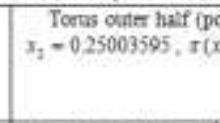 & $\begin{array}{l}\text { ositive curvature) } \\
\left.x_{7}\right)=3.14142623\end{array}$ \\
\hline \multicolumn{2}{|c|}{$\begin{array}{l}\text { Trimgle } B_{2}^{\prime} D^{\prime} O_{;} \text {on } \\
O_{1} H^{\prime} \text { crosis section }\end{array}$} & $\begin{array}{l}\text { Triangle } D^{\prime} E^{\prime} O \text {, on } \\
x-y \text { cross seetioe }\end{array}$ & $\begin{array}{l}\text { Tringle } A_{3} O_{1}^{\prime} O_{2} \text { on } \\
O_{2} G^{\prime} \text { cross section }\end{array}$ & $\begin{array}{l}\text { Triangle } \mathrm{GO} O \mathrm{O}_{4} \text {, th } \\
x-y \text { eross section }\end{array}$ \\
\hline \multirow{2}{*}{\multicolumn{2}{|c|}{$\begin{array}{l}b_{1}=0.24098647 \\
X_{1}^{\prime}=0.49274155 \\
Z_{2}^{\prime}=0.22833091\end{array}$}} & $a_{1}=0.02739495$ & $X_{7}^{\prime}=0.48844459$ & $X_{1}=1.49316821$ \\
\hline & & $\begin{array}{l}x_{0}=0.50576582 \\
Y_{0}^{\prime}=0.02896323\end{array}$ & $Z_{2}^{\prime}=0.62275234$ & $Y_{1}=0.02662385$ \\
\hline \multicolumn{2}{|c|}{$\alpha_{2}^{\prime}=65.30414182^{2}$} & $\alpha_{a}^{\prime}=35.00741245^{\prime}$ & $\theta_{2}=29.18776233^{\prime}$ & $\theta_{1}=206863135^{\prime}$ \\
\hline \multicolumn{2}{|c|}{$\beta_{1}=24.262+7720^{\circ}$} & $B_{1}=3.35382497^{*}$ & $\Phi_{2}=2270401238^{\circ}$ & $\phi_{1}=1.02150092^{\circ}$ \\
\hline \multicolumn{2}{|c|}{$\gamma_{1}=89.83338092^{*}$} & $Y_{3}^{\prime}=91.63576258^{\circ}$ & $v_{2}^{\prime}=128.10822529^{\prime \prime}$ & $V_{i}=176.90986773^{\circ}$ \\
\hline \multicolumn{2}{|c|}{$\varphi_{5}=24.23381334^{4}$} & $\phi_{0}-327752516^{\prime}$ & $6:-51.89177471^{\circ}$ & $S_{1}=3.09013227$ \\
\hline
\end{tabular}

* All data are from 16-digit numerical calculations, only 8-digit after the decimal point is presented.

** With different normalizations, the listed values of length parameters are the same for each branch and for taon as whole entity.

For SQS theory, the trefoil type is the real model for leptons. Otherwise, the vacancies in Table 13.1 cannot be filled. However, the study for leptons' torus model is not a waste effort. It serves as a rehearsal for the real show.

\section{Section 13: Gluons and Strong Interactions}

The strong interactions between quarks are mediated by eight gluons, which are gauge bosons with spin $h$ and zero mass. In this section, the gluons and the strong interactions are treated in terms of mathematics.

Definition 13.1: Eight gluons are made of eight pairs of quark and the same type anti-quark:

$$
\begin{aligned}
& g_{1}=d_{r} \bar{d}_{r}, g_{2}=d_{g} \bar{d}_{g}, g_{3}=u_{g} \bar{u}_{g}, g_{4}=d_{b} \bar{d}_{b}, \\
& g_{5}=u_{b} \bar{u}_{b}, g_{6}=s_{r} \bar{s}_{r}, g_{7}=s_{g} \bar{s}_{g}, g_{8}=s_{b} \bar{s}_{b} ; \\
& \text { or } \quad g_{i}=q_{j} \bar{q}_{j} ; i=1,2,3,4,5,6,7,8 ; \\
& \quad j=d_{r}, d_{g}, u_{g}, d_{b}, u_{b}, s_{r}, s_{g}, s_{b} .
\end{aligned}
$$

According to Definition 13.1:

$$
\begin{aligned}
& \bar{g}_{i}={\overline{q_{j} \bar{q}_{j}}}=\bar{q}_{i} q_{j}=q_{j} \bar{q}_{j}=g_{i} ; \\
& i=1,2,3,4,5,6,7,8, j=d_{r}, d_{g}, u_{g}, d_{b}, u_{b}, s_{r}, s_{g}, s_{b} .
\end{aligned}
$$

The anti-particle of gluon is itself:

$$
\bar{g}_{i}=g_{i}, i=1,2,3,4,5,6,7,8 .
$$

Definition 13.2: The gluons numerical parameters are defined as follows:

1 . The original $m$-parameter of gluon is defined as:

$$
m= \pm 2\left|m_{q}\right| \text {. }
$$

$m_{q}$ is the $m$-parameter of gluon's constituent quark.

2. The $n$ parameter of gluon is defined as:

$$
n= \pm \frac{|m|-2}{2} \text {. }
$$

3. The gluons handedness is defined as:

For right handed gluons:

$$
m>0 \text { and } n>0 \text {; }
$$

For left handed gluons:

$$
m<0 \text { and } n<0 \text {. }
$$

\begin{tabular}{|c|c|c|c|c|c|c|c|c|}
\hline Gluons & $g_{1}$ & $\boldsymbol{z}_{2}$ & 8 & E. & $g_{5}$ & $s_{t}$ & 5 & $\varepsilon_{1}$ \\
\hline Afade of & d, & $d_{g}, \overline{d_{n}}$ & $u_{2} R_{2}$ & $d, d_{2}$ & $u_{2} n_{2}$ & 3,2 & $x_{2}^{2}$ & $5,3,2$ \\
\hline$n-2 \times \omega_{r}$ & $2 \times 3=6$ & $2 \times 5=10$ & $2 \times 7=14$ & $2 n 11-22$ & $2 \times 13=20$ & $2 \times 17-34$ & $2 \times 25=15$ & $2 \times 3 I=62$ \\
\hline$\pi<-m-2 n$ & 2 & 2 & 2 & 2 & 2 & 2 & 2 & 2 \\
\hline$\pi=(n-2)$ & 2 & 4 & 6 & 50 & 12 & 16 & 22 & 30 \\
\hline$P$ & 0 & 0 & 0 & 0 & 0 & 0 & 0 & 0 \\
\hline $5 \sin (\alpha)$ & 1 & $t$ & 1 & 1 & 1 & 1 & 1 & 1 \\
\hline Mass & 0 & 0 & 0 & 0 & 0 & 0 & 0 & 0 \\
\hline Thoses & $\begin{array}{l}\text { The listed } \\
\text { The in, nt }\end{array}$ & $\begin{array}{l}\text { m. } m_{y, n}=1 \\
y, n \text { paras }\end{array}$ & parameter & $\begin{array}{l}\text { ate for gil } \\
\text { acons with }\end{array}$ & $\begin{array}{l}\text { tont ayh } n \\
\text { eft handedr }\end{array}$ & $\begin{array}{l}\text { ight habode } \\
\text { iest all have }\end{array}$ & negarive & \\
\hline
\end{tabular}

4. The gluon effective $m$-parameter as gauge boson with spin $\hbar$ is defined as:

$$
m_{E f f}= \pm(|m|-2|n|)= \pm 2 \text {. }
$$

5. The $p$-parameter of gluon equals to zero for zero mass: $p=0$.

The numerical parameters of eight gluons are listed in Table 13.1.

Table 13.1: The Numerical Parameters of Eight Gluons*

Explanation: The definitions of gluons numerical parameters are based on their geometrical model. For convenience, the following discussions are referring to gluons with right handedness. According to SQS theory, the model of a gluon as a boson without mass is a single loop with its $m$-parameter given by (13.3). As listed in Table 13.1, the original $m$-parameters for all eight gluons are $m \geq 6$ corresponding to spins of $s=m \hbar / 2 \geq 3 \hbar$, which is contrsdictory to gluon as gauge boson with spin $s=\hbar$. The problem can be solved by their model. As shown in Fig. 13.1(a), the gluon loop's two traces on opposite sides are merged into one and leave two small circles at two ends. Then the merged portion is twisted into $n=(m-2) / 2$ turns akin to a spring shown in Fig.13.1(b). The loop unmerged portion is evenly divided into two small circles. Each circle's circumferential length equals to $\lambda_{P}=L_{P}$ corresponding to spin $\hbar / 2$ with the same orientation. Two circles contribute the spin of $s=\hbar / 2+\hbar / 2=\hbar$ for the gluon. This scenario is consistent with the number parameters listed in Table 13.1. The effective $m$-parameter of 
$m_{E f f}=m-2 n=m-2\left(\frac{m}{2}-1\right)=2$ corresponds to the spin of $s=m_{E f f} \hbar / 2=\hbar$. The $n$-parameter equals to the number of turns shown in Fig.13.1 (b). The $p$-parameters of gluons all equal to zero for zero mass.

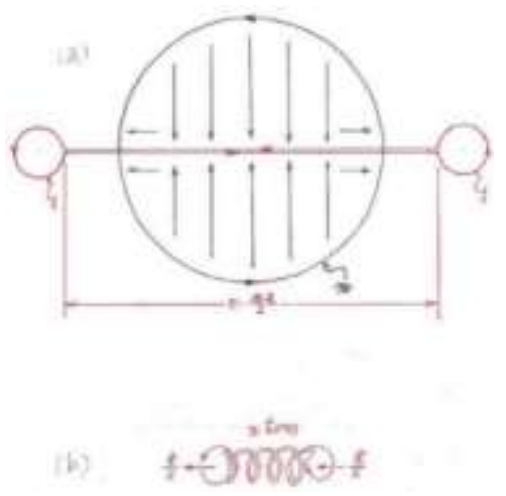

Fig.13.1: Gluon's model

Rule 13.1: The strong interaction between two quarks (two anti-quarks, a quark and an anti-quark) $q_{1}$ and $q_{2}$ with parameters $m_{1}, n_{1}$ and $m_{2}, n_{2}$ is mediated by a link made of gluons with numerical parameters $m_{i}, n_{i}$ satisfies the following equations:

$$
\begin{aligned}
& \left|m_{1}-m_{2}\right|=\sum_{i=1}^{N} a_{i} m_{i}, \\
& \left|n_{1}-n_{2}\right|=\sum_{i=1}^{N} a_{i} n_{i} .
\end{aligned}
$$

$N$ is the number of gluon types in the link; $a_{i}$ is the number of gluons of type $i$.

Explanation: In (13.8), $N>1$ means more than one types of gluons participating in the link; $a_{i}>1$ means more than one gluons of type $i$ participating in the link.

Definition 13.3: The strong interactions between two quarks (two anti-quarks, a quark and an anti-quark) are classified into two categories.

Regular type: All gluons in the link have the same handedness, i.e. all $m_{i}$ and all $n_{i}$ have the same sign.

Weakened type: Some gluons in the link have different handedness, i.e. $m_{i}$ and $n_{i}$ having different signs.

Explanation: In a link, all gluons share the same momentum orientation. Gluons are bosons. The gluons with same handedness and same spin orientation have a tendency of condensation, which represents a strong attractive force to enhance the link. The gluons with opposite handedness and opposite spin orientations weaken the link.

Unless stated otherwise, strong interactions are referring to the regular type.
Theorem 13.1: The regular type strong interaction between two members in a pair of quarks (anti-quarks, a quark and an anti-quark) with numerical parameters $m_{1}, n_{1}$ and $m_{2}, n_{2}$ satisfy:

$$
\left|n_{1}-n_{2}\right| \leq\left[\frac{1}{2}\left|m_{1}-m_{2}\right|-1\right] .
$$

Proof: For the regular type strong interaction, according to Definition 13.3, (13.9) is subjected to the condition that, all gluons participated in the link have the same handedness. According to (13.4), and (13.8):

$\left|n_{1}-n_{2}\right|=\sum_{i} a_{i} n_{i}=\frac{1}{2} \sum_{i} a_{i}\left(m_{i}-2\right)=\frac{1}{2}\left|m_{1}-m_{2}\right|-\sum_{i} a_{i} \leq \frac{1}{2}\left|m_{1}-m_{2}\right|-1 ; \sum_{i} a_{i} \geq 1$.

Formula (13.9) is proved for all gluons participated in the link with $m_{i}>0$ and $n_{i}>0$ or all gluons participated in the link with $m_{i}<0$ and $n_{i}<0$, which belong to the regular type. QED

Lemma 13.1: A pair of quarks (anti-quarks, a quark and an anti-quark) with numerical parameters $m_{1}, n_{1}$ and $m_{2}, n_{2}$ violating (13.9) is prohibited to have the regular type strong interaction between two members in the pair. The strong interaction belongs to the weakened type.

Proof: Lemma 13.1 is the reversed opposite of Theorem 13.1. QED

According to Theorem 13.1 and Lemma 13.1, the strong interactions among quarks (anti-quarks, a quark and an antiquark) have prohibitions meaning no regular type strong interactions between certain specific quarks (anti-quarks, a quark and an anti-quark). The selectivity of regular type strong interactions based on Theorem 13.1 and Lemma 13.1 plays an important rule for comparing the theoretical results with experimental facts.

The possible gluons links serving as mediators for the regular strong interaction among quarks (anti-quarks) are given in Table 13.2 and Fig.13.2. The form for numerical parameters used in Table 12.2 is:

$$
\begin{aligned}
& \frac{\left[\left|n_{1}\right| \pm\left|n_{2}\right|\right]_{q-\text { pair }}=\left[\sum_{i=1}^{N} a_{1}\left|n_{i}\right|\right]_{g-\text { link }}}{\left[\left|m_{1}\right| \pm\left|m_{2}\right|\right]_{q-\text { pair }}=\left[\sum_{i=1}^{N} a_{1}\left|m_{i}\right|\right]_{g-\text { link }}}, \sum_{i=1}^{N} a_{i} g_{i} ; \\
& \left(\left|m_{1}\right| \geq\left|m_{2}\right|,\left|n_{1}\right| \geq\left|n_{2}\right|\right) .
\end{aligned}
$$

In which, $m_{1}, n_{1}$ and $m_{2}, n_{2}$ are the $m$-parameter and $n$ parameter of two quarks (two anti-quarks or a quark and an anti-quark) involved; $N$ is the number of gluon types in the link; $a_{i}$ is the number of gluons for type $i ; m_{i}$ is the $m$ parameter of gluon type $i ; n_{i}$ is the $n$-parameter of gluon type $i$. In the "Facts" row of Table 13.2, the "+ /+ type" and "- I- type" represent the two \pm signs in numerator and denominator for the $q$-pair part in (13.10) take the same sign; the " $-1+$ type" and "+ $/$ - type" represent the two \pm signs in the numerator and denominator for the $q$-pair part in (13.10) take opposite signs. 
Table 13.2A: Gluons Links between Up Quarks

\begin{tabular}{|c|c|c|c|}
\hline 6. $\div$ & $x_{n}, \frac{1}{1}$ & $\alpha, \frac{1}{4}$ & $x_{1}, \frac{1}{11}$ \\
\hline$v_{i}, \frac{t}{1}$ & & $\frac{3-1-2}{1-1-6}, x_{4}$ & 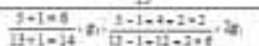 \\
\hline$u_{*}=\frac{3}{7}$ & $\left.\frac{5-1 \cdot 2}{7-1 \cdot 6}, 8\right\}$ & $\frac{3-9-6}{1+7-14} d$ & 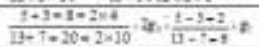 \\
\hline$u_{n} \cdot \frac{2}{15}$ & 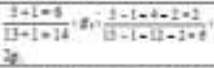 & $\frac{5+1-1-1 \times 4}{1-7+20+2 \times 10}, \geqslant, \frac{1-1-2}{10+7-9}, 6$ & 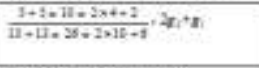 \\
\hline Fact & 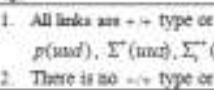 & seme wig at & 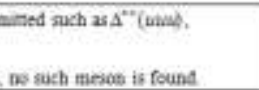 \\
\hline
\end{tabular}

Table 13.2B: Gluons Links between Down Quarks

\begin{tabular}{|c|c|c|c|}
\hline q. $\frac{\pi}{n}$ & $d_{*, \frac{1}{3}}$ & $d, \frac{3}{3}$ & $d i \frac{2}{11}$ \\
\hline$d,-\frac{1}{3}$ & $\frac{1+1-2}{3+5=6}, \varepsilon_{1}$ & & $\frac{1+1-1}{11-3-1 \cdot 4} \cdot x$ \\
\hline$d_{x}+\frac{3}{3}$ & & & $\frac{y-1-2}{11-9-4} \cdot x$ \\
\hline$d_{s} \frac{3}{4}$ & $\frac{3+1-6}{11+3-12} \cdot t$ & $\frac{3-3-2}{11-5 \cdot 6}, n$ & $\frac{3.5-10}{1+11-12} \cdot t$ \\
\hline Facts & \multicolumn{3}{|c|}{ 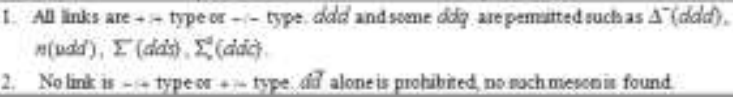 } \\
\hline
\end{tabular}

Table 13.2C: Gluons Links between Strange Quarks

\begin{tabular}{|c|c|c|c|}
\hline$q_{i}=\frac{\pi}{m}$ & $3,-\frac{7}{17}$ & $3, \frac{11}{21}$ & $5, \frac{11}{31}$ \\
\hline$I, \frac{7}{17}$ & 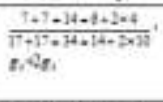 & $\frac{11+7 \cdot 19+12+6}{21+17-40-26-11}, \sin -2$ & 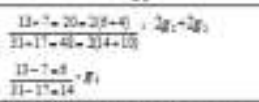 \\
\hline$s=\frac{11}{23}$ & 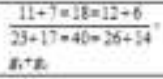 & $\frac{11-11-212}{23+33 *+6}, 8$ & 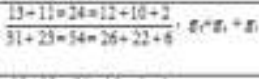 \\
\hline $5, \frac{11}{31}$ & 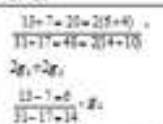 & 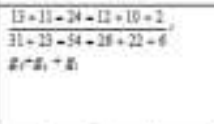 & 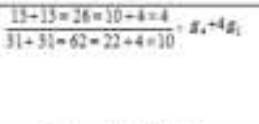 \\
\hline Fasth & \multicolumn{3}{|c|}{ 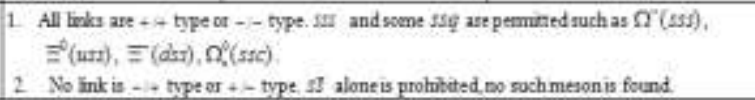 } \\
\hline
\end{tabular}

Table 13.2D: Gluons Links between Charm Quarks

\begin{tabular}{|c|c|c|c|}
\hline g. & $=1 \frac{7}{39}$ & $c_{1}, \frac{23}{9}$ & $=2, \frac{29}{37}$ \\
\hline c. $* \frac{7}{15}$ & 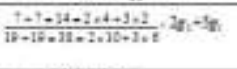 & $\frac{D-t-16-1-2}{3 p+15 * 4101 \times 6}$ & 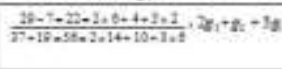 \\
\hline$f_{x}: \frac{23}{2 i}$ & 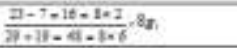 & & \\
\hline c). $\frac{2 \pi}{4}$ & 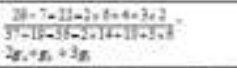 & & \\
\hline Fats & \multicolumn{3}{|c|}{ 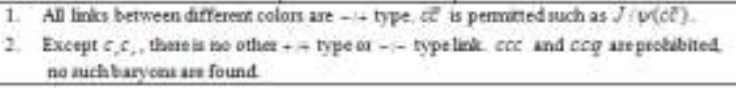 } \\
\hline
\end{tabular}

Table 13.2E: Gluons Links between Bottom Quarks

\begin{tabular}{|c|c|c|c|}
\hline$\frac{q}{n}$ & $b+\frac{17}{41}$ & $b_{0}, \frac{17}{47}$ & $b, n \frac{39}{59}$ \\
\hline$\frac{1}{b 1}$ & 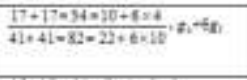 & 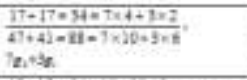 & $\frac{51-17+96 * 31-19 \times 7}{9+41-160-21+14 \times 6}, 2+236$ \\
\hline$\frac{1}{4}$ & 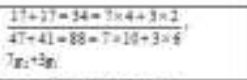 & $\frac{1+17-34-10+12 \times 2}{47+17-94=21+12 \times 6}, x+126$ & $\frac{59-17-56-4+16 \times 2}{59+47-106-10+16 \times 6}, 2,-164$ \\
\hline$\frac{6}{5}$ & $\frac{21-17-36-16+13 \times 2}{3-41-136-22+13+2}-x+13 x$ & 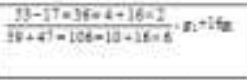 & \\
\hline Fath & 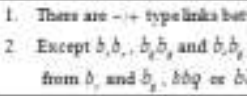 & 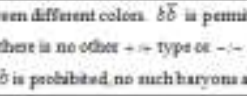 & 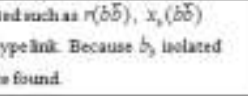 \\
\hline
\end{tabular}

Table 13.2F: Gluons Links between Up and Down Quarks

\begin{tabular}{|c|c|c|c|}
\hline a. $\frac{n}{n}$ & $u_{*} \cdot \frac{1}{1}$ & $x_{x} \cdot \frac{9}{7}$ & $u_{0}, \frac{3}{13}$ \\
\hline$\frac{d, \frac{1}{3}}{3}$ & & $\frac{3+1 \times 4}{7+1+10}=4$ & 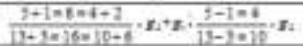 \\
\hline$d_{x} \cdot \frac{3}{3}$ & $\frac{5-1-2}{5=1-6} \cdot 2$ & & \\
\hline$d_{2, \frac{1}{1}}$ & $\frac{1-1-4}{11-1-10} \cdot$, $;: \frac{3-1-1-2 \times 2}{11+1-12-2 \times 6} ;$ in & & $\frac{3+5-10=6+4}{13+11-24-14+10} ; x$ \\
\hline Futs & 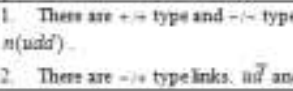 & 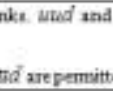 & 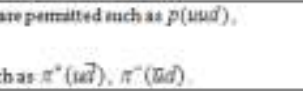 \\
\hline
\end{tabular}

Table 13.2G: Gluons Links between Up and Strange Quarks

\begin{tabular}{|c|c|c|c|}
\hline $9 . \frac{\pi}{n}$ & $a_{2}, \frac{1}{3}$ & $u_{x}+\frac{3}{7}$ & ${ }^{2}, \frac{1}{13}$ \\
\hline $2+\frac{7}{10}$ & 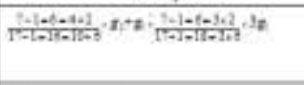 & 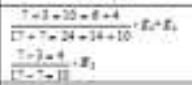 & 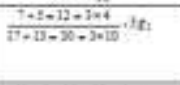 \\
\hline$x_{*}, \frac{11}{27}$ & 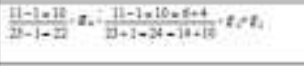 & & 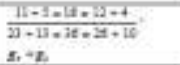 \\
\hline$s_{1}-\frac{13}{51}$ & 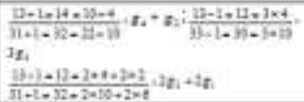 & 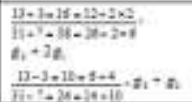 & 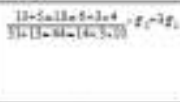 \\
\hline Facts & \multicolumn{3}{|c|}{ 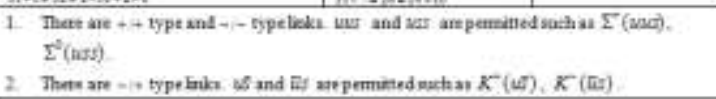 } \\
\hline
\end{tabular}

Table 13.2H: Gluons Links between Up and Charm Quarks

\begin{tabular}{|c|c|c|c|}
\hline$\phi_{1}=\frac{\pi}{n}$ & H,,$\frac{1}{1}$ & $u_{a}, \frac{3}{7}$ & $w_{k}=\frac{5}{13}$ \\
\hline$c_{*}=\frac{2}{15}$ & 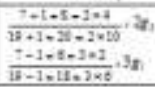 & 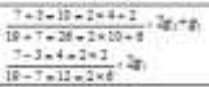 & 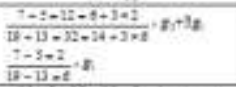 \\
\hline$\epsilon_{x}, \frac{19}{29}$ & & & $\frac{21-1-10-10+2 x+4}{25+13-42-22+2020}+2 ;-2 n$ \\
\hline$c, \frac{2 y}{37}$ & & & \\
\hline Fach & \multicolumn{3}{|c|}{ 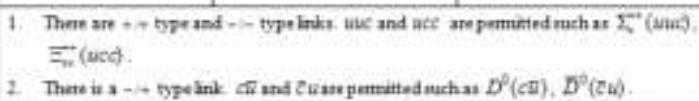 } \\
\hline
\end{tabular}

Table 13.2I: Gluons Links between Up and Bottom Quarks

\begin{tabular}{|c|c|c|c|}
\hline $\begin{array}{l}\text { 8. } \\
5\end{array}$ & $\mathrm{H}_{*+} \frac{1}{1}$ & $w_{3}, \frac{3}{3}$ & $u_{s} \cdot \frac{3}{3}$ \\
\hline$\frac{17}{4 !}$ & 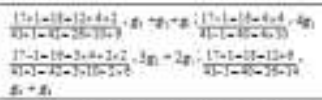 & 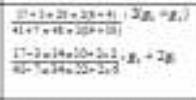 & 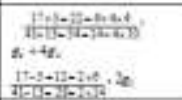 \\
\hline $\begin{array}{l}\frac{b}{4} \\
\frac{1}{4}\end{array}$ & 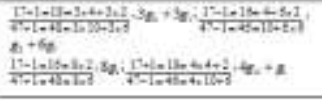 & 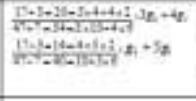 & 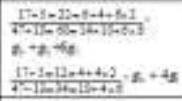 \\
\hline$\frac{b_{3}}{\frac{n}{n}}$ & & & \\
\hline Tacts & \multicolumn{3}{|c|}{ 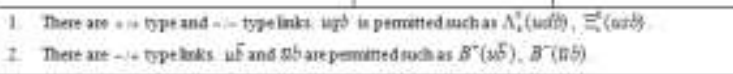 } \\
\hline
\end{tabular}

Table 13.2J: Gluons Links between Down and Strange Quarks

\begin{tabular}{|c|c|c|c|}
\hline 9. $\div$ & $\frac{d+1}{1}$ & $\frac{x_{1}^{2}}{x_{1}}+\frac{1}{2}$ & $d_{k, \frac{3}{12}}$ \\
\hline $3+\frac{7}{2}$ & 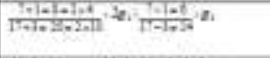 & 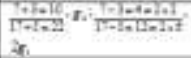 & 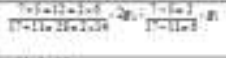 \\
\hline$x_{2}, \frac{11}{21}$ & 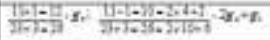 & & 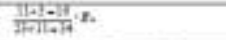 \\
\hline $3, \frac{23}{n}$ & 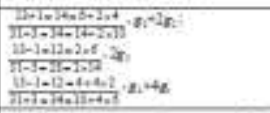 & 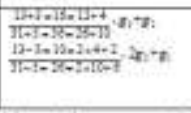 & 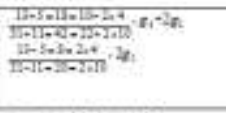 \\
\hline Fact & 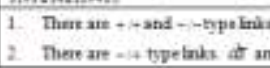 & 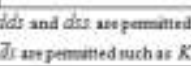 & 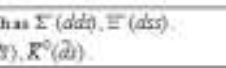 \\
\hline
\end{tabular}

Table 13.2K: Gluons Links between Down and Charm Quarks

\begin{tabular}{|c|c|c|c|}
\hline Q. $\frac{n}{2}$ & $d, \frac{1}{3}$ & $d_{1}=\frac{3}{2}$ & $d ; \frac{1}{n}$ \\
\hline $6, * \frac{1}{10}$ & 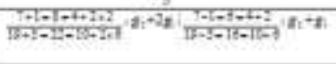 & 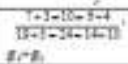 & 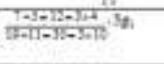 \\
\hline$c, \cdot \frac{29}{20}$ & & & 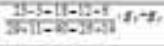 \\
\hline c, $\frac{29}{37}$ & & & \\
\hline Facit & \multicolumn{3}{|c|}{ 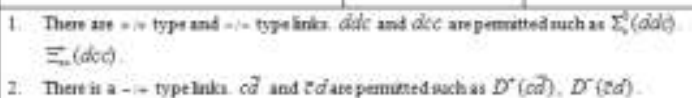 } \\
\hline
\end{tabular}

Table 13.2L: Gluons Links between Down and Bottom Quarks

\begin{tabular}{|c|c|c|c|}
\hline ต. $\frac{n}{n}$ & $\frac{d, n}{z}$ & $d_{n}, \frac{3}{7}$ & $d^{2}, \frac{3}{11}$ \\
\hline $2,+\frac{17}{41}$ & 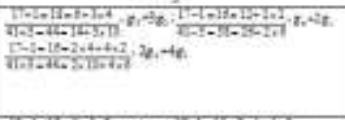 & 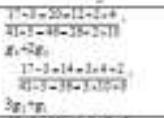 & 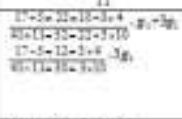 \\
\hline $\begin{array}{l}b_{y} \\
\frac{17}{4}\end{array}$ & 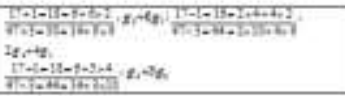 & 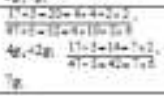 & 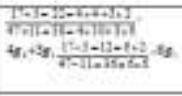 \\
\hline \multicolumn{4}{|l|}{$b_{6} \cdot \frac{39}{13}$} \\
\hline Futi & 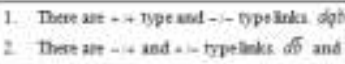 & depamatrod wach a: & 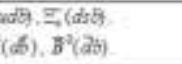 \\
\hline
\end{tabular}




\section{Z. Y. SHEN}

Table 13.2M: Gluons Links between Strange and Charm Quarks

\begin{tabular}{|c|c|c|c|}
\hline a. $\frac{\pi}{n}$ & $5+\frac{7}{17}$ & $x_{x}+\frac{11}{25}$ & $5,+\frac{13}{31}$ \\
\hline $6 \cdots \frac{7}{19}$ & 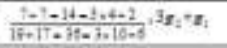 & 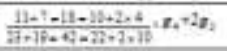 & 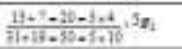 \\
\hline$c_{6}, \frac{23}{20}$ & 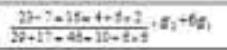 & & \\
\hline ch, $\frac{29}{37}$ & 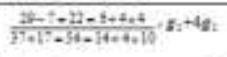 & & \\
\hline Facts & \multicolumn{3}{|c|}{ 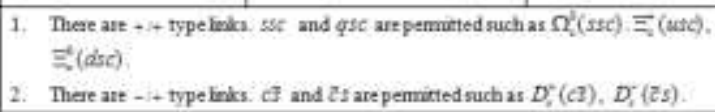 } \\
\hline
\end{tabular}

Table 13.2N: Gluons Links between Strange and Bottom Quarks

\begin{tabular}{|c|c|c|c|}
\hline$q \cdot \frac{n}{n}$ & $3.7 \frac{7}{17}$ & $s_{i} \cdot \frac{\pi}{23}$ & $s_{1}, \frac{13}{31}$ \\
\hline $0, \frac{17}{41}$ & 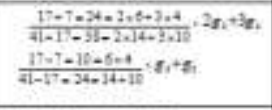 & 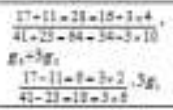 & 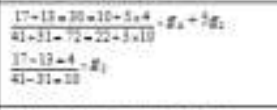 \\
\hline $\begin{array}{l}b_{b}= \\
\frac{17}{47}\end{array}$ & 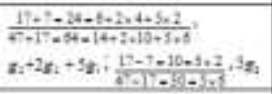 & $\frac{17+11-21-5,4}{47+27-50-9710}, 7 n_{6}$ & 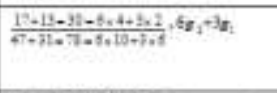 \\
\hline$b_{3}, \frac{99}{49}$ & & & 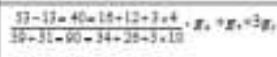 \\
\hline Facts & \multicolumn{3}{|c|}{ 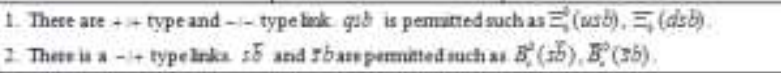 } \\
\hline
\end{tabular}

Table 13.20: Gluons Links between Charm and Bottom Quarks

\begin{tabular}{|c|c|c|c|}
\hline q. $\frac{n}{m}$ & c. $\frac{7}{19}$ & $c x=\frac{23}{25}$ & $\begin{array}{l}c_{3} \\
\frac{25}{37}\end{array}$ \\
\hline$\frac{b}{4 !}$ & 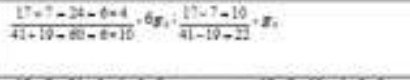 & & \\
\hline $\begin{array}{l}b_{8} \\
\frac{17}{47}\end{array}$ & 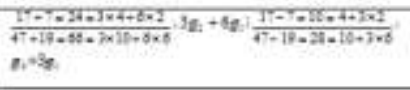 & & \\
\hline $\begin{array}{l}b_{1} \\
\frac{5 s}{39}\end{array}$ & & $\begin{array}{l}\frac{33-23-30-1+13=2}{59+29-81-16+19+6} \\
x+13 x\end{array}$ & \\
\hline Facts & 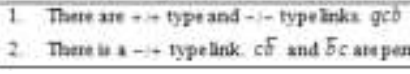 & 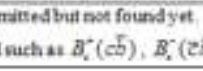 & \\
\hline
\end{tabular}

Three top quarks $t_{r}, t_{g}, t_{b}$ are not listed in Table 13.2, because there is no regular type strong interaction among them and with other types of quarks.

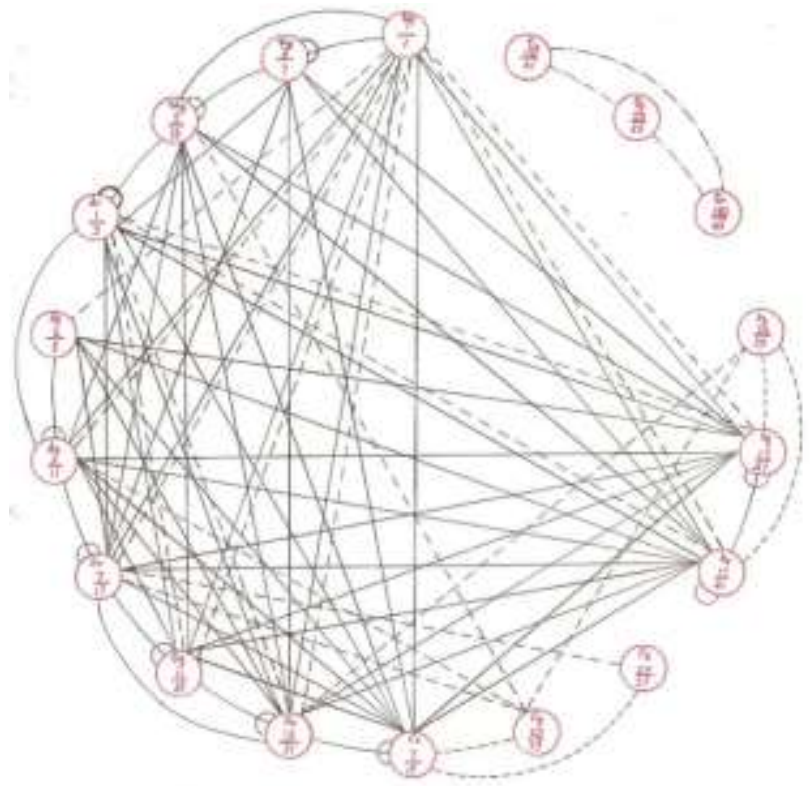

Fig.13.2: Regular type strong interactions among quarks. The multilink of the same type between two quarks listed in Table 13.2 is represented by a single line.
According to Table 13.2, Fig. 13.2 shows the regular strong interactions among quarks and anti-quarks. The solid line represents links between quark with quark or anti-quark with anti-quark (the "+ /+ type" and "- /- type" ). The dashed line represents links between quark with anti-quark (the "- /+ type" and "+/- type"). The dot-dashed lines represent the weakened links between top quark and top antiquark, which will be discussed later.

The strong interactions shown in Table 13.2 and Fig. 13.2 have following features:

1. In general, the hadrons consist of $u, \bar{u}, d, \bar{d}, s, \bar{s}, c$, $\bar{c}, b, \bar{b}$ can be constructed with the gluons links shown in Table 13.2 and Fig. 13.2, which are agreed with known experimental facts as shown in the "Facts" row of Table 13.2.

2. For the three lighter quarks $u, d, s$ shown in Fig. 13.2, there are only solid line links for quark with quark or antiquark with anti-quark among different colors in the same flavor. It means that, the same flavor quarks are permitted to form baryons such as $u u u$, ddd and sss. But the quark and anti-quark with same flavor are prohibited to form standalone mesons such as $u \bar{u}, d \bar{d}, s \bar{s}$. In fact, experiments confirmed these conclusions.

3. The neutral meson $\pi^{0}=(u \bar{u}-d \bar{d}) / \sqrt{2}$ is special. Table 13.3 provides a possible explanation for the formation of $\pi^{0}$. The gluons links consist of gluons with mixed "+" and "-" signs for $u \bar{u}$ and $d \bar{d}$ to form $\pi^{0}=(u \bar{u}-d \bar{d}) / \sqrt{2}$ via weakened strong interaction. In fact, the weakened strong interaction may explain why $\pi^{0}$ has a much shorter mean life $8.4 \times 10^{-17} \mathrm{~s}$ comparing to $\pi^{+}, \pi^{-}$mean life $2.6033 \times 10^{-8} \mathrm{~s}$.

Table 13.3A: Some Gluons Links between Up Quarks with Weakened Strong Interaction

\begin{tabular}{|c|c|c|c|}
\hline$p_{11} \div$ & $y_{;}, \frac{1}{1}$ & $u_{1}=\frac{3}{7}$ & $\alpha_{4} \cdot \frac{3}{7}$ \\
\hline$x_{i}, \frac{1}{1}$ & & 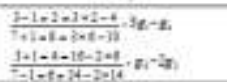 & 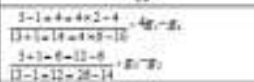 \\
\hline$u_{H}, \frac{3}{2}$ & 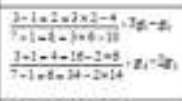 & & 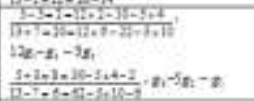 \\
\hline$H_{4}=\frac{3}{5}$ & 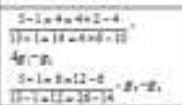 & 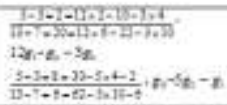 & \\
\hline Sotth & 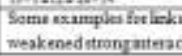 & 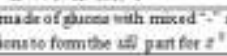 & 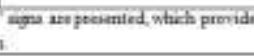 \\
\hline
\end{tabular}

Table 13.3B: Some Gluons Links between Down Quarks with Weakened Strong Interaction

\begin{tabular}{|c|c|c|c|}
\hline$v_{i} \frac{\pi}{n}$ & $d_{n}, \frac{1}{3}$ & $d_{,}, \frac{5}{3}$ & $d_{2} \frac{5}{12}$ \\
\hline d. $\frac{1}{y}$ & & 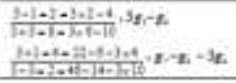 & 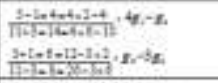 \\
\hline$d_{x}, \frac{3}{5}$ & 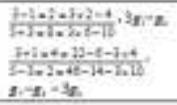 & & 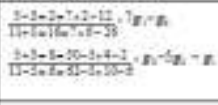 \\
\hline$d_{4} \cdot \frac{5}{11}$ & 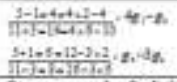 & 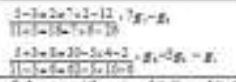 & \\
\hline Nonts & $\begin{array}{l}\text { Somit exumpies fortri } \\
\text { provide weakerend atror }\end{array}$ & $\begin{array}{l}\text { of douns with mased } \\
\text { ctomuto form the of past for }\end{array}$ & $\begin{array}{l}\text { ign arepresideted atich } \\
\text { monon }\end{array}$ \\
\hline
\end{tabular}


4. As shown in Fig. 13.2, for the two heavier quarks, $c$ quarks have only dashed line links between different colors, and $b$ qarck have only one solid line links between different colors. It means that, the same flavor quarks and anti-quark are permitted to form mesons such as $c \bar{c}$ and $b \bar{b}$; and the same flavor quarks are prohibited to form baryons such as $c c c$ or $b b b$. Experiments confirmed these conclusions.

5. There is no link from top quark or top anti-quark to any other quarks or anti-quarks, which means no strong interaction among them. It is confirmed by experiments. No hadrons made of top quark or top anti-quark with other type of quarks or anti-quarks have found. There is no regular link among three top quarks or three top antiquarks either. As mentioned in Section 11, top quark and top anti-quark are produced in pairs. Table 13.4 provides a possible explanation. In which, the links are weakened by the mixed "+" and "-" signs in gluons links. The result of such mixed links is a weakened strong interaction between $t$ and $\bar{t}$, which may contribute to $t \bar{t}$ pair's very short lifetime of $0.5 \times 10^{-24} \mathrm{~s}$.

Table 13.4: Weakened Gluons Links between Top Quarks and Top Anti-Quarks

\begin{tabular}{|c|c|c|c|}
\hline$Q_{1}+\frac{\pi}{n}$ & $t, \frac{783}{49}$ & $t_{t}+\frac{315}{51}$ & $t_{6} \cdot \frac{397}{81}$ \\
\hline $2,=\frac{28}{43}$ & & 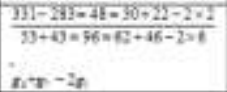 & 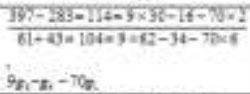 \\
\hline$\frac{3+1}{39}$ & 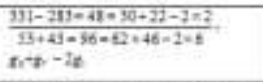 & & 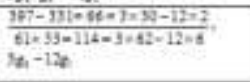 \\
\hline$t, \frac{385}{6 !}$ & 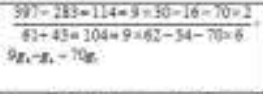 & 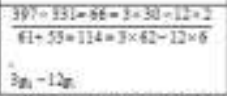 & \\
\hline Wates & 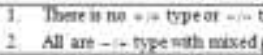 & 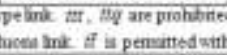 & the weakened strong \\
\hline
\end{tabular}

In essence, Table 13.2 and Fig. 13.2 provide mathematical explanations for strong interactions among quarks, which agreed with known experimental results. The reason for such agreement is due to careful selection of the $n$-parameters for quarks. According to definition 13.1 and Rule 13.1, there is still some room for alternative selections of $n$-parameters for quarks. But in order to meet all known experimental facts of strong interactions among quarks and anti-quarks to forming hadrons, the room for selecting correct $n$-parameters is limited.

High energy experiments have shown quarks confinement. When the gluons link between two quarks is broken, a quark and an anti-quark are created at the broken ends. This phenomenon can be explained naturally by SQS theory. According to Definition 13.1, all gluons are made of quark and anti-quark pairs. The broken parts of gluons link are naturally a quark and an anti-quark.

According to Definition 13.2, gluons have nonzero $n$ parameters corresponding to $n / m \neq 0$. Does $n / m \neq 0$ mean gluons having loop-2? The answer is: No. The $n$ parameter assigned to gluon does not represent loop-2, instead, $n$ is the number of turns of the "spring" made of the loop-1 merged portion as shown in Fig.13.1. However, there is a trick. When a gluon serve as the mediator of strong interactions, its $n$ does act like regular $n$-parameter as shown in (13.8b). But gluon doing loop-2 job is not necessary mean itself having loop-2.

Rule 13.2: The strong interaction between two gluons with parameters $m_{a}, n_{a}$ and $m_{b}, n_{b}$ by transmitting and receiving gluons with parameters $m_{i}, n_{i}$ satisfies the following equations:

$$
\begin{aligned}
& m_{a}+m_{b}=\sum_{i=1}^{M} a_{i} m_{i}, a, b, i=1,2,3 \cdots 8, \\
& n_{a}+n_{b}=\sum_{i=1}^{M} a_{i} n_{i}, a, b, i=1,2,3 \cdots 8 .
\end{aligned}
$$

In (13.11), $M$ is the number of gluon types in the link and $a_{i}$ is the number of gluons of type $i$.

According to Rule 13.2, there are trivial cases with $M=2$, $m_{1}=m_{a}, m_{2}=m_{b}$ and $n_{1}=n_{a}, n_{2}=n_{b}$. Besides these trivial cases, there are other possibilities, which serve as the mediator for strong interaction between gluons. Table 13.5 and Fig. 13.3 show some examples of strong interactions between gluons.

According to Rule 13.2 and as shown by Table 13.5 and Fig.13.3, the strong interactions among gluons have the following features.

1. The existence of strong interactions among gluons means that strong interactions are nonlinear in nature as expected.

2. In the second column from left, the strong interactions are regular type represented by all gluons in the link with the same "+" sign.

3. In the third column from left, the strong interactions are weakened type represented by the gluons in the link with mixed "+" sign and "-" sign.

The strong interaction mechanism introduced in this section is based on a link made of gluons sequence, which is a simplified concept. In reality, the scenario is more complicated. Inside a hadron, its valence quarks (valence anti-quarks) are surrounded by a network of gluons including many links. The strong interactions are dominated by the strongest link in the network.

As shown in Table.13.2A, in certain cases such as $u_{r} \leftrightarrow u_{g}$ there is only one link for regular strong interaction. In other cases such as $u_{g} \leftrightarrow u_{b}$ and $u_{b} \leftrightarrow u_{r}$, each has two links for regular strong interaction in parallel. Besides, there are weakened links among $u_{r}, u_{g}, u_{b}$ listed in Table 13.3A not shown in Table 13.2A. Among three top quarks $t_{r}, t_{g}, t_{b}$, there is no link for regular strong interaction. Under such circumstance, the next best option is to find the weakened link. So the overall scenarios are very rich and complicated, but the simplified concept does catch the essence of strong interactions evidenced by its results agreed with experiments. 
Z. Y. SHEN

Table 13.5: Some Examples of Strong Interactions between Gluons

\begin{tabular}{|c|c|c|c|}
\hline $\begin{array}{l}\text { Gluos } \\
\text { g. }, \frac{h}{m}\end{array}$ & $\begin{array}{l}\text { Regula strong interaction } \\
\text { between } g, * g \text {, }\end{array}$ & $\begin{array}{c}\text { Weakened strang interaction } \\
\text { between } g, \sim \varepsilon \text {. }\end{array}$ & $\begin{array}{l}\text { Gipon } \\
\text { s., } \frac{n}{m}\end{array}$ \\
\hline$g, \frac{2}{6}$ & $\frac{2+2-4-2+2}{6+5-12-6+6}+R_{i}+5_{i}$ & & $2=\frac{2}{6}$ \\
\hline $51 \cdot \frac{2}{6}$ & $\frac{1+2-6-4+2}{10+6=16-18+6}, s_{4}+s_{1}^{*}$ & $\frac{4-2-2-6-4}{20-6=d=16-10}, g_{5}-g_{2}$ & $8: \frac{4}{10}$ \\
\hline$g_{1}=\frac{2}{6}$ & $\frac{6+2-1=4+4}{14+3-20-10+10} \cdot 82+82$ & $\frac{6-2-4-10-6}{14-6-8-22-14} \cdot 8-5$ & $5, \frac{6}{14}$ \\
\hline$x_{*} \cdot \frac{2}{6}$ & $\frac{10+2-12=6+6}{22+6-23-14+14} \cdot g,+8$ & $\frac{16-2-8=12-t}{22-6-16-26-10}, g_{1}-g_{t}$ & c. $\frac{10}{22}$ \\
\hline $5, \frac{2}{6}$ & $\frac{12+2-14-10+4}{26+6-32-22+10}, 8+8$ & $\frac{12-2-10-16-6}{36-6-20-34-14}, 5,-8$ & $3=\frac{12}{28}$ \\
\hline s. $\frac{2}{6}$ & $\frac{16+2-11-12+6}{14+6=25=26+14} \cdot z_{3}+g_{3}$ & $\frac{16-2-14=38-16}{3-6-28=62-34} ; g_{5}-z_{4}$ & f.,$\frac{16}{34}$ \\
\hline$g, \frac{2}{6}$ & $\frac{22+7-74-12+12}{40+6-32-26+26} \cdot 8_{1}+5_{1}$ & $\frac{22-2-20-30-10}{48-6-40-62-22}, 54-54$ & $g, \frac{22}{46}$ \\
\hline $8=\frac{2}{6}$ & $\frac{30+2-32-16+16}{62+6-61-34+34}, 2+2$ & $\frac{30-2-25-30-2}{62-6-38-62-6}, B_{1}-8_{4}$ & 5. $\frac{30}{62}$ \\
\hline $5, \frac{4}{10}$ & $\frac{4+4-8=6+2}{10+10-20-14+6} \cdot 8 ;+\varepsilon$ & & $s_{2}, \frac{4}{10}$ \\
\hline $8: \frac{4}{10}$ & $\frac{8+4-10=6+4}{14+10-24=14+10} ; z_{5}+z_{3}^{*}$ & $\frac{6-4-2-4-2}{14-10-1-10-6}, 5_{2}-52$ & $g_{2} \cdot \frac{6}{16}$ \\
\hline $8: \frac{4}{10}$ & $\frac{10+4-14-12+2}{22+10-32-26+6} \cdot 8_{1}+g_{1}$ & $\frac{10-4-6-16-10}{22-10-12-34-22} \cdot g_{1}-g_{4}$ & $5, \frac{10}{22}$ \\
\hline$g: \frac{4}{10}$ & $\frac{12+4-16-10+6}{28+10-36-22+14}, 8+2$ & $\frac{12-4-1-30-22}{26-10-16-62-46} \cdot 52-87$ & $5, \frac{12}{26}$ \\
\hline$=2 \cdot \frac{4}{10}$ & $\frac{16+4=20=10+10}{34+10=44-22+22}, g_{t}+g_{t}$ & $\frac{16-4=12=22-15}{34-10-24-46-22}, z-\varepsilon$ & $\varepsilon_{1}, \frac{16}{34}$ \\
\hline$g: \cdot \frac{4}{10}$ & $\frac{22+4-26-16+10}{20+10-36-14+22}, 8,+g$ & $\frac{22-4-11-30-12}{46-10-56-62-16} \cdot g_{1}-\varepsilon_{3}$ & 4. $\frac{72}{46}$ \\
\hline $5:+\frac{4}{10}$ & $\frac{30+1-19-22+12}{62+10=72=26-26}, g+8 z$ & $\frac{30-1-26-30-4}{62-10=52=62-10}, 8_{5}-8_{2}^{*}$ & $g_{1} \cdot \frac{30}{62}$ \\
\hline$g_{N}=\frac{6}{14}$ & $\frac{6+6-12-15+2}{14+14-211-22+6}, g_{6}+z_{1}$ & & $g_{0}, \frac{6}{14}$ \\
\hline $5: \frac{6}{14}$ & $\frac{10-6=16=12+4}{22+14-36=26+10}, g_{7}+z_{7}$ & $\frac{10-6-4=6-2}{22-14=1-14-6}, z_{3}-3$ & a. $\frac{10}{22}$ \\
\hline$g_{5}=\frac{6}{14}$ & $\frac{12+8-11-16+2}{36+14-10-34+6}, 8_{1}+8_{1}$ & $\frac{12-6-6-10-4}{26-14-12-22-16}+56-5:$ & $5, \frac{12}{26}$ \\
\hline g: $: \frac{6}{14}$ & $\frac{10+6-21-12+10}{14+14=18=26-22}, B_{2}+8$ & $\frac{16-6-50-12-2}{34-14=25=26-6}, E_{2}-E_{1}$ & $g_{1} \cdot \frac{16}{34}$ \\
\hline$\varepsilon_{1} \cdot \frac{6}{14}$ & $\frac{22+5=3-16+12}{45-14-65-34+26}+82+82$ & $\frac{22-6-16-22-6}{46-14-32-46-14}, 97-85^{*}$ & $8 \cdot \frac{22}{46}$ \\
\hline $8+\frac{6}{14}$ & $\frac{30+6-36=30+6}{62+14=76-52+14}, g_{1}+g_{1}=$ & $\frac{30-5=24=35-6}{62-14-48-62-14} \cdot 5,-8_{3}{ }^{*}$ & $\varepsilon_{3}, \frac{30}{62}$ \\
\hline$z+\frac{10}{22}$ & $\frac{16+10-20-16+4}{22+22-14-34+20} \cdot E_{4}+B_{1}$ & & 8. $\frac{10}{22}$ \\
\hline $2 \cdot \frac{10}{22}$ & $\frac{12+10=22=16+6}{26+22=21=34+14}, g_{9}+g_{3}$ & $\frac{12-10-2-6-1}{26-22=1-14-16}, g_{5}-g_{1}$ & $g_{5} \cdot \frac{12}{26}$ \\
\hline $8 \cdot \frac{10}{22}$ & $\frac{16+10-26-22+4}{34+22-36-46+16}, R_{7}+R_{2}$ & $\frac{16-10-6-10-4}{34-22-12-22-10}, 84-8$ & $\varepsilon_{4}, \frac{10}{34}$ \\
\hline$s+\frac{10}{22}$ & $\frac{22+10-32-30+2}{45+22-61-62+6}, g_{t}+g_{2}$ & $\frac{22-10=12-16-4}{46-22-24-34-10}, 5,-5$ & $g_{1}, \frac{22}{46}$ \\
\hline $8+\frac{10}{22}$ & $\frac{30+10-20-30+16}{62+22-84-62+22} \cdot 51+26$ & $\frac{30-10-30-22-2}{12-22-40-40=6}: 81-8$ & $g_{8} \cdot \frac{30}{62}$ \\
\hline$g: \frac{12}{26}$ & $\frac{12+12=24-22+2}{25+26-52=46+6} \cdot g t+g_{1}$ & & $g_{1} \cdot \frac{12}{26}$ \\
\hline$E_{4} \cdot \frac{12}{26}$ & $\frac{16+12-21-22+6}{34+26-61-46+14} \cdot z_{1}+z_{2}$ & $\frac{10-12-4-6-2}{34-36-8-14-6} ; z_{1}-z_{1}$ & g. $\frac{10}{34}$ \\
\hline$g: \frac{12}{26}$ & $\frac{21+12=34-30+1}{36-26=72=62+20}, z_{1}+z_{2}$ & $\frac{22-12-10=16-6}{46-26-20=13-14}, g_{1}-g_{1}$ & $2, \frac{22}{46}$ \\
\hline g. $\frac{12}{26}$ & $\frac{30+12-12=30+12}{62+26-81-62+26}, h_{1}+g_{3}^{*}$ & $\frac{30-12-11-22-4}{62-28-36-46-10}, 57-5 ?$ & E*: $\frac{30}{62}$ \\
\hline$z_{4}: \frac{16}{34}$ & $\frac{16+16-32=30+2}{3+34-64-62-6}, g,+g$ & & $z_{1}, \frac{16}{34}$ \\
\hline$E_{6}=\frac{16}{34}$ & $\frac{22+16-34-22+16}{46+92=80=46+34}, z_{1}+z_{4}^{*}$ & $\frac{22-16-6-50-4}{46-34-12=22-10}, 24-s_{2}$ & g. $\frac{22}{45}$ \\
\hline$z_{1} \cdot \frac{16}{34}$ & $\frac{30+16=-86=36+16}{62+31=96=62+34}, g_{1}+z_{4}^{*}$ & $\frac{35-16=14-16-2}{5-34=25-34-6}, 8-8$ & $z_{1}, \frac{30}{62}$ \\
\hline si. $\frac{22}{46}$ & $\frac{22+22-44-22+21}{46+26-92-25+36}, 8_{t}+z_{7}+$ & & $5 r \cdot \frac{22}{46}$ \\
\hline$g_{1}=\frac{22}{46}$ & $\frac{30+22-52=30+22}{62+46-103-62+46}+g_{t}+g_{t}^{*}$ & $\frac{30-22-8-12-4}{62-46-16-25-10} \cdot g_{1}-g$ & $z_{1}, \frac{30}{62}$ \\
\hline$\varepsilon_{1} \cdot \frac{30}{62}$ & $\frac{30+30-50-30+30}{62+62-124-62+62}, g_{1}+g_{1}$ & & $\varepsilon_{*} \cdot \frac{30}{62}$ \\
\hline Notes. & \multicolumn{3}{|c|}{$\begin{array}{l}\text { 1. Only coe example is listed foe each colime itt each fow. } \\
2 \text {. In case of no pootrivial link, a trivial limk marked with * is presented }\end{array}$} \\
\hline
\end{tabular}

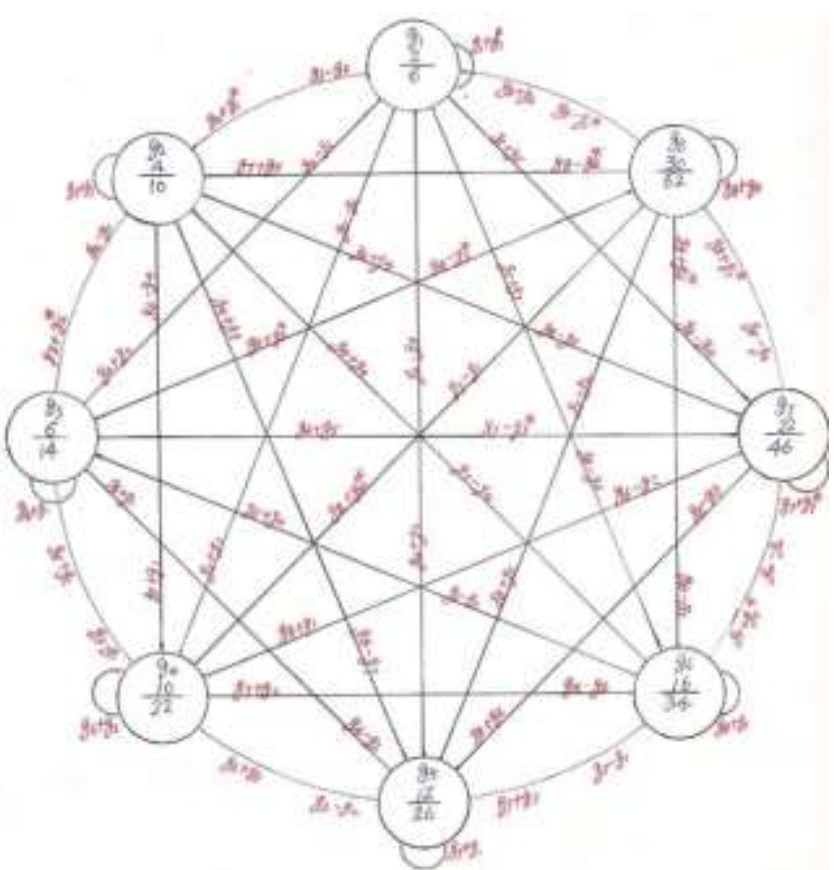

Fig.13.3: Some strong interactions among 8 gluons.

The strong interaction mechanism introduced in this section by SQS theory has some similarities as well as differences with quantum chromodynamics (QCD) of the standard model.

1. Both theories have eight gluons serving as the mediators for strong interactions.

2. Both theories explain the known experimental facts of strong interactions for hadrons.

3. Both theories indicate that strong interactions are nonlinear due to the fact that, there are strong interactions among gluons.

4. Both theories explain the confinement of quarks and anti-quarks.

5. According to QCD, gluons exhibit $S U$ (3) symmetry. According to Definition 13.1, the eight gluons are made of eight quark and anti-quark pairs. They also exhibit $S U$ (3) symmetries for flavors as well as colors like their constituent quarks.

6. According to QCD, the eight gluons are specifically assigned to a pair of quarks (anti-quarks) to transfer their colors. According to SQS theory, as described in this section, the eight gluons' function is not specialized. To serve as mediator for a specific strong interaction, a combination of gluons is lined up to make the link. This difference between two theories can be explained by proper combinations of gluons.

7. According to QCD, gluons are represented by complex parameters. On the other hand, as presented in this section, the gluons are represented by real numerical parameters. According to SQS theory, the phase of a complex number represents intrinsic time. Taking this factor into account, the difference is understandable. 
Theorem 13.2: For a hadron made of quarks (anti-quarks) and gluons, the gluons' $m$-parameters cancel out and the gluons' $n$-parameters also cancel out, which do not contribute to the hadron. In other words, only valence quarks (valence anti-quarks) $m$-parameters and $n$ parameters count for hadron as a composite particle.

Proof: The strong interactions are mutual in nature. For a pair of quarks (a pair of anti-quarks, or a quark and an anti-quark), $q_{a}$ and $q_{b}$, when $q_{a}$ sends a sequence of gluons with summed parameters $\sum_{i} m_{i}$ and $\sum_{i} n_{i}$ to $q_{b}$, in return $q_{b}$ sends the same sequence of gluons with summed parameters $\sum_{i} m_{i}$ and $\sum_{i} n_{i}$ back to $q_{a}$ along opposite direction. As a result, the net changes of parameters for the hadron are:

$$
\begin{aligned}
& \Delta\left[\sum_{i} m_{i}\right]=\sum_{i} m_{i}-\sum_{i} m_{i}=0, \\
& \Delta\left[\sum_{i} n_{i}\right]=\sum_{i} n_{i}-\sum_{i} n_{i}=0 .
\end{aligned}
$$

QED

The argument is also applicable to strong interactions between gluons and gluons.

Theorem 13.2 greatly simplifies the $m$-parameters and $n$ parameters of hadron as a composite particle. In essence, for hadron's $m$-parameters and $n$-parameters, only its valence quarks (valence anti-quarks) count, gluons do not count. Theorem 13.2 plays important roles for composite particles, which will be presented in Section 19.

The set of $n$-parameters for quarks used in Table 13.2 and Fig.13.2 is a specific selection cited from Table 11.2. It by no means the only selection. In fact, other selections are possible, which is worthwhile to explore further.

In this section, a framework is built for strong interactions based on mathematics. More works are needed for detailed quantitative results.

The basic idea of this section is to treat gluons and strong interactions in terms of mathematics and geometry. It is a step toward the final goal of SQS theory.

\section{Section 14: $W Z$ Bosons and Weak Interactions}

In this section, SQS theory provides a framework for weak interactions based on mathematics. It includes a model for gauge bosons $W^{ \pm}$and $Z^{0}$ along with other mediators associated with weak interactions.

$W^{ \pm}$and $Z^{0}$ particles are gauge bosons with mass heavier than $M_{\text {Max }}=4.973 \mathrm{GeV} / \mathrm{c}^{2}$ of (6.21). Their model should have topological structure similar to the model of top quarks. But top quarks are fermions, and $W^{ \pm}$and $Z^{0}$ are bosons. This problem can be solved by assuming two fermion states $Y_{1}$ and $Y_{2}$ as constituents. $W^{ \pm}$and $Z^{0}$ are treated as two mixed states of $Y_{1}$ and $Y_{2}$.
Postulation 14.1: Two fermion states $Y_{1}$ and $Y_{2}$ with spin $\hbar / 2$ and charges $|e| / 2$ serve as the components of $W^{ \pm}$ and $Z^{0}$.

The mass of $Y_{1}$ and $Y_{2}$ exceeds $M_{\text {max }}$, according to Rule 6.1, they must appear in pair serving as the constituents of $W^{ \pm}$and $Z^{0}$. In this way, the fractional charge does not show up.

According to 2010-PDG data, $W^{ \pm}, Z^{0}$ and top quark $t$ have a mass relation:

$$
\begin{gathered}
M_{W \pm}=80.399 \pm 0.023 \mathrm{GeV}, \\
M_{Z 0}=91.1876 \pm 0.0021 \mathrm{GeV}, \\
M_{t}=172 \pm 0.9 \mathrm{GeV} . \\
M_{W \pm}+M_{Z 0}=171.5866 \pm 0.0251 \mathrm{GeV} \approx M_{t}=172 \pm 0.9 \mathrm{GeV} .
\end{gathered}
$$

The mass relation implies that, they are correlated. This is the first clue to determine the parameters of $Y_{1}$ and $Y_{2}$.

The second clue is $W^{ \pm}$to $Z^{0}$ mass ratio correlated to Weinberg angle $\theta_{W}\left(M_{Z}\right)$ :

$$
\frac{M_{W \pm}}{M_{Z 0}}=\frac{80.399}{91.1876}=0.88169 \approx \cos \left(\theta_{W}\left(M_{Z}\right)\right)=\cos \left(28.757^{\circ}\right)=0.87667 \text {. }
$$

$\theta_{W}\left(M_{Z}\right)=28.757^{\circ}$ and $\sin ^{2}\left(\theta_{W}\left(M_{Z}\right)\right)=0.23146(12)$ are cited from 2010-PDS (p. 101). To combine (14.1d) and (14.2) yields an approximate mass relations for $W^{ \pm}, Z^{0}$ and top quark:

$$
M_{Z}=\frac{M_{t}}{1+\cos \theta_{W}}, M_{W}=\frac{M_{t}}{1+\cos \theta_{W}} \cos \theta_{W} .
$$

The third clue comes from an apparent symmetry in the Elementary Particles Table of Table 18.2 of Section 18, in which $Z^{0}, W^{ \pm}$are located at right end and photon $\gamma$, graviton $g$ at left end. According to SQS theory, $\gamma$ is correlated with electron and $g$ is correlated with up red quark. At right end of the table, $Z^{0}$ and $W^{ \pm}$should be correlated with taon and top blue quark.

Keeping these clues in mind, the number parameters are selected for $Y_{1}$ and $Y_{2}$ as:

For $Y_{1}$ :

$$
m_{1}=53, n_{1}=371, p_{1}=33102475,
$$

For $Y_{2}$ :

$$
m_{2}=61, n_{2}=427, p_{2}=38099075 .
$$

Formula (11.1b) is used to calculate $\theta_{2 O, Y}$ for $Y_{1}$ and $Y_{2}$ with $1 / 3$ replaced by $1 / 2$.

The calculated parameters of $Y_{1}$ and $Y_{2}$ are listed in Table 14.1.

$Y_{1}$ and $Y_{2}$ are not free standing particles, they serve as two branches of $W^{ \pm}$and $Z^{0}$. According to Table 14.1, except the original numerical parameters $m, n, p$ difference, 
all other parameters are the same for $Y_{1}$ and $Y_{2}$, which is similar to the three branches of charged leptons trefoil model.

$Y_{1}$ and $Y_{2}$ both have mass exceeding $M_{\text {Max }}$. Their model belongs to the spindle type torus akin to top quarks model shown in Fig. 11. 1. The $a_{2}=1.33406113$ is greater than $d=1$. The $f$-modification makes $a_{2}^{\prime}<1$, which is not fully applicable to $Y_{1}$ and $Y_{2}$.

Table 14.1: Parameters for Fermion States $Y_{1}$ and $Y_{2}$

\begin{tabular}{|c|c|c|c|}
\hline \multicolumn{2}{|l|}{$\begin{array}{l}\text { Modet: Spindte torus } \\
\text { Spin } h / 2\end{array}$} & \multicolumn{2}{|l|}{$\begin{array}{l}\text { Msodel: Sponsit terus } \\
\text { Spuz } \mathrm{A} / 2\end{array}$} \\
\hline \multicolumn{2}{|c|}{ Nase: $45: 5938774 \mathrm{GeV}: \quad$ Charge $/ / 2$} & \multirow{4}{*}{\multicolumn{2}{|c|}{$\begin{array}{l}\text { Mass } 4559387774 \mathrm{GeV} ; \quad \text { Charge H/2 } \\
\text { Original: } \frac{n}{m}=\frac{427}{61}, \frac{D}{n}=\frac{38099075}{427} \\
\text { Reduced: } \frac{n}{m}=\frac{7}{1}, \frac{E}{n}=\frac{624575}{7} \\
\frac{2 p}{m}=1249150\end{array}$}} \\
\hline \multirow{2}{*}{\multicolumn{2}{|c|}{$\begin{array}{l}\text { Original: } \frac{n}{m}=\frac{371}{53} \cdot \frac{D}{n}=\frac{33102475}{371} \\
\text { Rectoced: } \frac{n}{N}=\frac{7}{1} \cdot \frac{D}{n}=\frac{624575}{7}\end{array}$}} & & \\
\hline & & & \\
\hline \multicolumn{2}{|l|}{$27=1 \times 4 \%$} & & \\
\hline \multicolumn{4}{|c|}{$Y_{1}$ and $Y_{2}$} \\
\hline \\
\hline \multicolumn{2}{|c|}{$\begin{array}{l}\text { Torus Inner Hair (postive curvature) } \\
x_{1}=0.2499985 ; \pi\left(x_{1}\right)=3.14155362 \\
R=|1-a|=0.34406113\end{array}$} & \multicolumn{2}{|c|}{$\begin{array}{l}\text { Torus Ower Haif (jositvie curvature) } \\
x_{z}=0.2500014 ; \pi\left(x_{z}\right)=314154406\end{array}$} \\
\hline $\begin{array}{l}\text { Trangle } \$ O_{1} O_{3}^{\prime} \text { on } \\
O_{1} H C r o s s \text { Sertice }\end{array}$ & $\begin{array}{l}\text { Triangle } D O_{1} O_{1}^{\prime} \text { on } \\
x-y \text { Cross Section }\end{array}$ & $\begin{array}{l}\text { Triangle } A_{1} \sigma_{1} O_{1}, \infty \\
Q_{1} G \text { Cross Sertion }\end{array}$ & $\begin{array}{l}\text { Truesle } G 0, O, \infty 0 \\
x-y \text { Cross Section }\end{array}$ \\
\hline$x_{l}=\mathrm{t} 330838+3$ & $x_{0}=0.33404868$ & $X_{z}=133005099$ & $x_{2}=233395282$ \\
\hline$z_{1}=0.74664521$ & $Y_{0}=0.00288441$ & $Z_{2}=0.83276008$ & $r_{F}=0.02243538$ \\
\hline$\theta_{1}=36.30797806^{\circ}$ & $a_{1}=0.37063758^{\circ}$ & $b_{5}=12.38426492^{7}$ & Q -0.41372553 \\
\hline$A=113.89812807^{\circ}$ & $\phi=179.50523077^{2}$ & $a_{2}=19.66633629^{\circ}$ & $\phi=0.55197248^{2}$ \\
\hline$\psi_{1}=2929389387^{*}$ & $\psi_{0}=0.12388165$ & $v=14794884879:$ & $V_{1}=179.03430198^{\prime}$ \\
\hline $\mathrm{G}=6616187193^{\circ}$ & $6=0.49471923^{5}$ & $6=32.05115121^{2}$ & $\varepsilon_{1}=0.96569802^{4}$ \\
\hline
\end{tabular}

According to SQS theory, $W^{ \pm}$and $Z^{0}$ are two different combinations of $Y_{1}$ and $Y_{2}$. Their parameters are listed in Table 14.2.

Table 14.2: $W^{ \pm}$and $Z^{0}$ Parameters Based on $Y_{1}$ and $Y_{2}$

\begin{tabular}{|c|c|}
\hline 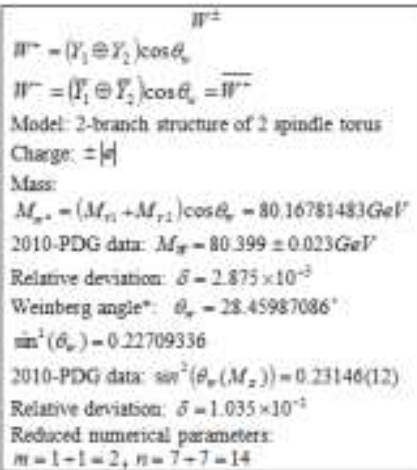 & 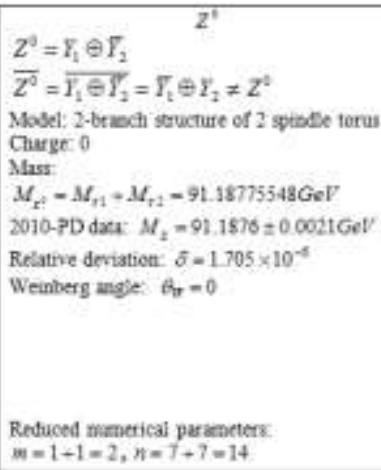 \\
\hline
\end{tabular}

According to SQS theory, $W^{ \pm}$or $Z^{0}$ serve as the intermediate state for weak interactions; there are other mechanisms and particles involved in weak interactions.

As indicated in Section 12, the reduced $n$-parameters of charged leptons $e, \mu, \tau$ is fractional: electron: $n_{e}=\frac{1}{2}$, muon: $n_{\mu}=1 \frac{5}{8}$, taon: $n_{\tau}=2 \frac{6}{7}$, which neither match to $W^{ \pm}$,
$Z^{0}$ with $n=$ even nor match to quarks with $n=$ odd. The solution for $n$-parameters mismatch problem is the key to treat weak interactions mathematically.

Rule 14.1: The Leptons Pairing Rule. To participate in weak interactions, charged leptons $e, \mu, \tau$ are paired with corresponding anti-neutrinos $\bar{v}_{e}, \bar{v}_{\mu}, \bar{v}_{\tau}$ and charged anti-leptons $e^{+}, \mu^{+}, \tau^{+}$are paired with corresponding neutrinos $v_{e}, v_{\mu}, v_{\tau}$ to form companion pairs.

For $e^{\uparrow} \& \bar{v}_{e}^{\uparrow}, \mu^{\uparrow} \& \bar{v}_{\mu}^{\uparrow}, \tau^{\uparrow} \& \bar{v}_{\tau}^{\uparrow}$ :

$$
\begin{aligned}
& m_{l}+m_{\bar{v}}=2, l=e, \mu, \tau ; \\
& n_{l}+n_{\bar{v} l}=0, l=e, \mu, \tau ;
\end{aligned}
$$

For $e^{+\downarrow} \& v_{e}^{\downarrow}, \mu^{+\downarrow} \& v_{\mu}^{\downarrow}, \tau^{+\downarrow} \& v_{\tau}^{\downarrow}$ :

$$
\begin{aligned}
& m_{\bar{l}}+m_{v l}=-2, \bar{l}=e^{+}, \mu^{+}, \tau^{+} ; \\
& n_{\bar{l}}+n_{v l}=0, \bar{l}=e^{+}, \mu^{+}, \tau^{+} .
\end{aligned}
$$

The arrows $\uparrow$ and $\downarrow$ indicate right and left handedness, respectively.

Explanation: Rule 14.1 serves as the basic rule for leptons participated in weak interactions. It solves lepton's fractional $n$-parameter problem and makes lepton pairs with $m=$ even, $n=0$ different from quarks with $m=$ odd,$n=$ odd . It lays the mathematical foundation for baryon number conservation and lepton number conservation including lepton family number conservation. Examples will be given later in this section.

\begin{tabular}{|c|c|}
\hline Particles involved in weak interistion & $n / m$ ratio types \\
\hline Quarks or ants-quarks & odd odd \\
\hline Pairs of quark and ant-gauts & even even \\
\hline$\partial, \mu, \mathrm{t}$ & fructinal fodf \\
\hline$\nabla_{n}, \nabla_{\mu}, \nabla_{n}$ & - fractina odd \\
\hline 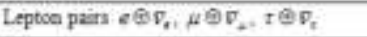 & $0 / 2$ \\
\hline$e^{*}, \mu^{*}, t^{*}$ & - fractinai odd \\
\hline$v_{n}, v_{\mu}, v_{n}$ & fractinal iodd \\
\hline Leptoen pairs $e^{*} \oplus v_{n}, k^{*} \oplus v_{*}, t^{*} \oplus v_{n}$ & $0\{-2\}$ \\
\hline II and $Z$ & $1 4 \longdiv { 2 }$ \\
\hline Gluets: & ever even \\
\hline Massons & newo 0 \\
\hline
\end{tabular}

In Table 14.3, different types of $n / m$ for particles involved in the weak interactions are listed.

Table 14.3: $n / m$ for Particles Involved in Weak Interactions

According to the types of $n / m$ listed in Table 14.3, except some rare events, in order to meet baryon number conservation and lepton number including lepton family number conservation, the mediators to make the links between the paired particles involved in weak interaction and $W^{ \pm}$or $Z^{0}$ must be the $n / m=e v e n / o$ type.

According to SQS theory, the link between quarkantiquark pair and $W^{ \pm}$or $Z^{0}$ is made of gluons. Gluons also participate in weak interaction! Is it true? No rule prohibits gluons participating in part of weak interaction, as long as the 
other part has specialized feature for weak interaction. The other part is the link between lepton pair and $W^{ \pm}$or $Z^{0}$, which is specialized for weak interactions.

Notice that, in Table 14.3, the $e \oplus \bar{v}_{e}, \mu \oplus \bar{v}_{\mu}, \tau \oplus \bar{v}_{\tau}$ pairs and $W^{ \pm}$or $Z^{0}$ both have $n / m=$ even $/ 2$. Since $2-2=0$ and even-even =even , the boson to make the link between $e \oplus \bar{v}_{e}, \mu \oplus \bar{v}_{\mu}, \tau \oplus \bar{v}_{\tau}$ and $W^{ \pm}$or $Z^{0}$ must have $m=0$ and $n=$ even. Gluons are not qualified for the job. A new type of scalar bosons with $m=0$ and $n=e v e n$ is introduced to do the job.

Definition 14.1: Eight scalar bosons called massons labeled as $G_{i}(i=1,2,3 \cdots 8)$ are made of eight pairs of quark and the same type of anti-quark as:

$$
\begin{aligned}
& G_{1}=c_{r} \bar{c}_{r}, G_{2}=c_{g} \bar{c}_{g}, G_{3}=c_{b} \bar{c}_{b}, G_{4}=b_{r} \bar{b}_{r}, \\
& G_{5}=b_{g} \bar{b}_{g}, G_{6}=b_{b} \bar{b}_{b}, G_{7}=t_{r} \bar{t}_{r}, G_{8}=t_{g} \bar{t}_{g} \\
& G_{i}=q_{j} \bar{q}_{j}, i=1,2,3 \cdots 8, j=c_{r}, c_{g}, c_{b}, b_{r}, b_{g}, b_{b}, t_{r}, t_{g} .
\end{aligned}
$$

Explanation: According to SQS theory, quarks have counterpart bosons. As shown in the Elementary Particles Table of Table 18.2, $u_{r}$ has its counterpart $g$; $t_{b}$ has its counterpart $W, d_{r}, d_{g}, u_{g}, d_{b}, u_{b}, s_{r}, s_{g}$, $S_{b}$ have their counterparts $g_{1}, g_{2}, g_{3}, g_{4}, g_{5}, g_{6}$, $g_{7}, g_{8}$, respectively. There are eight boson vacancies left in Table 18.2. To fill these vacancies, SQS theory introduces eight neutral scalar bosons with spin 0 as the building blocks to make the link between the lepton pairs $e \oplus \bar{v}_{e}, \mu \oplus \bar{v}_{\mu}, \tau \oplus \bar{v}_{\tau}$ or $e^{+} \oplus v_{e}, \mu^{+} \oplus v_{\mu}, \tau^{+} \oplus v_{\tau}$ on one hand and $W^{ \pm}$or $Z^{0}$ on the other for weak interactions.

According to Definition 14.1:

$$
\begin{aligned}
& \bar{G}_{i}={\overline{q_{j}}}_{\bar{q}}=\bar{q}_{j} q_{j}=q_{j} \bar{q}_{j}=G_{i}, i=1,2,3 \cdots 8, \\
& j=c_{r}, c_{g}, c_{b}, b_{r}, b_{g}, b_{b}, t_{r}, t_{g} .
\end{aligned}
$$

The anti-particle of a masson is itself:

\begin{tabular}{|c|c|c|c|c|c|c|c|c|}
\hline Massoms & $G_{1}$ & Gi & $G_{2}$ & $G$, & $G_{5}$ & $Q_{4}$ & $G$ & $G_{1}$ \\
\hline Conutituenta & $c, \oplus \varepsilon$ & $c_{r} \in \varepsilon_{r}$ & $c, \oplus \bar{z}$, & $b, \ominus 5$, & $b_{n} \oplus \bar{b}_{n}$ & $\bar{\alpha} \in \overline{\hat{a}}$ & $t, \oplus ?$ & $t_{r} \in t_{,}$ \\
\hline$m$ & 0 & 0 & 0 & 0 & 0 & 0 & 0 & 0 \\
\hline$|n|$ & 38 & 58 & 74 & 82 & 94 & 118 & 85 & 106 \\
\hline$|p|$ & $2 p_{0}$ & $2 p_{4}$ & $2 p_{k i}$ & $2 p_{k r}$ & $2 p_{w}$ & $2 p_{n}$ & $2 p p_{n}$ & $2 p_{x}$ \\
\hline $\begin{array}{l}\text { ALaA } \\
\text { GeV }\end{array}$ & $\begin{array}{l}2 M_{v} \\
2.194\end{array}$ & $\begin{array}{l}2 . M_{a} \\
2.756\end{array}$ & $\begin{array}{l}2 . M_{i} \\
2.084\end{array}$ & $\begin{array}{l}2 M_{11} \\
817 n\end{array}$ & $\begin{array}{l}2 M_{44} \\
8598\end{array}$ & $\begin{array}{l}2 M_{n g} \\
8381\end{array}$ & $\begin{array}{l}2 M_{g} \\
342,38\end{array}$ & $\begin{array}{l}2 M_{*} \\
34195\end{array}$ \\
\hline Charte & 0 & 0 & 0 & 0 & 0 & 0 & 0 & 0 \\
\hline Spin & 0 & 0 & 0 & 0 & 0 & 0 & 0 & 0 \\
\hline
\end{tabular}

$$
\bar{G}_{i}=G_{i}, i=1,2,3 \cdots 8 \text {. }
$$

The parameters of eight massons are listed in Table 14.4.

Table 14.4: The Parameters of Eight Masson $G_{i}(i=1,2 \cdots 8)$

The masson model is shown in Fig. 14.1. The twisted loop model is similar to gluon model with the following differences.
1. The loop is twisted in $n$ turns without the small loops at two ends.

2. The mass term longitudinal oscillation alone the loop is the standing-wave type with its zero amplitude point located at two ends. The mass term longitudinal oscillations instantaneous values along two branch paths have $180^{\circ}$ phase shift. But the loop path at end changes to opposite direction contributing another $180^{\circ}$ phase shift. The combination of two $180^{\circ}$ phase shifts yields $360^{\circ}$ phase shift meaning in phase. This is the reason for masson having double mass of its constituent quark as listed in Table 14.4.
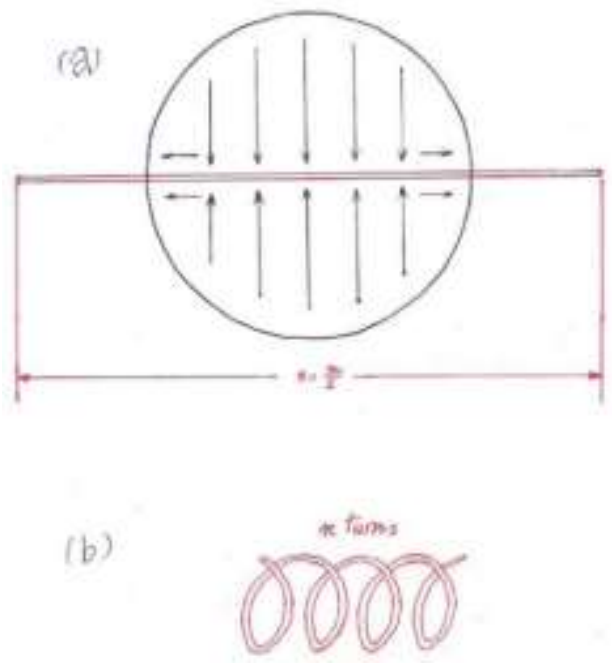

Fig. 14.1 Masson's model

The distinguish features of massons are: (1) They are neutral scalar bosons with spin $s=0$; (2) Their numerical parameters are $n / m=$ even $/ 0$; (3) They are pure mass stuff as the name implied.

According to SQS theory, there are three types of bosons involved in weak interactions, namely $W^{ \pm}$or $Z^{0}$, gluons and massons. In which, $W^{ \pm}$or $Z^{0}$ serves as the intermediate state; gluons and massons serve as the building blocks for two types of transitional links.

Take muon decay as an example to demonstrate the weak interaction between leptons. 2010-PDG data show that, muon decay mode $\mu^{-} \rightarrow e^{-}+\vec{v}_{e}+v_{\mu}$ has branching ratio

$\Gamma_{i} / \Gamma \approx 100 \%$. As shown in Section 12 and Rule 14-1, the reduced $m$-, $n$-parameters of electron, muon and associated anti-neutrinos are:

Electron $e^{-\uparrow}$ :

$$
m=1, n=1 / 2 \text {; }
$$

Electron anti-neutrino ${\overline{v_{e}}}_{e}^{\uparrow}$ :

Muon $\mu^{-\uparrow}$ : 


$$
m=1, n=1+5 / 8 ;
$$

Muon anti-neutrino $\bar{v}_{\mu}^{\uparrow}$ :

$$
m=1, n=-(1+5 / 8) .
$$

In (14.8), the arrows $\uparrow$ in particle symbols indicate these particles with right-handedness.

$W^{-}$serves as the intermediate state with reduced $m$-, $n$ parameters as:

$W^{-\uparrow}$ gauge boson:

$$
m=2, n=-14 \text {. }
$$

The "-" sign of $n$-parameter for $W^{-}$is due to the fact that $W^{-}$ is the anti-particle of $W^{+}$.

According SQS theory, $\mu^{-} \rightarrow e^{-}+\vec{v}_{e}+v_{\mu}$ decay mode includes two processes with two links: Process-1 with link-1: $\mu^{-}+\bar{v}_{\mu} \rightarrow W^{-}$; Process-2 with link-2: $W^{-} \rightarrow e^{-}+\bar{v}_{e}$. In process-1, $\bar{v}_{\mu}$ to replace $v_{\mu}$ is to represent the leptons pair of $\mu^{-} \oplus \bar{v}_{\mu}$ required by Rule 14.1. In presentation, $\bar{v}_{\mu}$ serves as an input. In reality of muon's decay, $v_{\mu}$ serves as an output. According to Feynman diagram, $\bar{v}_{\mu}$ as input and $v_{\mu}$ as output are equivalent. Look it the other way, for process-1, $\mu^{-}+\bar{v}_{\mu} \rightarrow W^{-}$takes the $\bar{v}_{\mu}$ as an input from a $v_{\mu} \& \bar{v}_{\mu}$ pair out of vacuum to avoid violation of lepton number conservation and leaves the $v_{\mu}$ as decay products. In this way, $W^{-}$boson status is justified and the lepton family number conservation law in process- 1 and process- 2 both are satisfied.

The two processes are illustrated in Table 14.5 to show

\begin{tabular}{|c|c|c|}
\hline $\begin{array}{c}\text { Process }-1 \text { ingut } \\
\mu^{*}+\bar{V}_{\alpha}\end{array}$ & $\begin{array}{c}\text { Ptocess- } 1 \text { with Link-1 } \\
\qquad \mu^{-}+\pi_{+} \rightarrow W^{-}\end{array}$ & $\begin{array}{c}\text { Intermediate State } \\
\mathbb{W}^{*}\end{array}$ \\
\hline$\frac{\frac{\pi_{-x p}}{m_{-\alpha i}}}{\frac{(1+5 / 8)+[-(1+5 / 8)]}{1+1}}=\frac{0}{2}$ & 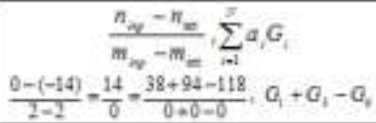 & $\begin{array}{l}\frac{n_{24}}{m_{m}} \\
\frac{-14}{2}\end{array}$ \\
\hline
\end{tabular}
the makeup of two links.

Table 14.5A: Process-1 for Muon Decay Mode $\mu^{-} \rightarrow e^{-} \vec{v}_{e} v_{\mu}$

\begin{tabular}{|c|c|c|}
\hline $\begin{array}{l}\text { Intermediate state } \\
\qquad W\end{array}$ & $\begin{array}{l}\text { Process } 2 \text { with Link-2 } \\
\qquad w^{-} \rightarrow e^{-}+v_{*}\end{array}$ & 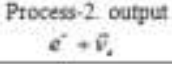 \\
\hline $\begin{array}{l}\frac{n_{m}}{m} \\
\frac{-14}{2}\end{array}$ & 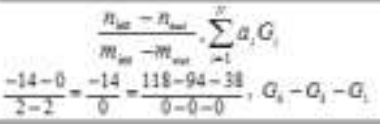 & $\frac{\frac{n_{\text {mid }}}{m_{m e}}}{\frac{1 / 2+(-1 / 2)}{1+1}}=\frac{0}{2}$ \\
\hline
\end{tabular}

Table 14.5B: Process-2 for Muon Decay Mode $\mu^{-} \rightarrow e^{-} \vec{v}_{e} v_{\mu}$

As shown in Table 14.5A and B, the weak interaction in the muon decay mode $\mu^{-} \rightarrow e^{-} \vec{v}_{e} v_{\mu}$ has two links and both links are made of three massons $G_{1}, G_{5}$ and $G_{6}$.

Take the free neutron decays to proton as an example to demonstrate the weak interaction involved baryons and leptons. According to 2010-PDG data, free neutron decay mode $n \rightarrow p+e^{-}+\bar{v}_{e}$ has branching ratio $\Gamma_{i} / \Gamma=100 \%$. The mechanism of such decay is a down quark in the free neutron transforms into an up quark changing neutron to proton plus an electron and an electron anti-neutrino: $d \rightarrow u+e^{-}+\bar{v}_{e}$. The decay mode also has two processes with two links and an intermediate state. As shown in Section 11, the $m-, n$ parameters of three up anti-quarks and three down quarks are: $\begin{aligned} \bar{u}_{r}^{\uparrow}: & m=1, n=-1 ; \bar{u}_{g}^{\uparrow}: m=7, n=-3 ; \bar{u}_{b}^{\uparrow}: m=13, \\ & n=-5 . \\ d_{r}^{\uparrow}: & m=3, n=1 ; d_{g}^{\uparrow}: m=5, n=3 ; d_{b}^{\uparrow}: m=11, \\ & n=5 .\end{aligned}$

In (14.10), $\bar{u}_{r}, \bar{u}_{g}, \bar{u}_{b}$ to replace $u_{r}, u_{g}, u_{b}$ has the same reason as $\bar{v}_{\mu}$ to replace $v_{\mu}$ in the first example.

The two processes are illustrated in Table 14.6 to show

\begin{tabular}{|c|c|c|}
\hline 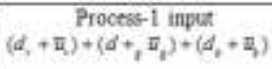 & $\begin{array}{l}\text { Process } 1 \text { with Link-1 } \\
\qquad d+\pi \rightarrow \pi^{*}\end{array}$ & $\begin{array}{l}\text { Intermediate state } \\
\qquad \pi^{-}\end{array}$ \\
\hline$\frac{\frac{\pi_{x y}}{w_{w}}}{\frac{(1-1)+(9-3)+(5-2)}{(1+3)+(6+5)+(11+13)}=\frac{9}{40}}$ & 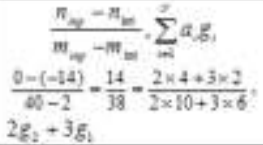 & $\begin{array}{l}\frac{m_{m}}{m_{m}} \\
\frac{-14}{2}\end{array}$ \\
\hline
\end{tabular}
the makeup of two links.

Table 14.6A: Process-1 for Down Quark Decay Mode $d \rightarrow u+e^{-}+\vec{v}_{e}$

\begin{tabular}{|c|c|c|}
\hline $\begin{array}{l}\text { Intermediate state } \\
\pi^{-}\end{array}$ & $\begin{array}{l}\text { Process-2 with Link-2 } \\
\qquad \mathbb{W}^{-} \rightarrow e^{-}-\bar{D}_{\text {. }}\end{array}$ & 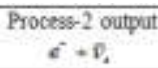 \\
\hline$\frac{n_{m}}{m_{m}}$ & $\frac{n_{m=1}-n_{m}}{m_{m=-1}-m_{\alpha}}, \sum_{=1}^{N} a, G$ & $\frac{n_{m a 1}}{m_{m}}$ \\
\hline$\frac{-14}{2}$ & $\frac{-14-0}{2-2}=\frac{-14}{0}=\frac{115-94-39}{0-6-0}, G_{2}-C_{2}-C_{2}$ & $\frac{1 / 2-1 / 2}{1+1}=\frac{0}{2}$ \\
\hline
\end{tabular}

Table 14.6B: Process-2 for Down Quark Decay Mode $d \rightarrow u+e^{-}+\vec{v}_{e}$

In this example, the process- 1 is quark and anti-quark transforms to $W^{-}$mediated by gluons. The process-2 is $W^{-}$ transforms to leptons mediated by massons.

These two examples serve as the typical cases for the regular weak interactions. The examples show that Rule 14.1 serves the purpose well. Other cases can be treated by the same way.

For the rarely occurred weak interactions, the violation of baryon number conservation and/or lepton number conservation will be treated differently.

Definition 14.2: The weak interactions are classified into two types.

Regular type: The weak interactions meet baryon number conservation and lepton number conservation including lepton family number conservation.

Rare type: The weak interactions violate baryon number conservation or lepton number conservation including lepton family number conservation.

The weak interaction mechanism proposed by SQS theory has following features.

1. It is based on mathematics. SQS theory provides a mathematic framework for weak interactions. 
2. In general, the rules introduced in this section meet the requirement of baryon number conservation. A single quark or anti-quark cannot participate in regular weak interaction, because its $n / m=o d d / o d d$. To participate in regular weak interaction, quark must pair with anti-quark to have combined parameters of $n / m=$ even / even .

3. In general, the rules introduced in this section meet the requirement for lepton number conservation including lepton family number conservation. A single lepton or anti-lepton cannot participate in the regular weak interaction, because its $n / m=$ fractional /odd . To participate, the lepton is paired with its companion antineutrino to form a pair. The pair' $s n_{p} / m_{p}=0 / 2$ along with $n_{W} / m_{W}= \pm 14 / 2$ of $W^{ \pm}$or $Z^{0}$ yield a combined $n_{\text {comb }} / m_{\text {comb }}=\left(n_{p}-n_{W}\right) /\left(m_{p}-m_{W}\right)=$ even $/ 0$, which naturally requires massons with $n / m=$ even $/ 0$ to serve as building blocks of the link between them. In this way, the lepton number and lepton family number are conserved, and the introduction of massons is justified.

4. For SQS theory, the conservation laws for baryon number and for lepton number including lepton family number are required by mathematics represented by Rule 14.1.

5. The rules introduced in this section are also applicable to some rare events. For instance, according to 2010-PDG data, the meson $\pi^{0}$ has two rare decay channels of $e^{+} e^{+} e^{-} e^{-}$and $e^{+} e^{-}$with $\Gamma_{i} / \Gamma=(3.34 \pm 0.16) \times 10^{-5}$ and $\Gamma_{i} / \Gamma=(6.46 \pm 0.33) \times 10^{-8}$, respectively. For these two decay channels, there is no anti-neutrino involved but lepton numbers including lepton family number are still conserved. Because $\pi^{0}$ is made of $\pi^{0}=(u \bar{u}-d \bar{d}) / \sqrt{2}$, the explanation for its quark and anti-quark part is similar to that of free neutron decay case. For the leptons part, the $n / m$ ratio of $e^{+}$is $n / m=(-1 / 2) / 1$, which is the same as $\bar{v}_{e}$. The explanation for the leptons part is the same as two previous examples. But $e^{+}$is not $\bar{v}_{e}$, Rule 14.1 is violated and the decay mode belongs to rare type.

6. It also provides possible mechanisms for the rare weak interaction events which violate lepton family number conservation. For instance, according to 2010-PDG data, $\pi^{0}$ has three other very rare decay channels: channel-A, $\pi^{0} \rightarrow \mu^{+}+e^{-}$with $\Gamma_{i} / \Gamma=3.8 \times 10^{-10} ;$ channel-B, $\pi^{0} \rightarrow \mu^{-}+e^{+}$with $\Gamma_{i} / \Gamma=3.4 \times 10^{-9}$; channel-C, $\pi^{0} \rightarrow \mu^{+} e^{-}+\mu^{-} e^{+}$with $\Gamma_{i} / \Gamma=3.6 \times 10^{-10}$. Let's take a look at channel-A. the $m$-parameters and $n$-parameters involved are:

For $\mu^{+\downarrow}: m_{\mu+}=-1, n_{\mu+}=1+5 / 8=13 / 8, \frac{n_{\mu+}}{m_{\mu+}}=\frac{13 / 8}{-1}$;

$$
\begin{aligned}
& \text { For } e^{-\uparrow}: m_{e}=1, n_{e}=1 / 2, \frac{n_{e}}{m_{e}}=\frac{1 / 2}{1} ; \\
& \text { For } Z^{0^{\uparrow}}: m_{Z 0}=2, n_{Z 0}=14 ; \frac{n_{Z 0}}{m_{Z 0}}=\frac{14}{2} \text {. }
\end{aligned}
$$

In this case, the problem is that, the $n / m$ values of (14.12) do not match as the way $e^{-}+\vec{v}_{e}$ did in Table 14.5B. To solve the problem, let's multiply numerator and denominator of $n / m$ with 8 and 10 for $\mu^{+}$and $e^{-}$, respectively.

$$
\begin{aligned}
& \text { For } \mu^{+\downarrow}: \quad \frac{n_{\mu+}^{\prime}}{m_{\mu+}^{\prime}}=\frac{n_{\mu+} \times 8}{m_{\mu+} \times 8}=\frac{13}{-8}, \\
& \text { For } e^{-\uparrow}: \quad \frac{n_{e}^{\prime}}{m_{e}^{\prime}}=\frac{n_{e} \times 10}{m_{e} \times 10}=\frac{5}{10}, \\
& \text { For } \mu^{+\downarrow}+e^{-\uparrow}: \frac{n_{\mu+}^{\prime}+n_{e}^{\prime}}{m_{\mu+}^{\prime}+m_{e}^{\prime}}=\frac{13+5}{-8+10}=\frac{18}{2} .
\end{aligned}
$$

The process- 2 with link- 2 can be carried out the same way as previous examples.

$$
\begin{aligned}
& \text { For } Z^{0} \rightarrow \mu^{+\downarrow}+e^{-\uparrow}: \\
& \qquad \frac{n_{z 0}-\left(n^{\prime}{ }_{\mu+}+n_{e}^{\prime}\right)}{m_{Z 0}-\left(m^{\prime}{ }_{\mu+}+m_{e}^{\prime}\right)}=\frac{14-18}{2-2}=\frac{-4}{0} .
\end{aligned}
$$

The link of (14.14) can be made of $3 G_{2}-G_{6}$ :

$$
\frac{-4}{0}=\frac{3 \times 38-118}{3 \times 0-0} \text {. }
$$

The same approach is applicable to channel-B and channelC. Is multiplication of same number to numerator and denominator of $n / m$ legitimate? From mathematic viewpoint, the answer is: Yes, of cause. If the reduction for the original $n / m$ is legitimate, so is the multiplication. From physics viewpoint, the multiplication of an integer $N$ to numerator and denominator of $n / m$ means that, the cyclic movements in loop- 1 and loop- 2 both take $N$ cycles instead of 1 cycle. For instance, in the case of (14.13), the process occurred at the moment that, $\mu^{\downarrow \downarrow}$ takes 8 cycles and $e^{-\uparrow}$ takes 10 cycles. The probability for such events occurred simultaneously determines the $\Gamma_{i} / \Gamma$ value for that decay channel, which explains the rarity of such decay channels.

7. The extremely rare "sphaleron" phenomenon [13] converts three baryons into three leptons which violate both baryon number conservation and lepton number conservation. Three baryons contain nine quarks. The combination has $n / m=o d d / o d d$, which serves as the mathematical origin for violating baryon number conservation. Three leptons combination has $n / m=$ fraction / odd, which serves as the mathematical origin for violating lepton number conservation. The regular rules introduced in this section are not valid. But as long as multiplication can apply to $n / m$, the "sphaleron" phenomenon can be interpreted mathematically. In fact, almost any weak interaction rare 


\section{Z. Y. SHEN}

event can be interpreted mathematically. The real difference is the probability of its occurrence.

These features indicate that, the weak interaction rules have the capability and potential to explain weak interactions in terms of mathematics. But there are some questions.

1. Is it possible that gluons and massons bypassing $W^{ \pm}$and $Z^{0}$ directly link quark-antiquark pair to lepton pairs $e \oplus \bar{v}_{e}, \mu \oplus \bar{v}_{\mu}$ or $\tau \oplus \bar{v}_{\tau}$ ?

2. Massons are scalar bosons. Are they qualified to serve as mediators for weak interactions?

3. Why gluons and massons are different? After all, both are quark anti-quark pairs and located in the same row in the Elementary Particles Table of Table 18.2.

Question-1 and Question-2 are correlated. If massons must attach to $W^{ \pm}$and $Z^{0}$, it answers both questions. In fact, there are clues for massons attachment to $W^{ \pm}$and $Z^{0}$. If the $S U$ (2) group symmetry associated with electroweak interaction is perfect, $W^{ \pm}$and $Z^{0}$ have no mass. The $S U(2)$ symmetry must be broken for $W^{ \pm}$and $Z^{0}$ to gain mass. Massons may play a role to provide mass for breaking the symmetry.

Regarding Question-3, the spin and mass differences of gluon and masson are originated from their models as explained previously.

Superficially, the introduction of eight massons seems to make theory complicated. In fact, it is just the opposite. The way SQS theory treated strong interaction and weak interaction is to reveal their mathematic nature in the simplest possible way. Gluons and massons both are made of quark and anti-quark pairs. This approach greatly simplified the theory. From SQS theory viewpoint, quarks and anti-quarks serve as the basic elements. Gluons and massons are composed with the basic elements in different ways to serve their specific purposes. Moreover, the mathematic framework of weak and strong interactions are self-consistent without artificial additions. The whole approach shows simplicity and elegance.

In Section 18, a new gauge boson will be introduced. It also plays some role for the weak interactions involved hadrons decay.

In this section, a framework of weak interactions is introduced. It has the capability and potential to explain weak interactions including rear events based on mathematics. But it only provides a framework; some details need to be finalized. For instance, the different ways $Y_{1}$ and $Y_{2}$ are combined to make $W^{ \pm}$and $Z^{0}$ with different masses, which need more works to nail down the details.

\section{Section 15: Unified Interactions}

Traditionally Grand Unification Theory (GUT) is to unify electromagnetic, weak, strong interactions, in which gravity is not included. For SQS theory, GUT means unification of all four interactions including gravity. The unification of interactions takes consecutive stages. Starts from electromagnetic interaction, weak interaction joints in, then strong interaction joints in, finally all interactions are unified with gravity. The GUT provided by SQS theory is based on mathematics.

In $E D S_{\dot{x}}(x)$ of (9.3), the second summation term not included in the original $D S$-function of (6.1) represents interactions mediated by bosons, which are originated from Fourier transformation of the added $\delta$-function terms in $E D S_{k}(k)$ of (9.1). For convenience, $E D S_{\dot{x}}(x)$ is divided into four summation terms labeled as $A, B, C, D$ :

$$
\begin{aligned}
& \operatorname{EDS}_{\dot{x}}(x)=\frac{1}{2} \sum_{j=-\infty}^{\infty} e^{-\pi(j-x)^{2}}+\frac{1}{2} \sum_{j=-\infty}^{\infty} e^{-\pi[j-(0.5-x)]^{2}} \\
& -\sum_{j=-\infty}^{\infty} e^{-4 \pi j^{2}} e^{-i 4 j \pi x}-\sum_{j=-\infty}^{\infty} e^{-4 \pi(j-0.5)^{2}} e^{-i 4(j-0.5) \pi x} \equiv A+B-C-D \\
& A=\frac{1}{2} \sum_{j=-\infty}^{\infty} e^{-\pi(j-x)^{2}}, B=\frac{1}{2} \sum_{j=-\infty}^{\infty} e^{-\pi[j-(0.5-x)]^{2}} \\
& C=\sum_{j=-\infty}^{\infty} e^{-4 \pi j^{2}} e^{-i 4 j \pi x}, D=\sum_{j=-\infty}^{\infty} e^{-4 \pi(j-0.5)^{2}} e^{-i 4(j-0.5) \pi x}
\end{aligned}
$$

Term- $A$ and term- $B$ represent fermions, while term- $C$ and term- $D$ represent bosons for interactions.

According to their variables $j$ and $(0.5-j)$ versus $x$ and $(0.5-x)$, term- $C$ and term- $D$ are related to term- $A$ and term- $B$, respectively. If the term- $A$ variable $x$ is in the $(0<x<0.25)$ range with $\pi(x)>\pi$; then the term- $B$ variable $(0.5-x)$ is in the $(0.25<(0.5-x)<0.5)$ range with $\pi(0.5-x)<\pi$. For the torus model, $\pi(x)>\pi$ and $\pi(0.5-x)<\pi$ correspond to its inner half and outer half, respectively. It implies that, term- $B$ and term- $D$ corresponding to torus outer half are related to the GWStriangle representing electroweak interaction. It is natural to assume term- $D$ related to electroweak interaction. With the same reason, term- $A$ and term- $C$ corresponding to torus inner half is related to the $C K M$-triangle representing hadrons decay. It is natural to assume term- $C$ related to electroweak and strong interactions. Let's take this argument as preassumption and verify it by its results later.

In the $S U(5)$ Grand Unified Theory [14, 15], a suppression factor $S F$ is introduced:

$$
S F=\left[\frac{M_{\text {proton }}}{M_{G U T}}\right]^{4} \text {. }
$$

$M_{\text {proton }}=0.938272013(23) \mathrm{GeV} / \mathrm{c}^{2}$ is the proton mass; and $M_{G U T} \sim 10^{15} \mathrm{GeV} / \mathrm{c}^{2}$ is the $S U(5)$ GUT mass scale for the unification of three interactions except gravitation. In a previous paper [16], the author borrowed this concept and utilized (15.2) to calculate the mass scale $M_{e w}$ for the unification of electromagnetic and weak interactions: 


$$
M_{e w}=\frac{M_{\text {proton }}}{\sqrt[4]{S F_{e w}}} .
$$

In which, $S F_{e w}$ is the redefined suppression factor for electroweak unification derived from the dominate term of term- $D$ in (15.1), in which $x$ is replaced by $x_{b}$ and $\pi$ is replaced by $\pi\left(x_{b}\right)$ :

$$
\begin{aligned}
& S F_{e w}=\left|e^{-4 \pi\left(x_{b}\right)(j-0.5)^{2}} e^{-i 4(j-0.5) \pi\left(x_{b}\right) x_{b}}\right|_{j=0 o r 1}=e^{-\pi\left(x_{b}\right)} . \\
& =e^{-20.37019456}=1.4234326 \times 10^{-9}
\end{aligned}
$$

The term with $j=0$ or 1 represents the dominate term, which has the maximum magnitude value in term- $D$. It dominates the suppression effect, because other terms with $|j|>1$ have much less value comparing to $j=0$ or 1 term.

In (15.4), the value of $x_{b}=1.1821861791847719 \times 10^{-5}$ is defined by (2.22), the value of $\pi\left(x_{b}\right)=20.37019456$ is cited from Table 3.1 , in which only 8 digits after the decimal point are taken.

Substituting (15.4) into (15.3) yields the mass of a scalar boson $U_{1}$ to unify electromagnetic and weak interactions:

$$
M_{e w}=\frac{M_{\text {proton }}}{\sqrt[4]{S F_{\text {ew }}}}=\frac{0.938272013}{\sqrt[4]{1.4234326 \times 10^{-9}}}=152.75469 \mathrm{GeV} / \mathrm{c}^{2} \text {. }
$$

$M_{e w}=152.75469 \mathrm{GeV} / \mathrm{c}^{2}$ is within LHC capability, it can be verified experimentally.

Corresponding energy $E_{U 1}=M_{e w} c^{2}=152.75469 \mathrm{GeV}$ of the scalar boson $U_{1}$ serves as the energy scale to unify electromagnetic and weak interactions.

The suppression factor $S F_{\text {ews }}$ for electroweak-strong unification is derived from the term- $C$ of (15.1). It is evaluated at $x_{a}=5.181994687988211 \times 10^{-10}$ of (2.21) and $\pi\left(x_{a}\right)=36.73631245$ cited from Table 3.1 with 8 digits after decimal point:

$S F_{\text {ews }}=\left|e^{-4 \pi\left(x_{a}\right) j^{2}} e^{-i 4 j \pi\left(x_{a}\right) x_{a}}\right|_{|j|=1}=e^{-4 \pi\left(x_{a}\right)}=e^{-4 \times 36.73631245}=1.52226012 \times 10^{-64}$

In (15.6), the $|j|=1$ term is taken as the dominate term instead of the $j=0$ term, because $j=0$ term represents the summation of all probabilities equal to 1 required by unitarity.

Using the value of $S F_{\text {ews }}$ giving by (15.6), the mass $M_{\text {ews }}$ of a scalar boson $U_{2}$ to unify electroweak and strong interactions is determined as:

$M_{\text {ews }}=\frac{M_{\text {proton }}}{\sqrt[4]{S F_{\text {ews }}}}=\frac{M_{\text {proton }}}{e^{-\pi\left(x_{a}\right)}}=\frac{0.938272013}{1.11076508 \times 10^{-16}}=8.44708 \times 10^{15} \mathrm{GeV} / \mathrm{c}^{2}$.

In the standard model, the electroweak-strong unification is called the grand unification. In SQS theory, the grand unification is reserved for the one including gravity.

Before dealing with the grand unification, let's look at the principles suggested by SQS theory.
1. There are two types of forces (force is synonymous to interaction). The long range force including electromagnetic force and gravitational force, both have unlimited effective range. The short range force including weak force and strong force has limited effective ranges. The first principle for grand unification is: All forces must be unified to a single force of the long range type. The reason is simple, long range includes short range, while short range does not include long range. According to the first principle, the weak force and strong force are not qualified as the final unified force.

2. Second principle: The force with selectivity is not qualified as the final grand unification force. Electromagnetic force is only for charged particles. It is not qualified as the final unified force. Otherwise, the forces between electrically neutral particles are left out after the grand unification.

3. According to the first and second principles, the only force qualified as the final unified force is the gravity.

The next question is: In the grand unification, which force is one finally unified with gravity? The answer comes from the Random Walk Theorem. In Section 4, the ratio of electrostatic force to gravity for a pair of electrons is

$$
R_{E / G}=\frac{f_{E}}{f_{G}}=\frac{\alpha}{4 \pi^{2}} N_{e}^{2} .
$$

In which, electron converting factor $N_{e}$ is interpreted as the ratio of long path to short path defined according to the Random Walk Theorem. It is natural to start with $R_{E / G}$ to explore grand unification. According to SQS theory, grand unification happens at:

$$
R_{E / G}=f_{E} / f_{G}=1 \text {. }
$$

Substituting (15.8) into (4.19) yields:

$$
N_{e}=\frac{2 \pi}{\sqrt{\alpha}} .
$$

In (15.9), the fine structure constant $\alpha$ as a running constant varies with energy. The closest value available at such high energy level from 2010-PDG $(p .126)$ is:

$$
\alpha\left(M_{Z}\right)=1 /(127.916 \pm 0.015) \text {. }
$$

$\alpha\left(M_{Z}\right)$ is the value of $\alpha$ at $Q^{2} \approx M_{Z}^{2}$ around $91 \mathrm{GeV}$.

Substituting (15.10) as $\alpha$ into (15.9) yields the converting factor at grand unification scale:

$$
N_{G U T}=\frac{M_{\text {Planck }}}{M_{G U T}} \approx \frac{2 \pi}{\sqrt{\alpha\left(M_{Z}\right)}}=71.06279805 .
$$

Corresponding length is the grand unification length scale: $L_{G U T}=N_{G U T} L_{P}=71.06279805 \times 1.61625 \times 10^{-35}=1.148552 \times 10^{-33} \mathrm{~m}$.

The mass of the scalar boson $U_{3}$ for grand unification is:

$$
\begin{aligned}
& M_{\text {GUT }}=\frac{M_{\text {Planck }}}{N_{G U T}}=\frac{\sqrt{\alpha\left(M_{Z}\right)}}{2 \pi} M_{\text {Planck }}=\sqrt{\frac{\alpha\left(M_{Z}\right) h c}{2 \pi G}} . \\
& =1.9243515 \times 10^{-9} \mathrm{Kg}=1.07948213 \times 10^{18} \mathrm{GeV} / \mathrm{c}^{2}
\end{aligned}
$$


For comparison purpose, the suppression factor $S F_{G U T}$ and $\pi\left(x_{G U T}\right)$ for grand unification are reversely calculated from $M_{G U T}$ as:

$S F_{G U T}=\left[\frac{M_{p}}{M_{G U T}}\right]^{4}=\left[\frac{0.938272013}{1.07948213 \times 10^{18}}\right]^{4}=5.70759593 \times 10^{-73}$,

$\pi\left(x_{G U T}\right)=-\frac{\ln \left(S F_{G U T}\right)}{4}=41.58672847$.

Table 15.1 listed the calculated parameters for three types of unifications proposed by SQS theory.

Table 15.1: The Parameters for Three Types of Unifications

\begin{tabular}{|c|c|c|c|c|}
\hline $\begin{array}{l}\text { Unification } \\
\text { Type }\end{array}$ & $\pi(x)$ & $S F$ & $\begin{array}{c}\text { Length Scale } \\
\text { (viv) }\end{array}$ & $\begin{array}{c}\text { Mass } \\
\text { (GeV/cices })\end{array}$ \\
\hline Electro-Weak & $\pi\left(x_{7}\right)=20.37019456$ & $1.42343 \times 10^{-4}$ & $8.11656 \times 10^{-71}$ & 152.7347 \\
\hline EW-Strong & $\pi\left(x_{4}\right)=36.73631245$ & $1.52226 \times 10^{-5}$ & $1.46778 \times 10^{-3 i}$ & $8.44708 \times 10^{15}$ \\
\hline Grand & $\pi\left(x_{6<7}\right)=41.58672847$ & $5.7076 \times 10^{-7}$ & $1.14855 \times 10^{-38}$ & $1.07948 \times 10^{14}$ \\
\hline
\end{tabular}

It is interesting to find out that, the mass ratio of $M_{G U T}$ to $M_{e w s}$ is very close to $\alpha\left(M_{Z}\right)^{-1}$ :

$$
\frac{M_{\text {GUT }}}{M_{\text {ews }}}=127.79353 \approx \alpha\left(M_{Z}\right)^{-1} .
$$

The number 127.79353 is so close to the medium value of $\alpha\left(M_{Z}\right)^{-1}=127.916$ cited from 2010-PDG (P.126) data with a relative deviation of $9.574 \times 10^{-4}$. Another way to check is to combine (15.13) and (5.15):

$$
\alpha\left(M_{Z}\right)^{-1}=\left[\frac{M_{\text {Planck }}}{2 \pi M_{\text {ews }}}\right]^{2 / 3}=127.83433738 .
$$

The relative deviation is reduced to $6.384 \times 10^{-4}$. With such high accuracy, it is very unlikely that (15.15) and (15.16) are by coincidence. In other words, these correlations are real and mean something worthwhile to dig in.

From theoretical perspective, (15.15) is an important finding, which has the following significances.

1. The parameters of grand unification and electroweakstrong unification are correlated with the fine structure constant $\alpha(M) . \alpha(M)$ as a running constant, its reversed value varies from $\alpha\left(M_{e}\right)^{-1}=137.035999084$ to $\alpha\left(M_{Z}\right)^{-1}=127.916$, a decrease of $6.655 \%$ for $\sim 10^{5}$ increase of energy scale. In the next $10^{16}$ increase of energy scale to $10^{18} \mathrm{GeV}$, the value of $\alpha(M)^{-1}$ is only decreased $6.384 \times 10^{-4}$. It shows a typical asymptotic behavior toward saturation: $\alpha\left(M>M_{Z}\right) \rightarrow \alpha\left(M_{G U T}\right)$. In other words, the majority of $6.384 \times 10^{-4}$ relative deviation is not necessarily caused by error.

2. Combining (15.15) and (15.16) and replacing $\alpha\left(M_{Z}\right)$ with $\alpha\left(M_{G U T}\right)$ yields the correlation of three masses:

$$
M_{e w s}=\alpha\left(M_{G U T}\right) M_{\text {Grand }}=\frac{\alpha^{3 / 2}\left(M_{G U T}\right)}{2 \pi} M_{\text {Planck }} .
$$

Due to the asymptotic nature of fine structure constant, the replacement of $\alpha\left(M_{Z}\right)$ with $\alpha\left(M_{G U T}\right)$ in (15.17) only has a minor effect.

3. More importantly, the finding proves an important evidence for the consistency of two very different methods used to deal with unifications for SQS theory. $M_{e w s}$ is calculated according to the suppression factor from (15.6) and formula (15.7) as a borrowed formula, $\pi\left(x_{a}\right)$ is originated from the $S$-equation. On the other hand, $M_{G U T}$ is determined by the equality of static electrical force and gravity along with the converting factor $N_{G U T}$ originated from Random Walk Theorem.

These two very different methods are consistent with a discrepancy less than $6.384 \times 10^{-4}$. It gives the legitimacy for both methods. After all, the borrowed formulas (15.3), (15.5) and (15.7) are legitimate; and the ways to determine suppression factors by $\pi\left(x_{a}\right)$ and $\pi\left(x_{b}\right)$ are legitimate as well. It gives more credit to the theoretical results listed in Table 15.1.

4. It proves a way to convert proton mass $M_{\text {proton }}$ and converting factor $N_{\text {proton }}$ to the Planck mass $M_{\text {Planck }}$. Substituting $M_{e w s}$ of (15.7) into (15.17) yields:

$$
\begin{aligned}
& M_{\text {proton }}=\frac{1}{2 \pi} \alpha\left(M_{G U T}\right)^{3 / 2} e^{-\pi\left(x_{a}\right)} M_{\text {Planck }}, \\
& N_{\text {proton }}=\frac{M_{\text {Planck }}}{M_{\text {proton }}}=\frac{2 \pi}{\alpha\left(M_{G U T}\right)^{3 / 2} e^{-\pi\left(x_{a}\right)}} .
\end{aligned}
$$

Proton is not an elementary particle. It composed of three quarks and many gluons. For such a complex system, its mass and converting factor can be derived from three mathematic constants, $\pi, e, x_{a}$ and a running constant $\alpha\left(M_{G U T}\right)$ by (15.18). It is a surprise. This correlation is important in two senses. First, SQS theory is based on three physics constants, $h, c, G$ and in principle no other physics inputs. The $M_{\text {proton }}$ in (15.3), (15.5), (15.7) is exceptional. With the help of (15.18a), $M_{\text {proton }}$ is replaced by $M_{\text {Planck }}$, proton mass is no longer a physics input for SQS theory. Second, most formulas and equations in this paper can be traced directly or indirectly back to the first principle of SQS theory. (15.3), (15.5), (15.7) are exceptions, which are borrowed from other theory. With the help of (15.18), the problem is solved. For instance, $M_{e w s}$ and $M_{e w}$ are expressed as:

$$
M_{\text {evs }}=\alpha\left(M_{G U T}\right) M_{G U T}=\frac{\alpha\left(M_{G U T}\right)^{3 / 2}}{2 \pi} M_{\text {Planck }}=\alpha\left(M_{G U T}\right)^{3 / 2} \sqrt{\frac{2 \pi h c}{G}} .
$$




$$
M_{e w}=\frac{\alpha\left(M_{G U T}\right)}{e^{\left[\pi\left(x_{a}\right)-\pi\left(x_{b}\right) / 4\right]}} M_{G U T}=\frac{\alpha\left(M_{G U T}\right)^{3 / 2}}{2 \pi e^{\left[\pi\left(x_{a}\right)-\pi\left(x_{b}\right) / 4\right]}} M_{\text {Planck }}=\frac{\alpha\left(M_{G U T}\right)^{3 / 2}}{e^{\left[\pi\left(x_{a}\right)-\pi\left(x_{b}\right) / 4\right]}} \sqrt{\frac{2 \pi h c}{G}} .
$$

Only mathematic constants and basic physics constants appear in (15.19), (15.20).

The value of converting factor $N_{G U T}$ given by (15.11) is twenty one orders of magnitudes less than electron converting factor:

$$
N_{e}=\lambda_{e} / L_{P}=1.501197 \times 10^{23} \text {. }
$$

The tremendous reduction of converting factor is the nature of random walk. As shown in Section 4, when the distance $l$ of two electrons is equal or greater than its Compton wavelength $\lambda_{e C}$, its converting factor is fixed at $N_{e}=\lambda_{e} / L_{P}=1.501197 \times 10^{23}$, which is represented by a flat straight line on the $N_{e}(l)$ versus $l$ diagram shown in Fig. 4.1. This is the macroscopic scenario. When the distance of two electrons is reduce to $l<\lambda_{e C}$, the converting factor $N_{e}(l)$ starts to vary. According to Random Walk Theorem, the number of steps along the random walk path $n_{r w}$ is

$$
n_{r w}=n_{s t}^{2} \text {. }
$$

$n_{s t}$ is the number of steps along the straight line distance between two electrons. The ratio of random path length and straight line distance is:

$$
R_{r w / s t}=n_{r w} / n_{s t}=n_{s t} .
$$

$R_{r w / s t}$ is linearly decreases with decreasing $n_{s t}$. In this region, $N_{e}(l)$ has a linear relation with $l$, which is represented by a straight line with $45^{\circ}$ angle to the $l$-axis in $N_{e}(l)$ versus $l$ diagram shown in Fig.4.1. The line stops at the grand unification length scale given by (15.12):

$L_{G U T}=N_{G U T} L_{P}=1.14855 \times 10^{-33} \mathrm{~m}$. This process has its deeper meanings, which will be discussed in Section 16.

As a summary of this section, let's look at the process of unifications.

1. The electrical force and magnetic force are unified by Maxwell equations with no specific length scale.

2. At the length scale $L_{e w}=8.11656 \times 10^{-18} \mathrm{~m}$, weak force joints with electromagnetic force to unify as electroweak force.

3. At length scale $L_{\text {ews }}=1.46778 \times 10^{-31} \mathrm{~m}$, strong force joints with electroweak force to unify as electroweakstrong force.

4. Finally, at length scale $L_{G U T}=1.14855 \times 10^{-33} \mathrm{~m}$,

electroweak-strong force is unified with gravity, and all four forces become one.

In the consecutive stages of the unifications process, the electromagnetic force, acting as the carrier, picks up other forces at different length scales and carries them to the final stage. At the final stage, all forces are united to gravity. This process follows the principles described at the beginning of this section.

The electromagnetic force acting as the carrier has a deep reason. It is the force has direct connection with gravity via the random walk process. This is another example to show the importance of the Random Walk Theorem and its origin, the SQS theory Fundamental Postulation of Gaussian Probability.

In some other source [17], the fine structure constant at $M_{Z}$ has a value $\alpha^{-1}\left(M_{Z}\right)=128.957 \pm 0.020$ different from PDG-2010 $\alpha\left(M_{Z}\right)^{-1}=127.916 \pm 0.015$. The discrepancy indicates a different asymptotic path from $\alpha\left(M_{Z}\right)^{-1}$ towards $\alpha\left(M_{G U T}\right)^{-1}$. It does not change the conclusion of the intrinsic link between $M_{e w s}$ and $M_{G U T}$.

In Section 22, the unification length scales $L_{e w s}$ and $L_{G U T}$ serving as milestones play important roles in cosmic history.

In Section 23, a universal formula for the fine structure constant $\alpha(M)$ will be given.

\section{Section 16: Logistic Equation and Grand Numbers}

In this section, an equation is discovered by 16-digit numerical calculation. It reveals the connections among logistic recurrence process, converting factor, Gaussian probability, random walk, $S$-equation and grand number phenomena. It also provides important clues related to vacuum structure, cosmic history and finite sporadic Lei group.

In Section 15, the converting factor of the scalar boson $U_{3}$ representing the grand unification is:

$$
N_{\text {GUT }} \approx 2 \pi \sqrt{\alpha^{-1}\left(M_{Z}\right)}=71.06279805 \text {. }
$$

An equation is discovered by using 16-digit numerical calculation, which provides connection of the electron converting $N_{e}$ to other constants:

$\sqrt{2} N_{e} e^{-\pi\left(x_{1}\right) r^{2}}-2 \pi \sqrt{\alpha^{-1}\left(M_{e}\right)}=4.2632564145606 \times 10^{-14} \approx 0$.

Equation (16.1) is not derived from the first principle. It is necessary to provide information in details. On (16.1) right side, the number $4.2632564145606 \times 10^{-14}$ is restricted by the resolution of 16-digit numerical calculation. It actually equals to zero. The values of two terms on left side of (16.1) are:

$$
\begin{aligned}
& \sqrt{2} N_{e} e^{-\pi\left(x_{1}\right) r^{2}}=73.5524601809382, \\
& 2 \pi \sqrt{\alpha^{-1}\left(M_{e}\right)}=73.5524601809382 .
\end{aligned}
$$

It proves the right side of (16.1) actually equal to zero, which serves as one of evidences that equation (16.1) is not by coincidence. 


\section{Z. Y. SHEN}

In (16.1), the following constants are cited from the medium value of 2010-PDG data, or derived from the $S$ equation.

Electron converting factor: $N_{e}=M_{\text {Planck }} / M_{e}$,

Planck mass: $M_{\text {Plank }}=\frac{h}{L_{P} c}$,

Planck length: $L_{P}=1.61625 \times 10^{-35} \mathrm{~m}$,

Electron mass from 2010-PGD data:

$$
M_{e}=9.10938215 \times 10^{-31} \mathrm{~kg}
$$

Planck constant from 2010-PDG data:

$$
h=6.62606896 \times 10^{-34} \mathrm{Js} \text {, }
$$

Speed of light in vacuum from 2010-PDG data:

$$
c=2.99792458 \times 10^{8} \mathrm{~m} / \mathrm{s},
$$

From 2010-PDG data (p.126):

$$
\alpha^{-1}\left(M_{e}\right)=137.035999084,
$$

From $S$-equation solution for electron:

$$
\pi\left(x_{1}\right)=\pi(0.125)=3.877102924420037 \text {. }
$$

In Appendix 5, a brief introduction of logistic equation is presented. $r$ is a parameter of the logistic equation:

$$
x_{i+1}=r x_{i}\left(1-x_{i}\right), i=0,1,2,3 \cdots \infty .
$$

\begin{tabular}{|c|c|c|}
\hline The rangt of ? & The behaviar of $x$ & Notes: \\
\hline $0<1 \leq 1$ & Monotonocaly couvergen to zeso & Process starts \\
\hline $1<r \leq 2$ & 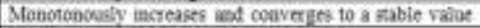 & \\
\hline $2 \times 753$ & Oscillates ad fuafly coeverges not $-1 / r$ & \\
\hline $3<x \leq 1+\sqrt{6}$ & Oscillites between 2 tumbers & \\
\hline $1+\sqrt{5}<r<r_{4}$ & Oscillates betainen $\left.2^{*}(1)=2,4 \cdots\right)$ mumbers & \\
\hline $72 x_{1}-3.5699457$ & Become fractal and chaobic & Process siops \\
\hline
\end{tabular}

Logistic equation represents a recurrence process with close connection to chaos theory. $x_{i}$ as a function of $r$ demonstrates different behaviors in different regions of $r$ shown in Table 16.1.

Table 16.1: The Typical Behaviors of $x_{i}$ in Different Ranges of

In (16.1), the logistic parameter is:

$$
\begin{aligned}
& r=r_{c}+\Delta r=3.5700363330324444 . \\
& r_{c}=3.5699457, \\
& \Delta r=9.06330324 \times 10^{-5} .
\end{aligned}
$$

$r_{c}$ is the threshold of parameter $r$. When $r \geq r_{c}$, the logistic recurrence process becomes chaotic.

In the equation (16.1), as shown by (16.5), the difference between parameter $r$ and $r_{c}$ is $\Delta r=9.06330327 \times 10^{-5}$. The relative deviation is:

$$
\Delta r / r_{c}=2.539 \times 10^{-5} .
$$

The value of $r$ just a litter bit more than the threshold of $r_{c}=3.5699457$. At that point and beyond, the logistic recurrent process becomes chaotic.

It is important to point out that, $\Delta r / r_{c}=2.539 \times 10^{-5}$ does not all contribute to the error of (6.1). In fact, $\Delta r$ could be interpreted as the logistic recurrent process going into chaotic region a coup of more steps. The error of (16.1) is less than $\Delta r / r_{c}=2.536 \times 10^{-5}$. With such high accuracy, equation (16.1) cannot be by coincidence. We should take it seriously and dig in deeply.

The second term on the left side of (16.1) is:

$$
2 \pi \sqrt{\alpha^{-1}\left(M_{e}\right)}=73.5524601809382 \text {. }
$$

Obviously, (16.2b) is the electron version of (15.11) with the $\alpha\left(M_{Z}\right)$ replaced by $\alpha\left(M_{e}\right)$ at electron mass scale as it should be.

Let's look at the logistic equation (16.1) as a progressive process with $r$ as a variable. To show $N_{e}(r)$ as a function of $r$. Rewrite (16.1) as:

$$
\sqrt{2} N_{e}(r) e^{-\pi\left(r_{1}\right) r^{2}}-2 \pi \sqrt{\alpha^{-1}(M)}=0 .
$$

In (16.7), $\alpha^{-1}(M)$ is a running constant. The problem is that $\alpha^{-1}(M)$ value varies with mass scale $M$. The experimental data of $\alpha^{-1}(M)$ are available only at a couple of discrete points. 2010-PDG ( $p .126)$ data provides:

$$
\alpha^{-1}\left(M_{e}\right)=137.035999084 \text {. }
$$

\begin{tabular}{|c|c|c|c|}
\hline 7 & $N,(r)$ & Length scaile ( $m$ ) & Noter \\
\hline 0.29898133 & 71.06279905 & $1.14855247 \times 10^{-417}$ & $\begin{array}{l}\text { Logiste recurreat peocess stat: } \\
\text { Graed mification }\end{array}$ \\
\hline 10 & $2.42622856 \times 10^{3}$ & $3.92139192 \times 10^{-11}$ & \\
\hline 1.15776991 & $9.08136684 \times 10^{3}$ & $1.46777592 \times 10^{-14}$ & Eloctro-weak-itcroeg unificatwo \\
\hline 28 & $2.73114605 \times 10^{1}$ & $4.41421485 \times 10^{-2}$ & \\
\hline 2.42319552 & $\sqrt{\overline{\mathrm{N}}}=3.874529 \times 10^{\mathrm{L}}$ & $6.26220719 \times 10^{-3}$ & $\begin{array}{l}\text { The eboese state correlated to } \\
\text { cosmic inflacion }\end{array}$ \\
\hline 3.0 & $7.16748773 \times 10^{11}$ & $1.1584452 \times 10^{-19}$ & \\
\hline 3.08255324 & $5,02184411 \times 10^{\text {I }}$ & $8.11655554 \times 10^{-41}$ & Electro-weak unification \\
\hline 357003633 & $\begin{array}{l}N_{0}=1.50119735 \times 10^{37} \\
\text { Fived value of } N_{s}\end{array}$ & $\begin{array}{l}2.426301022 \times 10^{-4 t} \\
\text { Electron Cocmpton } \\
\text { wrvelength }\end{array}$ & $\begin{array}{l}\text { Logistic tecurrent process stops } \\
\text { and gystem becomes chaotic }\end{array}$ \\
\hline
\end{tabular}

In Section 15, the value of $\alpha^{-1}\left(M_{Z}\right)$ is cited from 2010-PDG (p.126) data as:

$$
\alpha^{-1}\left(M_{Z}\right)=127.916 \pm 0.015 .
$$

Fortunately, as shown in Section 15 , above $M_{Z}$ mass scale the value of $\alpha^{-1}\left(M>M_{Z}\right)$ asymptotically approaches saturation. Table 16.2 lists the related parameters as a function of $r$. In which, $\alpha^{-1}\left(M_{e}\right)$ is used at $M_{e} c^{2}$ energy scale and $\alpha^{-1}\left(M_{Z}\right)$ medium value is used for at and beyond $M_{Z} c^{2}$ energy scale and its variation is ignored.

Table 16.2: Parameters of Electron Logistic Process

In Table 16.2, the logistic recurrent process belongs to the variable parameter type, in which parameter $r$ varies. It starts at $r=0.29898133$ and stops at $r=3.57003633$. In the process, besides the three mathematical milestones, $r=1$, $r=2, r=3$, the other milestones correspond to electroweak, electroweak-strong and grand unifications are also listed. The term with $N_{e}(r)=\sqrt{N_{e}}=3.874529 \times 10^{11}$ corresponds to electron's intermediate state, the $e$-boson state, related to cosmic inflation will be explained in Section 22.

Table 16.2 clearly demonstrates the dynamic nature of electron converting factor $N_{e}(r)$. It provides a convincing 
interpretation of $N_{e}(r)$ as a running constant. In essence, the variation of $N_{e}(r)$ is a random process originated from two correlated sources. One is the random walk process described in Section 4 and the other is the logistic recurrent process. In fact, $x_{i}$ and $\left(1-x_{i}\right)$ of (A5.3) can be interpreted as binary probabilities. In some way, $x_{i}$ and $\left(1-x_{i}\right)$ are related to the probabilities in the random walk. The details of the correlation are up to further exploration.

With the help of logistic process, the peculiar behavior of $N_{e}(l)$ in Section 4 is understood now. Why $N_{e}\left(l<\lambda_{e}\right)$ is a running constant? Because its logistic process belongs to the varying $r$ parameter type. Why $N_{e}\left(l \rightarrow \lambda_{e C}\right)$ does not show asymptotic behavior? Because the logistic process abruptly stops at $r=r_{c}+\Delta r$ and becomes chaotic.

This is the hidden scenario revealed in this section. Logistic recurrent process not only provides reasonable explanation for the peculiar behaviors of the converting factor $N_{e}(l)$, but also links it to other constants such as $r_{c}$ and $\alpha(M)$.

In Table 16.2, at the starting point of logistic process,

$$
N_{e}(r=0.29898133)=71.06279805 \text {. }
$$

It is the same as:

$$
N_{G U T}=\frac{M_{\text {Planck }}}{M_{G U T}} \approx \frac{2 \pi}{\sqrt{\alpha\left(M_{Z}\right)}}=71.06279805 \text {. }
$$

$N_{\text {GUT }}=71.06279805$ is the converting factor for scalar

boson $U_{3}$ representing grand unification, while

$N_{e}(r=0.29898133)=71.06279805$ is electron's converting

factor at GUT scale. Why are these two numbers equal? The apparent reason is that they use the same $\alpha\left(M_{Z}\right)=1 /(127.916)$. The deeper reason is that, at the $M_{G U T} c^{2} \sim 10^{18} \mathrm{GeV}$ energy scale, electron mass is in the same order of $U_{3}$ mass, which far exceeds $M_{\text {Max }}$. According to Rule 6.1, such heaver fermions must appear in pair; and a pair of fermions is a boson. It demonstrates the consistency of (15.11) from grand unification and (16.8) from logistic process of electron.

Equation (16.1) is for electron. The corresponding taon version is:

$$
\sqrt{2} N_{\tau} e^{-\pi\left(x_{1 \tau}\right) r_{1 \tau}^{2}}-2 \pi \sqrt{\alpha^{-1}\left(M_{\tau}\right)}=3.12638803734444 \times 10^{-13} \approx 0 .
$$

The data in (16.9) are from the following sources:

From 2010-PDG data:

$$
N_{\tau}=M_{\text {Planck }} / M_{\tau}=4.31732 \times 10^{19} .
$$

From 2010-PDG data: $\alpha\left(M_{\tau}\right)^{-1}=133.444 \pm 0.015$, take

$$
\alpha\left(m_{\tau}\right)^{-1}=133.444 \text {. }
$$

From $S$-equation:

$\pi\left(x_{1 \tau}\right)=\pi(0.249964052052628)=3.141671482326853$.
The numerical solution of (16.9) for $r_{1 \tau}$ is:

$$
\begin{aligned}
& r_{1 \tau}=r_{c}+\Delta r_{\tau}, \\
& \Delta r_{\tau}=5.4618773401173 \times 10^{-2}, \\
& \Delta r_{\tau} / r_{1 \tau}=1.53 \times 10^{-2} .
\end{aligned}
$$

Comparing to the electron case, the value of $r_{1 \tau}=r_{c}+\Delta r_{\tau}$ is increased. It means that for taon the logistic recurrent process goes further into the chaotic region before it stops.

Consider electron macroscopic converting factor $N_{e}$ and its square root $\sqrt{N_{e}}$ in orders of magnitude:

$$
\begin{aligned}
& \sqrt{N_{e}} \sim 3.87 \times 10^{11}, \\
& N_{e} \sim 1.5 \times 10^{23} .
\end{aligned}
$$

$\sqrt{N_{e}}$ and $N_{e}$ are grand numbers representing typical hierarchy phenomena, which deal with the ratio of two quantities having many orders of magnitude difference. There are other phenomena related to great numbers. For example, the total number of stars in a galaxy is close to $10^{11}$; the total number of galaxies in the visible universe is close to $10^{11}$. More examples will be given in later sections.

Definition 16.1: The rank- $G$ grand number is defined as:

$$
N_{G} \sim 10^{11 G} \text {. }
$$

A system consists of $N_{G} \sim 10^{11 G}$ elements is defined as a rank-G grand system. A grand system subjects to the following conditions:

1. The elements in the system belong to the some type;

2. The interaction of elements in the system is weak;

3. The elements in the system behavior stochastically;

4. The interaction of elements is nonlinear in nature, and it provides a growth mechanism with negative feedback. In fact, in (A5.1) and (A5.3), $r=\alpha>0$ represents growth and $\beta>0$ represents negative feedback to suppress growth.

Explanation: The grand number defined by (16.13) is based on decimal for convenience, which is not natural. The natural way to define it is:

$$
\begin{aligned}
& N_{G} \sim 2^{37 g}, \\
& 2^{37}=137438953472 \approx 1.374 \times 10^{11} .
\end{aligned}
$$

The relations between $g$ and $G$ are:

$$
\begin{aligned}
& g=\frac{11 \times \ln (10)}{37 \times \ln (2)} G=0.987600244425973 G, \\
& G=\frac{37 \times \ln (2)}{11 \times \ln (10)} g=1.012555439960666 g .
\end{aligned}
$$

For practical reasons, definition (16.13) is introduced and used throughout in the paper. Definition (16.14) serves as its natural origin. It is interesting to notice that, 2 and 37 both are prime numbers. 
According to Definition 16.1, galaxy is a $G=1$ grand system with $\sim 10^{11}$ stars and the visible universe is a $G=2$ grand system with $\sim 10^{22}$ stars.

The common feature of grand systems containing grand number of elements is related to the logistic recurrence process. When the random process in the system reaches the critical point $r_{c}=3.5699457$, the system becomes chaotic and stops growing. It can only grow further by organizing a next higher level, such as the visible universe on top of galaxies.

Since $N_{e} \sim 1.5 \times 10^{23}$ is close to a $G=2$ grand number, a natural question is: What is the electron's $G=1$ grand system with $\sqrt{N_{e}} \sim 3.87 \times 10^{11}$ ? This is an important question, which will be discussed in Section 22.

Notice that, $N_{G U T}=71.06279805 \approx 71$ deviates from a prime number 71 with a relative deviation of $8.837 \times 10^{-4}$. It is not by coincidence. In fact, it provides a clue with important physics and mathematics significances, which will be discussed in later sections.

The equation of (16.1) is correlated $N_{e}=M_{\text {Planck }} / M_{e}$ to other mathematic constants. It has important significance, which will be discussed in Section 20.

The only thing left unexplained is the numerical factor $\sqrt{2}$ in equation (16.1) and (16.9). It turns out that the factor $\sqrt{2}$ provides an important clue for the structure of space and much more. The details will be discussed in Section 21.

In summary, equation (16.1) is an important discovery. It reveals many important correlations including logistic recurrence process, converting factor, Gaussian probability, random walk, $S$-equation and grand number phenomena. Some of them are worthwhile to explore further.

\section{Section: 17 Neutrinos}

Neutrinos are puzzling particles with peculiar behaviors. Despite extensive efforts in recent years tried to find out neutrinos' properties, some of them are still not clearly known. In this section, only a framework is presented, in which many issues remain open.

Let's start with known facts.

Fact-1: Neutrinos have tiny mass evidenced by oscillations among three different types.

Fact-2: Neutrinos only have left-handedness and antineutrinos only have right-handedness.

Fact-3: Neutrinos have no electrical charge or tiny remnant electrical charge.

The main focus of this section begins with anti-neutrinos, because anti-neutrinos are the ones companioned with their changed leptons and play a pivotal role in most weak interactions.

Rule 17.1: According to the Leptons Pairing Rule in Section 14 , each charged lepton is paired with its companion anti-neutrino: $e \& \bar{v}_{e}, \mu \& \bar{v}_{\mu}, \tau \& \bar{v}_{\tau}$ with combined numerical parameters $m=e v e n, n=0$; each charged anti-lepton is paired with its companion neutrino: $e^{+} \& v_{e}, \mu^{+} \& v_{\mu}, \tau^{+} \& v_{\tau}$ with combined numerical parameters $m=-$ even, $n=0$. The $n$-parameters matching rules are:

$$
\begin{aligned}
& n_{l}+n_{\bar{v}_{l}}=0, \text { or } n_{\bar{v}_{l}}=-n_{l},, l=e, \mu, \tau, \bar{v}_{l}=\bar{v}_{e}, \bar{v}_{\mu}, \bar{v}_{\tau} ; \\
& n_{\bar{l}}+n_{v_{l}}=0 \text {, or } n_{v_{l}}=-n_{\bar{l}}, \bar{l}=e^{+}, \mu^{+}, \tau^{+}, v_{l}=v_{e}, v_{\mu}, v_{\tau} .
\end{aligned}
$$

Take electron anti-neutrino $\bar{v}_{e}$ as an example. As listed in Table 12.3, electron's after reduction $n$-parameter is $n_{e}=1 / 2$. According to (17.1a), the $n$-parameter of $\bar{v}_{e}$ is $n_{\overline{v e}}=-1 / 2$, to make the match:

$$
n_{e}+n_{\bar{v} e}=1 / 2+(-1 / 2)=0 .
$$

The $\bar{v}_{e}$ as an anti-fermion, according to Definition 11.2, its $m$-parameter must have opposite sign of its $n$-parameter:

$$
m_{\bar{v} e} n_{\bar{v} e}<0 \text {. }
$$

Because of $n_{\bar{v} e}=-1 / 2<0, \bar{v}_{e}$ must have $m_{\overline{v e}}>0$ corresponding to $\bar{v}_{e}$ right-handedness. The argument is also applicable to the other two types of anti-neutrinos, $\bar{v}_{\mu}$ and $\bar{v}_{\tau}$. It shows that, anti-neutrinos unique handedness is hidden in Rule 17.1 and originated from mathematics.

In reference [1] by the author, neutrinos were treated based on their companion leptons and the $W$ boson. The mass values of three types of anti-neutrinos were given as:

$$
\begin{aligned}
& M_{\overline{v e}}=M_{v e}=\left(\frac{\alpha}{2}\right)^{2}(1-\alpha) \frac{M_{e}^{2}}{M_{W}}=4.2922 \times 10^{-5} \mathrm{eV} / \mathrm{c}^{2}, \\
& M_{\bar{\nu} \mu}=M_{v \mu}=\left(\frac{\alpha}{2}\right)^{2}(1-\alpha) \frac{M_{e} M_{\mu}}{M_{W}}=8.8749 \times 10^{-3} \mathrm{eV} / \mathrm{c}^{2}, \\
& M_{\bar{v} \tau}=M_{v \tau}=\left(\frac{\alpha}{2}\right)^{2}(1-\alpha) \frac{M_{e} M_{\tau}}{M_{W}}=1.4925 \times 10^{-1} \mathrm{eV} / \mathrm{c}^{2} .
\end{aligned}
$$

In (17.4), the mass values, $M_{e}, M_{\mu}, M_{\tau}, M_{W}$ and the value of fine structure constant $\alpha=1 / 137.035999084$ are cited from 2010-PDG. $M_{\overline{v e}}, M_{\bar{v} \mu}, M_{\bar{v} \tau}$ are the mass values of three flavored anti-neutrinos $\bar{v}_{e}, \bar{v}_{\mu}, \bar{v}_{\tau}$, respectively, which are in principle different from the eigenstate neutrinos $\tilde{v}_{1}$, $\tilde{v}_{2}, \tilde{v}_{3}$. The difference will be discussed later in this section.

According to (17.4), the mass ratios of three antineutrinos to their companion charged leptons are:

$$
\begin{aligned}
& R_{\overline{v e} / e}=\frac{M_{\overline{v e}}}{M_{e}}=\left(\frac{\alpha}{2}\right)^{2}(1-\alpha) \frac{M_{e}}{M_{W}}=8.3996 \times 10^{-11}, \\
& R_{\bar{v} \mu / \mu}=\frac{M_{\bar{v} \mu}}{M_{\mu}}=\left(\frac{\alpha}{2}\right)^{2}(1-\alpha) \frac{M_{e}}{M_{W}}=8.3996 \times 10^{-11}, \\
& R_{\bar{v} \tau / \tau}=\frac{M_{\bar{v} \tau}}{M_{\tau}}=\left(\frac{\alpha}{2}\right)^{2}(1-\alpha) \frac{M_{e}}{M_{W}}=8.3996 \times 10^{-11} .
\end{aligned}
$$


The three ratios are identical, which indicate that three antineutrinos are closely related to their companion charged leptons as well as among themselves.

To determine anti-neutrinos models and parameters, two issues must be dealt with. One is mass and the other is electrical charge, which are very different from their companion charged leptons.

Let's start from electron torus model and look for the ways to transfer it into the model of its companion antineutrino $\bar{v}_{e}$. According to SQS theory, the mass $M_{\bar{v} e}$ of $\bar{v}_{e}$ is related to its model and numerical parameters in two ways.

1. According to (6.18) in Section 6, the distance between $x_{1}$ and $x_{2}$ on $x$-axis for $\bar{v}_{e}$ is related to its mass $M_{\bar{v} e}$ and electron mass $M_{e}$ as:

$$
4\left|x_{2}-x_{1}\right|=M_{e} / M_{\overline{v e}}=1 / R_{\bar{v} e / e} \text {. }
$$

According to (17.5a) and (17.6):

$$
\left|x_{2}-x_{1}\right|=1 /\left(4 R_{\overline{v e} / e}\right)=2.9763 \times 10^{9} .
$$

It indicates that, $x_{1}$ and $x_{2}$ are separated by a vast distance alone $x$-axis. The values of $\pi\left(x_{1}\right)$ and $\pi\left(x_{2}\right)$ are determined by $x_{1}$ and $x_{2}$ according to $S$-equation.

2. The numerical parameters $m, n, p$ and the mass oscillation term in $A T$-, $P S$-equations are related to the mass ratio. The mass ratio is defined as:

$$
R_{\bar{v} e / e}=\frac{p}{n}=\frac{M_{\bar{v} e}}{M_{e}}=8.3996 \times 10^{-11},
$$

The mass oscillation term is:

$$
a_{2}^{\prime} \sin [(2 p / m) \theta]=a_{2}^{\prime} \sin \left[\left(2 R_{\bar{v} e / e} n / m\right) \theta\right] .
$$

According to (17.8) and $m_{\bar{v} e}=1, n_{\bar{v} e}=-n_{e}=-1 / 2$, the way to reduce mass $M_{\bar{v} e}$ from $M_{e}$ is to reduce the value of the magnitude of $p$-parameter:

$$
p_{\bar{v} e}=n_{\overline{v e}} R_{\bar{v} e / e}=-\frac{1}{2} \times 8.3996 \times 10^{-11}=-4.1998 \times 10^{-11} .
$$

The numerical parameters of $\bar{\nu}_{\mu}$ and $\bar{v}_{\tau}$ can be determine by the same way. The results are summarized as: Electron anti-neutrino $\bar{v}_{e}$ :

$$
m_{\bar{v} e}=1, n_{\bar{v} e}=-1 / 2, p_{\bar{v} e}=-4.1998 \times 10^{-11} ;
$$

Muon anti-neutrino $\bar{\nu}_{\mu}$ :

$$
m_{\bar{v} \mu}=1, n_{\bar{v} \mu}=-(1+5 / 8), p_{\bar{v} \tau}=-1.3649 \times 10^{-10} ;
$$

Taon anti-neutrino ${\overline{v_{\tau}}}_{\text {: }}$

$$
m_{\bar{v} \tau}=1, n_{\bar{v} \tau}=-(2+6 / 7), p_{\bar{v} \tau}=-2.3999 \times 10^{-10} .
$$

For the electrical charge difference, the key is to let the electrical charge to vanish for $\bar{v}_{e}$ and keep all related rules valid. As discussed in previous sections, particle's electrical charge $q$ is related to Weinberg angle $\theta_{W}$ as $q \propto \sin \theta_{W} \cos \theta_{W}$. Let Weinberg angle $\theta_{W} \rightarrow 0$ or $\theta_{W}=0$, the electrical charge follows $q \rightarrow 0$ or $q=0$.
Based on these considerations, SQS theory provides two options for neutrinos model. The process starts from torus model and followed by trefoil model for easy to understand. Option-1: For Dirac Type Neutrinos

In the electron's torus model, move point $A$ and point $A^{\prime}$ to coincide with point $G$. As results, the torus model and trajectory for $\bar{v}_{e}$ are shown in Fig. 17.1.

When point $A$ and $A^{\prime}$ coincide with point $G, \xi_{2}=0$, $\psi_{2}=180^{\circ}, \phi_{2}=0, \theta_{2}=0$, and $\theta_{W}=\theta_{2}=0, q=0$. In the outer half of torus cross section, according to (8.8a), $A$ and $A^{\prime}$ coincided with point $G$ make $Z_{2}=0 \quad \xi_{2}=0$ and

$$
\begin{aligned}
& \frac{\int_{-\xi}^{\xi} \sqrt{\left(a_{2} \sin t\right)^{2}+\left(b_{2} \cos t\right)^{2}} d t}{2 Z_{2}}=1, \\
& \pi\left(x_{2}\right)=\pi .
\end{aligned}
$$

In the region $[0,0.5]$ of $x$-axis, the $S$-equation of (3.21) has only one solution at $x=x_{c}=0.24998715627302645$ :

$$
\pi\left(x_{c}\right)=\pi \text {. }
$$

According to $S(x)$ function's periodicity of (2.12) and symmetry of $(2.13),(17.12)$ is extended to the entire $x$-axis as:

$$
\begin{aligned}
& \pi\left(x_{c}+N\right)=\pi, N=0, \pm 1, \pm 2, \pm 3 \cdots \pm \infty \\
& \pi\left(1-x_{c}+N\right)=\pi, N=0, \pm 1, \pm 2, \pm 3 \cdots \pm \infty
\end{aligned}
$$
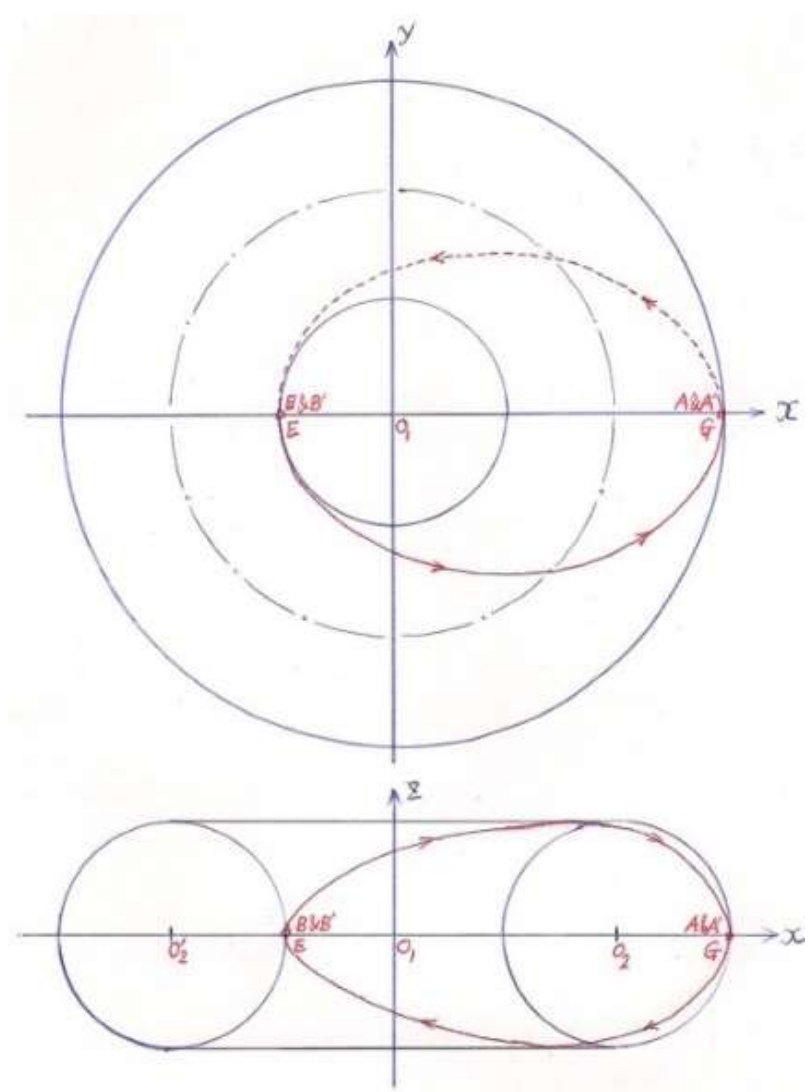

Fig.17.1: Torus model and trajectory for $\bar{v}_{e}$ according to Option-1. 
Z. Y. SHEN

According to (17.7) and (17.13), there are two ways to determine $x_{1}$ and $x_{2}$ for $\bar{v}_{e}$.

Way-1: $x_{1}=x_{c}, x_{2}=x_{c}+N ; \pi\left(x_{1}\right)=\pi\left(x_{c}\right)=\pi$,

$\pi\left(x_{2}\right)=\pi\left(x_{c}+N\right)=\pi$;

Way-2: $x_{1}=x_{c}, x_{2}=1-x_{c}+N ; \pi\left(x_{1}\right)=\pi\left(x_{c}\right)=\pi$,

$\pi\left(x_{2}\right)=\pi\left(1-x_{c}+N\right)=\pi$.

In both ways, $\pi\left(x_{1}\right)=\pi$ and $\pi\left(x_{2}\right)=\pi$, which are

required by probability matching. $\pi\left(x_{1}\right)=\pi$ means that, as point $A$ and point $A^{\prime}$ coincided with point $G$, point $B$ and $B^{\prime}$ also coincide with point $E$ as shown in Fig.17.1. The trajectory shown in Fig.17.1 is for $\bar{v}_{e}$. The trajectory for $v_{e}$ has the same projections as the trajectory for $\bar{v}_{e}$ with opposite direction along the switched solid line and dashed line on the $x-y$ plane. The differences of two ways are:

For Way-1:

$\left|x_{2}-x_{1}\right|_{\overline{v e}}=R_{\overline{v e} / e} / 4=M_{e} /\left(4 M_{\bar{v} e}\right)=|N|=$ integer,

For Way-2:

$\left|x_{2}-x_{1}\right|_{\overline{v e}}=R_{\overline{v e} / e}=M_{e} /\left(4 M_{\overline{v e}}\right)=\left|N+1-2 x_{c}\right|=$ non-integer .

Unfortunately, the accuracy of available data is not sufficient to choose which way to go. In the meantime, let's take Way-1.

In the above discussion, $\bar{v}_{e}$ serves as an example. The same principles are applicable to $\bar{v}_{\mu}$ and $\bar{v}_{\tau}$ based on the models of nuon and taon. Neutrinos $v_{e}, v_{\mu}, v_{\tau}$ share the some models as anti-neutrinos $\bar{v}_{e}, \bar{v}_{\mu}, \bar{v}_{\tau}$ respectively with different trajectories' directions.

Theorem 17.1: The Dirac type neutrino only with left handedness and the anti-neutrino only with right handedness must have zero electrical charge:

$q_{\bar{v}}=q_{v}=0$.

Proof: Let's starts from the opposite. If $q_{\bar{v}} \neq 0$ and $q_{v} \neq 0$, then points $A$ and points $A^{\prime}$ are not exactly coincided with point $G$; point $B$ and point $B^{\prime}$ are not exactly coincided with point $E$. In such case, there are four possible trajectories, in which two trajectories for $\bar{v}$ with right and left handedness and two trajectories for $v$ with right and left handedness. This scenario violates Fact-2, which must be not true. Then the opposite $q_{\bar{v}}=q_{v}=0$ must be true.

QED

The above discussions are based on torus model. The real model for $\bar{v}_{e}, \bar{v}_{\mu}, \bar{v}_{\tau}$ and $v_{e}, v_{\mu}, v_{\tau}$ are trefoil model with three branches. The way for three torus models combined into a trefoil type model is the same described in Section 12 for charged leptons. Fig.17.2 shows the trefoil type model and trajectory for $\bar{v}_{e}$.

The calculated parameters based on Option-1 trefoil type model are listed in Table 17.1, 17.2, and 17.3 for $\bar{v}_{e}, \bar{v}_{\mu}$ and $\bar{v}_{\tau}$, respectively. The listed data are based on $q_{\bar{v}}=0$ The after $f$-modification parameters are marked with '.

The torus model and the trefoil type model provided by Option-1 are only valid for Dirac type neutrinos and antineutrinos with two components. There is a reason to introduce Option-2 to provide a Majorana type model with only one component, because eigenstate anti-neutrinos belong to Majorana type as shown later in this section.

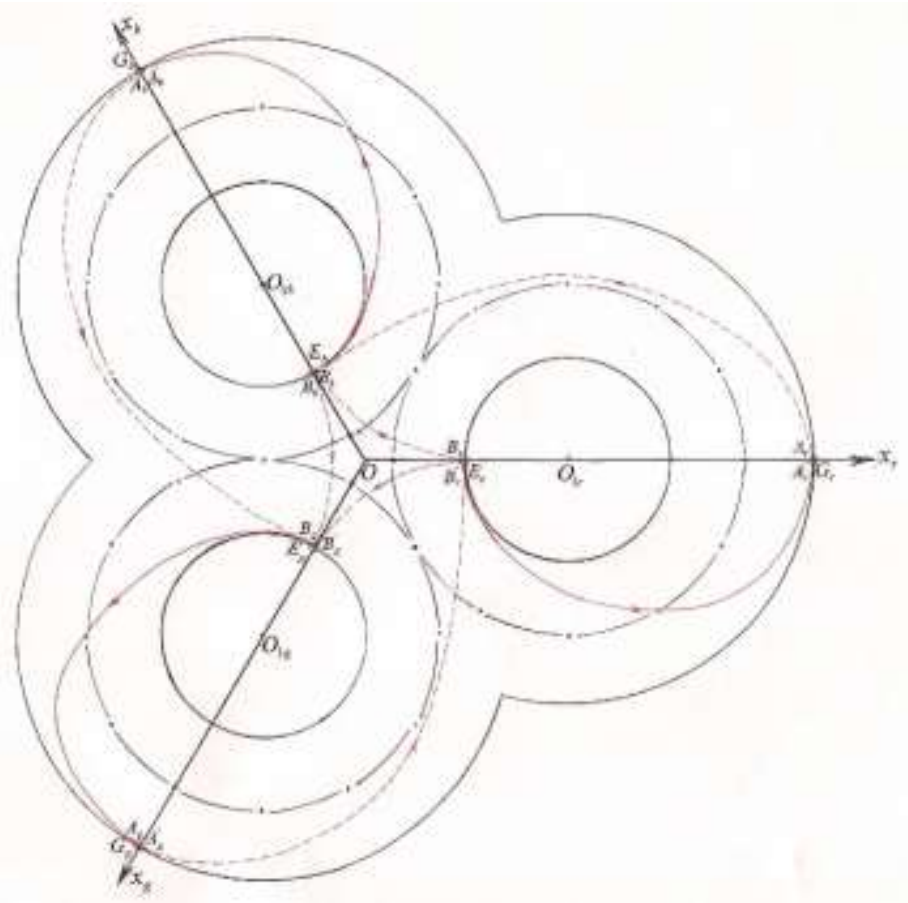

Fig.17.2: Trefoil type model and trajectory for $\bar{v}_{e}$ according to Option-1.

Table 17.1: Parameters for Electron Anti-Neutrino Based on Option-1

\begin{tabular}{|c|c|c|c|}
\hline \multicolumn{4}{|c|}{$\begin{array}{l}\text { Electron Anti-Neutrino as a Whole Entity } \\
\text { Symbot: } \nabla_{0}, \text { Mass: } M_{\mathrm{s}}=4.2922 \times 10^{-1} \mathrm{eV} \\
R_{\mathrm{s}},=M_{\mathrm{a}} / M_{s}=8.3996 \times 10^{-11}\end{array}$} \\
\hline \multicolumn{4}{|c|}{ Model: trefoil type sumilat to electron } \\
\hline \multicolumn{4}{|c|}{$\begin{array}{l}\text { Roduced number paraneter: } m_{m}=1, n_{n}=-1 / 2, p_{h}=-4.1998 \times 10^{-4} \\
d=1\end{array}$} \\
\hline \multicolumn{2}{|c|}{ 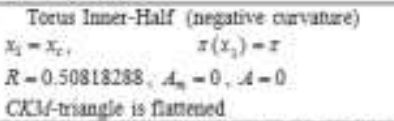 } & \multicolumn{2}{|c|}{$\begin{array}{l}x_{2}=x_{c}+N, \quad z\left(x_{1}\right)=\pi \\
\text { GFS - triangle and S-triangle are fattened }\end{array}$} \\
\hline $\begin{array}{l}\text { Triangle } B^{\prime}, D^{\prime} O^{\prime} \text {, on } \\
O_{1} H^{\prime} \text { eross sectiota }\end{array}$ & $\begin{array}{l}\text { Triangle } D^{\prime} E O_{7} \text { on } \\
x-y \text { coss section }\end{array}$ & $\begin{array}{l}\text { Triangle } d_{2}^{\prime}, O_{1} O_{2} \text { an } \\
O_{Q} G^{\prime} \text { ctoss section }\end{array}$ & $\begin{array}{l}\text { Triangie } G O O_{1} \text { on } \\
x-y \text { cross section }\end{array}$ \\
\hline $\begin{array}{l}b_{1}=0 \\
x_{1}^{n}=d_{2}-2.49181712 \\
z_{1}^{\prime}=0\end{array}$ & $\begin{array}{l}a_{1}=0 \\
x_{1}=2-0.5681828 s \\
Y_{0}=0\end{array}$ & $\begin{array}{l}x_{2}=d_{2}=0.49131712 \\
Z_{2}=0\end{array}$ & 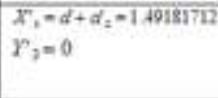 \\
\hline$\alpha_{1}=90^{\circ}$ & $\alpha_{c}^{\prime}=90^{\circ}$ & $\theta_{2}=0^{2}$ & $\theta_{1}=0^{2}$ \\
\hline$\theta_{1}=0^{\circ}$ & $\beta_{0}=\theta$ & $\phi_{2}=\theta^{\prime}$ & $\phi_{3}=0^{\circ}$ \\
\hline$Y_{1}=90^{\circ}$ & $r_{0}^{\prime}=90^{\prime}$ & $v_{2}^{\prime}=180^{\prime}$ & $v_{2}=180^{\circ}$ \\
\hline$\theta_{1}=0^{\circ}$ & $\phi_{0}=0^{*}$ & $\xi_{1}=0^{2}$ & $5,-0^{\circ}$ \\
\hline
\end{tabular}


Table 17.2: Parameters for Muon Anti-Neutrino Based on Option-1

\begin{tabular}{|c|c|c|c|}
\hline \multicolumn{4}{|c|}{ 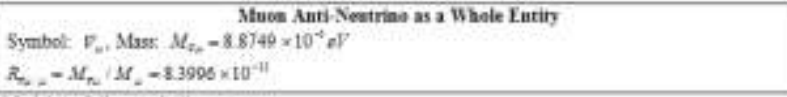 } \\
\hline \multicolumn{4}{|c|}{ Sodel trefoil type similar to ausal } \\
\hline \multicolumn{3}{|c|}{ 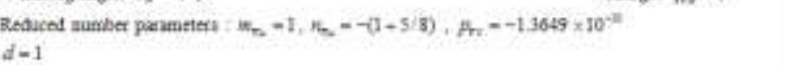 } & 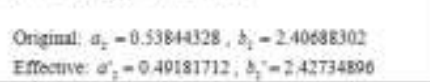 \\
\hline \multicolumn{2}{|c|}{ 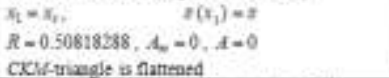 } & \multicolumn{2}{|c|}{ 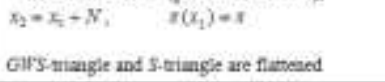 } \\
\hline $\begin{array}{l}\text { Trimgle } B_{1} D O_{y} \text { ou } \\
\text { OH'cross soctiou }\end{array}$ & 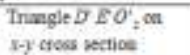 & 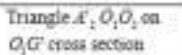 & $\begin{array}{l}\text { Trumgle } G O_{O} O_{2} \text { an } \\
x-y \text { anos section }\end{array}$ \\
\hline $\begin{array}{l}b_{1}=0 \\
x_{1}=a_{2}=0.49181712 \\
z_{1}^{\prime}=0\end{array}$ & $\begin{array}{l}4_{1}=0 \\
x_{2}^{\prime}=R-0 \operatorname{sos} 1828 \\
y_{5}^{\prime}=0\end{array}$ & $\begin{array}{l}x_{2}^{2}=d_{1}=0.4979172 \\
2:=0\end{array}$ & $\begin{array}{l}x_{1}=d+d_{2}=1 \text {. } \\
r_{3}=0\end{array}$ \\
\hline$\alpha_{1}^{\prime}=90^{\prime}$ & $\alpha_{1}^{\prime}=90^{\circ}$ & $\theta_{2}=0^{\circ}$ & $\theta_{1}=\theta^{\prime}$ \\
\hline$\hat{\theta}_{1}=\theta^{+}$ & $\beta_{i}=\theta$ & $\theta_{2}=\theta^{+}$ & $\phi_{i}=\theta^{\prime}$ \\
\hline$y_{1}^{\prime}=90^{\prime \prime}$ & $r_{1}=90^{2}$ & $v_{\mathrm{r}}=180^{\circ}$ & $V_{1}=180^{\circ}$ \\
\hline$\phi_{1}=\dot{\theta}^{2}$ & $\phi_{0}=0^{2}$ & $\xi_{2}=0^{\circ}$ & $\xi_{1}=0^{2}$ \\
\hline
\end{tabular}

Table 17.3: Parameters for Taon Anti-Neutrino Based on Option-1

\begin{tabular}{|c|c|c|c|}
\hline \multicolumn{4}{|c|}{ 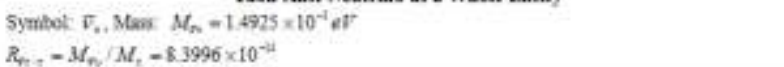 } \\
\hline \multicolumn{4}{|c|}{ Moder trefoel type similar to tase } \\
\hline Wenberg angle $\theta_{n}=0$ & ; & & Charge: ofer $=0$ \\
\hline \multicolumn{4}{|c|}{$\begin{array}{l}\text { Reduced sumber purametess }: m_{n}-1, n_{n}=-(2+6 / 7), p_{r}=-2.3999 \times 10^{-12} \\
d=1\end{array}$} \\
\hline \multicolumn{2}{|l|}{$f_{i}=0.94733344$} & \multicolumn{2}{|c|}{ Original: $a_{1}=0.5208362, b_{1}=4.39440915$} \\
\hline \multicolumn{2}{|l|}{$f_{i}=1.00187462$} & \multicolumn{2}{|c|}{ Effective $a_{1}^{\prime}=0,49340555, s_{1}^{\prime}=4,40264701$} \\
\hline \multicolumn{2}{|c|}{ Torus leser Half (negurive curvare) } & \multicolumn{2}{|c|}{ Torus Outer Haff (positivt curnature), } \\
\hline \multicolumn{2}{|c|}{ 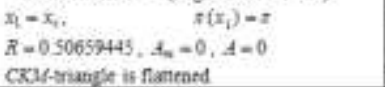 } & \multicolumn{2}{|c|}{$\begin{array}{l}x_{1}-x_{1}+N, \quad \quad r\left(x_{2}\right)-r \\
\text { Gils-triangle and S-triangle are fattmant }\end{array}$} \\
\hline $\begin{array}{l}\text { Triagie } S_{1}, \mathrm{DO}_{2} \text { on } \\
O_{1} H \text { ' cross sectioo }\end{array}$ & $\begin{array}{l}\text { Inangle } D \& O^{\prime}, \infty \text { a } \\
\text { sycross sectich }\end{array}$ & 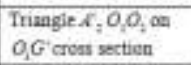 & $\begin{array}{l}\text { Trumgle } G^{\prime} O O_{;} \text {on } \\
\text { syranoss sectoon }\end{array}$ \\
\hline$\phi_{1}=0$ & $a_{1}=0$ & $x_{2}=a_{1}=0.49540559$ & $x_{1}=d+d_{1}=1.29340529$ \\
\hline$x_{1}=a_{7}^{\prime}=0.49340555$ & $X_{0}=R=0 \operatorname{so6} 59445$ & $z_{2}=0$ & $Y_{3}=0$ \\
\hline$Z_{1}^{\prime}=0$ & $r_{\mathrm{j}}^{\prime}=0$ & & \\
\hline$\alpha_{1}^{\prime}=90^{\prime}$ & $\alpha_{1}^{\prime}=90^{\prime}$ & $\theta_{1}=0$ & $\theta_{1}=0^{\prime}$ \\
\hline$\theta_{1}=0^{\circ}$ & $\theta_{n}=\theta^{2}$ & $\phi_{0}=0^{*}$ & $\phi_{1}=\sigma$ \\
\hline$y_{1}^{\prime}=90^{*}$ & $\gamma_{1}=90^{\circ}$ & $\nu r-180^{2}$ & $\psi_{1}^{\prime}=180^{\prime}$ \\
\hline$\theta_{1}=\theta^{\circ}$ & $\phi_{x}=\sigma^{\circ}$ & $5-\theta^{2}$ & $52=0$ \\
\hline
\end{tabular}

\section{Option-2: For Majorana type Neutrinos}

Start from Option-1 torus model for $\bar{v}_{e}$ with $q_{\bar{v}}=0$. Let loop-2 center $\mathrm{O}_{2}$ on right and center $\mathrm{O}_{2}{ }_{2}$ on left move toward loop-1 center $O_{1}$ and coincides with $O_{1}$. The torus surface becomes spherical surface for $b_{2} / a_{2}=1$ or elliptical surface for $b_{2} / a_{2} \neq 1$. In the new model, loop-2 is integrated into loop-1. It fits the one component Majorana type naturally. Spherical and elliptical surfaces belong to genus-0 topological manifold. The trajectory on torus surface is degenerated into a circle on the spherical surface or elliptical surface with points $G$ on its right and points $E$ on its left as shown in Fig.17.3.

The trajectory on trefoil type model is degenerated on sphere surface or elliptical surface as shown in Fig. 17.4. The model is degenerated from genus- 3 to genus- 0 without branches but the degenerated trajectory still retains its 3branch trefoil type with loop-2 integrated into loop- 1 .

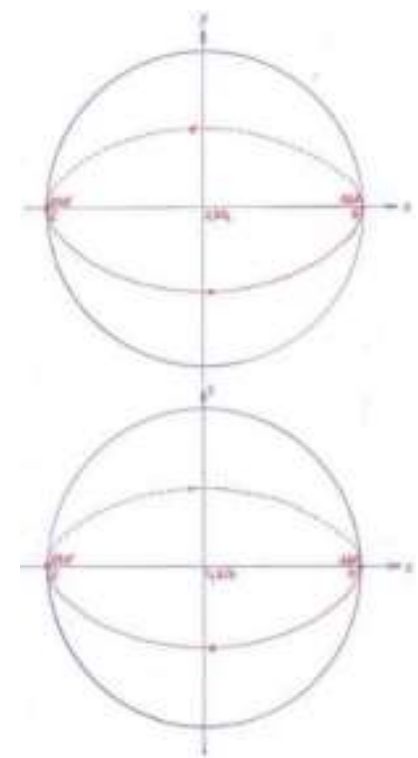

Fig 17.3: Torus model and trajectory degenerated to sphere for $\tilde{v}_{1}$ according to Option-2.

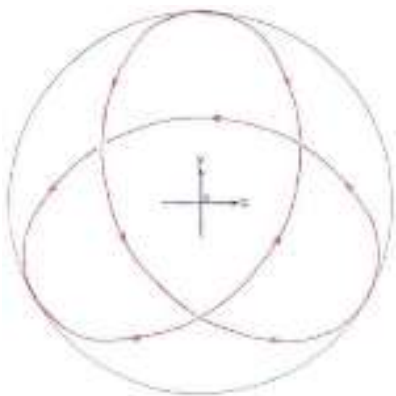

Fig 17.4: Trefoil type model and trajectory degenerated to sphere for $\tilde{v}_{1}$ according to Option-2.

In the process of weak interaction such as $n \rightarrow p+e+\bar{v}_{e}$, an anti-neutrino is created with a definitive flavor. The new born anti-neutrino flies with speed very close to the speed of light in vacuum. During it flying journey, the anti-neutrino is oscillating among different flavors. In other words, the flying anti-neutrino lost its flavor identity and becomes an oscillating system of eigenstate anti-neutrinos. When it is caught by a detector, the eigenstate anti-neutrino gets its flavor identity according to probability. The oscillating behaviors were found by experiments as the Fact-1, which serve as the evidence for anti-neutrinos having tiny mass.

It is important to point out that, during their free flying journey, the oscillation is among the members of eigenstate version, and the members of flavored version only show up at their birth or been detected. As soon as they start to fly, the flavored version converts to the eigenstate version.

Fine structure constant $\alpha$ is a running constant depending on energy scales. In (17.4) and (17.5), the $\alpha$ value is based on the 2010-PDG $(p .126)$ data:

$$
\alpha\left(M_{e}\right)=1 / 137.035999084 \text {. }
$$


Z. Y. SHEN

Take (17.4c) for $\bar{v}_{\tau}$ as an example, there are three different energy scales involved. If $\alpha$ is treated as a running constant, $(17.4 \mathrm{c})$ probably should be rewritten as:

$$
M_{\bar{\nu} \tau}=\left(\frac{\alpha\left(M_{e}\right)}{2}\right)\left(\frac{\alpha\left(M_{\tau}\right)}{2}\right)\left(1-\alpha\left(M_{Z}\right)\right) \frac{M_{e} M_{\tau}}{m_{W}}=1.5318 \times 10^{-1} e V / c^{2} .
$$

In (17.17), 2010-PDG ( $p .126)$ data medium values are used:

$$
\begin{aligned}
& \alpha\left(M_{\tau}\right)=1 / 133.444, \\
& \alpha\left(M_{Z}\right)=1 / 127.916 .
\end{aligned}
$$

The difference between (17.4c) and (17.17) is $2.6 \times 10^{-2}$.

The mass data listed in Table 17.1, 17.2 and 17.3 did not take $\alpha$ as a running constant into account, which may have up to $\sim 10^{-2}$ errors.

According to 2010-PDG data, three charged leptons have a mass relation:

$$
\sqrt{M_{e} M_{\tau}}=\frac{M_{\mu}}{3.50649} .
$$

According to (17.4) and (17.19), if ignore the effect of $\alpha$ as a running constant, three anti-neutrinos have a similar mass relation:

$$
\sqrt{M_{\bar{v} e} M_{\bar{v} \tau}}=\frac{M_{\bar{v} \mu}}{3.50649} .
$$

(17.19) and (17.20) indicate that, three types of neutrinos and anti-neutrinos are closely correlated.

The neutrino models and parameters proposed by SQS theory can be verified by checking on eigenstate neutrinos mass values. 2010-PDG provides the experimental data for the differences of eigenstates mass squares:

$$
\begin{aligned}
\Delta M_{21}^{2} & =\left(\begin{array}{c}
7.59^{+0.19} \\
-0.21
\end{array}\right) \times 10^{-5}\left(e V / c^{2}\right)^{2}, \\
\Delta M_{32}^{2} & =(2.43 \pm 0.13) \times 10^{-3}\left(e V / c^{2}\right)^{2} .
\end{aligned}
$$

Which are based on 2010PDG assumption:

$$
\Delta M_{32}^{2} \sim \Delta M_{31}^{2} \text {. }
$$

According to (17.4), the SQS theoretical values are:

$$
\begin{aligned}
& M_{\bar{v} \mu}^{2}-M_{\bar{v} e}^{2}=\left(7.876 \times 10^{-5}\right)\left(e V / c^{2}\right)^{2}, \\
& M_{\bar{v} \tau}^{2}-M_{\bar{v} \mu}^{2}=\left(2.22 \times 10^{-2}\right)\left(e V / c^{2}\right)^{2}, \\
& M_{\bar{v} \tau}^{2}-M_{\bar{v} e}^{2}=\left(2.227 \times 10^{-2}\right)\left(e V / c^{2}\right)^{2} .
\end{aligned}
$$

The $M_{\bar{v} \mu}^{2}-M_{\bar{v} e}^{2}$ theoretical value of (17.22a) is close to the experimental $\Delta M_{21}^{2}$ value of (17.21a). The other two are off by a factor of $\sim 9$ for mass square; for mass without square, they are off by a factor of $\sim 3$. The assumption $\Delta M_{31}^{2} \sim \Delta M_{32}^{2}$ of (17.21c) also fits (17.22b) and (17.22c) well. But they are not fair comparisons. In principle, $M_{\bar{v} e}, M_{\bar{v} \mu}, M_{\bar{v} \tau}$ as flavored anti-neutrinos mass are different from eigenstates mass $M_{1}, M_{2}, M_{3}$. Nevertheless, the 2010-PDG data provided some tentative information. For instance, one possible interpretation of experimental data given by (17.21) is:

$$
\begin{aligned}
& M_{\tilde{v} 1} \approx M_{\bar{v} e}, \\
& M_{\tilde{v} 2} \approx M_{\bar{v} \mu}, \\
& M_{\tilde{v} 3} \approx M_{\bar{v} \tau} / 3 .
\end{aligned}
$$

Future experiments will check the interpretation.

In this section, so far the focus is on anti-neutrinos. Now is the time to deal with neutrinos. Let's do it by the logical way to start from facts and to treat according to rules. The facts are Fact-1, Fact-2 and Fact-3. The rules are Rule 17.1 and Rule 17.2.

Rule 17.2: The right and left handedness defined by Definition 11.2 is universally valid for all fermions including two versions of neutrinos and anti-neutrinos.

According to Fact-1 and Fact-2, there is a paradox. A chaser is chasing an anti-neutrino with a speed slightly faster than its speed. It is possible, because anti-neutrino has mass and its speed must be less than the speed of light in vacuum. The chaser behind it sees an anti-neutrino with right handedness. When the chaser gets ahead of it, he or she sees a neutrino with left handedness. This scenario is impossible. An anti-particle cannot turn into a particle by just looking at it in different ways. The "chaser's paradox" must be eliminated. It serves as the key concept to introduce Hypothesis 17.1.

Notice that, the chaser's paradox is not applicable to flavored version neutrinos and anti-neutrinos, because they never fly. To avoid the paradox, the target is the flying eigenstate version.

Hypothesis 17.1: After their birth, the flavored neutrinos convert into corresponding eignstate neutrinos. The eigenstate neutrinos $\tilde{v}_{1}, \tilde{v}_{2}, \tilde{v}_{3}$ are Majorana type fermions with only one component and the anti-particles is the same as itself:

$$
\overline{\widetilde{v}}_{i}=\tilde{v}_{i}, i=1,2,3 \text {. }
$$

After their detection, the eigenstate neutrinos convert into corresponding flavored neutinos. The flavored neutrinos are Dirac type fermions with two components and have anti-particles:

$$
v_{j} \neq \bar{v}_{j}, j=e, \mu, \tau \text {. }
$$

Explanation: Hypothesis 17.1 suggests that, eigenstate neutrinos $\tilde{v}_{1}, \tilde{v}_{2}, \tilde{v}_{3}$ are Majorana type. SQS theory is not the first one to do so. Similar ideas were proposed earlier. But there are differences.

1. Hypothesis 17.1 is based on mathematics.

2. Hypothesis 17.1 clearly points out that, flavored version neutrinos and anti-neutrinos are Dirac type. This is necessary to avoid contradiction with experimental facts. According to 2010-PDG data book, muon decay mode $\mu \rightarrow e^{-} \bar{v}_{e} v_{\mu}$ has $\Gamma_{i} / \Gamma \approx 100 \%$; taon decay modes $\tau \rightarrow e^{-} \bar{v}_{e} \nu_{\tau}$ has $\Gamma_{i} / \Gamma=(17.85 \pm 0.05) \%$ and $\tau \rightarrow \mu^{-} \bar{v}_{\mu} \nu_{\tau}$ has $\Gamma_{i} / \Gamma=(17.36 \pm 0.05) \%$. Their charge conjugates $\mu^{+}$and $\tau^{+}$have corresponding decay 
modes. These facts clearly show that, the flavored version must have all flavored version neutrinos $v_{e}$, $v_{\mu}, v_{\tau}$ and anti-neutrinos $\bar{v}_{e}, \bar{v}_{\mu}, \bar{v}_{\tau}$ Therefore, three flavored neutrinos must be Dirac type fermions and have corresponding anti-particles.

Hypothesis 17.1 eliminates the chaser's paradox. For the frying Majorana type eigenstate anti-neutrino, when the chaser gets ahead of the anti-neutrino, he or she merely sees the anti-neutrino changed its right handedness to left handedness as it should be. The chaser's paradox is gone.

Rule 17.3: The conversion between the flavored version $v_{e}$, $\bar{v}_{e}, v_{\mu}, \bar{v}_{\mu}, v_{\tau}, \bar{v}_{\tau}$ and the eigenstate version $\tilde{v}_{1}, \tilde{v}_{2}$, $\tilde{v}_{3}$ are governed by converting probability matrixes:

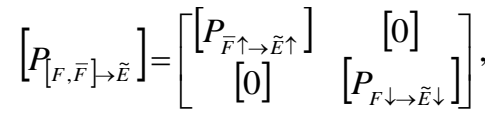

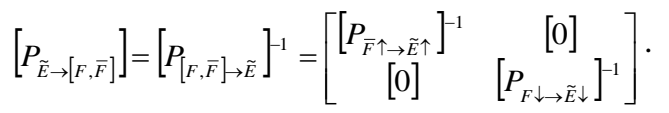

$\left[P_{\bar{F} \uparrow \rightarrow \tilde{E} \uparrow}\right]=\left[\begin{array}{lll}p_{\bar{v} e \uparrow \rightarrow \tilde{v} 1 \uparrow} & p_{\bar{v} e \uparrow \rightarrow \tilde{v} 2 \uparrow} & p_{\bar{v} e \uparrow \rightarrow \tilde{v} 3 \uparrow} \\ p_{\bar{v} \mu \uparrow \rightarrow \tilde{v} 1 \uparrow} & p_{\bar{v} \mu \uparrow \rightarrow \tilde{v} 2 \uparrow} & p_{\bar{v} \mu \uparrow \rightarrow \tilde{v} 3 \uparrow} \\ p_{\bar{v} \tau \uparrow \rightarrow \tilde{v} 1 \uparrow} & p_{\bar{v} \tau \uparrow \rightarrow \tilde{v} 2 \uparrow} & p_{\bar{v} \tau \uparrow \rightarrow \tilde{v} 3 \uparrow}\end{array}\right]$,

$\left[P_{F \downarrow \rightarrow \tilde{E} \downarrow}\right]=\left[\begin{array}{ccc}p_{v e \downarrow \rightarrow \tilde{v} 1 \downarrow} & p_{v e \downarrow \rightarrow \tilde{v} 2 \downarrow} & p_{v e \downarrow \rightarrow \tilde{v} 3 \downarrow} \\ p_{v \mu \downarrow \rightarrow \tilde{v} 1 \downarrow} & p_{v \mu \downarrow \rightarrow \tilde{v} 2 \downarrow} & p_{v \mu \downarrow \rightarrow \tilde{v} 3 \downarrow} \\ p_{v \tau \downarrow \rightarrow \tilde{v} 1 \downarrow} & p_{v \tau \downarrow \rightarrow \tilde{v} 2 \downarrow} & p_{v \tau \downarrow \rightarrow \tilde{v} 3 \downarrow}\end{array}\right]$.

In the subscripts, $F$ and $\bar{F}$ represent flavored version neutrinos and anti-neutrinos, respectively; $\tilde{E}$ represents eigenatate version neutrinos; the $\uparrow$ and $\downarrow$ represent right handedness and left handedness, respectively.

Explanation: The distinctive feature of Rule 17.3 is that, in the converting process, the handedness does not change evidenced by the non-diagonal sub-matrixes all equal to zero as shown by (17.26a) and (17.26b). According to Rule 17.3, the flavored anti-neutrinos only convert to the eigenstate neutrinos with right handedness, the flavored neutrinos only convert to the eigenstate neutrinos with left handedness, and vice versa. According to (17.24), when flavored neutrinos convert to eigenstate neutrinos, the particle versus anti-particle distinction is no longer valid. Nevertheless, the original distinction for flavored neutrinos and anti-neutrinos leave marks on their eigenstate version by different types of handedness. A new born flavored neutrino immediately converts to an eigenstate neutrino according to (17.26a), When caught by a detector it converts back to the flavored version according to (17.26b). The off-diagonal sub-matrixes equal to zero guarantee that, as the net result of two processes, the lepton numbers are conserved.

Conclusion 17.1: Eigenstate neutrinos $\tilde{v}_{1}, \tilde{v}_{2}, \tilde{v}_{3}$ have no electrical charge.
Proof: Assuming $\tilde{v}_{i}, i=1,2,3$ has charge $\Delta q$ and $v_{i}$ has charge $-\Delta q$. According to Hypothesis 17.1, $\overline{\widetilde{v}}_{i}=\tilde{v}_{i}$, then $\Delta q=-\Delta q$ and $\Delta q=0$.

QED

According to Hypothesis 17.1, the flavored neutrinos include six members $v_{e}, v_{\mu}, v_{\tau}, \bar{v}_{e}, \bar{v}_{\mu}, \bar{v}_{\tau}$ and the eigenstate neutrinos include three members $\tilde{v}_{1}, \tilde{v}_{2}, \tilde{v}_{3}$. The flavored anti-neutrinos and neutrinos are different particles. Their model is Option-1 type. The eigenstate neutrinos have no counter-particles, their model is Option-2 type. These two models look very different. In essence, they are closely correlated by degeneration as described previously.

According to Conclusion 17.1, the eigenstate neutrinos have no electrical charge. According to Theorem 17.1, it seems that the flavored neutrinos and anti-neutrinos also have no electrical charge. Look at it closely, there is an uncertainty. Theorem 17.1 is based on geometry of Option-1 model and Fact-2. The unique handedness stated in Fact-2 corresponds to particle's spin orientation with respect to its momentum. But the flavored neutrinos and anti-neutrinos never fly. Only the eigenstate neutrinos fly and have persist momentum. Therefore, the unique handedness stated in Fact-2 cannot refer to the flavored version. Whither the flavored version neutrinos and anti-neutrinos have remnant electrical charge or not, it cannot be determined by Theorem 17.1 along. On the other hand, the eigenstate version neutrinos are without electrical charge for sure according to Conclusion 17.1. Based on conservation of electrical charge, should the flavored version neutrinos and anti-neutrinos also have no electrical charge? Consider all these facts and factors, SQS theory intends to favor no remnant electrical charge for the flavored version neutrinos and anti-neutrinos as well.

On the experimental side, neutrino electrical charge data are not officially listed in 2010-PDG. Instead, it cites data from seven authors for the neutrinos charge upper limits ranging form $3 \times 10^{-4} e$ to $2 \times 10^{-15} e$. Neutrinos magnetic moment upper limit data from three authors are listed in 2010-PDG ranging from $3.9 \times 10^{-7} \mu_{B}$ to $0.54 \times 10^{-10} \mu_{B}$. These experimental data are not conclusive. More experimental works are needed.

In the visible universe, matters overwhelmingly dominate antimatters. On the other hand, when the universe was born, the big bang should produce equal amount of matters and antimatters. Over the years, physicists were puzzled by the "missing antimatters" question: Where are these antimatters? Hypothesis 17.2 may provide the answer.

Hypothesis 17.2: The eigenstate neutrinos $\tilde{v}_{1}, \tilde{v}_{2}, \tilde{v}_{3}$ flying around in the universe provide a possible solution for the "missing antimatters".

Explanation: Hypothesis 17.2 is based on two conditions: Condition-1: 2010-PDG data book stated that, the "baryon density of the universe" is $\Omega_{b}=0.044(4)$ and "neutrino density of the universe" is $0.0009<\Omega_{v}<0.048$. If 


\section{Z. Y. SHEN}

further observations confirm $\Omega_{v}=0.048 \approx \Omega_{b}=0.044(4)$, it will serve as the foundation of Condition- 2 .

Condition-2: If condition-1 is confirmed, then the question becomes: Are all these flying around eigenstate neutrinos with right handedness? Since the handedness of eigenstate neutrinos are not directly measurable, an indirect way is suggested to detect the cosmological originated neutrinos and measure the converted flavored neutrinos' handedness. According to Rule 17.3, the handedness of eigenstate neutrinos can be determined. There are three possible outcomes: 1, All anti-neutrinos have right handedness, the "missing antimatters" are found; 2 , More than $50 \%$ of anti-neutrinos have right handedness, part of the "missing antimatters" are found. 3 , Less than $50 \%$ of anti-neutrinos have right handedness, Hypothesis is disproved.

In this section, SQS theory provided a framework with the potential to explain neutrinos peculiar behaviors based on mathematics. Because of the complexity of the topic and limited available experimental data, some parameters haven't nailed down yet and many issues remain open. Some of them will be discussed in later sections.

\section{Section 18: Elementary Particles Table}

Elements periodic table not only is useful for understanding chemical elements but also valuable for exploring what's behind scene and for predicting new elements. Elementary particles table should do the same for physics at a deeper level.

For comparison purpose, Table 18.1 shows the Standard Model (SM) Elementary Particles Table.

Table 18.1: Standard Model (SM) Elementary Particles Table

\begin{tabular}{|c|c|c|c|c|c|c|}
\hline \multicolumn{4}{|c|}{ Fermions } & \multicolumn{2}{|c|}{ Bosons } & Summary \\
\hline \multirow[t]{2}{*}{ Quarks } & $d$ & c & $t$ & \multirow{4}{*}{$\begin{array}{l}\text { Gauge } \\
\text { bosons }\end{array}$} & $\gamma$ & \multirow{3}{*}{$\begin{array}{lr}\text { Fermion } & 12 \\
\text { Boson } & 12 \\
\text { Higgs boson } & 1\end{array}$} \\
\hline & $u$ & $s$ & $b$ & & 8 gluons & \\
\hline \multirow[t]{2}{*}{ Leptons } & $v_{\varepsilon}$ & $v_{\mu}$ & $v_{\varepsilon}$ & & $Z^{0}$ & \\
\hline & $e$ & $\mu$ & $\tau$ & & $W^{ \pm}$ & Total \\
\hline
\end{tabular}

In Table 18.1, there are 12 fermions and 12 bosons plus the higgs boson. The total number of elementary particles is 25 in which anti-particles are not included.

After introduced almost all elementary fermions and bosons in previous sections, SQS theory is ready to introduce the Elementary Particles Table as Table 18.2.

Table 18.2 does not list the six flavored neutrinos $v_{e}, v_{\mu}$, $v_{\tau}$ and anti-neutrinos $\bar{v}_{e}, \bar{v}_{\mu}, \bar{v}_{\tau}$, instead the three eigenstate neutrinos $\tilde{v}_{1}, \tilde{v}_{2}, \tilde{v}_{3}$ are listed. It is an important issue. The eigenstate version $\tilde{v}_{1}, \tilde{v}_{2}, \tilde{v}_{3}$ and the flavored version $v_{e}$, $v_{\mu}, v_{\tau}, \bar{v}_{e}, \bar{v}_{\mu}, \bar{v}_{\tau}$ are equivalent. SQS theory Elementary Particles Table must choose one version to list not both versions. The question is: Which version should be chosen?
As indicated in Section 17, the flavored version exist only in an extremely short time at their birth or been detected. On the other hand, the eigenstate version are flying in the universe all time, some of them since the big bang 13.7 billion years ago. Look at this way, the choice is justified.

Table 18.2: SQS Theory Elementary Particles Table

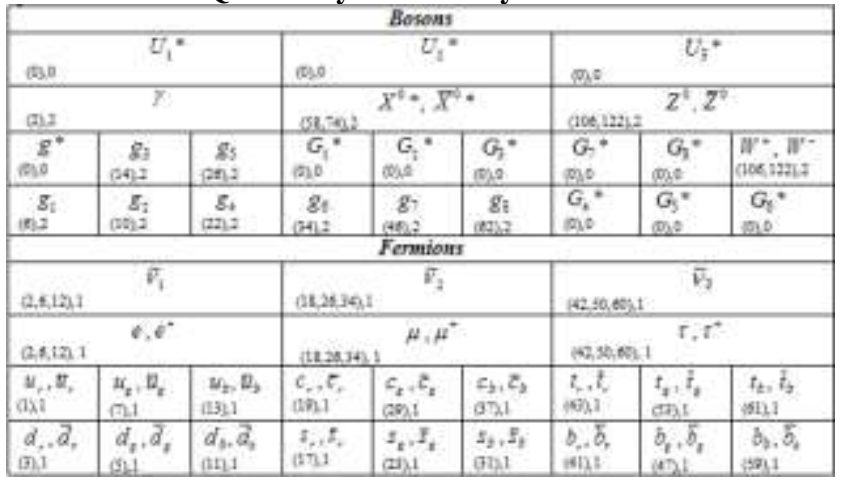

Notes: 1 . The particles marked with * are hypothetic particles.

2. Number in parenthesis is original $m$-parameter, number after parenthesis is effective $m$-parameter.

In previous sections, close correlations were established between bosons and fermions. The 8 gluons are made of 8 pairs of quark-antiquark shown by (13.1). The 8 hypothetic massons are made of the other 8 pairs of quark-antiquark shown by (14.6). These 16 bosons fit into 16 cells in the boson row of Table 18.2. On the upper left corner, the hypothetic graviton $g$ fits into the cell correlated to $u_{r}$; on the upper right corner, the $W$ boson fits into the cell correlated to $t_{b}$.

On the top row of table 18.2, three scalar bosons $U_{1}, U_{2}$, $U_{3}$ fit into the cells correlated to three eigenstate neutrinos $\tilde{v}_{1}, \tilde{v}_{2}, \tilde{v}_{3}$, respectively. In the next row, photon $\gamma$ and gauge boson $Z$ fit into the two cells correlated to leptons $e$ and $\tau$, respectively. Another hypothetical neutral gauge boson $X^{0}$ fits into the middle cell correlated to lepton $\mu$, which will be introduced later in this section.

Comparing these two Tables, there are some similarities, but the differences are obvious.

1. The major difference is the numbers of quarks. In the SM table, the number is 6. In the SQS table, the number is 18. The key is Postulation 11.1 based on Postulation 11.2 and Rule 11.1, which recognize quarks with the same flavor and different colors as different particles. It is a major step to open doors for new opportunities. It gives the hint that lepton has three branches to form the trefoil model. More importantly, it provided more cells for bosons. Otherwise, there is no room for 13 hypothetic bosons.

2. In the SM table, graviton is not included, because SM theory does not include gravity. The SQS table includes graviton $g$, because SQS theory aims at the grand 
unification for all four types of interactions, which should include graviton as the mediator of gravity.

3. In addition to graviton, the SQS table includes twelve other hypothetic bosons, in which $U_{1}, U_{2}, U_{3}$ and $G_{i},(i=1,2, \cdots 8)$ are scalar bosons, $X^{0}$ is a gauge boson. These hypothetic bosons are not included in the SM table.

4. SM theory includes the higgs boson. SQS theory does not need it.

5. In the SM table, there is no clear correlation between fermions and bosons. In the SQS table, the correlation between fermions and bosons is clear, which is important for predicting new particles.

In SQS theory, elementary particles are categorized into three types: particle, antiparticle and neutral (not necessary electrical neutral) particle. The neutral particle $p$ is defined as:

$$
\bar{p}=p \text {. }
$$

According to (13.2), gluons are neutral particles. According to (14.7), massons are neutral particles. According to SQS theory, photon is $\gamma=e^{-} e^{+}$and graviton is $g=u_{r} \bar{u}_{r}$ :

$$
\begin{aligned}
& \bar{\gamma}=\overline{e^{-} e^{+}}=\overline{e^{-}} \overline{e^{+}}=e^{+} e^{-}=e^{-} e^{+}=\gamma, \\
& \bar{g}=\overline{u_{r} \bar{u}_{r}}=\bar{u}_{r} u=u_{r} \bar{u}_{r}=g,
\end{aligned}
$$

$U_{1}, U_{2}, U_{3}$ are scalar bosons with mass and without charge, which also have no separate anti-particles:

$$
\bar{U}_{i}=U_{i}, i=1,2,3 \text {. }
$$

Therefore, photons $\gamma$, gravitons $g$ and $U_{1}, U_{2}, U_{3}$ are neutral particles.

There are three gauge bosons left: $W^{ \pm}, Z^{0}$ and the hypothetic $X^{0}$. According to PDG data, all properties of $W^{+}$ and $W^{-}$are the same except charge. Even the decay modes and branching ratios $\Gamma_{i} / \Gamma_{\text {total }}$ of $W^{+}$and $W^{-}$are charge conjugates. These experimental data clearly show that, $W^{+}$ and $W^{-}$are a pair of particle and anti-particle. According to SQS theory, all neutral elementary bosons $\gamma, g, g_{1}, g_{2}, g_{3}$, $g_{4}, g_{5}, g_{6}, g_{7}, g_{8}, G_{1}, G_{2}, G_{3}, G_{4}, G_{5}, G_{6}, G_{7}, G_{8}, U_{1}$, $U_{2}, U_{3}$ made of a pair of fermion and the same type antifermion have no separate anti-particles. $Z^{0}$ and $X^{0}$ are different, because they are made of a pair of fermion and different type anti-fermion. According to Table 14.2 and (18.12), they have separate anti-particles:

$$
\begin{aligned}
& \overline{Z^{0}}=\overline{Y_{1} \oplus \bar{Y}_{2}}=\bar{Y}_{1} \oplus Y_{2} \neq Z^{0}=Y_{1} \oplus \bar{Y}_{2} \\
& \overline{X^{0}}=\overline{X_{f 1} \oplus \bar{X}_{f 2}}=\bar{X}_{f 1} \oplus X_{f 2} \neq X^{0}=X_{f 1} \oplus \bar{X}_{f 2} .
\end{aligned}
$$

In the SQS theory Elementary Particles Table, the three scalar bosons $U_{1}, U_{2}, U_{3}$ for unifications are the heaviest particle in each column of the table. The three eigenstate neutrinos $\tilde{v}_{1}, \tilde{v}_{2}, \tilde{v}_{3}$ correlated to $U_{1}, U_{2}, U_{3}$ are the lightest fermion in each column of the table. Why is the lightest correlated to the heaviest? Because the mass of $\tilde{v}_{1}$, $\tilde{v}_{2}, \tilde{v}_{3}$ is in the same order as the mass of $\bar{v}_{e}, \bar{v}_{\mu}, \bar{v}_{\tau}$, respectively. Let's look at the formulas derived from (17.5) for orders of magnitudes comparison:

$$
\begin{aligned}
& {\left[\left(\frac{\alpha}{2}\right)^{2}(1-\alpha) \frac{M_{e w}}{M_{\bar{v} e}}\right]=\left(\frac{M_{e w} M_{W}}{M_{e}^{2}}\right)=4.703 \times 10^{10},} \\
& {\left[\left(\frac{\alpha}{2}\right)^{2}(1-\alpha) \frac{M_{e w s}}{M_{\bar{v} \mu}}\right]=\left(\frac{M_{e w s} M_{W}}{M_{e} M_{\mu}}\right)=1.258 \times 10^{22},} \\
& {\left[\left(\frac{\alpha}{2}\right)^{2}(1-\alpha) \frac{M_{G U T}}{M_{\bar{v} \tau}}\right]=\left(\frac{M_{G U T} M_{W}}{M_{e} M_{\tau}}\right)=9.559 \times 10^{22} .}
\end{aligned}
$$

In which $M_{e w}, M_{e w s}, M_{G U T}$ are the mass of $U_{1}, U_{2}, U_{3}$, respectively. It is interesting to notice that, the numbers of second and third formulas for $U_{2}, U_{3}$ versus $\bar{v}_{\mu}, \bar{v}_{\tau}$ are $G=2$ grand numbers, while the number of first formula for $U_{1}$ versus $\bar{v}_{e}$ is close to $G=1$ grand number. The apparent reason for such difference is that, $M_{e w}$ is $\sim 10^{-13}$ orders lighter than $M_{e w s}$. The mechanism of such difference is an open issue. Despite this difference, (18.6) implies that, the reason for the lightest correlated to the heaviest has something to do with grand numbers related to random walk. It usually is the origin of hierarchy phenomena.

\begin{tabular}{|c|c|c|c|c|c|c|c|c|c|c|c|}
\hline \multicolumn{4}{|c|}{ Particles } & \multicolumn{4}{|c|}{ Neutral Particles } & \multicolumn{4}{|c|}{ Antiparticles } \\
\hline \multicolumn{2}{|c|}{ Fermilons } & \multicolumn{2}{|c|}{ Bosons } & \multicolumn{2}{|c|}{ Fermions } & \multicolumn{2}{|c|}{ Bosoes } & \multicolumn{2}{|c|}{ Fermioas } & \multicolumn{2}{|c|}{ Bosons } \\
\hline Quant: & Lewax & $\begin{array}{l}\text { Gongt } \\
\text { benent: }\end{array}$ & $\begin{array}{l}\text { Salifif } \\
\text { bovenes }\end{array}$ & $\begin{array}{l}\text { Meneral } \\
\text { gearits }\end{array}$ & $\mid \begin{array}{l}\text { Nentar } \\
\text { leghes }\end{array}$ & 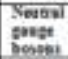 & 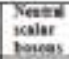 & \begin{tabular}{|l|} 
Ast: \\
quaita
\end{tabular} & $\begin{array}{l}\text { Teb } \\
\text { lephes }\end{array}$ & 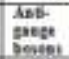 & 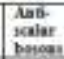 \\
\hline 18 & 3 & 3 & 0 & 0 & 3 & 9 & 12 & 18 & 3 & 3 & 0 \\
\hline \multicolumn{2}{|c|}{21} & \multicolumn{2}{|c|}{3} & \multicolumn{2}{|r|}{3} & \multicolumn{2}{|c|}{21} & \multicolumn{2}{|c|}{21} & \multicolumn{2}{|c|}{3} \\
\hline \multicolumn{4}{|c|}{24} & \multicolumn{4}{|c|}{24} & \multicolumn{4}{|c|}{24} \\
\hline \multicolumn{12}{|c|}{72} \\
\hline
\end{tabular}

If "the heaviest" is really made of a pair of "the lightest", massons may play the role to fill the tremendous mass gaps between $U_{1}, U_{2}, U_{3}$ and $\tilde{v}_{1}, \tilde{v}_{2}, \tilde{v}_{3}$. After all, the arrangement for $U_{1}, U_{2}, U_{3}$ correlated to $\tilde{v}_{1}, \tilde{v}_{2}, \tilde{v}_{3}$ in the SQS Elementary Particles Table is justified.

Table 18.3 shows the numbers of three types particles in SQS theory.

Table 18.3: Three Categories of Elementary Particles in Table 18.2

The three types particles are:

1. Particles: This type includes 18 quarks $u_{r}, u_{g}, u_{b}, d_{r}$, $d_{g}, d_{b}, s_{r}, s_{g}, s_{b}, c_{r}, c_{g}, c_{b}, b_{r}, b_{g}, b_{b}, t_{r}, t_{g}, t_{b}$, 3 leptons $e, \mu, \tau$, and 3 gauge bosons $W^{+}, Z^{0}, X^{0}$.

2. Antiparticles: This type includes 18 anti-quarks $\bar{u}_{r}, \bar{u}_{g}$, $\bar{u}_{b}, \bar{d}_{r}, \bar{d}_{g}, \bar{d}_{b}, \bar{s}_{r}, \bar{s}_{g}, \bar{s}_{b}, \bar{c}_{r}, \bar{c}_{g}, \bar{c}_{b}, \bar{b}_{r}, \bar{b}_{g}, \bar{b}_{b}$, $\bar{t}_{r}, \bar{t}_{g}, \bar{t}_{b}, 3$ anti-leptons $e^{+}, \mu^{+}, \tau^{+}$, and 3 anti-gaugebosons $W^{-}, \bar{Z}^{0}, \bar{X}^{0}$.

3. Neutral particles: This type includes 3 neutral leptons $\tilde{v}_{1}$, $\tilde{v}_{2}, \tilde{v}_{3} .9$ neutral gauge bosons $\gamma, g_{1}, g_{2}, g_{3}, g_{4}$, 
$g_{5}, g_{6}, g_{7}, g_{8}$, and 12 neutral scalar bosons $g, G_{1}$, $G_{2}, G_{3}, G_{4}, G_{5}, G_{6}, G_{7}, G_{8}, U_{1}, U_{2}, U_{3}$.

Definition 18.1: Graviton is a scalar boson with spin 0 .

Explanation: In other quantum gravity theories, graviton is assigned with spin $2 \hbar$. Since graviton has not been found, there is no experimental confirmation. In the SQS theory Elementary Particles Table, there is no place for graviton as a tensor boson with spin $2 \hbar$. According to the Random Walk Theorem in Section 4 and the discussion regarding electromagnetic force and gravitational force in Section 15, photon and graviton are two sides of the same coin. It is also shown in their models. According to SQS theory, photon model is a closed single loop with circumferential length of $2 L_{P}$ corresponding to spin $\hbar$; while graviton model is a cutoff loop with length of $2 L_{P}$. In essence, a graviton is a cutoff photon. A cutoff loop has zero spin. In terms of number parameters, as $q_{r}$ with $m_{q r}=1$, there is no way for $g=q_{r} \bar{q}_{r}$ to make graviton with $m_{g}=4$ required by spin $2 \hbar$. In short, graviton with spin $2 \hbar$ does not fit into SQS theory framework. There is also a philosophical reason. As shown in Section 15, gravity is the force to unify all forces. Gravity as the only force exerts to everything with mass or energy. In essence, gravity is the most fundamental and universal force, and so is graviton. According to natural philosophy, the most fundamental and universal thing should be the simplest one. Obviously, 0 fits this argument much better than $2 \hbar$. Is a scalar boson qualified as the mediator for interaction? Why not? $g$ with spin 0 is different from $\gamma, W^{ \pm}, Z^{0}$ and $g_{i}(i=1,2 \cdots 8)$ with spin $\hbar$. It just means $g$ is unique. Let's face it, gravity is unique in the first place. After all, there is no law forbidding the unique scalar boson $g$ serving as the mediator for the unique force gravity. Look at it the other way. A graviton center is at a discrete point $x_{i}$, the two ends stretch to $x_{i}+1$ and $x_{i}-1$. This scenario is closely related to the Random Walk Theorem. Starting from $x_{i}$ along the $x$-direction, the random walk has equal chance moving to $x_{i}+1$ or $x_{i}-1$ as the next step.

The same is for $y$-direction and $z$-direction. In fact, there is a hidden question in the Random Walk Theorem: Why the step length is 1 not 2 or 3 etc? The graviton's cutoff model provides the answer: Because the length of graviton only allows each step moving to \pm 1 .

Otherwise the Random Walk Theorem is in trouble. Graviton as a cutoff loop with length of $2 L_{P}$ fits the

Random Walk Theorem naturally. Assume that, the middle point of three orthogonal straight lines each with normalized length 2 is attached at each discrete point $p\left(x_{i}, y_{j}, z_{k}\right)$ with their six ends reaching to $x_{i} \pm 1$,

$y_{j} \pm 1, z_{k} \pm 1$ along $\pm x, \pm y, \pm z$ directions,

respectively. This arrangement not only fits the random walk process but also forms a network serving as the spatially quantized gravitational field. The three orthogonal straight lines represent three gravitons serving as the quanta of gravitational field. This scenario is much more natural than what graviton with spin $2 \hbar$ can offer. Moreover, a topological theorem provides a definitive support for graviton having spin 0 as the only option, which will be given in Section 25,

Most theories recognized graviton with spin $2 \hbar$. SQS theory probably is the only one recognizes graviton with spin 0 . It is a bold and risky undertaking. But within SQS theory framework there is no alternative. From SQS theory perspective, graviton with spin $2 \hbar$ is a misunderstanding. As shown in Fig. 18.1(a), two head-to-tail connected gravitons form a closed loop with loop length $4 L_{P}$ corresponding to spin $2 \hbar$, which might be mistakenly recognized as graviton. Actually it is merely a composite state made of two gravitons. Moreover, as shown in Fig. 18.1(b) and Fig. 18.1(c), when the loop area increases, the trajectory angular momentum increases in step of $\hbar$ not in step of $2 \hbar$. So graviton with spin $2 \hbar$ is a misunderstanding. Of cause, the final proof has to wait until the discovery of graviton.

Definition 18.1 serves as a prediction of SQS theory. Let's wait for graviton show up to say the final word.
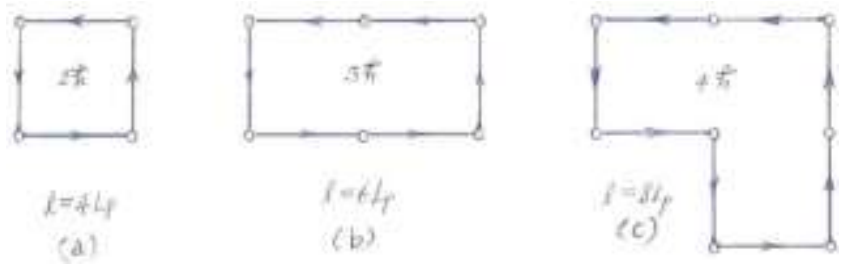

Fig. 18.1: Closed loops made of "head-to-tail" connected gravitons: (a) two gravitons, (b) three gravitons, (c) four gravitons.

There are thirteen hypothetic bosons listed in Table 18.2, in which twelve of them are introduced in previous sections. The only one left is the neutral gauge boson $X^{0}$.

Like the $W^{ \pm}$and $Z^{0}$, according to SQS theory, the gauge boson $X^{0}$ is also involved in weak interactions. As shown in Section $14, W^{ \pm}$and $Z^{0}$ are made of two fermion states $Y_{1}$ and $Y_{2}$, which share the same $m$-parameters with two top quarks $t_{g}, t_{b}: m_{Y 1}=m_{t g}=53$ and $m_{Y 2}=m_{t b}=61$. Similarly, the two fermion states $X_{f 1}$ and $X_{f 2}$ to make the $X^{0}$ boson share the same $m$-parameters with two charm quarks $c_{g}$ and $c_{b}: m_{X f 1}=m_{c g}=29$ and $m_{\mathrm{Zf} 2}=m_{c b}=37$. It is interesting to find out that, these two sets of $m$-parameters have something in common:

$$
\begin{aligned}
& 61-53=8=2^{3}, 61+53=114=2 \times 3 \times 19 \\
& 37-29=8=2^{3}, 37+29=66=2 \times 3 \times 11
\end{aligned}
$$


In fact, there are more similarities, which will be shown in Section 23.

The mass of $X^{0}$ boson is determined by the values of its $x_{1}$ and $x_{2}$ on the $x$-axis. Let's look at the special points on $x$-axis. The special point $x_{c}=0.249987156273026$ is a magic point. It is originated from the $S$-function of (2.11) and sets a slight deviation of anti-symmetry of $\Delta S(x)$ with respect to the center $x=0.25$ of region $[0 \leq x \leq 0.5]$. Point $x_{c}$ sets the boundaries of the gauge boson region $\left[x_{c} \leq x \leq x_{d}=0.5-x_{c}\right]$, and it sets a mass upper limit $M_{\text {Max }}=4.9732 \mathrm{GeV} / \mathrm{c}^{2}$ for standalone fermions. Moreover, $x_{c}$ defined other two characteristic points $x_{a}$ and $x_{b}$, which determined the mass of two scalar bosons $U_{1}$ and $U_{2}$ for electro-weak and electroweak-strong unifications. As shown in Section 15, this approach is closely related to the other approach based on random walk and unification of electrostatic force with gravity. It provided the legitimacy of both approaches. Point $x_{a}$ and point $x_{b}$ succeeded for finding two scalar bosons owe gauge boson a favor. It is the time for them to pay back. Definition 18.2: Based on $x_{a}=5.181994687988211 \times 10^{-10}$, $x_{b}=1.1821861791847719 \times 10^{-5}$ and the $S$-function of (2.11): $S(x)=\sum_{j=-\infty}^{\infty} e^{-\pi(x-j)^{2}}$, define two characteristic points $x_{f}$ and $x_{g}$ on the $x$-axis.

$$
\begin{aligned}
& \int_{x_{a}}^{x_{b}} S(x) d x-\int_{x_{f}}^{0.25} S(x) d x=0, \\
& x_{f}=0.24998715683601635, \\
& x_{g}=0.25001284316398365 .
\end{aligned}
$$

The summation index in $S(x)$ is truncated at $j= \pm 1000$, which is sufficient for convergence.

Notice that, $x_{f}$ and $x_{g}$ both are in the gauge boson region:

$$
\begin{aligned}
& x_{c}=0.249987156273026<x_{f}<0.25, \\
& 0.25<x_{g}<x_{d}=0.25001284372697355 .
\end{aligned}
$$

According to (6.18), the mass of two $X$-fermion states $X_{f 1}$, $X_{f 2}$ with $x_{1}=x_{f}, x_{2}=x_{g}$ is:

$M_{X f}=\frac{M_{e}}{4\left(x_{2}-x_{1}\right)}=\frac{M_{e}}{4\left(x_{g}-x_{f}\right)}=4.97345232 \mathrm{GeV} / \mathrm{c}^{2}$.

Because $x_{1}=x_{f}$ and $x_{2}=x_{g}$ are in the boson region and $M_{X f}>M_{M a x}$, the $X$-fermion states $X_{f 1}$ and $\bar{X}_{f 2}$ must appear in pair as a gauge boson $X^{0}$ with mass $M_{X}$ :

$$
M_{X}=2 M_{X f}=9.94690465 \mathrm{GeV} / c^{2} .
$$

The gauge boson $X^{0}$ is made of two fermion states $X_{f 1}$ and $\bar{X}_{f 2}$, which have the same mass and different before reduction numerical parameters. The gauge boson $X^{0}$ has its anti-particle $\bar{X}^{0}$ different from $X^{0}$.

$$
\begin{aligned}
& X^{0}=X_{f 1} \oplus \bar{X}_{f 2}, \\
& \overline{X^{0}}=\overline{X_{f 1} \oplus \bar{X}_{f 2}}=\bar{X}_{f 1} \oplus X_{f 2} \neq X^{0} .
\end{aligned}
$$

In the gauge boson region $\left[x_{c}, x_{d}\right], X^{0}$ boson has $\pi\left(x_{1}\right)<\pi$ and $\pi\left(x_{2}\right)<\pi$ :

$$
\begin{aligned}
& \pi\left(x_{1}=x_{f}\right)=3.14159265166911<\pi, \\
& \pi\left(x_{2}=x_{g}\right)=3.14150502884738<\pi
\end{aligned}
$$

It indicates that, the torus based model for $X^{0}$ boson is spindle type with two branches like $Z^{0}$ bosons model.

Look it closely, $X_{f 1}$ and $\bar{X}_{f 2}$ having mass around $4.97 \mathrm{GeV} / \mathrm{c}^{2}$ are originated from $c_{g}$ and $c_{b}$ having mass around $1.38 \mathrm{GeV} / \mathrm{c}^{2}$ and $1.34 \mathrm{GeV} / \mathrm{c}^{2}$, there is a mass gap between them. The gap is filled by massons.

The parameters of electroweak interaction are represented by the $G W S$-triangle in charged particles' model as shown by Fig. 8.4. Fig. 18.2 shows the $G W S$-triangle in extended region including the $C K M$-triangle. Besides the $G W S$-triangle of $\mathrm{AFO}_{2}$, there is another triangle $\mathrm{O}_{1} \mathrm{O}_{2} \mathrm{~F}$ similar to $\mathrm{AFO}_{2}$. It is called the $S$-triangle for SQS theory. The similarities of $G W S$-triangle and $S$-triangle are:

1. They both are compounded right-angled triangles including two small similar right-angled triangles.

2. They both share the common side $\mathrm{O}_{2} \mathrm{~F}$, which represents the $g^{\prime}$-type weak interaction.

3. They both have a long side: $\mathrm{O}_{2} \mathrm{~A}$ for $\mathrm{SWG}$-triangle and $\mathrm{O}_{1} \mathrm{O}_{2}$ for $\mathrm{S}$-triangle.

The similarities imply that the $S$-triangle also involves in some type of weak interactions. $W^{ \pm}$and $Z^{0}$ serve for electroweak interactions represented by $G W S$-triangle. It is natural to assume that, $X^{0}$ boson serves for the weak interactions represented by $S$-triangle. The $S$-triangle joints $G W S$-triangle on its right and links to $C K M$-triangle on its left. Noticed that, the $x_{a}$ and $x_{b}$ in the first integral term of (18.8a) served as two characteristic points to define $U_{1}$ and $U_{2}$ for electro-weak unification and electroweak-strong unification, respectively. Consider these factors as the clues to guess its function, $X^{0}$ boson is probably involved in some type of weak interactions responsible for hadrons decay. Of cause, more supportive evidences are needed for sure.

As shown in Fig. 18.2, besides the common side $\mathrm{O}_{2} \mathrm{~F}$ shared with $G W S$-triangle, $S$-triangle has two other sides: $O_{1} F$ labeled $g_{S}$ represents a $g$-like weak interaction and $F P$ labeled $q_{s}$ represents a charge of some kind.

According to trigonometry, the relations among $e, q_{S}, g^{\prime}$, $g_{S}$ and angle $\phi_{2}, \theta_{W}$ are: 


$$
\begin{aligned}
& g_{S} \tan \phi_{2}=g \tan \theta_{W}=g^{\prime}, \\
& \frac{q_{S}}{\cos \phi_{2}}=\frac{e}{\cos \theta_{W}}=g^{\prime}, \\
& e q_{S}=g^{\prime 2} \cos \theta_{W} \cos \phi_{2} .
\end{aligned}
$$

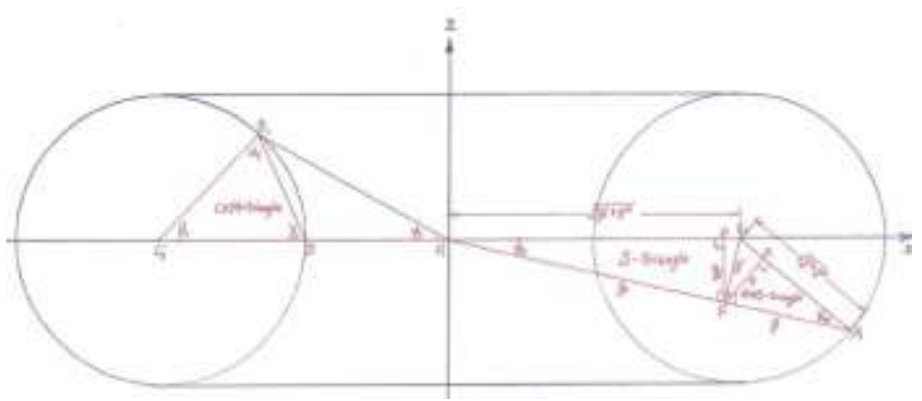

Fig.18.2: The $S$-triangle between $G W S$-triangle and $C K M$-triangle.

Based on the SQS theory Elementary Particles Table, the total number of particles and anti-particles is 72. Are all these particles elementary? At this level, yes! Is this the deepest level? It is a good question.

Look back to history, in the early twenty century, only four "elementary" particles were known, i.e. electron, proton, neutron, and photon. Since then, so many particles showed up; the numbers of particles kept growing. People started to think that so many particles cannot be all elementary; at a deeper level things could be simplified. The quark model was introduced, which reduced the numbers of elementary particles by more than tenfold at that time. Now, we probably are in the same situation.

Suppose there is a deeper level. What is it? To answer this question, let's go back to the fundamentals. As indicated by the name: Stochastic Quantum Space. SQS theory is a theory of space. Vacuum is the ground level of space. According to SQS theory, everything including all particles and interactions are originated from vacuum. Particles are excitations of vacuum, interactions are ripples through vacuum. In short, there is nothing but different states of vacuum. As emphasized in Section 3, SQS theory treats vacuum as a continuum with Planck scale grainy structure. Now let's take a step further to introduce the third fundamental postulation of SQS theory.

Postulation 18.1, The Vacuon Postulation: Vacuum is a quantum field. All different fields for particles and interactions are originated from this Mother Field. Ultimately, vacuum field is the only field, vacuon as the particle of vacuum field is the only elementary particle at the deeper level.

Explanation: Vacuon as the ultimate elementary particle has no dimension, no structure, no model, no mass, no spin, no charge of any kind, no anti-particle, no interaction, and no parameters except its location and movement. In short, vacuon is nothing but itself. Yet everything in the universe ultimately is made of vacuons. Vacuon creates everything from nothing. It is the ultimate elementary particle of SQS theory.

The closest thing for vacuon is a geometrical point. There are uncountable infinite numbers of it in the space continuum. Vacuons are free to move without interaction. It can be traced back to the first fundamental postulation of SQS theory, the Gaussian Probability Postulation. Gaussian probability is based on the precondition that, the events must be statistically independent. Otherwise, the probability is not Gaussian. Vacuons create events serving as the carriers of Gaussian probability. The events of Gaussian probability are independent, so are vacuons.

Models and trajectories of all elementary particles are different patterns of moving vacuons. Vacuon movement is to reach evenness. In Section 2, Gaussian probability function is assigned to each discrete point $x_{i}$. The superposition of all these probabilities is not evenly distributed. It has peak at $x_{i}$ and trough in between peaks. The unevenness drives vacuons moving from peak to trough for temporally even distribution. But the momentum keeps vacuons going and the temporally even distribution becomes uneven again. Like a pendulum, the vacuons oscillation goes on and on.

Is the unevenness acting as an interaction for vacuons? For particle physicist, interaction is synonymous to force. The tendency for vacuons reaching evenness is not an external force per se. But if one like to call it force, that is the only one.

Vacuon is the simplest thing you can think of, yet it has the capability to make all complex things in the universe. The key is Gaussian Probability Postulation, which laid the foundation.

Table 18.4 is the SQS theory Elementary Particle Table at the vacuon level. It serves as the foundation for all particles listed in the high level Elementary Particles Table of Table 18.2 .

Table 18.4: Elementary Particle Table at Vacuon Level

$$
\text { Vacuon }
$$

With the help of vacuon, SQS theory reduced the numbers of elementary particles from 72 to 1 at a deeper level.

Theorem 18.1: For a point particle such as vacuon moving with non-infinite speed, it has only 1-dimensional trajectories.

Proof: Assume that, a point particle moving with a noninfinite speed has a trajectory other than 1-dimensional such as a 2-dimensional surface. A surface, no matter how small it is, contains uncountable infinite 1dimensional lines. For the point particle with noninfinite speed to go through all lines on the surface, it requires infinite time to do so. That is impossible. Then the opposite must be true.

QED

Lemma 18.1: Theorem 18.1 is also valid for a set of point particles as long as all point particles in the set moving 
Journal of Modern Physics, 2013, 4.

along the same trajectory.

Proof: For a set of point particles moving in the same trajectory, the proof is the same as Theorem 18.1. QED

Lemma 18.2: The 1-dimensional trajectory for point particles can only change its location by discrete steps. In other words, trajectories are quantized in the space. Changing course is only allowed by jumping trajectories, continuously shifting trajectory is prohibited.

Proof: If the 1-dimentional trajectory were allowed to shift continuously, the shifting trajectory is no longer 1dimensional. It violates Theorem 18.1.

Theorem 18.1, Lemma 18.1 and Lemma 18.2 are based on geometry and point particle with non-infinite speed, which are universally valid. They serve as guidelines for vacuons movements, which have important implications shown in later sections.

In summary, SQS theory Elememtry Particles Table did its job. It provides a vacant cell for the gauge boson $X^{0}$ related to the $S$-triangle for some type of weak interaction. It confirms the eight massons and their scalar boson status. It reveals the correlations between fermions and bosons in general. In particular, without the table, the correlation of three scalar bosons $U_{1}, U_{2}, U_{3}$ to three eigenstate neutrinos $\tilde{v}_{1}, \tilde{v}_{2}, \tilde{v}_{3}$ is not so obvious. It helps to define graviton with spin 0 instead of $2 \hbar$. It counts the total numbers of elementary particles at this level to be 72 , no more and no less. Because 72 elementary particles are too many, it leads to the concept of vacuon at a deeper level.

\section{Section 19: Proton Neutron and Composite Particles}

In this section, proton, neutron and some simple composite particles such as helium nucleon, deuterium nucleon and tritium nucleon are discussed based on quarks models introduced in Section 11 and strong interactions introduced in Section 13.

Proton, neutron and some simple composite particles such as helium nucleon etc are made of up quarks and down quarks. According to the values listed in Table 11.2, the numerical parameters of up quarks and down quarks are summarized in Table 19.1.

Table 19.1: Numerical Parameters of Up Quarks and Down Quarks

\begin{tabular}{|c|c|c|c|c|c|c|c|c|c|}
\hline \multicolumn{9}{|c|}{ Up quarks } & \multicolumn{5}{c|}{ Down Quarks } \\
\hline Name & $u_{r}$ & $u_{\dot{z}}$ & $u_{b}$ & $u^{*}$ & Name & $d_{r}$ & $d_{z}$ & $d_{\dot{b}}$ & $d^{*}$ \\
\hline$m$ & 1 & 7 & 13 & 21 & $m$ & 3 & 5 & 11 & 19 \\
\hline$N$ & 1 & 3 & 5 & 9 & $n$ & 1 & 3 & 5 & 9 \\
\hline$P$ & 3 & 17.5 & 32.5 & 53 & $p$ & 13.5 & 20 & 49.5 & 83 \\
\hline$p / n$ & 3 & 5.833 & 6.5 & 15.333 & $p / n$ & 13.5 & 6.667 & 9.9 & 30.067 \\
\hline $2 p / m$ & 6 & 5 & 5 & 16 & $2 p / m$ & 9 & 8 & 9 & 26 \\
\hline
\end{tabular}

* Note: In the $u$ and $d$ columns, the numbers are the sum of three numbers in the same row.

A proton is made of $u u d$ and a neutron is made of $u d d$ and each flavored quark composed of three colored constituents with red, green and blue colors. The gluon connections diagrams of proton and neutron are shown in Fig.
19.1 and Fig. 19.2, respectively. The up quark and down quark with three colors are treated separately serving as the first level-colors level. The lines represent gluon connections for the regular type strong force. As shown in Fig.19.1, proton has 40 connections for 9 colored quarks corresponding to $4 \frac{4}{9}$ connections per colored quark. As

shown in Fig.19.2, neutron has 32 connections for 9 colored quarks corresponding to $3 \frac{5}{9}$ connections per colored quark. It is a fair comparison for proton and neutron, because they contain the some number (9) of colored quarks. Under these conditions, the number of connections per constituent serves as an index for the relative strength of the strong force to bind constituents. So proton is more tightly bound than neutron.

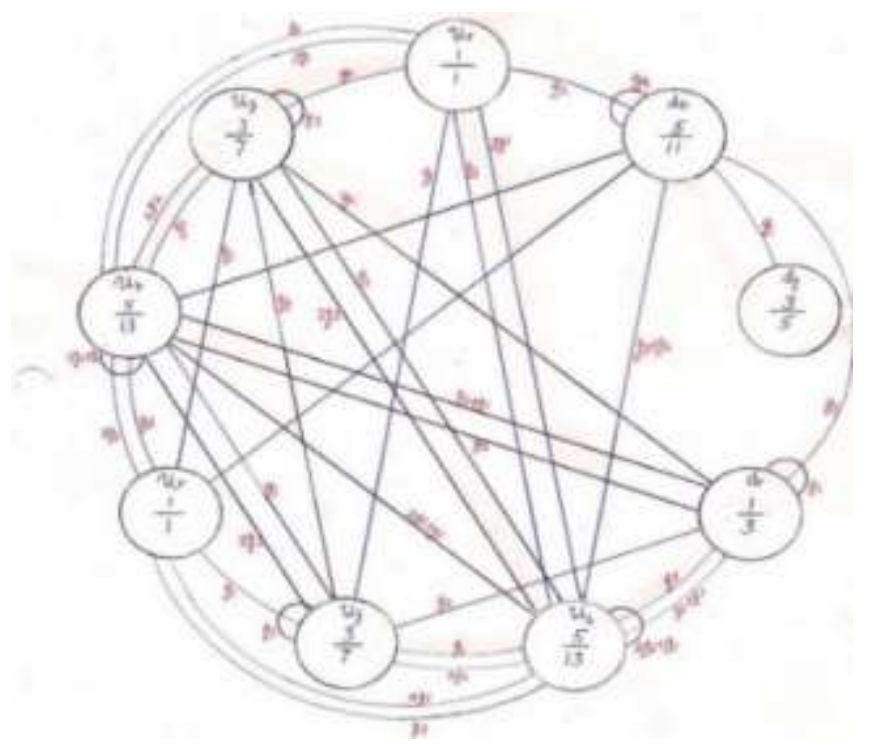

Fig.19.1: Gluon connections inside a proton at the first level.

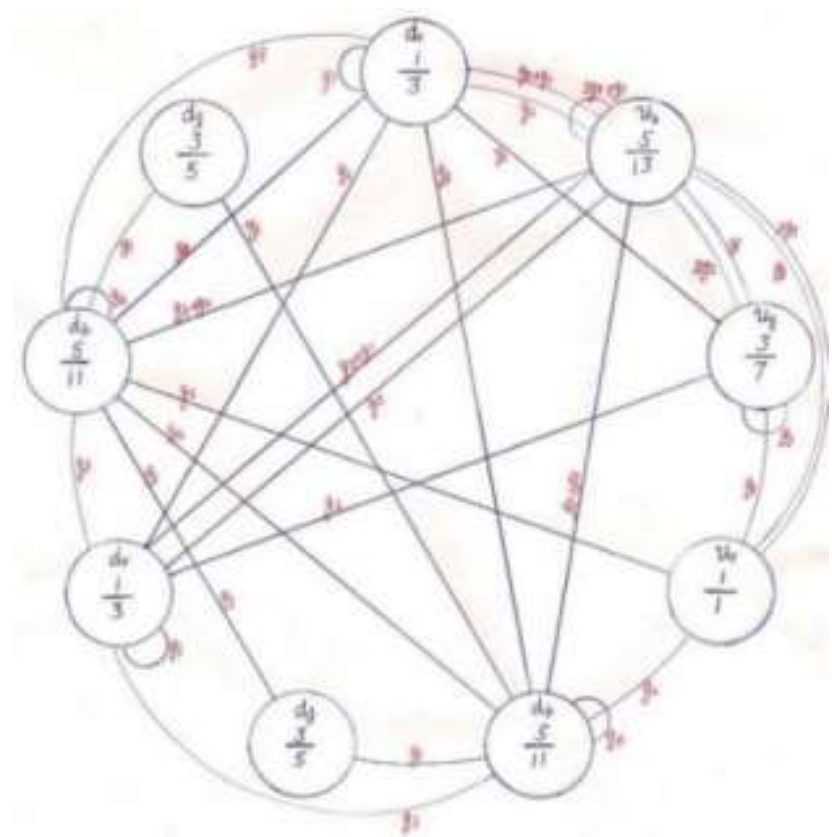

Fig.19.2: Gluon connections inside a neutron at the first level. 


\section{Z. Y. SHEN}

Helium nucleon is made of two protons and two neutrons, which include 36 quarks with tree different flavors and three different colors. The gluon connections diagram of helium nucleon is shown Fig. 19.3. To avoid over crowd lines, it only shows 86 connections for 6 different quarks as one sixth of total $86 \times 6=516$ connections.

As shown in Fig.19.3, $u_{r}$ has 24 connections, $u_{g}$ has 1+29 connections, $u_{b}$ has $1+47$ connections, $d_{r}$ has $1+29$ connections, $d_{g}$ has 6 connections, $d_{b}$ has $1+29$ connections.

The first number is for self-connection counted only once and the second number is for connections between two quarks counted twice. The total number of connections and connections per constituent for helium nucleon are:

$\left[(1+1+1+1)+\left(\frac{24+29+47+29+6+29}{2}\right)\right] \times 6=516$,

Connections per constituent: $\frac{516}{36}=14 \frac{1}{3}$.

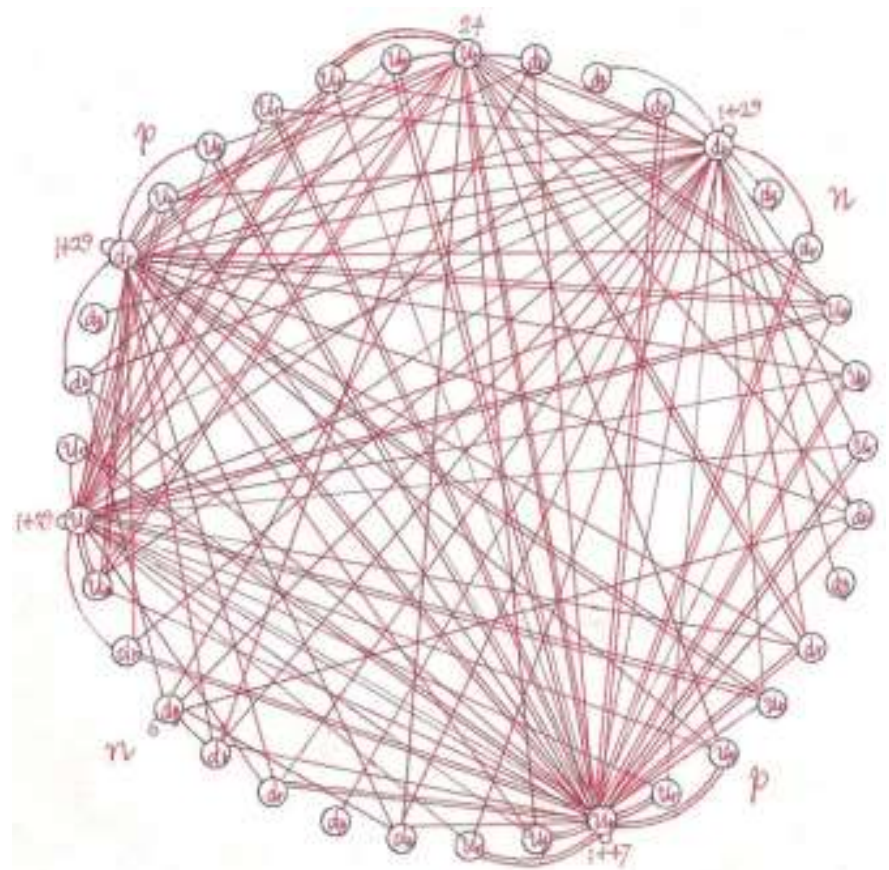

Fig.19.3: The 86 connections as one sixth of 516 total gluon connections inside a helium nucleon at the first level.

The connections per constituent $14 \frac{1}{3}$ for helium nucleon cannot directly compare to those for proton and neutron. It is not a fair comparison, because helium nucleon contains 36 colored quarks while proton and neutron each contains 9 colored quarks. In the case of gluon connections reaching the particle's entire region, the more constituents involved, the more are the number of connections per constituent. For fair comparisons, the number of connections per constituent for helium nucleon should be weighted lighter than proton and neutron. Assuming the weighted factor is inversely proportion to the number of constituents. The weighted binding strengths $(B S)$ of proton, neutron and helium nucleon for the first level are as follows.

Proton: $B S_{p}=\left(\frac{40}{9}\right) \times \frac{1}{9}=\frac{40}{81}=0.494$,

Helium nucleon: $B S_{H e}=\left(\frac{516}{36}\right) \times \frac{1}{36}=\frac{516}{1296}=0.398$,

Neutron: $B S_{n}=\left(\frac{32}{9}\right) \times \frac{1}{9}=\frac{32}{81}=0.395$,

Comparison: $B S_{p}>B S_{H e}>B S_{n}$.

The first level comparison of (19.2) shows the right order of binding strengths: Helium nucleon is weaker than proton and stronger than neutron.

Let's consider the second level-flavors level, which treats flavored quark as a whole entity. As shown in Table 19.1 , the corresponding numerical parameters are $m=21, n=9$ for $u$-quark, $m=19, n=9$ for $d$-quark. The second level gluon connections diagrams of proton and neutron are shown in Fig. 19.4. In which, proton has 5 connections for 3 quarks corresponding to $1 \frac{2}{3}$ connections per quark; neutron has 3 connections for 3 quarks corresponding to 1 connection per quark.

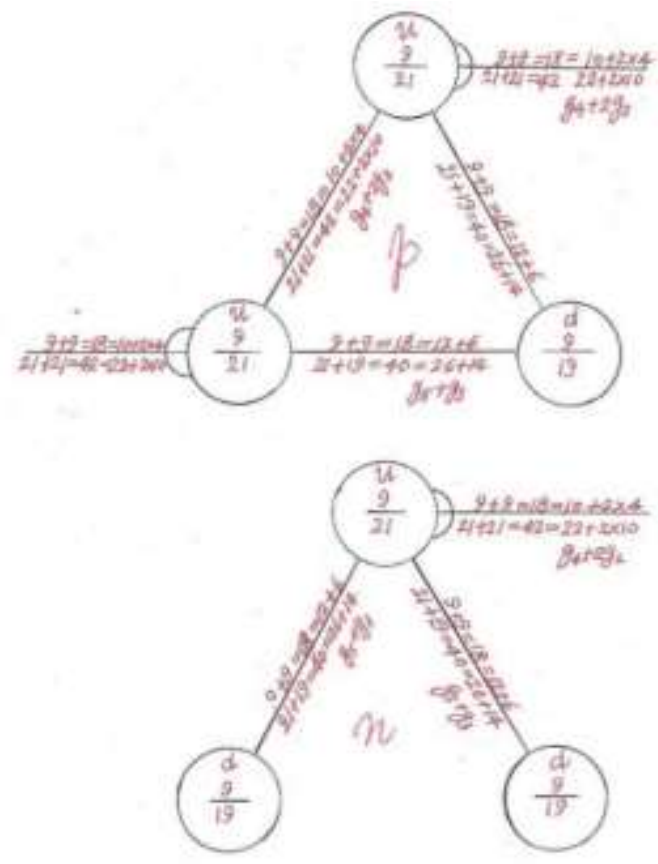

Fig.19.4: Gluon connections inside a proton and a neutron at the second level.

The corresponding gluon connections diagram of helium nucleon is shown in Fig. 19.5. There are 57 gluon connections for 12 quarks corresponding to $57 / 12=4 \frac{3}{4}$ connections per quark. Using the same weighted method as the first level, the weighted binding strengths $(B S)$ of proton, neutron and helium nucleon for the second level are as follows. 


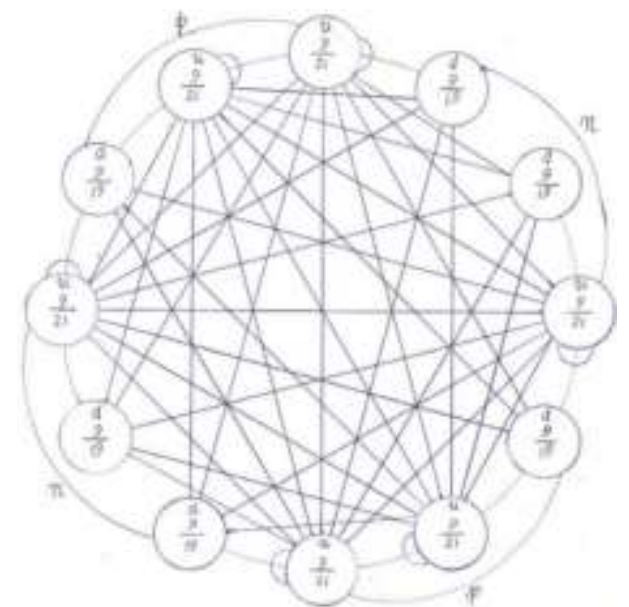

Fig.19.5: Gluon connections inside a helium nucleon at the second level.

Proton: $B S_{p}=\left(\frac{5}{3}\right) \times \frac{1}{3}=\frac{5}{9}=0.556$,

Helium: $B S_{H e}=\left(\frac{57}{12}\right) \times \frac{1}{12}=\frac{57}{144}=0.396$,

Neutron:, $B S_{n}=\left(\frac{3}{3}\right) \times \frac{1}{3}=\frac{3}{9}=0.333$,

Comparison: $B S_{p}>B S_{H e}>B S_{n}$.

The results of (19.2) and (19.3) both show the right orders: $B S_{p}>B S_{H e}>B S_{n}$, which indicate that, the binding strength of helium nucleon is weaker than proton and stronger than neutron. In reality, proton and helium nucleon are stable, while neutron in stable nucleons is stable and freestanding neutron is unstable with a mean life of $885.7 \pm 0.8 \mathrm{~s}$. The results at two levels are quite reasonable. Both show that helium nucleon is a very tightly bound composite particle.

Proton, neutron and helium nucleon also contain many gluons. These gluons $m$-parameters and $n$-parameters are not included in the diagrams. To ignore them is based on Theorem 13.2. It greatly simplifies the treatment for the numerical parameters of composite particles. The two level comparisons both make sense to explain proton, neutron and helium nucleon behaviors in terms of strong force binding strength, which serve as evidences to support Theorem 13.2.

Let's consider the third level-elementary particles level, which treats elementary particles such as proton and neutron as a whole entity serving as the constituents of the composite particles such as the helium nucleon, deuterium nucleon, tritium nucleon etc.

Proton is made of three flavored quarks $u^{+} u^{+} d^{-}$and neutron is made of three flavored quarks $d^{+} d^{+} u^{-}$. The "+" and "-"represent the sign of their numerical parameters. The numerical parameters are $m=21+21-19=23, n=9+9-9=9$ for proton, and $m=19+19-21=17, n=9+9-9=9$ for neutron. Fig.19.6 shows the gluons connections diagrams for helium nucleon, deuterium nucleon and tritium nucleon. For helium nucleon, there are 7 connections for 4 constituents corresponding to $7 / 4=1 \frac{3}{4}$ connections per constituent. For deuterium nucleon, there are 2 connections for 2 constituents corresponding to $2 / 2=1$ connections per constituent. For tritium nucleon, there are 3 connections for 3 constituents corresponding to $3 / 3=1$ connections per constituent. In case for the third level, the weighted method is different from the first and second levels. The difference is that, the nuclear force among protons and neutrons has limited range, which does not reach all constituents in the composite particles. As a first try, assuming the weighted factor for the third level is inversely proportional to the square root of constituents' number. The weighted binding strength $(B S)$ for helium nucleon, deuterium nucleon and tritium nucleon for the third level are as follows.

Helium nucleon: $B S_{H e}=\left(\frac{7}{4}\right) \times \frac{1}{\sqrt{4}}=0.875$,

Deuterium nucleon: $B S_{D e t}=\left(\frac{2}{2}\right) \times \frac{1}{\sqrt{2}}=0.707$,

Tritium nucleon: $B S_{T r i}=\left(\frac{3}{3}\right) \times \frac{1}{\sqrt{3}}=0.577$,

Comparison: $B S_{H e}>B S_{D e t}>B S_{T r i}$.

The results of (19.4) shows the binding strengths are in the right orders: $B S_{H e}>B S_{D e t}>B S_{T r i}$, which indicate deuterium nucleon is weaker than helium nucleon and stronger than tritium nucleon. In fact, helium and deuterium are stable, while tritium is unstable with a fairly long lifetime of 12.32 years. The results make sense. It shows that, Theorem 13.2 also valid for the third level composite particles.
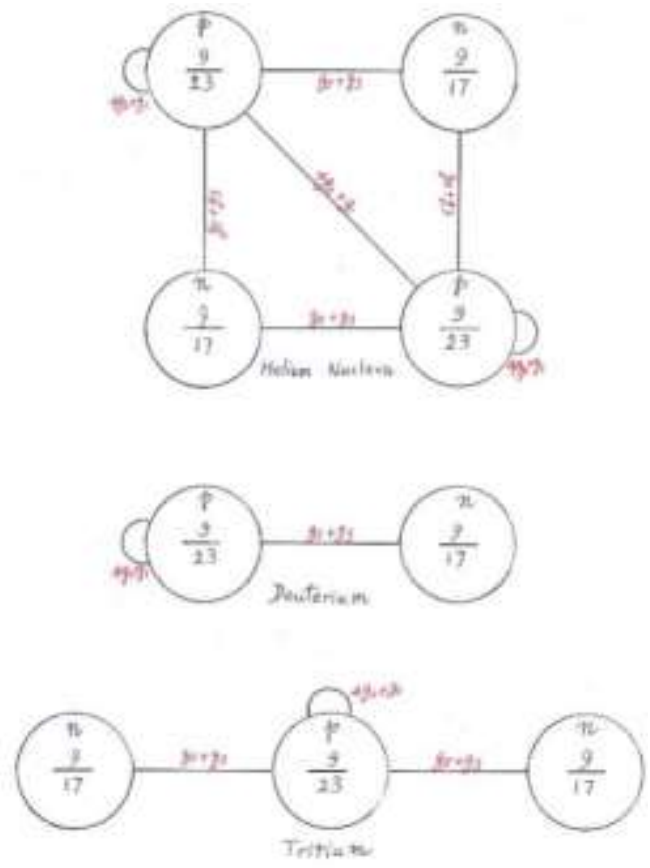

Fig.19.6: Gluon connections inside helium nucleon, deuterium nucleon and tritium nucleon at the third level. 


\section{Z. Y. SHEN}

The assumption of weighted factor for the third level used for (19.4) can be generalized as follows.

Helium nucleon: $B S_{H e}=\left(\frac{7}{4}\right) \times \frac{1}{4^{r}}$,

Deuterium nucleon: $B S_{D e t}=\left(\frac{2}{2}\right) \times \frac{1}{2^{r}}$,

Tritium nucleon: $B S_{T r i}=\left(\frac{3}{3}\right) \times \frac{1}{3^{r}}$,

Comparison: $B S_{H e}>B S_{D e t}>B S_{T r i}$ for $0<r \leq 0.5$.

For the generalized formulas (19.5), as long as $0<r \leq 0.5$, the results for these three composite particles all make sense. More examples of elements and isotopes are needed to narrow down the value of $r$ in formulas (19.5).

The reasonable results of binding strengths at three levels serve as strong supportive evidences for Theorem 13.2. Moreover, the different type of weighted factor used for the third level indicates that the binding strength is different from the first and second levels. It clearly shows the restrict range of nuclear force among protons and neutrons in a composite particle comparing to the strong force among quarks. It is well known in nuclear physics that, nuclear force is the fringing effect of strong force with exponential decay behavior from the edges of protons and neutrons.

Theorem 13.2 directly refers to $m$-parameters and $n$ parameters. Since gluons also contribute to composite particles' mass, quarks' $p / n$ ratio is no longer directly related to the mass of a composite particle. But quarks' $p$ parameter may play some other roles. It is the reason for the sum of $p$ values over three colors listed in the $u$ and $d$ columns of Table 19.1. Let's take a step further to see how it behaviors for composite particles.

Let's treat proton and neutron. According to Table 19.1, the $p / m$ and $2 p / m$ ratios of proton and neutron are:

For proton $\left(u^{+} u^{+} d^{-}\right)$:

$$
\frac{p}{m}=\frac{53+53-83}{21+21-19}=\frac{23}{23}=\frac{1}{1}, \frac{2 p}{m}=2 ；
$$

For neutron $\left(d^{+} d^{+} u^{-}\right)$:

$$
\frac{p}{m}=\frac{83+83-53}{19+19-21}=\frac{113}{17}, \frac{2 p}{m}=\frac{226}{17} \text {. }
$$

It shows that, the reduced $m$-parameters and $p$-parameters for proton and neutron are:

For proton: $m=1, p=1$;

For neutron: $m=17, p=113$.

The next step is to treat the composite particle the same way with proton and neutron as constituents.

For helium nucleon $\left(n^{+} p^{-} n^{+} p^{-}\right)$:

$$
\frac{p}{m}=\frac{113-1+113-1}{17-1+17-1}=\frac{224}{32}=\frac{7}{1}, \frac{2 p}{m}=14 ；
$$

For deuterium nucleon $\left(n^{+} p^{-}\right)$:

$$
\frac{p}{m}=\frac{113-1}{17-1}=\frac{112}{16}=\frac{7}{1}, \frac{2 p}{m}=14 ；
$$

For tritium nucleon $\left(n^{+} n^{+} p^{-}\right)$:

$$
\frac{p}{m}=\frac{113+113-1}{17+17-1}=\frac{225}{33}=\frac{75}{11}, \frac{2 p}{m}=\frac{150}{11} \text {. }
$$

For comparison, the $2 p / m$ value of electron is:

Electron: $\frac{2 p}{m}=\frac{2 \times(1 / 2)}{1}=1$,

Summarizing the data of (19.7) and (19.8) seemingly implies a rule: Simple composite particles with $2 \mathrm{p} / \mathrm{m}=$ integer are stable and with $2 p / m \neq$ integer are unstable. Which it valid for complex composite particles or not is an open issue. The rule is also valid for electron. However, it is not valid for other leptons and some quarks. Table 11.4 provides some clues for its selectivity.

The different behaviors of proton and neutron are originated from their difference in numerical parameters, which determine the gluons connections between them.

For $\left(p^{\uparrow} \leftrightarrow n^{\downarrow}\right)$ :

$$
\frac{n}{m}=\frac{9-(-9)}{23-(-17)}=\frac{18}{40}=\frac{12+6}{26+14}, g_{5}+g_{3} ;
$$

For $\left(p^{\uparrow} \leftrightarrow p^{\downarrow}\right)$ :

$$
\frac{n}{m}=\frac{9-(-9)}{23-(-23)}=\frac{18}{46}=\frac{4 \times 4+2}{4 \times 10+6}, 4 g_{2}+g_{1} ;
$$

For $\left(n^{\uparrow} \leftrightarrow n^{\downarrow}\right)$ :

$$
\frac{n}{m}=\frac{9-(-9)}{17-(-17)}=\frac{18}{34}=\frac{30-2 \times 6}{62-2 \times 14}, g_{8}-2 g_{3} ；
$$

It clearly indicates that, $p^{\uparrow} \leftrightarrow n^{\downarrow}$ and $p^{\uparrow} \leftrightarrow p^{\downarrow}$ are tightly bound, while $n^{\uparrow} \leftrightarrow n^{\downarrow}$ is loosely bound with weakened gluon connections indicated by the minus sign in $g_{8}-2 g_{3}$.

Dig it deeper, the difference is originated from the fact that, the up and down quarks have the same $n$-parameter, but they have different $m$-parameters. The sum of $m$-parameters is $1+7+13=21$ for up quark and $3+5+11=19$ for down quark. (19.10) shows that, the $m$-parameters difference weakens the binding of $n^{\uparrow} \leftrightarrow n^{\downarrow}$. Therefore, ultimately the different behaviors of proton and neutron are originated from mathematics.

Based on (19.10), some facts in nuclear physics can be explained. For instance: (1) Why proton is the most tightly bound particle? (2) Why helium nucleon is the most tightly bound composite particle? (3) Why the strong force bindings for $p^{\uparrow} \leftrightarrow n^{\downarrow}$ and $p^{\uparrow} \leftrightarrow p^{\downarrow}$ are tighter than $n^{\uparrow} \leftrightarrow n^{\downarrow}$ ? (4) Why proton is stable, free neutron is not stable? (5) Why neutrons in some nucleons are stable, in other nucleons are not stable? (6) Why deuterium is stable, tritium is not stable? (7) Why nucleons with $Z$ indexes equal to multiples of 4 have more binding energy than others? Of cause, these are the well known facts observed in experiments and explained by various nucleon theoretical models. SQS theory contribution is to provide a simple and clear explanation based on 
mathematics. Theorem 13.2 plays a pivotal role for the simplicity and clearness demonstrated in this section.

Gluons contribute to hadron's mass evidenced by proton and neutron. Does gluon also contribute to hadron's spin? It is an interesting question. According to Theorem 13.2, the two gluons sequences sent by a pair of quarks (a pair of antiquarks or a quark and an anti-quark) $q_{a}$ and $q_{b}$ are identical. In case of two gluons sequences sent by $q_{a}$ and $q_{b}$ simultaneously, their two aligned momentums with opposite directions cancel out and two opposite spins cancel out as well. So in this case, gluon does not contribute to hardron's spin. On the other hand, in case of two gluons sequences sent by $q_{a}$ and $q_{b}$ at different locations, the displacement of $q_{a}$ and/or $q_{b}$ between the two events causes the two gluons sequences' momentums no longer aligned, which produce a net angular momentum. It might contribute to a part of spin for the hadron.

Nuclear physics is very complicated. Whether SQS approach can make more contributions or not, it remains to be seen. What presented in the section is just a start. More work along this line is needed.

\section{Section 20: Basic Constants and Parameters}

SQS theory is a mathematic theory with physics significance. In principle, all equations, formulas and parameters are based on three fundamental Postulations and derived from mathematics. The mathematical results are interpreted by three basic physics constants, Planck constant $h$, speed limit of light in vacuum $c$, and gravitational constant $G$. To reach this goal takes steps.

Initially, SQS theory had two other physics inputs: electron mass and proton mass. In Section 15, a connection between proton mass and Plank mass via unified interactions was discovered. Proton mass as a physics input for SQS theory is no longer needed. In Section 16, a connection between electron mass and Plank mass via logistic equation was discovered. Electron mass as a physics input for SQS theory is no longer needed. After these two discoveries, SQS theory only needs three basic physics constants $h, c, G$ and in principle no other physics input is needed.

The three basic physics constants $h, c, G$ are related to Planck length $L_{P}$, Planck time $t_{P}$, Planck energy $E_{P}$ or Planck mass $M_{\text {Planck }}$ as:

$$
L_{P}=\sqrt{\frac{h G}{2 \pi c^{3}}}, t_{P}=\sqrt{\frac{h G}{2 \pi c^{5}}}, E_{P}=\sqrt{\frac{2 \pi h c^{5}}{G}}, M_{\text {Planck }}=\sqrt{\frac{2 \pi h c}{G}} ;
$$$$
c=\frac{L_{P}}{t_{P}}, h=E_{P} t_{P}, G=\frac{2 \pi L_{P}^{5}}{E_{P} t_{P}^{4}}=\frac{2 \pi L_{P}^{3}}{M_{\text {Planck }} t_{P}^{2}} .
$$

These two sets of basic constants are equivalent. From SQS theory standpoint, the set of $L_{P}, t_{P}, E_{P}$ or $M_{\text {Planck }}$ is preferred, because they are directly related to space, time, energy or mass. In fact, the Planck constants $L_{P}, t_{P}, E_{P}$ or $M_{\text {Planck }}$ are the basic length, basic time interval, basic energy or basic mass at Planck scale, respectively.

Besides three basic physics constants, there are other physics parameters such as the mass of elementary particles and various coupling constants etc. SQS theory standpoint is that, in principle all these physics parameters can be derived from particle's model and traced back to mathematic parameters with three basic physics constants as interpreters. So far, SQS theory did part of them, the others are still open issues.

The mass of a fermion is determined by its $p / n$ ratio based on electron mass. The interaction parameters are related to the $C K M$-triangle, $G W S$-triangle and $S$-triangle of the particle's model, which are determined by characteristic points on the model. The characteristic points are determined by $\pi\left(x_{1}\right)$ and $\pi\left(x_{2}\right)$ traced back to $S$-equation originated from Gaussian Probability Postulation. The derivation for other physics parameters from mathematical parameters is just a matter of time. The faith of SQS theory comes from this argument: If the geometrical models for elementary particles are real, everything should be derived from these models with characteristic points and triangles attached to them. If the physics parameters cannot be derived from these models, they are useless.

SQS theory introduced three sets of numerical parameters $m, n, p$ for elementary particles. The numerical parameters of elementary fermions are listed in Table 20.1. According to SQS theory, elementary bosons are made of pairs of elementary fermion and anti-fermion, their numerical parameters are derived from these fermions as shown in previous sections.

Table 20.1: The Numerical Parameters of Elementary Fermions

\begin{tabular}{|c|c|c|c|c|c|c|c|c|c|}
\hline $\begin{array}{l}\text { Eptope } \\
\text { quars }\end{array}$ & $\bar{a}$, & $u_{2}$ & $w_{4}$ & c. & $c_{i}$ & c) & $t$ & $t_{3}$ & t) \\
\hline in & 1 & 7 & 13 & 19 & 29 & 37 & 43 & 53 & 61 \\
\hline$n$ & 1 & 3 & 5 & 7 & 23 & 29 & 283 & 331 & 397 \\
\hline$p$ & 3 & 175 & 32.5 & 15029 & 62031 & 76183 & W01585:9 & $1707+8000$ & $132015 \times$ \\
\hline $\begin{array}{l}\text { Downotpe } \\
\text { quatis }\end{array}$ & $d$, & $d_{\text {, }}$ & $d_{j}$ & $x_{*}$ & $s_{s}$ & s) & $b$ & $b_{x}$ & $b_{3}$ \\
\hline$m$ & 3 & 5 & 11 & 17 & 23 & 31 & 41 & 47 & 59 \\
\hline$n$ & 1 & 3 & 5 & 7 & 11 & 13 & 17 & 17 & 53 \\
\hline$p$ & 13.5 & 20 & 49.5 & $124 !$ & 2277 & 2821 & 135915 & 143021 & 434653 \\
\hline $\begin{array}{l}\text { Chargod } \\
\text { lequonit }\end{array}$ & \multicolumn{3}{|c|}{$\theta$} & \multicolumn{3}{|c|}{$\mu$} & \multicolumn{3}{|c|}{$\mathrm{r}$} \\
\hline m & \multicolumn{3}{|l|}{1} & \multicolumn{3}{|l|}{1} & \multicolumn{3}{|l|}{1} \\
\hline$n$ & \multicolumn{3}{|l|}{$\frac{1}{2}$} & \multicolumn{3}{|l|}{$1 \frac{5}{3}$} & \multicolumn{3}{|l|}{$2 \frac{6}{7}$} \\
\hline D & \multicolumn{3}{|l|}{$\frac{1}{2}$} & \multicolumn{3}{|l|}{236} & \multicolumn{3}{|l|}{9935} \\
\hline Nentiaot & \multicolumn{3}{|c|}{$v_{t}$} & \multicolumn{3}{|c|}{$v_{\text {s }}$} & \multicolumn{3}{|c|}{$V_{\tau}$} \\
\hline m & \multicolumn{3}{|l|}{1} & \multicolumn{3}{|l|}{1} & \multicolumn{3}{|l|}{1} \\
\hline$n$ & \multicolumn{3}{|l|}{$\frac{1}{2}$} & \multicolumn{3}{|l|}{$1 \frac{3}{8}$} & \multicolumn{3}{|l|}{$2 \frac{6}{7}$} \\
\hline p* & \multicolumn{3}{|c|}{$\frac{1}{2}=R$} & \multicolumn{3}{|c|}{$336 \times 2.1$} & \multicolumn{3}{|c|}{$9939 \times R_{u}$} \\
\hline
\end{tabular}

* $R_{v / l}=8.3996 \times 10^{-11}$.

The total numbers of numerical parameters listed in Table 20.1 is 72 . In which the 9 numerical parameters of neutrinos is the same as those of their companion leptons except the some sign changes and the constant $R_{v / l}=8.3996 \times 10^{-11}$ 


\section{Z. Y. SHEN}

multiplication to $p$-parameters for neutrinos. The reduced $m$ parameters of leptons all equal to 1 for spin $\hbar / 2$. Substrate $9+3=12$ from 72 , the number of independent parameters is reduced to 60 . For the quarks, their $18 \mathrm{~m}$-parameters are uniquely determined by a set of 18 least odd prime numbers; their $n$-parameters are selected from prime numbers and subject to the tight constrain set by strong interactions leaving almost on room for alternative. Substrate $18+18=36$ from 60 , the number of independent numerical parameters is reduced to 24. In addition, according to definition $p / n=M / M_{e}$, electron's $p$-parameter must equal to its $n$-parameter. The number of independent numerical parameters for the current version of SQS theory is reduced to 23. It is interesting to notice that, the number 23 is close to the number of handpicked parameters in the standard model. But there is a difference. The twenty some parameters in standard model are physics in nature cited from experimental data, while the 23 left numerical parameters of SQS theory are mathematics in nature.

One of the final goals of SQS theory is to derive all physics properties of elementary particles and interactions from the first principle based on three fundamental postulations and three basic physics constants. We haven't got there yet. The major obstacle is the $p$-parameters. In the current version SQS theory, an elementary particle's $p$ parameter is determined by its mass. In principle, it should be the other way around: $p$-parameter determines mass. It indicates that, there is a rule missing in the current version of SQS theory, which is an important open issue.

Actually in the current version SQS theory, some particles' mass values are derived from the first principle already. In Section 15, the mass value of two scalar bosons $U_{1}$ and $U_{2}$ is derived from points $x_{a}$ and $x_{b}$ from special point $x_{c}$ originated from $S$-equation based on Gaussian Probability Postulation. The mass value of the scalar bosons $U_{3}$ is derived from the converting factor at the grand unification scale, which is originated from the Random Walk Theorem based on Gaussian Probability Postulation. More importantly, the results of these two methods are correlated to fine structure constant $\alpha\left(M_{Z}\right)$, which gives legitimacy to both methods. In Section 17, the mass value of the gauge boson $X$ is derived from points $x_{a}$ and $x_{b}$ also originated from special point $x_{c}$.

Is there a way to trace the mass value of other two gauge bosons $W^{ \pm}$and $Z^{0}$ back to the special point

$$
\begin{aligned}
x_{c}= & 0.24998715627302645 ? \text { Let's try: } \\
& \int_{x_{f}}^{0.25}\left[\sum_{n=-N}^{N} e^{-\pi(n-x)^{2}}\right] d x-\int_{0}^{0.25-x_{c}}\left[\left[\sum_{n=-N}^{N} e^{-\pi(n-x)^{2}}\right]-1\right] d x=0, \\
& x_{f}=0.249998889847477, \\
& x_{c}<x_{f}<0.25 .
\end{aligned}
$$

Point $x_{f}=0.249998889847477$ is used to determine two points on $x$-axis:

$$
\begin{aligned}
& x_{1}=x_{f}=0.249998889847477 \\
& x_{2}=0.5-x_{f}=0.250001110152523 .
\end{aligned}
$$

Using the points $x_{1}$ and $x_{2}$ to define a fermion state $f$, according to (6.18) its mass is:

$$
\begin{aligned}
& M_{f}=\frac{M_{e}}{4\left|x_{2}-x_{1}\right|}=57.5370162 \mathrm{GeV} / \mathrm{c}^{2} . \\
& 3 M_{f}=172.611049 \mathrm{GeV} / \mathrm{c}^{2} . \\
& \sqrt{2} M_{f}=81.3696287 \mathrm{GeV} / \mathrm{c}^{2} .
\end{aligned}
$$

To compare $3 M_{f}=172.611049 \mathrm{GeV} / \mathrm{c}^{2}$ with top quarks mass cited from 2010-PDG data $M_{q t}=(172.0 \pm 0.9) \mathrm{GeV} / \mathrm{c}^{2}$, the $3 M_{f}$ value is within its error range, and the relative deviation of $3 M_{f}$ from $M_{q t}$ medium value is $3.553 \times 10^{-3}$. According the correlations of $W^{ \pm}, Z^{0}$ and $q_{t}$ given by (14.2) and (14.3), the mass values of gauge bosons $W^{ \pm}, Z^{0}$ can be calculated from the value of $3 M_{f}$ and Weinberg angle $\theta_{W}$ as:

$$
\begin{aligned}
M_{Z} & =\frac{3 M_{f}}{1+\cos \theta_{W}}=91.7320308 \mathrm{GeV} / \mathrm{c}^{2}, \\
M_{W} & =\frac{3 M_{f}}{1+\cos \theta_{W}} \cos \theta_{W}=80.879018 \mathrm{GeV} / \mathrm{c}^{2}, \\
M_{Z} & +M_{W}=3 M_{f}=172.611049 \mathrm{GeV} / \mathrm{c}^{2}, \\
\theta_{W} & =\operatorname{arcos}\left(\left.\frac{M_{W}}{M_{Z}}\right|_{P D G \text { data }}\right)=28.15335581^{\circ} .
\end{aligned}
$$

The values $M_{Z}, M_{W}$ given by (20.8a), (20.8b) compare to 2010-PDG data, $M_{Z}=(91.1876 \pm 0.0021) \mathrm{GeV} / \mathrm{c}^{2}$, $M_{W}=(80.399 \pm 0.023) \mathrm{GeV} / \mathrm{c}^{2}$, both have relative deviation of $5.97 \times 10^{-3}$ from medium values. Comparing $\sqrt{2} M_{f}=81.3696287 \mathrm{GeV} / \mathrm{c}^{2}$ to $M_{W}=(80.399 \pm 0.023) \mathrm{GeV} / \mathrm{c}^{2}$, the relative deviation from its medium value is $1.2 \times 10^{-2}$. Notice that, $M_{f}$ and $M_{W}$ are the mass of a fermion state $f$ and gauge boson $W^{ \pm}$, respectively; while $\sqrt{2}$ is the numerical factor left unexplained in Section 16. It implies that, $\sqrt{2}$ may have something to do with the relation between fermions and bosons, which will be discussed in Section 21. In the meantime, it serves as a check point.

Prediction 20.1: There is an electrically neutral scalar boson as a composite state of $M_{S}=M_{f} \oplus \bar{M}_{f}$ with mass:

$$
M_{M s}=(2 \times 57.5370162) \mathrm{GeV} / \mathrm{c}^{2}=115.074032 \mathrm{GeV} / \mathrm{c}^{2}(20.9)
$$

If all of these are not by coincidence, the special point $x_{c}=0.24998715627302645$ did it again. It not only determined the mass value for two scalar bosons $W^{ \pm}, Z^{0}$, but also for the top quark $q_{t}$. It is the first quark with mass value traced back to the first principle. The three colored top quarks have slightly different mass values from their average value as 
listed in Table11.2, which are caused by color symmetry broken.

If the 3 colored top quarks' mass values are indeed traced back to the first principle, then the independent numerical parameters left is reduced from 23 to 20 . In which there are $15 p$-parameters of 15 quarks plus $2 p$-parameters of muon and taon, the rest 3 parameters are the $n$-parameters of 3 leptons.

Why does the special point $x_{c}$ determine top quark's mass not other type quarks? Because top quark with mass $M_{q t}>M_{M a x}$, it must appear with top anti-quark as boson state. Apparently the special point $x_{c}$ works only for bosons or boson states.

The remaining question is: for $3 M_{f} \approx M_{q t}$, where does the factor 3 come from? It is worthwhile to give a thought.

\section{Section 21: Space Structure and Symmetries}

The first fundamental postulation of SQS theory, Gaussian Probability Postulation, assigns Gaussian probability at each discrete point separated by Planck length, which makes continuous space with grainy structure. In previous sections, the Planck cube as the building block of space is based on 3-dimensional Cartesian coordinate. The adoption of Cartesian coordinate system implies a hidden a priori assumption: Space has cubic lattice structure, which has no proof. What is the real structure of space? What are the inherent symmetries of space? These are the two basic questions of this section.

To answer the first question, let's go back to the fundamental postulation. The 3-dimensional Gaussian probability distribution function has spherical symmetry like a ball with blurred boundary. The Gaussian probability distribution function converges rapidly toward zero with distance from its center. Let's ignore its blurredness and treat the 3-dimensional Gaussian distribution function as a hard ball called "Gaussian sphere" with definitive boundary. In Section 2, the radius of Gaussian sphere is defined as:

$$
R=\frac{1}{2 \sqrt{2}}=0.353553390593274 \text {. }
$$

From SQS viewpoint to consider the structure of space, the question is: What is the preferred way for Gaussian spheres to arrange themselves? As the Gaussian sphere treated as hard ball, the question becomes: What is the most compact packing for balls? It is a classic topic known as Kepler conjecture. Kepler conjectured that, the face-centered arrangement is the most compact packing for balls, which has an average density:

$$
D_{f-c}=\frac{\pi}{\sqrt{18}}=0.74048049 .
$$

Kepler conjecture was proved by Thomas Hales in 1998 using the exhaust method with extensive numerical calculations. For comparison, the cubic arrangement for balls has an average density:

$$
D_{c}=\frac{\pi}{6}=\frac{D_{f-c}}{\sqrt{2}}=0.52359878 .
$$

Nature has a tendency to reach the lowest energy for stability. As gravity dominates, the most compact packing is the one with lowest potential energy. The face-centered lattice is the preferred one for the space structure. This is the first supportive evidence for the face-centered lattice structure of space.

The ball packing argument is only a simulation. In reality, the spherical Gaussian probability distribution is not a hard ball with definitive boundary. Keep this in mind; let's take a close look at face-centered lattice structure.

Fig.21.1 shows a sketch of the face-centered lattice structure within a Planck cube. It shows that, the facecentered lattice can be viewed as an octahedron embedded in a cube. The lattice length of cube and octahedron are $l_{\text {cube }}=L_{P}$ and $l_{\text {octa }}=L_{P} / \sqrt{2}$, respectively. The lattice lengths ratio of cube to octahedron is:

$$
\frac{l_{\text {cube }}}{l_{\text {octa }}}=\sqrt{2} \text {. }
$$

In (21.2) and (21.3), the number $\sqrt{2}$ looks familiar. It appeared in front of electron converting factor $N_{e}$ of equation (16.1) in Section 16. The definition of electron converting factor is:

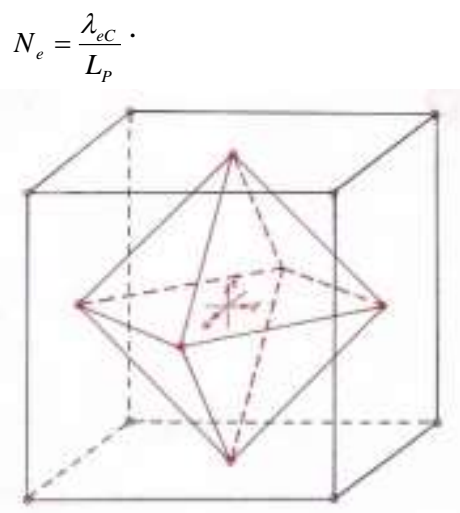

Fig.21.1: Face-centered lattice structure as an octahedron embedded in a cube.

According to (6.10), multiplying $\sqrt{2}$ to $N_{e}=\lambda_{e C} / L_{P}$ is equivalent to redefine Planck length $L_{P}^{\prime}$ based on the octahedral lattice length $l_{\text {octa }}=L_{P} / \sqrt{2}$ as:

$$
L_{P}^{\prime}=\frac{1}{\sqrt{2}} L_{P}=\frac{1}{\sqrt{2}} \sqrt{(\hbar) \frac{G}{c^{3}}}=\sqrt{\left(\frac{\hbar}{2}\right) \frac{G}{c^{3}}}=1.14286 \times 10^{-35} \mathrm{~m} .
$$

The $\sqrt{2}$ factor appeared in equation (16.1) is not by coincidence. It can be interpreted as $\sqrt{2}=L_{P} / L_{P}^{\prime}$. This is the second supportive evidence for the face-centered lattice structure of space.

Moreover, in Section 15 and Section 16, there are two related converting factor formulas: For the scalar boson of grand unification: 


$$
N_{G U T}=\frac{2 \pi}{\sqrt{\alpha\left(M_{Z}\right)}},
$$

For electron as a fermion in Section 16, letting $r=0$ in equation (16.1) yields:

$$
N_{e}(r=0)=\frac{1}{\sqrt{2}}\left[\frac{2 \pi}{\sqrt{\alpha\left(M_{e}\right)}}\right] .
$$

Despite the difference of $\alpha\left(M_{Z}\right)$ verses $\alpha\left(M_{e}\right)$

representing the difference of energy scales, the notable difference between (15.11) and (21.5) is the factor $\frac{1}{\sqrt{2}}$.

is for boson and (21.1) is for fermion. Notice that, in (21.4), under the square root sign, $\hbar / 2$ in the redefined $L_{P}^{\prime}$ is the basic spin for fermions, while $\hbar$ in the original $L_{P}$ definition is the basic spin for bosons. These comparisons imply that, the difference between fermions and bosons may have something to do with $\frac{1}{\sqrt{2}}$ and the difference between octahedral part and cubic part of the space face-centered structure. It serves a possible explanation for the difference between (15.11) and (21.5). It also serves as the third supportive evidence for the face-centered lattice structure of space.

Postulation 21.1: Space has face-centered lattice structure as shown in Fig. 21.1. It contains two parts: the cubic part with unit length of $L_{P}$ and the octahedral part with unit length of $L_{P}^{\prime}=L_{P} / \sqrt{2}$.

Explanation: Normalized to $L_{P}, L_{P}^{\prime}=L_{P} / \sqrt{2} \rightarrow 1 / \sqrt{2}$.

According to (2.9b):

$$
R=\frac{1}{2 \sqrt{2}} \rightarrow \frac{L_{P}^{\prime}}{2}=\frac{L_{P}}{2 \sqrt{2}} .
$$

It explains the mathematical reason for the definition of the radius of Gaussian sphere $R$. In reality, $R$ is determined by the balance of attractive force and repulsive force.

According to Postulation 21.1, space has crystal structure. It is well known in crystallography that, symmetry play an important rule to explain crystal properties. Let's try to answer the second basic question.

Definition 21.1: In the space with face-centered lattice structure, symmetry $O(r)$ is defined as a set of vertexes on the spherical surface centered at an octahedral vertex with radius $r$; symmetry $C(r)$ is defined as a set of vertexes on the spherical surface centered at a cubic vertex with radius $r$.

According to Definition 21.1, there are 18 symmetries for $O(r)$ with $r \leq 3$ and 18 symmetries for $C(r)$ with $r \leq 3$.

The parameters of 36 symmetries of $O(r)$ and $C(r)$ with $r \leq 3$ are listed in Table 21.1 and Table 21.2, respectively. In these tables, three sets of numbers are listed. In the column of "No. of vertexes Oct. + Cub. = Total", "Oct. and Cub." are the numbers of octahedral and cubic vertexes in the symmetry, respectively. In the column of "No. of equilateral triangles Conn.+ Separ. $=$ Total", "Conn. and Separ." are the numbers of connected and separated equilateral triangles in the symmetry, respectively. In the column of "No. of squares with center $O n+O f f=$ Total ", "On and Off" are the numbers of squares with center vertex of the symmetry on and off the square surface, respectively.

\begin{tabular}{|c|c|c|c|}
\hline $\begin{array}{l}\text { Symmetry name } \\
O(r)\end{array}$ & $\begin{array}{c}\text { No. of vertexes } \\
\text { Oct. }+C u b .=\text { Total }\end{array}$ & $\begin{array}{c}\text { No. of equilateral } \\
\text { triangles } \\
\text { Conn + Separ. }=\text { Total }\end{array}$ & $\begin{array}{c}\text { No. of squares with } \\
\text { center } \\
\text { On }+O f f=\text { Total }\end{array}$ \\
\hline$O(0)^{*}$ & $1+0=1$ & $0+0=0$ & $0+0=0$ \\
\hline$O(\sqrt{1 / 2})^{*}$ & $8+4=12$ & $8+0=8$ & $3+6=9$ \\
\hline$O(1)^{*}$ & $6+0=6$ & $8+0=8$ & $3+0=3$ \\
\hline$O(\sqrt{3 / 2})^{*}$ & $16+8=24$ & $0+8=8$ & $0+18=18$ \\
\hline$O(\sqrt{2})$ & $12+0=12$ & $8+0=8$ & $3+6=9$ \\
\hline$O(\sqrt{5 / 2})$ & $16+8=24$ & $0+0=0$ & $6+12=18$ \\
\hline$O(\sqrt{3})$ & $8+0=8$ & $8+0=8$ & $0+6=6$ \\
\hline$O(\sqrt{7 / 2})^{*}$ & $32+16=48$ & $0+0=0$ & $0+36=36$ \\
\hline$O(2)$ & $6+0=6$ & $8+0=8$ & $3+0=3$ \\
\hline$O(\sqrt{9 / 2})$ & $24+12=36$ & $8+8=16$ & $3+24=27$ \\
\hline$O(\sqrt{5})$ & $24+0=24$ & $0+0=0$ & $6+12=18$ \\
\hline$O(\sqrt{11 / 2})$ & $16+8=24$ & $0+8=8$ & $0+18=18$ \\
\hline$O(\sqrt{6})$ & $24+0=24$ & $0+8=8$ & $0+18=18$ \\
\hline$O(\sqrt{13 / 2})^{*}$ & $48+24=72$ & $0+0=0$ & $6+48=54$ \\
\hline$O(\sqrt{15 / 2})$ & $32+16=48$ & $0+0=0$ & $0+36=36$ \\
\hline$O(\sqrt{8})$ & $12+0=12$ & $8+0=8$ & $3+6=9$ \\
\hline$O(\sqrt{17 / 2})$ & $32+16=48$ & $0+8=8$ & $6+30=36$ \\
\hline$O(3)$ & $30+0=30$ & $8+8=16$ & $3+18=21$ \\
\hline 6 essentials total & $111+52=163$ & $16+8=24$ & $12+108=120$ \\
\hline Grand total & $347+112=459$ & $64+48=112$ & $45+294=339$ \\
\hline
\end{tabular}

Table 21.1: Symmetries Centered at an Octahedral Vertex in the Face-centered Space

Note: The symmetries marked with * are the 6 essential symmetries.

Table 22.2: Symmetries Centered at a Cubic Vertex in the Face-centered Space

\begin{tabular}{|l|l|l|l|}
\hline $\begin{array}{l}\text { Symmetry name } \\
C(r)\end{array}$ & $\begin{array}{c}\text { No. of vertexes } \\
\text { Oct. }+ \text { Cub. }=\text { Total }\end{array}$ & $\begin{array}{c}\text { No. of equilateral } \\
\text { triangles } \\
\text { Conn }+ \text { Separ. }=\text { Total }\end{array}$ & $\begin{array}{c}\text { No. of squares with } \\
\text { center } \\
\text { On+Off }=\text { Total }\end{array}$ \\
\hline$C(0)^{*}$ & $0+1=1$ & $0+0=0$ & $0+0=0$ \\
\hline$C(\sqrt{1 / 2})^{*}$ & $12+0=12$ & $8+0=8$ & $3+6=9$ \\
\hline$C(1)^{*}$ & $0+6=6$ & $8+0=8$ & $3+0=3$ \\
\hline$C(\sqrt{3 / 2})^{*}$ & $24+0=24$ & $0+8=8$ & $0+18=18$ \\
\hline$C(\sqrt{2})$ & $0+12=12$ & $8+0=8$ & $3+6=9$ \\
\hline$C(\sqrt{5 / 2})$ & $24+0=24$ & $0+0=0$ & $6+12=18$ \\
\hline$C(\sqrt{3})$ & $0+8=8$ & $8+0=8$ & $0+6=6$ \\
\hline$C(\sqrt{7 / 2})^{*}$ & $48+0=48$ & $0+0=0$ & $0+36=36$ \\
\hline$C(2)$ & $0+6=6$ & $8+0=8$ & $3+0=3$ \\
\hline$C(\sqrt{9 / 2})$ & $36+0=36$ & $8+8=16$ & $3+24=27$ \\
\hline$C(\sqrt{5})$ & $0+24=24$ & $0+0=0$ & $6+12=18$ \\
\hline$C(\sqrt{11 / 2})$ & $24+0=24$ & $0+8=8$ & $0+18=18$ \\
\hline$C(\sqrt{6})$ & $0+24=24$ & $0+8=8$ & $0+18=18$ \\
\hline$C(\sqrt{13 / 2})^{*}$ & $72+0=72$ & $0+0=0$ & $6+48=54$ \\
\hline$C(\sqrt{15 / 2})$ & $48+0=48$ & $0+0=0$ & $0+36=36$ \\
\hline$C(\sqrt{8})$ & $0+12=12$ & $8+0=8$ & $3+6=9$ \\
\hline$C(\sqrt{17 / 2})$ & $48+0=48$ & $0+8=8$ & $6+30=36$ \\
\hline$C(3)$ & $0+30=30$ & $8+8=16$ & $3+18=21$ \\
\hline 6 essentials total & $156+7=163$ & $16+8=24$ & $12+108=120$ \\
\hline Grand total & $336+123=459$ & $64+48=112$ & $45+294=339$ \\
\hline
\end{tabular}

Note: The symmetries marked with * are the 6 essential symmetries. 
Let's start from the octahedral part listed in Table 21.1. $O(0)$ is a basic symmetry represented by a single vertex, which has a rotational symmetry with any angle. It seems reasonable to identify $O(0)$ related to the $U(1)$ group. In the standard model, $U(1)$ represents electromagnetic interaction mediated by photons. From SQS theory viewpoint, photon and graviton are two sides of the same coin. So $O(0)$ is also related to graviton. In fact, the single vertex of $O(0)$ has dual identity like two sides of the same coin. It is the vertex on its shrunk sphere surface and it also is the center vertex. It seems reasonable to identify the former with electromagnetic interaction and the letter with gravitation. In fact, all symmetries have center vertex not included in Table 21.1 and Table 21.2, which corresponds to gravity's universality.

Symmetry $O(\sqrt{1 / 2})$ is an important basic symmetry having 12 vertexes located at following Cartesian coordinates with origin at the center vertex.

Cube vertexes:

$p_{1}\left(0, \frac{1}{2}, \frac{1}{2}\right), p_{2}\left(0, \frac{1}{2}, \frac{-1}{2}\right), p_{3}\left(0, \frac{-1}{2}, \frac{1}{2}\right), p_{4}\left(0, \frac{-1}{2}, \frac{-1}{2}\right)$,

Octahedral vertexes:

$p_{5}\left(\frac{1}{2}, \frac{1}{2}, 0\right) p_{6}\left(\frac{1}{2}, \frac{-1}{2}, 0\right), p_{7}\left(\frac{-1}{2}, \frac{1}{2}, 0\right), p_{8}\left(\frac{-1}{2}, \frac{-1}{2}, 0\right) ;$

$p_{9}\left(\frac{1}{2}, 0, \frac{1}{2}\right), p_{10}\left(\frac{1}{2}, 0, \frac{-1}{2}\right), p_{11}\left(\frac{-1}{2}, 0, \frac{1}{2}\right), p_{12}\left(\frac{-1}{2}, 0, \frac{-1}{2}\right)$.

In which 4 vertexes are cubic type and 8 vertexes are octahedral type. The hybrid of octahedral and cubic vertexes in $O(\sqrt{1 / 2})$ may serve as a link between fermions and bosons.

The 12 vertexes form 8 connected equilateral triangles, in which the 4 connected equilateral triangles in $x<0$ part are shown in Fig.21.2. $O(\sqrt{1 / 2})$ has 9 squares, in which 3 squares with center vertex on square surface and the other 6 squares with center vertex off square surface. The meaning of the equilateral triangles and the two types of squares will be given later in this section.

In essence, symmetry $O(\sqrt{2})$ is an enlarged version of $O(\sqrt{1 / 2})$. All symmetries are the same for $O(\sqrt{1 / 2})$ and $O(\sqrt{2})$.The only difference is the linear scale of $O(\sqrt{2})$ is enlarged by a factor of 2 comparing with $O(\sqrt{1 / 2})$.

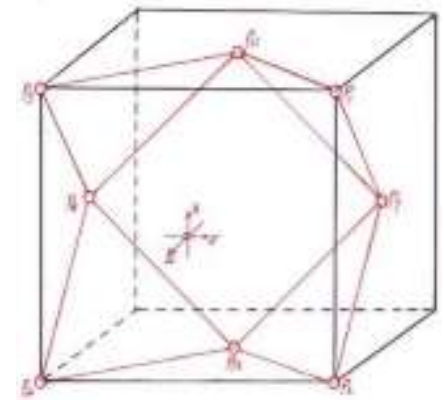

Fig.21.2: The vertexes of $o(\sqrt{1 / 2})$ with 4 vertexes located at $x>0$ not shown.
$O(1)$ is an important basic symmetry. As shown in Table 21.1 and Fig. 23.3, $O(1)$ has 6 octahedral vertexes, The 6 vertexes form an octahedron, which is one of two parts of the face-centered lattice structure of space with elongated length scale. The 6 vertexes are paired to form 3 orthogonal axes with $90^{\circ}$ span angles at center. It is identified as $S O(3)$ group. $S O(3)$ is closely related to the $S U(2)$ group. The elements of $S O(3)$ and the elements of $S U(2)$ are described by three parameters corresponding to the three Euler angles of a three dimensional rotation. The relation between $S O(3)$ and $S U(2)$ is that, each rotation in three dimensions of $S O(3)$ corresponds to two distinct elements of $S U(2)$. In some sense, $S U(2)$ is a dual version of $S O(3)$. It seems reasonable to relate $O(1)$ with the $S U(2)$ group. The 6 vertexes of $O(1)$ form 3 squares with center vertex on square surface. A square with center vertex on square surface is a part of $S O(3)$ and serves as a part of $S U(2)$ representing weak interaction.

As shown in Table 21.1 and Fig. 21.3, $O(1)$ has 8 connected equilateral triangles. The simplest representation of $S U(3)$ group is a triplet. It seems reasonable to relate the equilateral triangles in $O(1)$ with $S U(3)$ triplets such as

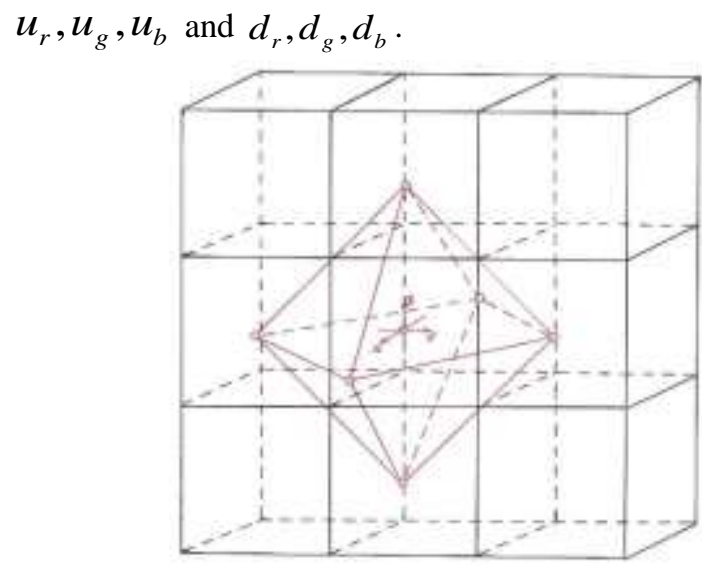

Fig.21.3: The 6 vertexes of $O(1)$.

SQS theory searching for the $S U(3)$ group comes from a long way. The first clue came from the 3-dimensional Gaussian probability standard deviation, $\sigma^{3}=1 / \sqrt{2 \pi}$. Its three roots provide one real number and two complex numbers, which define three axes separated by $\vartheta=120^{\circ}$ on a complex plane as shown in Fig. 3.2. The second clue came from the transportation route of electron moving in the zigzagging path named "zitterbewegung" having $\pm 120^{\circ}$ angle with $x$-axis on a complex plane shown in Fig. 3.4. The third clue is the fermions' loop-2 tiny tilt angle deviated from $\vartheta=120^{\circ}$. Now, all these clues point at one origin, which is the triplet symmetrical structure in the face-centered lattice. It 
Z. Y. SHEN

could serve as the geometrical explanation for $S U(3)$ group in the real space.

In essence, the $O(0) \oplus O(1)$ is related to three groups $U(1)$, $S U(2), S U(3)$ plus gravitation as the center vertex, which represent all four types of interactions. As shown in Section 15 , all four types of interactions are united into one. So it should not be a surprise to find out that, $O(0) \oplus O(1)$ related to all four types of interactions.

As shown in Table 21.1, symmetry $O(\sqrt{3})$ has 8 octahedral vertexes in which 4 of them in $x<0$ region are shown in Fug. 21.4. The 8 vertexes of $O(\sqrt{3})$ form a cube, which is one of the two parts of face-centered lattice space structure of space with elongated length scale. The 8 vertexes of $O(\sqrt{3})$ form 8 connected equilateral triangles and 6 squares with the center vertex off surface. Since the $S U(3)$ group has an eight-fold representation, octet, it seems reasonable to relate $O(\sqrt{3})$ to the eight-fold. The 6 off center squares represent strong interaction to hold the 8 equilateral triangles representing 8 fermions. A possible physics interpretation is: The octet of $O(\sqrt{3})$ represents the baryons octet as shown in Fig. 21.5. It includes 8 baryons: $p(u u d)$, $n(d d u), \Sigma^{+}(u u s), \Sigma^{-}(d d s), \Xi^{0}(u s s), \Xi^{-}(d s s), \Sigma^{0}(u d s)$, $\Lambda(u d s)$, in which each baryon is made of 3 quarks in the first and second generations.

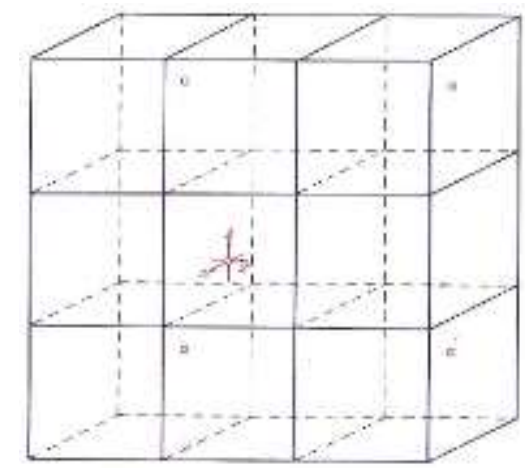

Fig. 21.4: The vertexes of $o(\sqrt{3})$ with 4 vertexes located at $x>0$ not shown.

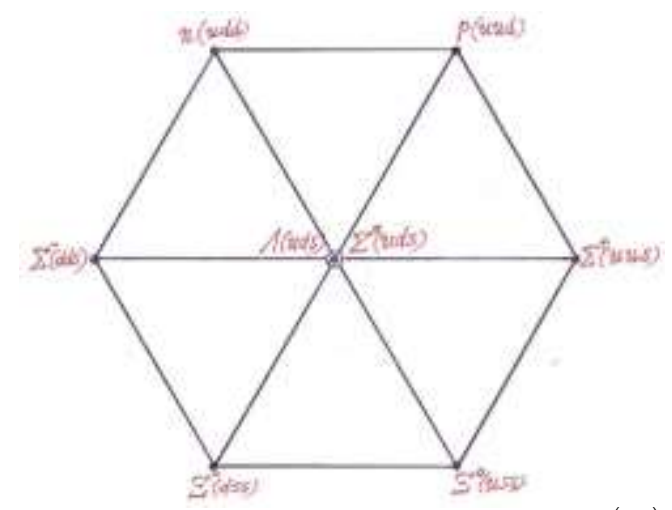

Fig. 21.5: The baryons octet represented by $O(\sqrt{3})$.
There is a difference between two different types of squares. The squares with center vertex on square surface such as those in $O(1)$ are related to part of $S U(2)$ representing weak interaction. The squares with center vertex off square surface such as those in $O(\sqrt{3})$ are related to a part of $S U(3)$ representing strong interaction. The ratio of center to square distance $D$ over square edge $E$ is defined as $R_{D / E}=D / E$.

For squares with center vertex on surface:

$$
R_{D / E}=\frac{D}{E}=0 \text {. }
$$

For the squares with center vertex off surface:

$$
R_{D / E}=\frac{D}{E}>0 \text {. }
$$

Back to the $O(\sqrt{1 / 2})$ symmetry, it has 3 squares with center vertex on surface and 6 squares with center vertex off surface. It seems that, both weak interaction and strong interaction are involved with its 8 equilateral triangles. A possible physics interpretation is: $O(\sqrt{1 / 2})$ represents 8 fermions, $u_{r}, u_{g}, u_{b}, d_{r}, d_{g}, d_{b}, e, v_{e}$ and their antiparticles of the first generation. The weak interactions among 8 fermions represented by 3 squares with center vertex on surface are understandable. The strong interactions among 6 quarks represented by squares with center vertex off surface are also understandable. But there are squares with center vertex off surface connecting to equilateral triangles representing leptons and quarks. What does such type connection mean?

Fig.21.6 shows the "Symmetries Family Tree" for $O(r)$ symmetries. It includes all 18 octahedral symmetries with radius $r \leq 3$.

In Fig.21.6, symmetries are illustrated by squares with names. The vertical location of the square is raised according to increasing $r$ values of $O(r)$. Inside the square from top down, the numbers in three rows, Oct. $\oplus$ Cub. $=$ Total, Conn.+ Separ.$=$ Total, On + Off $=$ Total are cited from Table 21.1. In Fig. 21.6, there are three types of connecting lines: A vertical single solid line indicates that the connected two squares have the same type symmetry with different scales. The forked solid lines indicate that the top symmetry is a combination of two symmetries below. The dashed line indicates that the two connected symmetries are somehow correlated.

In Table 21.1 and Fig. 21.6, the 6 symmetries marked with * belong to the essential type. In which, $O(0), O(1)$ $O(\sqrt{1 / 2})$ are basic symmetries as mentioned previously. The other three, $O(\sqrt{3 / 2}), O(\sqrt{7 / 2}), O(\sqrt{13 / 2})$, are the lowest symmetries having vertex numbers $24,48,72$, respectively.

As shown in Table 21.1, Fig. 21.6 and Fig. 21.7, symmetry $O(\sqrt{3 / 2})$ is the lowest one has 24 vertexes. The 24 vertexes of $O(\sqrt{3 / 2})$ form 8 separated equilateral triangles and 18 squares all with center vertex off surface, which 
indicate that the 8 triplets interact to each other via strong interactions.

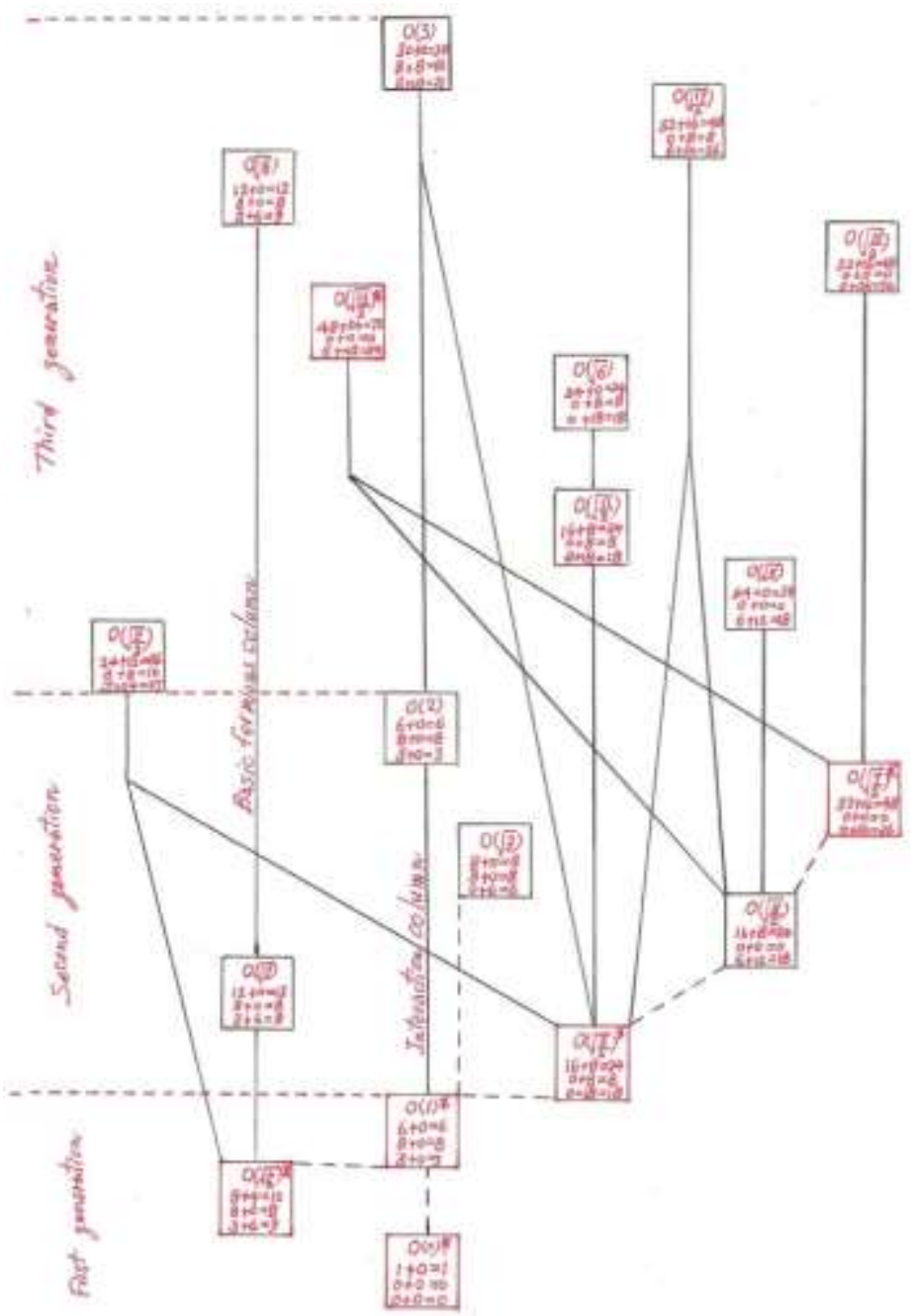

Fig. 21.6: The Symmetries Family Tree for $O(r)$ with $r \leq 3$.

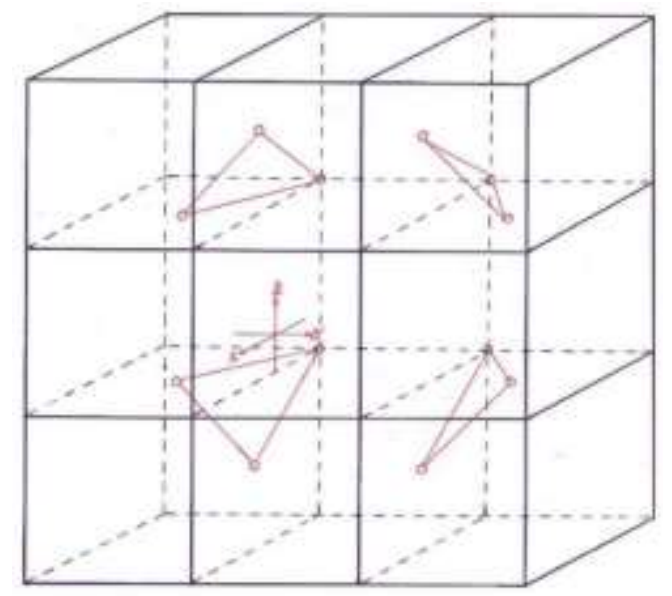

Fig. 21.7: The vertexes of $O(\sqrt{3 / 2})$ with 12 vertexes located at $x>0$ not shown.
As shown in Table 21.1, Fig. 21.6 and Fig. 21.8, symmetry $O(\sqrt{7 / 2})$ is the lowest one has 48 vertexes. In $O(\sqrt{7 / 2})$, the 48 vertexes form 36 squares all with center vertex off surface and no equilateral triangle.

As shown in Table 21.1, Fig. 21.6 and Fig. 21.9, symmetry $O(\sqrt{13 / 2})$ is the one has most vertexes for $r \leq 3$. The 72 vertexes of $O(\sqrt{13 / 2})$ form 54 squares, in which 6 squares are with center vertex on surface and 48 squares are with center vertex off surface. $O(\sqrt{13 / 2})$ has no equilateral triangle. In the Elementary Particle Table, the total number of elementary particles is 72 , which is relate to the vertex number 72 of $O(\sqrt{13 / 2})$.

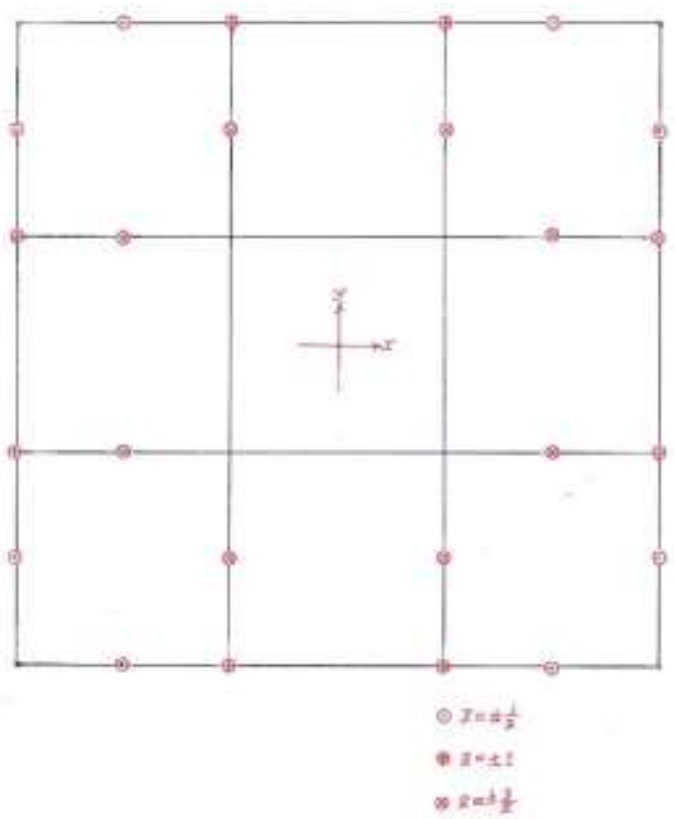

Fig. 21.8: The vertexes distribution of $O(\sqrt{7 / 2})$.

In the Symmetries Family Tree shown by Fig. 21.6, there are five columns; the vertical line connections indicate their heritage, which is originated from the bottom symmetry of each column. Out of five, two columns are significant. The one called "interactions column" is made of $O(0), O(1)$, $O(2)$ and $O(3)$; The other one called "basic Fermions column" is made of $O(\sqrt{1 / 2}), O(\sqrt{2})$ and $O(\sqrt{8})$.

The foundation of the interactions column is the $O(0)$ symmetry representing gravitation and electromagnetic interaction as mentioned previously. As shown in Section 15, all interactions are finally unified to gravitation. The $O(0)$ deserves to be the foundation of interactions column. From the foundation up, the next symmetry is $O(1)$. As mentioned previously, $O(0) \oplus O(1)$ represents gravitation,

electromagnetic, weak, and strong interactions. Fig. 22.6 clearly shows that, all four types of interaction are in this column. Up further, there are two more symmetries, $O(2)$, 
$O(3)$. In which $O(2)$ is an enlarged version of $O(1)$. As shown by the forked line, $O(3)=O(2) \oplus O(\sqrt{3 / 2})$, the combination nature of $O(3)$ is due to the fact that, its radius $r=3$ is sufficiently large to accommodate the additional 24 vertexes in the enlarged version of $O(\sqrt{3 / 2})$. But the core of $O(3)$ is an enlarged version of $O(1)$.

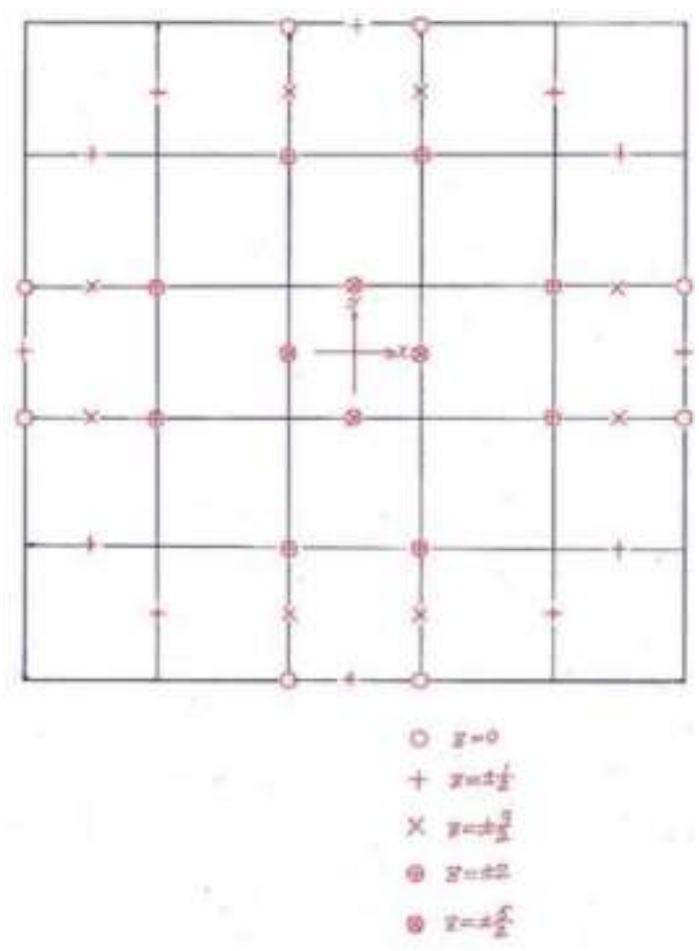

Fig. 21.9: The vertexes distribution of $O(\sqrt{13 / 2})$.

Overall, $O(1), O(2)$, and $O(3)$ are the interaction symmetries for the $1^{\text {st, }} 2^{\text {nd }}$, and $3^{\text {rd }}$ generations, respectively. It implies that, the radius values, 1, 2, 3 are related to the orders of three generations. $O(0)$ is related to all symmetries corresponding to gravity's universality, which was mentioned previously from another perspective.

The foundation of basic fermions column is $O(\sqrt{1 / 2})$, as mentioned previously, $O(\sqrt{1 / 2})$ are related to the first generation 8 fermions of $u_{r}, u_{g}, u_{b}, d_{r}, d_{g}, d_{b}, e, v_{e}$ and their anti-particles. Since $0 \leq \sqrt{1 / 2} \leq 1,1<\sqrt{2}<2$, and $2<\sqrt{8}<3$, it seems natural to relate $O(\sqrt{2})$ to the second generation 8 fermions of $s_{r}, s_{g}, s_{b}, c_{r}, c_{g}, c_{b}, \mu, v_{\mu}$ and their anti-particles; to relate $O(\sqrt{8})$ to the third generation 8 fermions of $b_{r}, b_{g}, b_{b}, t_{r}, t_{g}, t_{b}, \tau, v_{\tau}$ and their anti-particles. The arrangement confirms the correlation between the values of $r$ and the orders of generations.
The column made of $O(\sqrt{3 / 2}), O(\sqrt{11 / 2})$ and $O(\sqrt{6})$ is based on $O(\sqrt{3 / 2}) \cdot O(\sqrt{11 / 2})$ and $O(\sqrt{6})$ have similar properties as $O(\sqrt{3 / 2})$. They all have the same vertexes number 24, the same structure of 8 separate equilateral triangles and 18 squares with center vertex off surface.

The column made of $O(\sqrt{5 / 2})$ and $O(\sqrt{5})$ is based on $O(\sqrt{5 / 2})$. They all have 24 vertexes, and 18 squares in which 6 with center vertex on and 12 off surface. They have no equilateral triangle.

The column made of $O(\sqrt{7 / 2})$ and $O(\sqrt{15 / 2})$ are based on $O(\sqrt{7 / 2})$. They all have 48 vertexes, 36 squares with center vertex off surface. They also have no equilateral triangle.

The three columns based on $O(\sqrt{3 / 2}), O(\sqrt{5 / 2})$, $O(\sqrt{7 / 2})$ with foundations all start from the second generation. As shown in Fig.21.6, the columns based on $O(\sqrt{3 / 2}), O(\sqrt{5 / 2})$, and $O(\sqrt{7 / 2})$ have 2,1 , and 1 symmetries in the third generation, respectively.

The equilateral triangle and the square are two basic elements of the face-centered space lattice. The symmetries in columns based on $O(\sqrt{5 / 2}), O(\sqrt{7 / 2})$ and symmetry $O(\sqrt{13 / 2})$ have no equilateral triangle. But the symmetry with a definitive radius is not alone. The eighteen symmetries in the Symmetries Family Tree live together as a family. From family perspective, more equilateral triangles can be found. For instance, in the combined symmetry $O(\sqrt{3 / 2}) \oplus O(\sqrt{5 / 2})$ as shown in Fig. 21.10, besides the 8 equilateral triangles of $O(\sqrt{3 / 2})$, the combination adds 32 more equilateral triangles. $O(\sqrt{3 / 2}) \oplus O(\sqrt{5 / 2})$ as a whole has 40 equilateral triangles. The same is true for some other symmetry. For instance, the combined symmetries of $O(\sqrt{2}) \oplus O(\sqrt{5}), O(\sqrt{3 / 2}) \oplus O(\sqrt{7 / 2}), O(\sqrt{11 / 2}) \oplus O(\sqrt{13 / 2})$ and $O(\sqrt{9 / 2}) \oplus O(\sqrt{15 / 2})$ all add 8 more equilateral triangles. The numbers listed in Table 21.1 and Table 21.2 only count the equilateral triangles for each symmetry alone. Actually, the total number of equilateral triangles in $O(r)$ and $C(r)$ for $r \leq 3$ as two families are far more than the listed grand total of $112+112=224$.

The symmetries of $C(r)$ centered at cubic vertex have the same type of symmetries as $O(r)$. In fact, an $O(r)$ shifting its center along two orthogonal directions by $L_{P} / 2$ for each direction become a $C(r)$ and vice versa. $C(r), O(r)$ have the same "Conn.+ Separ. $=$ Total" and "On+Off $=$ Total". The difference is that, in the "Oct. $\oplus C u b .=$ Total "column, the numbers of "Oct." and "Cub." vertexes may change for $C(r)$ from those of $O(r)$ and keep the number of "Total" unchanged. Therefore, the Family Tree for $C(r)$ is the same 
type for $O(r)$ except the two numbers different in the "Oct. $\oplus$ Cub. $=$ Total".

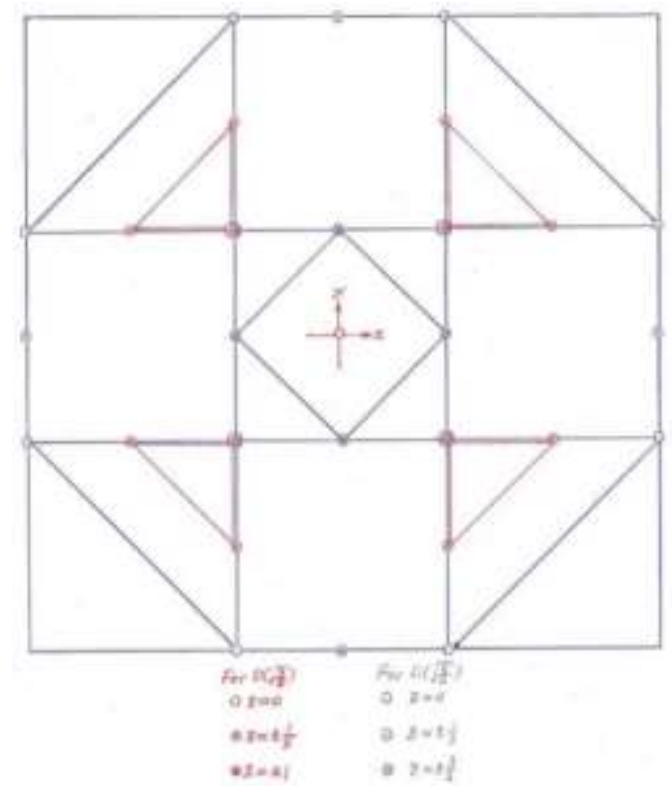

Fig. 21.10: Additional equilateral triangles of $O(\sqrt{3 / 2}) \oplus O(\sqrt{5 / 2})$.

The real difference between $C(r)$ and $O(r)$ is their physics interpretations. As mentioned previously, $O(r)$ based on the octahedral part is related to fermions and $C(r)$ based on the cubic part is related to bosons. This may provide a clue for the physics interpretation of $C(r)$ symmetries. For instance, the "basic fermions column" of $O(\sqrt{1 / 2}), O(\sqrt{2}), O(\sqrt{8})$ is interpreted to represent the basic fermions of three generations. Likewise, the column of $C(\sqrt{1 / 2}), C(\sqrt{2})$, $C(\sqrt{8})$ is the "basic bosons column" of three generations. The $C(\sqrt{1 / 2})$ are related to $g, g_{1}, g_{2}, g_{3}, g_{4}, g_{5}, \gamma, U_{1}$ of the first generation bosons. The $C(\sqrt{2})$ are related to $g_{6}, g_{7}$, $g_{8}, G_{1}, G_{2}, G_{3}, X, U_{2}$ of the second generation bosons. The $C(\sqrt{8})$ are related to $G_{4}, G_{5}, G_{6}, G_{7}, G_{8}, Z, W, U_{3}$ of the third generation bosons. As $O(\sqrt{3})$ is related to the baryons octet, likewise $C(\sqrt{3})$ is related to the mesons octet of $\pi^{+}(u \bar{d}), \pi^{-}(d \bar{u}), K^{+}(u \bar{s}), \bar{K}^{0}(s \bar{d}), K^{0}(d \bar{s}), K^{-}(s \bar{u})$, $\pi^{0}((d \bar{d}-u \bar{u}) / \sqrt{2}), \eta((d \bar{d}+u \bar{u}) / \sqrt{2})$ as shown in Fig. 21.11 .

In the $C(r)$ system, there are also 6 essential symmetries: $C(0), C(\sqrt{1 / 2}), C(1), C(\sqrt{3 / 2}), C(\sqrt{7 / 2})$ and $C(\sqrt{13 / 2})$, in which $C(0), C(\sqrt{1 / 2}), C(1)$ are basic symmetries.

Notice that, in Table 22.1 and Table 21.2, the number 163 is total number of vertexes in the 6 essential symmetries of $O(r)$ as well as the 6 essential symmetries of $C(r)$ :
$163=1+6+12+24+48+72$. In number theory, the number 163 has very special properties [18].

1 . Number 163 is a prime number.

2. Number 163 is the number of columns in the Monster group's character table to give the independent mini-jfunctions.

3. Number 163 is related to an irrational number very close to an integer:

$e^{\pi \sqrt{163}}=262537412640768743.99999999999925 \cdots$

4 , It was noticed by Euler that, the number 163 is in the solutions of an equation: $f(x)=x^{2}-x+41=0$ with solutions:

$$
x=\frac{1 \pm i \sqrt{163}}{2} .
$$

The values of $f(x)=x^{2}-x+41$ for $1 \leq x \leq 40$ give prime numbers.

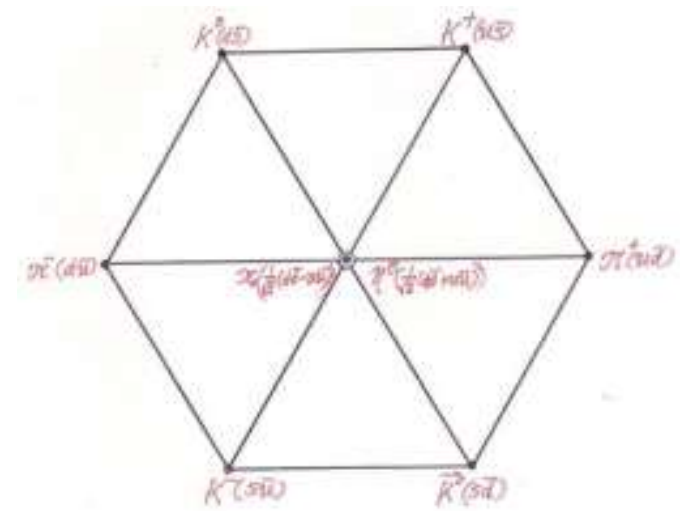

Fig.21.11: The mesons octet represented by $C(\sqrt{3})$.

Moreover, as shown by Fig. 21.12, the Number Tower, 163 is intrinsically related to the $m$-parameters of the first generation particles including 7 elementary fermions $e, u_{r}$, $u_{g}, u_{b}, d_{r}, d_{g}, d_{b}$ and 7 elementary bosons, $\gamma, g, g_{1}$, $g_{2}, g_{3}, g_{4}, g_{5}$.

The number of vertexes in the 6 essential symmetries in $O(r)$ system or $C(r)$ system equals to a very special number of 163 sitting on top of the Number Tower. It has important implications.

1. The set of 6 essential symmetries is not an arbitrary selection. It is based on mathematics.

2. The total number of elementary particles is 72 . It is the number of vertexes in $O(\sqrt{13 / 2})$ or $C(\sqrt{13 / 2})$. It also is the largest vertexes number in any symmetry of $O(r)$ or $C(r)$ with radius $r \leq 3$. The number 72 close relation with the magic number 163 is based on mathematics shown by the Number Tower. The SQS theory Elementary Particle Table given in Section 18 is based on mathematics. It contains 72 elementary particles including 24 particles, 24 anti-particles, 24 neutral particles. Notice that, $24,48=24+24,72$ are the 
number of vertexes for $O(\sqrt{3 / 2}), O(\sqrt{7 / 2}), O(\sqrt{13 / 2})$ and their counterparts in the $C(r)$ system, respectively.

3. There are only three generations of elementary particles. It was supported by Prime Number Postulation in Section 11 and Conclusion 12.1 in Section 12, which are based on the prime numbers property in number theory. Here we have the second independent support from number theory and space symmetry. As the number 72 backed by the magic number 163 , there is no room left for more generation beyond the existing three generations. Otherwise, the total number of elementary particles would exceed 72, which is not supported by the Number Tower and the magic number 163 sitting on top of it.

4. As shown in the Number Tower, the first generation is the base of all three generations. This is also supported by the two basic columns in the Symmetries Family Tree, in which the first generation particles and interactions serve as the foundations. The second and the third generations are the extensions of the first generation. This is also supported by Standard Model. SQS theory provides the mathematic interpretations based on number theory and symmetries of space.

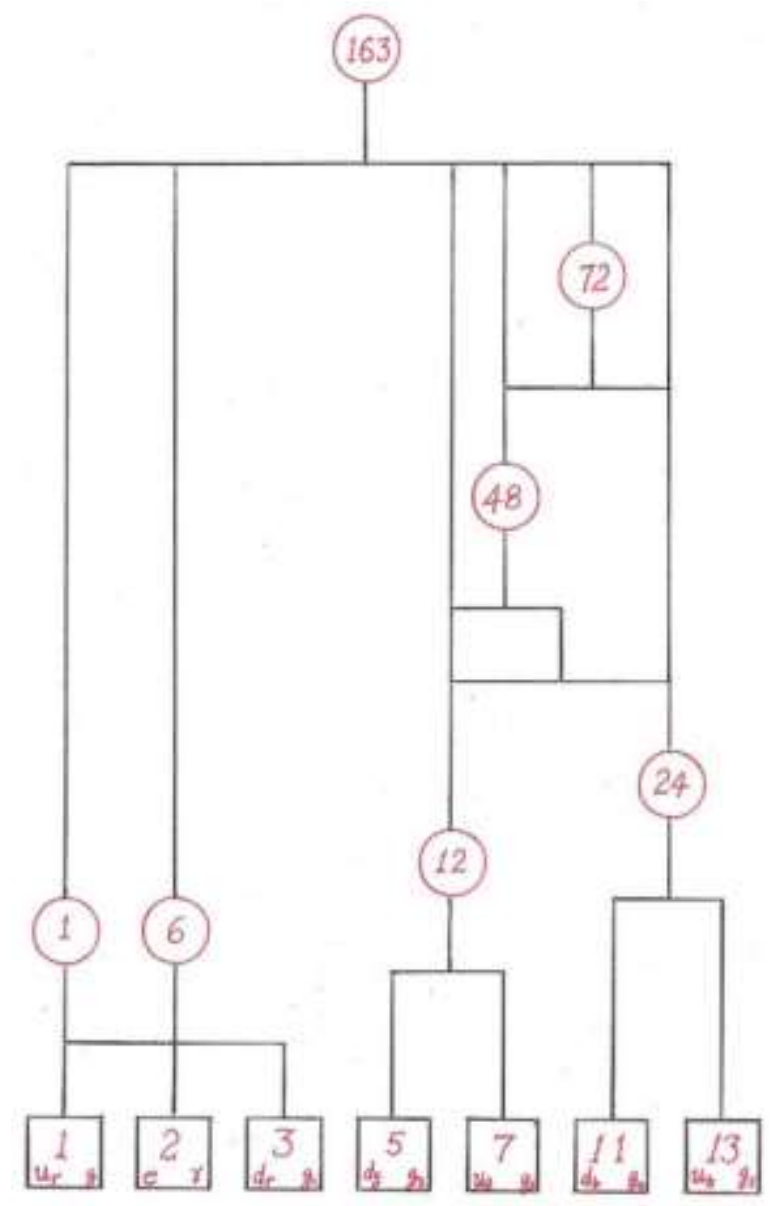

Fig. 21.12: The Number Tower.
According to the above discussion, the $18+18=36 O(r)$ and $C(r)$ symmetries with radius $r \leq 3$ cover all elementary particles and interactions for three generations. What about the symmetries with radius $r>3$ ? The answer will be given in Section 23.

Ideally, symmetries $O(r), C(r)$ all are perfect. In reality, the physics groups corresponding to $O(r), C(r)$ are not perfect caused by symmetry broken for particles to obtain mass. Take $O(\sqrt{1 / 2})$ as an example. In its perfect symmetry form, the 8 fermions $u_{r}, u_{g}, u_{b}, d_{r}, d_{g}, d_{b}, e, v_{e}$ all are mass-less to start with. Each of them obtains mass by broken symmetry in different ways.

The 12 vertexes of $O(\sqrt{1 / 2})$ are shown in Fig.21.13.

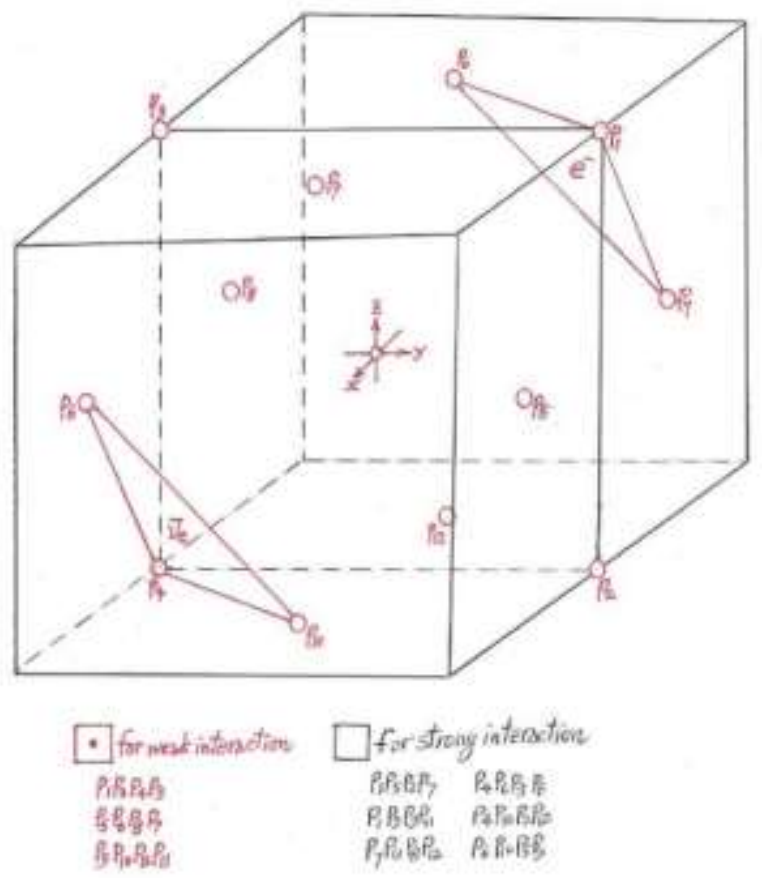

Fig. 21.13: The two triplets for $e^{-}$and $\bar{v}_{e}$ to demonstrate the retained symmetries and broken symmetries.

The equilateral triangle $p_{1} p_{7} p_{11}$ representing electron includes a cubic vertex $p_{1}$ and two octahedral vertexes $p_{7}$, $p_{11}$. There are three squares $p_{1} p_{2} p_{4} p_{3}, p_{5} p_{6} p_{8} p_{7}$, $p_{9} p_{10} p_{12} p_{11}$ with center vertex on surface representing weak interaction connect to $p_{1}, p_{7}, p_{11}$ respectively. There are three squares $p_{1} p_{5} p_{2} p_{7}, p_{1} p_{9} p_{3} p_{11}, p_{7} p_{11} p_{8} p_{12}$ with center vertex off surface representing strong interaction connect to $p_{1} \& p_{7}, p_{1} \& p_{11}, p_{7} \& p_{11}$ respectively. For electron to obtain mass, the symmetrical triplet $p_{1} p_{7} p_{11}$ must break under the conditions: (1) All three squares $p_{1} p_{5} p_{2} p_{7}, p_{1} p_{9} p_{3} p_{11}$, $p_{7} p_{11} p_{8} p_{12}$ with center vertex off center must break because electron has no strong interaction. (2) At least some of three 
squares $p_{1} p_{2} p_{4} p_{3}, p_{5} p_{6} p_{8} p_{7}, p_{9} p_{10} p_{12} p_{11}$ with center vertex on surface must retain because electron has weak interaction. There are two possible scenarios to meet these requirements.

Scenario-1: For an electron alone. Two vertexes $p_{7}, p_{11}$ shift angles to break the triplet $p_{1} p_{7} p_{11}$ for electron to obtain mass. The results are: (1) All three squares $p_{1} p_{5} p_{2} p_{7}, p_{1} p_{9} p_{3} p_{11}, p_{7} p_{11} p_{8} p_{12}$ with center vertex off center are broken for no strong interaction. (2) One square $p_{1} p_{2} p_{4} p_{3}$ with center vertex on center retains for weak interaction.

Scenario-2: For electron and electron anti-neutrino $\bar{v}_{e}$ as a matched pair. Equilateral triangle $p_{4} p_{6} p_{10}$ representing $\bar{v}_{e}$ includes a cubic vertex $p_{4}$ and two octahedral vertexes $p_{6}, p_{10}$. There are three squares $p_{1} p_{2} p_{4} p_{3}, p_{5} p_{6} p_{8} p_{7}, p_{9} p_{10} p_{12} p_{11}$ with center vertex on surface representing weak interaction connect to $p_{4}$, $p_{6}, p_{10}$ respectively. There are three squares $p_{4} p_{6} p_{3} p_{8}$, $p_{4} p_{10} p_{2} p_{12}, p_{6} p_{10} p_{5} p_{9}$ with center vertex off surface representing strong interaction connect to $p_{4} \& p_{6}$, $p_{4} \& p_{10}, p_{6} \& p_{10}$ respectively. Two vertexes $p_{1}, p_{7}$ shift angles to break the triplet $p_{1} p_{7} p_{11}$ for electron to obtain mass. At the same time, two vertexes $p_{4}, p_{6}$ shift the same angles with opposite directions to break the triplet $p_{4} p_{6} p_{10}$ for $\bar{v}_{e}$ to obtain mass. The results are: (1) All six squares $p_{1} p_{5} p_{2} p_{7}, p_{1} p_{9} p_{3} p_{11}$, $p_{7} p_{11} p_{8} p_{12}, p_{4} p_{6} p_{3} p_{8}, p_{4} p_{10} p_{2} p_{12}, p_{6} p_{10} p_{5} p_{9}$ with center vertex off center are broken for no strong interaction. (2) All three squares $p_{1} p_{2} p_{4} p_{3}, p_{5} p_{6} p_{8} p_{7}$, $p_{9} p_{10} p_{12} p_{11}$ with center vertex at center retain for weak interaction. This scenario shows that, besides their mathematical correlation of $n$-parameter matching, there is a geometrical correlation between electron and its anti-neutrino $\bar{v}_{e}$.

It is conceivable that, Scenario-2 is for regular type weak interactions with electron and $\bar{v}_{e}$ as a matched pair.

Scenario-1 is for rare type weak interactions with electron acting alone without $\bar{v}_{e}$. Since Scenario-1 has only one square retained with center vertex on surface and Scenario-2 has all three squares retained with center vertex on surface, which serve as an explanation for the rarity of the rare type weak interaction for electron without $\bar{\nu}_{e}$.

The similar scenarios are also valid for $\mu^{-} \& \bar{v}_{e}$ and $\tau^{-} \& \bar{v}_{\tau}$ which belong to the second and third generations, respectively.
In Fig. 21.13, the other two triplets $p_{2} p_{5} p_{12}$ and $p_{3} p_{8} p_{9}$ represent up quarks $u_{r}, u_{g}, u_{b}$ and down quarks $d_{r}, d_{g}$, $d_{b}$. For them to break symmetries to obtain mass and keep strong interaction as well as weak interaction, the way to shift angles is under the conditions: (1) At least some of the six squares $p_{1} p_{5} p_{2} p_{7}, p_{1} p_{9} p_{3} p_{11}, p_{7} p_{11} p_{8} p_{12}, p_{4} p_{6} p_{3} p_{8}$, $p_{4} p_{10} p_{2} p_{12}, p_{6} p_{10} p_{5} p_{9}$ with center off surface must retain for strong interaction. (2) At least some of three squares $p_{1} p_{2} p_{4} p_{3}, p_{5} p_{6} p_{8} p_{7}, p_{9} p_{10} p_{12} p_{11}$ with center on surface must retain for weak interaction. The same requirements are also valid for triplets representing strange, charm, bottom and top quarks.

These arrangements show the versatility and richness of the theory.

In the meantime, the above discussions regarding to the correlations of the symmetries in $O(r)$ and $C(r)$ systems to physics groups of elementary particles are hyperbolic. The real correlations between the symmetries listed in Table 21.1, Table 21.2 and the particles' groups such as $U(1), S U(2)$, $S U(3)$ require mathematical proof and more physical verification. But one thing is clear. If the face-centered space structure is the real space to accommodate all elementary particles and interactions, their symmetry groups must be originated from it.

It is important to point out that, all symmetries in the facecentered space structure are represented by real numbers. On the other hand, most of particles' groups are represented by complex numbers. This is a major difference. But the difference is only superficial. So far, SQS theory hasn't introduced intrinsic time as a variable yet. In essence, the intrinsic time can be represented by the phase angle of complex numbers. Once the intrinsic time variable is introduced, the major difference between these two systems will be resolved. In fact, intrinsic time represented by complex numbers' phase angle is the key to understand the relation between symmetries $O(r), C(r)$ in space and groups in particle physics.

Postulation 21.2: All elementary particles' groups are originated from the symmetries in $O(r)$ and $C(r)$ systems of space structure with face-centered lattice. Explanation: Lack of mathematical proof, this is the best thing one can offer. Table 21.1, Table 21.2 and the Symmetries Family Tree are useful for further investigations on this topic.

From SQS theory viewpoint, the physics groups of basic fermions and bosons are presented in Fig 21.14. Quarks with different colors are treated as different particles. Leptons are presented as trefoil model with three branches combined. The elementary fermions are represented by 12 equilateral triangles. The 22 elementary bosons except $\gamma$ and $g$ are represented by 8 equilateral triangles, in which the 6 


\section{Z. Y. SHEN}

equilateral triangles are combined into 2 hexagons. $\gamma$ and $g$ are located at the center of equilateral triangle representing $W$, $Z, X$. Fig. 21.14 is useful to identify the correlation between the symmetries in $O(r), C(r)$ systems and the groups in particle physics.

Postulation 21.1 and Postulation 21.2 are here to stay for SQS theory. Hopefully mathematic proofs and more physics evidences will follow. In fact, they have supports already. One is the Gaussian sphere and the Kepler-Hales theorem. If gravitation dominates in the Planck scale microscopic space, the face-centered lattice structure along with its symmetries is the only logical choice. The other is that, $O(r)$ and $C(r)$ symmetries with $r \leq 3$ fit physics groups well.

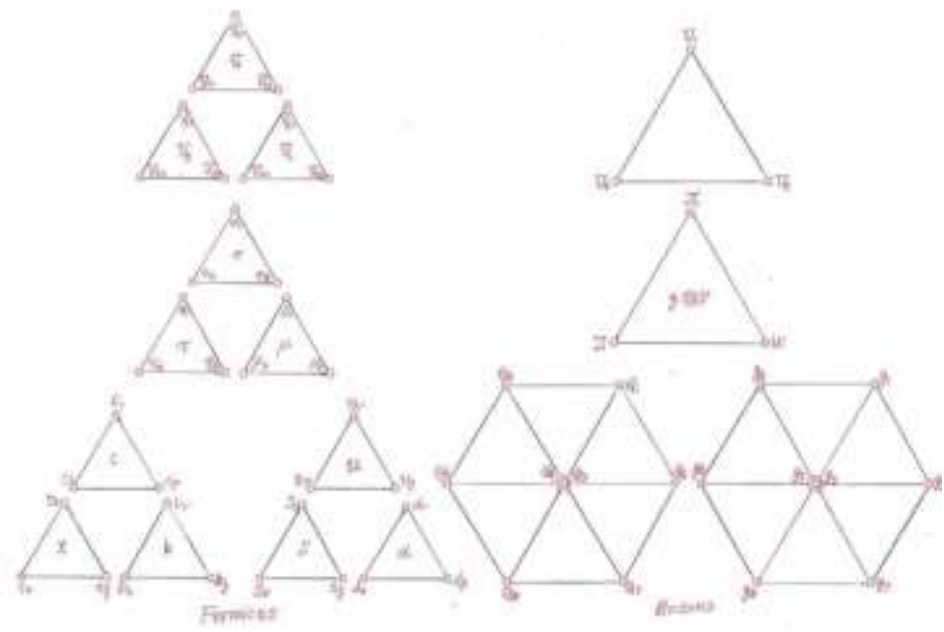

Fig.21.14: Elementary particles organized in groups.

Inherited from the face-centered lattice structure, space has intrinsic symmetries, which serve as the origin of all groups of elementary particles. Postulation 21.2 is waiting for proof. From SQS theory standpoint, it is the way Mother Nature selected.

In essence, space is like a crystal, but the macroscopic space is not a single crystal. Otherwise, the single crystal space with face-centered lattice is macroscopically anisotropic with preferred directions. That is impossible. Because theoretically space cannot be in such highly organized state with extremely low entropy; experimentally there is no evidence for such macroscopic space anisotropy. In fact, space is amorphous in large scale with single-crystal or poly-crystal domains. The system containing enormous identical Gaussian spheres with relative weak interactions fits well for the conditions of grand number phenomena. The number of Gaussian spheres in a typical domain can be estimated according to (16.13) as:

$$
N \sim 10^{11 G} .
$$

In which $G$ is the rank number. The $N \sim 10^{11 G}$ Gaussian spheres fit in a volume with length scale of

$$
L_{d o m}(G) \sim \sqrt[3]{10^{11 G}} L_{P} /(2 \sqrt{2}),
$$

For $G=1: L_{d o m}(1) \sim 10^{3} L_{P} \sim 10^{-32} \mathrm{~m}$,

For $G=2: L_{\text {dom }}(2) \sim 10^{6} L_{P} \sim 10^{-29} \mathrm{~m}$,

For $G=3: L_{d o m}(3) \sim 10^{10} L_{P} \sim 10^{-25} \mathrm{~m}$.

Such amorphous space is isotropic with grainy structures on top of the Planck scale grainy structure. In the multi-layer grainy structure, the lowest layer with Gaussian sphere as basic building block is single-crystal or near single-crystal, which serves as the home for elementary particles with their interactions. We will come back to this topic in later sections.

The sum of 3-dimensional Gaussian probability $S_{3}(x, y, z)$ for the cubic part alone was introduced by (2.24) with subscript changed for identification:

$S_{3, \text { cube }}(x, y, z)=S_{3}(x, y, z)=\sum_{x_{i}=-\infty}^{\infty} \sum_{y_{j}=-\infty}^{\infty} \sum_{z_{k}=-\infty}^{\infty} e^{-\pi\left[\left(x-x_{i}\right)^{2}+\left(y-y_{j}\right)^{2}+\left(z-z_{k}\right)^{2}\right]}$.

The octahedral part lattice can be treated as three Cartesian systems with 0.5 shifts along two orthogonal axes. In such coordinate system, the sum of the 3-dimensional Gaussian probability of the octahedral part alone is:

$S_{3, c t c}(x, y, z)=\frac{1}{3} S_{3}(x, y-0.5, z-0.5)+\frac{1}{3} S_{3}(x-0.5, y, z-0.5)+\frac{1}{3} S_{3}(x-0.5, y-0.5, z)$.

In which, the numerical factor $1 / 3$ is for unitarity.

According to (21.13) and (21.14), the overall sum of Gaussian probability for the 3-dimensional space with facecentered lattice structure is

$$
\begin{aligned}
& S_{3, f-c}(x, y, z)=\frac{1}{4} S_{3}(x, y, z)+\frac{1}{4} S_{3}(x, y-0.5, z-0.5) . \\
& +\frac{1}{4} S_{3}(x-0.5, y, z-0.5)+\frac{1}{4} S_{3}(x-0.5, y-0.5, z)
\end{aligned}
$$

In (21.15), the first term represents the cubic part and the other three terms combined represent the octahedral part. The numerical factor $1 / 4$ for unitarity has a deep meaning. In a Planck cube with the face-cantered lattice structure, there are 8 Gaussian spheres centered at 8 cubic vertexes at 8 corners and another 6 Gaussian spheres centered at 6 octahedral vertexes at 6 face centers. For the cubic part, the 8 spheres at corners each one sharing with 8 cubes contribute 1 Gaussian sphere to the Planck cube. For the octahedral part, the 6 spheres at 6 surface centers each one sharing with 2 cubes contribute 3 Gaussian spheres to the Planck cube. Therefore, the filling ratio for cubic part versus octahedral part is $1: 3$ corresponding to the unitarity factors $\frac{1}{4}$ and $\frac{1}{4}+\frac{1}{4}+\frac{1}{4}=3 \times \frac{1}{4}$.

It confirms the reason for selecting the numerical factor $1 / 4$ for unitarity in (21.15).

Actually formula (21.15) serves as a checkpoint for the consistency of the face-centered space structure. The facecentered structure is based on the most compact packing of Gaussian spheres. A hidden assumption is that, all Gaussian spheres in the space must be identical. In terms of profile and size, they are identical evidenced by $\sigma=1 / \sqrt{2 \pi}$ and 
$R=1 /(2 \sqrt{2})$ for all Gaussian spheres. The four identical numerical factor $1 / 4$ in (21.15) proved that, all Gaussian spheres also have the same vacuons density. It shows that, no matter where they are located, all Gaussian spheres have the same profile, the same size and the same vacuons density. In short, they are identical. Moreover, the four identical numerical factors also make the $S_{3, f-c}(x, y, z)$ symmetry with respect to interchanges of three variables $x, y, z$, which is required by the geometrical symmetry of the face-centered lattice structure. So everything is consistent.

According to Postulation 21.1, space has face-centered lattice structure, in which the cubic lattice structure serves as one of its two parts. The sums of probability data listed in Table 2.2 are based on $S_{3}(x, y, z)$ of (2.24) for the cubic lattice structure. For the space with face-centered lattice structure, the sum of Gaussian probability formula is (21.15). The calculated sums of probability data are listed in Table 21.3.

As shown in Table 21.3, the $\left.S_{3, f-c}(x, y, z)\right|_{\max }$ and

$\left.S_{3, f-c}(x, y, z)\right|_{\min }$ are:

$\left.S_{3, f-c}(x, y, z)\right|_{\max }=S_{3, f-c}(0,0,0)=1.00066522034066$,

$\left.S_{3, f-c}(x, y, z)\right|_{\min }=S_{3, f-c}(0.5,0.5,0.5)=0.999375328059491$ •

The difference between $\left.S_{3, f-c}(x, y, z)\right|_{\max }$ and $\left.S_{3, f-c}(x, y, z)\right|_{\min }$ is in $10^{-4}$ order. Compare to the values listed in Table 2.2:

$\left.S_{3, \text { cube }}(x, y, z)\right|_{\max }=S_{3}(0,0,0)=1.28236311585946$,

$\left.S_{3, \text { cube }}(x, y, z)\right|_{\min }=S_{3}(0.5,0.5,0.5)=0.762497670698562$ ( $\left.21.17 \mathrm{~b}\right)$ The difference between $\left.S_{3, \text { cube }}(x, y, z)\right|_{\max }$ and $\left.S_{3, \text { cube }}(x, y, z)\right|_{\min }$ is in $10^{-1}$ order. The comparison shows that, the sums of probability for the face-centered lattice structure are more evenly distributed due to more Gaussian probability distribution functions added at octahedral vertexes.

Table 21.3: $S_{3, f-c}(x, y, z)$ Values at 125 Points in a Planck Cube

\begin{tabular}{|c|c|c|c|}
\hline \multicolumn{4}{|c|}{ Based on $(21.15) *$} \\
\hline$z=0 \& z=1$ & $x=0 \& \& \mathrm{x}=1$ & $x=0.25 \& \mathrm{x}=0.75$ & $x=0.5$ \\
\hline$y=0 \& y=1$ & 1.00066652034066 & 1.00000697463607 & 0.999375328059491 \\
\hline$y=0.25 \& y=0.75$ & 1.00000697463607 & 0.999993025266642 & 1.00000697463607 \\
\hline$y=0.5$ & 0.999375328059491 & 1.00000697463607 & 1.00066652034066 \\
\hline$z=0.25 \& z=0.75$ & $x=0 \& \mathrm{x}=1$ & $x=0.25 \& \mathrm{x}=0.75$ & $x=0.5$ \\
\hline$y=0 \& y=1$ & 1.00000697463607 & 0.999993025266642 & 1.00000697463607 \\
\hline$y=0.25 \& y=0.75$ & 0.999993025266642 & 0.999979076091801 & 0.999993025266642 \\
\hline$y=0.5$ & 1.00000697463607 & 0.999993025266642 & 1.00000697463607 \\
\hline$z=0.5$ & $x=0 \& \mathrm{x}=1$ & $x=0.25 \& \mathrm{x}=0.75$ & $x=0.5$ \\
\hline$y=0 \& y=1$ & 0.999375328059491 & 1.00000697463607 & 1.00066652034066 \\
\hline$y=0.25 \& y=0.75$ & 1.00000697463607 & 0.999993025266642 & 1.00000697463607 \\
\hline$y=0.5$ & 1.00066652034066 & 1.00000697463607 & 0.999375328059491 \\
\hline
\end{tabular}

* The summation index in (21.15) is truncated at a sufficient large number $i, j, k= \pm 1000$.

Theorem 21.1: In the face-centered space structure, the Random Walk Theorem is valid only for the cubic part of face-centered structure. It is not valid for the octahedral part and the face-centered structure as a whole. In other words, all steps of the random walk zigzagging path only stop at the cubic vertexes.

Proof: As shown in Section 4, the key to prove Random Walk Theorem is based on the fact that, the numerical factor in front of the exponential part of (4.3) and (4.4) is 1 . Otherwise, the proof does not hold. The numerical factor in front of the exponential part in of (21.13) is 1 for the cubic part, which fits the requirement for random walk theorem proof. On the other hand, the numerical factors in front of the exponential part in $S_{3, o c t}(x, y, z)$ of (21.14) for the octahedral part and $S_{3, f-c}(x, y, z)$ of

(21.15) for the face-centered structure as a whole are $1 / 3$ and $1 / 4$, respectively, which do not fit the requirement for the random walk theorem. QED

Theorem 21.1 has important physics significance. It reveals more insights from the Random Walk Theorem. The random walk process is the mechanism for interactions between particles mediated by bosons without mass, i.e. $\gamma$ and $g$. Moreover, as shown in Section 4 and Section 15, gravitational force and electrostatic force are related to the long path and short path, respectively, which are based on the converting factor originated from the Random Walk Theorem. These two forces belong to the long range type with strengths inversely proportional to the square of distance. The tremendous difference between these two forces' strengths is also originated from the difference between long path and short path. All of these features are originated from the Random Walk Theorem, which is only valid for the cubic part of space. The adding of octahedral part in face-centered space structure does not make difference for these two long range forces.

Theorem 21.1 provides additional supports for Postulation 21.1 and to some extent for Postulation 21.2 as well. It indicates that, the theory based on face-centered space is consistent.

\section{Section 22: Cosmology}

Particle physics and cosmology are closely related. The topics of this section are cosmology and its correlation with particle physics based on prime numbers and the space structure introduced in Section 21.

Let's start from some related questions.

According to prime numbers table and the Prime Numbers Postulation, there are only three generations of elementary particles in the current period of universe. Is it the only period? This is the first question.

The electron converting factor $N_{e}=1.501197 \times 10^{23}$ is close to a $G=2$ grand number. Is there a $G=1$ grand number related to electron? This is the second question.

In Table 15.1, the mass or energy gap between $M_{e w}=152.7547 \mathrm{Gev} / \mathrm{c}^{2}$ and $M_{e w s}=8.44708 \times 10^{15} \mathrm{Gev} / \mathrm{c}^{2}$ is thirteen orders of magnitude. Is this tremendous gap really empty? This is the third question. 


\section{Z. Y. SHEN}

Let' start from the first question. The way to search for other periods is to look at the prime numbers table. The odd prime numbers less than 1000 is listed in Table 22.1.

To identify possible other periods and generations in a legitimate manner, the rules used to determine the three generations in the current period are summarized in a definition. Then a postulation is introduced to make the connections between generations of elementary particles and cosmic periods as well as space dimensions.

Table 22.1: The Odd Prime Numbers Less Than 1000*

\begin{tabular}{|c|c|c|c|c|c|c|c|c|c|c|c|c|c|c|c|c|c|c|c|c|}
\hline \multicolumn{1}{|c|}{3} & 5 & 7 & 11 & 13 & 17 & 19 & 23 & 29 & 31 & 37 & 41 & 43 & 47 & 53 & 59 & 61 & $\underline{67}$ \\
\hline$\underline{71}$ & 73 & 79 & 83 & $\underline{89}$ & $\underline{97}$ & 101 & 103 & 107 & 109 & 113 & 127 & 131 & 137 & 139 & 149 & 151 & 157 & $\underline{163}$ \\
\hline 167 & 173 & 179 & 181 & 191 & 193 & 197 & 199 & $\underline{211}$ & $\underline{223}$ & 227 & $\underline{229}$ & $\underline{233}$ & 239 & 241 & 251 & 257 & 263 & 269 \\
\hline 271 & 277 & 281 & 283 & 293 & 307 & 311 & 313 & 317 & 331 & 337 & 347 & $\underline{349}$ & $\underline{353}$ & 359 & 367 & 373 & 379 & 383 \\
\hline 389 & 397 & 401 & 409 & 419 & 421 & 431 & 433 & 439 & 443 & 449 & 457 & 461 & 463 & 467 & 479 & 487 & 491 & 499 \\
\hline 503 & 509 & 521 & 523 & 541 & 547 & 557 & 563 & 569 & 571 & 577 & 587 & 593 & 599 & 601 & 607 & 613 & 617 & 619 \\
\hline 631 & 641 & 643 & 647 & 653 & 659 & 661 & 673 & 677 & 683 & 691 & 701 & 709 & 719 & 727 & 733 & 739 & 743 & 751 \\
\hline 757 & 761 & 769 & 773 & 787 & 797 & 809 & 811 & 821 & 823 & 827 & 829 & 839 & 853 & 857 & 859 & 863 & 877 & 881 \\
\hline 883 & 887 & 907 & 911 & 919 & 929 & 937 & 941 & 947 & 953 & 967 & 971 & 977 & 983 & 991 & 997 & & & \\
\hline
\end{tabular}

*Note: The prime number pairing is for quarks only. 1 for $u_{r}$ is included and 2 for $e_{r}$ is excluded.

Definition 22.1: A set of three consecutive even pairs of prime numbers is defined as one generation. A set of six consecutive even pairs of prime numbers is defined as two generations. A set of nine consecutive even pairs of prime numbers is defined as three generations. This definition is also valid for other possible periods.

Postulation 22.1: The number of generations is intrinsically related to elementary particles, cosmic periods and space dimensions. The first cosmic period has one generation of elementary particles in 1-dimensional space. The second cosmic period has two generations of elementary particles in 2-dimensional space. The third cosmic period has three generations of elementary particles in 3-dimensional space.

Lemma 22.1: In case the number of consecutive even prime numbers pairs is not a multiple of 3 , it is defined as a period having fractional generations of elementary particles in the space with fractional dimensions.

Explanation: According to Definition 22.1 and Postulation 22.1 , the current period is the third cosmic period, in which there are three generations of elementary particles in 3-dimensional space. The other periods are hypothetical and subject to verification and confirmation.

With the help of Definition 22.1 and Postulation 22.1, let's look at the prime numbers table and search for other possible cosmic periods.

In Table 22.1, the $m$-parameters of 18 quarks started from $1 \& 3$ ended at $59 \& 61$ are listed in the first row. Since the before reduction $m$-parameters of leptons and bosons are related to their companion quarks' $m$-parameters, the meaning for numbers of even prime numbers pairs is not just for quarks. In fact, the nine even pairs of prime numbers represent three generations of elementary particles for the current period in 3-dimensional space. It is defined as the cosmic third period.
In the second row of Table 22.1, a set of six even pairs of prime numbers started from $101 \& 103$ ended at $151 \& 157$ is found to represent two generations of elementary particles. According to Definition 22.1 and Postulation 22.1, the two generations of elementary particles belong to the second cosmic period in 2-dimensional space.

In the third row of Table 22.1, a set of four even pairs of prime numbers started from $167 \& 173$ ended at $197 \& 199$ is found. According to Postulation 22.1 and Lemma 22.1, it is defined as the first period with $1 \frac{1}{3}$ generations of elementary particles in $1 \frac{1}{3}$-dimensional space.

In the third and fourth rows of Table 22.1, another set of nine even pairs of prime numbers started from $239 \& 241$ ended at $337 \& 347$ is found to represent three generations of elementary particles. The newly found three generations are different from the three generations of current period. They belong to the pre-big-bang period in 3-dimensional space.

The reason for the name of cosmic periods will be given later.

In Table 22.1, the odd pair immediately before and after a set of even pairs are underlined. They serve the function to start and to stop the set of even pairs. It is interesting to notice that, a single prime number, the special prime number 163 , separates two sets of even prime number pairs representing the first and second cosmic periods.

All pairs of prime members representing four periods are even pairs. The prime numbers in between periods are either a single prime number or pairs not qualified to form generation(s) represented by at least three consecutive even pairs.

The newly found three cosmic periods along with their generations meets all rules. It is legitimate in mathematic sense.

In the first cosmic period, the one generation is identified as the first generation including six quarks $u_{r}, u_{g}, u_{b}, d_{r}$, 
$d_{g}, d_{b}$, two leptons $e, v_{e}$ and their anti-particles along with eight bosons $\gamma, g, g_{i}(i=1,2,3,4,5)$ and $U_{1}$.

In the second cosmic period, the two generations are identified as the first generation and the second generation including twelve quarks $u_{r}, u_{g}, u_{b}, d_{r}, d_{g}, d_{b}, s_{r}, s_{g}, s_{b}$, $c_{r}, c_{g}, c_{b}$, four leptons $e, v_{e}, \mu, v_{\mu}$ and their anti-particles along with sixteen bosons $\gamma, g, g_{i}(i=1,2 \cdots 8), G_{i}(i=1,2,3)$, $X$ and $U_{1}, U_{2}$.

In the third cosmic period, the three generations are identified as the first generation, the second generation and the third generation including all elementary particles listed in the Elementary Particles Table.

This serves as an introduction for the first question, the details will be given later.

Let's turn attention to the second question, which concerns electron converting factor $N_{e}$ as $G=2$ grand number.

In essence, it is to search whether there is an electron intermediate state in existence corresponding to $G=1$ grand number $\sqrt{N_{e}} \sim 10^{11}$. Let's go back to the logistic equation of (16.1). Substituting $\sqrt{N_{e}}$ to replace $N_{e}$ and $\alpha\left(M_{Z}\right)$ to replace $\alpha\left(M_{e}\right)$, a numerical calculation found:

$$
\begin{array}{lr}
\sqrt{2} \cdot \sqrt{N_{e}} e^{-\pi\left(x_{1}\right) r^{2}}-2 \pi \sqrt{\alpha^{-1}\left(M_{Z}\right)}=2.8421709430404 \times 10^{-13} \approx 0 \\
r=2.4231955224637849, & (22.1) \\
N_{e, \text { int }}=\sqrt{N_{e}}=3.87452880945685 \times 10^{11}, & (22.2 \mathrm{a}) \\
\pi\left(x_{1}\right)=\pi(0.125)=3.877102924420037 . & (22.2 \mathrm{c})
\end{array}
$$

The $r$ value given by (22.2a) falls into the range of $2<r_{1} \leq 3$. According to Table 16.1, for $r$ value in this range, the logistic recurrent process oscillates and converges to:

$$
1-1 / r=0.587321786158118 \text {. }
$$

This scenario fits the intermediate state well, in which the logistic recurrent process is in progress and about half way through toward the threshold.

The $N_{e, \text { int }}=\sqrt{N_{e}}=3.87452880945685 \times 10^{11}$ given by (22.2b) is a $G=1$ grand number. The mass of the electron intermediate state is:

$M_{e, \text { int }}=M_{P} / N_{e, \text { int }}=1.97993553 \times 10^{8} \mathrm{GeV} / \mathrm{c}^{2}>M_{M a x}=4.97323432 \mathrm{GeV} / \mathrm{c}^{2}$.

According to Rule 6.1, it cannot be a standalone fermion state, two intermediate states must appear in pair to form a scalar boson called " $e$-boson" with mass $M_{e-b}$, converting factor $N_{e-b}$ and length scale $L_{e-b}$ :

$$
\begin{aligned}
& M_{e-b}=2 M_{e, \text { int }}=3.95987106 \times 10^{8} \mathrm{GeV} / \mathrm{c}^{2}, \\
& N_{e-b}=\frac{M_{P}}{M_{e-b}}=\frac{1}{2} N_{e, \text { int }}=1.9372644 \times 10^{11},
\end{aligned}
$$

$$
L_{e-b}=\frac{h}{M_{e-b} c}=3.13110359 \times 10^{-24} \mathrm{~m} .
$$

Now the second question regarding electron intermediate state is answered. The mass of $M_{e, \text { int }}$ and $M_{e-b}$ fall into the middle of the tremendous gap between $M_{e w}$ and $M_{e w s}$. The gap is not empty. So it answers the third question as well.

Let' turn attention to details of the first question.

According to the cosmology standard model, the universe started from a big bang with extremely high energy corresponding to extremely high temperature. From SQS theory perspective, there was a "pre-big-bang period" represented by a set of 9 prime number even pairs started from $239 \& 241$ and ended at $337 \& 347$ found in Table 22.1 as mentioned previously. The pre-big-bang universe was a 3dimensional "overheated liquid state". Its details will be given later.

The overheated liquid state is unstable, any random stimulation causes evaporation. The Gaussian spheres were evaporated and free to fly as 0-dimensional objects in space represented by the prime numbers 211, 223, 227, 229, 233 between the two sets of prime number even pairs representing the pre-big-bang period and the first period. Let's call it the "0-period".

The universe was born at the big bang. The cosmic time is set to zero: $t_{0}=0$, and the new born universe started to evolve.

During the 0-period, the flying around Gaussian spheres attracted each other by gravitational force and intended to gather as groups, which nurtured the first period.

The first cosmic period started at time scale $t_{1}$ with length scale $L_{1}$ :

$$
\begin{aligned}
& L_{1}=L_{G U T}=71 L_{P} \sim 1.1475 \times 10^{-33} \mathrm{~m}, \\
& t_{1}=t_{G U T}=L_{G U T} / c \sim 3.8278 \times 10^{-42} \mathrm{~s} .
\end{aligned}
$$

At time $t_{1}$, the first cosmic period started. The flying Gaussian spheres were gathered and organized into 1dimensional array in 1-dimensional space.

The 1-dimensional space was built as an array of Planck scale face-centered cubes. If the building block of array is a Planck cube centered at a cubic vertex including 12 octahedral vertexes, according to Table 21.1 and Table 21.2, only three symmetries $C(0), C(\sqrt{1 / 2}), O(0)$ are fully effective. There is no fermion in the three symmetries. This scenario is not acceptable. If the building block of array is a Planck cube centered at an octahedral vertex including 4 cubic vertex and 8 octahedral vertexes, according to Table 21.1 and Table 21.2, three symmetries $O(0), O(\sqrt{1 / 2})$, $C(0)$ are fully effective. It include 8 first generation fermions $u_{r}, u_{g}, u_{b}, d_{r}, d_{g}, d_{b}, e, v_{e}$ and 8 anti-fermions in $O(\sqrt{1 / 2})$ and 2 bosons $\gamma, g$ in $O(0)$ responsible for electromagnetic interaction and gravitation. As shown in Section 15, the grand unification is to unify electromagnetic 
interaction into gravity. In $(22.6 \mathrm{~b}), t_{1}$ is derived from $L_{G U T}$ originated from $M_{G U T}$. At time $t_{1}$, the reverse process of grand unification happened, electromagnetic interaction was separated from gravity. Therefore, in the first cosmic period after $t_{1}$, the two bosons $\gamma, g$ in $O(0)$ showed up is natural and fully expected.

For the symmetry $O(1)$ representing the other 6 first generation bosons $g_{i}(i=1,2,3,4,5)$ and $U_{1}$, out of its 6 vertexes, only 2 of them are in the array counting for one third of its constituents. It indicates that, 2 out of 5 gluons such as $g_{1}$ and $g_{2}$ would be in effect. According to Table $13.2 \mathrm{~A}$ and $13.2 \mathrm{~B}$, in the gluon links among $u_{r}, u_{g}, u_{b}, 9$ of the 12 links are made of $g_{1}$ and $g_{2}$; in the gluon links among $d_{r}, d_{g}, d_{b}, 3$ of the 6 links are made of $g_{1}$ and $g_{2}$. It means that strong interaction is partially in effect.

Actually, the first cosmic period was in $1 \frac{1}{3}$-dimensional space, which is a fractal space. The space dimension was not fixed, instead, it was a developing process from 1-dimension gradually toward 2-dimension. Accordingly, the cross section of the array gradually increases from $1 L_{P} \times 1 L_{P}$ to $1 L_{P} \times n L_{P}$. When the width number $n \geq 2$, the symmetry $O(1)$ has 4 out of its 6 vertexes in the wider array. As a result, 2 more gluons such as $g_{3}$ and $g_{4}$ would be in effect. According to Table $13.2 \mathrm{~A}, 13.2 \mathrm{~B}$ and $13.2 \mathrm{~F}$, the gluon links among $u_{r}, u_{g}, u_{b}$, all 12 links are made of $g_{1}, g_{2}$ and $g_{3}$; the gluon links among $d_{r}, d_{g}, d_{b}$, all 6 links are made of $g_{1}, g_{3}$ and $g_{4}$; the 7 gluon links between $u_{r}, u_{g}, u_{b}$ and $d_{r}, d_{g}, d_{b}$ are made of $g_{1}, g_{2}$. It means that strong interaction for the first generation quarks is in effect.

This scenario looks reasonable and is accepted by SQS theory.

During the first period and other early cosmic periods, all particles are in their extremely high energy states

corresponding to extremely high temperature, which are quite different from their ordinary states. For instance, according to Fig. 19.1 and Fig. 19.2, the gluon links binding quarks to form proton and neutron all are made of $g_{1}, g_{2}, g_{3}$ and $g_{4}$. But it does not mean that, proton, neutron were formed during the first cosmic period after the array cross section increased to $1 L_{P} \times 2 L_{P}$. Because of the extremely high temperature, it was impossible to form any hadron. Instead, "quark-antiquark liquid state" would be formed from these first generation quarks and anti-quarks bound by strong interaction mediated by these in effect gluons $g_{1}, g_{2}, g_{3}$ and $g_{4}$.
The second cosmic period started at $t_{2}$ with length scale $L_{2}$ :

$$
\begin{aligned}
& L_{2}=L_{e w-s} \sim 71 \alpha^{-1}\left(M_{e w-s}\right) L_{P} \approx 9082 L_{P} \sim 1.4678 \times 10^{-31} \mathrm{~m} . \\
& t_{2}=L_{e w-s} / c \sim 9082 t_{P} \sim 4.8963 \times 10^{-40} \mathrm{~s} .
\end{aligned}
$$

After $t_{2}$, the second cosmic period started and the space became 2-dimensional, The 2-dimensional space is a membrane. In order to accommodate major symmetries of the first and the second generations, the thickness of membrane should be $2 L_{P}$. As shown in Table 21.1 and Table 21.2, the 2-dimensional membrane with $2 L_{P}$ thickness centered at a cubic vertex accommodates 7 fully effective symmetries $C(0)$, $C(\sqrt{1 / 2}), C(1), C(\sqrt{3 / 2}), C(\sqrt{2}), O(0), O(\sqrt{1 / 2})$, which does not include the second generation fermions. This scenario is unacceptable. On the other hand, the 2-dimensional membrane with $2 L_{P}$ thickness centered at an octahedral vertex accommodates 7 fully effective symmetries $O(0)$, $O(\sqrt{1 / 2}), O(1), O(\sqrt{3 / 2}), O(\sqrt{2}), C(0), C(\sqrt{1 / 2})$, which include the first generation 8 fermions $u_{r}, u_{g}, u_{b}, d_{r}, d_{g}$, $d_{b}, e, v_{e}$ and their anti-particles represented by $O(\sqrt{1 / 2})$ plus second generation 8 fermions $s_{r}, s_{g}, s_{b}, c_{r}, c_{g}, c_{b}$, $\mu, v_{\mu}$ and their anti-particles represented by $O(\sqrt{2})$. Symmetry $O(2)$ is not fully effective. Among its 6 vertexes, only 4 are included. The effective bosons for the second period are the first generation 8 bosons $g, \gamma, g_{i}$ $(i=1,2,3,4,5), U_{1}$ plus second generation 6 bosons corresponding to two third of 9 bosons plus 1 anti-boson $\bar{X}^{0}$ in second generation. The second period started at length scale $L_{2}=L_{e w-s}$, which is the characteristic length of electroweak-strong unification. The scalar boson $U_{2}$ for that unification must be included. The strong interaction was separated and fully effective. All 8 gluons $g_{i}(i=1,2, \cdots, 8)$ must be included. There are two additional second generation bosons as candidates selected from the remain second generation bosons: 3 massons $G_{i}(i=1,2,3), X$ and antiboson $\bar{X}$. Because $X, \bar{X}$ cannot be produced without its counterpart, there are only two possible selections. One is $X$ and $\bar{X}$, the other is two massons.

The second period include the first and second generations 16 fermions: $u_{r}, u_{g}, u_{b}, d_{r}, d_{g}, d_{b}, s_{r}, s_{g}$, $s_{b}, c_{r}, c_{g}, c_{b}, e, v_{e}, \mu, v_{\mu}$ and 16 anti-particles along with 12 bosons for sure: $g, \gamma, g_{i}(i=1,2, \cdots, 8), U_{1}, U_{2}$, plus 2 additional bosons or 1 boson and 1 anti-boson from one out of two choices. Either way sounds reasonable. This scenario is accepted by SQS theory.

Conclusion 22.1: Weak interactions are not in effect in the second cosmic period with 2-dimensional space. 
Proof: The reason for Conclusion 22.1 is that, the necessary mediators and intermediate states of weak interactions are not available in the second cosmic period. $W^{ \pm}$and $Z^{0}$ as third generation gauge bosons are not available for the second cosmic period according to Postulation 23.1. $X$ and $\bar{X}$ or two out of three massons $G_{i}$ $(i=1,2,3)$ are available depending on the choice. But

the hypothetical gauge bosons $X$ and $\bar{X}$ serving as the intermediate state for some type of weak interactions have similar behavior as their counterpart $W^{ \pm}$and $Z^{0}$, they must have massons' assistance to make the $m$ parameters match and the $n$-parameters match as well as to fill the mass gap described in Section 14.

Unfortunately, the two choices can only provide either $X$ and $\bar{X}$ or two massons but not both. The necessary mediators and interstates are not in existence, weak interactions cannot perform.

Conclusion 22.1 is important. It leads to a prediction given later.

In the second comic period, all second generation particles except perhaps $v_{\mu}$ and $\bar{v}_{\mu}$ are unstable. Under normal conditions, they are subject to decay via weak interactions. But the second cosmic period did not provide normal conditions, in which things turned out quite differently. According to Conclusion 22.1, weak interactions are not in effect in second cosmic period. The unstable particles in the 2-dimensional membrane cannot decay via weak interactions. They must hold until the third period, when the gauge bosons $W^{ \pm}, Z^{0}, X^{0}$ and all massons are effective, then decay in the 3-dimensional space. Moreover, the big bang produced equal amount of elementary particle and anti-particles. Some of them remained in the second period. Whether they had the chance to annihilate in second cosmic period is an interesting question, which will be discussed later.

In (22.5c), the length scale $L_{e-b}$ is derived from the mass of $e$-boson originated from electron. As shown in Section 4, the factor $\alpha / 4 \pi^{2}$ is electrically originated. While the cosmic expension is driven by gravity. Take this factor into account, the third period started at $t_{3}$ with length scale $L_{3}$ :

$$
\begin{aligned}
L_{3} & =L_{e-b} \frac{\alpha}{4 \pi^{2}} \approx 3.843 \times 10^{7} L_{P} \approx 6.211 \times 10^{-28} \mathrm{~m}, \\
t_{3} & =L_{3} / c \approx 2.072 \times 10^{-36} \mathrm{~s} .
\end{aligned}
$$

After $t_{3}$, the space became 3-dimensional. All particles listed in the Elementary Particle Table showed up. All symmetries listed in Table 22.1 and Table 22.2 became fully effective. All four types of interactions were into full play. In fact, it is the universe started at 13.7 billion years ago we living in now.

The transition from the 2-dimensional space to the 3dimensional space was a phase transition, which triggered the cosmic inflation.
The $e$-boson played a pivotal role in the cosmic inflation. It sets the length scale $L_{3}$ and time scales $t_{3}$ of the cosmic inflation. In addition, the logistic recurrent processes started at time $t_{1}$, they reached about halfway at $t_{3}$, which corresponds to the birth of the $e$-boson. More importantly, the scalar $e$-boson with mass of $M_{s-b} \approx 3.9599 \times 10^{8} \mathrm{GeV}$ acted as the inflaton. According to the cosmology standard model, during the rapid expansion period, the inflation was driven by a scalar boson called inflaton. Physicists are looking for the inflaton for some time without satisfaction. Here is the $e$ boson. It is a heavy scalar boson. It was born at the very beginning of the cosmic inflation and drove the space expansion to the very end of the inflation. More importantly, the $e$-boson serving as inflaton provides solution for a problem for cosmology standard model. The $e$-boson has extremely short life due to its heavy mass. After it decayed, the inflation lost its driving force and stopped, which resolves the troublesome nonstop inflation problem. The $e$-boson is not tailor made for cosmic inflation; instead, it is the requirement of logistic process, grand number, and Rule 6.1. In short, the $e$-boson fits the inflaton perfectly.

The time scale of $t_{3}=L_{3} / c \approx 2.072 \times 10^{-36}$ is roughly agreed with starting time of the cosmic inflation proposed by cosmology standard model. It is a good thing, but it raises questions. If $t_{3}$ corresponds the time of the cosmic inflation, what about $t_{1}$ and $t_{2}$ ? Were there two more inflations before the big one? These are very interesting questions. Space dimensions change is equivalent to phase transition, which releases energy causing inflation. The scale of inflation is determined by the amount of released energy. In this case, the released energy depends on three factors: the number of Planck cubes involved, the contraction depth of space elements, the total energy involved. The numbers of Planck cubes involved are 71, 9082 , and

$L_{3} / L_{P} \approx 3.483 \times 10^{7}$ for the phase transitions occurred at $t_{1}, t_{2}$, and $t_{3}$, respectively. The ratios of contraction depth are estimated as $R_{1} \sim(71)^{0 / 1} \sim 1, R_{2} \sim(9082)^{1 / 2} \sim 95, \quad R_{3} \sim\left(3.483 \times 10^{7}\right)^{2 / 3} \sim 1.067 \times 10^{5}$ for the transitions occurred at $t_{1}, t_{2}$ and $t_{3}$, respectively. If the potential energy released is proportional to the square of $R_{i},(i=1,2,3)$, The ratios for the released energy is estimated as $R_{1}^{2}: R_{2}^{2}: R_{3}^{2} \sim 1: 9082: 1.138 \times 10^{10}$, for the phase transitions occurred at $t_{1}, t_{2}$ and $t_{3}$, respectively. In terms of scales, it seems no comparison between the two inflations occurred at $t_{1}$, $t_{2}$ and the big one at $t_{3}$. The two inflations at $t_{1}, t_{2}$ were just mini rehearsals of the big show at $t_{3}$. But there is another factor involved. According to the energy conservation law, the total energy of three cosmic periods and the 0-period should be the same. The big difference in the numbers of Planck cubes involved is partially compensated by energy per Planck cube. It 


\section{Z. Y. SHEN}

makes the released energy of three inflations quite different from $R_{1}^{2}: R_{2}^{2}: R_{3}^{2} \sim 1: 9082: 1.138 \times 10^{10}$. According to this argument, the difference of three inflations' scales could be closer. In other words, there were two comparable scale rehearsals before the big show.

But the three inflations scenario has a glitch. Phase transition is an abrupt event occurred in an extremely short time interval. Noticed that, the 1-dimensional space actually is $1 \frac{1}{3}$-dimensional, which has fractal behaviors. The transition from $1 \frac{1}{3}$-dimensional space to 2-dimensional space is not necessarily corresponding to a violent phase transition happened suddenly at $t_{2}$. Instead, it was a gradual process. If this argument holds, there were only two cosmic inflations occurred at $t_{1}$ and $t_{3}$.

According to SQS theory, the phase transition happened at $t_{3}$ corresponds to the big cosmic inflation proposed by cosmologist Ruth [19], which has been verified by astronomy observations. According to SQS theory, if there is one more phase transition happened at $t_{1}$ corresponding to a cosmic inflation before the big one happened at $t_{3}$. It should also leave some footprints somewhere. The most like place is the cosmic microwave background radiation (MBR).

From SQS theory perspective, the early cosmological history is the natural evolution of the space structure with Gaussian spheres as building blocks. The results derived from it fit the cosmology standard model pretty well. SQS theory contributions are: (1) The cosmic periods and the generations of elementary particles are correlated to space dimensions; (2) It is determined by the prime numbers. In essence, everything is traced back to mathematics.

Astronomical observations and measurements such as gravity lens effect and the cosmic MBR have found the evidence for dark matter. Astrophysics observations indicate that, the composition of universe is approximately $5 \%$ visible matter, $21 \%$ dark matter and 74\% dark energy. What is dark matter made of? Physicists are looking for the answer for quite some time. The favorite candidates for dark matters are hypothetical particles such as the WIMP (Weakly Interacting Massive Particle), axion and heavy neutrino etc. These dark matter candidates are hypothetic elementary particles and only interact with visible matter via gravitation and weak interaction. Around the world, many underground experiments have been carried out to detect dark matter candidate particles rare interaction with visible matters. Despite the extensive efforts, so far, there is no confirmed positive result. It is the time for a second thought.

Let's look at the dark matter issue from SQS theory perspective. The Elementary Particle Table includes several undiscovered neutral heavy particles: $G_{i},(i=1,2, \cdots 8), X, U_{1}$, $U_{2}, U_{3}$. But perhaps all of them are unstable particles, which do not fit the requirements for the dark matter. On the other hand, notice that, the reliable evidences for the existence of dark matter all are based on its gravitational effects. The idea for dark matter as elementary particle having weak interaction with visible matters is hypothetical without experimental support. There is no reason to reject other types of dark matter candidates interacting with visible matters only via gravity. Moreover, there is no reason to reject radical ideas such as: dark matter is made of something other than undiscovered elementary particles.

Hypothesis 22.1: At least part of dark matters in the universe is the debris left over from the cosmos inflation. The dominate part of this type dark matters is 2-dimensional membranes, which interact with visible matters only via gravity.

Explanation: The cosmos inflation was a gigantic violent event happened in an extremely short time interval with tremendous amount of energy involved. It is inconceivable to assume that, the phase transition only produced the 3-dimensional space out from a 2dimensional membrane. It is more natural to conceive that, the phase transition produced the 3-dimensional space along with many pieces of debris. Since the prestate of phase transition is a 2-dimensional membrane, the dominate part of debris is relatively small pieces of 2-dimensional membrane. The phrase "part of dark matters" in Hypothesis 22.1 leaves room for other possible dark matter candidates.

We haven't found any dark matter, because we were looking for the wrong candidates. Dark matter may not be undiscovered elementary particles. According to Hypothesis 22.1, dark matters are left over debris from the comic inflation and most of them are 2-dimentional membranes flooding around in 3-dimentional space.

The new type experiments for searching dark matter should be based on its gravitation effects. Astronomical observations have found that, dark matter is mixed with visible matter all over the universe. Therefore, it is possible to design experiments for searching dark matter based on its gravitational effects.

Suggestion 22.1: The new type of experiments for searching dark matter is to use extremely sensitive gravitation meter isolated from earthly interferences. As earth rotating around the sun, occasionally, a piece of 2dimensional membrane passes through the meter, which will produce a signal to indicate its gravitational effect as the evidence of its existence. The signal can be recorded by monitoring apparatus and analyzed by computer with recognition software.

There is another possibility to verify dark matter. A piece of relatively large 2-dimensional membrane is capable to attract other membranes via gravity. When sufficient membranes get together in the right way, it is possible to trigger a mini-inflation type of phase transition to transfer a piece of 2-dimensional dark mater into a chunk of 3dimentional visible matter. From our perspective, the event is like that, a chunk of 3-dimensional visible mater suddenly appears from void. According to Conclusion 22.1, in the 2- 
dimensional membrane weak interactions are not in effect. The second generation unstable elementary particles such as muon, hadrons made of $s$-quarks, $c$-quarks and their antiparticles in the membrane hold their decay and wait for the chance. Immediately after the mini-inflation, in the new born 3-dimensional chunk, gauge bosons $W, Z, X$ and massons become readily available and fully effective. These unstable particles are eligible to decay. As a result, a shower of high energy particles is released, which can be detected and serve as the evidence of the mini-inflation and dark matter. In addition, as mention previously, there were particles and antiparticles left in the 2-dimensional membrane. If they did not have a chance to annihilate in the membrane, these particles and anti-particles should release at once at the mini-inflation. If they were annihilated in the membrane already, the annihilation products remained in the membrane should release also. Either way, showers of high energy $\gamma$-rays and other particles and anti-particles should be detectable.

Suggestion 22.2: The way to verify the mini-inflation is to monitor the primary high energy cosmic rays. If the components fit the pattern of decay modes branching ratios of the second generation unstable particles or their annihilations products, these events serve as the evidence for the mini-inflation as well as for dark matter.

Explanation: In fact, occasionally cosmic rays with extremely high energy has been observed coming from places such as the center of galaxy. If the branching ratios and products fit the right pattern, they can serve as the evidence of mini-inflation and dark matter. The key is to analyze the components whether fits the right pattern or not.

So far, the cosmic history from big bang through inflation up to the current period is explained pretty well based on the prime numbers even pairs listed in Table 22.1 and the space structure and symmetries described in Section 21. It is natural to ask: What is the pre-big-bang history? What is the outlook for the cosmic future? The answers are also in the prime numbers table.

As mentioned previously, in the third row and fourth row of Table 22.1, there is another set of 9 even pairs starting from $239 \& 241$ and ended at $337 \& 347$. According to Definition 22.1, it is qualified to be a 3-dimensional space corresponding to the pre-big-bang period. Its $m$-parameters are much larger than those of current 3-dimensional universe. It indicates that the elementary particles in the pre-big-bang period had extremely high energy to form the overheated quark-antiquark liquid state. More details will be given in Section 23.

Now let's look at the future destiny of universe. The cosmic history from pre-big-bang period through the 0-period, first period, second period and the current third period indicate that, in the cosmic scale, the direction of time arrow is from the set with larger prime numbers toward the set with smaller prime numbers. It means that, to read the sets of prime numbers listed in Table 22.1 corresponding to cosmic history should be from bottom up and from right to left. In other words, the cosmic time sequence is from the period with the set of larger prime numbers to the period with the set of smaller ones.

Now we are in the current third period corresponding to a set of $18+1=19$ prime numbers, in which 18 prime numbers for quarks is listed in the first row of Table 22.1 plus the even prime number 2 for electron not listed. In the set of 19 prime numbers, the smallest one is 1 . At first glance, it seems no prime number smaller than 1 . If that is the case, our universe eventually will come to an end. But Mother Nature always has her ways. The number axis centered at 0 has two wings, the right wing points toward positive numbers and the left wing points toward negative numbers. There is a set of negative prime numbers, which is exactly the same as ordinary prime numbers set with minus signs. Taking the negative prime numbers into account, there is a future for our universe.

Definition 22.2: The negative prime numbers are defined as the negative value of the ordinary prime numbers, which correspond to the prime numbers listed in Table 23.1 with minus signs.

Hypothesis 22.2: In terms of cosmology and elementary particles, the negative prime numbers act the same way as their positive counterparts except that, all particle's $m$-parameters and $n$-parameters change signs.

Definition 22.2 and Hypothesis 22.2 lay the theoretical foundation for the future and the pre-big-bang history of the universe.

Astronomical observations found that, the universe currently is expanding with accelerating speed caused by the repulsive force of dark energy. Some cosmic models predicted that, the expansion will slow down and eventually turn to contraction. The contracting universe reverses its expansion process and finally back to a big crunch corresponding to the reverse of the big bang. Then the whole thing starts over again. The model is called "cyclic model". The universe cycles by itself over and over. Hypothesis 22.2 supports the cyclic model based on the negative prime numbers. The following is the scenario of universe future from SQS theory perspective based on Hypothesis 22.2.

The expansion of universe will slow down due to the dilution of dark energy density and eventually turn into a contraction. The evidence is in the extended prime numbers table. As time passing by, the corresponding prime numbers become smaller. Finally it reaches 1 , which is marked as the end of the current third period. But the cosmic evolution does not stop. It continues its journey. The number passes through 0 , which is the $m$-parameter for the graviton. Then it enters into the negative territory. According to Hypothesis 22.2, the negative prime numbers correspond to the $m$-parameter changing sign. Theoretically, here are two ways for the $n$ parameter to react: (1) The corresponding $n$-parameters also change its sign; (2) The corresponding $n$-parameters do not change sign. According to Definition 11.2, the second way means that all particles become anti-particles. All matters in 


\section{Z. Y. SHEN}

the universe suddenly become anti-matters! This scenario is impossible. Then the only possibility is the first way as Hypothesis 22.2 stated. The simultaneously changing signs of both $m$-parameter and $n$-parameter indicate that, all particles change their handedness corresponding to change the direction of their momentum. This scenario is supported mathematically and physically. The mathematic support comes from prime numbers. At the time universe stop expansion, the prime number sequence passes 0 and enters the negative territory causing $m$-parameters and $n$-parameters both changing their signs. The physical support is that, as universe stops expansion and starts to contract corresponding to all particles' momentum changing direction. This scenario is much easy to be accepted than the other scenario, all particles suddenly become anti-particles.

Sine the negative prime numbers table is the same as the positive prime number table except the minus signs, as the universe starts to contract, it basically follow the reversed process of the expansion universe. It is like to play a video in the reverse order. The consecutive cosmic events sequence is like that, as the negative third period coming to its end, the universe enters to the negative second period, then the negative first period, the negative 0 -period, the negative big bang, i.e. the big crunch, finally reaches the negative pre-bigbang period, i.e. the post-big-crunch period, One cycle of universe oscillation is completed. The post-big-crunch period is the same as pre-big-bang period except that all $\mathrm{m}$ parameters and all $n$-parameters change signs corresponding to time arrow in the opposite direction. To start the next cycle, the post-big-crunch period must transfer into the pre-bigbang period. This process can be realized through a "time tunnel". Since both periods possess extremely high energy, according to general relativity, the space-time is extremely curved providing the conditions to form time tunnel. Fig. 22.1 shows a diagram to illustrate the evolution of the cyclic universe.

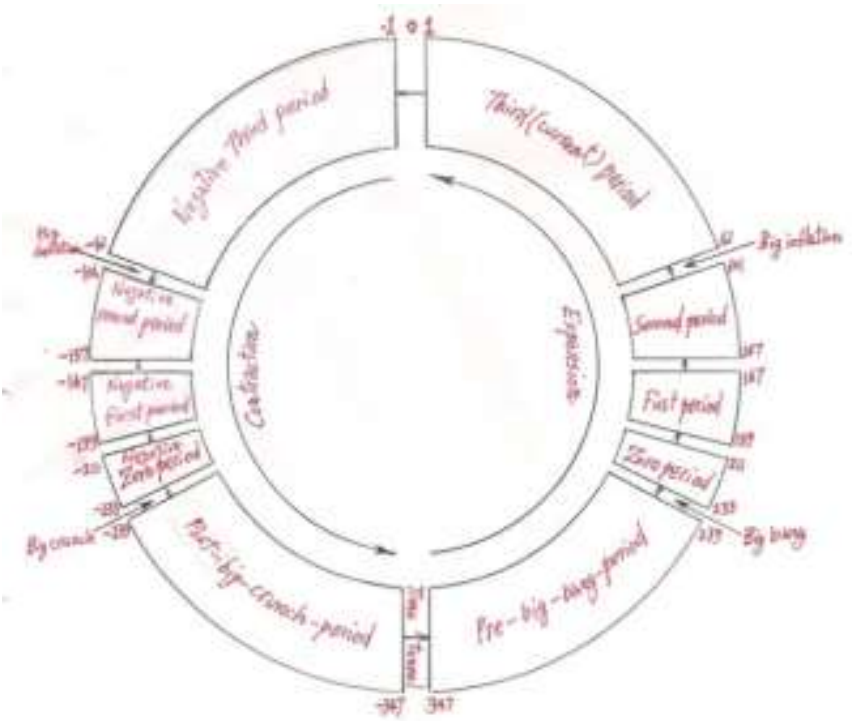

Fig. 22.1: Illustration of cosmic cycle based on prime numbers.
The repetitive cycles of cyclic model are in the cosmological sense. It does not mean that, everything in the universe will repeat exactly. In fact, according to SQS theory, space is stochastic in nature, which prohibits absolute determinism at the fundamental level and upper levels.

The cosmic history described in this section is based on the prime numbers table and the Prime Number Postulation. It especially depends on the discovery of three sets of consecutive prime number even pairs serving as the mathematical bases for the first period, the second period and the pre-big-bang period. The correspondence of the original set of $18+1$ prime numbers to the current third period is supported by many evidences. The question is: What is the mathematic relation between the newly found three sets of prime numbers and the original set of $18+1$ prime numbers?

Let's start from the first prime numbers of the three sets, according to Table 22.1, which are 167, 101, and 239 for the first, second, and per-big-bang period, respectively. A simple arithmetical calculation found the following formulas.

\section{Period-I:}

$167=1+2+3+5+7+11+13+17+19+23+29+31+(1+2+3)$

Period-II:

$101=1+2+3+5+7+11+13+17+19+23$,

Period-III*

$239=1+2+3+5+7+11+13+17+19+23+29+31+37+41$.

In which, Period-I, Period-II, and Period-III* are marked for first period, second period and pre-gig-bang-period, respectively.

It is interesting to find some rules in (22.9).

1. The first prime number 101 corresponding to Period-II equals to the sum of the ten consecutive prime numbers from 1 to 23 in the set of $18+1$ prime numbers for the current third period.

2. The first prime number 239 corresponding to Period-III* equals to the sum of the fourteen consecutive prime numbers from 1 to 41 in the set of $18+1$ prime numbers for the current third period.

3. The first prime number 167 corresponding to Period-I equals to the sum of the twelve consecutive prime numbers from 1 to 31 plus $(1+2+3)$ in the set of $18+1$ prime numbers for the current third period. The repeat of three prime numbers $(1+2+3)$ represents the fact that the space of Period-I is $1+\frac{1}{3}$-dimensional, which is different from Period-II and Period-III*.

The last prime numbers of the three sets corresponding to period-I, period-II, and period-III* are, 199, 157, 347, respectively.

Period-I: $199=61+59+53+(13)+(7)+(1+2+3)$,

Period-II: $157=61+59+(37)$,

Period-III*: $347=61+59+53+47+43+41+(43)$.

There are also some rules in (22.10). 
1. The order of summation in (22.10) is backwards from the last prime number 61 of the $18+1$ prime numbers set and consecutively takes the next one.

2. After the backwards consecutive summation ended, it jumps to the prime number(s) shown in parenthesis.

3. For Period-II, 37 is the prime number assigned to $c_{b}$ as the up type quark of the $2^{\text {nd }}$ generation. For Period-III*, 43 is the prime number assigned to $t_{r}$ as the up type quark of the $3^{\text {rd }}$ generation.

4. For Period-I, 13 and 7 are the prime numbers assigned to $u_{b}$ and $u_{g}$ as the up type quarks of the $1^{\text {st }}$ generation. $(1+2+3)$ indicates that the space of Period-I is $1+\frac{1}{3}$ dimensional, which is different from Period-II and Period-III*.

The rest of prime numbers $P_{i}$ in the three sets corresponding to Period-I, Period-II, and period-III* are expressed as follows.

Period-I: $P_{i}=167+x+y, i=2,3 \cdots 7$.

Period-II: $P_{i}=101+x+y, i=2,3 \cdots 11$.

Period-III*: $P_{i}=239+x+y, i=2,3 \cdots 17$.

In which $x$ and $y$ are two prime numbers selected from the set of 18 odd prime numbers from 1 to 61 corresponding to the current third period.

These rules answer the question. There are mathematical correlations between the three sets of prime numbers and the original set of $18+1$ prime numbers. It implies that, the three periods in cosmic history are closely related to the current periods. It also serves as another supportive evidence for the Prime Number Postulation and its roles in elementary particles and cosmology.

Conclusion 22.2: Based on (22.9), (22.10), (22.11), the three sets of prime numbers even pairs corresponding to Period- I, Period-II, Period-III* are based on the original set of $18+1=19$ prime numbers corresponding to the current Period-III.

The selection of four sets of prime number pairs according to Definition 22.1, Postulation 22.1 and Lemma 22.1 is based on the Prime Numbers Postulation and the even pairing rule. There are so many things depending on it. It is necessary to ask the question: Is it by coincidence? Let's try to answer.

The odd prime numbers are divided into two categories.

The $4 n+1$ category: $P_{1}=4 n+1, n=0,1,2,3 \cdots$

The $4 n-1$ category: $P_{2}=4 n-1, n=1,2,3 \cdots$

For a pair of two prime numbers, there are four possible combinations with average values as:

$$
\begin{aligned}
& A(+,-)=\left[\left(4 n_{1}+1\right)+\left(4 n_{2}-1\right)\right] / 2=2\left(n_{1}+n_{2}\right)=\text { even }, \\
& A(-,+)=\left[\left(4 n_{1}-1\right)+\left(4 n_{2}+1\right)\right] / 2=2\left(n_{1}+n_{2}\right)=\text { even }, \\
& A(+,+)=\left[\left(4 n_{1}+1\right)+\left(4 n_{2}+1\right)\right] / 2=2\left(n_{1}+n_{2}\right)+1=\text { odd }, \\
& A(-,-)=\left[\left(4 n_{1}-1\right)+\left(4 n_{2}-1\right)\right] / 2=2\left(n_{1}+n_{2}\right)-1=\text { odd },
\end{aligned}
$$

The distribution of prime numbers seems random. It is natural to assume that, the four combinations of (22.13) each has equal probability of occurrence. In other words, for two consecutive prime numbers being even pair or odd pair each has fifty-fifty equal chance. Based on this assumption, it is possible to give some estimation. As listed in Table 23.1, for the first set with 9 consecutive even pairs from $1 \& 3$ to $59 \& 61$ plus an odd pair $67 \& 71$ at end as a specific prime numbers sequence to occur randomly, the probability is $p_{9+1}=2^{-(9+1)}=2^{-10}=1 / 1024$. For the second set with 9 consecutive even pairs from $239 \& 241$ to $337 \& 347$ plus two odd pairs $229 \& 233$ and $349 \& 353$ at both ends as a specific prime numbers sequence to occur randomly, the probability is $p_{9+2}=2^{-(9+2)}=2^{-11}=1 / 2048$. Likewise, for the two shorter sequences, the one with two generations has probability of $p_{6+2}=2^{-(6+2)}=2^{-8}=1 / 256$, the one with one generation has probability of $p_{4+2}=2^{-(4+2)}=2^{-6}=1 / 64$. In average, $p_{9+1}=1 / 1024$ means that the event only occurs once per 1024 prime number pairs; for $p_{9+2}=1 / 2048$, it only occurs once per 2048 prime number pairs. These are the expected values according to statistics. But in fact, these two, not only one, sequences occurred in a set including only 71 odd prime numbers (35.5 pairs) from 1 to 353 . The 71 odd prime numbers (35.5 pairs) also include the other two shorter sequences, and all four sequences are in the right order. Are all of these by coincidence? It is virtually impossible.

Conclusion 22.3: The probability of four sets of prime number pairs occurred by coincidence in 71 least odd prime numbers sequence from 1 to 353 is in the order of

$$
p_{\text {coincid }}=\frac{71 / 2}{64 \times 256 \times 1024 \times 2048 \times(4 !)}=\frac{71}{3 \times 2^{39}} \approx 4.305 \times 10^{-11} \text {. }
$$

The four sets of consecutive prime number pairs found in the prime numbers table shown in Table 23.1 based on the Prime Numbers Postulation and the even pairing rule are not by coincidence.

Explanation: It is only a rough estimate. 71 divided by 2 is counting for prime number pairs. $(4 !)=(1 \times 2 \times 3 \times 4)$ in denominator is for the 4 sets in the right order.

The correlations of prime numbers to particle physics must have a deep origin. For instance, consider the question: Why the $m$-parameters and $n$-parameters of 18 quarks must be prime numbers? One possible reason is that, because prime numbers are not reducible, the 18 different prime numbers serving as $m$-parameters of 18 quarks prevent different quarks from mixed up by reductions; the $n$ parameters of 18 quarks with prime numbers different from corresponding $m$-parameters prevent reduction with $m$ parameters. In other words, the prime numbers serving as $m$ parameter and $n$-parameter give each quark unique mathematical identity to avoid mixed ups by reduction.

There is another stronger reason based on number theory. For the cyclic arithmetic theory, it is well known that, only the $p$-cyclic-arithmetic with $p$ as prime number is self- 


\section{Z. Y. SHEN}

consistent for multiplication and division [18]. Since $m$ and $n$ determines the lengths of loop-1 and loop-2, respectively, the $m$-parameter and the $n$-parameter equal to prime number have something to do with quark's internal cyclic movements related to the $p$-cyclic-arithmetic.

In terms of philosophy, prime numbers are the basic building blocks of numbers; likewise, quarks are the basic building blocks of matters. In fact, it was the author's original intuition to purposely look into the prime numbers searching for possible physics significance. However, the deeper reason for the roles of prime numbers in SQS theory is still an interesting open issue worthwhile to dig in.

In summary, the cosmic models and history provided in this section is based on prime numbers listed in Table 22.1 and its extended version to the negative territory. The finding of three cosmic periods has its significance. It confirms the importance of the Prime Numbers Postulation for dealing with elementary particles as well as for identifying cosmic periods. It provides a chance to recognize the meaning of intrinsic symmetries based on the geometry of the two parts of face-centered space structure introduced in Section 21. It reveals cosmic history and links it to elementary particles. It provides natural explanations for the big bang, inflations, dark matters etc. It predicts the future destiny of universe. It also provides two suggestions for verification.

The cosmic models and evolution according to SQS theory described in this section agreed with cosmological standard model pretty well. It serves as a supportive evidence of the face-centered space structure. Moreover, the classification of space symmetries as $O(r), C(r)$ and the Symmetries Family Tree provide the bases to identify the elementary particles and interactions in different cosmic periods, which are self-consistent and agreed well with particle physics and cosmological standard model. It cannot be by coincidence, which gives the credential for both. These agreements also serve as the supportive evidences for Definition 22.1 and Postulation 22.1 introduced at the beginning of this section.

There is a pending issue to think about it. The expansion of universe corresponds to entropy increase. Then the contraction of universe corresponds to entropy decrease. Is it a violation of the second law of thermodynamics?

\section{Section 23: The Monster and Two Other Sporadic Groups}

The finite Lie groups are classified into two categories, the classical groups and the sporadic groups. There are 26 sporadic groups in the second category [18]. Three sporadic groups $M$ (Monster, E8) $B$ (Baby monster) Suz (Suzuki) are closely related to the $m$-parameters and to some extent $n$ parameters of three generation elementary particles. It is a finding with important impacts on particle physics and cosmology.
The size of these three groups is factorized into prime numbers [18], which are listed in Table 23.1. The $m$ parameters of 18 quarks are also listed for comparison.

Let's take a closer look of the comparison between the $M$ group size factors and the $m$-parameters of 18 quarks.

The size for $M$-group is factorized into 15 prime numbers with different powers, in which 1 is not included. From SQS theory standpoint, the prime number 1 must be included with power $n=$ any integer:

$$
F_{M}=1^{n} \cdot 2^{46} \cdot 3^{20} \cdot 5^{9} \cdot 7^{6} \cdot 11^{2} \cdot 13^{3} \cdot 17 \cdot 19 \cdot 23 \cdot 29 \cdot 31 \cdot 41 \cdot 47 \cdot 59 \cdot 71 .
$$

Table 23.1: Factors of Three Sporadic Groups Size versus the $m$-Parameters of Quarks

\begin{tabular}{|c|c|c|c|c|c|}
\hline $\begin{aligned} F_{M S} & =808,0 \\
& =2^{45} \cdot 3\end{aligned}$ & $\begin{array}{r}\text { The Siz } \\
24,794,51 \\
5^{9} \cdot 7^{6} \cdot 11^{2}\end{array}$ & $\begin{array}{l}\text { ad Its Facto } \\
5,886,459,9 \\
3 \cdot 17 \cdot 19 \cdot 23\end{array}$ & $\begin{array}{l}\text { or the } M(\mathrm{M} \\
961,710,757,0 \\
-31 \cdot 41 \cdot 47 \text {. }\end{array}$ & $\begin{array}{l}\text { ster) Group } \\
754,368,000 \\
.71\end{array}$ & \\
\hline & The $m$ - & imeters for & Quarks of 3 & nerations ${ }^{*}$ & \\
\hline The firs & eneration & The sec & 1 generation & The thi & generation \\
\hline$u_{r}, u_{g}, u_{\mathrm{g}}$ & $1,7,13$ & $c_{n}, c_{p}, c_{b}$ & $19,29, \underline{37}$ & $t_{n}, t_{g}, t_{b}$ & $\underline{43}, \underline{53}, \underline{61}$ \\
\hline$d_{n}, d_{s}, d_{b}$ & $3,5,11$ & $s_{n}, s_{z}, s_{b}$ & $17,23,31$ & $b_{n}, b_{g}, b_{b}$ & $41,47,59$ \\
\hline & The Size a & Its Factors & the $B$ (Baby & onster) Grol & \\
\hline$F_{z}=4,154$, & $1,481,226,4$ & $91,177,580$, & $, 000,000$ & & \\
\hline$=2^{41} \cdot 3$ & $5^{6} \cdot 7^{2} \cdot 11$. & $17 \cdot 19 \cdot 23 \cdot 3$ & & & \\
\hline & le $m$-Paran & is for $12 \mathrm{Q}$ & $\mathrm{ks}$ of $1^{n t}$ and & Generati & \\
\hline The firs & eneration & The sec & 1 generation & The thi & generation \\
\hline$u_{r}, u_{g}, u_{b}$ & $1,7,13$ & $c_{n,}, c_{g}, c_{\mathrm{s}}$ & $19, \underline{29}, \underline{37}$ & & \\
\hline$d_{p}, d_{z}, d_{b}$ & $3,5,11$ & $s_{n}, s_{z}, s_{b}$ & $17,23,31$ & & \\
\hline & The Siz & ad Its Facto & or the $S u z(\mathrm{~S}$ & uki) Group & \\
\hline$F_{S t a x}=448$, & $497,600=$ & $3^{7} \cdot 5^{2} \cdot 7 \cdot 1$ & & & \\
\hline & The $m$ & ameters for & Quarks of $1^{x}$ & eneration & \\
\hline The firs & eneration & The sec & generation & The thi & zeneration \\
\hline$u_{p}, u_{g}, u_{b}$ & $1,7,13$ & & & & \\
\hline$d_{2,}, d_{z}, d_{b}$ & $3,5,11$ & & & & \\
\hline
\end{tabular}

* The prime numbers marked with under line are missing in the factors sequence of corresponding group.

Except the last prime number 71, which will be discussed later, there are 15 prime numbers left. In which 2 is the only even prime number assigned as the before reduction $m$ parameter of electron red branch $e_{r}$. To compare the 14 odd prime numbers with the 18 prime numbers assigned as the $m$ parameters of three generation quarks, there are 4 prime numbers $37,43,53,61$ missing in the $M$-group factors sequence. At first glance, the missing prime numbers seem a defect for the correlation between these two sets of prime numbers. Actually, it is just the opposite. The missing prime numbers have deep meanings. The missing prime numbers 53 and 61 are the $m$-parameters shared by $t_{g}, t_{b}$ and $Y_{1}, Y_{2}$, in which $Y_{1}$ and $Y_{2}$ are the two components of gauge bosons $W$ and $Z$. The missing prime number 37 is the $m$-parameter shared by $c_{b}$ and $X_{f 2}$, which is one of the two components for the gauge boson $X^{0}$. So the three prime numbers 37,53 , 61 all have the common reason for missing in $F_{M}$. They are the $m$-parameters of quarks serving as the constituents of bosons for weak interaction. The missing prime number 43 also has a meaning. The three prime number 43, 53, 61 are the $m$-parameters for three top quarks $t_{r}, t_{g}, t_{b}$, which have mass heavier than $M_{M a x}$. According to Rule 6.1, top quarks 
must appear in pair with anti-quarks as a boson state.

Therefore, for the four missing prime numbers in the $M$ group, they all are involved in something related to bosons or boson states with more than one quark (anti-quark) involved.

The size for the $B$-group is factorized into 12 prime numbers:

$$
F_{B}=1^{n} \cdot 2^{41} \cdot 3^{13} \cdot 5^{6} \cdot 7^{2} \cdot 11 \cdot 13 \cdot 17 \cdot 19 \cdot 23 \cdot 31 \cdot 47 .
$$

Except the last prime number 47 , which will be discussed later, there are 11 prime numbers left. The 10 odd prime numbers compare with the 12 prime numbers assigned as the $m$-parameters of first and second generation quarks, there are two prime numbers 29, 37 missing in the $B$-group. According to the some rule, 29,37 are the $m$-parameters shared by $c_{g}$, $c_{b}$ and $X_{f 1}, X_{f 2}$, which are the two components of gauge boson $X^{0}$.

The size for the Suz (Suzuki) group is factored into 7 prime numbers:

$$
F_{\text {Suz }}=1^{n} \cdot 2^{13} \cdot 3^{7} \cdot 5^{2} \cdot 7 \cdot 11 \cdot 13
$$

In which the 6 odd prime numbers are the $m$-parameters of first generation 6 quarks. There is no missing prime number in $F_{\text {Suz }}$.

Rule 23.1: The prime number factors sequence of three sporadic groups $M, B, S u z$ are closely related to the $m$ parameters of three generation elementary particles. The correlation rules are:

1. The even prime number 2 is the before reduction $m$ parameter of electron red branch.

2 . The odd prime numbers are related to quarks' $m$ parameters of corresponding generations, all three generations, first generation plus second generation, and first generation for $M$-group, $B$-group, and $S u z-$ group, respectively.

3. The missing prime number in the factors sequence corresponding to up type quark's $m$-parameter is related to boson or boson state with more than one types of quark (anti-quark) involved.

4. The missing prime number in the factors sequence corresponding to down type quark's $m$-parameter ends the previous generation(s).

Explanation: The rules from No.1 to No.3 have been explained. Let's talk about rule No.4. The $M$-group prime number factors sequence missed two prime numbers 61,67 , between 59 and 71 , in which the missing of 67 corresponding to the down type $m$ parameter ends all three generations. The $B$-group prime number factors sequence missed three prime numbers 37, 41, 43 between 31 and 47, in which the missing of 41 corresponding to the $m$-parameter of a down type quark $b_{r}$ ends two previous generations. The Suz-group prime number factor sequence ends at 13 , the missing of 17 corresponds to the $m$-parameter of a down type quark $s_{r}$ ends the first generation. In summary, the missing prime numbers in three sporadic groups' factored sequences are classified into two categories: (1) The missing prime number corresponding to the $m$ parameter of up type quark is related to boson or boson state with more than one types of quark (anti-quark) involved; (2) The missing prime number corresponding to the $m$-parameter of down type quark ends the previous generation(s). The correlation is one on one for every one without exception, which cannot be by coincidence.

Rule 23.1 reveals the intrinsic correlation between three sporadic groups and three generations of quarks and the electron red branch. It clearly shows that, the assignment of $18+1=19$ prime numbers as $m$-parameters of 18 quarks and electron red branch is supported by three sporadic finite Lie groups. More importantly, it provides the third mathematic evidence for the conclusion of only three generations of elementary particles. The first evidence is the Prime Numbers Postulation based on number theory. The second evidence is the magic number 163 also based on number theory. Here comes the third independent evidence based on group theory. The $M$-group is the largest group. Its correlation to all three generations leaves no room for more generation. No other group can change the conclusion, because no group is larger than the $M$-group.

Moreover, the No. 3 rule of Rule 23.1 supports the assignment of up type quark's $m$-parameter as $m$-parameter for the fermion constituents of gauge bosons $W, Z$ and $X$.

In addition, as shown in Table 23.2, the prime number factors of three sporadic groups also related to the $n$ parameters of quarks.

Table 23.2: Factors of Three Sporadic Group Size versus $n$ -

\begin{tabular}{|c|c|c|c|c|c|}
\hline \multicolumn{6}{|c|}{ The Factors of Size for the $M$ (Monster) Group } \\
\hline$F_{M}=2^{46} \cdot 3^{2}$ & $5^{9} \cdot 7^{6} \cdot 11^{2}$ & $\cdot 17 \cdot 19 \cdot 23$. & $\cdot 31 \cdot 41 \cdot 47 \cdot$ & & \\
\hline \multicolumn{6}{|c|}{ The $n$-Parameters for 18 Quarks of Three Generations* } \\
\hline \multicolumn{2}{|c|}{ The first generation } & \multicolumn{2}{|c|}{ The second generation } & \multicolumn{2}{|c|}{ The third generation } \\
\hline$u_{r}, u_{g}, u_{b}$ & $1,3,5$ & $c_{p}, c_{g}, c_{b}$ & $7,23,29$ & $t_{p}, t_{g}, t_{b}$ & $\underline{283}, \underline{331}, \underline{397}$ \\
\hline$d_{1}, d_{g}, d_{b}$ & $1,3,5$ & $s_{,}, s_{z}, s_{b}$ & $7,11,13$ & $b_{n}, b_{z}, b_{b}$ & $17,17, \underline{53}$ \\
\hline \multicolumn{6}{|c|}{ The Factors of Size for the $B$ (Baby monster) Group* } \\
\hline \multicolumn{6}{|c|}{$F_{B}=2^{41} \cdot 3^{13} \cdot 5^{6} \cdot 7^{2} \cdot 11 \cdot 13 \cdot 17 \cdot 19 \cdot 23 \cdot 31 \cdot 47$} \\
\hline \multicolumn{6}{|c|}{ The $n$-Parameters for 12 Quarks of $1^{* x}$ and $2^{* d}$ Generations } \\
\hline \multicolumn{2}{|c|}{ The first generation } & \multicolumn{2}{|c|}{ The second generation } & \multicolumn{2}{|c|}{ The third generation } \\
\hline$u_{v}, u_{s}, u_{b}$ & $1,3,5$ & $c_{n}, c_{s}, c_{b}$ & $19,23, \underline{29}$ & & \\
\hline$d_{v}, d_{b}, d_{b}$ & $1,3,5$ & $s_{,}, s_{z}, s_{b}$ & $7,11,13$ & & \\
\hline \multicolumn{6}{|c|}{ The Factors of Size for the Suz (Suzuki) Group } \\
\hline \multicolumn{6}{|c|}{$F_{S u z}=2^{13} \cdot 3^{7} \cdot 5^{2} \cdot 7 \cdot 11 \cdot 13$} \\
\hline \multicolumn{6}{|c|}{ The $n$-Parameters for 6 Quarks of $1^{n}$ Generation } \\
\hline \multicolumn{2}{|c|}{ The first generation } & \multicolumn{2}{|c|}{ The second generation } & \multicolumn{2}{|c|}{ The third generation } \\
\hline$u_{r}, u_{b}, u_{b}$ & $1,3,5$ & & & & \\
\hline$d_{v}, d_{z}, d_{b}$ & $1,3,5$ & & & & \\
\hline
\end{tabular}
Parameters of Quarks

As shown in Table 23.2, most quarks' $n$-parameters are selected from the prime number factors sequence of corresponding sporadic group. There are exceptions marked with underline. The exceptions for up type of quarks $t_{r}, t_{g}$, 


\section{Z. Y. SHEN}

$t_{b}$ and $c_{b}$ can be explained with the some rule for the $m$ parameters. There is one exception in a down type quark, which is $n_{b b}=53$ for $b_{b}$. What's the implication for this down type exception? It is an open issue.

In Table 23.3, the products of factors in orders of magnitude for three sporadic groups are listed. For comparison, the products of quarks' $m$-parameters for three generations along with the pre-big-bang period are also listed.

Table 23.3: The Products of Factors for $M, B, S u z$ groups and Products of $\boldsymbol{m}$-Parameters

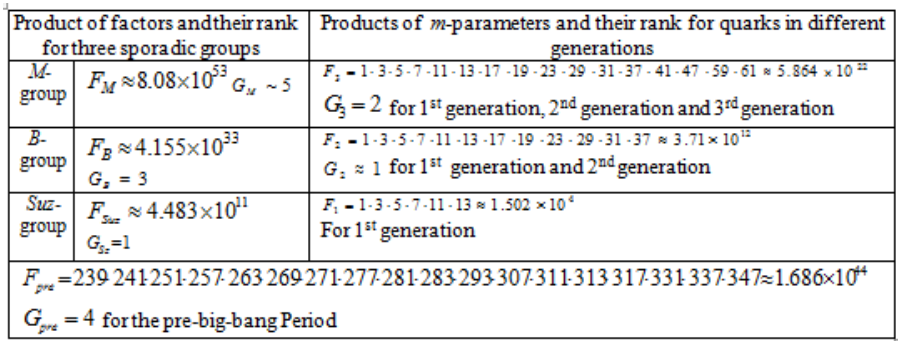

Note: $G$ is the rank of the grand number.

In the 7 products shown in Table 23.3, the 6 products either are grand numbers or close to a grand number. The only exception is the first generation. It shows that, grand numbers are common phenomena. They can be found in particle physics and cosmology as well as in mathematics such as group theory and number theory.

It is interesting to point out that, $G_{M} \sim 5, G_{B}=3$ and $G_{S u z}=1$ are odd numbers, while $G_{3}=2$ and $G_{p r e}=4$ are even numbers. Moreover, $G_{\text {pre }}=4$ is the double of $G_{3}=2$. It serves as a clue for the relation between these two periods.

So far in this section, all prime number factors of three sporadic groups $M, B, S u z$ are covered with two prime numbers 47 and 71 left, which are the last prime number after corresponded generation ended. Let's look at them closely.

For the largest prime number 71 in $F_{M}$, the first clue comes from GUT:

$$
N_{G U T}=\frac{M_{\text {Planck }}}{M_{G U T}} \approx \frac{2 \pi}{\sqrt{\alpha\left(M_{Z}\right)}}=71.06279805^{\circ} .
$$

The relative deviation of $N_{G U T}=71.0627805$ from the prime number 71 is $8.845 \times 10^{-4}$. In (15.11), $N_{G U T}$ is the converting factor for the grand unification scalar boson $U_{3}$ with mass $M_{G U T}=1.07948 \times 10^{18} \mathrm{GeV} / \mathrm{c}^{2}$, while $\alpha\left(M_{Z}\right)$ is the fine structure constant at $Z$ boson mass $M_{Z}=91.1876 \mathrm{GeV} / \mathrm{c}^{2}$. The two very different energy scales do not match. Consider the asymptotic behavior of $\alpha(M)$ as a running constant, it seems reasonable to use the prime number 71 to determine the value of $\alpha^{-1}\left(M_{G U T}\right)$ :

$$
\alpha^{-1}\left(M_{G U T}\right)=\left(\frac{71}{2 \pi}\right)^{2}=127.69002169
$$

This is a new way to determine the value of $\alpha$ at a particular mass scale, which is independent of the method used in Section 9. More details will be given later in this section.

Compare $\alpha^{-i}\left(M_{G U T}\right)=127.6900169$ with the experimental data from 2010-PDG (p.126): $\alpha^{-1}\left(M_{Z}\right)=127.916 \pm 0.016$, the value given by (23.4) seems reasonable. In other words, the grand unification of all interactions to gravity actually occurred at characteristic length scale:

$$
L_{G U T}=71 L_{P}=1.1475375 \times 10^{-33} \mathrm{~m} .
$$

This is the reason to use $L_{G U T}=71 L_{P}$ as $L_{1}$ in (22.6a) for the length scale of first period.

The prime number 71 serves as the characteristic length for the grand unification. It should also have other geometric and physics meanings.

Definition 23.1: The $M$-sphere is defined as a sphere in space centered at a vertex with radius of:

$$
R_{M}=L_{G U T}=71 L_{P}=1.14754 \times 10^{-33} \mathrm{~m} .
$$

It turns out that, the $M$-sphere is an important concept related to many issues.

The spherical surface of $M$-sphere is the boundary between microscopic region and the transitional region. According to SQS theory, space is divided into three regions: Inside of the $M$-sphere is the microscopic region, which is the territory of elementary particles, composite particles and their different states. The region with linear scale between $R_{M}=71 L_{P} \sim 1.148 \times 10^{-33} \mathrm{~m}$ and Compton scale $\lambda_{C}=h / M c$ is defined as the transitional region, which is the playground of the random walk and the logistic recurrent process discussed in Section 4 and Section 16, respectively. The region with linear scale larger than Compton scale is the macroscopic region.

Take $R_{M}=71 L_{P}$ as radius and draw the $M$-circle on the surface of $M$-sphere at the same center. The circumferential length of the $M$-circle is:

$$
L_{M}=2 \pi \times 71 L_{P}=446.10615681 L_{P} .
$$

Taking $L_{P}$ as basic length unit, $L_{M}$ becomes a number:

$$
L_{M}=2 \pi \times 71=446.10615681 \text {. }
$$

In the following discussions, all lengths are numbers with $L_{P}$ as the basic length unit.

In Fig. 23.1(a), the intersections of two adjacent Gaussian spheres to the $M$-sphere surface are shown as two circles with radius $r=1 /(2 \sqrt{2})$. The distance between the two centers is $d_{1}=1 / \sqrt{2}$. The two circles are either centered at two adjacent octahedral vertexes representing two fermions or one centered at an octahedral vertex and the other centered at an adjacent cubic vertex representing a fermion and a boson. The span angle of $d_{1}$ on the $M$-circle with respect to its center is:

$$
\Delta \phi_{M}=\frac{d_{1}}{L_{M}} \times 360^{\circ}=\frac{1}{2 \pi \times 71 \times \sqrt{2}} \times 360^{\circ}=0.57062302^{\circ} .
$$


As listed in Table 11.5, the average tilt angle for quarks and leptons from $\vartheta= \pm 120^{\circ}$ is:

$$
\overline{\Delta \vartheta}=0.53410359^{\circ} \text {. }
$$

Comparing $\overline{\Delta \vartheta}$ to $\Delta \phi_{M}$, the relative discrepancy is $\delta=6.4 \times 10^{-2}$. The near equality of $\Delta \phi_{M}$ and $\overline{\Delta \vartheta}$ has deep meanings. The arc length of $\overline{\Delta \vartheta}=0.53410359^{\circ}$ on the $M$-circle is:

$$
\Delta L_{M}=L_{M} \frac{\overline{\Delta \vartheta}}{360^{\circ}}=2 \pi \times 71 \frac{0.53410359^{\circ}}{360^{\circ}}=0.6618525 .
$$

As shown in Fig. 23.1(a), the center of the right Gaussian sphere shifts towards left and brings the Gaussian sphere with it to a new location shown by the red circle. $\Delta L_{M}$ is the distance between its original location and the new location after shifted. At its new location, the shifted Gaussian sphere overlaps with the Gaussian sphere at left. The distance between the centers of these two Gaussian spheres is:

$$
\Delta d_{M 1}=\frac{1}{\sqrt{2}}-\Delta L_{M}=0.04525428 \text {. }
$$

As shown in Fig. 23.1(a), these two Gaussian spheres are almost entirely overlapped.
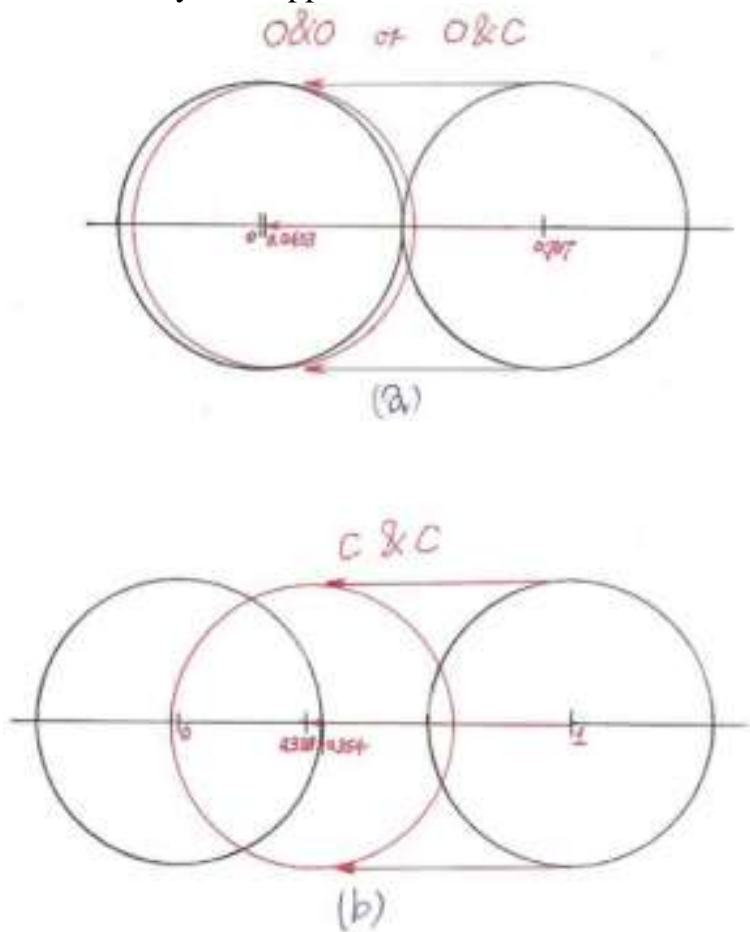

Fig. 23.1: Pairs of adjacent Gaussian spheres intersect with $M$ sphere surface.

Fig. 23.1(b) shows the case for two adjacent Gaussian spheres on the $M$-spherical surface centered at two adjacent cubic vertexes representing two bosons. The distance between them is $d_{2}=1$. The right Gaussian sphere shifts towards left with a distance of $\Delta L_{M}=0.6618525$. After shifted, its center is locates at a distance of $\Delta d_{M 2}$ from the center of its neighbor:

$$
\Delta d_{M 2}=1-\Delta L_{M}=0.3381475 \text {. }
$$

As shown in Fig. 23.1(b), the center of shifted Gaussian sphere just gets into its neighbor's boundary and overlaps with it about halfway.

The overlap of two Gaussian spheres means that, the vertexes at the center of Gaussian spheres are statistically no longer clearly distinguishable. As shown in Section 21, vertexes serving as elements of symmetries represent elementary particles. The overlapping Gaussian spheres are interpreted as two elementary particles represented by two vertexes no longer clearly distinguishable, which causes the symmetry broken.

In particle physics, at extremely high energy, particles are no long distinguishable. Quarks and anti-quarks with extremely high energy form the "quark-antiquark liquid state". The phenomenon has been demonstrated by physicists working on RHIC at Brookhaven National Laboratories and LHC at CERN. They found that, the quarks and anti-quarks are bound relatively tight to form a liquid like state.

The energy associated with particles increases rapidly with corresponding radius $r$ in the symmetrical space. As shown in Section 21, the first generation quarks, $u$ and $d$, with mass less than $10 \mathrm{MeV} / \mathrm{c}^{2}$ correspond to vertexes in the region with radius $r \leq 1$. The top quarks $t$ with mass of $1.72 \times 10^{5} \mathrm{MeV} / \mathrm{c}^{2}$ corresponds to vertexes in the region with radius $r \leq 3$. The 3 times radius increase causes the particles' mass more that $10^{4}$ times increase. Now, the $M$-sphere has a radius of $r=71$, comparing with $r \leq 3$ is more than 23 times increase in radius. It pushes the energy well into the quark-antiquark liquid state territory. In fact, the energy scale of grand unification happened on the $M$-sphere surface is $1.07948 \times 10^{18} \mathrm{GeV}$ as listed in Table 15.1.

Consider all of these facts, there are sufficient reasons to identify the region near the $M$-sphere inner surface as the region for the quark-antiquark liquid state.

Now let's deal with the largest prime number 47 in the $B$ group factors sequence.

Definition 23.2: The $B$-sphere is defined as a sphere in space centered at the same center of the $M$-sphere with radius:

$$
R_{B}=47 L_{P}=7.596375 \times 10^{-34} \mathrm{~m} \text {. }
$$

Take $R_{B}=47$ as radius and draw the $B$-circle on the $B$ sphere surface with the same center. The circumferential length is:

$$
L_{B}=2 \pi \times 47=295.30970944 .
$$

The arc length of $\overline{\Delta \vartheta}=0.53410359^{\circ}$ on the $B$-circle is:

$$
\Delta L_{B}=L_{B} \frac{\overline{\Delta \vartheta}}{360^{\circ}}=2 \pi \times 47 \frac{0.53410359^{\circ}}{360^{\circ}}=0.43812771 \text {. }
$$

Using the same method for calculation, the results on the $B$ spherical surface are:

$$
\begin{aligned}
& \Delta d_{B 1}=\frac{1}{\sqrt{2}}-\Delta L_{B}=0.26897907 . \\
& \Delta d_{B 2}=1-\Delta L_{B}=0.56187229 .
\end{aligned}
$$




\section{Z. Y. SHEN}

As shown in Fig. 23.2(a), the center of right Gaussian sphere is shifted towards left at $\Delta d_{B 1}=0.26897907$ from the center of its neighbor and overlapped with its neighbor about half way. As shown in Fig. 23.2(b), the center of right Gaussian sphere is shifted towards left at $\Delta d_{B 2}=0.56187229$ from the center of its neighbor, and two spheres are marginally overlapped.

It seems reasonable to call the region between $B$-sphere surface and $M$-sphere surface as the "quark-antiquark liquid region" or "liquid region" for short. But as shown by Fig. 23.2(b), the two Gaussian spheres are not detached yet. Inside the $B$-sphere, there are some liquid state remains left.

To find a clear cut for the liquid state region, let's search for another sphere. The $M$-sphere and $B$-sphere are defined by the prime numbers 71 and 47 of the $M$-group and $B$-group, respectively. The number 47 is the largest prime number in the $B$-group factors sequence corresponding to the $m$ parameter $m_{b g}=47$ of $b_{g}$ quark in the third generation. As shown in Table 23.1, for the Suz-group, there is no such prime number like 47 for the $B$-group. Look at it the other way, the first generation corresponding to $\mathrm{Suz}$-group does relate to a prime number at similar location as 47 . The number is $m_{s g}=23$ of $s_{g}$ quark in the second generation. Comparing with $m_{b g}=47$ for the $b_{g}$ quark in the third generation, they sit at similar locations with a generation difference. Before take $m_{s g}=23$ seriously, let's look at the three prime numbers in another way:

$$
\begin{aligned}
& 71+1=72, \\
& 47+1=48, \\
& 23+1=24,
\end{aligned}
$$
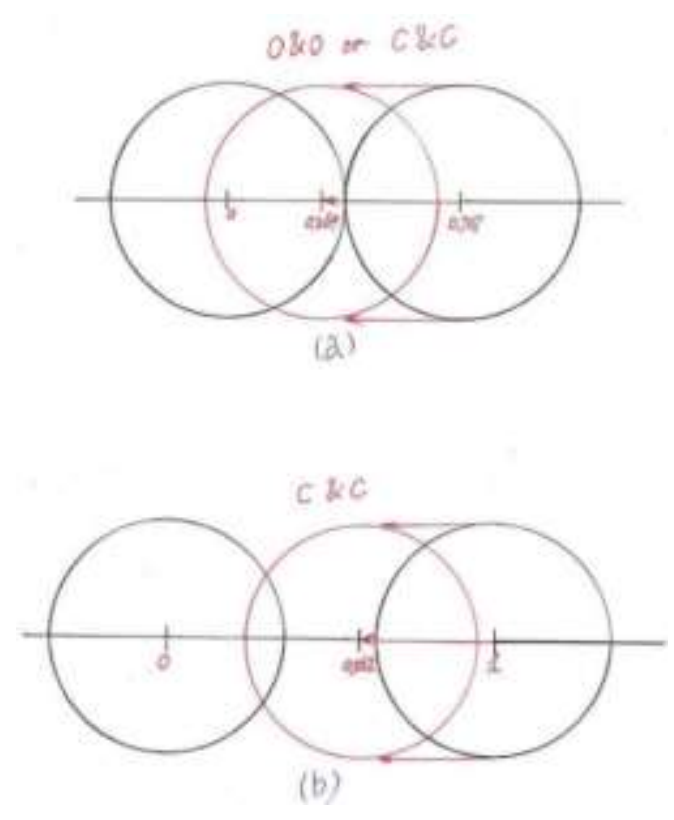

Fig. 23.2: Pairs of adjacent Gaussian spheres intersect with $B$-sphere surface.
The three numbers on the right look familiar as shown in Table 18.3. And these three numbers also serve as building blocks of the Number Tower to raise the magic number 163 to the top. The " +1 " term in (23.17) can be interpreted as adding the graviton, which is included in the Elementary Particle Table.

If the three formulas of (23.17) are not by coincidence, they provide two implications: (1) They serve as the second support for the Elementary Particle Table and its classification for particles; the first support is the Number Tower and the number 163 on top of it. (2) They provide the reason for using 23 to define a sphere like 47 to define the $B$ sphere and 71 to define the $M$-sphere.

Moreover, look closely at the factors sequence of size for other sporadic groups, there are three groups called Conway groups labeled $\mathrm{Co} 1, \mathrm{Co} 2, \mathrm{Co} 3$ with similar structure as the Suzuki group Suz. The factors sequences of $\mathrm{Co} 1, \mathrm{Co} 2, \mathrm{Co} 3$ are:

$$
\begin{aligned}
& F_{C o 1}=2^{21} \cdot 3^{9} \cdot 5^{4} \cdot 7^{2} \cdot 11 \cdot 13 \cdot 23, \\
& F_{C o 2}=2^{18} \cdot 3^{6} \cdot 5^{3} \cdot 7 \cdot 11 \cdot 23, \\
& F_{C o 3}=2^{10} \cdot 3^{7} \cdot 5^{3} \cdot 7 \cdot 11 \cdot 23 .
\end{aligned}
$$

The prime number 23 appears in $F_{C O 1}, F_{C O 2}, F_{C O 3}$ located at the right place corresponding to the down type quark $s_{g}$ with $m_{s g}=23$. These facts serve as the additional reasons for using the prime number 23 to define a sphere. Finally, there is another supportive fact:

$$
\frac{23+47+71}{3}=47 \text {. }
$$

Definition 23.3: The $S$-sphere is defined as a sphere in space centered at the center of the $M$-sphere with radius of:

$$
R_{S}=23 L_{P}=3.717375 \times 10^{-34} \mathrm{~m} \text {. }
$$

Take $R_{S}=23 L_{P}$ as radius and draw the $S$-circle on the $S$ sphere surface with the same center. The circumferential length of $S$-circle is:

$$
L_{S}=2 \pi \times 23=144.51326207 .
$$

The arc length of $\overline{\Delta \vartheta}=0.53410359^{\circ}$ on the $S$-circle is:

$$
\Delta L_{S}=L_{S} \frac{\overline{\Delta \vartheta}}{360^{\circ}}=2 \pi \times 23 \frac{0.53410359^{\circ}}{360^{\circ}}=0.21440292 \text {. }
$$

Using the same method for calculation, the results on the $S$ sphere surface are:

$$
\begin{aligned}
& \Delta d_{S 1}=\frac{1}{\sqrt{2}}-\Delta L_{S}=0.49270386, \\
& \Delta d_{S 2}=1-\Delta L_{S}=0.78559708 .
\end{aligned}
$$

As shown in Fig. 23.3(a), the center of right Gaussian sphere is shifted towards left at a distance $\Delta d_{S 1}=0.49270386$ from its neighbor and two spheres are overlapped marginally. As shown in Fig. 23.3(b), the center of right Gaussian sphere is shifted towards left at a distance $\Delta d_{S 2}=0.78559708$ from its neighbor and two spheres are detached.

Definition 24.4: The three regions inside the $M$-sphere are defined according to their radius ranges: 
The quark-antiquark liquid region:

$$
47<r_{q \bar{q} l} \leq 71,
$$

The intermediate region: $23<r_{\text {int }} \leq 47$,

The particles region: $0 \leq r_{\text {parti }} \leq 23$.

Explanation: In the quark-antiquark liquid region, the undistinguishable particles form the quark-antiquark liquid state having extremely high energy corresponding to extremely high temperature. In the transition region, individual particles are barely distinguishable, and some liquid states remains left near the $B$-spherical inner surface. In the particles region, most particles are clearly distinguishable. The detachment shown in Fig. 23.3(b) indicates that, the two Gaussian spheres centered at two adjacent vertexes representing two bosons are no longer mixed up. As shown in Fig. 23.3(a), the two adjacent Gaussian spheres with center to center distance $d_{1}=1 / \sqrt{2}$ always have some chance to mix up. In case the two Gaussian spheres centered at different types of vertexes, one at octahedral vertex presenting a fermion and the other at cubic vertex representing a boson, the mix up does not blur their identity because they belong to different types. In fact, these two Gaussian spheres are touched at their boundary to begin with. Any shift no matter how tiny causes overlapping. It indicates that, fermions and bosons are intrinsically linked. In case the two Gaussian spheres centered at two adjacent octahedral vertexes represents two fermions, they have chance to mix up and to blur their identity.
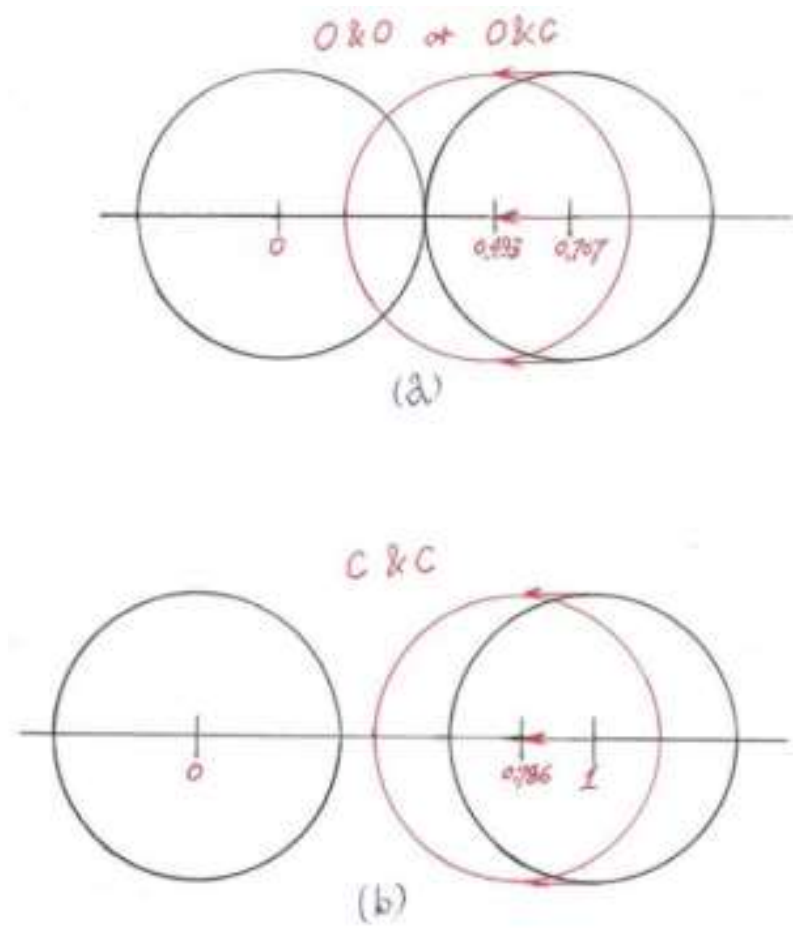

Fig. 23.3: Pairs of adjacent Gaussian spheres intersect with $S$-sphere surface.
The Gaussian spheres shifting locations are caused by the tilt angle $\overline{\Delta \vartheta} \approx 0.5341^{\circ}$, which breaks the symmetry and provides mass for particles. It is possible to give some interpretations for the Gaussian spheres shifting. The two bosons represented by two detached spheres are interpreted as bosons without mass. The one sphere representing a boson overlapped with the other sphere representing a fermion is interpreted as the boson gained mass from its component fermions. The two overlapped spheres representing two fermions are interpreted as fermions with mass. In fact, all fermions have mass, while bosons such as $\gamma, g$, $g_{i},(i=1,23 \cdots 8)$ have no mass; bosons such as $W, Z, X$, $G_{i},(i=1,23 \cdots 8), U_{1}, U_{2}, U_{3}$ gained mass from component fermions. So the interpretations seem reasonable within the particles region inside the $S$-sphere.

Let's look at the scenario shown in Fig. 23.2 for the intermediate region. The overlapped spheres shown in Fig. 23.2(a) are overlapped more, which means the particles with mass gained more mass. Since the intermediate region has much high energy than the particle region, the mass gain sounds reasonable. The detached spheres shown in Fig. 23.3(b) are overlapped, which means the original massless boson gained mass. Is that possible? Recall the $e$-boson made of a pair of electron and positron having mass

$M_{e-b}=3.95987106 \times 10^{8} \mathrm{GeV}$. Actually the $e$-boson is a "heavy photon" with zero spin. Why does the spin change? Because their numerical parameters combine in different ways.

Regular photon: $\gamma=e^{-\uparrow} e^{+\uparrow}, m=1+1=2, n=1 / 2-1 / 2=0$,

$p=1 / 2-1 / 2=0 ; M_{\gamma}=(p / n) M_{e}=0 \times M_{e}=0$,

$s_{\gamma}=m(\hbar / 2)=2(\hbar / 2)=\hbar$.

Heavy photon: $e$-boson $=e^{-\uparrow} e^{+\downarrow}, m=1-1=0$, $n=1 / 2+1 / 2=1, p=(1 / 2+1 / 2)=1$;

$M_{e-b}=2 \sqrt{N_{e}}(p / n) M_{e}=2 \sqrt{N_{e}} M_{e}=2 M_{e, \text { int }}, s_{e-b}=m \hbar / 2=0$.

So the $e$-boson as heavy photon with zero spin is explained and consistent with the rules. This argument also explains the fact that, gluons have zero mass and spin $\hbar$, while $U_{1}, U_{2}$, $U_{3}$ and massons have mass and spin 0 . So everything is consistent.

It is important to point out that, the $e$-boson as heavy photon is not another elementary particle. It is just a high energy state of the photon listed in the Elementary Particles Table, in which the heavy photon is not qualified to have its own seat.

Inside $S$-sphere, the number of vertexes is estimated as:

$$
N_{S}=\frac{V_{s}}{V_{0}} D_{f-c},
$$

The volume of Gaussian sphere:

$$
V_{0}=\frac{4 \pi}{3}\left(\frac{1}{2 \sqrt{2}}\right)^{3},
$$

The volume of $S$-sphere: 
Z. Y. SHEN

$$
V_{S}=\frac{4 \pi}{3}(23)^{3},
$$

The face-centered filling factor:

$$
D_{f-c}=\frac{\pi}{\sqrt{18}} \text {. }
$$

Substituting (23.27) and (21.1) into (23.26) yields:

$$
N_{S}=\frac{\pi \cdot(23 \cdot 2 \cdot \sqrt{2})^{3}}{\sqrt{18}}=2.039 \times 10^{5},
$$

For comparison:

$$
\begin{aligned}
& N_{B}=\frac{\pi \cdot(47 \cdot 2 \cdot \sqrt{2})^{3}}{\sqrt{18}}=1.74 \times 10^{6}, \\
& N_{M}=\frac{\pi \cdot(71 \cdot 2 \cdot \sqrt{2})^{3}}{\sqrt{18}}=5.997 \times 10^{6} .
\end{aligned}
$$

The $S$-sphere with $N_{S}=2.039 \times 10^{5}$ vertexes has sufficient room to accommodate all different types of elementary particles, composite particles and their different states.

The term "liquid" of quark-antiquark liquid state is not just symbolic, it has real implications. As mentioned in Section 21, space has its crystal structure with face-centered lattice. In the particles region, space structure is either singlecrystal or poly-crystal akin to the solid. As energy and temperature rising, space in the intermediate region corresponding to very hot solid is starting to melt. After temperature rose to "melting point", space becomes liquid. In the liquid region, even though temperature is extremely high, the binding force is strong enough to hold Gaussian spheres in the liquid state. It shows that, the term "liquid" is a good analogy to the space structure in quark-antiquark liquid region, which is also supported by experiments at RHIC and LHC.

Let's apply the analogy to the pre-big-bang period. As listed in Table 22.1, the pre-big-bang period prime numbers sequence starts at 239, which is about 3.366 times of 71 . It indicates that the lowest energy corresponding to 239 for the pre-big-bang period is many orders of magnitude higher than the highest energy in the liquid region of the $M$-sphere. In other words, the entire pre-big-bang sphere is in over heated liquid state. Any random stimulation causes the overheated pre-big-bang sphere to evaporate into "gaseous state" with free Gaussian spheres flying around. This is exactly the big bang scenario described in Section 22. The 3-dimensional over heated liquid sphere serves as the origin of universe corresponding to the pre-big-bang period. Then the big bang consecutively developed into the first period, the second period, and the current third period as described in Section 22.

It shows that, early cosmic history described in Section 22 is not only supported by the prime numbers table and the Prime Number Postulation but also supported by the category of regions for space based on three sporadic groups.

Back to the tilt angle of $\overline{\Delta \vartheta}=0.53410359^{\circ}$. The tilt angle $\Delta \vartheta$ deviated from $\vartheta= \pm 120^{\circ}$ is calculated according to (8.38) based on $A T$-equation and $P S$-equation with the data cited from particles' parameters of their models. Since $A T$ - equation and $P S$-equation are not derived from the first principle, their ad hoc nature requires verification and accuracy needs to be determined. As mentioned previously, the relative discrepancy of $\overline{\Delta \vartheta}=0.53410359^{\circ}$ from $\Delta \phi_{M}=0.57062302^{\circ}$ is $\delta=6.4 \times 10^{-2} \cdot \Delta \phi_{M}=0.57062302^{\circ}$ is the angle for totally overlapping of two Gaussian spheres shown in Fig.23.1(a). Use it as a criterion, the relative error caused by $A T$-formula and $P S$-equation is estimated no more than $\delta_{u p}=6.4 \times 10^{-2}$ on the upside. On the down side, as shown in Fig.23.1(b), use the right sphere center just touching to the left sphere surface as the criterion. The right sphere shifting distance is $D_{M 2}=1-1 /(2 \sqrt{2})=0.64644661$ corresponding to a span angle on the $M$-cycle of $\Delta \phi_{M 2}$ :

$$
\Delta \phi_{M 2}=\left(\frac{D_{M 2}}{2 \pi R_{M}}\right) \times 360^{\circ}=0.5216713^{\circ} .
$$

Compare to the tilt angle $\overline{\Delta \vartheta}=0.53410359^{\circ}$, the down side relative error is estimated as $\delta_{\text {down }}=-2.38 \times 10^{-2}$. It seems safe to say that, despite its ad hoc nature, the $A T$-equation and the $P S$-equation provide results in the ballpark. In Section 8, the $A T$-equation and the $P S$-equation as two independent equations with only one variable are satisfied simultaneously. It indicated that, there is something in it. Now the $A T$ equation and $P S$-equation have a second independent verification. These verifications are critical. Because so many things depend on it such as the $f$-modification, the effective parameters, and the significance of $M$-sphere, $B$-sphere, $S$ sphere etc. In fact, the $A T$-equation and the $P S$-equation catch the essence of elementary particles to break symmetry, to acquire mass and other related effects. This seems not an over statement.

So far the correlations and meanings of all prime numbers in the factors sequence of size for three sporadic groups are covered. There is one issue left. Besides electron's red branch with $m$-parameter $m_{e r}=2$, the other leptons' $m$-parameters are not related to the sporadic groups. The before reduction $m$-parameters for charged leptons are even numbers. Except $m_{e r}=2$ as the only even prime number, all others are not prime numbers. This is the reason for them not listed in Table 23.1. But an even number can be factorized into prime numbers. The factorized prime numbers sequence of three

\begin{tabular}{|c|c|c|c|}
\hline Name & Red branch & Green branch & Blue branch \\
\hline Electron & $F_{a r}=2$ & $F_{a g}=6=2 \cdot 3$ & $F_{a b}=12=2^{2} \cdot 3$ \\
\hline Muon & $F_{\mu}=18=2 \cdot 3^{2}$ & $F_{\mu t}=26=2 \cdot 13$ & $F_{j 6}=34=2 \cdot \underline{17}$ \\
\hline Taon & $F_{v}=42=2 \cdot 3 \cdot 7$ & $F_{\mathrm{s}}=50=2 \cdot 5^{2}$ & $F_{d}=60=2^{2} \cdot 3 \cdot 5$ \\
\hline$M$-group & \multicolumn{3}{|c|}{$F_{3 S}=2^{46} \cdot 3^{20} \cdot 5^{9} \cdot 7^{6} \cdot 11^{2} \cdot 13^{3} \cdot 17 \cdot 19 \cdot 23 \cdot 29 \cdot 31 \cdot 41 \cdot 47 \cdot 59 \cdot 71$} \\
\hline
\end{tabular}
charged leptons' $m$-parameter with their branches as shown in Table 23. 4. The factors sequence $F_{M}$ of the $M$-group is also listed at the bottom for comparison.

Table 23.4: The Factors of the before reduction $\boldsymbol{m}$-Parameters for Charged Leptons 
In the nine sets of prime number factors for three charged leptons with branches listed in Table 23.4, there are 6 prime numbers involved. In which 2 appears 11 times as $2^{11}, 3$ appears 6 times as $3^{6}, 5$ appears 3 times as $5^{3}$, and 7,13 , 17 each appears 1 time as $7^{1}, 13^{1}, 17^{1}$, respectively. To inspect the prime number factors in a symmetric manner, some criteria are needed.

The loose criterion: All prime number factors of charged leptons' before reduction $m$-parameters must be included in the prime number factors in $F_{M}$ of the $M$ group.

Notice that, 2, 3, 5, 7, 13, 17 all are included in the factors of $F_{M}$. Apparently they meet the loose criterion.

The strict criterion: All prime number factors of charged leptons' before reduction $m$-parameters must be included in the prime number factors in $F_{M}$ of the $M$ group under the condition that, the number of times used for a prime number in the $m$-parameters of quarks and charged leptons does not exceed that prime number's power number in $F_{M}$ factors.

In the $F_{M}=2^{46} \cdot 3^{20} \cdot 5^{9} \cdot 7^{6} \cdot 11^{2} \cdot 13^{3} \cdot 17 \cdots$ sequence, the power numbers for $2,3,5,7,11,13$ have sufficient room to accommodate $2^{1+1}, 3^{6+1}, 5^{3+1}, 7^{1+1}, 13^{1+1}$, in which " +1 " is to count they used once for the $m$-parameter of quarks already. But 17 in $F_{\mu b}$ is a problem, because 17 appears in $F_{M}$ sequence only once and it has been used for the $m$ parameter of strange red quark $s_{r}$ already. It does not meet the strict criterion.

There are ways to dealing with the problem.

1. The before reduction $m$-parameter of $m_{\mu b}=34=(31+37) / 2$ is derived from the $m$-parameter of two quarks $s_{b}$ and $c_{b}$ with $m$-parameters of $m_{s b}=31, m_{c b}=37$. To switch the $m$-parameters for $c_{b}$ and $c_{g}$, the new $m$-parameters of $\mu_{g}$ ad $\mu_{b}$ becomes:

$$
\begin{aligned}
& m_{\mu g}^{\prime}=\left(m_{s g}+m_{c g}^{\prime}\right) / 2=(23+37) / 2=30=2 \cdot 3 \cdot 5, \\
& m_{\mu b}^{\prime}=\left(m_{s b}+m_{c b}^{\prime}\right) / 2=(31+29) / 2=30=2 \cdot 3 \cdot 5 .
\end{aligned}
$$

If the $n$-parameters and $p$-parameters are switched with the $m$-parameters, nothing else is changed except the colors green and blue switched accordingly. The problem goes away. But it raises another question: Why switch number parameters for these two quarks? Besides, the switched $m$-parameters of these two quarks violate the order of prime number sequence. This approach is questionable.

2. The problem is originated from only one 17 in the factors of $F_{M}$. Multiplying 17 to the $F_{M}$ sequence yields a new sequence $F^{\prime}{ }_{M}$ :

$F^{\prime}{ }_{M}=17 \times F_{M}=2^{46} \cdot 3^{20} \cdot 5^{9} \cdot 7^{6} \cdot 11^{2} \cdot 13^{3} \cdot 17^{2} \cdot 19 \cdot 23 \cdot 29 \cdot 31 \cdot 41 \cdot 47 \cdot 59 \cdot 71$
The problem goes away. Multiplying $M$-group with another prime number sounds like a wild idea. But there is an additional merit:

$F^{\prime}{ }_{M}=17 \times F_{M} \approx 17 \times 8.08 \times 10^{53} \approx 1.374 \times 10^{55}$.

Back to Table 23.3, unlike the other two sporadic groups with $F_{B}$ and $F_{S u z}$ as $G=1$ and $G=3$ grand numbers,

$F_{M} \approx 8.08 \times 10^{53}$ is more than one order of magnitude away from $G=5$ grand number. $F^{\prime}{ }_{M} \approx 1.374 \times 10^{55}$ is a $G=5$ grand number, which puts it in line with $B$-group and $S u z$ group in terms of grand number ranks. The number 17 also appears two times for the $n$-parameter of $b_{r}$ and $b_{g}$ as shown in Table 23.2. $F^{\prime}{ }_{M}=17 \times F_{M}$ resolves the similar problem over there as well. So the multiplication of 17 to $F_{M}$ for the $M$-group may have some hiding reason in it.

After all, it seems not a waste effort for taking the strict criterion and deliberately looking for problems.

There is something special for the $M$-group. It defined the $M$-sphere, which provided many physics insights. Its largest factor 71 is a special prime number with many physics significances. The number 71 defined the $M$-sphere radius. The prime numbers table alone is not sufficient to make the decision. The $M$-sphere with radius 71 provided the span angle of $\Delta \phi_{M}=0.57062302^{\circ}$. This angle is checked with the average tilt angle $\overline{\Delta \vartheta}=0.53410359^{\circ}$ to verify the $A T$ equation and $P S$-equation. Moreover, as shown by (15.11), the relative deviation of $N_{G U T}=71.06529795$ from the prime number 71 is only $8.845 \times 10^{-4}$, which leads to a important clue for a formula to define the fine structure constant at GUT scale:

$$
\alpha^{-1}\left(M_{G U T}\right)=\left(\frac{71}{2 \pi}\right)^{2}=127.69002169 .
$$

The $\pi$ as a constant in (23.4) is a special case for the boson $U_{3}$ with extremely heavy mass $M_{G U T}$. For other particles, the general form of $\alpha^{-1}(M)$ is defined differently.

Definition 23.5: The $\alpha^{-1}(M)$ of a fermion or a fermion state with mass $M$ is defined as:

$$
\begin{aligned}
& \alpha^{-1}(M)=\left(\frac{71}{2 \bar{\pi}}\right)^{2} ; \\
& \bar{\pi}=\frac{1}{L_{2}} \oint \pi(l) d l .
\end{aligned}
$$

In (23.33), $L_{2}$ is the fermion's loop-2 length, $\bar{\pi}$ is the average value of $\pi(l)$ around loop-2, which is related to particle's mass.

Explanation: Definition 23.5 is a new way to define the fine structure constant based on a specific prime number 71 and geometry. It serves as an example to trace the mathematic origin of a physics constant, which fits SQS philosophy.

Formula (23.4) for $\alpha^{-1}\left(M_{G U T}\right)$ is a special case of the general definition (23.33). $M_{G U T}=1.07948 \times 10^{18} \mathrm{GeV} / \mathrm{c}^{2}$ is the 
mass of $U_{3}$ for grand unification. For the scalar boson $U_{3}$ made of two constituent fermion states with such extremely heavy mass, the value of $\bar{\pi}=\frac{1}{L_{2}} \oint \pi(l) d l$ is extremely close to $\pi$. The reason is that, $U_{3}$ constituent's spindle type torus model elliptic cross section on $x$-z plane with $b_{2} / a_{2}>>1$ is elongated so much to make $\bar{\pi}=\frac{1}{L_{2}} \oint \pi(l) d l \rightarrow \pi$ with negligible deviation.

$$
\alpha^{-1}\left(M_{G U T}\right)=(71 / 2 \pi)^{2}=127.69002169 \text { serves as an example }
$$

for the validity of Definition 23.5 at the heaviest mass of mass spectrum.

Take electron as another example. As shown in Section 8, the electron torus model loop-2 circular cross section on $x-z$ plane is divided into two halves. The outer half has positive curvature with $\pi(l)<\pi$; and the inner half has negative curvature with $\pi(l)>\pi$. According to (23.31b), $\bar{\pi}$ is calculated using the following formulas.

For outer half:

$\bar{\pi}_{o}=\frac{1}{2 m+1} \sum_{i=-m}^{m} \mid \frac{i b_{2} \pi}{m}\left[\left[\int_{0}^{\left|\frac{i_{2}}{m}\right|} \sqrt{1+\left(\frac{a_{2}}{b_{2}}\right)^{2} \frac{y^{2}}{b_{2}^{2}-y^{2}}} d y\right]^{-1}\right.$,

For inner half:

$\bar{\pi}_{i}=\frac{1}{2(2 m+1)} \sum_{i=-m}^{m} \int_{0}^{2 \pi} \sqrt{1+\left[2 a_{2} \mid \frac{i b_{2}}{m}\left[1-\sqrt{1-\left(\frac{i}{m}\right)^{2}}\right] \cos (2 \theta)\right]^{2}} d \theta$,

Overall average: $\bar{\pi}=\frac{\bar{\pi}_{o}+\bar{\pi}_{i}}{2}$.

In (23.34), each of the cross section two halves is divided into $2 m+1$ slices along $y$-axis and the values of $\pi(y)$ are calculated step by step. Then take a summation to get the average value of $\bar{\pi}_{o}$ and $\bar{\pi}_{i} \cdot(23.34 \mathrm{c})$ is used for the overall average of $\bar{\pi}$. In (23.34b) for inner half, the term $2 a_{2}\left|\frac{i b_{2}}{m}\right|\left[1-\sqrt{1-\left(\frac{i}{m}\right)^{2}}\right]$ is the variation amplitude in a circle on the saddle-shape surface with radius of $r=\left|i b_{2} / m\right|$, which is originated from $(8.12 \mathrm{a})$.

The results of 16-digit numerical calculation based on (23.33) and (23.34) for the electron's original version with $a_{2}=b_{2}=0.5$ are listed in the first row of Table 24.5.

As shown on Table 24.5 first row, the calculated value of $\alpha^{-1}\left(M_{e}\right)$ based on the original $a_{2}=0.5$ and $b_{2}=0.5$ is agreed with the 2010-PDG data $\alpha^{-1}=137.035999084$ with a relative deviation $8.487 \times 10^{-5}$. The agreement is an important verification in many senses.

1. Definition 23.5 is verified not only for $\alpha^{-1}\left(M_{G U T}\right)$ but also for $\alpha^{-1}\left(M_{e}\right)$. The mass $M_{G U T}$ of $U_{3}$ and the mass
$M_{e}$ of electron are at two ends of the mass spectrum. It is hopeful that Definition 23.5 is also valid for particles with mass in between.

2. $\alpha^{-1}(M)$ as a physics running constant is originated from the mathematic running constant $\bar{\pi}$. It serves as an example to support SQS theory philosophy. Ultimately, a valid unified theory should have no more than three basic physics constants and no other physics inputs. To convert $\alpha^{-1}(M)$ from a physics running constant to a mathematic one is an important step toward the right direction.

3. It confirms that, the prime number 71 originated from the $M$-group has many impacts on different issues. (23.33) is just one of them, but it is an important one. Because (23.33) is a very simple formula, it only has a prime number 71 and a geometrically originated mathematic running constant $\bar{\pi}$.

4. It confirms that, the electron torus model is correct in terms of its shape, sizes and the characteristic points. Sine electron serves as the base for other elementary particles, this confirmation has its significance.

To compare theoretical value of $\alpha^{-1}\left(M_{e}\right)=137.04762893$ with 2010-PDG data (p.126) $\alpha^{-1}\left(M_{e}\right)=137.035999084(51)$, the relative deviation of $8.487 \times 10^{-5}$ is out of its error range. In the formulas of (23.34), except the summation index truncation determined by convergence, there is no adjustable parameter. The index truncated at $m=10^{7}$ is sufficient for the converge with uncertainty less than $10^{-7}$. In fact, (23.34a) and (23.34b) are checked by taking integrals to replace the summations, which are agreed to each other with $\sim 10^{-7}$. Back to the electron model and parameters, there is no adjustable parameter either (for electron, before reduction number parameters $m=2, n=1, p=1$ are uniquely determined without alternative). Without adjustable parameter is a good thing for a theory. But it raises a question: Where does the $8.487 \times 10^{-5}$ relative deviation come from? One possible clue is that, formulas (23.34a) and (23.34b) are based on the torus model; the real model for electron is trefoil type with three torus as branches. As shown in Fig. 12.2, a part of torus outer half is in the combined region, which may have some deformation. (23.34a) does not take it into account. It may cause minor error.

Table 23.5: The Calculated $\alpha^{-1}(M)$ for Electron, $Z$ and $U_{3}$

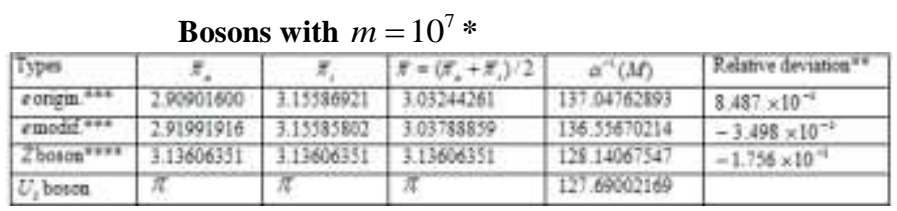

* The results are based on 16-digit calculation, only 8 digits after decimal point are listed. 
** Relative deviation for $\alpha^{-1}\left(M_{e}\right)$ is based on 2010-PDG data: $\alpha^{-1}=137.035999084$; Relative deviation for $\alpha^{-1}\left(M_{Z}\right)$ is based on 2010-PDG data: $\alpha^{-1}\left(M_{Z}\right)=127.916$.

*** The electron orig. data are based on $a_{2}=0.5, b_{2}=0.5$; the electron modif. data are based on $f$-modification with $f_{a}=0.9836342346444303$, $f_{b}=1.01623292003951$.

**** The data for $\alpha^{-1}\left(M_{Z}\right)$ are based on $a_{2}=1.3340611307962$, $b_{2}=10.748299405050495$.

It must point out that, formula (23.34b) is valid only for torus model with circular $x-z$ cross section. The inner half formula for elliptic cross section is complicated. The two equations (8.13a), (8.13b) with $b_{2} \neq a_{2}, b_{1} \neq a_{1}$ have two unknowns $a_{1}, b_{1}$, which are solved numerically by trial and error method. A tailor made program for calculating $\bar{\pi}_{i}$ is not available in the meantime. When adequate numerical method is available, it can be used to calculate $\alpha^{-1}(M)$ for other particles as well.

Fortunately, particles with mass $M>M_{M a x}=4.9732 \mathrm{GeV} / c^{2}$ have spindle type torus model, its loop-2 cross section inner half does not have negative curvature. The problem with $(23.34 \mathrm{~b})$ is irrelevant. For these particles, (23.34) is modified as: For outer half:

$$
\bar{\pi}_{o}=\frac{1}{2 m+1} \sum_{i=-n}^{m} \mid \frac{i b_{2} \pi}{m}\left[\int_{0}^{\left|\frac{b_{2}}{m}\right|} \sqrt{1+\left(\frac{a_{2}}{b_{2}}\right)^{2} \frac{y^{2}}{b_{2}^{2}-y^{2}}} d y\right]^{-1},
$$

For inner half: $\bar{\pi}_{i}=\bar{\pi}_{o}$,

Overall average: $\bar{\pi}=\frac{\bar{\pi}_{o}+\bar{\pi}_{i}}{2}=\bar{\pi}_{0}$

The modified formulas of (23.35) is used to calculate $\alpha^{-1}\left(M_{Z}\right)$ for $Z$ boson with mass $M_{Z}=91.18775548 \mathrm{GeV} / \mathrm{c}^{2}$ made of two fermion states $Y_{1}$ and $\bar{Y}_{2}$. The results are listed in Table 25.4 third row. The calculated value of $\alpha^{-1}\left(M_{Z}\right)=128.14067547$ is in between $\alpha^{-1}\left(M_{Z}\right)=127.916$ cited from 2010 PDG (p.126) and $\alpha^{-1}\left(M_{Z}\right)=128.957$ of reference [17] from Jegeriehner. It indicates that, the theoretical value is reasonable, which serves as the third checkpoint between $\alpha^{-1}\left(M_{e}\right)$ and $\alpha^{-1}\left(M_{G U T}\right)$. The results also serve as an indirect check for $Z$ boson related data listed in Table 14.1 and Table 14.2.

Since electron torus model cross section after $f$ modification is only slightly deviates from its original circular shape, formula (23.34b) serves as an approximation. It is used along with (23.34a) and (23.34c) to calculate the data for electron $f$-modified version. The results are listed in Table 23.2 second row. The relative deviation from 2010PDG data is $-3.498 \times 10^{-3}$ with an opposite sign compared to the original version relative deviation of $+8.487 \times 10^{-5}$. It indicates that, the $f$-modification is in the right direction with overshoot. Since the calculation is based on approximate formulas, it is difficult to find the reason for the overshoot and to analyze the errors. But a rough estimate is possible. Because of the overshot, the error of $A T$-equation and $P S$ equation used for the $f$-modification should be less than $\left|-3.498 \times 10^{-3}\right|=3.498 \times 10^{-3}$. It is less than the estimated errors $\delta_{u p}=6.4 \times 10^{-2}$ and $\delta_{\text {down }}=-2.38 \times 10^{-2}$ given previously. For ad hoc equations, to have multi-checkpoints on different bases is important. In fact, these are very important checkpoints for SQS theory in terms of symmetry broken and the origin of particles mass both based on $A T$ equation and $P S$-equation. The estimated errors $\delta_{u p}=6.4 \times 10^{-2}$ and $\delta_{\text {down }}=-2.38 \times 10^{-2}$ are from the $M$-circle which is defined based on a specific prime numbers 71 . In the derivation processes, there is no adjustable variable. The estimate error of less than $3.498 \times 10^{-3}$, is based on (23.33) and (23.34) according to electron parameters. As mentioned previously, there is no adjustment either. The results show that, despite their ad hoc nature, $A T$-equation and $P S$ equation yield reasonable results within adequate error range. It proves that the approach and framework are correct.

In summary, the three sporadic groups, $M, B, S u z$, provide important supports for SQS theory. The three groups' size factors sequences $F_{M}, F_{B}, F_{S u z}$ give strong support to Prime Number Postulation. The correlations between $F_{M}, F_{B}, F_{S u z}$ and the $m$-parameters of particles of three generations not only enhance their legitimacy but also reveal something behind scene such as missing prime number factors corresponding to bosons and boson states, the grand numbers with different ranks, the hidden meaning of three prime numbers 71, 47, 23 and the definition for the $M$-sphere, $B$ sphere, $S$-sphere etc. These types of information were discovered by the author after the third draft of this paper completed and this section was added after that.

There are some issues worthwhile to think about. Since the $M$-group is the largest finite Lie group, with no group in that category has larger size and all 26 sporadic groups' size factorized sequences have factors less than 71 , the question is: What is the group or groups corresponding to the pre-bigbang period? It is not just a mathematic issue; its answer might provide some insights for cosmology and particle physics like the three sporadic groups did. There is a clue in Table 23.3 to start with: $F_{3} \approx 5.864 \times 10^{22}$ of the third periods is a $G=2$ grand number, while $F_{\text {pre }} \approx 1.686 \times 10^{44}$ of the pre-bigbang period is a $G=4$ grand number. Further discussions along this line will be given in Section 25.

\section{Section 24: SQS Theory Basic Equations}

General relativity theory is not compatible with quantum theory. General relativity theory is deterministic without uncertainty, whereas quantum theory is stochastic with uncertainty. This is the main reason for their incompatibleness. From SQS theory standpoint, introducing uncertainty to original Einstein equations is the way to make general relative 


\section{Z. Y. SHEN}

theory compatible with quantum theory. It turns out as the basic equations of SQS theory, which not only reveal some new insights for gravitation but also serve as the primary basic equations for elementary particles and all interactions as well as things on top levels. In essence, SQS theory basic equations serve as a new version of unified field theory.

To reach the goal takes steps.

Einstein field equations in different terms are shown as follows.

The original with stress-energy term:

$$
R_{a b}-\frac{1}{2} g_{a b} R=\frac{8 \pi G}{c^{4}} T_{a b},
$$

The original without stress-energy term for vacuum:

$$
R_{a b}-\frac{1}{2} g_{a b} R=0,
$$

With stress-energy and cosmological terms:

$$
R_{a b}-\frac{1}{2} g_{a b} R+g_{a b} \Lambda=\frac{8 \pi G}{c^{4}} T_{a b} .
$$

In which, $G, g_{a b}, R_{a b}, R, \Lambda, c, T_{a b}$ are Newtonian gravitational constant, gauge tensor, Ricci tensor, Ricci scalar, cosmological constant, speed of light in vacuum, stressenergy tensor, respectively.

The parameters of Einstein equations (24.1) are:

$R=R(g)=g^{a b} R_{a b}(g)$,

$R_{a b}=R_{a b}(g)=R_{a c b}^{c}(g)=\partial_{c} \Gamma_{a b}^{c}(g)-\partial_{b} \Gamma_{a c}^{c}(g)+\Gamma_{a b}^{d}(g) \Gamma_{d c}^{c}(g)-\Gamma_{a c}^{d}(g) \Gamma_{d b}^{c}(g)$

$=\frac{1}{2} g^{c d}\left(\frac{\partial^{2} g_{a b}}{\partial x^{c} \partial x^{d}}+\frac{\partial^{2} g_{b d}}{\partial x^{c} \partial x^{a}}-\frac{\partial^{2} g_{a c}}{\partial x^{b} \partial x^{d}}-\frac{\partial^{2} g_{c d}}{\partial x^{b} \partial x^{a}}\right)+\Gamma_{a b}^{d}(g) \Gamma_{d c}^{c}(g)-\Gamma_{a c}^{d}(g) \Gamma_{d b}^{c}(g)$,

$\Gamma_{b c}^{a}=\Gamma_{b c}^{a}(g)=g^{a d} \Gamma_{d b c}(g)=\frac{g^{a d}}{2}\left(\frac{\partial g_{b c}}{\partial x^{d}}+\frac{\partial g_{c d}}{\partial x^{b}}-\frac{\partial g_{d b}}{\partial x^{c}}\right), a, b, c, d=0,1,2,3$.

In which, the " $g$ " in parenthesis indicates $g_{a b}$ serving as variables.

Postulation 24.1: For a collection of Gaussian spheres each centered at a vertex point $P_{i}$ in space with face-centered structure, gauge tensors $g_{a b}, g^{a b}$ at point $P$ are redefined as:

$$
\begin{aligned}
& G_{a b}=g_{a b} p(P), \\
& G^{a b}=\frac{g^{a b}}{p(P)}, \\
& G_{a b} G^{b c}=g_{a b} g^{b c}=\delta_{a}^{c} .
\end{aligned}
$$

The $p(P)$ at point $P$ is defined as:

$$
\begin{aligned}
& p(P)=\sum_{i} p_{i}\left(P ; P_{i}\right)=\sum_{i} e^{\frac{-L_{i}^{2}\left(P ; P_{i}\right) / L_{P}^{2}}{2 \sigma^{2}}}=\sum_{i} e^{-\pi \frac{L_{i}^{2}\left(P ; P_{i}\right)}{L_{P}^{2}}}, \\
& p_{i}\left(P ; P_{i}\right)=e^{-\pi \frac{L_{i}^{2}\left(P ; P_{i}\right)}{L_{P}^{2}}}, \\
& p_{w}(i)=\frac{p_{i}\left(P ; P_{i}\right)}{p(P)}=e^{-\pi \frac{L_{i}^{2}}{L_{P}^{2}}} / \sum_{i} e^{-\pi \frac{L_{i}^{2}}{L_{P}^{2}}}, \\
& \sum_{i} p_{w}(i)=\sum_{i} \frac{p_{i}\left(P ; P_{i}\right)}{p(P)}=\sum_{i} e^{-\pi \frac{L_{i}^{2}}{L_{P}^{2}}} / \sum_{i} e^{-\pi \frac{L_{i}^{2}}{L_{P}^{2}}}=1 .
\end{aligned}
$$

$L_{i}\left(P ; P_{i}\right)$ is the geodesic length connecting point $P$ and point $P_{i}$.
Definition 25.1: The space-time variables of point $P$ are defined as:

$$
\begin{aligned}
& P\left(x^{0}\left(\xi^{0}\right) ; x^{1}\left(\xi^{0}\right), x^{2}\left(\xi^{0}\right), x^{3}\left(\xi^{0}\right)\right), \\
& \xi^{0}=c \tau=\gamma^{-1} c t=\gamma^{-1} x^{0}=N^{-1} x^{0}, \\
& \gamma=N=\frac{1}{\sqrt{1-(v / c)^{2}}}=\frac{1}{\sqrt{1-\beta^{2}}} .
\end{aligned}
$$

Explanation: The $p(P)$ assigned to gauge tensors represents uncertainty. The $p(P)$ in denominator of (24.3b) is to satisfy rule $(24.3 \mathrm{c})$. So $(24.3 \mathrm{~b})$ is not a separate assumption. As shown in definition (24.5), all four space-time variables $x^{0}\left(\xi^{0}\right), x^{1}\left(\xi^{0}\right), x^{2}\left(\xi^{0}\right), x^{3}\left(\xi^{0}\right)$ are functions of intrinsic time $\tau=\xi^{0} / c \cdot \beta$ and $\gamma$ are the standard parameters of special relativity. $N$ is converting factor.

According to Postulation 24.1, the parameters of (24.2) are redefined as: $p\left(P ; P_{i}\right)$

$$
\begin{aligned}
& R(G)=G^{a b} R_{a b}(G), \\
& R_{a b}(G)=R_{a c b}^{c}(G)=\partial_{c} \Gamma_{a b}^{c}(G)-\partial_{b} \Gamma_{a c}^{c}(G)+\Gamma_{a b}^{d}(G) \Gamma_{d c}^{c}(G)-\Gamma_{a c}^{d}(G) \Gamma_{d b}^{c}(G), \\
& =\frac{1}{2} G^{c d}\left(\frac{\partial^{2} G_{a b}}{\partial x^{c} \partial x^{d}}+\frac{\partial^{2} G_{b d}}{\partial x^{c} \partial x^{a}}-\frac{\partial^{2} G_{a c}}{\partial x^{b} \partial x^{d}}-\frac{\partial^{2} G_{c d}}{\partial x^{b} \partial x^{a}}\right)+\Gamma_{a b}^{d}(G) \Gamma_{d c}^{c}(G)-\Gamma_{a c}^{d}(G) \Gamma_{d b}^{c}(G), \\
& \Gamma_{b c}^{a}(G)=G^{a d} \Gamma_{d b c}(G)=\frac{G^{a d}}{2}\left(\frac{\partial G_{b c}}{\partial x^{d}}+\frac{\partial G_{c d}}{\partial x^{b}}-\frac{\partial G_{d b}}{\partial x^{c}}\right), \quad a, b, c, d=0,1,2,3 .
\end{aligned}
$$

In which, the " $G$ " in parenthesis indicates gauge tensors redefined according to (24.3).

In the vacuum, there is no additional energy besides vacuum energy. Based on original Einstein equations (24.1b) for vacuum without the stress-energy tensor, $T_{a b}=0$, and according to Postulation 24.1, Einstein equations are redefined as:

$$
R_{a b}(G)-\frac{1}{2} G_{a b} R(G)=0 .
$$

Auxiliary equation is to determine geodesic length $L_{i}\left(P ; P_{i}\right)$ for $p_{i}\left(P ; P_{i}\right)$.

$$
\frac{d^{2} x^{a}}{d s^{2}}+\Gamma_{b c}^{a} \frac{d x^{b}}{d s} \frac{d x^{c}}{d s}=0, P \text { to } P_{i} .
$$

Substituting parameters of (24.6) into equations (24.7a), the derivation process is given in the Part A of Appendix 6.

According to the derivative process in Part-A of Appendixes 6, the redefined Einstein equations (24.7a) are presented as follows:

$$
R_{a b}-\frac{1}{2} g_{a b} R=\gamma^{2} \breve{E}_{a b}
$$

The emerging part on right side of (25.8a) is defined as:

$$
\gamma^{2} \breve{E}_{a b}==\gamma^{2}\left[\breve{R}_{a b}-\frac{1}{2} g_{a b} \breve{R}\right] .
$$

Auxiliary equations: $\frac{d^{2} x^{a}}{d s^{2}}+\Gamma_{b c}^{a} \frac{d x^{b}}{d s} \frac{d x^{c}}{d s}=0, P$ to $P_{i}$.

Explanation: The left side of (24.8a) is the kinematic part, which is the same and has the same contains as Einstein field equation (24.1b). The right side of (24.8a) is the emerging part, which is generated by derivative process for the $p(P)$ factor attached to 
gauge tensors. The emerging terms $\breve{R}_{a b}$ and $\frac{1}{2} g_{a b} \breve{R}$ are originated from terms $R_{a b}$ and $\frac{1}{2} g_{a b} R$ in the kinematic part, respectively. As shown in Part-A of Appendix 6, the kinematic part is deterministic, while the emerging part attached to probability is stochastic. Equations (24.8a) are hybrid stochastic differential equations. The mixed deterministic and stochastic nature has important physics implications, which will be explained later.

According to (A6.6), the contains of $\breve{R}_{a b}$ and $\frac{1}{2} g_{a b} \breve{R}$ for the emerging part are given as follows:

$\frac{1}{2} g_{a b} \breve{R}=\frac{1}{2} g_{a b} g^{c d} \breve{R}_{c d}$,

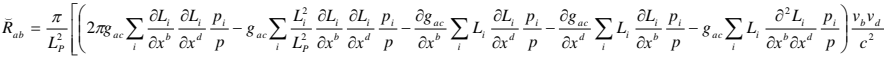

$$
\begin{aligned}
& +\left(2 \pi g_{d \alpha} \sum_{i} \frac{\partial L_{i}}{\partial x^{b}} \frac{\partial L_{i}}{\partial x^{a}} \frac{p_{i}}{p}-g_{d d} \sum_{i} \frac{L_{i}^{2}}{L_{p}^{2}} \frac{\partial L_{i}}{\partial x^{b}} \frac{\partial L_{i}}{\partial x^{a}} \frac{p_{i}}{p}-\frac{\partial g_{d d}}{\partial x^{b}} \sum_{i} L_{i} \frac{\partial L_{i}}{\partial x^{a}} \frac{p_{i}}{p}-\frac{\partial g_{d d}}{\partial x^{a}} \sum_{i} L_{i} \frac{\partial L_{i}}{\partial x^{b}} \frac{p_{i}}{p}-g_{d \alpha} \sum_{i} L_{i} \frac{\partial^{2} L_{i}}{\partial x^{b} \partial x^{a}} \frac{p_{i}}{p}\right) \frac{v_{b} v_{a}}{c^{2}} \\
& -\left(2 \pi g_{a b} \sum \frac{\partial L_{i}}{\partial x^{c}} \frac{\partial L_{i}}{\partial x^{d}} \frac{p_{i}}{p}-g_{a b} \sum \frac{L_{i}^{2}}{L_{p}^{2}} \frac{\partial L_{i}}{\partial x^{c}} \frac{\partial L_{i}}{\partial x^{d}} \frac{p_{i}}{p}-\frac{\partial g_{a b}}{\partial x^{c}} \sum L_{i} \frac{\partial L_{i}}{\partial x^{d}} \frac{p_{i}}{p}-\frac{\partial g_{a b}}{\partial x^{d}} \sum_{i} L_{i} \frac{\partial L_{i}}{\partial x^{c}} \frac{p_{i}}{p}-g_{a b} \sum L_{i} \frac{\partial^{2} L_{i}}{\partial x^{c} \partial x^{d}} \frac{p_{i}}{p}\right) \frac{v_{i} v_{d_{b}}}{c^{2}}
\end{aligned}
$$

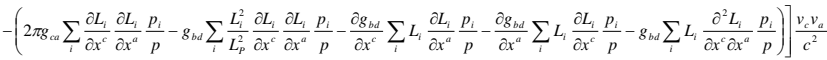

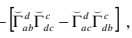

$$
\begin{aligned}
& \breve{\Gamma}_{b c}^{a}=g^{a d \Gamma_{d b c}}=\frac{\pi g^{a d}}{L_{p}^{2}}\left(g_{d b} \sum_{i} L_{i} \frac{\partial L_{c}}{\partial x_{c}} \frac{v_{c}}{c} \frac{p_{i}}{p}-g_{b c} \sum_{i} \frac{\partial L_{i}}{\partial x^{d}} \frac{v_{d}}{c} \frac{p_{i}}{p}-g_{\alpha d} \sum_{i} \frac{\partial L_{i}}{\partial x^{b}} \frac{v_{b}}{c} \frac{p_{i}}{p}\right), a, b, c, d=0,1,2,3, b
\end{aligned}
$$

According to (24.4c), all ratios $p_{i} / p$ in (24.9) are unitary weighted probability:

$$
\begin{aligned}
& p_{w}(i)=p_{i} / p=e^{-\pi\left(L_{j} / L_{P}\right)^{2}} / \sum_{j} e^{-\pi\left(L_{j} / L_{P}\right)^{2}}, \\
& \sum_{i} p_{w}(i)=\sum_{i} p_{i} / p=1 .
\end{aligned}
$$

SQS theory basic equations have three versions. One is for gravity, the other is for electromagnetic force, and the primary basic equation for all interactions and elementary particles as well as things on top levels.

\section{Step-1: SQS theory basic equations for gravity}

It starts from equation (24.8a):

$$
R_{a b}-\frac{1}{2} g_{a b} R=\gamma^{2} \breve{E}_{a b} \text {. }
$$

The emerging part on right side of (25.8) is:

$$
\gamma^{2} \breve{E}_{a b}==\gamma^{2}\left[\breve{R}_{a b}-\frac{1}{2} g_{a b} \breve{R}\right]=\left[\frac{8 \pi G}{c^{4}} T_{a b}-g_{a b} \Lambda\right] .
$$

In which, $G$ is the Newtonian gravitational constant, $T_{a b}$ and $\Lambda$ are the stress-energy tensor and cosmological constant respectively, which are generated from derivatives of $p(P)$ attached to redefined guarge tensor $g_{a b}$.

The question is: How to define the $T_{a b}$ and $\Lambda$ from the contains of the emerging part $\breve{E}_{a b}$ ? Since $T_{a b}$ is a tensor and $\Lambda$ is a scalar, the natural way to define $T_{a b}$ and $\Lambda$ is:

$$
\begin{aligned}
& T_{a b i}=\frac{\gamma^{2} c^{4}}{8 \pi G} \breve{R}_{a b}, \\
& \Lambda=2 \gamma^{2} \breve{R},
\end{aligned}
$$

The contains of $\breve{R}_{a b}$ and $\breve{R}$ in $T_{a b}$ and $\Lambda$ are given by (24.8a).
According to (24.10) (24.11) and (24.12), equation (24.8a) are presented as:

$$
R_{a b}-\frac{1}{2} g_{a b} R=\left[\frac{8 \pi G}{c^{4}} T_{a b}-g_{a b} \Lambda\right]
$$

Auxiliary equations are to determine geodesic lengths $L_{i}=L_{i}\left(P ; P_{i}\right)$ for $p_{i}\left(P ; P_{i}\right)$ :

$$
\frac{d^{2} x^{a}}{d s^{2}}+\Gamma_{b c}^{a} \frac{d x^{b}}{d s} \frac{d x^{c}}{d s}=0, P \text { to } P_{i} .
$$

It is important to point out that, in terms of space-time variables equation (24.13a) are inconsistent. On its right side the space-time variables are $x^{0}\left(\xi^{0}\right), x^{1}\left(\xi^{0}\right), x^{2}\left(\xi^{0}\right), x^{3}\left(\xi^{0}\right)$ defined by (24.5) and the derivative process carried out accordingly, on its left side the space-time variables are not. The inconsistence will be dialed later in this section.

To check the validity of redefined equation (24.13) is to convert into the form comparable to Einstein's original equations. The way to do so is to take the average of variables in equations (24.13). The average process irons out irrelevant microscopic details and retains their macroscopic contributions for comparison.

The redefined equations (24.13a) after average are presented as two equivalent forms:

$$
\begin{aligned}
& \bar{R}_{a b}-\frac{1}{2} \bar{g}_{a b} \bar{R}=\gamma^{2} \bar{E}_{a b} . \\
& \bar{R}_{a b}-\frac{1}{2} \bar{g}_{a b} \bar{R}=\left[\frac{8 \pi G}{c^{4}} \bar{T}_{a b}-\bar{g}_{a b} \bar{\Lambda}\right],
\end{aligned}
$$

The average process is taken on the elements of $\bar{R}_{a b}, \bar{R}$, $\bar{\Lambda}_{a b}, \bar{T}_{a b}, \bar{E}_{a b}$ as shown follows:

$$
\begin{aligned}
& \bar{R}_{a b}=R_{a b}\left(\overline{g^{a b}}, \overline{g_{a b}}, \overline{\frac{\partial g_{a b}}{\partial x^{e}}}, \overline{\frac{\partial^{2} g_{a b}}{\partial x^{e} \partial x^{f}}}\right), \\
& \bar{R}=R\left(\overline{g^{a b}}, \overline{g_{a b}}, \overline{\frac{\partial g_{a b}}{\partial x^{e}}}, \overline{\frac{\partial^{2} g_{a b}}{\partial x^{e} \partial x^{f}}}\right), \\
& \bar{\Lambda}=\Lambda\left(p_{w}(i), \overline{g^{a b}}, \overline{g_{a b}}, \overline{\frac{\partial g_{a b}}{\partial x^{e}}}, \overline{L_{i}}, \frac{\overline{\partial L_{i}}}{\partial x^{e}}, \frac{\overline{\partial^{2} L_{i}}}{\partial x^{e} \partial x^{f}}, \overline{v_{a}}\right), \\
& \bar{T}_{a b}=T_{a b}\left(p_{w}(i), \overline{g^{a b}}, \overline{g_{a b}}, \frac{\overline{\partial g_{a b}}}{\partial x^{e}}, \overline{L_{i}}, \frac{\overline{\partial L_{i}}}{\partial x^{e}}, \overline{\frac{\partial^{2} L_{i}}{\partial x^{e} \partial x^{f}}}, \overline{v_{a}}\right) \text {. } \\
& \overline{\bar{E}}_{a b}=E_{a b}\left(p_{w}(i), \overline{g^{a b}}, \overline{g_{a b}}, \bar{L}_{i}, \frac{\partial g_{a b}}{\partial x^{e}}, \frac{\partial L_{i}}{\partial x^{e}}, \frac{\partial^{2} L_{i}}{\partial x^{e} \partial x^{f}}, \overline{v_{a}}\right) .
\end{aligned}
$$

The average process is taken on individual particles. As show in Section 22 and Section 23, the three generations of elementary particles are represented by the activities in a sphere with radius $r=3 L_{P}$. However, to cover all particles in the universe under some extreme conditions such as the center of a star etc. The average area should be extended to a sphere with radius $r=71 L_{P}$ and volume $V=\frac{4 \pi}{3} r^{3}=\frac{4 \pi}{3}\left(71 L_{P}\right)^{3}$.The temporal average process should be taken in the range of $\pm \Delta t= \pm\left(71 t_{P}\right)$ corresponding to $\pm \Delta x^{0}= \pm\left(71 c L_{P}\right)$. The average value is assigned to the center vertex of the sphere. For all spheres centered at every vertexes, these spheres have large overlapped areas, the average process must avoid redundancy, which means a particle only 


\section{Z. Y. SHEN}

counted once in a sphere centered at the particle's center vertex. The average value is assigned to the center vertex of the sphere, which makes the space-time becoming discrete with discrete variables $\left(x_{i}^{1}, x_{i}^{2}, x_{i}^{3} ; x_{i}^{o}\right)$.

For the emerging terms $\bar{\Lambda}, \bar{T}_{a b}, \bar{E}_{a b}$ of (24.15c), (24.15d), (24.15e), the $p_{w}(i)$ is the weighted probability for the $i$-term. It will be explained later in this section, a particle has many different states corresponding to interactions to other particles with occurrence probability $p_{w}(i)$. According to $(24.4 \mathrm{~d})$, the weighted probability is unitary: $\sum_{i} p_{w}(i)=1$. The average process regarding $p_{w}(i)$ is to carry out the summation over all $i$-terms. After the average processes regarding $p_{w}(i)$ completed, all these emerging terms become deterministic without probability involved.

The average process for the variables and terms in (24.15) are given as follows.

$$
\begin{aligned}
& \overline{g_{a b}}=\frac{1}{2 \Delta x^{0} V} \int_{x_{i}^{0}-\Delta x^{0}}^{x_{i}^{0}+\Delta x^{0}} d x_{V}^{0} d v\left[g_{a b}\left(x, y, z ; x^{0}\right)\right]=g_{a b}\left(x_{i}, y_{j}, z_{k} ; x_{l}^{0}\right) \Rightarrow g_{a b} \text {, } \\
& \overline{g^{a b}}=\frac{1}{2 \Delta x^{0} V} \int_{x_{i}^{0}-\Delta x^{0}}^{x_{i}^{0}+\Delta x^{0}} d x_{V}^{0} d v\left[g^{a b}\left(x, y, z ; x^{0}\right)\right]=g^{a b}\left(x_{i}, y_{j}, z_{k} ; x_{l}^{0}\right) \Rightarrow g^{a b} \\
& \overline{\frac{\partial g_{a b}}{\partial x^{e}}}=\frac{1}{2 \Delta x^{0} V} \int_{x_{i}^{0}-\Delta x^{0}}^{x_{i}^{0}+\Delta x^{0}} d \int_{V}^{0} d v\left[\frac{\partial g_{a b}\left(x, y, z ; x^{0}\right)}{\partial x^{e}}\right]=g_{a b / e}\left(x_{i}, y_{j}, z_{k} ; x_{l}^{0}\right) \Rightarrow g_{a b / e}, \\
& \overline{\frac{\partial^{2} g_{a b}}{\partial x^{e} \partial x^{f}}}=\frac{1}{2 \Delta x^{0} V} \int_{x_{i}^{0}-\Delta x^{0}}^{x_{i}^{0}+\Delta x^{0}} d x_{V}^{0} d v\left[\frac{\partial^{2} g_{a b}\left(x, y, z ; x^{0}\right)}{\partial x^{e} \partial x^{f}}\right]=g_{a b l e f}\left(x_{i}, y_{j}, z_{k} ; x_{l}^{0}\right) \Rightarrow g_{a b l e f} \text {, } \\
& \overline{L_{i}}=\frac{1}{2 \Delta x^{0} V} \int_{x_{i}^{0}-\Delta x^{0}}^{x_{i}^{0}+\Delta x^{0}} d x^{0} \int_{V} d v\left[L_{i}\left(x, y, z ; x^{0}\right)\right]=L_{i}\left(x_{i}, y_{j}, z_{k} ; x_{l}^{0}\right) \Rightarrow L_{i} \text {, } \\
& \frac{\overline{\partial L_{i}}}{\partial x^{e}}=\frac{1}{2 \Delta x^{0} V} \int_{x_{i}^{0}-\Delta x^{0}}^{x_{i}^{0}+\Delta x^{0}} d x_{V}^{0} d v\left[\frac{\partial L_{i}\left(x, y, z ; x^{0}\right)}{\partial x^{e}}\right]=L_{i / e}\left(x_{i}, y_{j}, z_{k} ; x_{l}^{0}\right) \Rightarrow L_{i / e}, \\
& \overline{\frac{\partial^{2} L_{i}}{\partial x^{e} \partial x^{f}}}=\frac{1}{2 \Delta x^{0} V} \int_{x_{i}^{0}-\Delta x_{0}}^{x_{i}^{i}+\Delta x^{0}} d x_{V}^{0} d v\left[\frac{\partial^{2} L_{i}\left(x, y, z ; x^{0}\right)}{\partial x^{e} \partial x^{f}}\right]=L_{i / f f}\left(x_{i}, y_{j}, z_{k} ; x_{l}^{0}\right) \Rightarrow L_{i / e f} \text {, } \\
& \overline{v_{a}}=\frac{1}{2 \Delta x^{0} V} \int_{x_{i}-\Delta x^{0}}^{x_{i}+\Delta x^{0}} d x^{0} \int_{V} d v\left[v_{a}\left(x, y, z ; x^{0}\right)\right]=v_{a}\left(x_{i}, y_{j}, z_{k} ; x_{l}^{0}\right) \Rightarrow v_{a} \text {, } \\
& \bar{R}_{a b}\left(\overline{g^{a b}}, \overline{g_{a b}}, \frac{\overline{\partial g_{a b}}}{\partial x^{e}}, \overline{L_{i}}, \overline{\frac{\partial L_{i}}{\partial x^{e}}}, \overline{\frac{\partial^{2} L_{i}}{\partial x^{e} \partial x^{f}}}, \overline{v_{a}}\right)=R_{a b}\left(x_{i}, y_{j}, z_{k} ; x_{l}^{0}\right) \Rightarrow R_{a b}, \\
& \bar{R}\left(\overline{g^{a b}}, \overline{g_{a b}}, \overline{\frac{\partial g_{a b}}{\partial x^{e}}}, \overline{L_{i}}, \overline{\frac{\partial L_{i}}{\partial x^{e}}}, \overline{\frac{\partial^{2} L_{i}}{\partial x^{e} \partial x^{f}}}, \overline{v_{a}}\right)=R\left(x_{i}, y_{j}, z_{k} ; x_{l}^{0}\right) \Rightarrow R, \\
& \bar{\Lambda}\left(\overline{g^{a b}}, \overline{g_{a b}}, \frac{\overline{\partial g_{a b}}}{\partial x^{e}}, \overline{L_{i}}, \overline{\frac{\partial L_{i}}{\partial x^{e}}}, \overline{\frac{\partial^{2} L_{i}}{\partial x^{e} \partial x^{f}}}, \overline{v_{a}}\right)=\Lambda\left(x_{i}, y_{j}, z_{k} ; x_{l}^{0}\right) \Rightarrow \Lambda, \\
& \overline{T_{a b}}\left(\overline{g^{a b}}, \overline{g_{a b}}, \overline{\frac{\partial g_{a b}}{\partial x^{e}}}, \bar{L}_{i}, \frac{\overline{\partial L_{i}}}{\partial x^{e}}, \overline{\frac{\partial^{2} L_{i}}{\partial x^{e} \partial x^{f}}}, \overline{v_{a}}\right)=T_{a b}\left(x_{i}, y_{j}, z_{k} ; x_{l}^{0}\right) \Rightarrow T_{a b}, \\
& \overline{E_{a b}}\left(\overline{g^{a b}}, \overline{g_{a b}}, \frac{\partial g_{a b}}{\partial x^{e}}, \overline{L_{i}}, \frac{\overline{\partial L_{i}}}{\partial x^{e}}, \overline{\frac{\partial^{2} L_{i}}{\partial x^{e} \partial x^{f}}}, \overline{v_{a}}\right)=E_{a b}\left(x_{i}, y_{j}, z_{k} ; x_{l}^{0}\right) \Rightarrow E_{a b} .
\end{aligned}
$$

Space with face-centered lattice structure is symmetrical with respect to $\pm x^{1}, \pm x^{2}, \pm x^{3}$ and $\pm x^{0}= \pm c t$ axes. In the symmetrical space-time, integral of odd function over symmetrical range equals to zero by cancelation. Notice that, $g_{a b}, g^{a b}, L_{i}$ and their second order derivatives with respect to the same variable are even functions; their first order derivatives and second order derivatives with respect to different variables are odd functions. For integral over symmetrical range in symmetrical space-time, after average process only even functions retain and all odd functions equal to zero:

$$
\begin{aligned}
& g_{a b / e}=g_{a b / e}\left(x_{i}, y_{j}, z_{k} ; x_{l}^{0}\right)=0, \\
& g_{a b / e f}=g_{a b / e f}\left(x_{i}, y_{j}, z_{k} ; x_{l}^{0}\right)=0, e \neq f, \\
& L_{i / e}=p_{l e}\left(x_{i}, y_{j}, z_{k} ; x_{l}^{0}\right)=0, \\
& L_{i / e f}=L_{i / e f}\left(x_{i}, y_{j}, z_{k} ; x_{l}^{0}\right)=0, e \neq f .
\end{aligned}
$$

According (24.15), (24.16), (24.17), after average process, equations (24.14a) are presented as:

$$
R_{a b}-\frac{1}{2} g_{a b} R+g_{a b} \Lambda=\frac{8 \pi G}{c^{4}} T_{a b} .
$$

Cosmological term: $g_{a b} \Lambda=\gamma^{2}\left[\frac{1}{2} \bar{g}_{a b} \overline{\bar{R}}\right]$,

Stress-energy term: $\frac{8 \pi G}{c^{4}} T_{a b}=\gamma^{2} \overline{\bar{R}}_{a b}$,

Emerging part: $\gamma^{2} E_{a b}=\gamma^{2}\left[\overline{\widetilde{R}}_{a b}-\frac{1}{2} \bar{g}_{a b} \overline{\bar{R}}\right]=\frac{8 \pi G}{c^{4}} T_{a b}-\frac{1}{2} g_{a b} \Lambda$.

The Auxiliary equations (24.13b) are no longer needed, because the probabilities are averaged out.

The "bar" on top of parameters in equations (24.18) is omitted for simplicity. It is important to notice that, the differential derivatives in (24.13a) become differences in (24.18) evaluated at discrete cubic vertexes separated by Planck length.

As shown in formulas (24.19), the emerging terms $\frac{8 \pi G}{c^{4}} T_{a b}$ and $g_{a b} \Lambda$ contain $\gamma^{2}$ factor. It makes speeds $\mathcal{N}_{a},(a=1,2,3)$ in the emerging terms superluminal, which is not acceptable for macroscopic equations. Both sides of equations (24.18) are divided by $\gamma^{2}=N^{2}=\left(\lambda_{C} / L_{P}\right)^{2}$ factor. As results, the $\gamma^{2}$ factors in emerging part responsible for superluminal speed are canceled out and the separation between discrete points of difference equations (25.18) changes accordingly from Planck length $L_{P}$ to Compton length $\lambda_{C}$. In fact, this is a second average process and a transition. The second average process is taken in a cube with edges $L_{C} \times L_{C} \times L_{C}$ centered at each cubic vertex separated by a distance from neighboring cubic vertexes. Then it takes the summation of all relevant parameters assigned to the center vertex after the first average process over all vertexes in a cube divided by the cube volume $L_{C}^{3}$. The transition is to convert the Planck scale parameters to corresponding Compton scale parameters according to the converting rules given in Section 4. For instance, $L_{C}=N L_{P}, t_{C}=N t_{P}, m_{C}=m_{P} / N, E_{C}=E_{P} / N$.

Definition 24.1: SQS theory macroscopic basic equations for gravity are defined as the following difference equations with parameters evaluated at cubic vertexes separated by Compton scale $L_{C}=\gamma L_{P}=N L_{P}$ and.

$$
t_{C}=h_{P}=N t_{P} .
$$




$$
R_{a b}-\frac{1}{2} g_{a b} R+g_{a b} \Lambda=\frac{8 \pi G}{c^{4}} T_{a b} .
$$

Cosmological term: $g_{a b} \Lambda=\frac{1}{2} \bar{g}_{a b} \overline{\widetilde{R}}$,

Stress-energy term: $\frac{8 \pi G}{c^{4}} T_{a b}=\overline{\mathscr{R}}_{a b}$,

Emerging part: $\overline{\bar{E}}_{a b}=\overline{\widetilde{R}}_{a b}-\frac{1}{2} \bar{g}_{a b} \overline{\bar{R}}=\frac{8 \pi G}{c^{4}} T_{a b}-g_{a b} \Lambda$.

The Auxiliary equations are no longer needed,

Explanation: Basic equations (24.20) are converted from equations (24.13a) with two main differences: (1) Speeds in the emerging part are non-superluminal, because $\gamma^{2}$ factors in the emerging part are canceled out; (2) The $\gamma^{2}$ factor in denominator of the kinematic part $R_{a b}-\frac{1}{2} g_{a b} R$ elongates the separation for each direction by a factor of $\gamma=N=L_{C} / L_{P}$. All parameters in equations (24.20) are evaluated at cubic vertexes separated by Compton scale: $L_{C}=N L_{P}, t_{C}=N t_{P}$. Notice that, the bar on emerging terms $\overline{\bar{E}}_{a b}=\overline{\widetilde{R}}_{a b}-\frac{1}{2} \bar{g}_{a b} \overline{\bar{R}}$ of $(24.19 \mathrm{c})$ indicating they are the averaged values from two average process. This process should be carried out for all particles involved. The converting process is taken after the average process is completed, which converts the averaged parameters for each particle involved from Planck scale to Compton scale. The converting process is also applied to the kinematic part. Converting process is required by separation's scale change from Planck scale to Compton scale. After the converting process, equations (24.20) are in the microscopic sense comparable to Einstein equations (24.1c).

The features of basic equations (24.20) for gravity are as follows.

1. As results of average processes, the details of microscopic effects are ironed out and their macroscopic contributions are assigned to each cubic vertex separated by Compton length $L_{C}=N L_{P}$ and Compton time $t_{C}=L_{C} / c=N t_{P}$. Differential equations (24.13a) become difference equations (24.20) with the merit of no singularity.

2. As discussed in Section 21, particles gain mass via breaking space symmetry. For vacuum without "real matter", space-time is perfect symmetry. According to (24.17) all odd terms in equations (24.20), are canceled out by average and only even terms remain. According to (24.17a) and (24.17b), in the kinematic part of equations (24.20) only $\frac{\partial^{2} g_{a b}}{\partial x^{e} \partial x^{e}},(a, b, e=0,1,2,3)$ related terms remain. According to $(24.17 \mathrm{c})$, and $(24.17 \mathrm{~d})$, in the emerging part of equations (24.20) no term in the part of $\left[\overline{\bar{\Gamma}}_{a b}^{d} \overline{\bar{\Gamma}}_{d c}^{c}-\overline{\bar{\Gamma}}_{a c}^{d} \overline{\bar{\Gamma}}_{d b}^{c}\right]$ remain, and only $\frac{\partial^{2} L_{i}}{\partial x^{e} \partial x^{e}} v_{e} v_{e},(e=o, 1,2,3)$ related terms remain. The contents of cosmological term $g_{a b} \Lambda$ and stress-energy term $\left(8 \pi G / c^{4}\right) T_{a b}$ in equations (24.20) are significantly reduced. The remained terms still have nonzero values contributed by the even terms, which represent vacuum fluctuations. In essence, vacuum is matter with energy, there is no such thing as absolute void.

3. For the real world with matters around, as shown in Section 21, elementary particles obtain mass by breaking symmetry. As a result, space symmetry is broken. In equations (24.20), the kinematic part $R_{a b}-\frac{1}{2} g_{a b} R$ along with the cosmological term $g_{a b} \Lambda$ and stress-energy terms $\left(8 \pi G / c^{4}\right) T_{a b}$ have their full brown contents, which serve the function as original Einstein equations (24.1c). In essence, broken space symmetry provides mass for particles, which in return serves as the mechanism for space-time curvature and the origin of gravity. It shows the consistency of the theory.

4. Basic difference equations (24.20) are evaluated as discrete cubic vertex with Compton scale separations. It has important physics implications. For instance, hydrogen atom has a proton as its nucleon. The Compton length of proton is $L_{C}=h /\left(M_{\text {proton }} c\right)=1.321 \times 10^{-15} \mathrm{~m}$. Heavier atoms have even shorter Compton length. It shows that, according to equations (25.20), gravity has sufficiently fine resolution at atomic scale. More importantly, the Compton cube serves as a shield to isolate the effect of microscopic mass. Otherwise, without the shielding effect, everything will be crushed by tremendous gravitational force produced by Planck mass. It shows the physics impact of separations' elongation for difference equations (24.20) and the consistency of the theory.

5. As shown in (24.13a), the cosmological term $g_{a b} \Lambda=\gamma^{2}\left\lfloor\frac{1}{2} g_{a b} \breve{R}\right\rfloor$ contains $\gamma^{2}$ factor, which is eliminated in the cosmological term $g_{a b} \Lambda=\frac{1}{2} \bar{g}_{a b} \overline{\bar{R}}$ of macroscopic basic equations (24.20). It provides a way to dealing with the annoying dark energy problem. The one hundred twenty some orders of magnitude tremendous difference between theoretical value and observed data is a typical hierarchy problem. From SQS theory viewpoint, the way to dealing with such hierarchy problem is to apply appropriate converting factor. One is readily available. According (5.5), the converting factor for photon with wavelength $\lambda$ is:

$$
N=\frac{\lambda}{L_{P}}=\frac{1}{\sqrt{1-(v / c)^{2}}}=\gamma
$$

For orders of magnitude estimation, cosmic Microwave Background Radiation (MBR) frequency spectrum center wavelength $\lambda_{0}=1.08 \times 10^{-3} \mathrm{~m}$ is used to calculate the value of converting factor $N$. MBR photons are cosmically originated and their effects are elongated to cosmic scale by space expansion. As shown in Section 25, to consider their contribution should take long path wavelength to replace short wavelength. Based on cosmic MBR photons with $\lambda_{0}=1.08 \times 10^{-3} \mathrm{~m}$ and long path wavelength $\hat{\lambda}_{0}=N \lambda_{0}=\lambda_{0}^{2} / L_{P}$, the ratio of cosmological terms in microscopic equations 
Z. Y. SHEN

(24.13a) and cosmological term in macroscopic equations (24.20) is:

$$
R=\left(\frac{\hat{\lambda}_{0}}{L_{P}}\right)^{2}=\left(\frac{\lambda_{0}}{L_{P}}\right)^{4}=1.994 \times 10^{127}
$$

The ratio of theoretical mass density versus observed data is:

$$
R_{\rho}=\frac{\rho_{\text {Planck }}}{\rho_{\text {obserbed }}}=3.271 \times 10^{123 .}
$$

In which, $\rho_{\text {Planck }}=M_{P} / L_{P}^{3}=3.239 \times 10^{97} \mathrm{~kg} / \mathrm{m}^{3}$ is Planck density, $\rho_{\text {observed }}=9.9 \times 10^{-27} \mathrm{~kg} / \mathrm{m}^{3}$ is observed cosmic mass density. Notice that, there is a subtle difference between gravity and electromagnetic force with the factor $\alpha / 4 \pi^{2}$ shown in (4.19). Dark energy is related to gravity, while photon is generated by electromagnetic force. Taking this factor into account, the theoretical value of (24.23) with $\alpha \approx 1 / 137.036$. becomes:

$$
R^{\prime}=R\left(\frac{\alpha}{4 \pi^{2}}\right)=\left(\frac{\hat{\lambda}}{L_{P}}\right)^{2}\left(\frac{\alpha}{4 \pi^{2}}\right)=3.685 \times 10^{123} .
$$

Compare to $R_{\rho}=3.271 \times 10^{123}$ of (24.24), the theoretical result of (24.25) has a relative deviation of $12.6 \%$. Other estimations on different basis will be given in Section 25 with similar results. $R_{\rho}=3.271 \times 10^{123}$ is the largest grand number in physics and cosmology and the most annoying hierarchy problem. It can be reduced to $12.6 \%$ relative discrepancy by a theory not tailored for such purpose. There must be something in it.

6. It seems a surprise that started with Einstein equations (24.1b) for vacuum without stress-energy term and ended with basic equations (24.20) having stress-energy term automatically showed up from vacuum. After thought it over, it is really no surprise. By introducing probability, the stress-energy term generated by stochastic movement of vacuons in vacuum should be expected. Einstein reportedly said: "The left side of my equation is marble while the right side is straw." Now, both sides of basic equations (24.20) are marbles. Einstein in heaven should be happy to hear the news.

7. Started with Einstein equations (24.1b) for vacuum without cosmological term and ended with basic equations (24.20) having cosmological term automatically showed up from vacuum. The reason is the same as that for the stress-energy term. After the discovery of cosmic expansion, regarding his add-on cosmological constant $\Lambda$, Einstein said: "It was the biggest brander in my life." The authors of reference [20] correctly commented: "A great mistake $\Lambda$ was indeed! — not least because, had Einstein stuck by his original equation, he could have claimed the expansion of the universe as the most triumphant prediction of his theory of gravity." Now, Einstein's most triumphant prediction looks even more profound.

Cosmological term showed up in basic equations (24.20) but not by add-on. It is generated naturally from stochastic movement of vacuons in vacuum. More importantly, dark energy represented by cosmological term does exist evidenced by accelerating expansion of the universe. The stress-energy tensor of (24.21c) can be expressed as:

$$
T_{a b}=\frac{c^{4}}{8 \pi G}\left[\bar{E}_{a b}-g_{a b} \Lambda\right]=\frac{c^{4}}{8 \pi G}\left[\bar{X}_{a b} \frac{\bar{v}_{a} \bar{v}_{b}}{c^{2} L_{P}^{2}}\right] .
$$

In (24.26), the dimensionless function $\bar{X}_{a b}$ is defined as:

$$
\bar{X}_{a b}=\frac{c^{2} L_{P}^{2}}{\bar{v}_{a} \bar{v}_{b}}\left[\overline{\bar{E}}_{a b}-\bar{g}_{a b} \Lambda\right]=\frac{c^{2} L_{P}^{2}}{\bar{v}_{a} \bar{v}_{b}}\left[\overline{\bar{E}}_{a b}-\frac{1}{2} \bar{g}_{a b} \overline{\bar{R}}\right] .
$$

Substituting $G=\frac{2 \pi c^{3} L_{P}^{2}}{h}$ given by (2.1a) into $T_{a b}$ of (24.26) yields:

$$
\begin{aligned}
& T_{a b}=\frac{c^{4}}{8 \pi G}\left[\bar{X}_{a b} \frac{\bar{v}_{a} \bar{v}_{b}}{c^{2} L_{P}^{2}}\right]=\frac{h c^{4}}{16 \pi^{2} c^{5}}\left(\frac{\bar{v}_{a} \bar{v}_{b}}{L_{P}^{4}}\right) \bar{X}_{a b}=\frac{h c^{4}}{16 \pi^{2} c^{6} t_{P}}\left(\frac{\bar{v}_{a} \bar{v}_{b}}{L_{P}^{3}}\right) \bar{X}_{a b} . \\
& =\frac{h f_{P}}{16 \pi^{2} c^{2}}\left(\frac{\bar{v}_{a} \bar{v}_{b}}{L_{P}^{3}}\right) \bar{X}_{a b}=\frac{m_{P} c^{2}}{16 \pi^{2} c^{2}}\left(\frac{\bar{v}_{a} \bar{v}_{b}}{L_{P}^{3}}\right) \bar{X}_{a b}=\frac{m_{P}}{16 \pi^{2}}\left(\frac{\bar{v}_{a} \bar{v}_{b}}{L_{P}^{3}}\right) \bar{X}_{a b}
\end{aligned}
$$

In which, $t_{P}=L_{P} / c, f_{P}=1 / t_{P}, m_{P}=\sqrt{2 \pi h c / G}$ are the

Planck time, Planck frequency, Planck mass, respectively.

According to the converting rule in Section 4, from

Planck scale converting to the Compton scale: $m_{P} \Rightarrow m$ and $L_{P} \Rightarrow \lambda_{C},(24.28)$ is transformed as

$$
T_{a b}=\frac{m_{P}}{16 \pi^{2}}\left(\frac{\bar{v}_{a} \bar{v}_{b}}{L_{P}^{3}}\right) \bar{X}_{a b} \Rightarrow \frac{m}{16 \pi^{2}}\left(\frac{\bar{v}_{a} \bar{v}_{b}}{\lambda_{C}^{3}}\right) \bar{X}_{a b}=\frac{\bar{X}_{a b}}{16 \pi^{2}}\left(\frac{m \bar{v}_{a} \bar{v}_{b}}{\lambda_{C}^{3}}\right) .
$$

In which, $m, L_{P}$, and $\lambda_{C}$ are particle's regular mass,

Planck length, and Compton wavelength, respectively.

Express $T_{a b}$ of (24.29) in $4 \times 4$ matrix form:

$$
[T]=\frac{\bar{X}_{a b}}{16 \pi^{2}}[\mathrm{~T}]=\frac{\bar{X}_{a b}}{16 \pi^{2}}\left[\begin{array}{cccc}
\mathrm{T}_{00} & \mathrm{~T}_{01} & \mathrm{~T}_{02} & \mathrm{~T}_{03} \\
\mathrm{~T}_{10} & \mathrm{~T}_{11} & \mathrm{~T}_{12} & \mathrm{~T}_{13} \\
\mathrm{~T}_{20} & \mathrm{~T}_{21} & \mathrm{~T}_{22} & \mathrm{~T}_{23} \\
\mathrm{~T}_{30} & \mathrm{~T}_{31} & \mathrm{~T}_{32} & \mathrm{~T}_{33}
\end{array}\right]=\frac{\bar{X}_{a b}}{16 \pi^{2}}\left[\begin{array}{cccc}
\frac{m}{\lambda_{C}^{3}} & \frac{m \bar{v}_{1}}{\lambda_{C}^{3}} & \frac{m \bar{v}_{2}}{\lambda_{C}^{3}} & \frac{m \bar{v}_{3}}{\lambda_{C}^{3}} \\
\frac{m \bar{v}_{1}}{\lambda_{C}^{2}} & \frac{m \bar{v}_{1}^{2}}{\lambda_{C}^{3}} & \frac{m \bar{v}_{1} \bar{v}_{2}}{\lambda_{C}^{3}} & \frac{m \bar{v}_{1} \bar{v}_{3}}{\lambda_{C}^{3}} \\
\frac{m \bar{v}_{2}}{\lambda_{C}^{2}} & \frac{m \bar{v}_{2} \bar{v}_{1}}{\lambda_{C}^{3}} & \frac{m \bar{v}_{2}^{2}}{\lambda_{C}^{3}} & \frac{m \bar{v}_{2} \bar{v}_{3}}{\lambda_{C}^{3}} \\
\frac{m \bar{v}_{3}}{\lambda_{C}^{2}} & \frac{m \bar{v}_{3} \bar{v}_{1}}{\lambda_{C}^{3}} & \frac{m \bar{v}_{3} \bar{v}_{2}}{\lambda_{c}^{3}} & \frac{m \bar{v}_{3}^{2}}{\lambda_{C}^{3}}
\end{array}\right]
$$

The macroscopic gravity equation (24.20) is difference equation with Compton wavelength as difference:

$$
\Delta x=\Delta y=\Delta z=\lambda_{C} .
$$

Let's look at some elements of matrix $[\mathrm{T}]$ to see the physics meaning.

The $(0,0)$ element of matrix $[\mathrm{T}]$ is:

$$
(0,0)=\frac{m}{\lambda_{C}^{3}}=\frac{m}{\Delta x \Delta y \Delta z}=\frac{m}{\Delta V}=\rho_{m} .
$$

In which $\rho_{m}$ is the mass density.

The off diagonal elements $(0, a)$ and $(a, 0)$ of matrix $[\mathrm{T}]$ is:

$$
(0, a)=(a, 0)=\frac{m \bar{v}_{a}}{\lambda_{e C}^{3}}=\frac{m \bar{v}_{a}}{\Delta x \Delta y \Delta z}=\frac{p_{a}}{\Delta V}=\rho_{p_{a}}, \mathrm{a}=1,2,3 .
$$

In which $\rho_{p_{a}}$ is the density of the momentum's $a$-direction component.

The diagonal element $(1,1)$ of matrix $[\mathrm{T}]$ is the stress force $f_{x}$ perpendicular to the $y-z$ surface $\Delta S_{y z}$ :

$$
(1,1)=\frac{m \bar{v}_{1}^{2}}{\lambda_{e C}^{3}}=\frac{m \bar{v}_{x}^{2}}{\Delta x \Delta y \Delta z}=\frac{m \frac{\Delta \Delta \Delta x}{\Delta \Delta \Delta t}}{\Delta x \Delta y \Delta z}=\frac{m \frac{\Delta x}{\Delta t \Delta t}}{\Delta y \Delta z}=\frac{m a_{x}}{\Delta S_{y z}}=\frac{f_{x}}{\Delta S_{y z}} .
$$


The off diagonal element $(1,2)$ of matrix $[\mathrm{T}]$ is the stress force along the $x$-direction on $x-z$ surface $\Delta S_{x z}$.

$$
(1,2)=\frac{m \bar{v}_{1} \bar{v}_{2}}{\lambda_{e c}^{3}}=\frac{m \bar{v}_{x} \bar{v}_{y}}{\Delta x \Delta y \Delta z}=\frac{m \frac{\Delta \Delta \Delta y}{\Delta \Delta \Delta t}}{\Delta x \Delta y \Delta z}=\frac{m \frac{\Delta x}{\Delta \Delta \Delta t}}{\Delta x \Delta z}=\frac{m a_{x}}{\Delta S_{x z}}=\frac{f_{x}}{\Delta S_{x z}} .
$$

Let's discuss the meaning of these results

1. According to (24.32) through (24.35), the matrix $[T]$ fits

the stress-energy tensor well.

2. The value of dimensionless number factors $\bar{X}_{a b} /\left(16 \pi^{2}\right)$ is

less than 1 , which is multiplied to corresponding elements of $[\mathrm{T}]$ serving as the purpose of converting speeds product $v_{a} v_{b}$ from the microscopic Planck scale into the macroscopic scale.

The above derivations and results are rough estimations. The accurate ones will be available after the solutions of the microscopic basic equation for gravity (24.20) is obtained.

Basic equations (24.20) are valid for gravity as one of two long range forces. The other one is electromagnetic force. SQS theory macroscopic basic equations also have a version for electromagnetic force. Let's start from the basic equations for gravity (24.20) and take steps to convert to the basic equations for electromagnetic force.

As the first step, let's move the cosmological term $g_{a b} \Lambda$ in (24.20) from left to right to build the basic equation of electromagnetic force.

$$
R_{a b}-\frac{1}{2} g_{a b} R=\frac{8 \pi G}{c^{4}} T_{a b}-g_{a b} \Lambda=8 \pi G\left[\frac{T_{a b}}{c^{4}}-\frac{g_{a b}}{8 \pi G} \Lambda\right] .
$$

According to (4.19), the ratio of static electrical force and gravitational force is:

$$
R_{E / G}=\frac{f_{E}}{f_{G}}=\frac{\alpha}{4 \pi^{2}} N^{2},
$$

Multiplying $R_{E / G}=\frac{\alpha}{4 \pi^{2}} N^{2}$ to both sides of equations (24.36) yields:

$$
\left(\frac{\alpha}{4 \pi^{2}} N^{2}\right)\left(R_{a b}-\frac{1}{2} g_{a b} R\right)=\left(\frac{\alpha}{4 \pi^{2}} N^{2}\right) 8 \pi G\left[\frac{T_{a b}}{c^{4}}-\frac{g_{a b}}{8 \pi G} \Lambda\right] .
$$

According to (A4.1), (20.1), (4.15), $\alpha=\frac{e^{2}}{2 \varepsilon_{0} h c}, G=\frac{2 \pi h c}{M_{P}^{2}}, N=\frac{M_{P}}{M}$,

the right side of equations (24.38) becomes:

$$
\begin{aligned}
& \left(\frac{\alpha}{4 \pi^{2}} N^{2}\right) 8 \pi G\left[\frac{T_{a b}}{c^{4}}-\frac{g_{a b} \Lambda}{4 \pi \varepsilon_{0} G}\right]=\frac{e^{2}}{M^{2}}\left(\frac{2 T_{a b}}{\varepsilon_{0} c^{4}}-\frac{g_{a b} \Lambda}{4 \pi \varepsilon_{0} G}\right) \\
& =\frac{e^{2}}{M^{2}} T_{a b}^{E M} \Rightarrow\left(\frac{q_{1}}{M_{1}}\right)\left(\frac{q_{2}}{M_{2}}\right) T_{a b}^{E M} .
\end{aligned}
$$

In (24.39), the factor $e^{2} / M^{2}$ is split to two factors $q_{1} / M_{1}$ and $q_{2} / M_{2}$ for two charged particles with electrical charge $q_{1}$ mass $M_{1}$ and charge $q_{2}$ mass $M_{2}$, respectively. The equivalence of stress-energy tensor for electromagnetic ( $E M)$ force is defined as:

$$
T_{a b}^{E M}=\frac{2 T_{a b}}{\varepsilon_{0} c^{4}}-\frac{g_{a b} \Lambda}{4 \pi \varepsilon_{o} G}=\frac{c^{4}}{4 \pi \varepsilon_{0} G}\left[\overline{\bar{R}}_{a b}-\frac{1}{2} \bar{g}_{a b} \overline{\bar{R}}\right]=\frac{c^{4}}{4 \pi \varepsilon_{0} G} \bar{E}_{a b} .
$$

On left side of equations (24.38), the multiplied factor $\left(\frac{\alpha}{4 \pi^{2}} N^{2}\right)$ is absorbed by the kinematic part $R_{a b}-\frac{1}{2} g_{a b} R$. The factor $N^{2}$ shrinks the Compton scale separation $L_{C}=N L_{P}$, $t_{C}=N t_{P}$ back to the Planck scale $L_{P}=L_{C} / N, t_{P}=t_{C} / N$. According to (23.4), the factor $\frac{\alpha}{4 \pi^{2}}=\frac{1}{71 \times 71}$ elongates the shrunk separation from Planck scale to GUT scale:

$$
L_{G U T}=71 N L_{P}, t_{G U T}=L_{G U T} / c=71 t_{P} .
$$

In which $L_{G U T}=71 L_{P}$ and $t_{G U T}=71 t_{P}$ are the GUT scale length and time, respectively.

Definition 24.2: SQS theory macroscopic basic equations for electromagnetic force are defined as the following difference equations with parameters evaluated at cubic vertexes separated by GUT scale, $L_{G U T}=71 L_{P}$,

$$
\begin{aligned}
t_{G U T}= & 71 t_{P}: \\
& R_{a b}-\frac{1}{2} g_{a b} R=\frac{q_{1}}{M_{1}} \frac{q_{2}}{M_{2}} T_{a b}^{E M} .
\end{aligned}
$$

Explanation: Left side of (24.42) is the kinematic part representing charged particles movements. Right side is the dynamic part serving as electromagnetic driving force for charged particles movements similar to the function of stress-energy term in equations (24.20) for gravitational force. Similar to the converting process for equations (24.20), the parameters of equations (24.42) are subject to a converting process from the Planck scale to the GUT scale $L_{P} \Rightarrow L_{G U T}=71 L_{P}$ and $t_{P} \Rightarrow t_{G U T}=71 t_{P}$.

The basic equations (24.42) for electromagnetic force have following features.

1. Spatial and temporal separations of difference equations (24.42) at GUT scale $L_{G U T}=71 L_{P}, t_{G U T}=L_{G U T} / c=71 t_{P}$ are originated from the dynamic part converted from gravity to electromagnetic force. As shown in Section 15, $L_{G U T}=71 L_{P}$ is the length scale for electromagnetic force unified with gravity. For distance longer than $L_{G U T}=71 L_{P}$, electromagnetic force separated from gravity becomes an independent force. It shows that, the adjustment of separation is valid and the theory is consistent.

2. The factor $N^{2}=\gamma^{2}$ responsible for superluminal speed in the emerging part of equations (24.18) is expelled to build the basic equations (24.20) for gravitational force. As a result, the separations of difference equations were elongated from Planck scale to Compton scale for gravity. Then the factor $N^{2}=\gamma^{2}$ is taken back along with the factor $\frac{\alpha}{4 \pi^{2}}=\frac{1}{71 \times 71}$ to build the basic equations (25.42) for electromagnetic force. As a result, the separation of difference equations is shrunk back to the Planck scale then elongated to the GUT scale. These 
Z. Y. SHEN

processes are mathematically consistent and physically make sense.

3. The correlation between two versions of macroscopic basic equations (24.20) and (24.42) serves as a support of the concept: Gravitational force and electromagnetic force as two long range forces are two sides of the same coin. The microscopic effects of weak force and strong force as two short range forces are averaged out and their macroscopic contributions are included in cosmological term $g_{a b} \Lambda$ and stress-energy term $8 \pi G T_{a b}$ of equations (24.20) or the $\frac{q_{1}}{M_{1}} \frac{q_{2}}{M_{2}} T_{a b}^{E M}$ term of equations (24.42). It shows the richness and rigorousness of the theory.

4. The distinctive feature of equations (24.42) is the mass factor $M_{1} M_{2}$ in denominator of the dynamic part at right. Actually the same mass factor also appeared in the dynamic part's denominator of equations (24.20) for gravity, but it cancels out by the mass factor in nominator according to principle of initial mass equal to gravitational mass. Therefore, a fair comparison of the effects for electrical force $f_{E}$ and gravitational force $f_{G}$ should be $f_{E} / M_{1} M_{2}$ versus $f_{G} / M_{1} M_{2}$.

Take the electromagnetic force between a pair of electron as an example to show the two long range forces' profiles varying with distance $l$. In (24.42), let $M_{1} M_{2}=M_{e}^{2}$ and $q_{1} q_{2}=e^{2}$. As shown in Fig. 4.1 and discussed in Section 16, the converting factor $N_{e}$ as a function of distance $l$ has two types of variation profiles in two regions.

Region-I:

$$
N_{e}=\frac{M_{P}}{M_{e}}=\text { const. } \text {, for } l \geq \lambda_{e C},
$$

Region-II:

$$
N_{e}(l)=\frac{M_{P}}{M_{e}(l)}=N_{e}\left(L_{G U T}\right) \frac{l}{L_{G U T}}<N_{e}, \text { for } L_{G U T}=71 L_{P}<l<\lambda_{e C} .
$$

In Region-II, the average length scale for the discrete variables of difference equations (24.20) is changing with $l$.

Fig. 24.1 shows the profiles of $\left[f_{G} / M_{e}^{2}\right]$ and $\left|f_{E} / M_{e}^{2}\right|$ verses $l$ in broad regions.

In the macroscopic Region-I $l \geq \lambda_{e C}$, both $\left|f_{E} / M_{e}^{2}\right|$ and $\left[f_{G} / M_{e}^{2}\right]$ are proportional to $1 / l^{2}$ with tremendous strengths ratio $\left|f_{E}\right| / f_{G}=N_{e}^{2} \alpha / 4 \pi^{2} \sim 10^{42}$ as expected according to general relativity and Newtonian gravity equation. In the transitional Region-II, $L_{G U T}=71 L_{P}<l<\lambda_{e C}$, electron mass square $M_{e}^{2}$ is proportional to $1 / l^{2}$; static electrical force $\left|f_{E}\right|$ is also proportional to $1 / l^{2}$. As a result, $\left|f_{E} / M_{e}^{2}\right|$ becomes constant, while $\left[f_{G} / M_{e}^{2}\right]$ keeps its $1 / l^{2}$ variation profile. At GUT length scale, electromagnetic force is unified into gravitational force as shown by two profiles merged into one at $L_{G U T}=71 L_{P}$. In the microscopic RegionIII, $l<L_{G U T}=71 L_{P}$, The unified force $\left[f_{G} / M_{e}^{2}\right]$ stars to saturate, when it passes its peak then starts to decrease. At $l=x_{c} L_{P}=0.2998715627302645 L_{P},\left[f_{G} / M_{e}^{2}\right]=0$. In the subregion $0 \leq l<x_{c} L_{P},\left[f_{G} / M_{e}^{2}\right]<0$, gravity is converted from attractive to repulsive. The profile of $\left[f_{G} / M_{e}^{2}\right]$ in this subregion is according to data listed in Table 3.1 based on 1dimensional $S$-equation with $\pi(x)$ as a running constant.

As shown in Fig.24.1, at the vicinity of $l=L_{G U T}=71 L_{P}$ and $l=\lambda_{e C}$, the two intersections of straight line and curves are slightly rounded by variation of the running constant $\alpha(M)$ as mentioned in Section 4.

Fig. 24.1 reveals important physics implications:

1. The tremendous difference between $\left|f_{E} / M_{e}^{2}\right|$ and $\left[f_{G} / M_{e}^{2}\right]$ in Region-I is caused by $\left|f_{E} / M_{e}^{2}\right|=$ const in region-II.

2. The $\left|f_{E} / M_{e}^{2}\right|=$ const in region-II plays a pivotal rule for the unification of electromagnetic force and gravitational force at GUT scale.

3. Now we understand the physics meaning for difference equations (24.20) with separation of Compton length scale $L_{C}$. When $l \geq L_{C}$, the mass of the two objects involved becomes constant, which put the basic equations (24.20) on the same base to compare with Einstein's equations (24.1c). The average process is for macroscopic comparison purpose. The redefined equation (24.20) for gravity are universally applicable for all three regions, I, II, and III.

4. Gravity and electromagnetic force are indeed two sides of the same coin, but gravity is the dominate side.

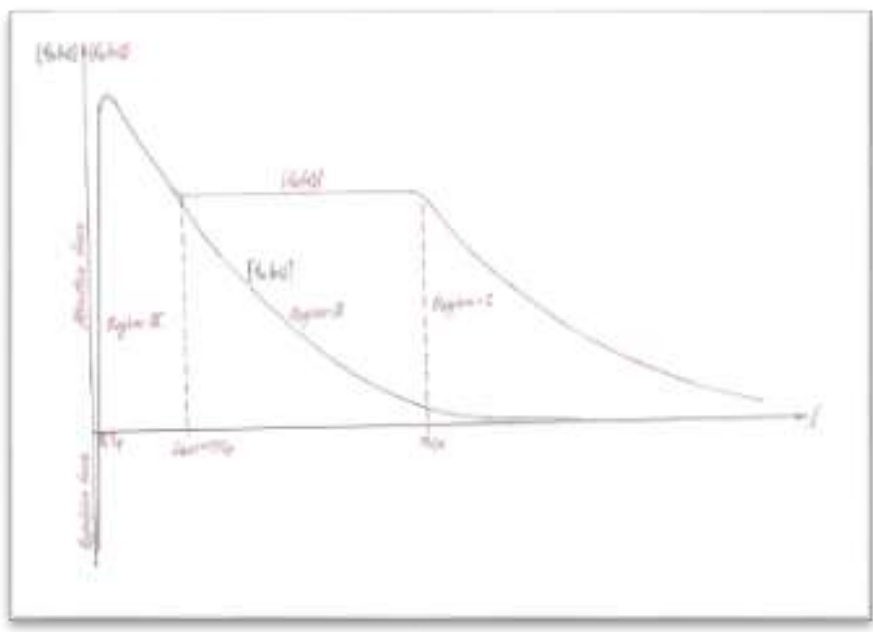

Fig. 24.1: The profiles of $\left[f_{G} / M_{e}^{2}\right]$ and $\left|f_{E} / M_{e}^{2}\right|$ versus $l$ in broad regions. (Scales are not in proportion.)

The profile shown in Fig.24.1 are informative. It supports the basic difference equations (24.20) and (24.42) and 
corresponding separations' adjustments. It shows that, in the macroscopic Region I, equations (24.20) are equivalent to

Einstein's equations (24.1c) as expected.

Conclusion 24.1: SQS theory macroscopic basic equations with two versions are derived from redefined Einstein equations (24.14) by average, which focus on the big picture for two long range forces. The version with basic equations (24.20) for gravitational force is equivalent to Einstein equations (24.1c) with two main differences: (1) The stress-energy term and the cosmological term are naturally generated from stochastic vacuum; (2) Equations (24.20) are difference equations with Compton scale separation, which have the merits of shielding effect and no singularity. The version for electromagnetic force is difference equations (24.42) with GUT scale separation having the merit of no singularity. The right side of (24.42) serves as the dynamic part. The left side of (24.42) serves as the kinematic part, which is virtually the same as kinematic part of (24.20) with different scale separations. The separations' adjustments are valid. The theory is consistent. It is conceivable that, the solutions of these two sets of macroscopic basic equations will contribute to cosmology and black hole physics with the advantage of no singularity.

After the macroscopic basic equations establishment completed, the next goal is to look for SQS theory microscopic basic equations representing elementary particles and interactions.

It starts from equations (24.8). According to Postulation 24.1 , the emerging part at the right side of equations (24.8) is converted to space-time variables $x^{0}\left(\xi^{0}\right), x^{1}\left(\xi^{0}\right), x^{2}\left(\xi^{0}\right), x^{3}\left(\xi^{0}\right)$ as functions of intrinsic temporal variable $\xi^{0}=c \tau$, while the kinematic part of the left side is not converted. The difference in space-time variables makes two parts of equations (24.8) imbalanced. For the macroscopic basic difference equations (24.20) and (25.42), the imbalance is rebalanced by proper adjustments of separation scales. Microscopic equations (24.8) are differential equations, which have no separation to adjust. Other measure must be taken to rebalance the kinematic part and the emerging part. The logical way is to convert spacetime variables of the kinematic part the same way as for the emerging part. It puts the two parts on the same footing. The derivation process is given in Part-B of Appendix 6.

According to (A6.10), the redefined Einstein equations (24.8) are presented as:

$$
R_{a b}-\frac{1}{2} g_{a b} R=E_{a b},
$$

Emerging term in (25.56a) is:

$$
E_{a b}=\gamma^{2} \breve{E}_{a b}=\gamma^{2}\left[\breve{R}_{a b}-\frac{1}{2} g_{a b} \breve{R}\right] \text {. }
$$

Auxiliary equations are to determine geodesic lengths $L_{i}=L_{i}\left(P ; P_{0}\right)$ for $p_{i}\left(P ; P_{i}\right)$ :

$$
\frac{d^{2} x^{a}}{d s^{2}}+\Gamma_{b c}^{a} \frac{d x^{b}}{d s} \frac{d x^{c}}{d s}=0, P \text { to } P_{i} .
$$

According to (A6.9), the kinematic part of (24.44a) has the following contents:

$$
\begin{aligned}
& \frac{1}{2} g_{a b} R=\frac{1}{2} g_{a b} g^{c d} R_{c d}, \\
& R_{a b}=\gamma^{2}\left[\frac{1}{2} g^{c d}\left(\frac{\partial^{2} g_{a b}}{\partial x^{c} \partial x^{d}}\left(\frac{v_{c} v_{d}}{c^{2}}\right)+\frac{\partial^{2} g_{b d}}{\partial x^{c} \partial x^{a}}\left(\frac{v_{c} v_{a}}{c^{2}}\right)-\frac{\partial^{2} g_{a c}}{\partial x^{b} \partial x^{d}}\left(\frac{v_{b} v_{d}}{c^{2}}\right)-\frac{\partial^{2} g_{c d}}{\partial x^{b} \partial x^{a}}\left(\frac{v_{b} v_{a}}{c^{2}}\right)\right)\right] \\
& +\gamma^{2}\left[\Gamma_{a b}^{d} \Gamma_{d c}^{c}-\Gamma_{a c}^{d} \Gamma_{d b}^{c}\right] \\
& \Gamma_{b c}^{a}=\frac{g^{a d}}{2}\left(\frac{\partial g_{b c}}{\partial x^{d}}\left(\frac{v_{d}}{c}\right)+\frac{\partial g_{c d}}{\partial x^{b}}\left(\frac{v_{b}}{c}\right)-\frac{\partial g_{d b}}{\partial x^{c}}\left(\frac{v_{c}}{c}\right)\right), a, b, c, d=0,1,2,3 .
\end{aligned}
$$

In which, the $\gamma^{2}$ factors are included and no probability attached.

According to (A6.11), the speed product matrix in kinematic part of equations (24.44a) is:

$$
\left[V_{i}\right]=\left[V_{i}\right]^{\mathrm{T}}=\gamma^{2}\left[\begin{array}{cccc}
1 & v_{1, i} & v_{2, i} & v_{3, i} \\
v_{1, i} & v_{1, i} v_{1, i} & v_{1, i} v_{2, i} & v_{1, i} v_{3, i} \\
v_{2, i} & v_{2, i} v_{1, i} & v_{2, i} v_{2} & v_{2, i} v_{3, i} \\
v_{3, i} & v_{3, i} v_{1, i} & v_{3, i} v_{2, i} & v_{3, i} v_{3, i}
\end{array}\right], i=1,2,3 \cdots
$$

In which, the $\gamma^{2}$ factors are included and no probability attached.

The emerging terms of equations (24.44a) are the same of (24.9), in which, the $\gamma^{2}$ factors are included. The weighted probability ${ }_{p_{w}}(i)=p_{i} / p=\exp \left(-\pi L_{i}^{2} / L_{P}^{2}\right) / \sum_{i} \exp \left(-\pi L_{i}^{2} / L_{P}^{2}\right)$ serves as the weighted factor for parameters with subscript $i$ as mentioned previously.

According to (A6.7), the speed product matrix of the emerging part in equations (24.44a) is:

$$
\left[V_{i}\right]=\left[V_{i}\right]^{\mathrm{T}}=\gamma^{2}\left[\begin{array}{cccc}
1 & v_{1, i} & v_{2, i} & v_{3, i} \\
v_{1, i} & v_{1, i} v_{1, i} & v_{1, i} v_{2, i} & v_{1, i} v_{3, i} \\
v_{2, i} & v_{2, i} v_{1, i} & v_{2, i} v_{2} & v_{2, i} v_{3, i} \\
v_{3, i} & v_{3, i} v_{1, i} & v_{3, i} v_{2, i} & v_{3, i}, v_{3, i}
\end{array}\right], i=1,2,3 \ldots
$$

In which, the $\gamma^{2}$ factor is included. The reason for the speed products matrix $\left[V_{i}\right]$. and its elements having subscript $i$, because their value depends on $i$ from the weighing probability $p_{w}(i)=p_{i} / p=\exp \left(-\pi L_{i}^{2} / L_{P}^{2}\right) / \sum_{i} \exp \left(-\pi L_{i}^{2} / L_{P}^{2}\right)$.

\section{Definition 25.3: SQS theory primary basic equations are} defined as:

$$
R_{a b}-\frac{1}{2} g_{a b} R=E_{a b},
$$

Emerging part:

$$
E_{a b}=\gamma^{2} \breve{E}_{a b}=\gamma^{2}\left[\breve{R}_{a b}-\frac{1}{2} g_{a b} \widetilde{R}\right] .
$$

Auxiliary equations are needed to determine geodesic lengths $L_{i}=L_{i}\left(P ; P_{i}\right)$ for $p_{i}=p_{i}\left(P ; P_{i}\right)$.

$$
\frac{d^{2} x^{a}}{d s^{2}}+\Gamma_{b c}^{a} \frac{d x^{b}}{d s} \frac{d x^{c}}{d s}=0, P \text { to } P_{i} .
$$

Explanation: The primary basic equations (24.48) include ten independent differential equations for ten independent variables $g_{a b}=g_{b a},(a, b=0,1,2,3)$. The contents of kinematic part $R_{a b}-\frac{1}{2} g_{a b} R$ and emerging part $E_{a b}$ are given by (24.45) and (24.9), respectively. 
Einstein's general relativity is based on two principles, the covariance principle and the equivalence principle.

Acceleration is equivalent to gravitation and both are represented by space-time curvature. SQS theory primary basic equations (24.48) are based on Einstein equations (24.1b) along with two principles of general relativity. According to Theorem 18.1, Lemma 18.1 and Lemma 18.2, vacuons movement is restricted in 1-dimensional discrete trajectories. Along a trajectory, variations of vacuons' movement are represented by space-time curvature in terms of gauge tensors. General relativity is background independent, which is suitable for different coordinate systems.

For a given discrete trajectory, the vacuons with superluminal speed move along a 1-dimensional closed trajectory. For the 1-dimensional closed trajectory, one spatial variable $x^{3}$ along trajectory's longitudinal direction and temporal variable $x^{0}$ are selected as the effective variables for the selected coordinate system. The speed product matrix (24.46) for the kinematic part and the speed product matrix (24.47) for the emerging dynamic part in the selected coordinate system both are reduced as:

$$
[V]=[V]^{\mathrm{T}}=\gamma^{2}\left[\begin{array}{cccc}
1 & 0 & 0 & v \\
0 & 0 & 0 & 0 \\
0 & 0 & 0 & 0 \\
v & 0 & 0 & v^{2}
\end{array}\right] .
$$

According to (24.50), the contents of kinematic part and the dynamic part of primary basic equations (24.48) are significantly reduced. The original ten independent equations of (24.48) for 10 independent variables, $g_{a b}=g_{b a},(a, b=0,1,2,3)$, are reduced to 3 independent equations for three independent variables, $g_{a b}=g_{b a},(a, b=0,3)$. The reduction significantly simplifies primary basic equations (24.48) and the solutions.

The features of the primary basic equations (24.48) are as follows.

1. The primary basic equations (24.48a) are hybrid stochastic differential equations. The kinematic part $R_{a b}-\frac{1}{2} g_{a b} R$ with no probability attached is deterministic, while the emerging part $E_{a b}$ as dynamic part with probabilities attached is stochastic. The mixed states have important physics implications.

2. Uncertainty shows up in the emerging part of primary basic equations (24.48) evidenced by weighted probability $p_{w}(i)=p_{i} / p$ in the emerging part (24.9). The uncertainty comes from more than one geodesic lengths $L_{i}\left(P ; P_{i}\right)$ involved representing interactions.

3. Interactions are effective only in the close vicinity. As shown in Section 21, The two sets of symmetries $O(r)$, $C(r)$ with radius $r \leq 3$ are sufficient to serve as the origin of the groups representing all three generations particles and interactions. Question: Why $r \leq 3$ ? The answer is hidden in primary basic equations (24.48). The next effective radius longer than $r=3$ is $r=\sqrt{19 / 2}$. For orders of magnitude estimation, assume $\left.L_{i}\left(P ; P_{i}\right)\right|_{\substack{P=0 \\ P_{i}=r}} \approx r$ and ignore temporal part's effect. The derivative of $p_{i}=p\left(P ; P_{i}\right)=e^{-\pi L_{i}^{2}}$ is: $D(i)=\left|\frac{\partial p_{i}}{\partial x^{a}}\right|=\left|\frac{\partial e^{-\pi t_{i}^{2}}}{\partial x^{a}}\right| \approx\left|\frac{\partial e^{-\pi r^{2}}}{\partial x^{a}}\right|=\left|-2 \pi r \frac{\partial r}{\partial x^{a}} e^{-\pi r^{2}}\right|=\frac{2 \pi r}{\sqrt{3}} e^{-\pi r^{2}}$ $a=1,2,3$.

The ratio of $D(r=\sqrt{19 / 2})$ over $D(r=\sqrt{1 / 2})$ represents their relative contributions:

$\frac{D(r=\sqrt{19 / 2})}{D(r=\sqrt{1 / 2})}=\frac{\left(r e^{-\pi r^{2}}\right)_{r=\sqrt{19 / 2}}}{\left(r e^{-\pi r^{2}}\right)_{r=\sqrt{1 / 2}}}=\frac{\sqrt{19 / 2} e^{-\pi(\sqrt{19 / 2})^{2}}}{\sqrt{1 / 2} e^{-\pi(\sqrt{1 / 2})^{2}}} \sim 2.29 \times 10^{-12}$.

As shown in (24.51b), the relative contribution of $r=\sqrt{19 / 2}$ term is in the order of $10^{-12}$, which is negligible in most cases. Now we know the reason for $r \leq 3$, because the Gaussian spheres centered at vertexes with $r>3$ all have too long $L_{i}\left(P ; P_{i}\right)$ and too low weighted probability $p_{w}(i)=p_{i} / p$ with negligible effects on interactions. In fact, the result of (24.51b) sets the background noise floor for all elementary particles and interactions at $\sim 2.29 \times 10^{-12}$. The reciprocal of $2.29 \times 10^{-12}$ is $4.37 \times 10^{11}$ as a $G=1$ grand number. Is it a coincidence?

4. Primary basic equations (24.48a) include two parts: the kinematic part $R_{a b}-\frac{1}{2} g_{a b} R$ and the dynamic part $E_{a b}$. The dynamic part serves as the cause of vacuons' movement. The kinematic part represents state of vacuons' movement as the result produced by the cause. These two parts are intrinsically correlated to causality. But they are different in terms of deterministic versus stochastic. The obvious question is: How does the stochastic cause of dynamic part produce the deterministic result for the kinematic part? According to Theorem 18.1, Lemma 18.1 and Lemma 18.2, vacuons' movement is confined in discrete trajectory and change of movements is by jumping trajectories. In essence, vacuons' movement along a trajectory is deterministic and uncertainty occurs only at jumping trajectories. The deterministic kinematic part is to represent the state of movement in a trajectory without uncertainty involved, while the stochastic dynamic part causes jumping to different trajectories with uncertainty. It answers the question naturally.

5. The hybrid state of primary basic equations (25.48a) has a deeper meaning. The quantization of vacuons' trajectories is a necessity required by introduction of uncertainty in the first place. Otherwise, if there is no quantization of discrete trajectories, the stochastic dynamic part as cause and the deterministic kinematic 
part as result would be head-on contradictory with no way out.

6. Take a close look at the geodesics. According to Lagrange principle, particle takes the path with shortest time interval from point $A$ to point $B$. In general relativity, geodesic is the shortest distance connecting two points in curved space. As shown in previous sections, particle's trajectory on torus model is determined by geodesics connecting characteristic point $A$ and point $B$. All of these should come from the same origin. As shown in Fig. 24.2, in which Fig. 24.2(a) and Fig. 24.2(b) are for a single vertex $V$ and for two vertexes $V$ and $C$, respectively. Vertex $V$ represents a particle with torus model, while vertex $C$ is the center vertex representing interactions. Fig. 24.2 shows that the particle's trajectory $T$ and the roundtrip geodesic lengths $2 L_{i}$ are integrated into an overall closed loop with characteristic points $A, B$, vertex $V$ and center vertex $C$ serving as junctions. The diagram for trefoil type model is similar to Fig. 24.2 with 3 branches and 6 characteristic points plus 3 vertexes representing trefoil type model.

a. The $\gamma$ factor in the speed product (24.46), (24.47) and (24.50) indicates that, the speed $\mathcal{w}=\hat{v}$ is superluminal. It is expected, because the vacuons movement in the overall loop is similar to the movement in the long path

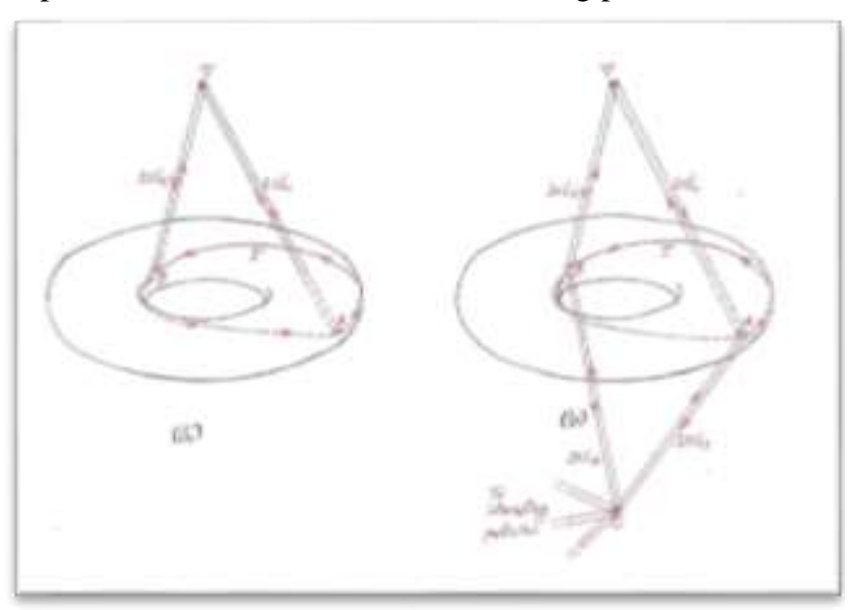

Fig. 24.2: Demonstration of particle's overall loops based on torus mode: (a) Only vertex $V$ is included; (b) Vertex $V$ and center vertex $C$ are included for interactions.

In general, the solutions of primary basic equations (25.48) cover all elementary particles and interactions, which are classified into three types.

\section{Type-1: To cover all elementary particles and all interactions.}

In this case, all relevant vertexes inside the sphere with radius $r \leq 3$ should be included. As listed in Table 21.1 or Table 21.2, the number of vertexes is $V=459$. Besides 3 independent equations of (24.48a), $M=V N_{c}=459 N_{c}$ auxiliary equations to determine $M=V N_{c}=459 N_{c}$ geodesic lengths connecting $N_{c}$ characteristic points on particle's model are added. The total number of equations involved is $3+459 N_{c}$. It seems complicated. But to determine properties of all three generations elementary particles and interactions in details, the complication seems reasonable.

Fortunately, for practical purposes, there is no need to solve all $3+459 N_{c}$ equations.

\section{Type-2: To cover elementary particles and interactions in generations.}

As shown in Table 21.1, Table 21.2 and Fig. 21.6, symmetries $O(r), C(r)$ with $r \leq 1, r \leq 2$ and $r \leq 3$ correspond to elementary particles and interactions of the $1^{\text {st }}$ generation, the $1^{\text {st }}$ generation plus the $2^{\text {nd }}$ generation and all three generations, respectively. The number of vertexes involved are 19,141 and 459 for the $1^{\text {st }}$, the $1^{\text {st }}$ plus the $2^{\text {nd }}$ and all three generations, respectively. According to the procedure used for derive formulas (24.51), values of $r, V$ and background noise level for different generations are listed in Table 24.1.

Table 24.1: The $r, V$, Numbers of Symmetries and Back Ground Noise Level for Generations

\begin{tabular}{|l|c|l|l|l|}
\hline \multicolumn{1}{|c|}{ Generations } & $r^{*}$ & $V^{* *}$ & No. of Symmetries & $B_{\text {G noise level }} * * *$ \\
\hline All three & $\sqrt{19 / 2}$ & 459 & $18 O(r) \oplus 18 C(r), r \leq 3$ & $2.29 \times 10^{-12}$ \\
\hline First \& second & $\sqrt{9 / 2}$ & 141 & $9 O(r) \oplus 9 C(r), r \leq 2$ & $1.05 \times 10^{-5}$ \\
\hline First & $\sqrt{3 / 2}$ & 19 & $3 O(r) \oplus 3 C(r), r \leq 1$ & $7.49 \times 10^{-2}$ \\
\hline
\end{tabular}

* The $r$ value takes the next one higher the number of generation as shown in Fig. 21.6.

** The $V$ value is counted the number of vertexes listed in Tables 21.1.

*** The background noise value is calculated according to (24.51) with corresponding $r$ values.

The first generation with $V=19$ is relatively simple. Table 24.1 shows that, the background noise level value increases rapidly with decreasing number of generations. The first generation's high back ground noise $7.49 \times 10^{-2}$ indicates that, to calculate interaction related parameters for first generation particles accurately, the effects of second generation should be taken into account. In fact, first generation does not include the $W^{ \pm}, Z^{0}$ and $X^{0}$ for weak interactions. For strong interactions, three gluons $g_{6}, g_{7}, g_{8}$ belong to second generation. Moreover, baryon octet represented by $O(\sqrt{3})$ and mason octet represented by $C(\sqrt{3})$ both are crossovers of first and second generations. These facts indicate that, first generation and second generation are interconnected, which can be explained by the first generation's background noise is too high.

\section{Type-3: To cover a single elementary particle.}

As shown in Section 21, symmetry $O(\sqrt{1 / 2})$ includes 8 equilateral triangles representing 2 leptons $e, v_{e}$ and 6 quarks $u_{r}, u_{g}, u_{b}, d_{r}, d_{g}, d_{b}$. Take the equilateral 


\section{Z. Y. SHEN}

triangle with 3 vertexes in $O(\sqrt{1 / 2})$ representing electron as an example. The number of auxiliary equations is reduced from $M=459 N_{c}$ to $M=3 N_{c}=3 \times 2=6$ for 3 branches of electron's trefoil type model, in which $N_{c}=2$ counts for two characteristic points $A$ and $B$ of each branch. Since electron's properties can be derived from its torus model represented by one $O(\sqrt{1 / 2})$ vertex, the number of auxiliary equations is reduced from $M=3 N_{c}=6$ to $M=1 \times N_{c}=2$,

Three independent primary basic equations plus two auxiliary equations, the five equations provide solutions for electron's trajectories on torus model and related parameters. It is surprisingly simple.

A vertex in $O(\sqrt{1 / 2})$ at different locations represents other member particles of $O(\sqrt{1 / 2})$. Question: How to differentiate these particles from the same set of equations applied to one vertex? For one vertex, space is spherical symmetry. It is conceivable that, primary basic equations (24.48) have a set of so many solutions. To select the solutions representing trajectories on electron torus model, some selecting rules are needed. One is readily available. The set of electron's numerical parameters can be used to select the solutions of primary basic equations (24.48) representing the trajectories on electron's torus model. The way to do so is based on the following steps learned from previous sections.

1. Use the set of unique numerical parameters $m_{e}=1$, $n_{e}=1 / 2, p_{e}=1 / 2$ for electron to select a set of relevant solutions of equations (24.48).

2. Use characteristic point $A$ and point $B$ to determine the overall loop including trajectory and roundtrip geodesic lengths as shown in Fig. 24.2(a) to represent electron.

3. Rotate point $A$ and point $B$ to form circle- $A$ and circle- $B$, the trajectory rotates with point $A$ and point $B$ to form the torus model supporting a set of discrete trajectories on its surface.

4. Add center vertex $C$ representing interactions and additional roundtrip geodesic lengths as shown in Fig.24.2(b), then apply $f$-modification based on solutions of $P S$-equation to change the torus surface with slightly different $a_{2}^{\prime}$ and $b_{2}^{\prime}$ for a set of discrete surfaces.

5. Electron's all physics parameters are derived from characteristic point $A$, point $B$ and associated triangles: $C K M$-triangle, $G W S$-triangle and $S$-triangle.

The same procedure is valid for electron's trefoil type model represented by a triplet with three vertexes in $O(\sqrt{1 / 2})$. Comparing to torus model, the difference is that, there are 6 characteristic points involved instead of only 2 for torus model.

Electron is just an example. The same procedure is valid for other member particles of $O(\sqrt{1 / 2})$. Applying primary basic equations (24.48) to different vertexes representing $u_{r}$, $u_{g}, u_{b}, d_{r}, d_{g}, d_{b}$ or $v_{e}$, solutions are selected according to their unique set of numerical parameters and characteristic points.

In general, there are three type interactions for elementary particles. Does the center vertex represent all three type interactions? As shown in Section 15 and Section 22, all interactions are unified with gravity at GUT scale with characteristic length $L_{G U T}=71$. Inside the $M$-sphere with radius $R_{M}=L_{G U T}=71$, gravity is the only effective interaction. The center vertex represents gravitational interaction. So the answer is: Yes. But it raises another question: How to distinguish three different type interactions? Section 21 already provided the answer: $O(0)$ symmetry corresponding to $U(1)$ group represents electromagnetic interaction; the square with center vertex on surface represents weak interaction; the square with center vertex off surface represents strong interaction. So these interactions are distinguishable. More details will be given later in this section.

A particle ignored interaction is represented by a set of trajectories on a surface of its model corresponding to a set of selected solutions of primary basic equations (24.48). A particle with interactions is represented by a set of trajectories on a set of its model's surfaces corresponding to another set of selected solutions of primary basic equations (24.48). In essence, trajectory is primary and model is secondary.

If symmetry is perfect, the member particles all are massless. Particle's mass is obtained by symmetry broken caused by vertex shifting from its original location to break the symmetry as shown in Section 21.

Symmetry $O(\sqrt{1 / 2})$ is picked as an example. The same procedure is valid for other symmetries. It is conceivable that, primary basic equations (24.48) applying to different vertexes of symmetries $O(\sqrt{1 / 2}), O(\sqrt{2}), O(\sqrt{8})$ and $C(\sqrt{1 / 2}), C(\sqrt{2})$, $C(\sqrt{8})$ cover all three generation elementary particles and interactions. It shows the versatility and richness of primary basic equations (24.48). The details will be revealed when solutions are available.

The primary basic equations (24.48) are based on SQS theory three fundamental Postulations. The first one provides Gaussian probability and Gaussian spheres. The second one correlates prime numbers to elementary particles' numerical parameters. The third one provides vacuons. All seem reasonable. But look closely, there are open issues.

One important open issue is the meaning of intrinsic time $\tau=\gamma^{-1} t$. The introduction of intrinsic time by (24.5) is phenomenological. It is conceivable that, intrinsic time serves as the "clock" for vacuons. It might play a pivotal rule for oscillation along the closed geodesic loop. Further work along this line is needed.

Another important open issue is the origin of selecting rules. As mentioned previously, the set of numerical 
parameters and characteristic points are used as selecting rules to choose the relevant solutions for a particle from primary basic equations (24.48). But if everything is ultimately originated from primary basic equations, so are these rules. For instance, numerical parameters $m, n, p$ are used to determine loop-1, loop-2 lengths and the oscillation pattern along the closed loop. Their value should be determined by primary basic equations (24.48) not the other way around. The same argument is also valid for characteristic points. This is a very important open issue. Since the solution of primary basic equations (24.48) is not available yet, to get some ideas, let's look at three special cases.

\section{Case-1: Mechanism of emitting and absorbing a photon by charged particles.}

For photon with spin $\hbar$, its trajectory $T_{p}$ is a circular loop with circumference length $C_{p}=2 \lambda_{P}=2 L_{P} \Rightarrow 2$ and radius $R=C_{p} / 2 \pi \Rightarrow 1 / \pi$. All lengths are normalized with respect to Planck length (Planck wavelength) $L_{P}=\lambda_{P}$. In Fig.24.3, $V$ is the vertex representing photon and $C$ is the center vertex, $a, b, c$ are characteristic points, $L_{j}$ and $L_{k}$ are geodesic lengths connected to vertex $V$ and vertex $C$, respectively.

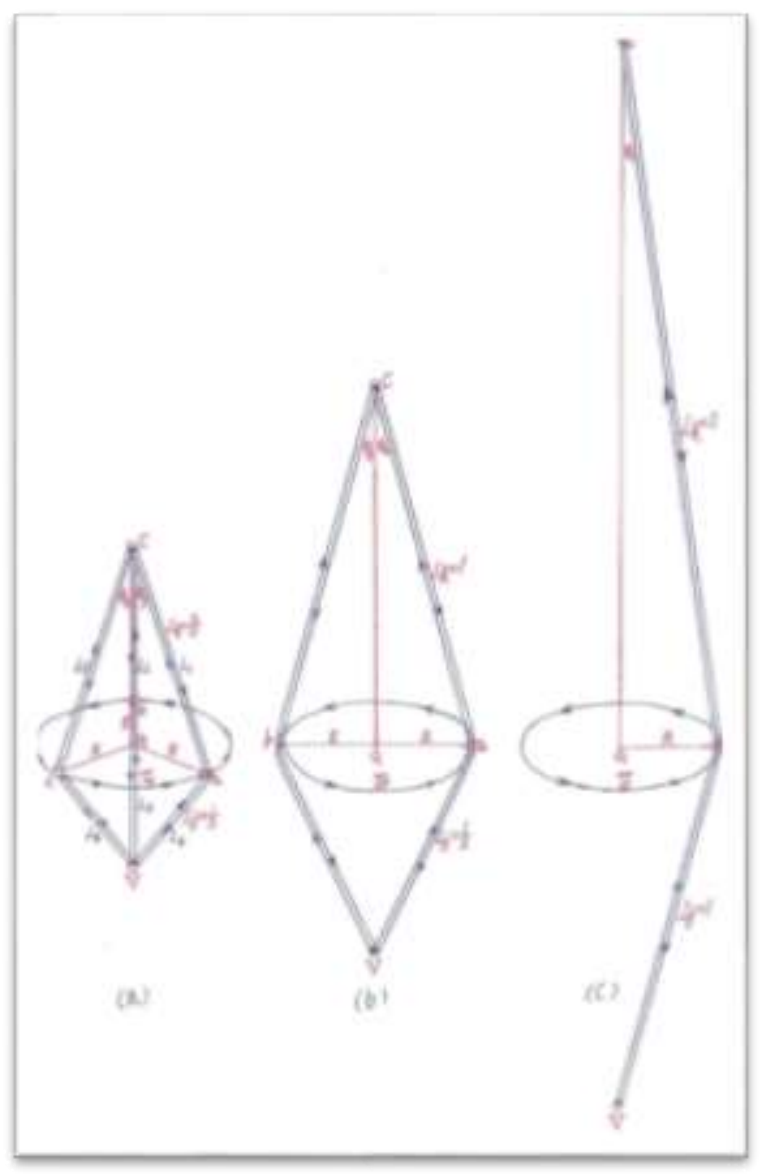

Fig.24.3: Overall closed loops represent photon's emitting and absorbing mechanism: (a) $k=3$, (b) $k=2$, (c) $k=1$.
Photon as a stable particle, its internal movement in the overall loop including trajectory and roundtrip geodesic lengths is exactly synchronized. If not, sooner or later, internal movement will fade by cancelation. The synchronization condition of photon for trajectory and all roundtrip geodesic lengths included is:

$$
\begin{aligned}
& L_{\text {overall, } p}=T_{p}+2 j L_{j}+2 k L_{k}=2+2 j L_{j}+2 k L_{k}=I_{p}, \\
& L_{j}=1 / k, L_{k}=2 L_{j}=2 / k, k=1,2,3 .
\end{aligned}
$$

$L_{\text {overall, }}$ is the length of overall loop including trajectory length $T_{p}$ and roundtrip geodesic lengths. The factor 2 is for counting roundtrip. $I_{p}$ is an integer. $k=3, k=2, k=1$ corresponds to Fig.24.3(a), (b),(c), respectively

As shown in Fig. 24.3, the span angle of line $O_{1} a$ at center vertex $C$ is:

$$
\theta_{i}=\arcsin \left(\frac{R}{L_{k}}\right), k=1,2,3 .
$$

For a first trial, take $k=3, L_{k}=L_{3}=2 / k=2 / 3$ as shown in Fig.24.3(a). for $k=3, L_{3}=2 / k=2 / 3$, formula (24.53) yields:

$$
\theta_{3}=\arcsin \left(\frac{R}{L_{3}}\right)=\arcsin \left(\frac{3}{2 \pi}\right)=28.51995651^{\circ} \text {. }
$$

As listed in Table 8.1 and Table 8.2, electron's original and effective Weinberg angles are $\theta_{W}=28.45987086^{\circ}$ and $\theta^{\prime}{ }_{W}=28.47948454^{\circ}$, respectively. The relative deviations of $\theta_{W}$ and $\theta_{W}^{\prime}$ from $\theta_{3}=28.51995651^{\circ}$ are $2.11 \times 10^{-3}$ and $1.419 \times 10^{-3}$, respectively. Formula (24.53) is based on flat space. In curved space, $L_{3}$ is the length of geodesic, which is slightly different from straight line $a C$. Taking this fact into account, the $\sim 10^{-3}$ relative deviation is justified. It gives credit to identify $\theta_{3}=28.51995651^{\circ}$ as the approximate value of Weinberg angle for electron.

According to (24.53) and shown in Fig. 24.3(a), the total length $L_{\text {overall, } p}$ of overall loop including a full circle of the trajectory $T_{p}$ plus roundtrips along six geodesic lengths connecting three characteristic points $a, b, c$ to 2 vertexes $V$ and $C$ is:

$L_{\text {overall, }}=2+2 j L_{j}+2 k L_{k}=2+2 \times 3 \times(1 / 3)+2 \times 3 \times(2 / 3)=8=I_{p}$.

$L_{\text {overall, } p}=8$ is an integer, which satisfies synchronization condition (24.52).

Encouraged by the first trial positive results, let's take more trials as shown in Fig. 24.3(b) and Fig.24.3(c).

For $k=2, L_{2}=2 / k=1$ :

$$
\theta_{2}=\arcsin \left(\frac{R}{L_{2}}\right)=\arcsin \left(\frac{1}{\pi}\right)=18.56074472^{\circ}, \frac{3}{2} \theta_{2}=27.84111708^{\circ} \text {, }
$$


Z. Y. SHEN

For $k=1, L_{1}=2 / k=2$ :

$\theta_{1}=\arcsin \left(\frac{R}{L_{1}}\right)=\arcsin \left(\frac{1}{2 \pi}\right)=9.15784951^{\circ}, 3 \theta_{1}=27.47354853^{\circ}$.

For $k=2, L_{2}=2 / k=1$, corresponding synchronization conditions are:

$L_{\text {overall, }, p}=2+2 \times(2 \times 1 / 2)+2 \times(2 \times 1)=8=I_{P}$,

For $k=1, L_{1}=2 / k=1$ :

$L_{\text {overall, },}=2+2 \times(1 \times 1)+2 \times(1 \times 2)=8=I_{P}$.

The results of (24.57) also satisfy synchronization condition (24.52).

As shown in Section 11, formula (11.1) is used to calculate Weinberger angles for particles with fractional charges. Besides the factor $\sin \theta_{W}$, another factor $\cos \theta_{W}$ is involved. Therefore, the values of $\frac{3}{2} \theta_{2}<\theta_{3}$ and $3 \theta_{1}<\theta_{3}$ in (24.54) are understandable.

Formula (11.1) is derived from (10.1b) and originated from (8.44) based on normalization $\sqrt{g^{2}+g^{\prime 2}}=1$, which is optional. According to (24.56a) and (24.56b), corresponding results are:

$\frac{\sin \theta_{2} \cos \theta_{2}}{\sin \theta_{3} \cos \theta_{3}}=0.71927448$, instead of $2 / 3=0.66666667,(24.58 \mathrm{a})$

$\frac{\sin \theta_{1} \cos \theta_{1}}{\sin \theta_{3} \cos \theta_{3}}=0.37453391$, instead of $1 / 3=0.33333333$.

This approach not only provides results more accurate than those from formula (11.1) but also serves as a way to determine other parameters for charged fermions without normalization involved. According to (8.43) and (8.44), $\sin \theta_{w}=e / g$, $\cos \theta_{w}=e / g^{\prime}$, and $\sin \theta_{i} \cos \theta_{i}=q_{i} / \sqrt{g^{2}+g^{\prime 2}}$. With the value of electrical charge $q_{i}$ determined from fine structure constant $\alpha$, the value of $g, g^{\prime}$ and $\sqrt{g^{2}+g^{\prime 2}}$ can be derived from $\theta_{i}$ given by (24.58a) or (24.58b). Then the value of all parameters of $G W S$-triangle and $S$-triangle is determined without normalization involved.

Based on the positive results of Weinberg angles, it is conceivable that, the cases for $k=1, k=2$ and $k=3$ represent the mechanism of emitting or absorbing photons by charged particles' with electrical charges $q= \pm e / 3, q= \pm 2 e / 3$ and $q= \pm e$, respectively.

Let's take a closer look at $k=3$ case shown by Fig. 24.3(a), which represents the mechanism of emitting or absorbing photon by electron.

As mentioned previously, the geodesic length $L_{i}$ and related parameters are weighted by the weighed probabilities:

$$
\begin{aligned}
& p_{w}(i)=p_{i} / p=e^{-\pi L_{i}^{2}} / \sum_{j} e^{-\pi L_{j}^{2}}, \\
& \sum_{i} p_{w}(i)=\sum_{i} p_{i} / p=1 .
\end{aligned}
$$

In which $L_{i}$ and $L_{j}$ are normalized to the Planck length $L_{P}$. The weighted probability concept is also valid for the mechanism of emitting or absorbing photons, which represents these processes' stochastic nature. Taking stochastic nature into consideration, the synchronization condition (24.52) is just a "snap shot" cut from the whole "movie". It is a specific case for all roundtrip geodesic lengths are included in the overall loop. In reality, the six roundtrip geodesic lengths included or not included in the overall loop are determined by probabilities. In essence, the scenario is dynamic and stochastic in nature. Taking the stochastic effect into consideration, the synchronization condition of (24.52) is generalized as:

$$
\begin{aligned}
& L_{\text {overall }, p}=\left[T_{p}+\sum_{i} 2 L_{i}\right] \text { via } p_{w}(i)=I_{p}, \\
& p_{w}(i)=p_{i} / p=e^{-\pi L_{i}^{2}} / \sum_{j} e^{-\pi L_{j}^{2}} .
\end{aligned}
$$

In (24.59), the six geodesic lengths $L_{i},(i=1,2 \cdots 6)$ are renamed as shown in Fig. 24.3(a). In which, three $L_{i},(i=1,2,3)$ attach to center vertex $C$ and other three $L_{i},(i=4,5,6)$ attach to vertex $V$. The subscript "via $p_{w}(i)$ " in $(25.59 \mathrm{a})$ indicates that, synchronization condition is stochastic with probability $p_{w}(i)$ involved. $p_{w}(i)$ is the probability for roundtrip geodesic lengths $2 L_{i}$ included in overall loop, while $\left[1-p_{w}(i)\right]$ is the probability for $2 L_{i}$ not included in overall loop.

As shown in Fig. 24.3(a), there are 6 roundtrip geodesic lengths $2 L_{i}$ involved. For each one of the 6 roundtrip geodesic lengths included or not included in the overall loop, the total number of overall loops' different patterns is $N_{p}=2^{6}=64$.

According to (24.59b), $p_{w}(i)$ is determined by its geodesic length $L_{i}$ in the nominator and all geodesic lengths in the denominator.

For $L_{i},(i=1,2,3)$ attached to vertex $C$ :

$$
p_{i}=e^{-\pi l_{i}^{2}}=e^{-\pi\left(\frac{2}{3}\right)^{2}}=0.24752012,
$$

For $L_{i},(i=4,5,6)$ attached to vertex $V$ :

$$
p_{i}=e^{-\pi L_{i}^{2}}=e^{-\pi\left(\frac{1}{3}\right)^{2}}=0.70534668 .
$$

Summation of all $L_{i},(i=1,2 \cdots 6)$ :

$$
\sum_{j=1}^{6} e^{-\pi L_{i}^{2}}=3\left[e^{-\pi\left(\frac{2}{3}\right)^{2}}+e^{-\pi\left(\frac{1}{3}\right)^{2}}\right]=2.85860041 .
$$

According to (25.10) and (25.60), the probabilities for the two types of roundtrip geodesic lengths included in the overall loop are given as follows:

$L_{i},(i=1,2,3)$ included in $L_{\text {overall, }, p}$ :

$$
p_{w}(1,2,3)=e^{-\pi\left(\frac{2}{3}\right)^{2}} / \sum_{j=1}^{6} e^{-\pi L_{j}^{2}}=0.08658787,
$$


$L_{i},(i=4,5,6)$ included in $L_{\text {overall }, p}$ :

$p_{w}(4,5,6)=e^{-\pi\left(\frac{1}{3}\right)^{2}} / \sum_{j=1}^{6} e^{-\pi L_{j}^{2}}=0.246745463$,

Check for unitarity:

$\sum_{j=1}^{6} p_{w}(i)=3 \times[0.08658787+0.246745463]=1$.

To get the idea, let's look at some typical cases.

1. For the case of all 6 roundtrip geodesics $L_{i},(i=1,2 \cdots 6)$ included in the overall loop, the occurrence probability is: $p_{\text {case- } 1}=\left[p_{w}(1,2,3)\right]^{3}\left[p_{w}(4,5,6)\right]^{3}=9.75256159 \times 10^{-6} . \quad(24.62 \mathrm{a})$ For the case, synchronization condition (25.59) is satisfied as:

$$
L_{\text {oveall, }}=\left[C_{p}+\sum 2 L_{i}\right]_{\text {ria }} p_{p_{x}(i)}=2+3 \times\left(2 \times \frac{2}{3}\right)+3 \times\left(2 \times \frac{1}{3}\right)=8=I_{p} .
$$

2. For the case of all 6 roundtrip geodesics $L_{i},(i=1,2 \cdots 6)$ not included in the overall loop, the occurrence probability is: $p_{\text {case }-2}=\left[1-p_{w}(1,2,3)\right]^{3}\left[1-p_{w}(4,5,6)\right]^{3}=0.32570587 . \quad$ (24.63a)

For the case, synchronization condition (24.59) is satisfied: $L_{\text {overall, } 2}=\left[C_{p}+\sum_{i} 2 L_{i}\right]_{\text {via } P_{w}(i)}=2+0 \times\left(2 \times \frac{2}{3}\right)+0 \times\left(2 \times \frac{1}{3}\right)=2=I_{p}$.

3. For the case of only 1 roundtrip geodesic $L_{i},(i=1,2,3)$ connected to $C$ and only 1 roundtrip geodesic $L_{i},(i=4,5,6)$ connected to $V$ included in the overall loop, the occurrence probability is:

$p_{\text {case }-3}=p_{w}(1,2,3)\left[1-p_{w}(1,2,3)\right]^{2} p_{w}(4,5,6)\left[1-p_{w}(4,5,6)\right]^{2}=0.01011401$.

For the case, synchronization condition (24.59) is satisfied:

$L_{\text {overall, }}=\left[C_{p}+\sum_{i} 2 L_{i}\right]_{\text {via } p_{w}(i)}=2+1 \times\left(2 \times \frac{2}{3}\right)+1 \times\left(2 \times \frac{1}{3}\right)=4=I_{p}$.

4. For the case of only 2 roundtrip geodesic $L_{i},(i=1,2,3)$

connected to $C$ and only 2 roundtrip geodesic $L_{i},(i=4,5,6)$

connected to $V$ included in the overall loop, the occurrence probability is:

$p_{\text {case- } 4}=\left[p_{w}(1,2,3)\right]^{2}\left[1-p_{w}(1,2,3)\right]\left[p_{w}(4,5,6)\right]^{2}\left[1-p_{w}(4,5,6)\right]$.

$=3.14066054 \times 10^{-4}$

For the case, synchronization condition (24.59) is satisfied:

$L_{\text {overall, }, 4}=\left[C_{p}+\sum_{i} 2 L_{i}\right]_{\text {via } p_{w}(i)}=2+2 \times\left(2 \times \frac{2}{3}\right)+2 \times\left(2 \times \frac{1}{3}\right)=6=I_{p} \cdot$

5. For the case of only 1 roundtrip geodesic $L_{i},(i=1,2,3)$

connected to $C$ and no roundtrip geodesic $L_{i},(i=4,5,6)$

connected to $V$ included in the overall loop, the occurrence probability is:

$p_{\text {case }-5}=p_{w}(1,2,3)\left[1-p_{w}(1,2,3)\right]^{2}\left[1-p_{w}(4,5,6)\right]^{3}=0.03087563$.

For the case, synchronization condition (24.59) is not satisfied:

$L_{\text {overall, }}=\left[C_{p}+\sum_{i} 2 L_{i}\right]_{\text {via } p_{w}(i)}=2+1 \times\left(2 \times \frac{2}{3}\right)+0 \times\left(2 \times \frac{1}{3}\right)=3 \frac{1}{3} \neq I_{p}$.

6. For the case of only 1 roundtrip geodesic $L_{i},(i=1,23)$

connected to $C$ and only 2 roundtrip geodesic $L_{i},(i=1,23)$

connected to $V$ included in the overall loop, the occurrence probability is:

$$
p_{\text {cas }-6}=p_{w}(1,2,3)\left[1-p_{w}(1,2,3)\right]^{2} p_{w}(4,5,6)^{2}\left[1-p_{w}(4,5,6)\right]=3.31307079 \times 10^{-3} .
$$

For the case, synchronization condition (25.59) is not satisfied as:

$$
L_{\text {overall, },}=\left[C_{p}+\sum_{i} 2 L_{i}\right]_{\text {via } p_{w}(i)}=2+1 \times\left(2 \times \frac{2}{3}\right)+2 \times\left(2 \times \frac{1}{3}\right)=4 \frac{2}{3} \neq I_{p} .
$$

The synchronization condition (24.59) must be satisfied, for cases like No. 5 and No. 6, the way to do so is to change the values of $C_{p}$ and $L_{i}$ by adjusting gauge tensors $g_{a b}$ for reinstalling synchronization condition (24.59). It is legitimate according to background independence principle. Space structure is not a priori determined, it emerges naturally with gauge tensors $g_{a b}$ determined by primary basic equations (25.48). In this case, synchronization condition is a necessary condition for primary basic equations (24.48) to have stable solutions. After the adjustment of tensors $g_{a b}$ completed, the probabilities change accordingly.

Not only cases like No. 5 and No. 6 request adjusting gauge tensors $g_{a b}$, in fact all $N_{p}=2^{6}=64$ cases request adjusting gauge tensors $g_{a b}$. For instance, case No. 3 and case No.4 both satisfy synchronization condition (24.59), but these two overall loops have different patterns with different $g_{a b}$ distributions in space. In general, for all $N_{p}=2^{6}=64$ overall loops' different patterns, each one has its unique $g_{a b}$ distribution in space. The total number of different $g_{a b}$ distributions is $N_{p}=2^{6}=64$.

It is very important to point out that, the diagrams shown in Fig. 24.3, the synchronization conditions of (24.59) and all related formulas are only for the mechanism of photon's emitting and absorbing not for free flying photon. For free flying photon, the scenario is very simple. A free flying photon is represented by a circular trajectory with circumstance of $2 L_{P}$ with nothing attached. It is conceivable that, as shown in case No. 2, when all 6 roundtrip geodesics $L_{i},(i=1,2 \cdots 6)$ are disconnected, the overall loop $L_{\text {overall, } p}=2$ with nothing attached becomes the trajectory of a photon free to fly. The solution of primary basic equations (24.48) representing free flying photon should be very simple.

In this case, the overall loop concept and the synchronization concept are verified for photon's emitting and absorbing, which provide some insights for photon as well as for other particles.

\section{Case-2: Electron}

As shown in Section 12, electron is represented by trefoil trajectory with loop-1 and loop- 2 combined movements on genus-3 trefoil type model. The situation is more complicated than photon. Details have to wait until the solutions of primary basic equations (24.48) available. But it is possible to take a bird-eyes view to get some ideas. 
Z. Y. SHEN

For electron with spin $\hbar / 2$, its loop- 1 is a circle with circumferential length $L_{1}=m=1$. Its loop- 2 is a closed loop with circumferential length $L_{2}=n=1 / 2$.

For electron as a stable particle, the length of overall loop including trajectory and the roundtrip lengths of geodesics involved is determined by synchronization condition:

$$
\begin{aligned}
& L_{\text {overall }}=\left[T_{e}+2 \sum_{i} L_{i}\right]_{\text {via } p_{w}(i)}=I_{e}, \\
& p_{w}(i)=\frac{p_{i}}{\sum_{i} p_{i}}=\frac{e^{-t t_{i}^{2}}}{\sum_{i} e^{-\pi l_{i}^{2}}}
\end{aligned}
$$

In which, $L_{\text {overall,e }}$ is overall loop length, $T_{e}$ is electron's trajectory length, $2 L_{i}$ is roundtrip geodesic length, $I_{e}$ is an integer. When multiple geodesic lengths are involved, weighted probabilities $p_{W}(i)=p_{i} / p$ are attached to parameters in the emerging part (24.9) of primary basic equations (24.48). In (24.68), $p_{w}(i)$ is the probability for the roundtrip geodesic length $2 L_{i}$ included in $L_{\text {overall,e }}$, while $\left[1-p_{w}(i)\right]$ is the probability for the roundtrip geodesic length $2 L_{i}$ not included in $L_{\text {overall,e }}$. The roundtrip length $2 L_{i}$ of each geodesic included or not included in the overall loop changes spontaneously by chances, the overall loop length $L_{\text {overall,e }}$ changes accordingly. It shows that, electron's internal movements are dynamic and stochastic in nature.

Electron trefoil model with 3 branches has $2 \times 3=6$ characteristic points. Electron is represented by 3 vertexes of $O(\sqrt{1 / 2})$. The total number of roundtrip geodesics $2 L_{i}$ connecting 6 characteristic points to 3 vertexes is $M_{1}=6 \times 3=18$. The number of possible overall loops is determined by each geodesic roundtrip $2 L_{i}$ included or not included in the overall loop as:

$$
N_{1}=2^{M_{1}}=2^{18}=2.621 \times 10^{5} .
$$

$N_{1}=2.621 \times 10^{5}$ is number of solutions for primary basic equation (24.48) representing electron. As mentioned in previous Section, jumping trajectories on electron model's same surface corresponds to emit and absorb pseudo-photons. $N_{1}=2.621 \times 10^{5}$ is the number of trajectories on electron's trefoil type model's same surface.

This is the case for an electron ignored interactions except interacting with pseudo-photons.

Electron is involved in electromagnetic interaction and weak interaction. Taking electromagnetic interaction into consideration, the center vertex $C$ and the vertex $V$ representing photon with its overall loop including relevant roundtrip geodesic lengths as shown in Fig. 25.3(a) is added to the diagram representing electron trefoil model.

For the electron part, total number of geodesics involved including those connected to center vertex $C$ is $M^{\prime}{ }_{e}=6 \times(3+1)=24$. The number of possible overall loops determined by each roundtrip geodesic included or not included is:

$$
N^{\prime}=2^{M_{e}^{\prime}}=2^{24}=1.678 \times 10^{7} .
$$

For the photon part, according to Fig. 24.3(a) in Case-1, total number of geodesics involved is $M_{p}=6$. The number of overall loops determined by each roundtrip geodesic included or not included is:

$$
N_{p}=2^{M_{p}}=2^{6}=64 .
$$

As shown in Case-1 for photon, the number of different gauge tensors $g_{a b}$ distributions is $N_{p}=2^{6}=64$. Since electron and photon share the same space, as photon adjusts gauge tensors $g_{a b}$ around its surrounding, which inevitably changes the gauge tensors $g_{a b}$ around electron's surrounding. Electron trefoil type model's $M^{\prime}{ }_{e}=6 \times(3+1)=24$ geodesic lengths $L_{i}$ change accordingly. Taking this effect into account, $N^{\prime}$ of (24.70) is changed to $N^{\prime \prime}$ as:

$$
N^{\prime \prime}=N^{\prime} \times N_{p}=2^{24} \times 2^{6}=2^{30}=1.074 \times 10^{9} .
$$

It is important to notice that, the contribution of $N_{p}=2^{6}=64$ in $N^{\prime \prime}$ is not to combine photon's overall loop to electron's overall loop. It is counting the effects of photon's $g_{a b}$ adjustments upon electron. Because of $N_{p}=2^{6}=64$ different $g_{a b}$ distributions generated by photon's overall loop different patterns, electron's each original overall loop becomes 64 different loops caused by the values of geodesics $L_{i}$ changed according to 64 different $g_{a b}$ distributions. The same is true for other particle involved in interactions.

Taking weak interaction into consideration, as shown in Section 14, electron $e^{-}$paired with electron anti-neutrino $\bar{v}_{e}$ via gauge boson $Z$ for the regular type weak interactions. As shown in Fig. 17.2, $\bar{v}_{e}$ has trefoil type model with 3 branches similar to electron's trefoil type model. Each branch has 2 characteristic points, total number of geodesic lengths is $M_{\bar{v} e}=(3 \times 2) \times(3+1)=24$ for the $\bar{v}_{e}$ part. Gauge boson $Z=Y_{1} \oplus \bar{Y}_{2}$ is made of two companion fermion states, which has ginus-2 type torus model with two branches. Each branch has 2 characteristic points, total number of geodesic lengths for the $Z$ part is $M_{Z}=(2 \times 2) \times(2+1)=12$. The numbers of possible overall loops are $N_{\bar{v} e}=2^{M_{\overline{v e}}}=2^{24}$ and $N_{Z}=2^{M_{Z}}=2^{12}$ for the $\bar{v}_{e}$ part and the $Z$ part, respectively. Taking these effects into account, $N^{\prime \prime}$ of (24.72) is changed to $N$ for counting electromagnetic and weak interactions as: $N=N^{\prime \prime} \times N_{\overline{v e}} \times N_{Z}=2^{30} \times 2^{24} \times 2^{12}=2^{66}=7.379 \times 10^{19}$. $N=7.379 \times 10^{19}$ is the number of primary basic equations' solutions for electron with interactions. According to (24.69) and (24.73), on top of a set of $N_{1}=2^{18}$ solutions, primary 
basic equations have another set of $N_{2}=N / N_{1}$ solutions for electron:

$$
N_{2}=N / N_{1}=2^{66} / 2^{18}=2^{48}=2.815 \times 10^{14} .
$$

Physics interpretation is that, electron's trefoil type model has $N_{2}=N / N_{1}=2.815 \times 10^{14}$ different surfaces with slightly different $a_{2}^{\prime}$ and $b_{2}^{\prime}$ caused by $f$-modifications. According to (8.41b), electron's torus model is estimated having $N==1.509 \times 10^{14}$ solutions of $P S$-equation, which is also valid for the trefoil model. The number $N_{2}=2.815 \times 10^{14}$ is in the same order of $N==1.509 \times 10^{14}$ given by $(8.41 \mathrm{~b})$, The orders of magnitude agreement serves as another theoretical verification for the $P S$-equation as well as for the validity of analysis in this case for primary basic equations (24.48) representing electron in terms of numbers of solutions.

An electron has $N=7.379 \times 10^{19}$ solutions of primary basic equations (24.48). Is this scenario too complicated? The answer is: Not at all. For the $N=7.379 \times 10^{19}$ solutions, in which $N_{1}=2.621 \times 10^{5}$ solutions represent a set of the same type trajectories rotating around $A$-circle and $B$-circle to form trefoil type model with the same parameters represented by the same $C K M$-triangle, $G W S$-triangle and $S$-triangle. The $N_{1}=2.621 \times 10^{5}$ solutions have the same form with a parameter representing rotation angle. The $N_{2}=2.815 \times 10^{14}$ solutions represent a set of trefoil type surfaces caused by $f$ modification. The $N_{2}=2.815 \times 10^{14}$ solutions have the same form with slightly different values of parameters for $a_{2}^{\prime}$ and $b_{2}^{\prime}$. This scenario is consistent with electron's two sets of discrete trefoil trajectories and jumping trajectories. Primary basic equations (24.48) are stochastic differential equations representing stochastic processes. Corresponding to members of statistic ensemble, multiple solutions are natural and fully expected. The situation is similar to quantum mechanics. The mixed state is a superposition of a set of enormous number of wave functions as solutions of Schrodinger equation; each one has probability for its occurrence. The mixed state of quantum mechanics is commonly accepted, so should be the multi-solution of equations (24.48).

The superposition of $N=7.379 \times 10^{19}$ solutions represents electron in stochastic sense, which corresponds to the "electron clouds" concept in Section 8.

The vacuons moving in trajectory with superluminal speed $\hat{v}=N_{e} v \sim 1.5 \times 10^{23} c$ have the capability to go through all $N=7.379 \times 10^{19}$ overall loops many times within Planck time scale. It indicates that $N=7.379 \times 10^{19}$ overall loops are in the ballpark. In fact, vacuons' speed must be superluminal, otherwise there is no sufficient time for vacuons to go through all these possible paths.

Each geodesics included or not included in the overall loop is a binary discrete event, which supports the discrete trajectory concept.

Gauge tensors along overall loop adjust automatically to satisfy synchronization condition (24.68) under all circumstance. It shows that, the space-time continuum determined by primary basic equations (24.48) is dynamic and stochastic in nature. In essence, microscopic space-time structure is not a priori determined. Background independent is a natural request by primary basic equations (24.48) at Planck scale.

The center vertex $C$ serves as the junction to connect all particles involved in interactions. It confirms that, center vertex $C$ represents all interactions as mentioned previously. Moreover, as shown in this case, center vertex $C$ serves another function to distinguish different particles involved in interactions. If there is no center vertex $C$ serving as partition, the overall loops representing photon, $\bar{v}_{e}, Z$ and electron are directly connected to each other, the distinctions of these particles would be blurred. The center vertex $C$ not only serves as junction but also as partition.

In this case, the overall loop concept and the synchronization concept for particle's stability are verified for electrons in principle. Hopefully, when solutions of primary basic equations (24.48) for electron are available, these concepts can be confirmed.

\section{Case-3: Unstable Particles}

The difference between unstable and stable particles is synchronization. As shown in Case-1 and Case-2, photon and electron as stable particles, synchronization conditions are exact with phase difference equal to zero precisely. Otherwise, any nonzero phase difference no matter how tiny, as cyclic movement goes on, sooner or later it will accumulate to $180^{\circ}$ phase difference for cancelation corresponding to particle's decay.

For unstable particles, the quasi-synchronization condition is:

$$
\begin{aligned}
& L_{\text {overall }}=\left[T_{u}+2 \sum_{i} L_{i}\right]_{\text {via } p_{w}(i)}=I_{u}+k \delta \\
& p_{w}(i)=\frac{p_{i}}{\sum_{i} p_{i}}=\frac{e^{-\pi L_{i}^{2}}}{\sum_{i} e^{-\pi L_{i}^{2}}}
\end{aligned}
$$

In which, $k$ is the number of turns around the overall loop, $I_{u}$ is integer. When $k \delta=0.5$ corresponding to $180^{\circ}$ phase difference, the particle has a chance to decay. Depending on each $2 L_{i}$ included or not included in $L_{\text {toverall,u }}$, the values of $L_{\text {overall,u}}$ are stochastic in nature, so are decay times. The scenario is consistent with the stochastic multiple decaytimes for muon as an example described in Section 7.

It is important to point out that, the synchronization condition and quasi-synchronization condition are not externally imposed; they belong to primary basic equation (24.48) serving as the condition of stable solutions for stable particle or quasi-stable solutions for unstable particle. The same is true for numerical parameters and characteristic points, they are not externally imposed, instead, they are requested by primary basic equations (25.48) to have meaningful solutions. In essence, all selecting rules 
Z. Y. SHEN

ultimately are originated from the set of primary basic equations (24.48). So it does tell the whole story.

In principle, some information provided in the previous twenty three sections before this section based on three fundamental postulations can be obtained from corresponding solutions of primary basic equations (24.48). Even though, these efforts are not wasted. They serve as very helpful and informative rehearsals. Imaging without these rehearsals, putting the set of primary basic equations (24.48) as the real show on the stage, one would be very hard to recognize what it is.

The set of primary basic equations (24.48) tells the whole story. What is the whole story? From SQS theory perspective, it includes three parts.

Bottom Part: Information of all elementary particles and interactions include trajectories, models, characteristic points, triangles and related parameters.

Top Part: Information of universe, multiverse and anything on top of them include space dimensions, cosmic periods and cosmic evolution.

In-Between Part: Information of things in between the bottom part and the top part.

The bottom part is governed by primary basic equations (24.48). The top part is governed by basic equations (24.20) and (24.42), which are originated from basic equations via average and converting processes. The in-between part needs some explanation. If the set of primary basic equations (24.48) does unify general relativity with quantum theory including standard model and quantum mechanics, it should provide information up to the molecules level. Further up to the upper levels, uncertainty plays a pivotal rule evidenced by the existence of freewill. The set of primary basic equations (24.48) is based on probability serving as the ultimate origin of uncertainty. It lays the foundation for things in upper levels. But, of cause, it cannot provide deterministic information for things in upper levels. In fact, no theory can, because it violates freewill.

In principle, the set of primary basic equations (24.48) cover all elementary particles and interactions in microscopic world and things on higher levels. In essence, it tells the whole story.

The above statement is the final goal of SQS theory. We just get started. There is a long interesting journey to go. With the joint efforts of so many talented physicists and mathematicians, sooner or later we will get there.

Conclusion 24.2: Equations (24.48) serve as the primary basic equations for SQS theory. The solutions of equations (24.48) under different circumstances provide information of all elementary particles and interactions as well as things at higher levels. Basic equations (24.20) for gravity and basic equations (24.42) for electromagnetic forces are macroscopic versions for two long range forces.

Summary: The goal of this section is to establish SQS theory basic equations based on Einstein original equations (24.1b) for vacuum with Gaussian probability assigned to gauge tensors. What turn out are basic equations which cover things from universe down to elementary particles' internal movements. The key is to introduce probability. It makes general relativity automatically quantized. Einstein original equations for vacuum are the right ones to begin with. The only thing lacking was probability. Unfortunately, Einstein did not like it with his famous saying: "God does not play dice." But if God wants to create the world, he must play dice.

Put it casually: $M r$. SQS borrowed an equation from $D r$. Einstein and added a rolling dice to develop the basic equation. It paid back with interests: marbles for straws and everything in the universe.

In this section, the foundation and framework of SQS theory basic equations are established based on Einstein equations for vacuum with Gaussian probability assigned to gauge tensors. Since the solutions of basic equations are not available yet, more works along this line are needed. To reach the goal wouldn't be easy, but it is definitely worth the effort.

\section{Section 25: Discussions}

This section provides an overview of SQS theory with emphasis on open issues.

Originally, SQS theory was intended to be a theory of space. It turns out to cover many aspects of particle physics and cosmology.

SQS theory as a mathematic theory with physics significances includes four parts.

Part-1: The Foundation. It includes three fundamental postulations: (1) Gaussian Probability Postulation; (2) Prime Number Postulation; (3) Vacuon Postulation. These three fundamental postulations serve as the first principle for SQS theory.

Part-2: The Framework. Based on the foundation, SQS theory built a framework including a series of definitions, additional postulations, theorems, lemmas, hypothesis, rules, equations, formulas and conclusions.

Part-3: The Results. Based on Part-1 and Part-2, SQS theory produced many results in terms of space structure, symmetries, and elementary particles with their trajectories, models, parameters, interactions as well as cosmic structure and evolution. SQS theory provided twenty five predictions for experimental verifications.

Part-4: SQS Theory Primary Basic Equations. Based on Einstein Equations for vacuum of general relativity and introduction of probability to redefine gauge tensors, SQS theory established the primary basic equations.

Fig. 25.1 shows the SQS Theory Family Tree. Three fundamental postulations shown by three triangles serve as the roots of the Tree shown on the bottom. SQS theory primary basic equations (24.48) along with macroscopic basic equations (24.20) and (24.42) shown in red hexagons on the bottom serve as the foundation. The three basic 


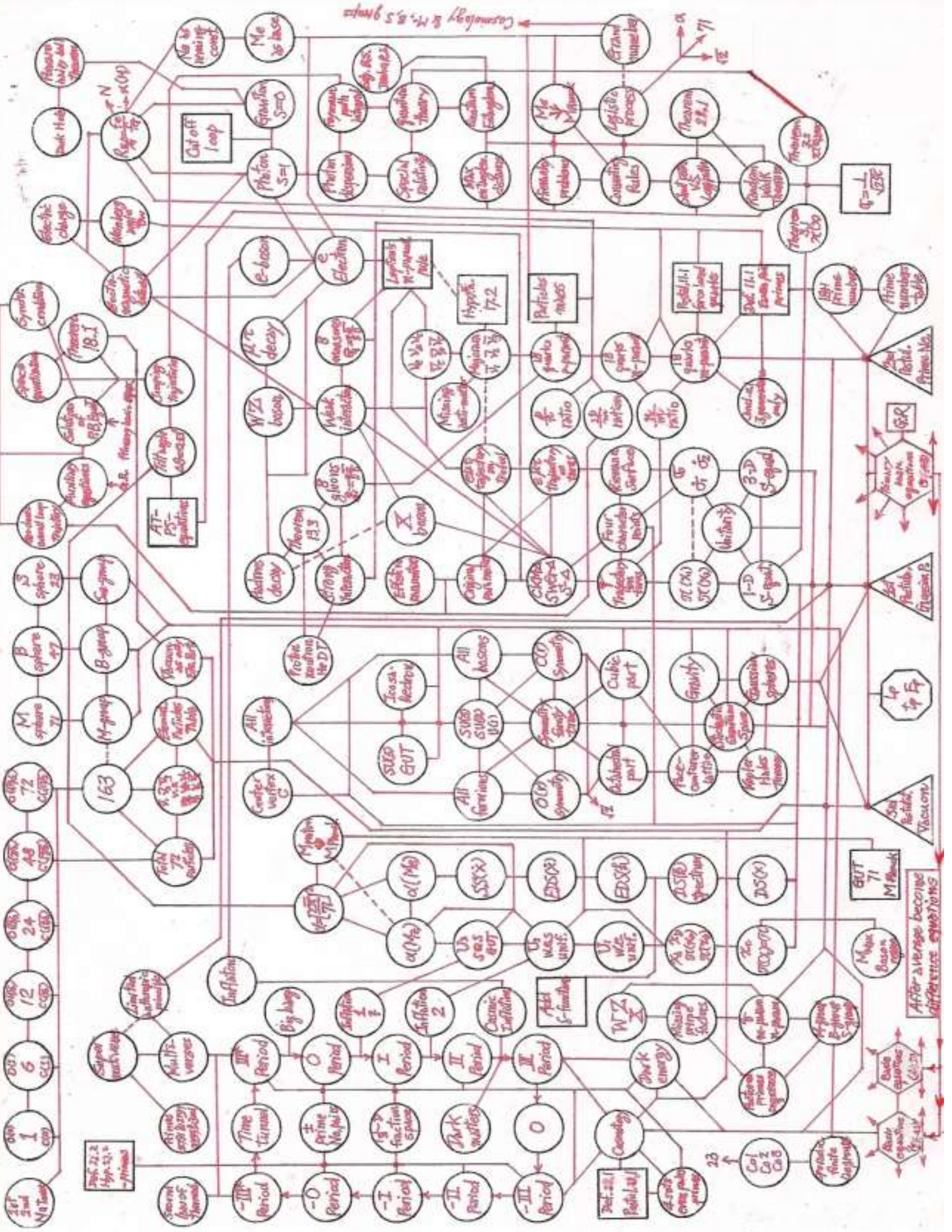

Fig.25.1: SQS theory family tree. 


\section{Z. Y. SHEN}

constants $L_{P}, t_{P}, E_{P}$ in an octagon serving as the vocabulary to translate the mathematic results into physics are placed on the bottom alongside the three roots and basic equations. The major additional postulations, hypothesizes and rules are shown by 13 squares. The results and some intermediate steps serving as elements of the Family Tree are shown by 156 circles. The solid line indicates that, the linked elements are based on logic deduction, mathematic derivation or factual correlation. The dashed line indicates that, the linked elements are somehow correlated.

As shown in the Family Tree, $S$-equations play important roles for SQS theory. The 1-dimensional $S$-equation, $S(x)-1=0$, is the most important one serving as the foundation. Other $S$-equations are derived or extended from this one. Its solutions $\pi\left(x_{1}\right), \pi\left(x_{2}\right)$ determine the location of characteristic points on particles' model, which provide physics properties for the particle and its interactions with others represented by three triangles, $G W S$-triangle, $C K M$ triangle and $S$-triangle. More works along this line are needed to complete the task. What is the function of $S$-triangle? What does the triangle $O_{1} B_{1} D$ between $C K M$-triangle and $S$ -

triangle mean? These are open issues.

The 1-dimensioanal $S$-equation is a growing point. Many branches of Family Tree grow from it. The $D S(x)$-equation is defined as an extension of the $S$-equation and Fourier transformed into $D S_{k}(k)$-equation. The $D S_{k}(k)$-equation on complex $k$-plane serves as the spectrum of particles. Fermions and bosons correspond to local minimums and local maximums of $\left|D S_{k}(k)\right|$, respectively. The $k$-plane spectrum provides information of mass and a series of possible decay times for particles. But it leaves two open issues: The mass value is not uniquely determined; The correlation between decay times and lifetime is not clearly known.

The $D S_{k}(k)$-equation is extended by adding two sets of delta functions to define the $\operatorname{EDS}_{k}(k)$-equation, which is Fourier transformed back to complex $\dot{x}$-plane as the $\operatorname{EDS}_{\dot{x}}(\dot{x})$-equation. Comparing it to the $D S(x)$-equation, the additional two summation terms are identified to represent interactions. In Section 15, these two terms are used for calculating suppression factors to determine the characteristic mass values $M_{e w}, M_{e w s}$ for two unifications based on $\pi\left(x_{a}\right), \pi\left(x_{b}\right)$ and proton mass $M_{\text {proton }}$. It was a risk undertaking, because (15.2) is a borrowed formula not from SQS theory first principle and proton is a composite particle. Fortunately, it worked out well to provide the mass scales for two unifications. Moreover, formula (15.15) was found to link $M_{\text {ews }}$ and $M_{G U T}$ with $\alpha\left(M_{Z}\right)$. It not only provider legitimacy to the borrowed formula (15.2) but also reveals the correlation between $M_{\text {proton }}$ and $M_{\text {Planck }}$. It eliminates $M_{\text {proton }}$ as a physics input of SQS theory. In addition, it serves as an independent confirmation for the interaction terms in $E D S_{\dot{x}}(\dot{x})$-equation.

The $D S(x)$-equation has a solution at $x_{1}=0.125$ on the $\mathrm{x}$-axis corresponding to electron, while $\operatorname{EDS}_{\dot{x}}(\dot{x})$-equation no longer has the solution at $x_{1}=0.125$. It is expected, because the adding of two interaction terms altered its function. To define $S S_{\dot{x}}(\dot{x})$ equation based on $\operatorname{EDS}_{\dot{x}}(\dot{x})$-equation is an attempt to restore that function. A solution of the $S S_{\dot{x}}(\dot{x})$-equation is found at a new location $\dot{x}=x_{1}^{\prime} e^{i \theta_{W}}, x_{1}^{\prime}=0.12408112557821315$ and $\theta_{W}=28.4598708641138^{\circ}$. The fine structure constant $\alpha\left(M_{e}\right)=1 / 137.03599908345$ is derived from the value of $x_{1}^{\prime}$. The importance is the form of solution shows that, electron is a changed particle with Weinberg angle $\theta_{W}$ as a phase angle and the contribution of electromagnetic energy to its mass by (9.8) related to $\alpha\left(M_{e}\right)$ of (9.9). The theory is consistent and the extension of $E D S_{k}(k), E D S_{\dot{x}}(\dot{x})$ and $S S_{\dot{x}}(\dot{x})$ from $D S_{k}(k)$ are justified.

The $\Delta S$-function, $\Delta S(x)=S(x)-1$, did an excellent job to find the special point $x_{c}=0.249987156273026$ and the slightly broken anti-symmetry of $\Delta S(x)$ in the region $[0,0.5]$ from its center $x=0.25$. It is a very important finding with many impacts. Point $x_{c}$ sets the boundary of the boson states region $\left[x_{c}, x_{d}=0.5-x_{c}\right]$. Fermions' $x_{1}$ and $x_{2}$ falling into this region must appear in pair with anti-particle as a boson state, which is verified by top quarks' pair production and $e$-boson serving as the inflaton. Point $x_{c}$ also defined two other special points $x_{a}$ and $x_{b}$, which are used to calculate the values of $\pi\left(x_{a}\right), \pi\left(x_{b}\right)$ from the $S$-equation to determine the mass value for two unifications as mention previously.

The deviation of $x_{c}=0.249987156273026$ from $x=0.25$ is only $\sim 1.28 \times 10^{-5}$, but its impacts are huge. It shows the sensitivity, accuracy and power of $\Delta S$-function and its origin, the $S$-equation based on SQS theory first principle.

For the 3-dimensional Gaussian probability, its standard deviation's three values show $\vartheta= \pm 120^{\circ}$ symmetry on the complex plane and set the three branch points on the Riemann surface to define the two cuts on two layers. The former provides the first clue for $S U$ (3) group and the latter leads to the torus model and the four characteristic points on its surface.

An important trunk of the Family Tree is rooted from Gaussian Probability Postulation. The Random Walk Theorem plays a critical role for many important issues including converting rules, origin of hierarchy problems, photon dispersion, the route to GUT etc. In the converting factor transition region, logistic recurrent process and random walk process both are in action. The former has a variable binary probability, while the latter has six probabilities corresponding to \pm directions along three dimensions for each step. A strict relation of these two processes is needed to reveal 
more physics insights. It is an open issu.

The Prime Number Postulation based on even-pairing rule is the key to correlate prime numbers with elementary particles. It provides the mathematic foundation to recognize quarks with different colors as different particles and to identify leptons trefoil type model with three branches. It serves as a backbone of the Elementary Particle Table. In return, The Elementary Particles Table did its job including prediction of the boson $X$ along with other 12 bosons, $g, G_{i}(i=1,2 \cdots 8), U_{1}, U_{2}, U_{3}$. Its most important contribution is to determine 72 as total number of elementary particles at this level. The number 72 is supported by the Number Tower especially the magic number 163 on its top. In fact, there is a Second Number Tower shown in Fig. 25.2.

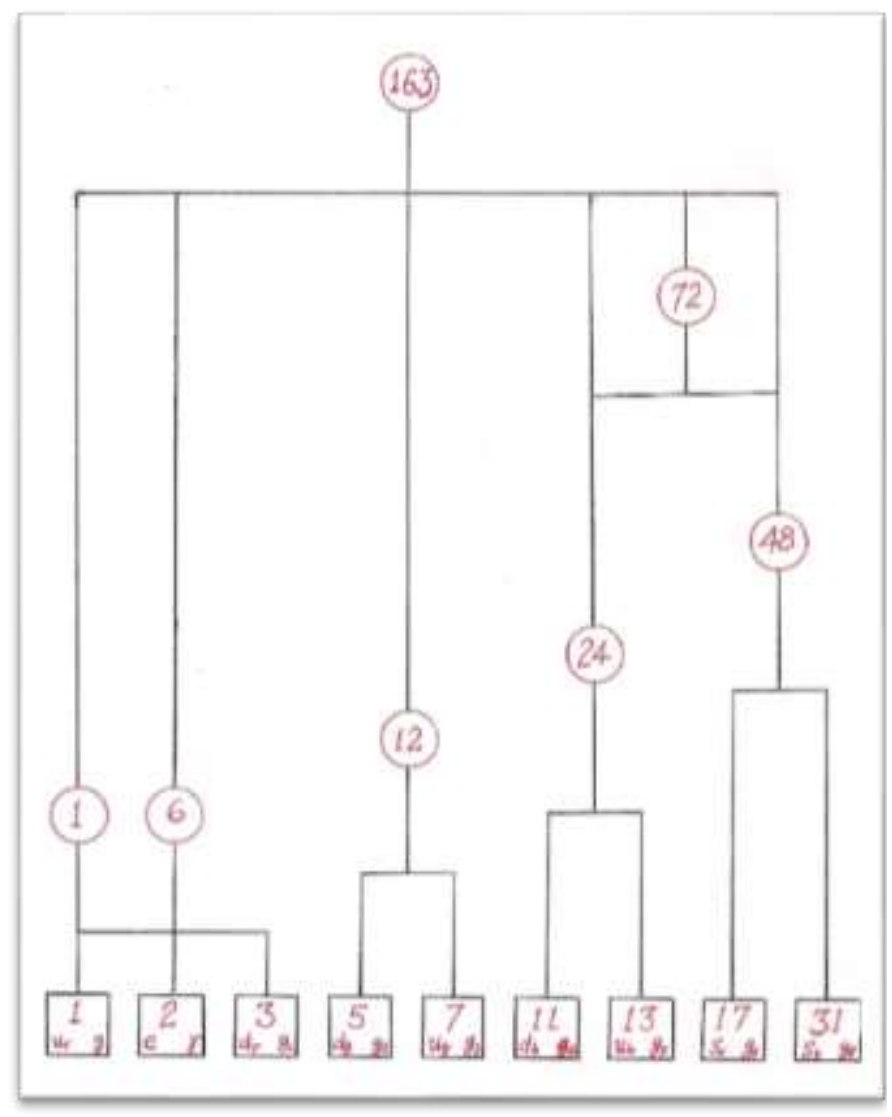

Fig. 25.2: The second Number Tower.

Two number towers produce the number 48 in different ways. First number tower is based on seven prime numbers from the $m$-parameters of first generation quarks and electron with "up+down" pairing. Second number tower includes two $m$-parameters of strange quarks to produce number 48 in a different way: $m_{s r}+m_{s b}=17+31=48$ with "red+blue" pairing. The prime number $m_{s g}=23$ used to define the $S$-sphere is left out. Is it a coincidence? In the second number tower, the prime numbers assign to quarks with three flavors $u, d$, $S$, which are the members of isospin group $S U(3)$. Is it a coincidence? Both number towers provide the mathematic basis for six special numbers, 1, 6, 12, 24, 48, 72, and the magic number $163=1+6+12+24+48+72$ as well as the classification of elementary particles in the Elementary Particles Table.

In essence, SQS theory is a mathematic theory. If there is a mathematic inconsistency, it must be taken very seriously. For example, from "common sense", the three cells for electrical neutral leptons in Elementary Particles Table should be filled with the flavored version 3 neutrinos and 3 anti-neutrinos. But it makes the total number of elementary particles equal to 75 instead of 72 not supported by two number towers especially the number 163 on top of them. The author tried many ways to resolve the mathematic inconsistency without success. Then the only way out is to take eigenstate version three anti-neutrinos as Majorana type fermions to fill the three cells. Fortunately, it works well with a bonus - the flying around eigenstate neutrinos $\tilde{v}_{1}, \tilde{v}_{2}, \tilde{v}_{3}$ provide an opportunity to resolve the missing antimatters mystery.

Another trunk of Family Tree is also rooted from fundamental postulations. Gaussian sphere as an assembly of vacuons is defined based on Gaussian Probability Postulation and Vacuon Postulation. With the help of Kepler-Hales theorem, the face-centered lattice is identified as the structure of 3-dimensional space. The two sets of symmetries $O(r), C(r)$ with $r \leq 3$ are identified and their vertexes are related to three generations elementary particles and interactions as shown by the Symmetries Family Tree. The two sets of 6 basic symmetries based on the vertexes numbers 1, 6, 12, 24, 48, 72 have important physics significances. The symmetries $O(r), C(r)$ with $r \leq 3$ are correlated to physics groups such as $U(1), S U(2), S U(3)$, which give elementary particles another geometrical origin besides their models. But the strict mathematic proof of correlation between symmetries $O(r), C(r)$ with $r \leq 3$ and groups $U(1)$,

$S U(2), S U(3)$ remains an open issue.

In SQS theory current version, the selection of the $p$ parameter is from physics to mathematics. According to SQS theory philosophy, it should be the other way around. This controversy implies that, at least one rule is missing in the current version. When the missing rule is found, it will provide the way to select $p$ - parameter from first principle to determine particle's theoretical mass value. It remains as an important open issue. The solution is hidden in primary basic equations (24.48).

Similar fluctuation behaviors are found in different areas of SQS theory. The first one is muon's decay times determined by $k_{i}$ values at local minimums of $\left|D S_{k}(k)\right|$. 


\section{Z. Y. SHEN}

There are so many local minimums corresponding to so many possible decay times for muon originated from the fluctuation nature of $\left|D S_{k}(k)\right|$. The second one is the

fluctuation nature of the complex $\dot{x}$-plane, on which the value of $\alpha\left(M_{e}\right)$ is calculated from electron's mass and

Weinberg angle. The value of $\alpha\left(M_{e}\right)=1 / 137.03599908345$

listed in Table 9.1 is from one root of $|S S(\dot{x})|=0$. In fact, there is a serious of roots corresponding to different $\alpha$ values, which indicates $\alpha$ as a running constant. The third one is the fluctuation nature of the $P S$-equation. There is a series of roots or minimums corresponding to slightly different tilting angles of loop-2 with slightly different effective parameters' values. The three types of fluctuations have something in common. They all are random in nature, all vary in small steps, and all correspond to real physics parameters. These facts imply that, they have the same mechanism and come from the same origin. The mechanism is particles' discrete trajectories proved by Theorem 18.1 and related lemmas. A particle and its parameters are represented by its trajectory on model surfaces. Jumping trajectories in discrete manner causing parameters change is responsible to these fluctuation behaviors. Ultimately it is originated from Gaussian Probability Postulation and stochastic nature of the quantum space.

In Section 22, the finding of three more sets of prime number even pairs in prime numbers table is very important. Formulas (22.9), (22.10), (22.11) and Conclusion 22.2 indicate that, they are based on the original set of $18+1$ prime numbers. With the help of Postulation 22.1, cosmic history and periods are correlated to elementary particles and traced back to prime numbers table. It provides a mathematical explanation for cosmic evolution from the big bang through inflation(s) up to the current period. It also predicts the future of universe. Finally it reaches a conclusion: A cyclic universe oscillates with alternate expanding and contracting periods. All of these are built in prime numbers table based on even-pairing rule. It shows mathematics at work.

As mentioned at the end of Section 22, the universe during its contracting period, entropy decrease seems contradictory to the second law of thermodynamics. The second law is such a fundamental physics law; any violation is going to shake the foundation of physics. It must be dealt with. The precondition for applying second law is that, the statistic ensemble for second law to apply must be an isolated one. Then the questions become: Is the universe an isolated statistic ensemble? Is there anything on top of the universe? There are clues from grand numbers, as listed in Table $23.3, F_{3}=5.864 \times 10^{22}$ as a $G=2$ grand number is for universe current third period, $F_{\text {pre }}=1.686 \times 10^{44}$ as a $G=4$ grand number is for prebig-bang period. For reference, the universe containing $\sim 10^{11}$ galaxies with $\sim 10^{22}$ stars correspond to $G=1$ and $G=2$ grand numbers, respectively.

Hypothesis 25.1: There is a multiverse including $\sim 10^{22}$ universes organized in two levels, each level has $\sim 10^{11}$ members. Our universe is one member of the lower level sub-multiverse.

SQS theory is not the first one to propose the multiverse concept. Other theory such as superstring theory did years ago. Despite the same name, there are differences. The motivation of SQS theory to propose its multiverse concept is trying to find a way to resolve the second law problem during cosmic contracting period. The clues are from two grand numbers. The $\sim 10^{11}$ universes in a sub-multiverse or the $\sim 10^{22}$ universes in the multiverse correctively form a statistic ensemble for the second law to apply. Our universe is just an element akin to a molecule in the air. The entropy is counted for entire ensemble not for one element. Moreover, each universe in the sub-multiverse started from Gaussian spheres evaporated from pre-big-bang over heated liquid state at different times in a random fashion. From SQS perspective, the overall scenario is like that, at a given time, different universes are in their different periods. Some are expanding and others are contracting akin to six cylinders in a combustion engine. It provides a possible solution for the second law problem. But it raises a question: What is the physics links among member universes in the sub-multiverse serving as the statistic ensemble? It may have something to do with neutrinos and photons.

Table 25.1 shows mass, Compton wavelength, converting factor, long-path Compton wavelength and maximum entanglement distance for three types of neutrinos. Mass values are sited from (17.4), converting factor and long-path values are calculated according to rules introduced in Section 4. Wavelength $\lambda$ for photon as boson is replaced by Compton wavelength $\lambda_{v C}$ for neutrinos as fermions. Maximum entanglement distance is changed accordingly to $d_{\max }=(2+1 / 4) \hat{\lambda}_{v C}=(2+1 / 4) \lambda_{v C}^{2} / L_{P}$ for neutrinos.

Table 25.1: Three Type Neutrinos Related Parameters*

\begin{tabular}{|c|c|c|c|c|c|}
\hline Nam & $\begin{array}{l}M, \\
\Delta \forall i e^{2}\end{array}$ & 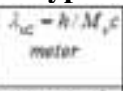 & $N_{v}=A_{s e} L_{p}$ & 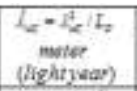 & $\begin{array}{c}\alpha_{-}=(2+1 / 4) L_{1}^{1} / I_{3} \\
\text { moter } \\
\text { (Aghityear) }\end{array}$ \\
\hline$v_{0} \%$, & $4.2922 \times 10^{-7}$ & $2539 \times 10^{-1}$ & $1.787 \times 10^{4}$ & $\begin{array}{l}5.163 \times 10^{15} \\
\left(5.457 \times 10^{-4}\right)\end{array}$ & $\begin{array}{l}1.152 \times 10^{11} \\
\left(1.228 \times 10^{4}\right)\end{array}$ \\
\hline$v_{j i}$ & $3.5749 \times 10^{-4}$ & $1.397 \times 10^{-4}$ & $8.644 \times 10^{15}$ & $\begin{array}{l}1.208 \times 10^{27} \\
\left(1.276 \times 10^{11}\right)\end{array}$ & $\begin{array}{l}2.717 \times 10^{2} \\
\left(2.872 \times 10^{14}\right)\end{array}$ \\
\hline$y_{,}, \bar{V}$ & $1.4925 \times 10^{-1}$ & $3.307 \times 10^{-1}$ & $5.14 \times 10^{3}$ & $\begin{array}{l}4.27 \times 10^{12} \\
\left(4.513 \times 10^{2}\right)\end{array}$ & $\begin{array}{l}9.507 \times 10^{54} \\
\left(1015 \times 10^{2}\right)\end{array}$ \\
\hline
\end{tabular}

According to (17.23), the mass of $\tilde{v}_{1}$ and $\tilde{v}_{2}$ are close to the mass of $v_{e}$ and $v_{\mu}$, respectively, the mass of $\tilde{v}_{3}$ is 
close to one third of $v_{\tau}$ mass. For $\tilde{v}_{1}$ with mass close to $v_{e}$, the $d_{\max } \sim 1.228 \times 10^{16}$ light years maximum entanglement distance for a pair of entangled $\tilde{v}_{1}$ is $\sim 10^{5}$ times longer than the $\sim 8.4 \times 10^{10}$ light years visible universe size. It is capable to reach neighboring member universe territory in the sub-multiverse. For $\tilde{v}_{2}$ with mass close to $v_{\mu}$, the $d_{\max } \sim 2.872 \times 10^{11}$ light years maximum entanglement distance is marginal to reach neighboring members of the sub-multiverse. For $\tilde{v}_{3}$ with one third of $v_{\tau}$ mass, the $d_{\max } \sim 3^{2} \times\left(1.015 \times 10^{9}\right)=9.139 \times 10^{9}$ light years maximum entanglement distance is less than visible universe size. It is not capable to reach neighboring members of sub-multiverse. If this is the case, a pair of entangled $\tilde{v}_{1}$ is eligible to serve as the physics link between adjacent members of the sub-multiverse. There is an interesting twist: cross universes connected $\tilde{v}_{1}$ might only oscillate with $\tilde{v}_{2}$, which is a distinctive feature different from its non-cross-universes counterpart. It provides a chance to verify the possible link between our universe and neighboring universes.

Accordingly, a pair of entangled photons with wavelength longer than millimeter is also capable to do the job. The long wavelengths portion of the cosmic microwave background radiation (MBR) spectrum is in this range. It may leave some traces there.

In ordinary flat space, $d_{\max }$ sets a limit of the distance between two entangled particles. But under extraordinary circumstance, things turn out differently. The edge of visible universe is like the event horizon of a black hole. The long path link between two entangled particles is capable to pass through event horizon. It is possible, because event horizon is defined based on speed does not exceed the speed of light, the superluminal speed along long path link does not subject to this restriction.

In Section 24, cosmic MBR photons long-path wavelength $\hat{\lambda}_{0}=\lambda_{0}^{2} / L_{P}$ at frequency spectrum center wavelength $\lambda_{0}=1.08 \times 10^{-3} \mathrm{~m}$ is used to deal with the dark energy hierarchy problem. For a double check, let's use eigenstate anti-neutrino $\tilde{v}_{2}$ with long path wavelength $\hat{\lambda}_{\widetilde{v} 2} \approx \hat{\lambda}_{\tilde{v} \mu}=1.208 \times 10^{27} \mathrm{~m}$ listed in Table 25.1 for an independent estimation:

$$
\left(\frac{\hat{\lambda}_{\tilde{v} 2}}{L_{P}}\right)^{2}=5.582 \times 10^{123} \text {. }
$$

Since anti-neutrino $\tilde{v}_{2}$ is electrically neutral, so the additional factor $\alpha / 4 \pi^{2}$ is not applicable. Comparing theoretical result $5.582 \times 10^{123}$ of (25.1) with observed data $R_{\rho}=3.271 \times 10^{123}$ of $(24.24)$, the relative discrepancy is $70.6 \%$. The agreement from two independent sources provides additional support for using converting rule to deal with dark energy problem. Question: Why use long path wavelength of $\tilde{v}_{2}$ not $\tilde{v}_{1}$ in (26.1)? Answer: For an oscillating pair, the one with shorter wavelength sets the limit. The result of (25.1) is important in another sense. It confirms that, the factor $\alpha / 4 \pi^{2}$ is applicable only for the case with electromagnetic force and gravity involved.

As universe keeps expanding in an accelerating rate, sooner or later, the distance between universe and its closest neighboring universe becomes too long exceeding the maximum entanglement distance of these particles made the cross universes physics link. Then the second law ensemble is in trouble. But look at it the other way, it might provide the cause to trigger cosmic contraction. It is a wild idea. But it doesn't hurt to give a thought.

There is another possible scheme to resolve the second law problem based on the definition of isolated statistic ensemble. During cosmic contracting period, the universe boundary is shrinking. Does an ensemble with shrinking boundary qualified to be an isolated one for the second law to apply? It deserves a thought. But in any case, the second thermodynamic law always holds.

In case the second law does not need entanglements to hold during cosmic contracting period, the multiverse concept still has a support from grand numbers: $F_{3}=5.864 \times 10^{22}$ and $F_{\text {pre }}=1.686 \times 10^{44}$. It indicates that, there is something $\sim 10^{22}$ times bigger on top of our universe. Except the multiverse, what else can be?

SQS theory provides a way to resolve the black hole information paradox. As Hawking suggested, a pair of virtual photons pops out from vacuum with one outside and the other inside event horizon. The outside one becomes a real photon carrying out part of black hole's energy/mass known as the Hawking radiation. Eventually, the black hole loses its entire energy/mass and vanishes. The paradox is that, after the black hole vanished, information in the back hole is lost. It contradicts to the conservation of information according to quantum theory. From SQS theory standpoint, the two photons entangled as coherent states interact to each other with superluminal speed along long path link. Like the cosmic entanglement case, long path link is capable to pass through event horizon and transmitting information along with energy/mass out of black hole via Hawking radiation. In this way, black hole does not lose any information. Information paradox is resolved. The key is the long path with superluminal speed.

SQS theory supports its own version of the limited anthropic principle. Compare to the strong anthropic principle, it has an important difference. The strong anthropic principle is based on the assumption: Physics laws and constants are different in different universes. According to SQS theory, there are two different types of physics laws and constants. The first type based on mathematics such as prime numbers does not change; 


\section{Z. Y. SHEN}

while the second type based on geometry may change as the geometry changes. Any universe as a member of the multiverse in its third period is governed by 18 prime numbers serving as the $m$-parameters of 18 quarks and in some extent the $n$-parameters as well. These parameters cannot change, because they are based on the same sets of 18 prime numbers. SQS theory standpoint is that, for any universe as a member of the multiverse in its third cosmic period corresponding to our current universe, the physics laws and constants may change, but they subject to strict limitations imposed by a set of unchangeable 18 prime numbers. Our universe nurtured human being on earth, some other universe in the multiverse with $\sim 10^{22}$ members should be capable to do the same. Superficially this argument seems to restrict the power of anthropic principle; actually it is to enhance the power of anthropic principle. If everything including all physics laws and constants can change arbitrarily, the $\sim 10^{22}$ member universes in multiverse are not sufficient to include even one universe having the set of physics laws and constants for human being to exist. Then the anthropic principle loses its power entirely. Mother Nature may change her mind but not arbitrarily. No one, not even God, can change the prime numbers.

Back to the multiverse issue, $\alpha^{-1}(M)=(71 / 2 \bar{\pi})^{2}$ is defined by (23.33), its values depend on a prime number 71 and a running constant $\bar{\pi}$. For any universe in its third period, 71 as a special prime number in the $M$-group does not change, while $\bar{\pi}$ depending on geometry of particles model may change making $\alpha(M)$ as a running constant. It supports the SQS theory limited anthropic principle.

Prime number 71 defined the $M$-sphere for the current universe corresponding to a set of 9 even pairs of prime numbers listed in the first row of Table 22.1. The pre-bigbang period corresponds to another set of 9 even pairs of prime numbers listed in the third and fourth rows of Table 22.1. The prime number located at similar location as 71 is 353. Let's look at (23.33a) for the pre-big-bang period universe, if 71 is replaced by 353 and kept $\bar{\pi}$ unchanged, the value of $\alpha^{-1}(M)$ is increased about $5^{2}=25$ times. Sine fine structure constant $\alpha$ is related to electrical charge, such big change is very unlikely. The alternative is: $\bar{\pi}$ in denominator increases approximately 5 times to $\pi(0.25) \approx 5 \pi$ for compensation. Since the 1-dimensional $\pi(x)$ carries information from the 3 -dimensional space, $\pi(0.25) \approx 5 \pi>\pi$ indicates that pre-big-bang space has much larger area of negatively curvature corresponding to tremendous repulsive force pushing everything outwards. It is a white hole. As mentioned in Section 22, after-bigcrunch universe transfers into pre-big-bang universe via a time tunnel. The outlet of time tunnel is a white hole. Here is the white hole! The two comedies are matched so well. It was conducted by Mother Nature using mathematic language, the actress and actors were prime numbers. If this argument holds, it serves as another evidence for the roles played by 71 and 353 in specific and for the correlation between prime numbers and cosmic history in general.

In fact, 71 is a special prime number in many senses. It is the largest prime number factor in the $M$-group factors sequence. It is in the non-even prime numbers pair $67 \& 71$ to end the three generations. It is the radius to define $M$-sphere and $M$-circle. It is the prime number in formula (23.33a) to define the running fine structure constant. Noticed that, the sum of three $m$-parameters for three strange quarks is:

$$
m_{s r}+m_{s g}+m_{s b}=17+23+31=71 .
$$

Prime number 71 is also related to the Euclid number $n=7$ for $(n !+1)$ :

$$
71 \times 71=5041=7 !+1=(1 \cdot 2 \cdot 3 \cdot 4 \cdot 5 \cdot 6 \cdot 7)+1 .
$$

This was a mathematic formula introduced by Greeks two thousand some years ago. Finally, $71+1=72$ is the total number of particles listed in Elementary Particles Table. All of these are based on mathematics; no wander 71 played an important role in physics.

Some mathematicians do not recognize 1 as a prime number. Their definition of prime number is like that: A prime number is a natural number that has exactly two distinct natural number divisors: 1 and itself.

For SQS theory, this definition is unacceptable. The first natural number 1 must be a prime number. There are mathematical reasons. (1) If 1 is not recognized as a prime number, the first even pair $1 \& 3$ does not exist. As a result, the first number tower no longer holds. (2) The second number tower no longer holds either. (3) The magic number 163 loses its foundation. (4) The symmetries family trees lose their foundation. (5) Definition 22.1 and Postulation 22.1 no longer hold. Cosmic history and future based on it lose their foundation. There are physics reasons. (1) If 1 is not recognized as a prime number, up quark $u_{r}$ and down quark $d_{r}$ do not exist. The total number of quarks would be 16 instead of 18. A flavor triplet is in trouble and two color triplets no long exist. (2) Graviton as $g=u_{r} \bar{u}_{r}$ does not exist. (3) Electron trefoil type model loses its red branch, because the only even prime number $m_{e r}=2=(1+3) / 2$ corresponding to electron red branch lost its foundation. (4) Photon as $\gamma=e^{-} e^{+}$is "handicapped" due to the "wound" of $e^{-}$and $e^{+}$. (5) For the current cosmic period we live in, the space is $2 \frac{2}{3}$-dimensional! What kind of world is that?

SQS theory is obligated to provide a prime number definition.

Definition 25.1, The Prime Number Definition: A prime number is a natural number that only has 1 and itself as its natural number divisor or divisors. 
Explanation: Definition 25.1 recognizes 1 as a prime number. For 1 as a prime number, two divisors happen to be the same. This kind of degenerations is common in mathematics as well as in physics.

Quantum theory is known for its uncertainty nature such as Heisenberg uncertainty principle. But the basic equation of quantum theory such as Schrodinger equation is deterministic. The uncertainty comes later from the statistic interpretation of its solution, the square of wave function's magnitude $\psi \psi^{*}$, serving as probability. SQS theory introduced uncertainty in the first place with the Gaussian Probability Postulation. Quantization comes later. After vacuon introduced in Section 18, Theorem 18.1 and Lemmas 18.1, 18.2 are proved to confirm trajectories' discrete nature. It serves as the basic quantization for SQS theory. In essence, quantum theory is from quantization to probability, while SQS theory is from probability to quantization. These two approaches are quite different.

According to SQS theory, particle's spin is the angular momentum of its loop-1 internal cyclic movement. It is based by the fact that conservation of angular momentums is the sum of spin and trajectory angular momentums combined. Apples can't add to oranges. Two addable terms must belong to the same type. The formula to calculate a particle's spin is:

$$
s=\frac{L_{1}}{\lambda_{P}}\left(\frac{h}{4 \pi}\right)=\frac{L_{1}}{L_{P}}\left(\frac{h}{4 \pi}\right)=m\left(\frac{h}{4 \pi}\right)=m \hbar / 2 \text {. }
$$

In which, $L_{1}=m \lambda_{P}$ is loop-1 circumferential length, $\lambda_{P}=L_{P}$ is Planck wavelength, $m$ is reduced $m$ parameter.

For bosons without mass, photon and gluons with $m=2$ have spin $s=\hbar$; graviton and massons with $m=0$ have spin $s=0$. For charged leptons' trefoil model three branches together with reduced $m$-parameter, $m=3 \times(1 / 3)=1, s=3 \times(\hbar / 6)=\hbar / 2$. Formula (25.3) is valid for these particles.

For quarks, all 18 quarks have reduced $m$-parameter $m=1$, formula (25.3) is also valid. But all 18 quarks' original $m$-parameters are greater than 1 , reduction means $m-, n-, p$-parameters divided by the some number $m$ for a quark, which makes the quark's $m=1$ and its $n$ - and $p$ parameters changes accordingly. As shown in Section 13, strong interactions are based on quarks' original $m$ parameter and $n$-parameter versus gluons' $m$-parameter and $n$-parameter. After quarks' $m$ - and $n$-parameters reductions, how does gluon "recognize" quark's original parameters? There is a possible solution. When a quark interacts with gluons, its reduced $m-, n$-, and $p$ - parameters are multiplied by the same number $m$ to recover their original value for gluons to recognize. The multiplication of $m$ physically means that, the quark's two cyclic movements go through $m$ cycles, which is acceptable. There is any question. Reduction makes quark's $n$ - parameter fractional. As shown in Section 14, leptons' $n$ parameters are fractional. Does the reduction for quarks blur the distinction between quarks and leptons and the distinction between strong and weak interactions? The answer is: No. Because quarks' $m-, n-$, and $p$ - parameters are different from leptons'.

SQS theory does not use operators. It is not the first one to do so. Feynman's path integral theory did it decades ago. Feynman's path integral equation is equivalent to Schrodinger's equation, which shows that quantum theory can serve its functions without operators. It gives confidence to SQS theory to go its own way without operators.

In Section 5, SQS theory provided the dispersion equation (5.7) as a modification for special relativity based on Planck length $L_{P}$. Later in Section 23, the $M$ sphere with radius $r=71 L_{P}$ is introduced, which serves as a domain with linear scale longer than Planck length. According to grand number phenomena, large domains with linear scale of $\sim 10^{11 N} L_{P}$ are possible as shown by (21.12). The multi-layer domain possibility raises a question: Should dispersion equation (5.7) change accordingly? The answer is: Yes. At least the $M$-sphere is legitimate evidenced by the fact that grand unification occurred on its surface. The dispersion equation (5.7) are generalized as:

$$
\begin{aligned}
& v(f)=c \sqrt{1-\left(f L_{\text {domain }} / c\right)^{2}}, \\
& v(\lambda)=c \sqrt{1-\left(L_{\text {domain }} / \lambda\right)^{2}} .
\end{aligned}
$$

$L_{\text {domain }}$ is the length scale of the effective domain. Compare to (5.7), equation (26.4) makes dispersion stronger and relatively easier for experimental or observational verification. The results will provide information for effective domain size. It is important to point out that, if an effective domain with $L_{\text {domain }}>L_{P}$ is found, it does not mean equation (5.7) is abolished and replaced by equation (26.4). Photon dispersion equation (5.7) is fundamental based on space basic grainy structure, which is always valid no matter higher level domain exists or not. This is the reason why equation (25.4) is defined as a generalization not a replacement of original dispersion equation (5.7).

Quantum mechanics is a very successful theory in terms of extremely high accuracy and very broad practical applications. But it has many contradict versions of interpretation and seemingly none of them is commonly accepted. SQS theory provides an opportunity for a new interpretation. The key is the meaning of locality. As discussed in Section 5, if locality means interactions and information transmission are restricted by speed of $v \leq c$ under any circumstances, the superluminal phenomena found by many experiments between entangled particles inevitably lead to "spooky action at a distance". The long 


\section{Z. Y. SHEN}

path provides a way out. Entangled particles are linked by long path. It is a physical entity for interactions and information transmission along it with superluminal speed $\hat{v}=N c>c$ seen by stationary observers. In this way, locality is reserved and many other "spooky actions" in quantum mechanics can be interpreted with common sense. This is a topic with very important physics and philosophical significances for further investigations. SQS theory standpoint is clear: Einstein was right — "No spooky action at a distance."

General relativity is a beautiful theory. Based on two principles, general relativity provides a set of Einstein equations for gravity in terms of space-time curvature. It has been verified by many experiments and observations without even one failure. General relativity serves as one of two pillars for modern physics. The problem is that, general relativity is not compatible with quantum theory. For decades, there were many attempts to quantize general relativity and none of them is commonly accepted. From SQS theory viewpoint, it is the time to rethink the issue. In fact, this is the initial inspiration for the author to search SQS theory basic equations.

As mentioned in Section 24, the key concept for SQS basic equations based on Einstein original equations (24.1b) is to introduce uncertainty to gauge tensors by Postulation 24.1. From SQS theory perspective, the concept is very clear to begin with: To unify general relativity with quantum theory, uncertainty is primary and quantization is secondary. The other key concept is to introduce the intrinsic time by (24.5), which naturally leads to the superluminal speed for vacuons movements inside elementary particles and the appropriately adjustment of separation for the variable in difference equations of (24.20) and (24.42) etc. Both concepts paid off tremendously as shown in Section 24.

Standard Model (SM) is proved to be a successful effective theory with enormous theoretical results agreed very well with experimental data. As shown in previous sections, many results from SQS theory are agreed well with experimental data. There must be a strict link between SM and SQS theory. When the link is found, some open issues will settle down.

On the other hand, there are some differences between SM and SQS theory.

The first one is the difference ways to treat particles. SQS theory provides trajectories on models to represent elementary particles, while SM treats them as points. This is the reason that, SQS theory does not have divergence problem and does not need renormalization. There are deeper reasons for SQS theory to avoid divergence problem. For the long range force such as electrostatic force, its strength is inversely proportional to the square of distance. As distance approaches to zero, its strength and energy density approach to infinity causing divergence. According to SQS theory, electromagnetic force is unified into gravity at length scale $L_{G U T}=71 L_{P}$ on $M$-sphere surface. So there are no infinity and no divergence for electromagnetic force. For gravity, its strength is also inversely proportional to the square of distance. It keeps that way until near saturation as shown in Fig.24.1. At $l=x_{c} L_{P}=0.2998715627302645 L_{P}$, gravitational force vanishes. In the region $0 \leq l<x_{c} L_{P}$, it becomes repulsive. So there is no divergence for gravitational force. In fact, this is the way SQS theory eliminated singularity. Within the $M$-sphere, two short range forces are unified into gravity, so there is no divergence either.

The second one is the different ways to introduce parameters. SM has twenty some handpicked parameters from experimental data, while SQS theory has three sets of mathematical parameters. In which two sets mostly are determined prime numbers and the other set is determined by particle's mass in the current version. In essence, the second difference is originated from the first one. It is understandable that, one can derive parameters from a geometric model with trajectory and characteristic points, but no one can derive any parameter except its location and movement from a point.

The third one is SM does not include gravity, SQS theory does. In fact, SQS theory unified all interactions to gravitational interaction as shown in Section 15.

The fourth one is the number of elementary particles. As shown in Table 18.1 and Table 18.2, SM has 25 particles not including anti-particles, while SQS theory has 72 particles. The difference is stemmed from SQS theory recognized quarks with different colors as different particles. After the vacuon introduced, the difference becomes the other way around with only one ultimate elementary particle for SQS theory.

SM is a well-developed theory. With decades of cooperative efforts, it is capable to calculate the cross sections and branching ratios for particles and interactions from Feynman diagrams, which are agreed with experiments very well. SQS theory is a developing theory. It just gets started. It hasn't done these type calculations yet but has the potential to do so. The potential is based on properties of SQS theory. One is in the 1-dimensional $S$ equation, in general the probability at $x_{1}$ has excess and the probability at $x_{2}$ has deficit. For most particles, the excess does not match the deficit exactly. The mismatch provides the mechanism for the particle to interact with others or transfer to others. The other one is jumping trajectories, which also provide the opportunity for the particle interacting with others or transferring into others. These two properties are intrinsically correlated based on primary basic equations (24.48). For instance, as shown in Section 24, different elementary particle represented by different vertexes in $O(\sqrt{1 / 2})$ share the same center vertex. It serves as a junction of their overall closed geodesic loops, which provides the mechanism for interactions 
among particles. So SQS theory does have the potential to provide the method for calculating cross sections and branching ratios. This is an important open issue. It wouldn't be easy and may require some tricks and extensive number crunching. But in principle it is feasible. Hopefully, it can be done in the near future.

SQS theory does not intend to compete with SM. It provides geometrical models and mathematical interpretations to support SM at a deeper level. It also provides a way to make SM as a quantum theory compatible with general relativity.

There are similarity between super-symmetry theory and SQS theory. Both theories require fermions and bosons somehow matching to each other. But there is an important difference. All hypothetic particles predicted by super-symmetry theory such as the "s-" for bosons and the "-o" for fermions are not discovered yet. In SQS theory Elementary Particles Table, there are no undiscovered fermions; for the 24 bosons, 13 of them are waiting to be discovered. If the 8 massons are indeed attached to $W, Z, X$ bosons, the number of undiscovered bosons is reduced to 5 . The difference between supersymmetry theory and SQS theory is stemmed from the different ways to match fermions and bosons. Supersymmetry theory does not look for possible matches in existing elementary particles, while SQS theory does. In fact, all fermions and bosons in Table 18.2 are matched, in which only 13 bosons are hypothetical.

There are some similarities between SQS theory and string theory [21]. After all, strings and trajectories both are 1-dimensional objects representing elementary particles. In this regard, these two theories do have some common grounds. In addition, for SQS theory the way to introduce mass by adding oscillating mass terms in the $A T$ - and $P S$ - equations is inspired by string theory. But there are major differences.

The first difference is the number of space dimensions. Superstring theory is based on 9-dimensional or 10dimentional space (the early version of string theory was based on 25-dimensional space), while SQS theory is based on 3-dimentional space. As shown in Sections 21, the physics groups are related to two set of symmetries, $O(r)$ and $C(r)$ with $r \leq 3$, which are the intrinsic property of the 3-dimensional space with face-centered lattice structure. It includes two parts, the cubic part and the octahedral part. The face-centered lattice structure can be viewed as an octahedron imbedded in a cube. Someone may interpret the imbedded octahedral part as the hidden space. For instance, $O(\sqrt{1 / 2})$ symmetry centered at 1 octahedral vertex has 12 vertexes on the spherical surface. The $12 \div 2=6$ pairs of vertexes related to the center vertex form 6 non-orthogonal axes, which might be interpreted as a 6-dimensional space hidden in a 3dimensional space represented by the cubic part. From SQS theory perspective, it is an illusion of the face- centered space structure. The argument is to state SQS theory viewpoint and by no means to criticize string theory. After all, what is the number of space dimensions? 9? 10 ? 25? or 3? Only experiments can answer.

The second difference is the nature of string and trajectory. String theory treats string as a vibrating thread with mass and elasticity. SQS theory treats trajectory as a path of vacuons movement. This difference makes the other difference. For some version of the string theories, except graviton, all other elementary particles are represented by open strings; only graviton is represented by closed string. For SQS theory, except graviton, all other elementary particles' trajectories are closed loops. The reason is that, in general, vacuons movement along trajectory cannot stop suddenly and revise directions abruptly. As the only exception, graviton stops at cubic vertex $x_{i}$ and changes its directions. It has specific reasons based on Theorem 3.2, Theorem 4.1 and Theorem 21.1.

The third difference is the topological structures of elementary particles. The particle models proposed by SQS theory are topological manifolds with genus numbers of zero, one, two and three. String theory has so many different Calabi-Yao manifolds with possible numbers up to $10^{500}$. If the model does represent an elementary particle, it should provide particle's all physics parameters from its geometrical parameters. SQS theory did so with models having genus number not exceeding three. The key is model having definitive shape and size plus characteristic points and related triangles. Model's shape and size are determined by particle's $m-, n$-, $p$-parameters; its characteristic points' location and related triangles are determined by $\pi\left(x_{1}\right)$ and $\pi\left(x_{2}\right)$ as messengers carrying information from the $S$-equation. In principle, the particle's all physics parameters can be derived from these geometry parameters. On the other hand, if the topological manifold has no definitive shape, size and lack of characteristic points, the only way for it to represent an elementary particle with all physics parameters is to increase its genus number. Again, it is by no means to criticize string theory. What type of strings or trajectories and models elementary particles really have, only experiments can tell.

The fourth difference is fermions versus bosons. The original string theory based on 26-dimensional space-time had only bosons. Fermions were introduced later via super-symmetry to form the superstring theory. For SQS theory, fermions are primary, bosons made of a pair of fermion and anti-fermion are secondary. Fermion or boson, which one is primary? This is the question. A basic theory should answer.

Over the years, string theory has accumulated so many mathematic achievements and some physics insights. It takes time for SQS theory to learn. Hopefully, more mutual understandings will benefit both theories. 


\section{Z. Y. SHEN}

There are similarities between SQS theory and the loop theory [22]. One similarity is obvious. Both theories are basically dealing with loops. There is another important similarity. One of the major merits of loop theory is background independence. Space structure is not a priori determined; instead, it emerges naturally. SQS theory supports background independence. In the transitional and macroscopic scales, the moving around $M$-spheres arrange themselves according to distribution of mass and energy to satisfy basic equations (24.20) the same way as general relativity. Inside the $M$-sphere, Gaussian spheres arrange themselves in face-centered lattice structure to reach minimum potential energy. Inside the Planck cube, the primary basic equations (24.48) provide a mechanism for background independence based on the stochastic behavior of geodesics adjusting gauge tensors as shown in Section 24.

Composite preons theory is based on preons triplets to form models for elementary particles [23,24]. In SQS theory, charged leptons and neutrinos trefoil trajectories have three branches. For quarks, there are flavor triplets and color triplets. In its current version, composite preons theory does not provide detail information regarding elementary particles' parameters for further comparison.

Striking similarities between crystallography and particle physics were found [25]. For SQS theory, it is not only similarity; the microscopic space is a crystal with face-centered lattice structure. All elementary particles, interactions and symmetries are originated from it. This area deserves further investigation based on the facecentered lattice structure. Hopefully, they will give SQS theory more supports and inspirations.

Technicolor theory is proposed as an alternative of higgs mechanism to provide mass for particles with mass [26]. There are some similarities between technicolor theory and SQS theory. The eight hypothetic massons $G_{i}$ $(i=1,2 \cdots 8)$ introduced in Section 14 are pure mass stuff. Massons contribute a portion of mass for gauge bosons $W$, $Z$ and $X$. If $U_{1}, U_{2}, U_{3}$ are indeed made neutrino and anti-neutrino pairs, the tremendous mass gap between the "heaviest" and the "lightest" would be also filled by vast numbers of massons. From SQS theory perspective, massons provide a portion of mass to bosons with mass. Whither massons also play a role to provide a portion of mass for some fermions, it is an open issue. According to SQS theory, particles' mass is determined by $p / n=M / M_{e}$ ratio and generated by sinusoidal oscillation of mass term $\sin [(2 p / m) \theta]$ along trajectory, which is ultimately originated from solutions of primary basic equations (24.48). It serves as the universal mechanism of particles' mass for SQS theory. But it does not necessarily mean no common grounds for Higgs mechanism, technicolor theory and SQS theory. There are possible correlations among these theories, which deserve a close look.
The original Grand Unification Theory (GUT) intended to unify three interactions excluding gravity was based on $S U(5)$ group [27, 28]. Despite the same name, it is different from SQS theory GUT including gravity. The GUT based on $S U(5)$ is an elegant theory. According to the minimal $S U(5)$ model [28], protons are not stable and decay with lifetime of $\tau \sim 10^{29 \pm 1.7}$ years. Unfortunately, this prediction was disproved by Irvine-MichiganBrookhaven $(I-M-B)$ experiment and later by SuperKamiokande $(S-K)$ experiment. It was such a disappointment, afterwards physicists moved on other directions. From SQS theory perspective, there are reasons to believe the original GUT based on $S U(5)$ group might have a chance for revival.

The protons predicted lifetime of $\tau \sim 10^{29 \pm 1.7}$ years is based on $S U(5)$ group. The obvious precondition is that, $S U$ (5) group must be in existence in the first place. It is well known in crystallography that, 5 -fold symmetry does not exist in single-crystal structure; it exists in the quasicrystal structure with quasi-periodic lattice lengths [29, 30]. The quasi-crystal lattice must have at least two different spatial periods with irrational ratio. The facecentered space structure in its single-crystal form does not support $S U(5)$ group. For $S U(5)$ group to exist, the facecentered space structure must have defect to accommodate 5-fold spatial symmetry.

As show in Fig. 25.3, icosahedron has 5-fold spatial symmetry. Like $O(\sqrt{1 / 2})$ symmetry, icosahedron also has 12 vertexes on a spherical surface with radius:

$$
r_{\text {icos }}=\left[\frac{1}{4} \sqrt{10+2 \sqrt{5}}\right]\left[\frac{L_{P}}{\sqrt{2}}\right]=\left(\frac{L_{P}}{\sqrt{2}}\right) \sin \left(\frac{2 \pi}{5}\right)=0.95105652\left(\frac{L_{p}}{\sqrt{2}}\right),
$$

The relative deviation of $r_{i \text { cos }}=0.95105652\left(L_{p} / \sqrt{2}\right)$ from $r_{O(\sqrt{1 / 2})}=L_{P} / \sqrt{2}$ is $4.9 \%$.

The icosahedron has 20 connected equilateral triangles on its surface. It has sufficient room to accommodate quarks $u\left(u_{r}, u_{g}, u_{b}\right), u\left(u_{r}, u_{g}, u_{b}\right), d\left(d_{r}, d_{g}, d_{b}\right)$ and leptons participated in the proton $p(u u d)$ decay process.

According to Kepler-Hales theory, space based on Gaussian spheres with face-centered lattice structure is the ground state of vacuum with lowest potential energy. A icosahedron as defect in face-centered space structure is in a quasi-stable state with higher energy. To accommodate 5 -fold symmetry, the 12 vertexes of $O(\sqrt{1 / 2})$ must shift locations converting to icosahedron structure. The conversion process is governed by probability. Assume a vertex stays in $O(\sqrt{1 / 2})$ and shifts to icosahedron with equal probability $1 / 2$. The probability for 12 vertexes of $O(\sqrt{1 / 2})$ all shift to icosahedron is $p=(1 / 2)^{12}=1 / 4096$. For a tank of water containing $N \sim(M=10) \times 10^{31}=10^{32}$ 
protons with lifetime $\tau \sim 10^{31}$ years, one originally expected to detect $M=10$ decay events per year. This expectation is based on the assumption that, $S U(5)$ group is fully applicable to all $N$ protons involved. But it is not true, because for $N$ protons only $p N$ protons are eligible for $S U(5)$ group to apply. Take this effect into account, for the water tank containing $N \sim(M=10) \times 10^{31}=10^{32}$ protons, the expected number of proton decay events should be multiplied by probability $p$ and become $10 p=10 \times(1 / 4096) \approx 0.00244$ per year. Instead 10 decay events per year, the real expectation is $\sim 2.4$ events per 1000 years. According to this argument, $I-M-B$ type and $S$ $K$ type experiments should increase the number of protons in tank by at least 1000 times.

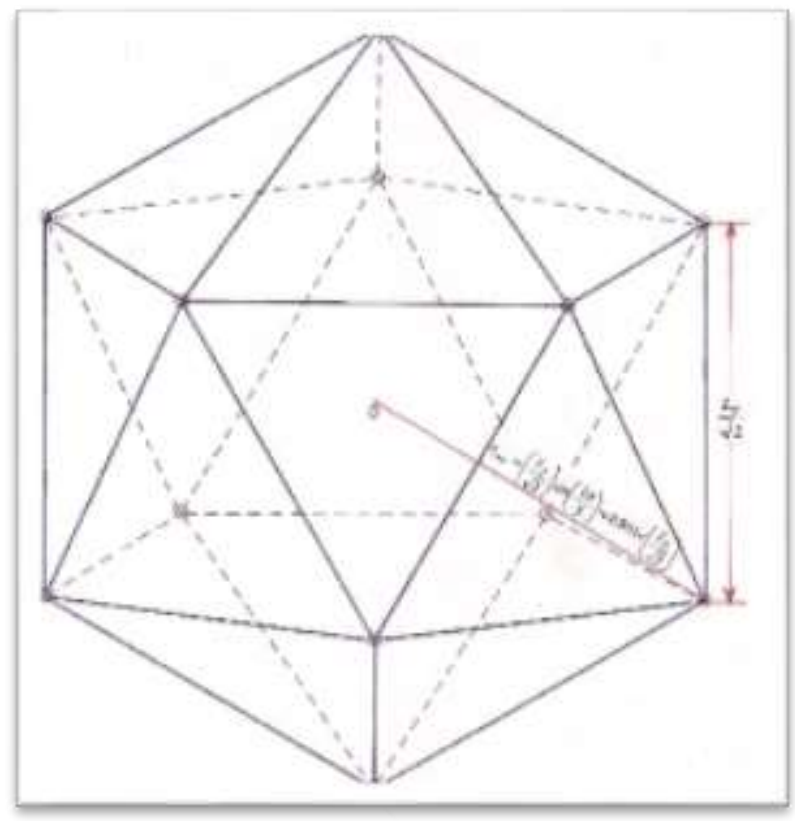

Fig. 25.3: Icosahedron with 12 vertexes and 20 equilateral triangles on its surface has 5-fold symmetry.

There are also questions regarding the validity of $I-M$ $B$ type and $S$ - $K$ type experiments. As shown in Section 7 , in the time interval $0<t<t_{\min }=2.075 \times 10^{-13} s$, muons have zero probability to decay. What is proton's $t_{\min }$ ? If proton's $t_{\min } \sim 10^{11}$ years, even all protons were born at big bang $1.37 \times 10^{10}$ years ago, none of them is eligible for decay yet. If this is the case, to increase the number of protons for $I-M-B$ type and $S-K$ type experiments would not help at all, the only way is to wait until $t>t_{\min }$.

In Section 15, SQS theory borrowed a method from the GUT based on $S U(5)$ group to calculate the characteristic mass for two unifications. This method is proved to be equivalent to the one with more credibility. It shows that, there is some truth in $S U(5)$ group. For instance, $S U(5)$ group breaks down to $S U(3) \otimes S U(2) \otimes U(1)$, which are the right ones for elementary particles and interactions in the standard model. Moreover, $S U(5)$ group contains 24 bosons, in which 13 of them called " $X$-bosons" are hypothetic. As shown in the Elementary Particles Table, SQS theory also has 24 bosons, in which 13 are hypothetical. Is this a coincidence? It is worthwhile to investigate $S U(5)$ group to find out its relations with $O(r)$ and $C(r)$ symmetries. The bottom line is that, as long as $O(\sqrt{1 / 2})$ can convert to icosahedron with 5-fold symmetry, proton has a chance to decay. The question is: Under what condition and what's the probability? This is an interesting open issue worthwhile to explore.

Elementary particles' models proposed by SQS theory are $\mathrm{s}$ in Table 25.2.

Most models listed in Table 25.2 have been explained in previous Sections. A few models need some explanation. The spindle type torus models are listed as genus- 0 , because their center hole(s) are covered.

Topological manifold is allowed to continuously deform, but the heavy mass $M>M_{\operatorname{Max}}$ requires $a_{2}^{\prime}>d$ preventing the center hole to be uncovered. For gauge bosons $W, Z$, $X$ and scalar bosons $U_{1}, U_{2}, U_{3}$ with heavy mass $M>M_{\text {Max }}$, their models belong to two joint spindle type torus with genus- 0 .

Table 25.2: Summary of Elementary Particles Models

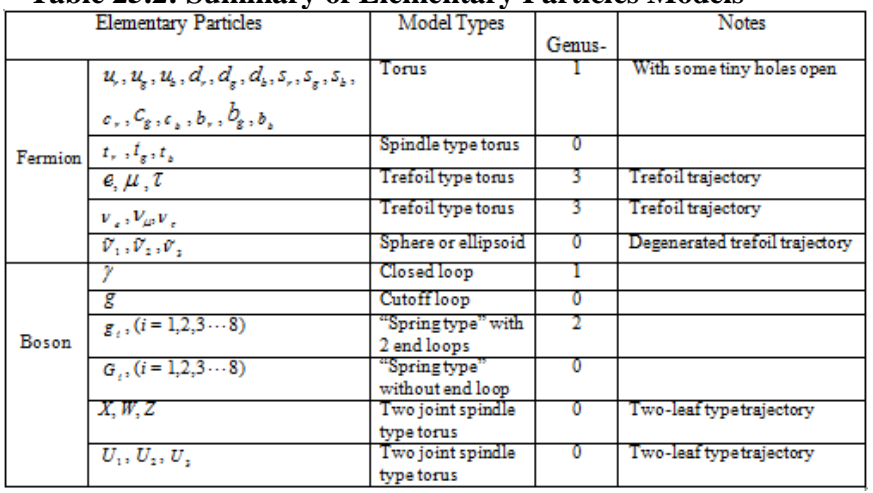

In algebraic topology, Henri Poincare discovered the "hairy ball theorem" [31]. Imagine a ball with a hair growing out from every point on its surface. One tries to comb the hairs flat and smoothly around the ball. Put in mathematic terms. "Hairs" correspond to nonzero tangent vector field made of a set of tangent vectors. "Comb the hairs around the ball" is to arrange the tangent vectors around the closed surface. "Flat" means tangent vectors pointing only at tangential direction of the closed surface. "Smoothly" means tangent vectors arranged with continuity without abruption.

Poincare theorem proved that, no matter how to arrange these tangent vectors (hairs), it always leaves some crown (bundle of hairs) stretched out from the ball surface. Poincare theorem is valid for any genus- 0 closed 


\section{Z. Y. SHEN}

surface topologically equivalent to the ball surface. Fig. 25.4 shows two crowns on a spherical surface.

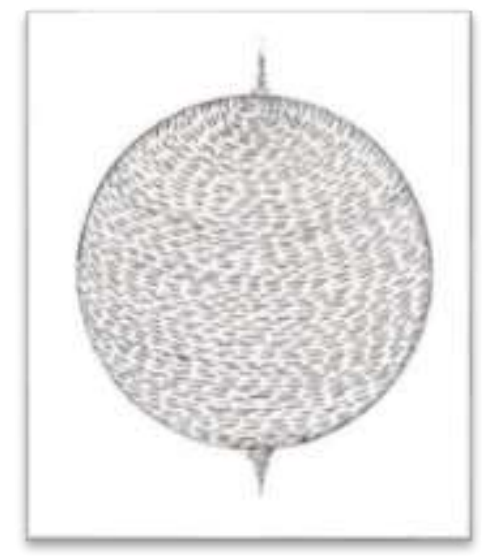

Fig. 25.4: "Combed hairs" on spherical surface as genus-0 manifold with two "crowns".

There are two closed surfaces, on which hairs can be combed flat and smoothly without crown. One is genus-1 torus with one center hole and the other is Klein bottle.

In 3-dimensional space, closed surfaces are classified into two types: Type-1 surface with genus number equal to 1 and Type- 2 surface with genus number other than 1 . According Poincare theorem and related rules, Type-1 closed surface does not have crown and Type- 2 closed surface has at least two crowns.

From SQS theory perspective, Poincare theorem and related rules provide important clues for elementary particles' models, trajectories and interactions. As shown in previous sections, particle's trajectories are on its model's closed surface (the small holes on torus surface are closed by filling points according to Penrose [2]). For a trajectory on model surface, the tangent vectors along its path are flat and smooth. For a set of trajectories on the model surface, the tangent vectors along these trajectories are arranged like combed hairs. According to Poincare theorem and related rules, for the set of trajectories on the model's Type-2 closed surface, there are always crowns for trajectories to jumping out or jumping in. Jumping literarily means vector must leave the surface, which makes it no longer tangential and abruptly changing directions. This is what happens at the crowns.

As described previously, jumping trajectories are equivalent to interactions. Therefore, a particle having interactions must be capable to jump trajectories and its model surface must have crowns. The requirement for crowns is met for Type- 2 model with genus number other than 1 . The problem is the fifteen quarks with genus-1 torus model: $u_{r}, u_{g}, u_{b}, d_{r}, d_{g}, d_{b}, s_{r}, s_{g}, s_{b}, c_{r}, c_{g}, c_{b}$, $b_{r}, b_{g}, b_{b}$. The genus-1 torus belongs to Type-1, which has no crown. If this is really the case, there would be no electromagnetic interaction, no strong interaction and no weak interaction for these fifteen quarks. Obviously, it is not true. One possible way to solve the problem is to leave same of four tiny holes open on torus surface, which makes their model as open surface to have crowns. For instance, leaving two tiny holes open at characteristic points $A$ and $B$ creates two crowns. It serves as a working assumption for SQS theory.

In topological terms, free flying photon with genus-1 model of Type- 1 has no crown meaning no interaction among photons. In fact, electromagnetic interactions are linear and photons do not interact with each other. It is also evidenced by the fact that, Maxwell equations are linear equations. It serves as a supportive evidence for the effectiveness of Poincare theorem and related rules in particle physics.

Gluon's genus- 2 model of Type- 2 has crowns indicating that, there is interaction among gluons. In fact, strong interaction mediated by gluons is nonlinear and gluons interact with each other as shown in Table 13.5. It also serves as another supportive evidence for the effectiveness of Poincare theorem and related rules in particle physics.

Graviton's genus-0 model of Type- 2 has crowns. They should interact with themselves. In fact, as the mediator of gravity, graviton interacts with anything having mass and energy. The flying around gravitons have energy and dynamic mass for gravity to act upon. It is also evidenced by the fact that, Einstein equations of general relativity are nonlinear equations and nonlinearity represents selfinteraction. The gravity among gravitons is extremely feeble, but it does exist. It supports graviton with genus-0.

Conclusion 25.1: Graviton is a scalar boson with spin 0. Explanation: Poincare theorem and related rules serve as the topological evidence for Conclusion 25.1, graviton must have spin 0 . Let's consider the opposite. If graviton has spin 2 or any other nonzero spin values, its closed loop model belongs to genus-1 of Type-1 without crown corresponding to no interaction among gravitons. It is obviously not true. This is a conclusive evidence for graviton having spin 0 as stated in Definition 18.1 based on other mathematic reasons.

Black hole with closed event horizon is a genus-0 manifold of Type-2. According to Poincare theorem, black hole must have crowns and hairs. Therefore, Hawking radiation is not only a possibility but also a necessity. It serves as a mathematic support for Hawking radiation and the solution of information paradox suggested by SQS theory.

Poincare theorem and related rules also support an "absolute black hole" with no stretched out hair.

Definition 25.2: Absolute Black Hole. A chuck of matter with total mass exceeding critical mass to form a manifold with genus-1 torus event horizon is defined as an absolute black hole.

Explanation: The name "absolute black hole" is chosen to differentiate it from black hole. Black hole has 
Hawking radiations as stretched out hairs. Absolute black hole has no radiation and no stretched out hair.

Theorem 25.1: No Radiation Theorem. Absolute black hole has no radiation of any kind.

Proof: According to Poincare theorem with related rules and Definition 25.2, absolute black hole with genus1 torus event horizon has no crown and no stretched out hair which means no radiation. QED

Lemma 25.1: Hawking Mechanism. Absolute black hole has no Hawking radiation, but it has Hawking mechanism. A pair of virtual photons pops up in the vicinity of absolute black hole's event horizon. One photon falls into absolute black hole, and the other photon is kept in the event horizon. This Hawking mechanism process keeps going on. As results, more and more photons are circulating in absolute black hole's event horizon like "combed hairs".

Proof: The Hawking mechanism is the same as that occurred in the vicinity of ordinary black hole. The only difference is that absolute black hole has no "crown", therefore photon cannot radiate. QED

Lemma 25.2: When two absolute black holes collide in proper ways, they transfer into a regular black hole with genus-0 event horizon or a special black hole with genus-2 event horizon. Both subject to Hawking radiation.

Proof: According to Poincare theorem with related rules and Theorem 25.1, the regular black hole with genus- 0 event horizon or the special black hole with genus- 2 event horizon has crowns and stretched out hairs - radiation.

QED

The boundary of visible universe like black hole's event horizon is a genus-0 manifold of Type-2. According to Poincare theorem, it must have crowns. Therefore, the cross universes connections via entangled $\tilde{v}_{1}, \tilde{v}_{2}$ and cosmic MBR photons not only are possible but also are necessary. It serves as an independent mathematic support for the multiverse concept and the physics connections among its member universes.

So far everything is consistent. It indicates that, Poincare theorem in particular and topology in general play critical role for elementary particles' models, trajectories and interactions as well as for cosmology. It is mathematics at work.

According to Green et al [21], spinors may relate to the tangent vectors in Poincare theorem. Spinor is the basic concept of spinor theory [32]. The relation between spinor theory and SQS theory is an open area. Spinor theory is based on complex space, while SQS theory is based on real space except the abstract 3 complex planes associated with 3 axes introduced in Scheme-2 of Section 3. What is the implication of real space versus complex space is an interesting topic.

SQS theory provided a framework for cosmology based on prime numbers with results agreed to cosmology standard model plus some new insights. But some important issues left open such as: (1) How many cosmic inflations did happen, one, two or three? (2) What is the mechanism of $1 \frac{1}{3}$-dimensional space in the first period transferred into 2-dimensional space in the second period? (3) Based on mathematics, is there a way to reveal cosmic history and to predict cosmic future in more details? (4) When will the current accelerating cosmic expansion end? (5) Are there more cosmic "secretes" hidden in prime numbers? In fact, there is one.

It is interesting to find another set of 13 consecutive even paired prime numbers listed in Table 25.3. It starts from $2791 \& 2797$ and ends at $2999 \& 3001$. The two prime number odd pairs are $2777 \& 2789$ and $3011 \& 3019$ marked with underline to start and to stop the even paired prime number sequence at two ends.

Table 25.3: The Prime Numbers between 2707 and 3083 and 13 Consecutive Even Pairs

\begin{tabular}{|r|l|l|l|l|l|l|l|l|l|l|l|}
\hline 2707 & 2711 & 2713 & 2719 & 2729 & 2731 & 2741 & 2749 & 2753 & 2767 & $\underline{2777}$ & $\underline{2789}$ \\
\hline 2791 & 2797 & 2801 & 2803 & 2819 & 2833 & 2837 & 2843 & 2851 & 2857 & 2861 & 2879 \\
\hline 2887 & 2897 & 2903 & 2909 & 2917 & 2927 & 2939 & 2953 & 2957 & 2963 & 2969 & 2971 \\
\hline 2999 & 3001 & $\underline{3011}$ & $\underline{3019}$ & 3023 & 3037 & 3041 & 3049 & 3061 & 3067 & 3079 & 3083 \\
\hline
\end{tabular}

The set of 13 even prime numbers pair represents $4 \frac{1}{3}-$ dimensional space without temporal dimension. The product of 26 prime numbers in this set is:

$F_{S}=2791 \cdot 2797 \cdot 2801 \cdot 2803 \cdot 2919 \cdot 2833 \cdot 2837 \cdot 2843 \cdot 2851 \cdot 2857$

$2861 \cdot 2879 \cdot 2887 \cdot 2897 \cdot 2903 \cdot 2909 \cdot 2917 \cdot 2927 \cdot 2939 \cdot 2953$

$2957 \cdot 2963 \cdot 2969 \cdot 2971 \cdot 2999 \cdot 3001=9.642 \times 10^{89}$.

In the 13 consecutive even pairs representing $4 \frac{1}{3}-$

dimensional space, the one additional even pair served as fractal mechanism to change dimensions.

Based on $F_{S}$, two prime number products are defined as:
$F_{S 4}=2791 \cdot 2797 \cdot 2801 \cdot 2803 \cdot 2819 \cdot 2833 \cdot 2837 \cdot 2843=3.948 \times 10^{27}(25.7 \mathrm{a})$

$F_{S 9}=F_{S} / F_{S 4}=2851 \cdot 2857 \cdot 2861 \cdot 2879 \cdot 2887 \cdot 2897 \cdot 2903 \cdot 2909 \cdot 2917 \cdot 2927$.

$2939 \cdot 2953 \cdot 2957 \cdot 2963 \cdot 2969 \cdot 2971 \cdot 2999 \cdot 3001=2.442 \times 10^{62}$.

In which, $F_{S 4}$ is the product of 4 consecutive even pairs in the beginning part of $F_{S}$ and $F_{S 9}$ is the product of the other 9 consecutive even pairs.

As shown in Section 23, the product $F_{3}=5.864 \times 10^{22}$ as a $G=2$ grand number corresponds to the universe in current period. The product $F_{\text {pre }}=1.686 \times 10^{44}$ as a $G=4$ grand number corresponds to the multiverse in pre-big-bang period. 


\section{Z. Y. SHEN}

It seems natural to assume that, the product $F_{S}=9.642 \times 10^{89}$ as a $G \approx 8$ grand number corresponds to the precursor of the "super-multiverse".

Hypothesis 25.2: Super-Multiverse. There is a supermultiverse including $\sim F_{S} / F_{\text {pre }} \approx 10^{44}$, multi-universes organized in two levels, each level has $\sim 10^{22}$ member multiverse. Our multiverse is one member of the lower level. The total number of universes in the super-multiverse is $\sim F_{S} / F_{3} \approx 10^{66}$.

Hypothesis 25.2 serves as a foundation to explore possible physics implications of $F_{S}$ related issues.

Hypothesis 25.3: Big Start. The super-multiverse started at the big start, which is the beginning of time. Before the big start, everything was static without any variation.

Time started at the big start, everything started to develop.

Before the big-start, the $4 \frac{1}{4}$ - dimensional space corresponding to $F_{S}$ without temporal dimension was static, in which everything was at a standstill without any movement. The mass/energy was "frozen".

The big start was the beginning of time, temporal dimension split from the $4 \frac{1}{4}$-dimensional space. The $(3+1)$-dimensional space-time corresponding to $F_{S 4}$ was born. The mass/energy carried out by $F_{S 4}$ became dynamic and the super-multiverse started to evolve. The mass/energy corresponding to $F_{S 9}=F_{S} / F_{S 4}$ served as the precursor of dark energy and dark matter. The mass/energy carried out by $F_{S 4}$ served as the precursor of ordinary matter/energy, which was the "raw material" of the ordinary elementary particles and energy to build the planets, stars and galaxies in the super-multiverse.

The concept of changing space-time dimensions is checked with two ways. One way is given by $F_{S 9}$ in (25.7b). The other comparative way is to treat spatial dimensions as the exponentials of $F_{S}$. As space changed from $4 \frac{1}{3}$ dimensional to 3-dimensional:

$$
\left[F_{S}\right]^{\frac{3}{4 \frac{1}{3}}}=\left[9.42 \times 10^{89}\right]^{\frac{3}{4 \frac{1}{3}}}=1.98 \times 10^{62} .
$$

Comparing to $F_{S 9}=2.442 \times 10^{62}$, the result of (25.8) has a relative deviation of $18.9 \%$. For two numbers' difference as large as 62 orders of magnitude, the $18.9 \%$ relative deviation serves as a check for the validity of changing space-time dimensions based on three products $F_{S 4}, F_{S 9}$ and

$F_{S}=F_{S 4} F_{F 9}$.

After the big-start, according to Hypothesis 25.2, the super-multiverse kept expanding and splitting to form multilevel multiverses and universes. The mechanism of space expansion is Planck cubes' splitting. As the original one super Planck cube split into $N$ Planck cubes, the mass/energy in a Planck cube decreases according to $N^{-1}$ rate, and the mass/energy density decreases according to $N^{-2}$ rate. Correspondingly, the ratio of the super Planck density at bigstart versus the ordinary mass/energy density in the current universe is estimated as:

$$
R=\left[\frac{F_{S}}{F_{S 4}}\right]^{2}=\left[F_{S 9}\right]^{2}=5.965 \times 10^{124} .
$$

Notice that, in the two previous estimations, the theoretical values of (24.25), (25.1) and the observed ratio

$R_{\rho}=\frac{\rho_{\text {Planck }}}{\rho_{\text {obserbed }}}=3.271 \times 10^{123}$ of (24.24) all are based on

Planck density versus overall density including the contributions of ordinary matter, dark matter and dark energy. According to cosmic observation and cosmic MBR data, the universe contains $4 \%$ ordinary matter, $23 \%$ dark matter and $73 \%$ dark energy. Taking this factor into account, the value of (25.9) should be adjusted as:

$$
\cdot R^{\prime}=R\left(\frac{4 \%}{73 \%}\right)=\left[\frac{F_{S}}{F_{S 4}}\right]^{2}\left(\frac{4 \%}{73 \%}\right)=\left[F_{S 9}\right]^{2}\left(\frac{4 \%}{73 \%}\right)=3.268 \times 10^{123}
$$

Compare the value of (25.10) with $R_{\rho}=3.271 \times 10^{123}$ of (24.24), the theoretical result $R^{\prime}=3.268 \times 10^{123}$ has a relative deviation of $9.17 \times 10^{-4}$.

Three theoretical results for the dark energy hierarch problem are listed in Table 25.4 to compare with observed data.

Table 25.4: Theoretical Results for Dark Energy Hierarch Problem Compare to Observed Data

\begin{tabular}{|l|l|l|l|l|}
\hline Formulas & $\begin{array}{l}\text { Formula } \\
\text { numbers }\end{array}$ & Results & $\begin{array}{l}\text { Relative } \\
\text { discrepay }\end{array}$ & Theoretical basis \\
\hline$\left(\frac{\hat{\lambda}_{0}}{L_{P}}\right)^{2}\left(\frac{\alpha}{4 \pi^{2}}\right)$ & $(24.25)$ & $3.685 \times 10^{123}$ & $12.6 \%$ & $\begin{array}{l}\text { cMBR frequency spectrum } \\
\text { center wavelength }\end{array}$ \\
\hline$\left(\frac{\hat{\lambda}_{\bar{V} 2}}{L_{P}}\right)^{2}$ & $(25.1)$ & $5.582 \times 10^{123}$ & $70.6 \%$ & $\begin{array}{l}\text { Eigenstate anti-neutrino } \\
\bar{v}_{2} \text { Compton wavelength }\end{array}$ \\
\hline$\left(\frac{F_{S}}{F_{S 4}}\right)^{2}\left(\frac{4 \%}{73 \%}\right)$ & $(25.10)$ & $3.268 \times 10^{123}$ & $9.17 \times 10^{-4}$ & $\begin{array}{l}\text { Prime numbers products } \\
F_{S} \text { and } F_{S 4}\end{array}$ \\
\hline$R_{\rho}=\frac{\rho_{\text {Planck }}}{\rho_{\text {observed }}}=3.271 \times 10^{123}$ & \multicolumn{3}{|c|}{$\begin{array}{l}\text { Original discrepancy: } \\
123 \text { orders of magnitude }\end{array}$} \\
\hline
\end{tabular}

The comparison says all.

As shown in Section 22, the legitimacy of three cosmic periods, Period-I, Period-II and Period-III*, were verified by (22.9), (22.10), (22.11) and summarized in Conclusion 22.2. It shows that, the prime number corresponding to these three periods are closely correlated to the prime number corresponding to current period, Period-III. Let's verify the legitimacy of the big start with prime number products $F_{S}$.

The following formulas are based on the prime numbers corresponding to cosmic periods listed in Table 22.1 and Table 25.3.

The first prime number in product sequence $F_{S}$ is 2791 , which is closely correlated to the prime numbers 
corresponding to Period-III, Period-II, Period- $\mathrm{I}^{-}$and PeriodIII*.

$$
\begin{aligned}
2791 & =[1+2+3+5+7+11+13]_{\text {Period-III }} \\
& +[101+103+107+109+113]_{\text {Period-II }} \\
& +[179+181+191+193+197]_{\text {Period-I }^{-}} \\
& +[239+241+251+257+263]_{\text {Period-III }} \\
& +(1+2+3)+(7+11) .
\end{aligned}
$$

The rules in (25.11) are similar to the rules in (22.9). As shown on right of (25.11), prime number 2791 is the sum of 4 sets of beginning prime numbers in parenthesis for Period-III,

Period-II, Period- $\mathrm{I}^{-}$and Period-III* plus $(1+2+3)$ and $(7+11)$. The repeat of three prime numbers $(1+2+3)$ represents the fact that, the space corresponding to product sequence $F_{S}$ is $4+\frac{1}{3}$ dimensional. The repeat of two prime numbers $(7+11)$ represents the fact that, there is a temporal dimension hidden in the product sequence $F_{S 4}$. For the Period- $\mathrm{I}^{-}$, the two prime numbers 167 and 173 at beginning of the sequence are omitted to transfer Period-I with $\left(1+\frac{1}{3}\right)$-dimension space into

Period- $\mathrm{I}^{-}$with 1-dimension space to avoid redundancy. Otherwise, the sum of Period-I and Period-II would be $\left(3+\frac{1}{3}\right)$ -dimension space contradictory to Period-III with 3dimensional space.

The last prime number in product sequence $F_{S}$ is 3001, which is closely correlated to the prime numbers corresponding to Period-III, Period-II, Period- $\mathrm{I}^{-}$and PeriodIII*.

$$
\begin{aligned}
3001 & =[61+59+53+47]_{\text {Period-III }} \\
& +[157+151+149+139]_{\text {Period-II }} \\
& +[199+197+193+191]_{\text {Period-I- }} \\
& +[347+337+331+317]_{\text {Period-III* }} \\
& +(43)+(19)+(5)+(1+2+3) .
\end{aligned}
$$

The rules in (25.12) are similar to the rules in (22.10). The order of summation in (25.12) is backwards from the last prime numbers $61,157,199,347$ of prime numbers sets corresponding to Period-III, Period-II, Period- I $^{-}$, Period-III* and consecutively takes the next ones. After the backwards consecutive summation ended, it jumps to the prime number(s) shown in parenthesis. For Period-III, 43 is the prime number assigned to $t_{r}$ as the up type quark of the $3^{\text {rd }}$ generation, 19 is the prime number assigned to $c_{r}$ as the up type quark of the $2^{\text {nd }}$ generation. Prime number 5 and the set of three prime numbers $(1+2+3)$ is similar to the prime number 7 and $(1+2+3)$ in formula (22.10).

Besides 2791 and 3001, the rest 24 prime numbers $P_{i}$ in product sequence $F_{S}$ are expressed as the same form as (22.11).

$$
P_{i}=2791+x+y, i=2,3 \cdots 25 .
$$

In which $x$ and $y$ are two prime numbers selected from the set of odd prime numbers from 1 to 147 in two prime numbers sequences corresponding to Period-III and Period-II.

Conclusion 25.2: Based on (25.11), (22.12), (25.13), the set of even paired prime numbers started from $2791 \& 2797$ ended at $2999 \& 3001$ corresponding to the big start are based on the original 4 sets prime numbers corresponding to Period-III, Period-II, Period- I $^{-}$and Period-III*.

Conclusion 25.2 supports Hypothesis 25.2 and related issues of the big start related to $F_{S}$.

From SQS theory perspective, some mathematical issues may have important physics implications. Examples are given as follows.

1. Besides the three finite sporadic Lie groups, $M$-group, $B$ group, and Suz-group, are there any other finite sporadic Lie groups also related to SQS theory?

2. What type group, sub-group or something else correlates to three sets of prime numbers corresponding to the first period, second period, pre-big-bang period and the precursor of the big-start? In fact, there are clues. One clue is related to $F_{S}$ and other even paired prime numbers sequences $F_{I I I}=F_{3}, F_{I I}, F_{I}, F_{p r e}$ :

$$
A=F_{S} \cdot F_{I I I} \cdot F_{I I} \cdot F_{I} \cdot F_{p r e}=1.979 \times 10^{200} .
$$

In which, $F_{S}=9.642 \times 10^{89}$ for pre-big-start is given by (25.6), $F_{I I}=F_{3}=5.864 \cdot 10^{22}$ for current period and $F_{\text {pre }}=1.686 \cdot 10^{44}$ for pre-gig-bang period are listed in Table 23.3, $F_{I}$ and $F_{I I}$ are the products for two sets of prime numbers sequences corresponding to cosmic Period-I and Period-II:

$F_{I}=167 \cdot 173 \cdot 179 \cdot 181 \cdot 191 \cdot 193 \cdot 197 \cdot 199=1.353 \times 10^{18},(25.15 \mathrm{a})$

$F_{\text {II }}=101 \cdot 103 \cdot 107 \cdot 109 \cdot 113 \cdot 127 \cdot 131 \cdot 137 \cdot 139 \cdot 149 \cdot 151 \cdot 157$

$=1.534 \times 10^{25}$

The prime numbers product sequences for three sporadic groups $S u z$-group, $B$-group and $M$-group as listed in Table 23.3 have the following products square:

$$
B=\left(F_{S z} \cdot F_{B} \cdot F_{M}^{\prime}\right)^{2}=6.547 \times 10^{200} .
$$

In which $F^{\prime}{ }_{M}=17 \times F_{M}=1.374 \times 10^{55}$ is given by (23.32) based on an argument to multiply an additional prime number factor 17 to the product sequence of $M$-group. The results of (25.14) and (25.16) only deviate by a factor of $A / B=0.302$ for two grand numbers in the orders of $10^{200}$. If this is not a coincidence, it supports the cosmological roles played by newly found 13 even pairs of prime numbers corresponding to $F_{S}=9.642 \times 10^{89}$.

Moreover, there are three additional clues.

$$
\begin{array}{ll}
A: & F_{I I I} \cdot F_{I I} \cdot F_{I} \cdot F_{p r e}=2.052 \times 10^{110}, \\
B: & \left(F^{\prime}{ }_{M}\right)^{2}=1.887 \times 10^{110},
\end{array}
$$



$A / B:\left(F_{\text {III }} \cdot F_{I I} \cdot F_{I} \cdot F_{p r e}\right) /\left(F^{\prime}{ }_{M}\right)=1.087$.
A: $\quad F_{\text {III }} \cdot F_{\text {pre }}=9.887 \times 10^{66}$,
$B: \quad\left(F_{B}\right)^{2}=1.726 \times 10^{67}$,
$A / B:\left(F_{\text {III }} \cdot F_{\text {pre }}\right) /\left(F_{B}\right)^{2}=0.573$.
A: $\quad F_{\text {III }}=5.864 \times 10^{22}$,
$B: \quad\left(F_{S z}\right)^{2}=2.01 \times 10^{23}$,
$A / B:\left(F_{I I I}\right) /\left(F_{S z}\right)^{2}=0.292$.

In which, $A$ is the products of prime numbers sequence for different cosmic period(s) shown in Section 22; $B$ is the squared product of corresponding sporadic Lie group(s) shown in Section 23; $A / B$ is the ratio serving as the relative discrepancy factor. If all these are not by coincidence, there are some implications: (1) There is a general relation between the products of prime numbers sequence(s) corresponding to cosmic period(s) and squared product(s) of sporadic Lie group(s); (2) Grand number phenomena show up in many different areas; (3) It seems reasonable to modify $F_{M}$ by multiplying 17 as $F^{\prime}{ }_{M}=17 \times F_{M}$. But it raises interesting questions: Why the prime number 17 is so special? There is a clue: $a^{b}+b^{a}=c^{2}, a=2, b, c$ are prime numbers. (25.20) Checked for $b \leq 53$, only two sets of prime numbers fit (25.20).

$a=2, b=1, c=3: \quad 2^{1}+1^{2}=3$;

$a=2, b=3, c=17: 2^{3}+3^{2}=17$.

So 17 is a special prime number.

3. What is the physics implication of the $j$-function closely related to $M$-group?

4. The Riemann conjecture has some hidden physics significance. Physicists have speculated: The zeros of the $\zeta$-function might relate to some particles' spectrum. For SQS theory, $\left|D S_{k}(k)\right|$ serving as particles' spectrum for mass and decay times also has a series of local minimums. Are they somehow correlated to $\zeta$ function? Riemann hypothesis is based on the zeta function:

$$
\zeta(s)=\sum_{n=1}^{\infty} \frac{1}{n^{s}} .
$$

In which, $s$ are complex numbers. A connection between zeta function and prime numbers was discovered by Euler, who proved the identity:

$$
\sum_{n=1}^{\infty} \frac{1}{n^{s}}=\prod_{p=p \text { rimes }} \frac{1}{1-p^{-s}} \text {. }
$$

In which, $p$ are prime numbers greater than 1 . Does it mean something for SQS theory?

5. Mathematics helped SQS theory tremendously. Are there any other mathematics related to SQS theory? For instance, golden ratio, fractals, Fibonacci numbers, Morley theorem etc.
6. Beyond the set of consecutive even pairs listed in Table 25.3, are there other sets of even pairs qualified for cosmological periods? If they are, what do they mean? These are important issues for mathematicians, physicists and cosmologists to find out and thank about.

It is important to point out that, except electron, the way to derive parameters from models for other particles is only for demonstration purpose. It is by no means the final version. In fact, the selection of $p$-parameters is still an open issue. Moreover, other than electron, the way to calculate particles geometrical parameters on the outer half of torus $x-z$ cross section based on normalization $\sqrt{g^{2}+g^{\prime 2}}=1$ is optional. For the inner half, there are also some optional issues. For the primary basic equations (24.48), solutions are not available yet. In essence, SQS theory is still a developing theory. The final version is waiting for these open issues and options to settle down. What this paper did is only scratching the surface. There are many open areas left to be explored.

Simplicity is the guideline to develop SQS Theory. The phenomena in Physics world are complicated. The physics basic principle is simple. The deeper the level the simpler it is. At the deepest level, the basic principle should be the simplest. SQS Theory serving as a version of unified field theory has three fundamental postulation: the Gaussian Probability Postulation, the Prime Numbers Postulation and the Vacuon Postulation to begin with. After the establishment of SQS theory basic equation, the basic principles become even simpler. The Prime Number Postulation actually should derive from the set of primary basic equations (24.48) as the necessary condition for its stable or quasi-stable solutions. Moreover, since the vacuon serving as the basic unit of space and the carrier of Gaussian probability, the Vacuon Postulation can be combined with the Gaussian Probability Postulation. In other words, SQS theory ultimately has only one first principle, the Gaussian Probability Postulation, which provides our basic understanding of the space. The three basic physics units: Planck length, Planck time and Planck energy (Planck mass) are another example of simplicity. In essence, SQS theory is a mathematic theory. Its physics meaning is interpreted in terms of these three basic units. In principle, no more physics input is needed. In this (25.22) regard, Planck contributed most. He not only is the one found the Planck constant $h$, but also is the one to point out that three Planck units can be defined by three basic physics constants: $h, c$ and $G$.

The final goal for SQS theory is to unify all interactions and all elementary particles into a self-consistent theory based on the first principle. It tells the whole story. It is exciting, but there is a long journey to go. A gold mine is out there. Let's go for it.

\section{Section 26: Suggested Experimental Verifications for SQS Theory Predictions}


SQS theory is such a different theory from other particle physics and cosmology theories. Extraordinary claims require extraordinary evidence. SQS theory provides twenty five predictions for experimental and/or observational verifications.

1. SQS theory predicts the mass of the scalar boson $U_{1}$ responsible for electroweak unification as:

$$
M_{e w}=152.754(5) \mathrm{GeV} / \mathrm{c}^{2} \text {. }
$$

The uncertainty of (15.5) comes from two sources: one is the relative accuracy of proton mass $M_{p}=938.272013$ (23) (PDG 2010 data) in $10^{-8}$ order, the other one is the relative accuracy of $\pi\left(x_{b}\right)=20.3701945(6)$ limited by the 16-digit numerical calculation, which is in $10^{-9}$ order. So the uncertainty of $M_{e w}$ given in (15.5) is a conservative one. The predicted value of $M_{e w}$ is within the energy range of LHC.

2. According to (18.11), the hypothetical neutral gauge boson $X^{o}$ with mass $M_{X}=9.94690465 \mathrm{GeV} / \mathrm{c}^{2}$ probably has a very narrow width. It is worth the effort to find it; after all it is an elementary particle. After the $X^{o}$ boson is found, the next thing is to find out the value of two parameters $g_{s}$ and $q_{s}$ defined as two sides of the $S$-triangle, which may provide some insights related to hadrons decay with the $X^{o}$ boson involved.

3. The hypothetical neutral scalar boson $M_{M s}=M_{f} \oplus \bar{M}_{f}$ is a composite particle with predicted mass

$$
M_{M s}=115.074032 \mathrm{GeV} / \mathrm{c}^{2} \text {. }
$$

The uncertainty of the mass value depends on the accuracy of electron's mass in the order of $\sim 10^{-8}$.

4. In Section 14, SQS theory predicted 8 hypothetical neutral scalar boson massons, $G_{i},(i=1,2,3 \cdots 8)$. The mass values listed in Table 14. 4 are within LHC capability. But there are some concerns of how to identify them. It is possible that, massons are attached to $W, Z, X$ gauge bosons serving as part of their mass. If this is the case, massons are not detectable as individual particles. Nevertheless, there is a chance providing an indirect way to test the effects of two heavy massons, $G_{7}$ and $G_{8}$ each with mass values around $342 \mathrm{GeV} / \mathrm{c}^{2}$. If they indeed attach to $W$ or $Z$, it is conceivable to find two resonances around or higher than $342 \mathrm{GeV} / \mathrm{c}^{2}$ as the high energy states of $W$ or $Z$. So there are two possibilities: One is to find all eight massons with their predicted mass listed in Table 14.4, the other is to find the effects of two heavy massons $G_{7}$ and $G_{8}$ attached to $W$ or $Z$. Either way serves as the evidence of hypothetical massons.

5. More accurate experiments are needed to determine the mass and mixing angle of eigenstate neutrinos, which can be used to verify the three flavored neutrinos' mass values of (17.4) as well as the estimated mass relations of (17.23) predicted by SQS theory.

6. As discussed in Section 5, photons' speed varies with its frequency (energy) predicted by dispersion equation (5.7). Since the dispersion effect is in the $\sim 10^{-56}$ order for visible lights, it is impossible to test by experiments in that wavelength range. The generalized dispersion equation (25.4) is relatively easier for experimental verification. Hopefully GRB090510 type of $\gamma$-ray bursts with much higher energy and longer distance can provide chances for verification.

7. The long path concept originated from the Random Walk Theorem is very important in many senses. In case of entanglement, the extended long path makes the physics link between entangled particles. Along the long path, interactions and information are transmitted with superluminal speed many orders of magnitude faster than $c$, which was recently confirmed by experiment [9]. It serves as evidence for the superluminal speed. For direct evidence of the long path, one way is to confirm the maximum distance $d_{\max }=(2+1 / 4) \lambda_{0}^{2} / L_{P}$ between a pair of entangled photons. For an experimentally manageable distance $d_{\max }=1 \mathrm{~km}$, the required wavelength is in $8.475 \times 10^{-17} \mathrm{~m}$ range corresponding to $\gamma$-ray with energy of $14.629 \mathrm{GeV}$. The alternative way is to use a pair of entangled charged nucleons. The manageable distance can be increased by circulating the entangled nucleons in a ring with extremely high vacuum to prevent de-coherency. Such types of experiments are not easy, but it is worth the effort. Because so many things are based on the long path concept.

8. According to SQS theory, entangled photons have entanglement red shift and de-coherent blue shift. If some photons of the cosmic microwave radiation (MBR) were entangled to begin with, their entanglement red shifts and de-coherent blue shifts provide opportunities to verify them as well as for the stretched out long path for cross universes links. The cosmic MBR is the relic of high temperature radiations in the early universe with blackbody radiation spectrum. Due to the expansion of space, the MBR spectrum went through tremendous red shift and its temperature is lowered to $2.725 k$. The measured cosmic MBR kept the blackbody spectrum. According to the discussion in Section 5, the entanglement red shift is frequency (wavelength) dependent causing distortion to the blackbody spectrum. Comparing to the tremendous red shift caused by space expansion, the distortion is extremely small even for the maximum red shift of $\lambda_{\max }=1.5 \lambda_{0}$ according to (5.19). The de-coherent blue shift is unique. According to (5.22) and (5.24), the range of blue shifts for the photon far away from decoherence location is from 1.5 times to 2 times of its 
frequency. The red shifts and blue shifts have cancelation effect in the spectrum. It causes the difficulty to verify them. Fortunately, the highly raised peak of blackbody spectrum provides a mechanism to reduce the cancelation effect. Over all, the combination of entanglement red shifts and de-coherent blue shifts causes the cosmic MBR spectrum deviated from the blackbody spectrum such that, its lower frequency portion has tiny gain due to entanglement red shifts and its higher frequency portion also has tiny gain due to de-coherent blue shifts, while its middle portion has deficit to pay for these gains. There are two ways for verification. One way is to compare the measurement cosmic MBR data with blackbody radiation theoretical spectrum to find the deviation in different portions. The other way is based on statistic analysis of the measured cosmic MBR data. Since the stochastic nature of blackbody radiation and the randomness of measurements' errors, the measured cosmic MBR data should have no statistic correlation among different frequency components. That is the scenario without counting the effects of entanglement red shift and de-coherent blue shift. The entanglement red shifts produce a correlation with gain for low frequency component and deficit for high frequency component in the spectrum's lower and middle portions. The de-coherent blue shifts produce a correlation with deficit for low frequency component and gain for high frequency component in the spectrum's middle and higher portions. The deviation overall effect upon the spectrum is to slightly lift up its two end portions and to slightly press down its middle portion. Due to the overwhelm effect of the space expansion red shift, the correlations and deviations are extremely small. It requires extremely sensitive and extremely high precision measurements to verify. If the entanglement red shift deviation does exist, it indicates that some photons in the cosmic MBR are still entangled. If the de-coherent blue shift deviation does exist, it indicates that some originally entangled photons in the cosmic MBR were de-coherent already. If none of these two deviations exist, it indicates that, either the photons in cosmic MBR were not entangled to begin with or the entangled photons were de-coherent shortly after their entanglement. In any case, it is worth the effort.

9. As shown in Section 17, The PDG-2010 data listed the upper limit $\Omega_{v}<0.048$ of "neutrino density" is close to the upper limit $\Omega_{b}<0.044(4)$ of baryon density in the universe, but the lower limit $0.0009<\Omega_{v}$ is far less than

$\Omega_{b}<0.044(4)$. From SQS theory standpoint, it is very important to narrow error range of the value $\Omega_{v}$ to determine the amount of $\tilde{v}_{1}, \tilde{v}_{2}, \tilde{v}_{3}$ comparing to the amount of $\Omega_{b}$. In addition, it is very important to measure the cosmology originated neutrinos' handedness to determine the percentage of $\tilde{v}_{1}, \tilde{v}_{2}, \tilde{v}_{3}$, contribute to the "missing antimatters". In case $\tilde{v}_{1}, \tilde{v}_{2}, \tilde{v}_{3}$ contribute only a part of the "missing antimatters", it provides the opportunity for other types of antimatter candidates.

10. SQS theory would like to see more accurate experimental data of the upper limit for flavored version neutrinos and anti-neutrinos electrical charge and magnetic moment to verify whether they have remnant electrical charge or not. According to Conclusion 17.1, the three eigehstate antineutrinos $\tilde{v}_{1}, \tilde{v}_{2}, \tilde{v}_{3}$ have no electrical charge. According to Theorem 17.1 and related discussions, for the flavored version $v_{e}, v_{\mu}, v_{v}$ and $\bar{v}_{e}, \bar{v}_{\mu}, \bar{v}_{v}$, SQS theory intends to favor no electrical change. Hopefully, further experiments will press the upper limits lower.

11. As discussed in Section 25, the cross-universes entangled eigenstate anti-neutrinos $\tilde{v}_{1}$ oscillates only with $\tilde{v}_{2}$ not with $\widetilde{v}_{3}$, while the regular anti-neutrinos oscillate among all three members $\tilde{v}_{1}, \tilde{v}_{2}, \tilde{v}_{3}$. The different oscillation patterns provide an opportunity for verification. Of cause, $\tilde{v}_{1}$ and $\tilde{v}_{2}$ are not directly detectable, but their information can be extracted from the detected flavored neutrinos or anti-neutrinos based on the probability matrix of (17.26) and (17.27). The key is to make sure that, the neutrinos $\tilde{v}_{1}, \tilde{v}_{2}$ are cross universes originated.

12. According to SQS theory, part of dark matter is the debris left over from the cosmos inflation. In which the majority is 2-dimensional membranes flooding around in space. The right way to detect such dark matter should base on gravitation effects. As suggested in Section 22, gravity-meters with extremely high sensitivity isolated from local interferences fit the job.

13. SQS theory predicts the mini-inflation caused by a piece of 2-dimensional dark matters membrane occasionally converting into a chunk of visible matters in the 3dimensional space. As discussed in Section 22, the unstable particles' decay modes and branching ratios are the same as the second generation particles'. In other words, the decay events happen in ordinary 3 -

dimensional space according to the same laws and rules. The way to verify this prediction is to detect the decay products in the original cosmic ray and to see whether they fits the decay modes and branching ratios of second generation particles. The most likely target of these cosmic rays source is the center region of galaxies, where the dark matter has higher density. Samuel Ting's Alfa Magnetic Spectrometer fits this job perfectly. In addition, the 2-dimensional membrane contains equal amount of particles and anti-particles, their annihilations produce a boost of high energy $\gamma$ ray. The most likely place to look for is the center region of galaxies.

14. SQS theory suggested that, there were more than one cosmic inflations happened after the big bang. These cosmic inflations should leave some traces on cosmic MBR. As shown in Section 22, the first cosmic inflation 
and the third cosmic inflation happened at $t_{1} \sim 3.8278 \times 10^{-42} \mathrm{~s}$ and $t_{3} \sim 2.072 \times 10^{-36} \mathrm{~s}$ after the big bang, respectively. It is worthwhile to look for possible traces.

15. SQS theory predicts that cosmic history went through three periods each with different dimensional spaces, $1 \frac{1}{3}$-dimensional, 2-dimensional, 3-dimensional for the $1^{s t}$ period, $2^{\text {nd }}$ period, $3^{\text {rd }}$ periods, respectively. The space with different numbers of dimensions should leave some traces on cosmic MBR map or somewhere else. When more detailed data with better precision and resolution are available, that is the place to look for.

16. According to the logistic recurrent process described in Section 16, the transition from Compton length to GUT scale, the mass of a particle such as electron or muon increases continuously. This effect may be testable. For instance, Randolf et al found that, to replace electron with muon in the hydrogen atom, some peculiar behaviors were observed including different values for proton's size and the Rydberg constant [33]. The changing mass effect due to random walk theorem may be responsible for these anomalies. It is worthwhile to check with existing experiments data and the future ones.

17. As shown in Section 7, SQS theory predicts that, muon has zero probability to decay in the time intervals $0<t<t_{\text {min }}=2075 \times 10^{-13} s$ and the probability of decay at time $t=8.0933 \times 10^{-3} \mathrm{~s}$ or longer is less than $10^{-7}$, in which time $t=0$ is set at the birth of the muon. When the muon factory is in operation, the two predictions should be relatively easy to check with experiments. If these predictions are confirmed, which serve as supportive evidences for $\left|D S_{k}(k)\right|$ as particles spectrum.

18. SQS theory suggested that, quarks with the same flavor and different colors are different elementary particles having different masses. The difficulty to confirm is due to the fact that, there is no standalone individual quark in existence. Fortunately, there is an indirect way. Numerical calculation methods such as the lattice QCD to analyze hadrons experimental data are capable to extract the mass values of their component quarks. Comparison of PDG2010 data with PDG-2008 data found more distinctive multi-peak behaviors of flavored quark's mass curves, which support SQS theory. Hopefully more experimental data and more powerful numerical computation capability for lattice QCD will finally verify the mass values of 18 quarks listed in Table 11.2.

19. SQS theory proposed the multiverse with $\sim 10^{22}$ member universes organized into two levels. There are two possible ways to verify this hypothesis. One way is to look at the cross-universe entangled eigenstate anti-neutrino $\tilde{v}_{1}$ and $\tilde{v}_{2}$ oscillation pattern as suggested in Prediction No. 11. The other way is to look at the cosmic MBR map to search for the trace of cross universes entangled photons. If such photon is found, it serves as evidence for cross universes entangled photons pair as well as for the neighbor universe in the multiverse. But one must make sure that the entanglement is cross universes. One possible way to verify is to observe the de-coherent blue shift. According to (5.24), for the de-coherence located at $d=d_{\max }$ in a neighbor universe, the originally entangled photon in our universe shows a large blue shift as its frequency suddenly doubled to $f^{\prime}{ }_{0, \max }=2 f$. It also serves as evidence for the existence of long path.

20. According SQS theory, graviton is scalar boson with spin 0 instead of tensor boson with spin 2 . The decisive proof is to find the graviton and to measure its spin. Graviton having spin 0 is based on mathematics supported by two Number Towers and independently verified by Poincare's "hairy ball" theorem. SQS theory takes conclusion 25.1 seriously. There are many existing experimental setups designed to look for gravitational wave and gravitons. Perhaps all of them are based on the assumption of graviton having spin 2 . Whether these types of setups eligible to find graviton with spin 0 or not? It is a question for experimenters and general relativity theorists to think about. The urgency is enhanced by the fact that, despite decades of extensive efforts, no gravitation wave or graviton have found. It is the time for a second thought.

21. According to Definition 25.2, Theorem 25.1 and Lemma 25.1, Lemma 25.2, when two absolute black holes collide in the proper ways, they transfer into a regular black hole with genus- 0 event horizon or a special black hole with genus- 2 event horizon. Both are eligible to have Hawking radiations. As discussed in Section 25, absolute black hole with ginus-1 event horizon has Hawking mechanism without Hawking radiation. The absolute black hole holds half photons of virtual photon pairs produced by Hawking mechanism in its event horizon. After the collision, these held photons suddenly have the chance to release. The burst of Hawking radiations caused by collision is detectable.

22. Since the gravity distribution pattern of absolute black hole with genus-1 event horizon is quite different from that of black hole with genus-0 event horizon, the different patterns provide another way to indentify the absolute black hole.

23. As shown in Section 25, GUT based on $S U$ (5) group may have a chance to revival. The key is to reinterpret the results of $I-M-B$ type and $S-K$ type experiments. It is worthwhile to consider redo these experiments with larger water tank. For instance, if the linear size of water tank is increased by 20 -fold corresponding to 8000 times more protons involved, it would be possible to see some proton decay events. But before doing that, one must make sure proton's $t_{\min }<10^{10}$ years from theoretical estimation. 
Z. Y. SHEN

24. A remote hope is to find the $e$-boson with predicted mass $M_{e, b}=3.959754 \times 10^{8} \mathrm{GeV}$, which is roughly $10^{4}$ times higher than the full scale energy of LHC. It is far beyond the current accelerator capability. But there is another way. Occasionally, a cosmic ray with extremely high energy hits earth. There is a chance that, it contains the trace for the leftover $e$-boson or its decay products from the big cosmic inflation. It is worthwhile to monitor and observe. The verification of the $e$-boson is significant. It not only will confirm electron having a high energy intermediate boson state but also will shed lights on details of the cosmos inflation. Let's keep hoping.

25. The author is confident to voluntarily offer a falsified test for SQS theory. In our universe current period, if any experiment and/or observation find additional space dimension or dimensions beyond the existing three space dimensions, SQS theory is proved to be false.

Hopefully some experimental physicists will be interested and figure out the ways to carry out these proposed experiments. The author is standing by for assistance.

\section{Section 27: Conclusions}

The conclusions of SQS theory are summarized as follows.

1. For the current cosmic period, space is a 3-dimensional continuum with Planck scale face-centered lattice as the basic building block.

2. Space is stochastic in nature with Gaussian probability distribution function attached at each discrete point, which serves as the ultimate origin of all physics uncertainties and stochastic behaviors.

3. Space is made of a collection of infinite point particles called vacuons corresponding to infinite geometrical points. Vacuons serve as the event carriers of the Gaussian probability distribution function.

4. The Gaussian sphere is defined as a sphere with radius $r=L_{P} /(2 \sqrt{2})$. The separation between two adjacent Gaussian spheres is $2 r=L_{P} / \sqrt{2}$ determined by the balance of gravity attractive and repulsive forces.

5. SQS theory is background independent. Space structure is not a priori determined. At upper level, redefined gauge tensors adjust simultaneously in according with mass and energy distribution governed by macroscopic basic equations (24.20). Inside the $M$-sphere with radius $R_{M}=71 L_{P}$, Gaussian spheres governed by gravity arrange themselves to reach minimum potential energy. At the Planck scale, redefined gauge tensors adjust simultaneously to satisfy microscopic primary basic equations (24.48).

6. Space is classified into three regions: (1) The microscopic region (2) The transitional region (3) The macroscopic region. The classification is based on mathematics and physics. The boundary of the microscopic region is set by the GUT scale of $71 L_{P}$, The boundary of the transitional region is set by the Compton scale $\lambda_{C}$, which have deep physics meanings and mathematical foundation.

7. Inside the $M$-sphere, space has Planck scale facecentered lattice structure including two parts, the cubic part and the octahedral part. Space symmetries based on its face-centered lattice structure are classified into two types, $O(r)$ and $C(r)$, each has 18 symmetries within $r \leq 3$, which serve as the origin of the symmetrical groups for elementary particles and interactions.

8. The Random Walk Theorem is proved based on Gaussian Probability Postulation. It serves as the foundation of many important issues for SQS theory. For the two long rang forces, according to Theorem 21.1, the random walk zigzagging path each step stops only at cubic vertexes.

9. Based on the Random Walk Theorem, the short path and long path are defined. Short path is the distance between two points; long path is the random walk zigzagging path connecting two points. The ratio of the long path and the short path is defined as the converting factor.

10. Converting rules based on the Random Walk Theorem are used to treat hierarchy problems. The effectiveness of these converting rules serves as physics evidence for the Random Walk Theorem.

11. SQS theory supports locality. Einstein was right: "No spooky action at a distance". A pair of entangled particles is physically linked by the stretched out long path. The transmitting speed of information and interactions along the long path between the entangled particles is superluminal but not infinite.

12. Photons have dispersion. Its speed varies with frequency (energy). The real meaning of $c$ is a basic physics constant. No photon travels with speed exactly equal to $c$. For the short path, photon travels with speed $v$ slightly less than $c$. For the long path, interactions and information transmit with superluminal speed

$\hat{v}=N v=\left(\lambda / L_{P}\right) v>>c$. Dispersion equation is given by (5.7) and generalized by (25.4) serving as revision of special relativity.

13. The 1-dimensional $S$-equation is defined based on Gaussian Probability Postulation. It serves as the base for a series of secondary $S$-equations with impacts on different areas.

14. In $x$-axis region [0,0.25], the $S$-equation's solution $x_{c}=0.24998715627302645$ is a very special point. It provides the mass value of two scalar bosons $U_{1}, U_{2}$, and to some extent the mass value of top quark and three gauge bosons $X, W^{ \pm}, Z^{0}$.

15. For the 3-dimensional Gaussian probability distribution function, its standard deviation has three values, $\sigma_{0}=1 / \sqrt{2 \pi}, \sigma_{2}=e^{i 2 \pi / 3} / \sqrt{2 \pi}, \sigma_{3}=e^{i 4 \pi / 3} / \sqrt{2 \pi}$, 
corresponding to the three branch points on Riemann surface. According to Roger Penrose, the three branch points plus another one at infinity serve as the ends of the two pairs of cuts on Riemann surface two sheets. The edges of two pairs of cuts are glued together, it makes the two-sheet Riemann surface topologically equivalent to a torus with four tiny holes. The torus serves as the basic building block of models for Dirac type fermions and some bosons with mass.

16. Dirac type fermions have two components geometrically represented by two loops. Loop-1 is the torus center cycle, and loop-2 is perpendicular to loop-1 and has its center at the circumference of loop-1.

17. According to theorem 18.1, Lemma 18.1 and Lemma 18.2, the vacuons as point particles with non-infinite speed only take discrete 1-dimensional trajectories. It serves as the basic quantization of space for SQS theory.

18. Dirac type elementary fermion is represented by a set of trajectories on its torus based model. Along a trajectory, there are a combination of three types of internal movements: a cyclic movement along loop-1, another cyclic movement along loop-2, and an oscillation along the trajectory path.

19. According to Theorem 18.1 Lemma 18.1 and Lemma 18.2, particle's trajectory must be 1-dimensional and it changes trajectory only by jumping not by shifting. The movements in a trajectory are totally deterministic and the uncertainty only comes from jumping trajectories.

20. A set of three numerical parameters, $m, n, p$ is assigned to each Dirac type fermion. The ratio of $n / m$ equals to the length ratio of loop-2 to loop-1 for the fermion's torus model. The ratio $p / n$ equals to the ratio of fermion's mass to electron's mass. The value of numerical parameters, $m, n, p$ are ultimately determined by corresponding solutions of primary basic equations (24.48).

21. According to Theorem 3.1, the 1-dimensional $S$ equation defined $\pi\left(x_{1}\right)$ and $\pi\left(x_{2}\right)$ serve as messengers carrying curvature information to the torus model to define the locations of characteristic points on its surface.

22. On the $x$-axis, region $x_{c}<x<x_{d}=0.5-x_{c}$ is defined as the gauge boson region. Fermion's $x_{1}$ and $x_{2}$ in this region with mass $M>M_{\text {Max }}=4.97323432 \mathrm{GeV} / \mathrm{c}^{2}$ must be paired with anti-fermion as a boson state.

23. Elementary particles are represented by trajectories on models with genus numbers of $0,1,2,3$ as listed in Table 25.2.

24. On its model surface, fermion's trajectory is made of two connected geodetics between two characteristic points $A$ and $B$ defined by $\pi\left(x_{2}\right)$ and $\pi\left(x_{1}\right)$, respectively. The value of $\pi\left(x_{1}\right)$ and $\pi\left(x_{2}\right)$ are determined by fermion's mass and the 1-dimensional $S$ equation. The values of these geometrical parameters are ultimately determined by corresponding solutions of primary basic equations (24.48).

25. On fermion's torus model outer half surface, the characteristic points $A$ defines the $G W S$-triangle and $S$ triangle, which provide geometrical parameters for electromagnetic and weak interactions. On fermion's torus model inner half surface, the characteristic points $B$ defines the $C K M$-triangle corresponding to hadrons decay modes. These characteristic points and triangles represent the geometric parameters for the particle, which serve as the origin of particle's physics parameters. The values of these geometrical parameters are ultimately determined by corresponding solutions of primary basic equations (24.48).

26. The $A T$-equation and $P S$-equation with the mass term $\sin \left(\frac{2 p}{m} \theta\right)$ to break the $\vartheta= \pm 120^{\circ}$ symmetry, which serves as the mechanism to provide particle's mass. Despite their ad hoc origin, the results derived from $A T$ equation and $P S$-equation are agreed to experiment data with reasonable accuracy.

27. According to Postulation 11.1, quarks with the same flavor and different colors are different elementary particles with different parameters. Accordingly, there are eighteen quarks instead of only six. It is supported by two independent experimental evidences.

28. The Prime Numbers Postulation along with even pairing rule plays pivotal roles in many areas including elementary particles and cosmology. The 18 least odd prime numbers $1,3,5,7,11,13,17,19,23,29,31,37$, $41,43,47,53,59,61$ paired as even pairs are assigned as the $m$-parameters of 18 quarks, $u_{r}, d_{r}, d_{g}, u_{g}, d_{b}$, $u_{b}, s_{r}, c_{r}, s_{g}, c_{g}, s_{b}, c_{b}, b_{r}, t_{r}, b_{g}, t_{g}, b_{b}, t_{b}$, respectively. Quarks $n$-parameters are also selected from prime numbers.

29. There are only three generations of elementary particles. The fourth generation and beyond are strictly forbidden by mathematics.

30. Lepton's before reduction $m$-parameter equals to the average value of corresponding up type and down type quarks $m$-parameters. All leptons reduced $m$-parameters equal to 1 corresponding to spin $\hbar / 2$. Leptons' reduced $n$-parameters are fractional, which play a pivotal rule for weak interactions and serve as mathematical distinction between leptons and quarks.

31. The $n$-parameters for the flavored version neutrinos and anti-neutrinos, $v_{e}, v_{\mu}, v_{\tau}, \bar{v}_{e}, \bar{v}_{\mu}, \bar{v}_{\tau}$, are determined by the matching rule of (17.1), which matches the $n$ parameter of corresponding charged lepton. The weak interactions are classified into two types. The ordinary type meets the matching rule and satisfies the lepton family numbers conservation law. The rare type violates the matching rule and the lepton family numbers conservation law. 
32. According to SQS theory, the eigenstate neutrinos $\tilde{v}_{1}$, $\tilde{v}_{2}, \tilde{v}_{3}$ all are Marjorana type, which are the ones flying around in space. The flavored version neutrinos and anti-neutrinos $v_{e}, v_{\mu}, v_{\tau}, \bar{v}_{e}, \bar{v}_{\mu}, \bar{v}_{\tau}$ as Dirac type only exist at the birth or been detected. The relation between the eigenstate version and the flavored version neutrinos is determined by converting probability matrixes of (17.26) and (17.27). This scheme eliminated the chaser's paradox and provided a candidate for the missing antimatters.

33. Photon as a gauge boson with spin $\hbar$ has single closed loop structure.

34. The eight gluons are made of corresponding quark and anti-quark of the same type: $g_{i}=q_{j} \bar{q}_{j},(i=1,2, \cdots 8)$, $\left(j=d_{r}, d_{g}, u_{g}, d_{b}, u_{b}, s_{r}, s_{g}, s_{b}\right)$. Strong interactions are mediated by eight gluons and classified into two types. The ordinary type mediated by gluons with the same handedness and the weakened type mediated by gluons with different handedness.

35. Based on Theorem 13.2, SQS theory provided a simplified way to treat certain composite particles such as proton, neutron, and some light nucleons. It shows some promises in terms of interpretation for the binding forces for these composite particles based on simple mathematic rules.

36. Graviton has spin 0 represented by cutoff loop with length $2 L_{P}$, which is supported by Poincare's "hairy ball" theorem. The cutoff loops move through the longpath and only stops at cubic vertexes serving as the building blocks for space with cubic lattice structure.

37. Bosons with mass are made of combination of their fermions constituents, and so are their trajectory and model.

38. The unifications for electro-weak, electroweak-strong, and grand unification of all four interactions including gravitation occur at energy scales of $152.7547 \mathrm{GeV}$, $8.44708 \times 10^{15} \mathrm{GeV}, 1.07948 \times 10^{18} \mathrm{GeV}$, respectively.

39. Logistic recurrent process plays an important role to drive the converting factor as a running constant from grand unification scale to Compton scale with important impacts on elementary particles and interactions.

40. Grand number phenomena with rank- $G$ based on $\sim 10^{11 G}\left(\sim 2^{37 g}\right)$ are found in many areas including elementary particle physics and cosmology. Grand number phenomena are intrinsically related to logistic process, random walk, group theory and Gaussian probability.

41. SQS theory provides the Elementary Particles Table including 72 particles. The number 72 is supported by two Number Towers with the special prime number 163 on top. It is an indication of mathematics at work.

42. In the deeper level, there is only one elementary particle, vacuon. All elementary particles and interactions are ultimately originated from different patterns of vacuons movements.

43. SQS theory provides a simple formula $\alpha(M)=[\bar{\pi} / 71]^{2}$ to determine the values of fine structure constant $\alpha(M)$ at different mass scales. At three mass scale, $M_{e}, M_{Z}$ and $M_{G U T}$, it provided reasonably accurate results. $\alpha(M)$ as a physics running constant is originated from $\bar{\pi}$ as a mathematic running constant.

44. According to the Prime Numbers Postulation and Postulation 22.1, cosmic history is intrinsically related to elementary particles and both are based on prime numbers. Three more sets of prime numbers were found corresponding to three cosmic periods, the $1^{\text {st }}$-period, the $2^{\text {nd }}$-period, and the before-big-bang-period. The legitimacy of three sets of prime numbers has additional support from the correlations given by (22.9), (22.10), (22.11) and Conclusion 22.2.

45. According to SQS theory, started from the big bang, three space phase transitions occurred at $t_{1}=3.8278 \times 10^{-42} s, t_{2}=4.8963 \times 10^{-40} s$, and $t_{3}=2.072 \times 10^{-36} \mathrm{~s}$. The one occurred at $t_{3}$ caused the big cosmic inflation suggested by cosmology standard model.

46. According to SQS theory, electron has an intermediate boson state. The $e$-bosom, with mass

$M_{e-b}=3.95987106 \times 10^{8} \mathrm{GeV} / \mathrm{c}^{2}$ serves as the inflaton to drive the cosmic inflation. When the inflaton decayed, the inflation stopped.

47. Based on the $O(r)$ and $C(r)$ symmetries in the facecentered space, the elementary particles and interactions in the cosmic periods are explained naturally. The $O(r)$ and $C(r)$ symmetries also serve as the guideline for the solutions of primary basic equations (24.48).

48. At least part of dark matters is 2-dimensional debris left over from cosmic inflation, which only interact with ordinary matters via gravity. Two suggestions are given for verifications.

49. Based on Definition 22.2 and Hypothesis 22.2 and the "negative prime numbers table", SQS theory proposed a cyclic universe model. In which the negative prime numbers sequences represent a cosmic contracting period ended up with the big crunch. The post-big-crunch universe transfers via a time tunnel into the pre-big-bang universe, and a new round of cosmic cycle starts.

50. SQS theory supported the multiverse concept as a possible way to resolve the second thermodynamic law problem during the cosmic contracting period. Based on grand numbers and the two prime numbers sequences, number of universes in the multiverse is estimated around $10^{22}$ organized in two levels.

51. SQS theory proposed the limited anthropic principle. For any member universe in the multivers during its third period, 
the change of physics laws and the variation of physics constants are not arbitrary; they subject to strict restrictions imposed by the same set of $18+1$ prime numbers of our current universe.

52. Close correlation between the prime numbers sequence of three finite sporadic Lie groups, $M$-group, $B$-group, Suzgroup and the $18+1=19$ prime numbers assigned as the $m$ parameters for the 18 quarks and electron was found, in which the missing prime numbers in the sequence are correlated to some top type quark's boson states. The correlation provides additional support to the Prime Numbers Postulation.

53. Based on the largest prime number 71 in the prime numbers sequence of $M$-group, the $M$-sphere with radius $r=71 L_{P}$ is defined. Inside the $M$-sphere, two more spheres, the $B$-sphere with radius $r=47 L_{P}$ and the $S$ sphere with radius $r=23 L_{P}$ are defined. Between $M$ sphere and $B$-sphere is the "quark-antiquark liquid state" region; inside the $S$-sphere all elementary particles are distinguishable.

54. Poincare's "hairy ball" theorem and related rules play important roles for elementary particles in terms of trajectories, models and interactions as well as for cosmology. As shown in Section 25, black hole has hairs and must have crowns, cross universes connections are necessary, and graviton must have spin 0 . These are the conclusions supported by Poincare's "hairy ball" theorem and related rules. It is mathematics at work.

55. Based on Poincare's "hairy ball" theorem, SQS theory proposed the absolute black hole with no radiation of any kind and the suggestions for verification.

56. Another set of 13 even pairs of prime numbers as listed in Table 25.3 is found. According to Hypothesis 25.2, it corresponds to a super-multiverse including $\sim 10^{44}$ member multiverses organized in two levels, each level has $\sim 10^{22}$ member multiverse. Hypothesis 25.2 is supported by grand numbers as well as its estimates for dark energy hierarchy problem agreed with other estimates and observed data as listed in Table 25.4.

57. Based on Einstein equation (24.1b) for vacuum with redefined gauge tensors attached to probability, SQS theory established two sets of basic equations. The macroscopic set includes equations (24.20) for gravity and equations (24.42) for electromagnetic force. The microscopic set is equations (24.48), which serve as the primary basic equations representing all elementary particles and interactions as well as things on upper levels.

58. In essence, SQS theory is a mathematic theory with physics interpretations. Based on three basic physics constants $c, h, G$ or equivalently $L_{P}, t_{P}, E_{P}\left(M_{p}\right)$. In principle, all physics parameters can be derived from mathematics with $c, h, G$ or $L_{P}, t_{P}, E_{P}\left(M_{p}\right)$ as "interpreters".
Let's end this paper with two famous ancient statements, one from East and one from West.

East philosopher:

“道生一, 一生二, 二生三, 三生万物。” 老子

English translation:

"Tao generates one, one generates two, two generates three, three generates everything." Laozi

West philosopher:

"Everything is numbers." Pythagoras

\section{Acknowledgement}

First and foremost the author would like to thank the authors, editors and all contributors of Review of Particle Physics, Particle Data Group (PDG). The experimental data listed in PDG biannual report are extensively cited in this paper as supportive evidences for SQS theory. Without these experimental results, it is impossible to develop SQS theory.

The author would like to thank Roger Penrose for his book The Road to Reality - A Complete Guide to the Laws of the Universe. It provides extensive mathematic background knowledge. Especially in Chapter 8, a short but comprehensive description of Riemann surface and correlations to torus gives the author confidence finally to nail down the torus model and the four characteristic points on its surface.

The author would like to thank Mark Ronan for his book Symmetry and the Monster-One of the Greatest Quests of Mathematics, and the mathematicians involved in study of the sporadic groups. The book provides an overall and easy to understand summary of the sporadic groups with emphasis on $M$-group and other sporadic groups. Without reading this book, the author would miss the opportunity to find the correlations between $M$-group, $B$-group, Suz-group and three sets of prime number $m$-parameters for three generations of quarks, which serve as important checkpoints for the Prime Number Postulation and many related issues.

The author would like to thank Leo Birenbaum, Charlie Charter III, Xiao-Chun Gao, William Holstein, Fan Jin, Alan Lauder, Guang-Juang Ni, Author Oliner, Chengyi Pan, John Radomsky, ShaoXue Ren, Kemin Sheng, Jian Song, Xiang-Bai Tan, Qidong Wang, Shun-Jin Wang, Qingdong Yao, Wen Zhou, and Xue-Tian Zhu for their helps, supports and encouragements. During the eight years long journey to develop SQS theory, their helps, supports and encouragements were very important to the author especially in difficult times.

\section{Reference}

All experimental data cited in this paper unless stated otherwise are cited from Review of Particle Physics, Particle Data Group (PDG) 2010.

[1] Shen Zhiyuan (2007), "On Stochastic quantum space and related topics", Frontier Science, 4, 4-35. In Chinese with English abstract. 


\section{Z. Y. SHEN}

[2] Roger Penrose, The Road to Reality: A Complete Guide to the Laws of the Universe, Vintage Books, New York, 2007, 135-138.

[3] Nanneke D., Fogwell S. and Gabrielse G. (2008), Phys. Rev. Lett. 100, 120801.

[4] Aoyama T., Hayakawa M., Kinoshita T. and Nio M. (2008), Phys. Rev. D77, 053012.

[5] Feynman R. P. and Hibbs A. R., Quantum mechanics and path integrals, McGraw-Hill, 1965.

[6] Abdo A. A. et al. "Testing Einstein's special relativity with Fermi's short hard $\gamma$-ray burst GRB090510", Aug. 13, 2009, available at: http://arxiv.org/abs/0908.1832v1, with supplementary material available at: http://gammaray.nsstc.nasa.gov/gbm/grb/GRB090510/sup porting_material.pdf

[7] Abdo A. A. et al. "A limit on the variation of the speed of light arising from quantum gravity effects", Nature Letter, published on line, Oct. 28, 2009.

[8] Supplementary Information, available at http://www.nature.com/nature/journal/v462/n7271/extref/ nature08574-s1.pdf

[9] Salart D., et al. (2008), Testing the speed of 'spooky action at a distance', Nature, $\mathbf{4 5 4}, 861$.

[10] David Hestenes, (1990), "The Zitterbewegung Interpretation of Quantum Mechanics", Found. Physica, 20, No. 10, 1213-1232.

[11] M. B. Green, J.H. Schwarz and E. Witten, String Theory, Cambridge Press, Vol.1, 27-42.

[12] Roger Penrose, The Road to Reality: A Complete Guide to the Laws of the Universe, Vintage Books, New York, 2007, 943-946.

[13] Klinkhamer, F. R., Manton, N. S., (1984), “A SaddlePoint Solution of the Weinberg-Salam Theory", Phys. Rev., D30, 2212-2220.

[14] Lerner R. G., and Trigg G. L. (1990), Encyclopedia of Physics, Second edition, VCH Publishers, 445-448.

[15] Georgi, H., Glashow, S. L., (1974), "Unity of All Elementary Particle Forces", Phys. Rev. Lett. 32, 438441.

[16] Shen Zhiyuan (2008), "Correction and Prediction", Frontier Science, 8, 86-87. In Chinese.

[17] Jegeriehner, F., (2008), "The running fine structure constant via the Adler function", Nuclear Physics BProceedings Supplements Volumes 181-182, 135-140.

[18] Ronan Mark, Symmetry and the Monster, One of the Greatest Quest of Mathmatics, Oxford University Press, 2006, 227-228.

[19] Guth, Alan, The Inflationary Universe, Reading, Addison-Wesley, (1997).

[20] Misner, C. W., Thorne, K. S. and Wheeler, J. A., Gravitation, Freeman and Company, New York, 2003.

[21] M. B. Green, J. H. Schwarz and E. Witten (1987), Superstring Theory, Cambridge University Press.

[22] Abhay Ashtekar (1986), "New variables for classical and quantum gravity”, Phys. Rev. Lett. 57 (18): 2244-2247.
[23] Sundance O. Bilson-Thompson, "A topological model of composite preons", arXiv:hep-ph /0503213v2 27 Oct 2006 and a preprint for Elsevier Science, May 19, 2006.

[24] Sundance O. Bilson-Thompson, Fotini Markopoulou, Lee Smolin, "Quantum gravity and the stardand model", arXiv:hep-th/0603022.

[25] Frank Wilczek, "Why are analogies between condensed matter and particle physics?" Physics Today, July 1998, 11-13.

[26] Christopher T. Hill and Elizabeth H. Simmons, (2003), "Strong dynamics and electroweak symmetry breaking", Physics Reports, 381, 235-402.

[27] Howard Georgi and S.L. Glashow, (1974), "Unity of all elementary-particle Forces", Phys. Rev. Lett. 32, 4.

[28] Rita G. Lerner and George L. Trigg, (1991), Encyclopedia of Physics, second edition, $\mathrm{VCH}$ Publishers, 446-448.

[29] Freeman Dyson, (2008) "Birds and frogs", Notice of AMS, 56 (2), 212-223.

[30] Dov Levine, Paul J. Steinhardt, (1984) "Quasicrystals: a new class of ordered structure", Phys. Rev. Lett. 53, 2477-2480.

[31] Richard Elwes, Mathematics 1001, Firefly Books, 2010.

[32] Roger Penrose, The Road to Reality: A Complete Guide to the Laws of the Universe, Vintage Books, New York, 2007. 958-1003.

[33] Randolf, P., et al. (2010), "The size of proton", Nature Letters, 466 (8), 213-217. Doi: 10.1038/Nature09250.

\section{Appendixes}

\section{Appendix 1}

The 1-dimensional Gaussian probability function of (3.1) is:

$$
\begin{aligned}
& p\left(x ; x^{\prime}\right)=e^{-\pi\left(x-x^{\prime}\right)^{2}} ; \\
& x=-\infty, \cdots, 0, \cdots, \infty ; x^{\prime}=-\infty, \cdots, 0, \cdots, \infty .
\end{aligned}
$$

Take Fourier transform of $p\left(x ; x^{\prime}\right)$ with respect to variable $x$ :

$$
\begin{aligned}
& p\left(k ; k^{\prime}\right)=\frac{1}{2 \pi} \int_{-\infty}^{\infty} p\left(x ; x^{\prime}\right) \exp (i k x) d x=\frac{1}{2 \pi} \int_{-\infty}^{\infty} \exp \left\{-\pi\left[\left(x-x^{\prime}\right)^{2}-\frac{i k}{\pi} x\right]\right\} d x . \\
& =\frac{1}{2 \pi} \exp \left[-x^{x^{2}}+\left(x^{\prime}+\frac{i k}{2 \pi}\right)^{2}\right]_{-\infty}^{\infty} \int_{-\infty}^{\infty} \exp \left[-\pi\left(x-x^{\prime}-\frac{i k}{2 \pi}\right)\right] d\left(x-x^{\prime}-\frac{i k}{2 \pi}\right) \\
& =\frac{1}{2 \pi} \exp \left(-\frac{k^{2}}{4 \pi}\right) \exp \left(i k x^{\prime}\right) \int_{-\infty}^{\infty} \exp \left[-\pi \xi^{2}\right] d \xi=\left[\frac{1}{2 \pi} \exp \left(-\frac{k^{2}}{4 \pi}\right)\right]\left[\exp \left(i k x^{\prime}\right)\right]
\end{aligned}
$$

In (A1.1), the real function $P(k)$ is the probability distribution function in the $k$-space:

$$
P(k)=\frac{1}{2 \pi} e^{-\frac{k^{2}}{4 \pi}} .
$$

The phase factor is:

$$
Q\left(k ; x^{\prime}\right)=e^{i k x^{\prime}} \text {. }
$$

As shown by (A1.2), $P(k)$ is also Gaussian as expected, and the unitarity requirement is satisfied:

$$
\int_{-\infty}^{\infty} P(k) d k=\frac{1}{2 \pi} \int_{-\infty}^{\infty} e^{-\frac{k^{2}}{4 \pi}} d k=1 .
$$


The standard deviation of $P(k)$ is

$$
\sigma_{k}=\sqrt{2 \pi} \text {. }
$$

Multiplying $\sigma_{k}$ with $\sigma$ given by (2.3) yields:

$$
\sigma_{k} \cdot \sigma=1 \text {. }
$$

\section{Appendix 2}

3-dimensional geodesic coordinators in parametric form:

$$
x^{\alpha}=x^{\alpha}(s) ; \alpha=a, b, c .
$$

Here $x^{a}, x^{b}, x^{c}$ are 3-dimensional spatial coordinators, and $s$ is the affine parameter. The solution $x^{\alpha}=x^{\alpha}(s)$ of following differential equation represents geodesic:

$$
\frac{d^{2} x^{a}}{d s^{2}}+\Gamma_{b c}^{a} \frac{d x^{b}}{d s} \frac{d x^{c}}{d s}=0 .
$$

In which, the Christoffel symbol of second type is:

$$
\Gamma_{b c}^{a}=g^{a d} \Gamma_{b c d}=\frac{1}{2} g^{a d}\left(\frac{\partial g_{b c}}{\partial x^{d}}+\frac{\partial g_{c d}}{\partial x^{b}}-\frac{\partial g_{d b}}{\partial x^{c}}\right) .
$$

The components of a symmetrical gauge tensor satisfy:

$$
g_{a b}=g_{b a} \text {. }
$$

\section{Appendix 3}

Let's evaluate the effect on $\left|D S_{k}(k)\right|$ of disregarding the factor:

$$
\frac{1}{4 \pi} e^{-\pi k^{2}}=\frac{1}{4 \pi} e^{-\pi\left(k_{r}+i k_{i}\right)^{2}}=\frac{1}{4 \pi} e^{-\pi\left(k_{r}^{2}-k_{i}^{2}\right)} e^{-2 i \pi k_{r} k_{i}} .
$$

For the local minimums or local maximums of $D S_{k}(k)$ are evaluated by its absolute value $\left|D S_{k}(k)\right|$, the second factor $e^{-i 2 \pi k_{r} k_{i}}$ of (A3.1) does not have effect, because $\left|e^{-i 2 \pi k_{r} k_{i}}\right|=1$ regardless the values of $k_{r}$ and $k_{i}$. Since mass and decay times are determined by the location of local minimums or local maximums of $\left|D S_{k}(k)\right|$ on complex $k$-plane, the effect of $e^{-\pi\left(k_{r}^{2}-k_{i}^{2}\right)}$ is only related to the variation of $k_{r}$ and $k_{i}$.

$$
\begin{aligned}
& d\left(e^{-\pi\left(k_{r}^{2}-k_{i}^{2}\right)}\right)=\frac{\partial}{\partial k_{r}}\left(e^{-\pi\left(k_{r}^{2}-k_{i}^{2}\right)}\right) d k_{r}+\frac{\partial}{\partial k_{i}}\left(e^{-\pi\left(k_{r}^{2}-k_{i}^{2}\right)}\right) d k_{i} \\
& =\left(-2 \pi k_{r} e^{-\pi\left(k_{r}^{2}-k_{i}^{2}\right)}\right) d k_{r}+\left(2 \pi k_{i} e^{-\pi\left(k_{r}^{2}-k_{i}^{2}\right)}\right) d k_{i}
\end{aligned}
$$

Substitute the muon data from Table 7.1 into (A3.2):

$$
\begin{aligned}
& -2 \pi k_{r} e^{-\pi\left(k_{r}^{2}-k_{i}^{2}\right)} \sim-10^{3} \times e^{-100000} \rightarrow 0 ; \\
& 2 \pi k_{i} e^{-\pi\left(k_{r}^{2}-k_{i}^{2}\right)} \sim 10^{-14} \times e^{-100000} \rightarrow 0 .
\end{aligned}
$$

Substitute the taon data from Table 7.1 into (A3.2): $-2 \pi k_{r} e^{-\pi\left(k_{r}^{2}-k_{i}^{2}\right)} \sim-10^{4} \times e^{-10000000} \rightarrow 0 ;$

$2 \pi k_{i} e^{-\pi\left(k_{r}^{2}-k_{i}^{2}\right)} \sim 10^{-7} \times e^{-10000000} \rightarrow 0$.

In both cases, the errors caused by disregarding the factor $e^{-\pi\left(k_{r}^{2}-k_{i}^{2}\right)}$ are negligible.

\section{Appendix 4}

The fine structure constant is:

$$
\alpha=\frac{e^{2}}{2 \varepsilon_{0} h c} .
$$

$\varepsilon_{0}$ is permittivity of free space. Electron's Compton wavelength

of electron is: $\lambda_{e C}=\frac{h}{M_{e} c}$.

Classical electron radius is: $r_{e}=\frac{e^{2}}{4 \pi \varepsilon_{0} M_{e} c^{2}}$,

Electron mass is: $M_{e}=\frac{e^{2}}{4 \pi \varepsilon_{0} r_{e} c^{2}}$.

According to the converting rule given in Section 4, Compton wavelength $\lambda_{e C}$ is originated from Planck wavelength $\lambda_{P}=L_{P}$, which is the circumferential length of circular loop-1. On the other hand, $r_{e}$ is defined as radius. To be compare on the same base, $\lambda_{e c}$ should compare to $2 \pi r_{e}$.

Since mass is inversely proportional to corresponding wavelength, the ratio of electron's electromagnetic mass $M_{e E M}$ to its total mass $M_{e E M}$ is:

$$
\frac{M_{E M}}{M_{e}}=\frac{2 \pi r_{e}}{\lambda_{e C}}=\frac{2 \pi\left[\frac{e^{2}}{4 \pi \varepsilon_{0} M_{e} c^{2}}\right]}{\frac{h}{M_{e} c}}=\frac{e^{2}}{2 \varepsilon_{0} h c}=\alpha .
$$

The electromagnetic modification factor for mass is:

$$
\frac{M_{e}-M_{E M}}{M_{e}}=1-\frac{M_{E M}}{M_{e}}=1-\alpha .
$$

(A4.5) is valid to other charged particles as well.

\section{Appendix 5}

The origin of logistic equation is the following nonlinear difference equation:

$$
x_{j+1}=\alpha x_{j}-\beta x_{j}^{2}, j=1,2,3 \cdots .
$$

In which parameters $\alpha>0$ and $\beta>0$ represent the positive action (Type- $P$ ) for positive feedback and negative action (Type$N$ ) for negative feedback, respectively. Convert the variables in (A5.1) as:

$$
\begin{aligned}
& x_{i}=\frac{\beta}{\alpha} x_{j} ; i=1,2,3 \cdots ; j=1,2,3 \cdots, \\
& x_{i+1}=\frac{\beta}{\alpha} x_{j+1} ; i=1,2,3 \cdots ; j=1,2,3 \cdots .
\end{aligned}
$$

Substituting (A5.2) into (A5.1) yields the logistic equation with parameter $r$ :

$$
\begin{aligned}
& x_{i+1}=r x_{i}\left(1-x_{i}\right), i=1,2,3 \cdots . \\
& r=\alpha .
\end{aligned}
$$

The logistic equation of (A5.3) represents a recurrent process. In (A5.3), $r=\alpha$ and $\beta$ is absorbed. As $r$ value increases, Type- $P$ action overwhelms Type- $N$ action.

(A5.3) is the logistic equation with constant parameter $r$. In some cases, parameter $r$ also varies, which belongs to the varying $r$ type logistic equation. 


\section{Appendix 6 \\ Part A: The derivative process for the basic equations based on Postulation 24.1.} are:

Based on Postulation 24.1, the redefined Ricci parameters

$R(G)=G^{a b} R_{a b}(G)$,

$R_{a b}(G)=R_{a c b}^{c}(G)=\partial_{c} \Gamma_{a b}^{c}(G)-\partial_{b} \Gamma_{a c}^{c}(G)+\Gamma_{a b}^{d}(G) \Gamma_{d c}^{c}(G)-\Gamma_{a c}^{d}(G) \Gamma_{d b}^{c}(G)$

$=\frac{1}{2} G^{c d}\left(\frac{\partial^{2} G_{a b}}{\partial x^{c} \partial x^{d}}+\frac{\partial^{2} G_{b d}}{\partial x^{c} \partial x^{a}}-\frac{\partial^{2} G_{a c}}{\partial x^{b} \partial x^{d}}-\frac{\partial^{2} G_{c d}}{\partial x^{b} \partial x^{a}}\right)+\Gamma_{a b}^{d}(G) \Gamma_{d c}^{c}(G)-\Gamma_{a c}^{d}(G) \Gamma_{d b}^{c}(G)$,

$\Gamma_{b c}^{a}(G)=G^{a d} \Gamma_{d b c}(G)=\frac{G^{a d}}{2}\left(\frac{\partial G_{b c}}{\partial x^{d}}+\frac{\partial G_{c d}}{\partial x^{b}}-\frac{\partial G_{d b}}{\partial x^{c}}\right), a, b=0,1,2,3$.

The redefined gauge tensors $G_{a b}=g_{a b} p$ and $G^{a b}=g^{a b} / p$ have $p(P)=\sum_{i} p_{i}\left(P ; P_{i}\right)=\sum_{i} e^{-\pi L_{i}^{2}\left(P ; P_{i}\right) / L_{P}^{2}}$ attached. Simplified notations $p=p(P)$ and $p_{i}=p_{i}\left(P ; P_{i}\right)$ are used from now on. As shown in (A6.1), the terms containing derivatives of $G_{a b}=g_{a b} p$ have two forms: (1) Product of two first order derivatives $\frac{\partial G_{a b}}{\partial x^{e}} \frac{\partial G_{c d}}{\partial x^{f}},(a, b, c, d, e, f=0,1,2,3) \cdot(2)$ Second order derivatives $\frac{\partial^{2} G_{a b}}{\partial x^{e} \partial x^{f}},(a, b, e, f=0,1,2,3)$. For the first order derivatives, the derivation process yields:

$$
\begin{aligned}
& G^{c d} \frac{\partial G_{a b}}{\partial x^{e}}=G^{c d} \frac{\partial}{\partial x^{e}}\left(g_{a b} p\right)=G^{c d}\left[p \frac{\partial g_{a b}}{\partial x^{e}}+g_{a b} \frac{\partial p}{\partial x^{e}}\right] \\
& =G^{c d}\left[p \frac{\partial g_{a b}}{\partial x^{e}}+g_{a b} \frac{\partial}{\partial x^{e}}\left(\sum_{i} e^{-\pi L_{i}^{2} L_{p}^{2}}\right)\right]=\frac{g^{c d}}{p} p\left[\frac{\partial g_{a b}}{\partial x^{e}}-\frac{2 \pi g_{a b}}{p} \sum_{i} \frac{L_{i}}{L_{p}^{2}} \frac{\partial L_{i}}{\partial x^{e}} e^{-\pi t_{i}^{2} / L_{p}^{2}}\right] \\
& =g^{c d}\left[\frac{\partial g_{a b}}{\partial x^{e}}-2 \pi g_{a b} \sum_{i} \frac{L_{i}}{L_{p}^{2}} \frac{\partial L_{i}}{\partial x^{e}} \frac{p_{i}}{p}\right] .
\end{aligned}
$$

In (A6.2), the first term serves as the term of kinematic part, which is in the $\Gamma_{a b}^{d}(g) \Gamma_{d c}^{c}(g)-\Gamma_{a c}^{d}(g) \Gamma_{d b}^{c}(g)$ part of kinematic part $R_{a b}-\frac{1}{2} g_{a b} R$. The second term is in the $\breve{\Gamma}_{a b}^{d}(g) \breve{\Gamma}_{d c}^{c}(g)-\breve{\Gamma}_{a c}^{d}(g) \breve{\Gamma}_{d b}^{c}(g)$ part of emerging part $\breve{R}_{a b}-\frac{1}{2} g_{a b} \breve{R}$.

For the second order derivatives, the derivation process yields:

$$
\begin{aligned}
& G^{c d} \frac{\partial^{2} G_{a b}}{\partial x^{e} \partial x^{f}}=G^{c d} \frac{\partial}{\partial x^{e}}\left(\frac{\partial}{\partial x^{f}}\left(g_{a b} p\right)\right)=G^{c d} \frac{\partial}{\partial x^{e}}\left[p \frac{\partial g_{a b}}{\partial x^{f}}+g_{a b} \frac{\partial p}{\partial x^{f}}\right] \\
& =G^{c d} \frac{\partial}{\partial x^{e}}\left[p \frac{\partial g_{a b}}{\partial x^{f}}+g_{a b} \frac{\partial}{\partial x^{f}}\left(\sum_{i} e^{-\pi \frac{L_{i}^{2}}{L_{p}^{2}}}\right)\right]=G^{c d} \frac{\partial}{\partial x^{e}}\left[p \frac{\partial g_{a b}}{\partial x^{f}}+g_{a b} \sum_{i}\left(-2 \pi \frac{L_{i}}{L_{L}^{2}} \frac{\partial L_{i}}{\partial x^{f}} e^{-\pi}\right)\right] \\
& =G^{c d}\left\{p \frac{\partial^{2} g_{a b}}{\partial x^{e} \partial x^{f}}+\frac{\partial g_{a b}}{\partial x^{f}} \frac{\partial}{\partial x^{e}}\left(\sum_{i} e^{-\pi t \frac{L_{i}^{2}}{L_{p}^{p}}}\right)+\frac{\partial g_{a b}}{\partial x^{e}} \sum_{i}\left(-2 \pi \frac{L_{i}}{L_{P}^{2}} \frac{\partial L_{i}}{\partial x^{f}} e^{-\pi \frac{L_{i}^{2}}{L_{p}^{p}}}\right)\right. \\
& \left.+\frac{g_{a b}}{L_{P}^{2}} \sum_{i}\left[\left(-2 \pi \frac{\partial L_{i}}{\partial x^{e}} \frac{\partial L_{i}}{\partial x^{f}}-2 \pi L \frac{\partial^{2} L}{\partial x^{e} \partial x^{f}}+4 \pi^{2} \frac{L_{i}^{2}}{L_{P}^{2}} \frac{\partial L_{i}}{\partial x^{e}} \frac{\partial L_{i}}{\partial x^{f}}\right) e^{-\pi \frac{L_{i}^{2}}{L_{P}}}\right]\right\} \\
& =\frac{g^{c d}}{p}\left\{p \frac{\partial^{2} g_{a b}}{\partial x^{e} \partial x^{f}}-2 \pi \frac{\partial g_{a b}}{\partial x^{f}} \sum_{i} \frac{L_{i}}{L_{P}^{2}} \frac{\partial L_{i}}{\partial x^{e}} e^{-\pi \frac{L_{i}^{2}}{L_{P}^{2}}}-2 \pi \frac{\partial g_{a b}}{\partial x^{e}} \sum_{i} \frac{L_{i}}{L_{P}^{2}} \frac{\partial L_{i}}{\partial x^{f}} e^{-\pi \frac{L_{i}^{2}}{L_{P}^{2}}}\right. \\
& \left.-\frac{2 \pi g_{a b}}{L_{P}^{2}} \sum_{i} \frac{\partial L_{i}}{\partial x^{x}} \frac{\partial L_{i}}{\partial x^{f}} e^{-\pi \frac{L_{i}^{2}}{L_{p}^{2}}}-2 \pi g_{a b} \sum_{i} \frac{L_{i}}{L_{L}^{2}} \frac{\partial^{2} L}{\partial x^{e} \partial x^{f}} e^{-\pi \frac{L^{2}}{L_{p}^{2}}}+4 \pi^{2} g_{a b} \sum_{i} \frac{L_{i}^{2}}{L_{P}^{4}} \frac{\partial L_{i}}{\partial x^{e}} \frac{\partial L_{i}}{\partial x^{f}} e^{-\pi \frac{L_{i}^{2}}{L_{p}^{2}}}\right\} \\
& =g^{c d} \frac{\partial^{2} g_{a b}}{\partial x^{e} \partial x^{f}}+\left\{2 \pi g ^ { c d } \left[2 \pi g_{a b} \sum_{i} \frac{L_{i}^{2}}{L_{P}^{4}} \frac{\partial L_{i}}{\partial x^{e}} \frac{\partial L_{i}}{\partial x^{f}} \frac{p_{i}}{p}-\frac{g_{a b}}{L_{P}^{2}} \sum_{i} \frac{\partial L_{i}}{\partial x^{e}} \frac{\partial L_{i}}{\partial x^{f}} \frac{p_{i}}{p}\right.\right. \\
& \left.\left.-\frac{\partial g_{a b}}{\partial x^{e}} \sum_{i} \frac{L_{i}}{L_{P}^{2}} \frac{\partial L_{i}}{\partial x^{f}} \frac{p_{i}}{p}-\frac{\partial g_{a b}}{\partial x^{f}} \sum_{i} \frac{L_{i}}{L_{P}^{2}} \frac{\partial L_{i}}{\partial x^{e}} \frac{p_{i}}{p}-g_{a b} \sum_{i} \frac{L_{i}}{L_{P}^{2}} \frac{\partial^{2} L}{\partial x^{e} \partial x^{f}} \frac{p_{i}}{p}\right]\right\} \text {. }
\end{aligned}
$$

For the second order derivative $G^{c d} \frac{\partial^{2} G_{a b}}{\partial x^{e} \partial x^{f}}$ of (A6.3), the first term serves as the term of kinematic part, which is in the $\frac{1}{2} g^{c d}\left(\frac{\partial^{2} g_{a b}}{\partial x^{c} \partial x^{d}}+\frac{\partial^{2} g_{b d}}{\partial x^{c} \partial x^{a}}-\frac{\partial^{2} g_{a c}}{\partial x^{b} \partial x^{d}}-\frac{\partial^{2} g_{c d}}{\partial x^{b} \partial x^{a}}\right)$ part of $R_{a b}$ and $R$. The five terms in parenthesis serve as the terms in the emerging part $\breve{R}_{a b}-\frac{1}{2} g_{a b} \breve{R}$.

According to (24.6) and (A6.3), the terms in the emerging part generated by gauge tensor attached to probability are:

$\frac{1}{2} g_{a b} \breve{R}=\frac{1}{2} g_{a b}\left(g^{c d} \breve{R}_{c d}\right)$,

(A6.4a)

$\breve{R}_{a b}=$

$=\frac{2 \pi}{L_{P}^{2}}\left[\left(2 \pi g_{a c} \sum_{i} \frac{\partial L_{i}}{\partial x^{b}} \frac{\partial L_{i}}{\partial x^{d}} \frac{p_{i}}{p}-g_{a c} \sum_{i} \frac{L_{i}^{2}}{L_{p}^{2}} \frac{\partial L_{i}}{\partial x^{b}} \frac{\partial L_{i}}{\partial x^{d}} \frac{p_{i}}{p}-\frac{\partial g_{a c}}{\partial x^{b}} \sum_{i} L_{i} \frac{\partial L_{i}}{\partial x^{d}} \frac{p_{i}}{p}-\frac{\partial g_{a c}}{\partial x^{d}} \sum_{i} L_{i} \frac{\partial L_{i}}{\partial x^{b}} \frac{p_{i}}{p}-g_{a c} \sum_{i} L_{i} \frac{\partial^{2} L_{i}}{\partial x^{b} \partial x^{d}} \frac{p_{i}}{p}\right)\right.$

$+\left(2 \pi g_{c d} \sum_{i} \frac{\partial L_{i}}{\partial x^{b}} \frac{\partial L_{i}}{\partial x^{a}} \frac{p_{i}}{p}-g_{c d} \sum_{i} \frac{L_{i}^{2}}{L_{P}^{2}} \frac{\partial L_{i}}{\partial x^{b}} \frac{\partial L_{i}}{\partial x^{a}} \frac{p_{i}}{p}-\frac{\partial g_{c d}}{\partial x^{b}} \sum_{i} L_{i} \frac{\partial L_{i}}{\partial x^{a}} \frac{p_{i}}{p}-\frac{\partial g_{c d}}{\partial x^{a}} \sum_{i} L_{i} \frac{\partial L_{i}}{\partial x^{b}} \frac{p_{i}}{p}-g_{c d} \sum_{i} L_{i} \frac{\partial^{2} L_{i}}{\partial x^{b} \partial x^{a}} \frac{p_{i}}{p}\right)$

$-\left(2 \pi g_{a b} \sum_{i} \frac{\partial L_{i}}{\partial x^{c}} \frac{\partial L_{i}}{\partial x^{d}} \frac{p_{i}}{p}-g_{a b} \sum_{i} \frac{L_{i}^{2}}{L_{P}^{2}} \frac{\partial L_{i}}{\partial x^{c}} \frac{\partial L_{i}}{\partial x^{d}} \frac{p_{i}}{p}-\frac{\partial g_{a b}}{\partial x^{c}} \sum_{i} L_{i} \frac{\partial L_{i}}{\partial x^{d}} \frac{p_{i}}{p}-\frac{\partial g_{a b}}{\partial x^{d}} \sum_{i} L_{i} \frac{\partial L_{i}}{\partial x^{c}} \frac{p_{i}}{p}-g_{a b} \sum_{i} L_{i} \frac{\partial^{2} L_{i}}{\partial x^{c} \partial x^{d}} \frac{p_{i}}{p}\right)$

$\left.-\left(2 \pi g_{c a} \sum_{i} \frac{\partial L_{i}}{\partial x^{c}} \frac{\partial L_{i}}{\partial x^{a}} \frac{p_{i}}{p}-g_{b d} \sum_{i} \frac{L_{i}^{2}}{L_{P}^{2}} \frac{\partial L_{i}}{\partial x^{c}} \frac{\partial L_{i}}{\partial x^{a}} \frac{p_{i}}{p}-\frac{\partial g_{b d}}{\partial x^{c}} \sum_{i} L_{i} \frac{\partial L_{i}}{\partial x^{a}} \frac{p_{i}}{p}-\frac{\partial g_{b d}}{\partial x^{a}} \sum_{i} L_{i} \frac{\partial L_{i}}{\partial x^{c}} \frac{p_{i}}{p}-g_{b d} \sum_{i} L_{i} \frac{\partial^{2} L_{i}}{\partial x^{c} \partial x^{a}} \frac{p_{i}}{p}\right)\right]$

$-\left[\widetilde{\Gamma}_{a b}^{d} \breve{\Gamma}_{d c}^{c}-\breve{\Gamma}_{a c}^{d} \breve{\Gamma}_{d b}^{c}\right]$,

$\breve{\Gamma}_{b c}^{a}=g^{a d} \breve{\Gamma}_{d b c}=\frac{\pi g^{a d}}{L_{P}^{2}}\left(g_{d b} \sum_{i} L_{i} \frac{\partial L_{i}}{\partial x^{c}} \frac{p_{i}}{p}-g_{b c} \sum_{i} L_{i} \frac{\partial L_{i}}{\partial x^{d}} \frac{p_{i}}{p}-g_{c d} \sum_{i} L_{i} \frac{\partial L_{i}}{\partial x^{b}} \frac{p_{i}}{p}\right)$,

$a, b, c, d=0,1,2,3$.

According to Definition 24.1, the space-time variables $x^{0}\left(\xi^{0}\right), x^{1}\left(\xi^{0}\right), x^{2}\left(\xi^{0}\right), x^{3}\left(\xi^{0}\right)$ as functions of intrinsic time $\xi^{0}=c \tau=\gamma^{-1} c t$, The derivation process should be carried out further such as:

$\frac{\partial}{\partial x^{a}}=\frac{\partial}{\partial x^{a}\left(\xi^{0}\right)} \Rightarrow \frac{\partial}{\partial x^{a}} \frac{\partial x_{a}}{\partial \xi^{0}}=\frac{\partial x_{a}}{\partial\left(\gamma^{-1} c t\right)} \frac{\partial}{\partial x^{a}}=\gamma \frac{\partial x_{a}}{\partial t} \frac{1}{c} \frac{\partial}{\partial x^{a}}=\gamma \frac{v_{a}}{c} \frac{\partial}{\partial x^{a}}$,

$a=0,1,2,3$.

Utilizing (A6.5) to carry out the derivative process with respect to space-time variables $x^{0}\left(\xi^{0}\right), x^{1}\left(\xi^{0}\right), x^{2}\left(\xi^{0}\right), x^{3}\left(\xi^{0}\right)$ as functions of intrinsic time $\xi^{0}=c \tau=\gamma^{-1} c t$ for the emerging terms given by (A6.4a) and (A6.3b) yields:

$\frac{1}{2} g_{a b} \breve{R}=\frac{1}{2} g_{a b}\left(g^{c d} \breve{R}_{c d}\right)$,

(A6.6a)

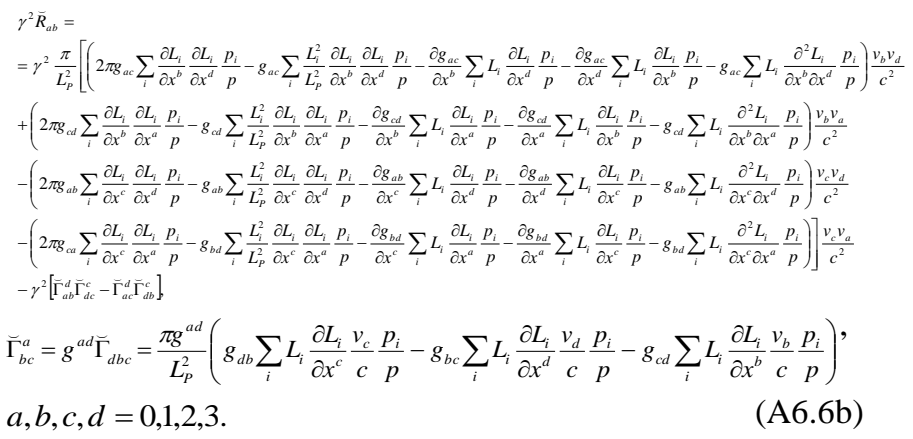

In (A6.6), the emerging terms $\breve{R}_{a b}$ and $\breve{R}$ are originated from the kinematic terms $R_{a b}$ and $R$, respectively.

The speed products the emerging terms can be presented in $4 \times 4$ matrix form as: 
http://dx. doi. org/10.4236/jmp. 2013.4.410165 Published 0nline 0ctober 2013 (http://www. scirp. org/journal/jmp)

$\left[V_{i}\right]=\left[V_{i}\right]^{\mathrm{T}}=\gamma^{2}\left[\begin{array}{cccc}1 & v_{1, i} & v_{2, i} & v_{3, i} \\ v_{1, i} & v_{1, i} v_{1, i} & v_{1, i} v_{2, i} & v_{1, i} v_{3, i} \\ v_{2, i} & v_{2, i} v_{1, i} & v_{2, i} v_{2} & v_{2, i} v_{3, i} \\ v_{3, i} & v_{3, i} v_{1, i} & v_{3, i} v_{2, i} & v_{3, i} v_{3, i}\end{array}\right], i=1,2,3 \ldots$

Part-B: The spatial and temporal derivatives of gauge tensor $g_{a b}$ for the kinematic part of basic equations based on Postulation 24.1.

According to (24.5), the space-time variables are $\left(x^{1}\left(\xi^{0}\right), x^{2}\left(\xi^{0}\right), x^{3}\left(\xi^{0}\right) ; x^{0}\left(\xi^{0}\right)\right)$ functions of the intrinsic time $\xi^{0}=c \tau=\gamma^{-1} c t$, the derivative process should go further as: $\frac{\partial g_{a b}}{\partial x^{e}} \Rightarrow \frac{\partial g_{a b}}{\partial x^{e}} \frac{\partial x^{e}}{\partial \xi^{0}}=\gamma\left(\frac{\partial g_{a b}}{\partial x^{e}} \frac{\partial x^{e}}{\partial x^{0}}\right)=\gamma\left(\frac{\partial g_{a b}}{\partial x^{e}}\right)\left(\frac{v_{e}}{c}\right)$,

$\left(\frac{\partial g_{a b}}{\partial x^{e}}\right)\left(\frac{\partial g_{c d}}{\partial x^{f}}\right) \Rightarrow \gamma^{2}\left(\frac{\partial g_{a b}}{\partial x^{e}}\right)\left(\frac{\partial g_{c d}}{\partial x^{f}}\right)\left(\frac{v_{e} v_{f}}{c^{2}}\right)$,

$\frac{\partial^{2} g_{a b}}{\partial x^{e} \partial x^{f}} \Rightarrow \frac{\partial}{\partial x^{e}}\left(\gamma \frac{\partial g_{a b}}{\partial x^{f}}\left(\frac{v_{f}}{c}\right)\right)=\gamma \frac{\partial}{\partial x^{e}}\left(\frac{\partial g_{a b}}{\partial x^{f}}\right)\left(\frac{v_{f}}{c}\right)$

$\Rightarrow \gamma\left(\frac{\partial^{2} g_{a b}}{\partial x^{e} \partial x^{f}} \frac{\partial x^{e}}{\partial \xi^{0}}\right)\left(\frac{v_{f}}{c}\right)=\gamma^{2}\left(\frac{\partial^{2} g_{a b}}{\partial x^{e} \partial x^{f}} \frac{\partial x^{e}}{\partial x^{0}}\right)\left(\frac{v_{f}}{c}\right)=\gamma^{2}\left(\frac{\partial^{2} g_{a b}}{\partial x^{e} \partial x^{f}}\right)\left(\frac{v_{e} v_{f}}{c^{2}}\right)$.

Based on (A6.8), the parameters of kinematic part $R_{a b}-\frac{1}{2} g_{a b} R$ change as:

$\frac{1}{2} g_{a b} R=\frac{1}{2} g_{a b} g^{c d} R_{c d}$,

$$
\begin{aligned}
& R_{a b}=\gamma^{2}\left[\frac{1}{2} g^{c d}\left(\frac{\partial^{2} g_{a b}}{\partial x^{c} \partial x^{d}}\left(\frac{v_{c} v_{d}}{c^{2}}\right)+\frac{\partial^{2} g_{b d}}{\partial x^{c} \partial x^{a}}\left(\frac{v_{c} v_{a}}{c^{2}}\right)-\frac{\partial^{2} g_{a c}}{\partial x^{b} \partial x^{d}}\left(\frac{v_{b} v_{d}}{c^{2}}\right)-\frac{\partial^{2} g_{c d}}{\partial x^{b} \partial x^{a}}\left(\frac{v_{b} v_{a}}{c^{2}}\right)\right)\right] \\
& +\gamma^{2}\left[\Gamma_{a b}^{d} \Gamma_{d c}^{c}-\Gamma_{a c}^{d} \Gamma_{d b}^{c}\right] \\
& \Gamma_{b c}^{a}=\frac{g^{a d}}{2}\left(\frac{\partial g_{b c}}{\partial x^{d}}\left(\frac{v_{d}}{c}\right)+\frac{\partial g_{c d}}{\partial x^{b}}\left(\frac{v_{b}}{c}\right)-\frac{\partial g_{d b}}{\partial x^{c}}\left(\frac{v_{c}}{c}\right)\right), a, b, c, d=0,1,2,3 .
\end{aligned}
$$

As a result, equations (24.44a) are presented as:

$$
R_{a b}-\frac{1}{2} g_{a b} R=E_{a b},
$$

Emerging part:

$$
E_{a b}=\gamma^{2} \breve{E}_{a b}=\gamma^{2}\left[\breve{R}_{a b}-\frac{1}{2} g_{a b} \breve{R}\right] .
$$

The contents of emerging part are the same of (A6.6). The contents of the kinematic part are given by (A6.9). The speed product matrix of the kinematic part is:

$$
\left[V_{i}\right]=\left[V_{i}\right]^{\mathrm{T}}=\gamma^{2}\left[\begin{array}{cccc}
1 & v_{1, i} & v_{2, i} & v_{3, i} \\
v_{1, i} & v_{1, i} v_{1, i} & v_{1, i} v_{2, i} & v_{1, i} v_{3, i} \\
v_{2, i} & v_{2, i} v_{1, i} & v_{2, i} v_{2} & v_{2, i} i_{3, i} \\
v_{3, i} & v_{3, i} v_{1, i} & v_{3, i} v_{2, i} & v_{3, i} i_{3, i}
\end{array}\right], i=1,2,3 \cdots
$$

The speed product matrix of emerging part is the same as (A6.11). 